\title{
Robert LOWIE (1936)
}

\section{TRAITÉ DE SOCIOLOGIE PRIMITIVE}

\author{
(Traduction et présentation de E. Métraux)
}
Un document produit en version numérique par Jean-Marie Tremblay, professeur de sociologie au Cégep de Chicoutimi
Courriel: jmt_sociologue@videotron.ca
Site web: http://pages.infinit.net/sociojmt

Dans le cadre de la collection: "Les classiques des sciences sociales"

Site web: http://www.uqac.uquebec.ca/zone30/Classiques_des_sciences_sociales/index.html

Une collection développée en collaboration avec la Bibliothèque

Paul-Émile-Boulet de l'Université du Québec à Chicoutimi

Site web: http://bibliotheque.uqac.uquebec.ca/index.htm 
Cette édition électronique a été réalisée par Jean-Marie Tremblay, professeur de sociologie au Cégep de Chicoutimi à partir de :

\section{Robert Lowie (1883-1957)}

Traité de sociologie primitive (1936)

Une édition électronique réalisée à partir du livre de Robert Lowie (1936), Traité de sociologie primitive. Paris : Union générale d'Éditions, 1969, 443 pages. Collection : Petite bibliothèque Payot, $\mathrm{n}^{\mathrm{0}} 137$.

Polices de caractères utilisée :

Pour le texte: Times, 12 points.

Pour les citations : Times 10 points.

Pour les notes de bas de page : Times, 10 points.

Édition électronique réalisée avec le traitement de textes Microsoft Word 2001 pour Macintosh.

Mise en page sur papier format

LETTRE (US letter), 8.5'” x 11'’)

\section{\& Fait avec}

Macintosh 


\section{Table des matières}

PRÉFACE

Carte indiquant la situation géographique de la plupart des tribus mentionnées

Répertoire des tribus pour la carte géographique

1. - INTRODUCTION

2. - LE MARIAGE

a Prohibitions de mariage

b Moyens d'obtenir un conjoint

c L'union préférentielle

Références

3. - LA POLYGAMIE
a Polygynie
b Polyandrie
c Communisme sexuel
d Communisme sexuel hypothétique $\underline{\text { Références }}$

4. - LA FAMILLE

a La parenté bilatérale

b Élasticité du lien familial

c Résidence patrilocale et matrilocale

d Division du travail selon les sexes

e Ségrégation des célibataires

f Ségrégation sexuelle

g Adoption

h Résumé

Références

5. - RÈGLES DE PARENTÉ

a Parenté maternelle et paternelle

b Tabous des beaux-parents

c Autres tabous

d Privilèges de familiarité

e Tabou et licence

f Teknonymie $\underline{\text { Références }}$

6. - LE CLAN
a Types d'organisation clanique
b Origine ou origines
c Clans d'un ordre supérieur 
d Totémisme

Références

\section{7. - HISTOIRE DU CLAN}

a Antériorité de la famille

b Origine du clan

c Le clan et la terminologie dakota

d Clans utérins et clans consanguins Références

8. - CONDITION DE LA FEMME
a Théorie et pratique
b Matriarcat
c Résidence matrilocale
d Interprétation économique e Corrélation avec les diverses phases de la civilisation

\section{9. - LA PROPRIÉTÉ}
a Communisme primitif
b Propriété du sol
c Biens mobiliers
d Propriété immatérielle
e Héritage Références

10. - LES ASSOCIATIONS
a Îles Andaman
b Australie
c Massaï
d Îles Banks
e Indiens Pueblo
f Crow
g Hidatsa
h Résumé
Références

\section{1. - THÉORIE DES ASSOCIATIONS}
a Système de Schurtz
b Dichotomie sexuelle
c Classes d'âge
d Types d'associations
e Les sociétés d'âge chez les Indiens des Prairies
f Conclusions générales Références 
12. LE RANG
a Bravoure
b Shamanisme : richesse
c Castes
d Conclusion
Références

\section{3. - LE GOUVERNEMENT}
a $\underline{\text { Australie }}$
b Polynésie et Micronésie
c Mélanésie et Nouvelle-Guinée
d Afrique
e Amérique du Nord
f La démocratie et les organisations primitives
g Organisation tribale et territoriale $\underline{\text { Références }}$

14. - LA JUSTICE
a Responsabilité collective
b Intention criminelle
c Wehrgeld (composition)
d Preuves
e $\underline{\text { Australie }}$
f Ifugao
g Esquimaux
h Indiens des Prairies
i Polynésie
j Afrique
$\mathrm{k}$ Conclusion Références

15.- CONCLUSION

APPENDICE. - LA FAMILLE, UNITÉ SOCIALE

BIBLIOGRAPHIE 
Professeur d'anthropologie à l'Université de Californie, Robert-H. LOWIE fut l'un des principaux représentants de l'anthropologie culturelle américaine.

Lors de la première publication en France de ce Traité de sociologie primitive, Alfred MÉTRAUX écrivait : « Cet ouvrage est venu combler une lacune importante dans les sciences sociologiques et a permis à un large public d'accéder au monde des institutions primitives, tout en constituant pour les spécialistes une vue d'ensemble sur des questions auxquelles on n'avait guère consacré jusqu'ici que des études partielles.

« Peu d'ethnologues modernes étaient aussi bien qualifiés que R.-H. LOWIE pour nous tracer un tableau général de la science de l'homme [...] L'enquête directe est la seule voie pour parvenir à ce « sens de l'humain » qui doit caractériser un bon ethnologue.

« Ces qualités, M. LOWIE les possède à un très haut degré. Les recherches qu'il a poursuivies chez les Indiens Crow l'ont classé parmi les observateurs les plus fins et les plus pénétrants de l'homme primitif et ont développé chez lui cet instinct qui lui permet d'interpréter les faits sans avoir recours à des systèmes absolus et rigides. »

\section{ROBERT LOWIE}




\section{Carte indiquant la situation géographique de la plupart des tribus mentionnées}

$\underline{\text { Retour à la table des matières }}$

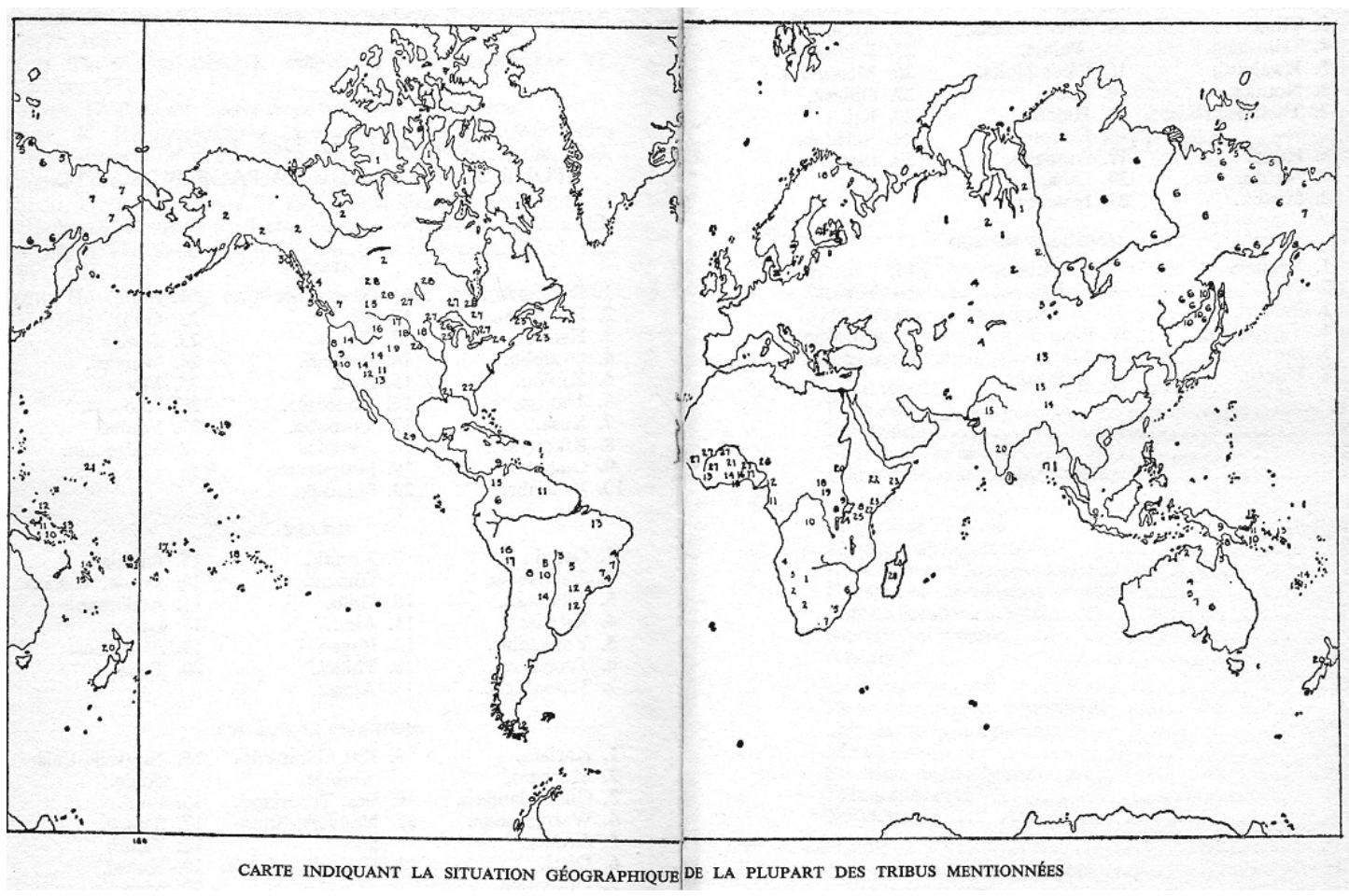




\section{Répertoire des tribus}

\section{pour la carte de la page suivante}

$\underline{\text { Retour à la table des matières }}$

\section{AFRIQUE}

1. Bochimans.

2. Hottentots.

3. Herero.

4. Ovambo.

5. Zoulou.

6. Thonga.

7. Xosa.

8. Kikouyou.

9. Ouganda.

10. Bakouba.
11. Mpongwe.

12. Ekoi.

13. Kpelle.

14. Ashanti.

15. Ewé.

16. Dahomey.

17. Yorouba.

18. Azande.

19. Mangbettu.

20. Shillouk.
21. Lobi.

22. Galla.

23. Somali.

24. Watussi.

25. Massai.

26. Haoussa.

27. Foulbé.

28. Malgaches.

\section{EURASIE}

1. Ostiak.

2. Samoyèdes.

3. Altaïques.

4. Kirghiz.

5. Youkaghir.

6. Tongouzes.

7. Tchouktche.
8. Koriak.

9. Ghiliak.

10. Gold.

11. Ainu.

12. lfugao.

13. Thibet.

14. Assam.
15. Pundjab.

16. Vedda, Ceylan.

17. Andaman.

18. Lapons.

19. Albaniens.

20. Toda.

\section{AUSTRALIE ET OCÉANIE}

1. Kariera.

2. Murngin.

3. Queenslandais.

4. Warramunga.

5. Arunta.

6. Dieri.

7. Urabunna.
8. Insulaires du détroit de Torrès.

9. Kaï, Nouvelle-Guinée.

10. Îles Trobriand.

11. Nouvelle-Bretagne

12. Nouvelle-Irlande.

13. Îles Salomon.
14. Îles Banks.

15. Nouvelle-Calédonie.

16. Fidji.

17. Samoa.

18. Tahiti.

19. Hawaï.

20. Maori. 
21. Îles Marshall. 


\section{AMÉRIQUE DU NORD}

1. Esquimaux.

2. Athapaskan du nord.

3. Tlingit.

4; Tsimshian.

5. Kwakiutl.

6. Noutka.

7. Thompson River.

8. Hupa.

9. Maidu.

10. Miwok.

\section{AMÉRIQUE DU SUD}

1.

Yaghan.

2. Ona.

3. Bakaïri.

4. Tupinamba.

5. Bororo.

6. Uitoto.
11. Navaho.

12. Hopi.

13. Zuñi.

14. Ute, Paviotso, Paiute.

15. Pieds Noirs.

16. Crow.

17. Hidatsa.

18. Dakota.

19. Arapaho.

20. Omaha.
21. Natchez.

22. Yuchi.

23, Algonkin du nord-est.

24. Iroquois.

25. Winnebago.

26. Menomini.

27. 0jibwa.

28. Kri.

29. Aztèques.

30. Maya.
7. Botokudo.

8. Chiriguano.

9. Goajiro.

10. Chané.

11. Macusi.

12. Kaingang.
13. Canella.

14. Toba.

15. Chibcha.

16. Quichua.

17. Aymara 


\section{PRÉFACE}

$\underline{\text { Retour à la table des matières }}$

Dans l'édition originale de ce livre, j'ai tenté de résumer les faits essentiels de l'organisation sociale. et de mettre en garde contre des conclusions générales qu'on a tirées à une époque où l'ethnographie ne reposait pas encore sur une base empirique suffisante. Un critique anglais anonyme m'a comparé, non sans raison, à une servante qui enlève les toiles d'araignée dans une chambre fermée depuis longtemps. Je dois dire que cette humble tâche n'était pas le seul but que je me fusse proposé, mais c'est celui qui s'est imposé en premier lieu à l'attention de beaucoup de lecteurs. Quelquesuns ont jugé mon effort honnête, mais ennuyeux et stérile. D'autres m'ont, de plus, accusé du crime de lèse-majesté : j'aurais péché contre le nom sacro-saint de Lewis H. Morgan. Des critiques plus favorablement disposés ont déclaré que mon livre n'était qu'un compendium utile de faits qui n'avaient pas encore été réunis en un seul volume. Je ne tenterai pas ici de réfuter ces critiques dans le détail, car la réaction des lecteurs dépend nécessairement de leurs goûts et de leurs besoins individuels. Mon livre représente l'attitude de beaucoup de sociologues actuels, lesquels ne s'opposent pas du reste à toute interprétation raisonnable, c'est-à-dire à la confrontation des faits les uns avec les autres. En principe, je n'ai certes pas évité de tels rapprochements. Ai-je réussi à en démontrer le bien-fondé, ceci est une autre question. Refuser des généralisations prématurées n'équivaut pas à rejeter toute espèce de généralisation.

Au sujet de Morgan, je voudrais dissiper tout doute. Les crItiques, parfois plus que sévères, que j'ai adressées à cet auteur,le visent moins lui-même que ses trop serviles défenseurs actuels. Un pionnier a beaucoup d'excuses pour lui, surtout lorsqu'il a des résultats aussi sérieux à son actif. Si j'avais à écrire une histoire de la 
sociologie, j'exposerais en détail ce que l'ethnographie doit à Morgan. Le but que je me proposais dans ce livre m'a conduit à souligner ses erreurs. Cependant, si je n'ai pas rendu pleine justice à un aîné, j'ai voulu rendre sensibles les absurdités auxquelles on s'expose en tenant un savant, quel qu'il soit, pour une autorité infaillible. Cela est d'autant plus vrai qu'à l'époque où Morgan écrivait, on manquait presque totalement d'informations sur de vastes régions.

Pour ceux qui liront cet ouvrage, une réponse exhaustive à toutes les critiques qui m'ont été adressées serait moins utile qu'un exposé de mon attitude à l'égard de certaines écoles sociologiques. Je désirerais plus particulièrement exprimer mon opinion relative au prétendu conflit entre l'école « historique » (diffusionniste) et l'école « fonctionnelle ».

A mon avis, cette antithèse est purement artificielle. Puisque c'est de la " culture » que nous avons à nous occuper, notre devoir idéal est de projeter le maximum de lumière sur chaque parcelle de réalité qui se range sous cette rubrique. Je suis entièrement d'accord avec Malinowski lorsqu'il dit que chaque culture n'est pas un assemblage de faits dispersés, mais un tout complet, du moins dans une mesure considérable. Il s'ensuit qu'il est indispensable de déterminer de quelle façon se coordonnent les éléments qui ont été observés sur le terrain. C'est poursuivre un but esthétique bien plus que scientifique que de se contenter d'une série de tableaux isolés, si brillants soient-ils, des diverses cultures. Chaque élément, chaque ensemble culturel est conditionné par l'espace et par le temps, facteurs qu'une science de la culture ne saurait négliger. Si les peuples de la Mélanésie et du nord-ouest de l'Amérique présentent en commun le clan matrilinéaire, l'avunculat et l'héritage de la veuve de l'oncle, ces deux cultures ne peuvent pas être traitées comme deux systèmes fermés et sans relations l'un avec l'autre. Quiconque les étudie sans idées préconçues, est frappé par les ressemblances qu'ils présentent et cherche à se les expliquer.

L'explication peut revêtir deux formes. Historiquement l'analogie s'éclaire pleinement si les tribus que nous comparons ne formaient à l'origine qu'un seul peuple, ou si elles ont dérive leurs éléments culturels d'une source unique. Fonctionnellement, la ressemblance peut être attribuée au fait qu'un de ces éléments serait apparenté organiquement à un autre, si bien que la présence du premier rendrait celle du second plus probable que ne le voudraient les lois du hasard. Je suis fermement convaincu que les deux interprétations sont a priori également valables et que chacune doit trouver sa justification dans les conditions particulières du cas à considérer. S'il est prouvé que les tribus ont été en contact à un moment donné, on peut à bon droit invoquer l'argument historique. Lorsqu'il ne semble pas qu'il y ait eu contact, l'analogie s'explique par le principe de corrélation, selon la loi bien connue qui veut que les mêmes causes produisent les mêmes effets.

Donnons un exemple concret. Une tribu du centre de la Californie, les Miwok, et une peuplade de Sioux méridionaux, les Omaha, possèdent en commun plusieurs particularités relatives à la terminologie de parenté, ainsi que certaines règles matri- 
moniales. Or ils ne sont nullement apparentés, ils sont même séparés par une immense région où ces caractéristiques n'apparaissent pas; et les Omaha ne partagent pas ces éléments avec les autres tribus du groupe Sioux, tels les Dakota et les Crow. Si ces éléments se sont transmis des Omaha aux Miwok, pourquoi les Dakota qui vivent tout près ne les ont-ils pas adoptés? S'ils viennent d'une autre direction, pourquoi n'ont-ils laissé aucune trace dans le territoire intermédiaire? La conclusion inévitable est que certaines particularités des termes de parenté et certaines règles matrimoniales sont en relation fonctionnelle les unes avec les autres, ce qui explique la diffusion observée ainsi que l'absence de diffusion relevée également, point tout aussi important logiquement.

Par le rôle considérable que j'attribue aux relations fonctionnelles, mon point de vue me rapproche de Radcliffe-Brown dont la parenté avec l'école sociologique de Mauss et de Durkheim rend l'œuvre particulièrement intéressante aux lecteurs français. En fait, je suis pleinement d'accord avec Radcliffe-Brown en ce qu'il fait, mais je cesse de le suivre lorsqu'il expose ce qui, selon lui, devrait être fait. Son étude systématique de l'organisation sociale en Australie, sa connaissance précise et critique des anciens auteurs, sa définition bien établie des divers concepts, le rapport qu'il établit entre les terminologies de parenté et les règles matrimoniales, tout cela suscite mon admiration la plus vive. Ce qui plus est, je trouve chez ce savant un sens inné, quoique peut-être peu apparent, des problèmes historiques. Le simple fait que dans son livre sur les îles Andaman il ait compilé toutes les indications que nous possédons sur les autres Pygmées, est fort significatif. Il est évident qu'il considère comme concevable qu'il y ait quelque corrélation historique entre leurs cultures et que cette corrélation est potentiellement importante.

C'est seulement lorsque Radcliffe-Brown nous expose quels devraient être les buts de la sociologie que je me trouve en sérieux désaccord avec lui. Tout d'abord, avec Radcliffe-Brown ethnographe et contre Radcliffe-Brown philosophe, je suis fermement convaincu de la valeur de l'argument historique. Les phénomènes culturels ont entre eux des rapports chronologiques dont doivent tenir compte ceux qui les étudient. Il va sans dire que nous préférons une connaissance exacte de la chronologie à de simples conjectures. Cependant, lorsque les conjectures expliquent les faits observés, lorsqu'elles peuvent s'appuyer sur des lois plus ou moins prouvées, elles sont aussi naturelles qu'inoffensives. Elles ne deviennent dangereuses que lorsqu'elles se cristallisent en dogmes. Secondement, je m'insurge contre l'idée que Radcliffe-Brown ou quelque autre savant aurait découvert une " loi sociologique universelle ». Aujourd'hui que les sciences physiques insistent moins qu'auparavant sur la valeur éternelle de leurs généralisations, c'est proclamer en somme la supériorité des sciences sociales sur les sciences naturelles.

Pour en venir aux historiens qui se reconnaissent tels, j'admire profondément les tentatives que font Graebner et le Père Wilhelm Schmidt pour représenter l'ensemble de l'évolution humaine comme un tout organique. Schmidt en particulier a fait une magnifique oeuvre de pionnier en reliant les unes aux autres nos connaissances en 
préhistoire et en ethnographie. En principe, il a tenté ce que toute étude culturelle idéale devrait se proposer : assigner à chaque culture et à chaque élément culturel sa propre position chronologique. En pratique, son système présente deux défauts qui le rendent à mes yeux inadmissible. D'un côté, comme beaucoup d'autres auteurs, il est trop prompt à accepter pour leur valeur courante les concepts traditionnels de l'ethnologie, créant ainsi de pseudo-problèmes. J'ai suffisamment critiqué cette tendance dans ce volume. Par exemple, si, comme je le crois, le totémisme n'est pas une réalité historique, les questions relatives à la diffusion à travers le monde de cette prétendue entité disparaissent dans une large mesure; il ne reste plus que des problèmes spécifiques ayant trait aux rapports entre elles des formes évidemment identiques que revêt ce phénomène à l'intérieur de limites étroites. Ma seconde objection porte sur l'application d'un système qui ne se base que sur les faits océaniens et africains et qui prétend reconstruire n'importe quelle culture. La seule méthode qui s'impose ici, est celle qui s'inspire de la méthode géologique : traiter provisoirement chaque zone un peu importante en elle-même, y déterminer les phases des Kulturkreise, et, en dernier lieu seulement, confronter les diverses reconstructions. Cependant le Père Schmidt s'y refuse absolument. A la vérité, il a amélioré dans le détail le plan originel de Graebner, mais il a conservé l'erreur fatale qui consiste à appliquer à l'Amérique et à la Sibérie les classifications valables pour l'Océanie, sans même essayer l'opération inverse. Néanmoins, sur quelques points essentiels, on verra que les conclusions du Père Schmidt et les miennes concordent.

Mon point de vue est donc éclectique, non par besoin de concilier toutes les tendances, mais parce que les diverses opinions actuellement en cours semblent toutes contenir une part de vérité et se compléter mutuellement. Si je n'étais pas limité par le temps, l'espace et les moyens dont je dispose, ce volume deviendrait une encyclopédie, un recueil de tableaux graphiques dépeignant les sociétés avec toute la verve et la vivacité d'impression d'un Malinowski. Je mettrais alors soigneusement en évidence un grand nombre de corrélations qui, selon moi, rem. placeraient avantageusement les « lois » de Radcliffe-Brown. Puis j'établirais également des rapports historiques entre les faits, je reconstruirais le cours de l'évolution sociale dans chaque continent pour tenter en fin de compte une synthèse mondiale.

Toutefois, s'il m'est permis de modifier un mot de Pasteur, « le devoir cesse où le pouvoir manque », et mes lecteurs français devront se contenter d'une oeuvre beaucoup plus modeste. J'ai corrigé les fautes typographiques de l'édition américaine ainsi que certaines erreurs de fait, et, pour autant que le temps l'a permis, j'ai pris connaissance des derniers travaux parus. J'ai aussi joint à mon livre une carte indiquant la position des peuplades mentionnées les plus importantes.

Je dois beaucoup de reconnaissance à Mme Métraux qui a excellemment traduit ce traité quelque peu pesant. Elle m'a redonné confiance en la traduction.

Robert-H. Lowie. 


\section{1}

\section{INTRODUCTION}

$\underline{\text { Retour à la table des matières }}$

Dans un certain sens, la société et la civilisation primitive ont la même extension. En effet, la civilisation, ou la culture pour employer le terme ethnographique, c'est, selon la célèbre définition de Tylor, « ce tout complexe qui inclut les connaissances, la foi, l'art, la morale, la loi, les coutumes ainsi que toutes les autres facultés et habitudes acquises par l'homme en tant que membre d'une société ». Il s'ensuit qu'une description complète de la société implique l'étude de toutes les phases de la civilisation. Il ne saurait être question ici d'une tâche aussi énorme. Je me limiterai aux aspects de la culture, qu'on appelle organisation sociale, c'est-à-dire que je traiterai des groupes qui constituent la société, de leurs fonctions, de leurs relations mutuelles et des facteurs qui déterminent leur croissance.

Pourtant les différents aspects de la civilisation sont si étroitement liés les uns aux autres qu'il est en pratique impossible de se concentrer sur l'un d'eux à l'exclusion des autres. De récents événements nous ont familiarisés avec l'interdépendance mutuelle de certaines branches de la culture qui sembleraient disparates. Des opérations militaires ne sauraient être conduites avec succès sans la collaboration des savants de laboratoire et des cultivateurs. Ce principe est également applicable aux stades moins développés. Si nous voulons étudier l'organisation sociale, il ne nous est pas permis 
de négliger les facteurs industriels, car la société est souvent organisée sur le mode industriel précisément, en corporations de forgerons et d'architectes, de constructeurs de bateaux et de tatoueurs. Ce qui nous importe ici, cependant, ce ne sont point les procédés techniques appliqués par ces artisans, même s'ils sont caractéristiques de la société à laquelle ils appartiennent; nous traiterons plutôt de la situation de chaque corps au sein de la communauté, de son état d'infériorité ou de supériorité, de ses prérogatives et de ses devoirs comme unité parmi d'autres agrégats analogues ou opposés. De même, si nous avons l'occasion de nous occuper des corporations religieuses, nous ne concentrerons pas notre intérêt sur des croyances ou des rites, mais sur la situation des divers groupes dans l'organisme en général. Si nous avions à considérer le christianisme sous cet angle, les différences relatives à la confession auriculaire ou à la théorie de la transsubstantiation ne figureraient que comme des étiquettes de groupe, tandis que la prééminence progressive de l'une des catégories de croyants, la décadence d'une autre, l'impuissance d'une troisième seraient les phénomènes qui attireraient surtout notre attention. Néanmoins, il est impossible d'établir par avance la somme des connaissances qui seraient nécessaires pour éclairer l'essentiel du problème, et, si nous n'y prenions garde, nous pourrions bien nous trouver plongés jusqu'au cou dans les subtilités de la dispute scolastique. Il n'en va pas autrement avec les peuples primitifs, et, pour jauger avec exactitude le caractère de l'organisation sociale, il est souvent de toute importance de tenir compte de faits représentant tous les autres aspects de l'activité indigène.

Au point de vue scientifique, l'étude des sociétés primitives ne demande pas à être justifiée. Elles existent, font partie de la réalité-et la science est tenue de les prendre en considération. Mais l'esprit dans lequel on les a observées jusqu'ici n'a pas toujours été le même et il ne sera pas inutile de signaler les divers buts qu'on a poursuivis en étudiant ces sociétés.

Tout d'abord, on peut adopter une attitude essentiellement monographique. Quelques observateurs fixent leur attention sur un peuple déterminé à un moment précis de son existence et travaillent à décrire sa culture avec la plus grande fidélité possible. S'il réussit dans son effort, ce type d'ethnographe devient un artiste qui pénètre par sympathie dans l'esprit latent de cette civilisation; il crée alors un tableau à la façon de la Renaissance de Gobineau. Tel est l'idéal de recherche humaniste préconisé par le philosophe Windelband et son école. Pour eux, chaque manifestation de l'histoire humaine représente un phénomène unique, un ensemble de valeurs absolument indéfinissable qui ne peut être perçu que par l'intuition d'un visionnaire et transmis ensuite au public en colorations plus pâles. L'effort ethnographique, dirigé selon cet esprit, aboutirait à une collection de portraits culturels, dont chacun serait un tout complet et isolé des autres.

Une telle attitude vis-à-vis des phénomènes de la civilisation n'est en aucune manière incompatible avec un but scientifique, et, pour autant qu'elle met en évidence les phases subtiles d'une culture, elle peut même apporter des éléments indispensables à une description exhaustive de la réalité. Mais il est également vrai que la science ne 
saurait se contenter de cette immersion esthétique dans les diverses manifestations de la société humaine. En fait, un ethnographe qui passerait successivement de l'un de ces tableaux à l'autre, obéirait peu à peu imperceptiblement à un exercice mental tout à fait différent de l'impulsion qui pousse le peintre à reproduire l'individualité de son modèle et qui ne rappellerait plus son propre effort initial de re-création. Des comparaisons spontanées avec des tableaux antérieurs se mêleraient aux processus d'absorption pure. En face de l'allure martiale d'une culture viendrait contraster la déformation religieuse d'une autre civilisation ou l'avarice forcenée d'une troisième. On noterait les analogies aussi bien que les divergences et on chercherait inévitablement à en expliquer les causes. En d'autres termes, les phénomènes ne seraient plus perçus en eux-mêmes, mais en relation les uns avec les autres.

Il s'agirait partiellement d'un problème de relations causales. Il est bien naturel de supposer que les mêmes causes produisent les mêmes effets et, en conséquence, il incomberait à l'ethnologue de déterminer quelles sont ces causes. On pourrait a priori les chercher dans des affinités raciales, dans la similitude du milieu géographique ou encore dans quelque autre condition fondamentale commune aux cultures comparées. Pratiquement, ainsi qu'on le verra plus loin, il est malaisé d'isoler de telles déterminantes au milieu de l'infinie complexité des faits culturels et de démontrer qu'elles sont des facteurs d'importance. Quelques ethnologues ont même abandonné tout espoir de les démêler jamais. Mais que la recherche des relations causales soit une entreprise vaine ou non, il est un ordre de relations que celui qui étudie les civilisations ne peut ignorer, c'est la chronologie. Supposons que notre galerie de tableaux renferme une représentation de toutes les cultures imaginables. Il faudrait qu'elle présentât aussi différentes descriptions des cultures successives d'un même peuple. L'observateur-esthète devrait se contenter de noter que le tableau représentant le Japon en l'an 1000 et celui du Japon de 1900 figurent des incarnations disparates de deux idéaux culturels aussi éloignés l'un de l'autre qu'ils le sont tous deux de la Renaissance italienne. Cependant, l'homme de science ne saurait adopter une telle attitude. Pour lui, le fait qu'une culture est sortie d'une autre, qu'une même civilisation a varié selon les époques, est de toute importance. S'il n'introduit pas la notion de temps dans les relations entre des cultures qui ne sont que les anneaux d'une même chaîne, le savant perçoit bientôt qu'il a négligé la part essentielle de la réalité. Pour mieux nous exprimer, celui qui veut étudier une civilisation doit être un historien, quels que soient les autres devoirs qui lui incombent.

Mais à quelle catégorie d'historiens doit appartenir l'ethnographe? Quelques savants éminents dont la pensée s'est formée dans les laboratoires d'expérimentation, ont décrété avec emphase quelle est la seule méthode historique valable. Accoutumés à voir les phénomènes physiques décrits en de brèves équations mathématiques, ils ne peuvent concevoir qu'une autre science puisse présenter une valeur quelconque si elle ne se conforme pas au modèle des mécaniques célestes. Selon le professeur Pearson dans The Grammar of Science, «l'histoire ne deviendra jamais une science et restera un catalogue de faits énumérés d'une façon plus ou moins agréable tant que ces faits ne pourront être réduits à des séries que résumeront brièvement des formules scien- 
tifiques ». Et, appliquant son dogme à la civilisation plus spécialement, cet auteur soutient que, dans ses grandes lignes, le développement de l'homme a suivi le même cours en Europe, en Afrique et en Australasie; qu'il peut se résumer en quelques principes fondamentaux; et que toute préoccupation de l'historien étrangère à celle-ci ne saurait mériter d'être prise en considération. Des opinions analogues ont été exprimées par le professeur Ostwald, chimiste, et le Dr Driesch, zoologue.

Cette attitude fait preuve d'une étonnante naïveté. Il est hors de doute que les ethnologues et autres historiens seraient grandement coupables s'ils ne parvenaient pas à découvrir les lois qui sont à la base de la civilisation et à donner ainsi aux faits le plus haut degré de coordination dont ils soient susceptibles. Mais la première question à se poser est l'existence de ces lois et la mesure dans laquelle cette coordination est possible. On ne peut pas adopter l'hypothèse de l'uniformité de l'histoire culturelle simplement parce qu'elle nous semble pratique. Même en physique, le chercheur n'a pas toujours la chance de pouvoir réduire ses phénomènes à une formule newtonienne. Il doit théoriquement accepter le fait que l'eau atteint son point de densité maximum à 40 centigrades, ainsi que le commun des hommes s'en aperçoit pratiquement, sans s'attendre à ce que l'eau adopte les propriétés des autres liquides. L'ethnologue, de même, ne saurait admettre que sa tâche lui soit prédéterminée. Si l'évolution sociale obéit à des lois, il doit à coup sûr les découvrir, mais seulement si des indices en sont discernables, et sa position scientifique n'est en rien modifiée si ces lois n'existent pas. Son devoir est de tracer le cours qu'a suivi en fait la civilisation; la synthèse qu'il opérera devra dépendre de la nature des faits. Chercher à atteindre l'idéal d'une autre branche de connaissances humaines peut être positivement néfaste, car cela conduit facilement à une simplification artificielle, c'est-à-dire à une falsification. Cela équivaut à soutenir que l'eau doit se condenser en gelant. Si chaque peuple du globe avait une histoire culturelle complètement différente de celle des autres peuples, la tâche de l'historien serait de noter ces singularités, d'en tirer le meilleur parti possible, et, contribuant ainsi pour sa part à la somme des connaissances, sa dignité scientifique ne serait en rien diminuée du fait du manque de malléabilité de son matériel. C'est pourquoi, sans renoncer de prime abord à la recherche des lois de l'évolution sociale, nous proclamons fortement notre indépendance devant ce dogmatisme pseudo-scientifique qui s'obstine à formuler tous les phénomènes selon un mode qui n'a été utile que dans une aire restreinte de la connaissance humaine. Sans nous laisser influencer par des préjugés pour ou contre la régularité historique, nous essaierons de déterminer quels sont les faits et dans quel ordre ils se succédèrent.

Ici, cependant, l'ethnologue rencontre un obstacle qui est épargné à l'historien des civilisations supérieures. La succession des événements dans les communautés primitives est rarement notée, sauf pour la période la plus récente, et, lorsque les informations positives remontent à quelques siècles en arrière, l'ethnographe se considère comme privilégié. Voilà donc une difficulté bien réelle mais non insurmontable. En effet, outre les rares sources documentaires, le savant possède tout un stock de faits ethnographiques et linguistiques bien établis et, lorsqu'il les combine avec les rensei- 
gnements que lui apporte la distribution géographique, il lui est souvent possible de reconstruire l'histoire avec une certitude presque totale. En ce qui concerne les phénomènes de l'organisation sociale, des exemples nous en seront fournis dans les chapitres suivants. Je vais donc illustrer la méthode par un exemple tiré de la technologie : pour fondre le fer, les indigènes de Madagascar emploient un soufflet à piston d'un type tout différent des soufflets qu'utilisent les forgerons nègres sur le continent africain tout proche. En un magnifique exemple de reconstruction historique, Tylor a fait remarquer que le soufflet à piston se rencontre aussi à Sumatra, en d'autres points de l'archipel malais ainsi que dans la partie voisine du continent asiatique, et que, d'autre part, les Malgaches de Madagascar sont anthropologiquement et linguistiquement membres de la famille malaise. Le soufflet à piston est donc sans aucun doute une invention malaise que les Malgaches apportèrent à différentes régions au cours de leurs migrations. En combinant ainsi l'anthropologie générale et l'étude de la distribution géographique d'un objet caractéristique, Tylor réussit à établir avec certitude l'histoire d'une invention mécanique.

En effet, dans la reconstruction historique d'une culture, les phénomènes de distribution géographique jouent un rôle extraordinaire. Si une caractéristique se retrouve partout, elle peut réellement être le produit de quelque loi sociale universellement opérante. Si on ne la rencontre qu'en certains cas limités en nombre, elle peut cependant avoir évolué par l'action de quelque contingence agissant dans des conditions spéciales qui doivent être déterminées par l'analyse des cultures où cette caractéristique apparaît. D'autre part, comme dans le cas du soufflet des Malgaches, il se peut qu'aucune loi ne soit impliquée, sinon des questions de parenté. Enfin ceux chez qui on retrouve le même trait peuvent être de familles différentes mais, par des contacts et des emprunts, posséder en commun certaines particularités culturelles.

Ainsi les indices fournis par la distribution géographique demandent à être expliqués, soit par quelque facteur causal, soit par des affinités tribales, soit encore par des échanges internationaux; et la réponse obtenue à l'aide d'informations étrangères à l'ethnologie sera nécessairement moulée dans une forme historique. Si nous faisons l'historique du travail du fer, nous assignerons au soufflet malais une date relativement tardive, car il représente une forme spécialisée et évoluée dans une région de l'Asie distante des anciens centres de métallurgie; et il nous faudrait considérer le soufflet malgache comme une importation assez récente, car Madagascar est le point le plus éloigné qu'ait atteint la civilisation malaise.

Comme les analogies culturelles abondent entre peuples d'origine différente, leur interprétation se réduit en général à un choix entre les deux termes d'une alternative. Eues sont dues aux mêmes causes, que celles-ci puissent être déterminées ou non; ou bien elles sont le résultat d'emprunts. On retrouve à la base de plus d'une discussion ethnologique une prédilection pour l'une ou l'autre de ces explications. Et, à l'heure actuelle, des écoles influentes, aussi bien en Angleterre que sur le continent européen, démontrent avec une bruyante insistance que tous les parallèles culturels remontent à un seul centre de diffusion. Il est nécessaire d'envisager dès le début ce problème 
épineux, car la défense acharnée de l'une ou l'autre de ces alternatives a des conséquences pratiques lointaines. En effet, si tout parallélisme est dû à un emprunt, les lois sociologiques qui ne peuvent être déduites que d'analogies se développant indépendamment, n'existent pas. L'histoire des religions, de la vie sociale ou de la technologie consiste alors uniquement à énoncer le lieu d'origine des croyances, coutumes ou instruments, et à décrire leur trajet vers différents points du globe. D'autre part, si la théorie des emprunts n'explique qu'une partie seulement des parallèles observés, celle des causes semblables devient le but idéal à atteindre dans l'élucidation des autres phénomènes tout au moins. Il est donc convenable d'établir dès le début quelle position on est incliné à adopter dans le conflit entre les théories rivales de la diffusion et de l'évolution indépendante.

La grande force de la théorie diffusionniste repose dans l'abondance de preuves indiquant que les échanges ont joué un rôle important dans la croissance des civilisations. On ne S'en aperçoit pas seulement par déduction, mais aussi par observation, tel le cas de l'influence de l'Égypte sur la Grèce ou de l'Arabie sur l'Europe du Moyen Age. A tout ce vaste ensemble de témoignages, il faut ajouter de nombreux exemples d'emprunts établis par déduction, mais d'une manière qui ne laisse subsister aucun doute. Chaque fois qu'une caractéristique bien définie se rencontre dans une zone continue, la conclusion qui s'impose est qu'elle s'est développée en un point déterminé de cette zone et de là s'est déplacée jusqu'à ses limites. Cette conclusion est souvent corroborée par une épreuve quantitative : le trait en question se rencontre à un haut degré de développement à son centre d'origine et va en dégénérant vers la périphérie. Ainsi Boas a élégamment démontré que le cycle du corbeau dans la mythologie canadienne a son origine dans la partie septentrionale de la Colombie britannique et de là s'est déplacé vers le sud. Plus on s'éloigne du point d'origine, moindre est le degré de développement du cycle jusqu'à ce qu'il finisse par se perdre complètement. Cette combinaison d'aventures légendaires ne serait pas restée confinée à une étroite bande côtière si elle était le produit de quelque loi créatrice de mythes; et l'on ne remarquerait pas cette simplification progressive s'il ne s'agissait d'un cas de transmission successive vers des zones de plus en plus distantes de la source d'origine.

La diffusion doit donc être saluée comme une vera causa. Mais est-ce bien la seule? Comment nous expliquerons-nous l'apparition de caractéristiques semblables dans des régions fort éloignées dont les populations ne sont pas racialement apparentées et n'ont eu, à notre connaissance, aucun contact? Dans ces conditions, le diffusionniste devra avoir recours à l'hypothèse auxiliaire selon laquelle le contact a dû exister autrefois. Il est convaincu que chaque élément culturel est dû à un concours de circonstances si extraordinaire que jamais des conditions semblables ne pourront se présenter une seconde fois. Tel est, en effet, le dogme essentiel du credo diffusionniste.

Il nous faut tout de suite reconnaître que plusieurs des arguments apportés à l'appui de cette proposition n'ont pas toujours été particulièrement heureux. Ainsi le 
dédoublement des découvertes scientifiques a été cité comme preuve de ce qu'une même particularité peut se développer indépendamment, argument qui n'est guère convaincant. Un examen historique attentif nous montre que, d'ordinaire, les coinventeurs ont puisé largement au même matériel culturel, ainsi que le firent Newton et Leibnitz lors de la découverte de leurs formules. Un tel cas ne saurait donc être comparé à la création indépendante d'éléments culturels dans des zones complètement séparées, En outre, lorsque des savants modernes aboutissent simultanément à des résultats semblables, ils ne construisent pas simplement sur les mêmes fondements, mais ils se comportent en travailleurs entraînés qui cherchent consciemment à apporter telle ou telle pierre à l'édifice. Cet effort délibéré sur la base d'une formation spéciale n'existe absolument pas dans les civilisations primitives et la vraisemblance d'une invention répétée en est d'autant diminuée.

La faiblesse de la théorie diffusionniste sous sa forme extrême repose dans son manque de discrimination. Rares sont ceux qui affirment qu'une invention hautement compliquée puisse se renouveler plusieurs fois, mais, lorsque ce principe s'étend aux conceptions et aux découvertes les plus simples, il va à l'encontre du bon sens. Il est vrai que l'homme souffre d'une certaine pauvreté d'invention et qu'il préfère même suivre la voie la plus facile des emprunts; cependant ses déficiences ne sont pas aussi grandes qu'on le prétend. Sinon, cette admirable adaptation au milieu que nous remarquons dans des zones fort distantes, n'aurait jamais pu se produire. Les Micronésiens n'auraient jamais appris à substituer la coquille du peigne géant à la pierre qu'on ne trouvait plus sur leurs îles, afin d'en faire des tranchants de hache; les habitants des îles Andaman et les indigènes de l'Amérique du Sud n'auraient pas su stupéfier les poissons avec des plantes vénéneuses, et aucun de ces innombrables et ingénieux procédés industriels peu répandus n'aurait vu le jour. Nous faudra-t-il croire que les Indiens des Prairies, qui étaient capables de confectionner ces broderies extraordinairement compliquées en piquants de porc-épic, n'auraient pu découvrir par eux-mêmes que les excréments de buffle pouvaient être utilisés comme combustible, et auraient dû l'apprendre d'une autre source? Les Hidatsa du Nord Dakota traversent encore le Missouri dans des bateaux qui ressemblent à ceux des pêcheurs du Pays de Galles, avec une armature en forme de parapluie recouverte d'une peau. Devons-nous expliquer cette analogie par quelque connexion qui aurait existé autrefois et qui eût été oubliée au cours des temps? Nous ne nous rangerons certainement pas à cette opinion si, parmi les tribus voisines, nous trouvons le prototype du bateau Hidatsa, un radeau improvisé en peaux de tente supportées par un entrecroisement de pièces de bois, prouvant le caractère autochtone de l'embarcation. Puis il y a le cas des Australiens et des Fuégiens; ces deux peuplades remarquèrent bientôt, en faisant la connaissance du verre, que cette matière se substitue avantageusement à la pierre dans la fabrication de certains instruments. Nous pourrions ainsi dresser une longue liste de toutes les idées simples dont on sait positivement qu'elles ont été conçues plus d'une fois ou qui tout au moins, selon toute probabilité, ont une origine indépendante en deux points ou davantage. Il se rencontre même des indices prouvant que des notions plus complexes ont été en quelques cas inventées une seconde fois. Rien n'est plus remarquable que la présence du zéro dans la notation des Maya du Yucatan, 
invention à laquelle les Grecs et les Romains ne sont pas arrivés et qui double celle des Hindous, sans la moindre possibilité d'influences mutuelles.

Cependant les exemples cités se rattachent à une section limitée du domaine culturel, celle dans laquelle les problèmes mécaniques ou théoriques sont résolus par des moyens intellectuels. Les aspects religieux, sociologiques et esthétiques de la culture obéissent à des motifs totalement différents. On peut concevoir que pour ceux-ci qui échappent davantage au contrôle rationnel et où, en d'autres termes, la faculté analogique fonctionne avec une force que rien ne limite, les possibilités d'évolution indépendante soient diminuées ou même supprimées. En fait, un observateur admettrait plus facilement que les découvertes les plus importantes du génie mécanique puissent avoir une origine multiple, plutôt que de croire que l'esprit humain aurait remonté indépendamment plusieurs fois le sentier tortueux qui aboutit à quelque grotesque conception mythologique. Néanmoins les départements non rationnels de la culture ne manquent pas d'exemples prouvant l'origine indépendante de particularités analogues. Un seul cas nous suffira. On ne saurait faire à un Australien Kurnai plus grave affront que de l'appeler orphelin; il en va de même pour l'Indien Crow du Montana. Il nous paraît étrange qu'un terme aussi inoffensif puisse être considéré comme la plus insultante des imprécations, mais la chose est explicable. Parmi les peuples primitifs, l'influence dérive souvent directement du nombre plus ou moins grand de parents dévoués. L'orphelin sans famille est, par conséquent, condamné à l'impuissance sociale et, si l'on tient compte de la vanité indigène, il est naturel que le vocabulaire injurieux ne renferme pas d'épithète plus dégradante. Donc, non seulement il est clair que les Kurnai ne l'ont pas empruntée aux Crow, ni inversement, mais encore la raison de ce parallélisme nous est rendue évidente par ce que nous connaissons de la vie primitive.

Il n'est pas nécessaire de multiplier les exemples de ce type, car il est une catégorie culturelle qui pourrait nous fournir des informations en abondance, c'est le langage. Les phénomènes linguistiques appartiennent à la même classe psychologique que ceux qui ont donné naissance aux aspects non intellectuels de la culture et les mêmes observations peuvent s'y appliquer. L'anglais a fini par se rapprocher du chinois par la simplicité de sa grammaire, non point en raison d'influences directes exercées par la Chine sur le parler des Iles Britanniques, mais pour des causes internes. Si les langues indiennes Shoshoni du Grand Bassin de l'Amérique du Nord connaissent le duel, ce n'est point parce qu'elles ont emprunté cette notion aux anciens Grecs, mais parce que c'est un concept qui peut surgir indépendamment. On pourrait énumérer ainsi une multitude d'exemples démontrant qu'une même manière de classifier ou de décrire les phénomènes s'est développée dans des langues sans aucune relation à l'origine et qui ne sauraient avoir eu de points de contact. Puisqu'il en est ainsi, et quand bien même la raison de ces ressemblances nous demeurerait à jamais cachée, c'est donc un fait établi que le raisonnement et la classification par analogie peuvent produire des résultats analogues. C'est du pur dogmatisme que de décréter que de tels résultats ne sauraient se produire dans le cas des coutumes et des mythes. 
Bref, il n'y a aucune raison pour exclure la possibilité d'un développement indépendant lorsqu'on étudie l'organisation sociale. Je traiterai donc chaque cas d'analogie en particulier et je ne me prononcerai pas entre les théories rivales avant d'être arrivé au terme de mes recherches.

Les remarques qui précèdent nous expliquent pourquoi une place aussi considérable a été accordée dans les chapitres suivants aux questions de distribution géographique. En étudiant la diffusion d'un phénomène, nous pouvons tout de suite nous rendre compte s'il peut se ranger, même à titre d'hypothèse, parmi les conséquences nécessaires de l'esprit grégaire des hommes, tandis qu'une comparaison des particularités qui l'accompagnent nous révélera quelles sont les conditions favorables à son apparition. La distribution géographique d'une institution peut nous démontrer qu'elle a été propagée, et, lorsque d'autres informations viennent s'y ajouter, elle peut aider à reconstruire presque complètement un processus historique. Lorsque nous ne connaissons que la fréquence d'une institution, nous n'en savons pas encore beaucoup, mais nous possédons du moins un point à partir duquel amplifier notre information. Lorsque nous ignorons la distribution d'un phénomène dont l'histoire s'est perdue, nous ne connaissons rien de théoriquement important.

La science de la société primitive a une valeur éducative qui recommanderait son étude même à ceux qui ne s'intéresseraient pas de prime abord aux phénomènes de l'histoire culturelle. Nous sommes tous nés dans un ensemble d'institutions traditionnelles et de conventions sociales que nous tenons non seulement pour naturel mais encore comme la seule réponse concevable aux nécessités sociales. Lorsque les étrangers n'observent pas les mêmes conventions que nous, nos préjugés nous les font considérer comme nettement inférieurs. Contre ce provincialisme étroit, il n'est pas de meilleur antidote qu'une étude systématique des civilisations étrangères. En nous familiarisant avec les particularités de sociétés diverses, reposant sur des fondements totalement différents de ceux auxquels nous sommes habitués, nous élargirons notre notion des virtualités sociales, de la même manière que l'espace à dimension n élargit la vision du géomètre non euclidien. Nous jugerons alors notre ensemble d'opinions et de coutumes simplement comme l'une des nombreuses variantes possibles; et nous nous enhardirons à les modifier selon nos nouvelles aspirations. 


\section{2}

\section{LE MARIAGE}

$\underline{\text { Retour à la table des matières }}$

Si l'organisation sociale n'est qu'une phase de la culture et ne peut être comprise qu'en connexion avec d'autres phases, cela est encore bien plus vrai des divers aspects de l'organisation sociale. Si c'est par l'étude de la famille primitive que nous débutons, nous nous apercevrons bientôt que, pour comprendre ses phénomènes, il nous faut faire intervenir des faits qui sembleraient de prime abord sans aucun rapport. Dans certaines parties de l'Océanie, l'homme se rend régulièrement à son club pour manger et pour dormir; une habitude de ce genre modifie si profondément la vie de famille qu'on est obligé de tenir compte de ces deux aspects à la fois si l'on veut décrire l'un ou l'autre. Si, d'autre part, nous commençons par étudier les clubs, nous nous trouverons bientôt entraînés à discuter des notions de propriété, car le fait d'être membre de ces organisations équivaut à la possession d'un titre de propriété. Mais toute étude de la propriété inclut les notions de parenté qui déterminent l'héritage. Et ainsi de suite. Toutes ces différentes questions sont si étroitement liées les unes aux autres qu'il est impossible d'en considérer une comme essentielle et d'en déduire logiquement les autres. Par quelque bout que nous commencions, il nous faut avoir recours à de constants recoupements et anticipations, car, pour les nécessités de l'exposé, nous sommes obligés d'examiner l'un après l'autre les divers fragments d'un tout organique.

Puisqu'il en est ainsi, il importe peu d'arrêter par quoi nous débuterons. Je choisirai la famille comme première unité particulière à considérer et je commencerai naturellement par décrire les conditions en face desquelles se trouve un individu qui désire fonder une nouvelle famille, les prohibitions et les prescriptions sociales 
auxquelles il doit se soumettre dans le choix d'un conjoint et les moyens traditionnels d'en obtenir un.

Chapitre 2

\section{a. Prohibitions de mariage.}

$\underline{\text { Retour à la table des matières }}$

Dans toutes les parties du monde, il existe quant au choix du conjoint des restrictions basées sur la proximité de la parenté. Ceux qui transgressent ces règles sont coupables du crime mortel d'inceste. A l'intérieur du cercle de famille restreint, les relations sexuelles sont universellement tabou. Il n'existe pas de tribu qui approuve l'accouplement des parents et de leurs enfants, et, là où des unions entre frères et sœurs se produisent, elles semblent être le produit d'un extrême raffinement plutôt qu'un signe de primitivité. Dans les communautés où l'orgueil du sang prend une forme hypertrophiée, ainsi qu'autrefois en Égypte ou au Pérou, le souverain ne peut trouver aucune compagne de rang suffisamment élevé, excepté sa plus proche parente. De tels cas sont cependant rares et ne modifient pas les habitudes de la grande masse.

Il n'appartient pas à l'ethnographe mais au biologiste et au psychologue d'expliquer pourquoi l'homme ressent aussi profondément l'horreur de l'inceste. L'observateur d'une société se contente du fait que la crainte de l'inceste limite le nombre des unions biologiquement possibles. Il doit de plus noter les diverses conceptions des différentes communautés sur l'inceste. Car, tandis qu'il est universellement défendu aux parents et aux enfants, aux frères et aux sœurs de s'unir, beaucoup de tribus favorisent et même prescrivent les mariages entre certains parents plus éloignés. Ainsi, alors que l'aversion pour l'union entre membres d'un cercle de famille restreint peut être instinctive, l'extension de ce sentiment en dehors du cercle de famille restreint est conventionnelle, quelques tribus traçant la ligne de démarcation avec plus de rigueur que d'autres. Les Pieds Noirs du Montana, par exemple, non seulement désapprouvent le mariage entre cousins, mais voient de mauvaise grâce toute union à l'intérieur du groupe local, « car il y a toujours quelque doute qu'il ne s'y glisse une parenté proche dont on n'aurait pas tenu compte ». Les Shuswap et les Indiens de Thompson River en Colombie britannique interdisent les unions entre cousins issus de germains et les Nez Percés de l'Idaho prohibent même celles entre cousins au troisième degré. Selon une Indienne de la tribu des Paviotso du Nevada, les cousins les plus éloignés sont encore considérés par les siens comme des parents et le mariage est, en conséquence, défendu.

Cette répugnance à l'endroit de l'union entre parents doit se rattacher à un usage primitif commun. Un Paviotso, par exemple, s'adresse à tous ses cousins, de quelque 
degré qu'ils soient, en les traitant de frères et de sœurs. Or, chez les primitifs, le nom a une grande importance, et la distance qui sépare le fait d'appeler une jeune fille sa sœur et celui de la considérer réellement comme sa sœur pour tout ce qui a trait au mariage, est infime, grâce à l'identification superstitieuse des choses qui portent une même appellation. Il suffit donc qu'une cousine du sixième degré soit désignée du même terme qu'une autre du premier degré pour que toutes deux soient considérées avec la même horreur.

En fait, l'extension de ce sentiment est encore beaucoup plus surprenante. Fréquemment la prohibition ne s'applique pas seulement aux alliances entre véritables parents, mais même à celles entre individus qu'aucun lien n'unit et qui ne sont apparentés que par une fiction légale selon laquelle tous les membres d'un certain groupe social descendent d'un même ancêtre. La puissance magique du nom du groupe joue sans doute ici un rôle important. Ainsi, dans une tribu australienne, un homme appartenant au groupe Emou ne s'alliera pas à une femme Emou d'une tribu étrangère vivant à cent milles de là, quoique la parenté de sang soit tout à fait exclue.

La règle qui prescrit qu'un individu doit trouver son conjoint en dehors de son propre groupe, que ce groupe soit la famille, le village ou quelque autre unité sociale, a reçu le nom d'exogamie. La règle contraire qui oblige un homme à se marier à l'intérieur de son groupe s'appelle endogamie. L'endogamie fleurit partout où les distinctions sociales en sont venues à prendre une importance de premier plan. Le système des castes chez les Hindous en est le meilleur exemple; l'aristocratie européenne nous en fournit un autre. A un degré inférieur de civilisation, les Tsimshian de la Colombie britannique désapprouvent l'alliance entre le parent d'un chef et quelqu'un de sa suite ou entre l'un de ces derniers et les gens du commun. C'est seulement la notion de l'obligation du mariage à l'intérieur de l'unité sociale qui constitue l'endogamie et non l'occurrence de facto d'unions dans le groupe. Par exemple, les jeunes gens de Kalamazoo choisissent d'ordinaire leur femme dans leur propre ville, mais rien ne les empêche d'aller la chercher à Ottumwa ou à Przemysl. Ce n'est guère que lorsque nous décelons la notion de prohibition qu'il peut être question de tendance endogamique. Nous en rapprocherons le sentiment de désapprobation soulevé par les unions entre catholiques et protestants.

L'endogamie et l'exogamie ne s'excluent pas mutuellement, sauf en ce qui concerne la même unité. Les Toda des Nilgire Hills, dans le sud de l'Inde, sont divisés en deux groupes, les Tartharol et les Teivaliol, entre lesquels le mariage légal est défendu. Mais chacun se subdivise en groupes exogames. Un homme de la section Pan du groupe Tartharol ne peut pas prendre pour femme une jeune fille Teivaliol, mais doit la choisir parmi les Tartharol d'une autre section que la sienne.

Chapitre 2

\section{b. Moyens d'obtenir un conjoint.}


Il est dangereux de généraliser sur les tribus primitives. Cependant l'assertion selon laquelle le mariage représente pour elles une institution civile plutôt que sacramentelle ne rencontrera que peu d'exceptions. Il diffère toutefois notablement du mariage en faveur dans la civilisation blanche moderne en ce que, chez les primitifs, le contrat lie souvent des familles et non des individus. Ce phénomène apparaît clairement dans deux formes de mariage qu'on appelle le mariage par échange et le mariage par achat. Dans ces deux cas, la jeune fille est traitée comme un bien dont les siens ne se déferont pas sans avoir reçu une compensation adéquate.

Parmi les Kariera de l'Australie occidentale, l'acquisition d'une épouse est compliquée par certaines règles d'union préférentielle. C'est-à-dire qu'un homme non seulement ne doit pas épouser sa sœur et certaines proches parentes, mais est pratiquement obligé de s'unir à une cousine d'un type déterminé ou à quelque parente plus éloignée désignée du même terme (voir plus loin : Union préférentielle). En même temps que cette limitation, l'échange se pratique couramment. Un individu, A, ayant une ou plusieurs sœurs, trouve un individu $\mathrm{B}$, qui présente le degré de parenté voulu et qui a également une sœur. Chacun prendra pour femme la sœur de l'autre. Cette méthode semble avoir une distribution géographique nettement définie. Elle est commune en Australie et aux îles du détroit de Torrès, mais elle est rare et même inexistante dans la région voisine de Mélanésie.

A part ces échanges de sœurs, chez les Kariera, les parents arrangent des unions du type orthodoxe entre de jeunes cousins. La mort prématurée de l'un d'eux peut amener un changement, mais le nouvel arrangement se conformera à la norme matrimoniale : les futures épouses seront des cousines du type prescrit, quoique peut-être plus éloignées. Dans ces cas de fiançailles entre enfants, un jeune homme grandit avec la notion qu'un tel sera probablement son beau-père, qu'il convient de lui faire à l'occasion des présents et de lui rendre des services. Mais comme sa fiancée peut mourir, il existe tout un groupe de beaux-pères potentiels qui ont les mêmes droits, quoique à un moindre degré; ces attentions peuvent se concevoir comme une forme de compensation équivalente au phénomène de l'achat dans d'autres zones. Lorsqu'une jeune fille atteint l'âge fixé, elle est simplement remise à son fiancé. Le fait que nous avons en réalité à faire à un contrat entre familles appert clairement de quelques autres éléments de la vie matrimoniale des Kariera. « Lorsqu'il y a plusieurs sœurs dans une famille, elles sont considérées comme les femmes de celui qui épouse l'aînée. » Nous sommes ici en présence d'une coutume fort répandue qu'on appelle le sororat. D'autre part, les femmes d'un individu passent automatiquement après sa mort à un jeune frère ou à quelque parent du même rang, usage qu'on désigne de l'appellation technique de lévirat. Enfin un homme peut renoncer à son droit sur la jeune sœur de sa femme en faveur d'un frère cadet. 
En comparaison de l'échange, l'achat a une distribution géographique extraordinairement vaste. Cependant, il importe de distinguer les diverses variétés d'achat qui diffèrent tout autant psychologiquement que légalement. Dans quelques régions, la femme est à tous égards un effet mobilier qu'on peut transférer et léguer; en d'autres, on ne trouve que l'apparence de l'achat, puisque le prix offert est compensé ou même dépassé par un don équivalent ou dot.

Commençons par l'achat dans le sens le plus strict du terme. Parmi les Kirghiz, tribu musulmane du sud-ouest de la Sibérie, un père fiancera son fils âgé de dix ans à une fillette et se mettra dès lors à amasser le prix de la fiancée qui atteint parfois 81 têtes de bétail. La somme se verse par acomptes; c'est seulement lorsque la famille de la fiancée en a reçu une partie importante que le prétendant peut rendre visite à la jeune fille; et le mariage a lieu lors du paiement intégral. A cause de la forte somme exigée par la famille de la jeune fille, il est rare qu'on ait plus d'une épouse, et il n'est guère fréquent de trouver une femme divorcée, quoique sous la loi musulmane, l'autorité du mari ne connaisse pas de limites. Ici la femme est nettement conçue comme la propriété de son conjoint et perd tout contact avec sa propre famille.

Chez les Ho, une tribu Ewé de l'intérieur du Togo, dans l'Afrique occidentale, il existe une série de paiements et de prestations qui finissent par donner un titre de propriété sur la femme, mais celle-ci n'est pas complètement séparée de sa famille et son status social est bien supérieur à celui de la femme Kirghiz. Ici l'arrangement initial a souvent lieu avant la naissance de l'enfant : un homme qui aime une femme a l'habitude de se faire réserver la prochaine fille dont elle accouchera. Si la proposition est acceptée, le fiancé doit faire don d'un présent préliminaire à ses futurs beauxparents, puis il continue à apporter mensuellement à la petite fille des coquillages cauris, à prêter assistance à ses parents dans les travaux des champs et à rendre toutes sortes d'autres services. A sa puberté, la jeune fille est remise au fiancé, mais si les compensations qu'il a offertes ne sont pas suffisantes, les parents annuleront le contrat en lui renvoyant une somme stipulée en coquillages. Il ne peut faire aucun doute que, chez les Ho, la femme ne soit en un certain sens l'objet d'un droit de propriété. Elle peut servir de gage, être remise à un créancier; elle passe en héritage à un frère du mari, alors qu'elle-même n'a aucun droit à la succession. En pratique, néanmoins, la rigueur théorique de ces conceptions est souvent atténuée. Les femmes peuvent exercer une grande influence sur leurs maris, se soulèvent parfois contre eux et les quittent; elles ont droit en outre à une compensation pour fournir leurs époux en coton.

La fausseté des notions que pourrait suggérer le mot consacré d' « achat » ressort clairement de l'attitude des Kai, une peuplade papou de Nouvelle-Guinée. Ici le prix de la fiancée consistant en une défense de sanglier, un cochon et autres objets précieux, est payée aux oncles maternels et aux frères de la jeune fille, alors que le père n'a droit qu'à une certaine redevance en travail. Dans un sens, le mari devient par transaction le possesseur de sa femme; elle passe en héritage à ses frères et oncles, est punissable par le mari pour adultère; en cas d'enlèvement, sa perte est compensée par 
la restitution du prix de la fiancée. Mais, bien que la femme ne soit rien, juridiquement parlant, sa personne n'est pas remise complètement par les siens. Si un homme brise de la poterie appartenant à sa femme, il doit indemniser la famille de celle-ci; il n'a pas plus droit à hériter de ses biens qu'elle à devenir sa légataire; les enfants appartiennent définitivement à la femme et à sa famille. Bref, ce que l'homme acquiert par achat est une prérogative exclusivement sexuelle ; et même, en retour des services rendus par son épouse dans le ménage, il est tenu à fournir une somme de travail équivalente au profit du ménage.

La coutume dite lobola des Thonga de l'Afrique du Sud contraste avec celle des Kai. Il est vrai qu'ici aussi le prétendant et sa famille acquièrent une femme en fournissant la somme stipulée par la fiancée (lobola) - en bétail ou en houes - et la veuve passe naturellement en héritage à quelque membre de la famille de son mari, à l'un de ses frères cadets, aux frères de sa sœur ou à ses fils d'un autre lit. Le concept de propriété est donc appliqué ici à un plus haut degré encore. Lorsqu'un homme a fourni le prix ordinaire de la fiancée, la famille de sa femme l'emploie dorénavant à acquérir une fiancée pour un fils adulte. Or, si la femme du premier s'enfuit, son mari peut réclamer la lobola; comme celle-ci a déjà été dépensée pour l'achat d'une femme au beau-frère, la parenté insolvable de la femme qui s'est enfuie peut être obligée à livrer l'épouse qu'on vient d'acheter au mari de la fugitive. Jusqu'ici nous n'avons à faire qu'à un cas particulier du principe d'achat rigoureusement appliqué. Mais, en un point, il diverge fondamentalement de la pratique des Kai : la lobola, et j'insiste sur ce point, sert à acheter non seulement la femme mais aussi toute sa progéniture, si bien qu'un mari peut se faire restituer le prix de la fiancée si sa femme meurt sans lui laisser d'enfants; d'autre part la descendance d'une femme appartient à sa famille à elle aussi longtemps que la lobola n'aura pas été payée.

C'était une conception toute différente qui prédominait chez les Hidatsa du Nord Dakota. L'achat était ici la forme de mariage la plus honorable pour une femme et on n'achetait que les jeunes filles qui n'avaient jamais été mariées auparavant. Mais, quoiqu'on reconnût quelque droit de propriété, ainsi que le prouve la règle du lévirat, celui-ci était relativement peu développé. Le mari n'avait aucun pouvoir absolu ni sur sa femme ni sur ses enfants. Il est vrai qu'il pouvait épouser les sœurs cadettes de sa femme, mais, d'autre part, au début de sa vie conjugale, il faisait souvent figure de domestique dans la maison de ses beaux-parents. Il importe aussi de noter que fréquemment l'achat n'était qu'un échange de cadeaux, ceux du fiancé étant parfois de moindre valeur que les présents offerts par la famille de la jeune fille.

Il n'est pas rare du tout en Amérique du Nord de voir ainsi s'équilibrer la dot et le prix de la fiancée. C'était une caractéristique des unions matrimoniales des Tlingit du sud de l'Alaska. Dans de tels cas évidemment, il nous est difficile de parler encore d'achat. Les concepts légaux se rattachant à ces usages sont intéressants. Chez les Tlingit, les enfants appartenaient toujours à la mère en cas de divorce. Si le mari se séparait de sa femme pour des raisons d'incompatibilité, il était obligé de restituer la dot à ses parents à elle qui, cependant, gardaient les cadeaux. Toutefois, si la sépa- 
ration était motivée par l'adultère de la femme, le mari avait le droit de garder la dot et de se faire restituer ses présents.

Nous en avons dit suffisamment pour montrer la grande variabilité du concept d'achat et la portée des notions juridiques qui s'y rattachent. Avant d'en finir avec les cas où une compensation quelconque est offerte en échange de la femme, il convient de faire observer que la notion de prestation en travail pour une fiancée, qui se substitue souvent à l'achat ou qui le complète, peut avoir une tout autre signification!

Ainsi, chez les Koriak du nord-est de la Sibérie, la prestation est la méthode établie pour obtenir une femme. Même lorsque le beau-fils vit chez les parents de sa femme qui non seulement ne perdent pas l'aide de leur fille, mais bénéficient encore des services permanents de son mari, la règle ne se relâche pas. Non seulement le prétendant doit se rendre utile, mais encore il est obligé de supporter des privations et un traitement humiliant; sa période de service est une épreuve de caractère et d'aptitudes plutôt que l'équivalent du prix de la fiancée.

Les épreuves imposées aux prétendants jouent un rôle de premier plan dans le folklore indigène qui se plait à dépeindre les héros surmontant les obstacles les plus extraordinaires. La réalité est moins romantique; cependant il ne manque pas d'exemples d'épreuves bien définies. Ainsi, parmi les Arowak de la Guyane britannique, un futur mari doit prouver qu'il est un bon tireur en plaçant, d'un bateau en mouvement, une flèche dans un nid de pic; il doit en outre faire preuve de son ardeur au travail en débroussaillant un champ et en remplissant un grand nombre de paniers pendant un laps de temps fixé. L'idée qui est à la base de ces corvées est de s'assurer que le jeune homme est capable de pourvoir à la subsistance d'une famille. Ce motif revient constamment, tel un refrain, dans les propos des Indiens de l'Amérique du Nord où le chasseur habile apparaît comme le gendre idéal.

Si commune que soit la notion qu'une compensation quelconque est exigible en échange de la fiancée, elle n'est aucunement universelle. Dans quelques-uns des cas cités plus haut, un examen plus attentif nous a indiqué que la forme même sous laquelle on fournit un équivalent peut renfermer des notions essentiellement diverses. Il ne saurait y avoir achat réel quand la dot est égale aux présents du fiancé, ni lorsque ces présents se réduisent, comme c'est le cas des Indiens au nord-ouest de l'Amazone, à un pot de tabac et de coca. Il nous faut cependant mentionner un certain nombre de formes matrimoniales où l'on ne retrouve même pas l'apparence d'une compensation.

Il y a tout d'abord le mariage par rapt. Bien qu'occupant une place exagérée dans les ouvrages anciens traitant de cette matière, il est au fond d'importance assez secondaire. Par exemple, les belliqueux Indiens des Prairies enlevaient souvent les femmes des tribus ennemies et les épousaient; mais, dans la grande majorité des cas et pour des raisons qui sont évidentes, les unions se faisaient au sein même de la 
tribu. La prise d'une femme par violence n'était pas, en effet, chose si simple et pouvait déchaîner des haines de famille. Il est vrai que parmi les Athapaskan méridionaux de la région du Mackenzie, il y avait des assauts de lutte entre rivaux, le plus fort étant autorisé à emmener la femme, même lorsque celle-ci avait déjà été mariée; et l'on nous a signalé des pratiques analogues chez les Esquimaux de la côte occidentale de la Baie d'Hudson. Mais, en général, il est peu de peuples qui admettent que la sanction sociale dans la communauté repose sur la force brutale. Dans bien des cas, il est vrai, nous trouvons une sorte de représentation dramatique du rapt de la fiancée. Ainsi parmi les Koriak, celle-ci, souvent avec l'aide de ses amis, résiste aux avances du jeune homme qui risque même d'être vigoureusement battu avant qu'il puisse la soumettre. De tels usages étaient interprétés par les sociologues de l'ancienne école comme des survivances d'un état où les mariages se seraient effectués normalement par rapt. Mais il est bien plus simple et moins hypothétique de les rattacher aux autres particularités des expériences pré-matrimoniales des Koriak et de ne voir dans le rapt symbolique que l'épreuve finale du savoir-faire et de la bravoure du prétendant. Ailleurs nous pouvons supposer avec Hobhouse que la dramatisation du rapt symbolise l'appropriation et qu' « elle n'est pas nécessairement la survivance de quelque chose de plus réel mais plutôt l'expression légale de la nature du fait accompli ».

Secondement il se rencontre des cas où le jeune couple, défiant les désirs de leurs parents ou les règles conventionnelles, se marie par consentement mutuel et tourne par la fuite les obstacles éventuels. On trouve partout des exemples de ces cas; cependant la portée de ces mariages d'amour varie énormément. En Amérique actuellement, le mariage basé sur l'affection mutuelle représente théoriquement le plus haut type atteint, mais il est notoire qu'il n'en va pas de même pour les couches supérieures de la société en Europe où, comme chez les Kai, le mariage légal et la satisfaction de l'instinct sexuel sont deux choses distinctes. Ce dernier trouve abondamment à se satisfaire en dehors du mariage, lequel est dissocié de l'amour sous sa forme la plus haute comme la plus basse pour se baser sur des considérations d'ordre économique, social ou politique. Le type d'union qui nous paraît, à nous Américains, le plus élevé, peut donc être considéré par d'autres comme nettement inférieur en valeur sociale. Parmi les Indiens Crow du Montana, l'occasion ne manquait pas de faire des conquêtes amoureuses lors des excursions, pique-niques et autres festivités et quelquesunes des liaisons ainsi nouées se transformaient dans la suite en unions durables. Pourtant, du point de vue de la tribu, de telles alliances ne pouvaient se comparer aux mariages par achat qui passaient pour beaucoup plus honorables et plus susceptibles de durée. Cela était dû sans doute au fait qu'un homme n'achetait une femme ou une jeune fille que si celle-ci jouissait d'une réputation de chasteté bien établie. L'histoire matrimoniale typique d'un Crow pouvait ainsi se décomposer en plusieurs liaisons d'amour et en un seul mariage orthodoxe par achat, lequel, en raison du sororat, devenait souvent polygamique. Une femme ne se déclassait pas à proprement parler lorsqu'elle s'unissait à un homme par inclination, mais, ce faisant, elle s'éloignait de la perfection idéale. Sa conduite ne soulevait même aucun commentaire défavorable, à moins qu'elle ne changeât trop souvent de compagnons. Un homme brave et bien fait 
pouvait avoir des aventures en nombre indéfini, ne ressemblant en rien à des alliances maritales. Les liaisons amoureuses durables jouissaient de plus de considération que la simple aventure ou le concubinage, mais n'atteignaient jamais au prestige du mariage par achat.

Ces coutumes des Crow nous suggèrent un principe d'application plus vaste. En général, il y a plus d'une manière de se procurer un conjoint permanent, quoique les diverses méthodes soient plus ou moins élevées dans l'échelle de l'estime publique. Un Crow peut se procurer une femme en l'achetant, ou en héritant de la femme de son frère; il peut contracter une union de sentiment sans rien payer, ou acquérir des épouses supplémentaires grâce au sororat après avoir acheté la fille aînée de la famille; enlever une femme étrangère en attaquant un camp Dakota, ou enfin, dans certaines conditions, prendre la femme d'un des membres de sa tribu si elle a été auparavant sa maîtresse. En d'autres régions les moyens peuvent différer, mais leur multiplicité est assez fréquente. C'est ce qui rend particulièrement difficile une évaluation numérique des diverses formes de mariage et la tâche est encore compliquée par les différentes significations de termes tels qu'achat ou prestation. Rangeronsnous les Crow parmi ceux qui achètent leur fiancée parce que l'alliance par achat représente chez eux la méthode idéale et quand bien même soixante pour cent peutêtre des unions stables ne sont pas orthodoxes? Est-il admissible de classer sans distinction les Kai, les Kirghiz et les Hidatsa parmi ceux qui achètent leur femme? Hobhouse a tenté un recensement des peuples qui pratiquent l'achat, indiquant le pourcentage par populations de chasseurs, de bergers et d'agriculteurs qui fournissent une compensation en échange de leurs femmes. Mais, quoiqu'il nous faille saluer avec joie tout effort vers une plus grande précision des affirmations sociologiques, dans le cas présent il nous semble condamné à l'échec à cause de la variabilité des formes matrimoniales au sein de la même tribu et des différentes nuances de signification que comporte l'achat.

Chapitre 2

\section{c. L'union préférentielle.}

Retour à la table des matières

Alors que les sociétés primitives interdisent fréquemment des unions qui nous paraissent naturelles, il arrive qu'elles favorisent ou même prescrivent des mariages que notre civilisation actuelle ignore. Nous en avons déjà vu plusieurs exemples : un certain type de mariage entre cousins, le lévirat et le sororat.

Quand des peuples primitifs favorisent le mariage entre cousins, celui-ci se limite presque toujours à cette parenté qu'on désigne techniquement du terme de cousins 
croisés, alors qu'aux cousins parallèles ou identiques la loi de l'inceste interdit de s'unir. Les enfants de deux frères sont des cousins parallèles ou identiques ainsi que ceux de deux sœurs. D'autre part, les enfants d'un frère sont cousins croisés avec ceux de sa sœur et réciproquement. La question se présente donc de la façon suivante : les enfants de frères ou de sœurs du même sexe sont des cousins parallèles et les langues primitives les appellent d'ordinaire frères et sœurs également; les enfants de frères ou sœurs de sexe différent sont cousins croisés et se désignent généralement par un terme exprimant une parenté plus éloignée. Les mariages entre cousins croisés appartiennent en théorie à deux types : un homme peut épouser soit la fille du frère de sa mère, soit celle de la sœur de son père. Pratiquement ces deux formes peuvent coïncider grâce au fait que le frère de la mère épouse d'ordinaire, selon la coutume tribale, la sœur du père. Lorsque tel n'est pas le cas, le mariage d'un homme avec la fille de l'oncle maternel, représente la variété la plus fréquente.

Le mariage entre cousins croisés a une très intéressante distribution géographique. Loin d'être universel, il a cependant été signalé dans presque toutes les grandes subdivisions du globe. Dans l'Australie occidentale et près du lac Eyre, les tribus prescrivant le mariage avec la fine de l'oncle maternel coudoient celles qui le prohibent. Cette coutume fleurit dans plusieurs îles de la Mélanésie, les Fidji notamment, mais est désapprouvée par des groupes polynésiens voisins, tels les habitants de Samoa. L'Asie du sud est peut-être le centre où cette institution atteint son plus haut développement; en tous cas, elle nous a été décrite dans tous ses détails chez les Toda et les Vedda; elle se rencontre chez diverses peuplades de l'Inde ainsi que parmi les Mikir tibéto-burmans d'Assam et à Sumatra. Elle existe également en Sibérie; les Ghiliak prescrivent l'union d'un homme avec la fille du frère de sa mère et il est pour le moins probable que les mariages entre cousins permis par les Kamchadal et les Tongouzes se conforment au même type. Bien qu'il soit relativement rare en Amérique, cet usage est signalé sur la côte septentrionale de la Colombie britannique, dans le centre de la Californie et au Nicaragua; le fait que les Indiennes Chibcha en Amérique du Sud ont un seul mot pour mari et fils de la sœur du père nous donne à croire qu'elles s'unissent fréquemment avec des cousins croisés. Dans la zone des forêts tropicales de l'Amérique du Sud, il est actuellement hors de doute que les indigènes ont pratiqué le mariage entre cousins croisés, souvent en même temps que d'autres unions préférentielles. Nous ne savons pas avec certitude si ce type d'union préférentielle était approuvé par les nègres du Soudan, mais il est orthodoxe en certaines parties de l'Afrique du Sud et de l'Est, chez les Hottentots, les Herero, les Bassouto et les Makondé.

On peut se demander ce qui arrive dans ces tribus quand un homme n'a pas de cousins croisés, lorsque sa mère n'a pas de frère ou son père pas de sœur. D'après nos meilleures sources, il semblerait qu'en de tels cas aurait lieu la substitution d'un parent plus éloigné jouissant du même statut. C'est ce qui se passait, nous dit-on, chez les Kariera de l'Australie occidentale; c'était tout d'abord la fille du frère de la mère qui entrait en ligne de compte, mais, s'il n'avait pas d'oncle maternel, le jeune homme s'adressait au cousin parallèle de sa mère ou, en cas de nécessité, à quelque parent 
éloigné du côté maternel, à la condition toutefois qu'il fût aussi appelé oncle maternel.

A ce propos, une question importante se pose. Si les primitifs étendent la notion de cousins croisés de manière à y faire entrer des parents plus éloignés, n'y aura-t-il pas là la source de plus d'une erreur? Ne se peut-il pas que nos observateurs aient mal compris la signification du terme indigène et donnent l'impression qu'on encourage le mariage entre Proches Parents alors qu'on réalité la forme d'alliance préconisée est celle entre parents beaucoup plus éloignés ou même fictifs ? Les rapports des investigateurs modernes sont par bonheur si précis qu'il nous est possible de nous prononcer. Les Toda, les Vedda et les Fidjiens prescrivent nettement le mariage entre véritables cousins croisés dans le sens le plus strict du terme. Trente pour cent des mariages fidjiens étudiés par Thompson appartiennent à ce type. Parmi les Toda, ce n'est que le douze pour cent, mais cette différence s'explique par le transfert ultérieur des femmes qui n'obéit à aucune règle définie et qui trouble les dispositions originelles des fiançailles entre enfants. On trouve pourtant chez eux des familles observant rigidement la norme, avec six unions sur huit conformes à la théorie. La différence d'âge n'est pas un obstacle, une femme de vingt ans peut épouser un petit garçon de deux ans. D'autre part, Gifford nous signale que, parmi les Miwok de Californie, il s'est produit des variations locales relatives au degré de parenté préconisé pour les alliances entre cousins croisés. Quelques districts recommandent les mariages entre véritables cousins croisés alors que d'autres admettent ceux entre cousins de même statut mais plus éloignés.

L'ensemble de nos informations suffit à prouver que dans une génération donnée le sentiment de l'inceste ne peut être instinctif en ce qui concerne les cousins au premier degré, mais est tout conventionnel. S'il était instinctif, pourquoi les unions entre cousins parallèles seraient-elles généralement tabou et celles entre cousins croisés souvent prescrites? Pourquoi une tribu permet-elle le mariage des cousins croisés alors que ses voisins les plus proches jettent l'anathème sur cette institution? Pourquoi quelques communautés autorisent-elles l'alliance avec la fille d'un oncle maternel, mais jamais avec l'autre variété de cousins croisés (les Miwok par exemple)?

Relativement à cette institution, on peut encore se demander jusqu'à quel point elle est non seulement permise ou même recommandée, mais encore obligatoire. Us faits recueillis étant trop peu nombreux pour autoriser une réponse généralisée, il semblerait que les tribus varient beaucoup de l'une à l'autre à cet égard. Les Kariera, si mon interprétation des faits est correcte, rendent le mariage entre cousins croisés pratiquement obligatoire; les Fidjiens accordent des dispenses dans les cas d'antipathie individuelle, tandis que chez les Toda et les Miwok d'autres formes de mariage orthodoxes coexistent avec les unions entre cousins croisés.

Peut-on éclairer de quelque manière la signification essentielle et l'origine de cette institution? Tylor, se rangeant à l'avis de Fison, nous explique de façon assez plausible pourquoi les mariages entre cousins croisés sont admis, alors que ceux entre 
cousins parallèles sont tabou. Il suppose que cette coutume a pris naissance dans des communautés divisées en moitié exogames avec des règles fixes de descendance. Dans de tels cas, les cousins parallèles appartiennent toujours à la même moitié et la loi de l'exogamie leur défendra toujours de s'unir, tandis que les cousins croisés se rattachent à des moitiés différentes et ne seront pas affectés par les restrictions exogamiques. $\mathrm{Si}$, par exemple, la filiation est paternelle, il s'ensuit la situation suivante : un individu ainsi que ses frères et sœurs appartiennent à la moitié de la tribu $\mathrm{A}$ à laquelle se rattache leur père. Les enfants de cet individu et ceux de ses frères seront aussi $\mathrm{A}$, donc il leur est défendu de se marier entre eux. Mais ses sœurs doivent épouser des hommes de B et leurs enfants seront tous B, appartenant donc au groupe dans lequel les enfants du frère doivent chercher leur conjoint.

Nous aurions là une explication idéale si les mariages de cousins croisés n'étaient que des alliances entre les membres de certains groupes, quel que soit leur degré de parenté. Mais, comme Rivers l'a fait remarquer et ainsi que le prouvent les renseignements recueillis chez les Fidjiens, les Toda et d'autres, cette hypothèse est contraire aux faits. C'est souvent le cousin croisé du premier degré qui est considéré comme le conjoint préféré, alors que des parents plus éloignés ne lui sont substitués qu'en cas de nécessité. Et c'est ce que Tylor ne nous explique pas. Tout ce qu'il nous démontre, c'est que, lorsqu'il y a organisation bipartite, les cousins croisés au sens strict du terme sont parmi les conjoints potentiels. Il ne nous dit pas pourquoi les cousins les plus proches parmi ces conjoints potentiels sont considérés comme les véritables, Et pourquoi les autres ne représentent qu'un pis aller. Une autre difficulté réside dans le fait que les tribus pratiquant le mariage entre cousins croisés ne sont pas toujours organisées en moitiés exogames. Les Toda sont divisés en deux groupes mais endogames, tandis que les Ghiliak, les Tsimshian et les tribus de l'Afrique du Sud mentionnées plus haut ne connaissent pas du tout ce dualisme de l'organisation. Bien plus, Rivers a prouvé qu'en Mélanésie ce sont précisément les tribus chez lesquelles n'existe pas une telle organisation qui pratiquent le mariage entre cousins croisés, alors que cette dernière institution ne se retrouve pas là où la double organisation fonctionne dans toute sa rigueur; elle ne peut donc être simplement la conséquence d'un double système exogamique.

En ce qui concerne la Mélanésie, Rivers nous présente une autre hypothèse qui, ainsi qu'il le reconnaît, n'est valable que pour les faits océaniens. Il suppose qu'à l'origine les hommes âgés occupant le pouvoir s'adjugeaient les femmes disponibles, mais qu'ils transmirent dans la suite leurs privilèges maritaux aux fils de leurs sœurs, substituant finalement leurs filles à leurs femmes. Une explication analogue, mais d'un caractère moins hypothétique, est fournie par Gifford. Celui-ci avance des preuves indiquant que le mariage entre cousins croisés chez les Miwok est une institution relativement récente que précédait un régime de droits maritaux détenus par un individu sur la fille du frère de sa femme. Ces droits, ainsi que le suppose assez vraisemblablement Gifford, passaient en héritage au fils de cet homme, d'où s'ensuivait le mariage avec la fille de l'oncle maternel. Il importe d'observer que, selon les phénomènes dont ils traitent respectivement, Rivers et Gifford présupposent différentes 
descendances, question sur laquelle nous reviendrons dans la suite. En Mélanésie, c'est ou c'était le fils de la sœur, en Californie le fils qui occupait la place d'héritier présomptif; or, si le mariage entre cousins croisés est la conséquence de règles de succession, comme le croient nos deux auteurs, l'une et l'autre de leurs explications sont satisfaisantes, mais applicables seulement aux tribus chez lesquelles existent des lois de succession correspondantes.

On peut naturellement concevoir que des cousins croisés en soient venus à se marier par une méthode plus directe. Lorsque la possession de biens joue un rôle prédominant dans la conscience tribale, comme en Colombie britannique, le désir de garder à l'intérieur du cercle de famille des biens souhaitables peut avoir conduit au mariage avec la fille de la sœur du père ou avec la fille du frère de la mère, ainsi que le suggère Swanton. Une cause différente, quoique apparentée avec la précédente, est à rechercher dans le sentiment de caste qui réprouve l'union avec une personne de rang inférieur. Il est vrai qu'on parviendrait aux mêmes fins avec le mariage entre cousins parallèles. Mais ces derniers, ainsi qu'on l'a fait observer, sont communément appelés frères et sœurs et, grâce à la tendance primitive à identifier ce qui porte une désignation similaire, passent souvent pour tels, participant ainsi au même sentiment d'inceste. Les cousins croisés seraient donc les parents les plus proches dont le mariage, permis par le droit coutumier, pourrait conserver la propriété et le prestige social dans la même famille.

Il nous faut enfin remarquer que toutes les explications qui viennent d'être avancées supposent des conditions d'existence spéciales. Le mariage entre cousins croisés est, selon toute probabilité, un phénomène qui n'a pas évolué à partir d'une cause unique, mais qui a pris naissance indépendamment en des points et pour des motifs différents.

Avant de quitter cette intéressante institution, consacrons encore quelques mots à son influence sur les classifications de parenté. Lorsqu'un homme épouse régulièrement la fille du frère de sa mère ou celle de la sœur de son père, l'oncle maternel sera normalement le beau-père et la tante paternelle la belle-mère. Il n'est donc pas étonnant que dans beaucoup de tribus pratiquant cette forme de mariage, l'on désigne le frère de la mère et le beau-père par le même terme et qu'on n'ait aussi qu'une seule appellation pour la sœur du père et pour la belle-mère. Tel est le cas aux îles Fidji et chez les Vedda, mais les effets de ce type de mariage peuvent ne pas s'arrêter là. Puisque l'épouse est normalement la cousine croisée, nous trouvons parfois qu'il n'y a pas de mot spécial pour « mari » et pour « femme », mais que le même terme désigne à la fois le mari et le cousin croisé ou la femme et la cousine croisée. De plus, un beau-frère peut recevoir la même appellation qu'un cousin croisé, tandis qu'une femme appellera sa belle-sœur du même nom que sa cousine croisée. Cependant les tribus ne procèdent pas toujours à cet égard avec une conséquence rigoureuse et, là où d'autres formes d'union préférentielle coexistent avec le mariage entre cousins croisés (comme chez les Miwok), l'influence de ce dernier peut être affaiblie ou même disparaître complètement. 
Si vaste que soit la distribution géographique du mariage entre cousins croisés, il paraît insignifiant à côté de deux autres formes d'union préférentielle : le lévirat et le sororat. Quoique fréquemment associées, ces coutumes se rencontrent aussi séparément ; il vaut donc mieux les traiter l'une après l'autre plutôt que comme un phénomène unique. Tylor a trouvé le lévirat dans un tiers des tribus sur lesquelles on était renseigné de son temps. Actuellement la proportion serait encore bien plus grande sans doute. Il est en effet plus aisé de compter les cas où l'on sait positivement que cette coutume fait défaut plutôt que d'énumérer ceux où elle apparaît. En Amérique du Nord, elle ne semble nettement réprouvée que parmi les groupes Pueblo du sudouest, alors que chez les Navaho voisins l'usage exige qu'une veuve épouse le frère de son mari ou quelque proche parent du défunt. Cependant le lévirat n'apparaît pas partout sous la même forme. Tout d'abord beaucoup de tribus limitent les seconds époux de la veuve aux frères cadets du mari décédé, comme le font les Koriak et les Andamènes. Quoique ce soit en Asie qu'il est le plus souvent signalé, le lévirat des cadets ne se restreint aucunement à ce continent, car il est mentionné à Santa Cruz (Mélanésie) et en Australie occidentale. A cause de l'insuffisance des informations fournies d'ordinaire par les observateurs sur cette question, nous ne pouvons même pas être certains que le lévirat n'appartienne pas communément à ce type. Néanmoins, pour quelques régions, les faits sont suffisamment explicites pour prouver que le lévirat y existe sous toutes ses formes. C'est le cas, par exemple, des îles Banks et du détroit de Torrès.

Secondement, les conséquences juridiques et psychologiques peuvent différer totalement. Chez les Indiens de Thompson River, le frère du défunt a des droits incontestables sur la veuve; en beaucoup d'autres endroits, comme chez les Thonga, la femme est autorisée à faire son choix parmi un nombre considérable de parents de son mari; ailleurs encore, cette disposition n'apparaît nullement obligatoire. En fait, l'attitude indigène est parfois à l'opposé de ce à quoi on aurait pu s'attendre : la veuve est moins réclamée de droit que reçue en legs obligatoire. Le beau-frère est donc tenu de fournir protection et appui à la veuve et à ses enfants. Telle est souvent la nature de cette institution chez les Tchouktche, ainsi que parmi les Gournditch-Mara dans le sud-ouest du Victoria (Australie).

Ces conceptions variables et l'insuffisance d'informations exactes nous empêchent d'édifier une théorie valable pour tous les faits connus. La seule observation générale que nous puissions avancer en toute sécurité est celle de Tylor, à savoir que le lévirat résulte du fait que le mariage est envisagé comme un contrat entre groupes plutôt qu'entre individus. Il découle de ce principe que lorsqu'une union prend fin par la mort d'un des conjoints, le défunt est automatiquement remplacé par un membre du groupe auquel il appartenait. C'est à partir de ce point qu'il nous faudra étudier les conditions spécifiques du milieu social, le statut de la femme à l'intérieur de la communauté et les méthodes en usage pour se procurer une épouse. 
Un grand nombre de cas s'expliquent par les règles observées lors de l'achat d'une femme. Lorsque celle-ci est nettement achetée, au sens strict du terme, elle forme naturellement un bien transmissible. Ainsi, parmi les Kirghiz, un frère cadet hérite de la veuve, même s'il est mineur. De même, une veuve Kai devient la propriété du frère célibataire du mari défunt; si un homme d'une autre famille désire l'épouser, il doit offrir un certain paiement. Ces conceptions sous-jacentes apparaissent avec évidence dans la loi des Shasta. Étant donné que, dans cette tribu californienne, les frères et les autres parents d'un individu apportent presque toujours leur contribution au prix de la fiancée, ils acquièrent un droit secondaire sur la femme et, à la mort du mari, celle-ci passe naturellement à la garde du frère ou, à son défaut, d'un parent plus éloigné.

Le fait que la notion de propriété se retrouve souvent à la base du lévirat apparaît dans d'autres formes d'union préférentielle qui coexistent avec le lévirat ou qui s'y substituent. Dans un ménage polygame Thonga de cinq femmes, la veuve principale devient généralement le lot de celui des frères cadets du défunt auquel revient le domaine, la deuxième et la troisième passent aux autres frères, la quatrième à un neveu (fils de la sœur) du défunt, la cinquième à l'un des fils du défunt, qui naturellement ne doit pas être son fils à elle. C'est dire que ceux qui héritent d'un mort ont aussi droit à la veuve. De même, nous trouvons dans les îles Banks en Mélanésie et sur la côte nord-ouest de l'Amérique du Nord, outre le lévirat, une règle qui veut que la veuve passe au fils de la sœur du mari défunt. Ce ne peut être pure coïncidence si, dans ces deux dernières régions, c'est aussi le fils de la sœur qui est l'héritier présomptif des biens de l'oncle.

Ce principe, bien qu'il nous éclaire sur beaucoup de points, n'explique pas tout. Pourquoi rencontrons-nous souvent le lévirat sous une forme limitée? Comme Jochelson nous le fait remarquer, les frères plus âgés aussi bien que les plus jeunes héritent des propriétés du mort et pourtant seuls les cadets ont droit à épouser la veuve. Ici se présente à nous une explication fournie par le bon sens, mais qui n'a d'autre valeur que celle d'une hypothèse. Toutes choses égales d'ailleurs, le fils aîné a bien des chances de se marier avant le cadet qui peut ensuite avoir des difficultés à se procurer une épouse, soit à cause du prix de la fiancée, très onéreux, soit parce que les femmes disponibles sont rares. Dans de telles conditions, le lévirat du cadet peut avoir pour origine le principe qu'il faut donner à celui qui n'a pas, et cette tendance peut s'être généralisée en coutume.

Une autre limitation du concept de propriété inclus dans le lévirat réside dans le fait qu'il existe des peuples le pratiquant qui n'achètent pas leurs femmes et qui ne considèrent pas celles-ci comme une propriété, strictement parlant. En beaucoup de ces cas, cependant, nous pouvons faire appel au principe général de Tylor sur le mariage primitif en tant que contrat entre familles : puisque du point de vue indigène l'union de deux individus symbolise une alliance entre groupes, les droits maritaux du conjoint défunt passent naturellement à un membre du même groupe. 
Cette hypothèse est encore étayée par le fait qu'elle explique admirablement la coutume complémentaire du sororat. Nous avons décrit plus haut le lévirat des Shasta, qui se joint au sororat d'une manière révélatrice. De même que les frères du jeune homme s'unissent pour lui payer sa fiancée, ainsi c'est à la famille de cette dernière dans son ensemble qu'incombent les services qu'on est normalement en mesure d'attendre d'une épouse. Si elle reste stérile, ses parents doivent fournir gratuitement une sœur ou une cousine comme épouse supplémentaire, et la même règle est applicable lors de la mort de la première femme. Il s'agit ici non seulement d'un droit mais d'une obligation de la part du mari : il ne peut se remarier en dehors de sa bellefamille que par autorisation spéciale de celle-ci.

Sur la base de la théorie de Tylor, nous pouvons nous attendre à voir coexister le lévirat et le sororat et c'est bien ce qui arrive dans une très large mesure, alors qu'inversement les Indiens Pueblo, chez qui on n'a pas retrouvé le lévirat, ne pratiquent pas non plus le sororat. Cette corrélation intime est soulignée à juste titre par Frazer qui a trouvé des exemples de sororat dans toutes les régions du globe. Les rapports entre ces deux institutions seraient sans aucun doute beaucoup plus étroits encore si nos informations sur les règles matrimoniales dans les tribus primitives n'avaient pas un caractère aussi décousu. En des cas assez nombreux, on peut affirmer avec assurance que c'est par pure négligence que les auteurs ont relevé l'un de ces deux phénomènes plutôt que l'autre. Il existe pourtant quelques cas notables où la corrélation n'existe pas et qui doivent être envisagés dans toute leur importance. Tandis que les Koriak pratiquent ces deux coutumes, leurs voisins, les Tchouktche, n'ont que le lévirat. Il en va de même chez les Massaï de l'Afrique orientale qui défendent expressément le mariage avec deux femmes unies par les liens du sang. De telles exceptions, cependant, ne sont pas suffisamment nombreuses pour nous empêcher de considérer le lévirat et le sororat comme deux institutions se rattachant étroitement l'une à l'autre.

Le sororat ainsi que le lévirat existent sous deux formes essentielles, quoique le principe de différenciation ne soit pas le même. Un homme peut avoir le droit d'épouser la sœur de sa femme pendant que celle-ci est en vie; ou bien ce droit peut être limité au mariage avec la sœur de l'épouse défunte. Granet a justement dénommé la première de ces coutumes polygynie sororale. Il nous est à l'heure actuelle impossible de délimiter nettement la distribution géographique de ces deux variantes; cependant, en Amérique du Nord, le sororat restreint au décès semble fleurir à l'ouest des Montagnes Rocheuses, alors que le mariage simultané avec plusieurs sœurs est fréquent à l'est de cette chaîne. Un parallèle au lévirat des cadets nous est fourni par la règle, probablement universelle, du sororat autorisant l'homme à épouser exclusivement les jeunes sœurs de sa femme. Cette limitation se comprendra facilement si l'on se souvient que dans une société primitive les jeunes filles se marient presque toujours lorsqu'elles atteignent la puberté ou immédiatement après, ce qui fait que la sœur aînée d'une jeune fille nubile est déjà la femme d'un autre. 
De même que le mariage entre cousins croisés, le lévirat et le sororat tendent à faire naître une terminologie de parenté bien définie. Comme Sapir l'a fait remarquer, leur influence peut s'exercer dans deux directions. D'une part, puisque ces formes de mariage conduisent à une identification du beau-père (parâtre) et de l'oncle paternel, de la marâtre et de la tante maternelle, il est naturel de désigner chacun de ces couples de parents du même terme. Inversement, le fils du frère devient l'enfant du premier lit pour un homme, l'enfant de la sœur celui du premier lit pour une femme. Ces identifications ont été relevées par Sapir chez les Wishram du Washington méridional. La seconde manière de formuler dans le langage les coutumes sociales que nous étudions, est plus intéressante encore. Puisque l'oncle paternel peut épouser la mère et ainsi occuper le statut du père, il est appelé simplement père; et, pour une raison correspondante, les sœurs de la mère sont désignées du même terme que la mère. En outre, il est normal qu'un homme range les enfants de ses frères avec les siens et qu'une femme traite de même ceux de ses sœurs. Bien plus, puisqu'un homme épouse souvent les sœurs de sa femme, il n'est pas étonnant qu'un même mot désigne à la fois la femme et les belles-sœurs, alors qu'une femme n'aura qu'une seule appellation pour son mari et pour le frère de celui-ci qui est son époux potentiel. Ces désignations se rencontrent en effet parmi les Yahi du nord de la Californie. La méthode usitée par les Yahi pour désigner les divers parents est répandue sur toute la terre et, comme le lévirat et le sororat sont des institutions à large diffusion, elle offre une explication satisfaisante de ce qui nous semble à première vue un phénomène embarrassant, à savoir qu'un homme peut avoir à la fois une douzaine de " pères » et autant de « mères ». Nous aurons l'occasion de revenir plus tard sur cette question.

Le mariage entre cousins croisés, le lévirat et le sororat ne sont aucunement les seules formes d'unions préférentielles. En fait, nous en avons déjà rencontré d'autres à l'occasion - l'héritage de la veuve par le fils de la sœur ou fils d'un premier lit (Thonga), le mariage d'un individu avec la fille du frère de sa femme (Miwok). Cette dernière variété est intéressante du fait de ses conséquences sur la terminologie de parenté des Miwok. Comme nous le montre Gifford, il y a jusqu'à douze termes qui reflètent cette institution. Par exemple, le mot wokli est appliqué non seulement au frère ou à la sœur de la femme, mais aussi au fils ou à la fille de son frère à elle; car, puisqu'un homme épouse la fille du frère de la femme, les frères et sœurs de cette seconde femme deviendront ses beaux-frères et belles-sœurs. C'est bien parce que tant de termes de parenté décèlent ce type de mariage alors qu'aucun ne suggère l'union entre cousins croisés que Gifford en conclut de façon convaincante à la plus grande ancienneté de la première forme chez les Miwok. Parfois plusieurs types de mariage préférentiel coexistent. Dans la région de l'Orénoque, chez les Tamanak actuellement disparus, un homme pouvait épouser non seulement sa cousine croisée, mais aussi la fille de sa sœur ainsi que la sœur ou la nièce de sa femme, soit la fille de la sœur de sa femme. En outre, grâce au lévirat et à l'héritage de la veuve par le fils, il pouvait épouser la veuve d'un frère ou celle de son père, alors que le sororat lui donnait des droits sur la sœur de sa femme. 
Quoique nous ne tentions pas ici d'épuiser les variétés existantes de mariages orthodoxes, il nous faut en citer encore un type, caractérisé par le mariage d'un homme, non avec la fille du frère de sa mère, mais avec la fille de la fille du frère de la mère de la mère. Cette dernière forme de mariage nous rappelle l'union entre cousins croisés, mais en diffère en ce que l'étroitesse de la parenté est diminuée. Cet usage, limité à l'Australie, se rencontre à la fois dans les zones centrale et occidentale et son rayon de distribution est voisin de celui du mariage entre cousins croisés.

Chapitre 2

\section{d. Références.}

$\underline{\text { Retour à la table des matières }}$

Note. - Tous les titres sont cités en détail dans la bibliographie où les auteurs sont rangés par ordre alphabétique et leurs diverses publications disposées par ordre chronologique; deux titres paraissant la même année sont différenciés par des lettres. Les références de chapitres citent le nom de l'auteur, s'il est représenté par un seul ouvrage, son nom et la date de parution si on a eu recours à plusieurs de ses livres ou articles; et si deux livres sont publiés la même année, une lettre est adjointe. L'indication des pages est entre virgules. Exemples : RADCLIFFE-BROWN : 156. JOCHELSON, 1908 : 739. RIVERS, 1914 (b) :1, 123.

1) HOBHOUSE : 145. WISSLER, $1911:$ 19. TEIT, $1900:$ 325; id., 1909 : 591. SPINDEN, 1908 : 250. HOPKINS : 45. BOAS, 1916 (a) : 498. RIVERS, $1906: 34$ et suiv.

2) TYLOR, 1889 : 253. RADCLIFFE-BROWN : 156. RIVERS, 1914 (b) 1, 123. RADLOFF : 476485. SPIETH : 120, 182-198, 61-66. KEYSSER 85-92. JUNOD : 1, 102-125, 194, 232, 258-266, 480. LOWIE, 1917 (a) : 46, 74 et suiv. ; id., 1912 - 220 et suiv. KRAUSE : 220 et suiv. JOCHELSON, 1908 : 739 et suiv. ROTH, 1915 ,315. WHIFFEN, 163. HFARNE : 104 et suiv. BOAS, 1907 : 466 . HOBHOUSE : 153, 158.

3) RADCLIFFE-BROWN : 190-194. RIVERS, 1914 (b) : 1, 48, 184, 257, 270, 294; 11, 24, 121 et suiv.; id., 1906 : 512 et suiv. THOMSON : 182-201. SELIGMAN : 64, 75. STACK et LYALL : 17. FRAZER, J. G., 1910 : 11, 188; IV, 141-149. CZAPLICKA : 89, 98 et suiv., 107. BOAS, 1916 (a) : 440. SWANTON, 1905 (a) : 50 et suiv., 63, 68. GIFFORD, $1916: 187$ et suiv. MORGAN, 1871 : 265. RIVERS, 1915. SCHINZ : 177. JUNOD : I, 200, 207, 243. WEULE, 1908 : 96. TYLOR, $1889: 262$ et suiv., 253. FRANCISCAINS : 432. JOCHELSON, $1908: 748$ et suiv. MAN : 71. RADCLIFFE-BROWN : 158, 190-194. REPORTS, V :245. TEIT, $1900: 325$. BOGORAS 608. HOWITT : 250. RADLOFF 485. KEYSSER : 88. DIXON, 1907 463. MERKER : 47. SAPIR, 1916: 327-337. GIFFORD, 1916 :190. KIRCHHOFF (a) et (b). 


\section{LA POL YGAMIE}

\section{$\underline{\text { Retour à la table des matières }}$}

Quoique le nom de polygamie soit en général appliqué au mariage avec deux ou plusieurs femmes, il désigne en réalité l'union soit d'un homme, soit d'une femme avec plus d'un conjoint. Ce qu'on appelle d'ordinaire polygamie, se nomme en réalité polygynie, l'institution complémentaire étant la polyandrie. Il nous faut en outre étudier l'alliance d'un groupe d'hommes avec un groupe de femmes, coutume connue sous le nom de mariage par groupe.

Chapitre 3

\section{a. Polygynie.}

$\underline{\text { Retour à la table des matières }}$

La polygynie est un de ces mots dangereux qui demandent à être étudiés avec soin sous peine d'entraîner une méconnaissance totale des conditions qu'ils doivent désigner. Dans chaque société humaine, le nombre des naissances de garçons et de filles est à peu près égal. Or, pour que la polygynie ou la polyandrie deviennent de pratique courante, la nécessité s'impose d'un facteur non biologique modifiant 
l'équilibre naturel. La première chose à faire lorsqu'on est en présence d'une peuplade polygame, est de se procurer un recensement des membres nubiles des deux sexes. Parmi les Esquimaux, la vie du chasseur arctique est si dure que la population mâle adulte est sérieusement diminuée, ce qui rend arithmétiquement possible la polygynie. Holm signale un village dans le sud-ouest du Groenland comptant vingt et un habitants dont cinq hommes seulement. Mais la moyenne qu'il a généralement trouvée est de cent quatorze femmes pour cent hommes. Parmi les Esquimaux du Centre, les Kinipetu (en 1898) étaient au nombre de trente-cinq hommes, quarante-six femmes, trente-huit garçons et vingt-sept filles; chez les Aiwilik, vingt-six hommes, trente-quatre femmes, vingt-sept garçons, quinze filles. A l'ouest de la baie d'Hudson, le capitaine Comer a trouvé, en 1902, cent dix-neuf hommes, cent vingt-trois femmes, cent trente-huit garçons et soixante-six filles chez les Netchilik; et quarante-six hommes, cinquante-huit femmes, quarante et un garçons et trente-trois filles parmi les Samniktumiut. Ces chiffres nous indiquent que, dans tous ces cas, sauf dans le premier village signalé par Holm, la bigamie elle-même ne peut être de pratique générale. En effet, à l'époque de Crantz, un Groenlandais sur vingt à peine possédait deux femmes; le capitaine Holm n'en a jamais vu qui en eût trois et même un Aiwilik particulièrement influent se contentait de deux épouses seulement. Bref, même parmi les Esquimaux, qui constituent un cas a fortiori, la monogamie est pratiquement de règle, quoique la polygynie soit permise; et l'union avec plus de deux femmes est sans aucun doute exceptionnelle.

Il est vrai qu'en Afrique on a mentionné des exemples d'une extraordinaire multiplicité d'épouses. Même si l'on fait abstraction d'anomalies comme celles de la cour du Dahomey où toutes les Amazones sont considérées fictivement comme les épouses du roi, nous trouvons des cas bien nets d'hommes possédant cinq, dix, vingt et même soixante femmes et cela assez fréquemment, du moins en ce qui concerne le premier de ces chiffres. Malheureusement, aucun des auteurs ne daigne nous fournir des indications quant au rapport numérique des sexes. D'après les remarques qu'ils ont laissé tomber incidemment, il semble certain que seuls les personnages riches et éminents peuvent avoir des ménages polygynes. Ainsi, chez les Kikouyou de l'Afrique orientale, M. et Mme Routledge ont trouvé la monogamie « tout à fait courante »; il était commun d'avoir deux ou trois femmes et, chez les riches, six ou sept. Il est clair que même une pratique de la polygynie aussi modérée de la part des puissants et des riches devait rendre très difficile à beaucoup de jeunes gens de se procurer une épouse. Mais les difficultés découlant de cette situation sont mitigées par deux éventualités. Tout d'abord, on peut recevoir une femme en héritage au moyen du lévirat ou d'une des autres méthodes orthodoxes. Secondement, on a généralement tendance à fermer les yeux sur ce qu'on pourrait appeler des irrégularités pré-matrimoniales chez les jeunes gens. Un Thonga est ainsi en mesure de satisfaire son appétit sexuel bien avant que sa parenté ait amassé la somme nécessaire à l'achat d'une épouse légitime.

Les exemples africains nous montrent qu'à la limitation biologique de la polygamie viennent s'ajouter des restrictions imposées par les conditions économiques. 
Lorsque le prix de la fiancée atteint une valeur considérable, la bigamie elle-même devient hors de portée pour la moyenne des hommes, alors même qu'elle est sanctionnée en théorie. Les Kirghiz, bien que convertis à l'Islam, ne peuvent pas, en règle générale, s'offrir une seconde femme et il est rare qu'ils fassent usage de ce privilège, à moins que la première épouse ne soit stérile. De même pour la grande majorité des Kai, la bigamie est impraticable, quoique permise, et seuls les chefs l'observent.

Il est un autre facteur purement social, limitant la polygynie, qui requiert notre attention. Certains peuples pratiquent ce qu'on appelle la résidence matrilocale, c'està-dire que le mari s'établit chez les parents de sa femme. A moins que le sororat ne soit en faveur, l'alliance avec une seconde femme dépend ainsi de l'autorisation des parents de la première. Ainsi les Zuñi et les Hopi, qui pratiquent la résidence matrilocale mais non le sororat, sont strictement monogames. On ne saurait dire que cette coutume exclut absolument la polygynie, mais il est certain qu'elle tend à la limiter fortement. Les Youkaghir de la Sibérie septentrionale mentionnent des cas où un homme vivait une partie de l'année comme gendre dans une famille et le reste du temps chez un autre ménage, mais toujours en qualité de gendre; cependant la monogamie était la forme de mariage prédominante.

Si nous voulons comprendre la psychologie de la polygynie primitive, il nous faut au préalable éliminer les préjugés conventionnels à ce sujet. La polygynie n'est aucunement un indice d'infériorité féminine et n'est pas ressentie comme une dégradation pour les intéressées. Le mari peut se déterminer à prendre une seconde femme, non point poussé par une libido excessive, mais au contraire à cause du désir exprimé par sa première épouse de se décharger sur une autre d'une partie des soins du ménage. « Pourquoi est-ce à moi à faire tout le travail? Pourquoi ne t'achètes-tu pas une autre femme? » se plaint l'épouse Kikouyou. Dans le même esprit, la femme d'un chef Kai a tant d'obligations sociales à remplir qu'elle salue avec joie l'arrivée d'une aide; et de même une femme Tchouktche peut demander avec insistance à son mari qu'il lui procure une collaboratrice supplémentaire. Chez les Kai, en effet, il est si facile de satisfaire ses besoins sexuels par l'adultère que, dans le mariage légal avec une seconde femme, le motif sexuel est inexistant. On peut dire qu'en général ce sont les facteurs économiques qui sont les plus puissants. Chez les Thonga, seuls les gens fortunés peuvent acheter plusieurs femmes, mais cet investissement de capitaux est d'un rapport considérable, le travail des femmes non seulement subvenant aux besoins du mari, mais encore lui permettant d'entretenir généreusement des amis et d'accroître son prestige social. Dans ce sens, la polygynie devient une marque de distinction. Dans un milieu tout différent, sur le Mackenzie au nord du Canada, les Athapaskan faisaient effectuer des transports par leurs femmes et le chef Matonabbee avait jusqu'à sept ou huit de ces épouses-esclaves. Un autre motif qui peut déterminer un homme à prendre des femmes supplémentaires est le désir universel de procréation. Lorsque la première épouse est stérile, il est de pratique courante que le mari prenne une seconde femme dans l'espoir qu'elle lui donnera des enfants. Le facteur sexuel pur et simple ne doit naturellement pas être écarté non plus, mais tout tend à prouver que son influence sur le développement de la polygynie est faible. 
L'analyse des mariages polygynes dans une tribu déterminée va se montrer révélatrice. Parmi les Koriak à rennes, Jochelson a trouvé que $6 \%$ seulement des hommes avaient deux ou plusieurs femmes, un seul en avait trois. Dans ce dernier cas, la première femme avait bien eu des enfants, mais la maladie l'avait défigurée et la seconde était restée stérile. Dans quelques-uns des autres cas, on avait eu recours à la polygamie à cause de la stérilité de la première femme; dans d'autres encore, la seconde femme avait été reçue en héritage selon le lévirat et était considérée comme trop âgée.

Il nous reste à voir les relations qu'entretiennent entre elles les différentes femmes d'une famille polygyne. En Afrique où leur nombre est fréquemment considérable, chacune occupe d'ordinaire avec ses enfants une hutte séparée et a son ménage indépendant. Le Thonga dispose les huttes de ses épouses en arc de cercle et son idéal est d'avoir assez de femmes pour fermer le cercle autour de son habitation. Quant aux sentiments des différentes femmes, les témoignages varient. M. JUNOD, peut-être par préjugé de missionnaire, nous brosse un tableau sombre des escarmouches domestiques entre femmes, mais rien de tel n'a été observé par M. et Mme Routledge en Afrique orientale où, ainsi qu'ils le font justement remarquer, la séparation des femmes dans des huttes indépendantes contribue à maintenir la paix et « rappelle un village dirigé par un seul chef ». Il va sans dire qu'il faut s'attendre à rencontrer partout des cas sporadiques de jalousie, mais, lorsque c'est sur la demande de la première femme que le mari en prend une seconde, ce danger est bien réduit. Le sororat peut avoir le même résultat; les Hidatsa, du moins, estiment que, mariées à un seul homme, des sœurs sont moins susceptibles de se quereller que d'autres femmes. Parmi les Koriak et les Tchouktche, on a en général trouvé de l'harmonie dans les ménages polygynes avec çà et là des cas de mauvais caractère, ce qui doit représenter l'état normal de cette institution.

Un facteur important contribue sans doute à faire naître l'entente, c'est la supériorité universellement reconnue de la première femme. Ainsi, dans les tribus sibériennes que nous venons de citer, la seconde épouse est à tous égards la servante de la première. La même situation se retrouve chez les Kai de la Nouvelle-Guinée où la première femme envoie les autres chercher l'eau et le bois et leur fait préparer les repas pour les invités. on nous signale des conditions analogues chez les Massaï; ici aussi la première femme surveille les autres, a à sa disposition une plus grande partie du bétail du mari et se distingue des suivantes par le nombre et la valeur des cadeaux reçus.

Ainsi, une étude attentive de la réalité nous conduit à une tout autre opinion de la polygynie que celle que nous donnaient de cette institution les préjugés modernes. Cependant il est un fait qu'il importe de rappeler encore une fois : alors que la majorité probablement des tribus primitives permet la polygamie, les conditions biologiques et, dans une certaine mesure, sociales, empêchent la plupart des hommes de faire usage de leurs prérogatives théoriques. 
Chapitre 3

\section{b. Polyandrie.}

\section{$\underline{\text { Retour à la table des matières }}$}

La polyandrie est beaucoup moins répandue que la polygynie. On peut en effet en compter sur les doigts d'une main les cas non douteux. Elle se rencontre dans quelques communautés d'Esquimaux, mais non dans toutes, occasionnellement chez les Wahuma (Bahima) de l'Afrique orientale; elle atteint son plus haut point de développement au Tibet et dans l'Inde méridionale. Pour ceux qui veulent expliquer la vie sociale par des motifs purement économiques, il sera intéressant d'observer les différences dans les conditions d'existence parmi les peuples en question. Les Esquimaux sont des chasseurs de mer, les Wahuma et les Toda des peuplades pastorales; parmi les Tibétains, seuls ceux qui pratiquent l'agriculture, et non les nomades, connaissent la polyandrie. Dans un certain sens pourtant, pour les Wahuma et les Esquimaux, le facteur économique entre en ligne de compte.

La polyandrie des Wahuma est un phénomène unique. Quoique légale, ce n'est pas une institution permanente et elle n'est appliquée que dans des circonstances spéciales et pour une période limitée. Lorsqu'un homme est trop pauvre pour se payer une femme à lui seul, il reçoit l'assistance de ses frères qui partagent ses droits maritaux jusqu'à ce que la femme devienne enceinte; elle appartient alors uniquement au mari.

Sous cette forme, la polyandrie n'amène aucun trouble dans l'équilibre naturel entre sexes, mais, lorsqu'elle représente la coutume générale, elle présuppose une majorité artificiellement obtenue d'hommes en âge d'être mariés. On peut atteindre ce but de deux manières différentes. Dans certaines communautés d'Esquimaux, les conditions de vie sont si rudes que les petites filles sont considérées comme une charge et fréquemment tuées peu après leur naissance; ainsi se trouve enrayée la tendance à la polygynie due aux dangers auxquels les hommes sont exposés. L'infanticide des filles, quoique apparemment non fondé sur une nécessité économique, est de même à l'origine de la polyandrie des Toda. Mais les Tibétains agriculteurs ne pratiquent pas cet infanticide, sauf lorsqu'ils sont directement influencés par les Chinois; ils sont pourtant polyandres, alors que les Chinois ne le sont pas. Par malheur, nos informations sur les Tibétains sont loin d'être explicites à cet égard.

Chez les Tibétains, la polyandrie est de la variété fraternelle, c'est-à-dire que plusieurs frères se partagent une femme. Il est intéressant de noter que, dans les cas de stérilité, on choisit une seconde femme qui peut être la sœur de la première. Quoique nous ne soyons pas renseignés d'une façon aussi précise qu'il serait souhaitable, il 
semble bien que les considérations économiques aient été toute puissantes dans la formation des coutumes matrimoniales tibétaines. Pourquoi, par exemple, la polyandrie est-elle restreinte aux indigènes cultivateurs et pourquoi l'est-elle également au type fraternel? Rockhill suppose qu'il faut en chercher la cause dans le désir de trans. mettre indivise la propriété.

C'est sur les Toda que nous sommes de beaucoup le mieux renseignés et c'est eux qui nous donnent un aperçu de ce qu'est la vie dans une communauté polyandre. Tout d'abord, aussi haut que peuvent remonter les rapports dignes de foi, nous trouvons qu'il y a toujours une majorité marquée de mâles, jointe à la pratique de l'infanticide des filles. Mais cette dernière coutume s'est de plus en plus perdue sous l'influence des Blancs et la majorité masculine en a été d'autant diminuée. «En 1871, il y avait 140,6 hommes pour 100 femmes; en 1881, 130,4 hommes contre 100 femmes; en 1891, 1359 hommes et au recensement de 1901, 127,4 hommes pour 100 femmes. » Ces recensements officiels sont confirmés par les rapports généalogiques de Rivers. Celui-ci nous montre que, pour trois générations successives, le nombre des hommes pour 100 femmes était respectivement de 159,7, 131,4 et 129,2 dans l'un des groupes Toda et dans un autre groupe plus conservateur de 259, 202 et 171. Les raisons de l'infanticide des filles chez les Toda demeurent obscures, car rien dans leur histoire présente ou passée ne donne à croire qu'ils y aient été poussés par les nécessités économiques. Sa disparition progressive a affecté les coutumes matrimoniales de la façon intéressante que nous allons voir.

Fort communément, mais non toujours, la polyandrie des Toda est de la variété fraternelle, c'est-à-dire que, lorsqu'un homme se marie, il est entendu que sa femme devient aussi l'épouse de ses frères et ils vivent en général tous ensemble. Même un frère né ultérieurement sera considéré comme partageant les droits de ses aînés. Dans les cas de polyandrie fraternelle, il ne s'élève jamais de dispute parmi les maris, et les Toda sourient même à l'idée d'une telle éventualité. Lorsque la femme est enceinte, l'aîné des frères célèbre la cérémonie de l'arc et de la flèche qui, dans cette tribu, établit conventionnellement la paternité légale, mais tous les frères sont considérés comme les pères de l'enfant.

La situation est plus compliquée lorsqu'une femme épouse plusieurs hommes qui ne sont pas frères et qui peuvent vivre dans des villages différents. Dans de tels cas, l'établissement de la paternité légale est extrêmement intéressante. Socialement parlant, le mari qui célèbre la cérémonie de l'arc et de la flèche durant la grossesse de la femme établit sa qualité de père, non seulement du premier enfant, mais aussi de tous ceux qui naîtront dans la suite jusqu'à ce qu'un autre mari accomplisse le rite requis. D'ordinaire, il est entendu que les deux ou trois premiers enfants appartiennent au premier mari et qu'à la grossesse suivante ce sera un autre des époux qui fera valoir ses droits paternels et ainsi de suite. La paternité biologique est complètement négligée; un homme mort depuis longtemps peut être considéré comme le père si aucun autre homme n'a célébré depuis le rite essentiel. 
Les statistiques que nous avons citées plus haut montrent que les chiffres tendent à se rapprocher de l'équilibre normal entre sexes et cela grâce à la diminution de l'infanticide des filles. On pourrait croire que cette évolution a conduit les Toda directement à la monogamie, mais ce serait méconnaître la force conservatrice qui règle les relations sociales. Les Toda sont restés attachés à la polyandrie et l'ont tempérée de polygynie. Là où autrefois trois frères se partageaient une épouse, ils en ont maintenant deux et s'adaptent ainsi au nombre croissant des femmes.

Les faits relatifs à la polyandrie sont fort instructifs en ce qu'ils font apparaître la faiblesse de la théorie unilinéaire de l'évolution, théorie qui veut qu'une loi interne fasse évoluer les coutumes de toutes les sociétés vers les mêmes conséquences. La polyandrie des Toda et celle des Esquimaux sont toutes deux en relations causales avec l'infanticide des filles et, dans ce sens, il y a parallélisme, les mêmes causes conduisant aux mêmes effets. Mais qu'y a-t-il derrière l'infanticide des filles? Dans le cas des Esquimaux, ce sont les rigueurs économiques de l'existence sur le continent arctique; chez les Toda, la cause de l'infanticide des filles est obscure, mais nous savons d'une façon positive qu'il ne dépend pas de la vie économique. Nous pouvons encore comparer les conditions chez les anciens Toda et chez les Tibétains. Dans ces deux peuplades, la rareté des filles nubiles rend la polyandrie possible, mais cette rareté même se produit de diverses manières puisque l'infanticide n'est pas pratiqué par les Tibétains polyandres. Dans le développement de leurs coutumes polyandriques, les Esquimaux, les Toda et les Tibétains n'ont pas passé par les mêmes phases, quoique l'une de ces phases, le manque de femmes, conduise naturellement à la polyandrie. Le fait que les Toda sont entrés en contact avec une civilisation étrangère qui prohibe l'infanticide, a exercé sur l'histoire de leurs coutumes matrimoniales une influence plus grande que toute loi inhérente d'évolution sociale. Ces influences étrangères peuvent en fin de compte obliger les Esquimaux, les Toda et les Tibétains à se plier à une monogamie imposée, mais si ce résultat est obtenu, ce sera parce que ces trois peuplades auront emprunté cette coutume au même centre culturel et non à cause de quelque tendance mystérieuse de la polyandrie à aboutir à la monogamie, après avoir traversé des phases identiques.

Avant de passer à un autre type de mariage, il est nécessaire de distinguer la véritable polyandrie des coutumes selon lesquelles des hommes peuvent se désister temporairement en faveur d'autres de leurs droits maritaux. Cet usage découle de la notion de propriété du mari sur les faveurs de sa femme dont il peut disposer à son gré, soit pour se concilier un supérieur, soit en gage d'amitié. Ainsi, parmi les Crow, un jeune homme prêtera pour un temps sa femme à un camarade ou à un homme plus âgé que lui dont il désire partager les pouvoirs surnaturels. En fait, un tel prêt faisait normalement partie des transactions par lesquelles les Indiens des Prairies de diverses tribus acquéraient certains privilèges cérémoniels. Comme simple marque d'hospitalité, cette coutume se retrouve sur toute la surface du globe. Un Massaï, en visite dans un village étranger, va voir un des membres de la même classe d'âge que lui, qui abandonne immédiatement au visiteur sa femme et sa hutte; et, pour ne citer qu'un 
autre exemple, dans diverses tribus australiennes, les hommes considèrent de leur devoir de fournir des compagnes à leurs hôtes de marque.

Chapitre 3

\section{c. Communisme sexuel.}

$\underline{\text { Retour à la table des matières }}$

Supposons que la tendance récente des Toda vers une combinaison de la polyandrie et de la polygynie se développe et finisse par prédominer, nous aurons alors plusieurs groupes de frères unis à des groupes correspondants de deux ou de plusieurs femmes. Les diverses unités entrant dans ce qu'on appelle le mariage par groupe peuvent cependant varier considérablement en ce qui concerne leur importance numérique, leur constitution et les restrictions imposées sur les rapports maritaux. Si, dans une communauté, les lois de l'inceste faisaient complètement défaut et que, non seulement les frères et les sœurs, mais aussi les parents et les enfants pussent s'unir sans encourir de réprobation, nous aurions les conditions d'un état de promiscuité parfaite. A l'autre bout de l'échelle, il y aurait le mariage des Toda modernes, soumis à des règles définies. Comme le terme de mariage s'applique malaisément aux états désignés du nom de mariage par groupe, je suivrai l'exemple de Rivers et j'y substituerai comme équivalent générique celui de communisme sexuel. Avant d'ouvrir une discussion théorique de ce phénomène, voyons d'abord quels sont les faits concrets.

Tout d'abord, il nous faut reconnaître que le communisme sexuel peut exister parallèlement au mariage individuel, c'est-à-dire qu'une partie de la communauté peut vivre selon le premier de ces principes et l'autre se conformer au second. Notre propre civilisation, qui encourage la prostitution, nous en fournit un exemple parfaitement clair. Les sociétés primitives diffèrent surtout de la nôtre en ce que le communisme sexuel est publiquement sanctionné à l'intérieur de certaines limites. Les Bororo du Matto Grosso sont divisés en deux groupes : les hommes mûrs mariés selon les règles et vivant dans des huttes séparées, et les célibataires habitant une maison spéciale où ils possèdent en commun les jeunes filles qu'ils enlèvent au village, dédommageant les frères ou les oncles de leurs maîtresses par des présents de flèches ou d'ornements personnels. Alors que nos informations sur cet état de choses au Brésil ne nous permettent malheureusement pas une compréhension parfaite des règles sociales impliquées, la situation chez les Massaï est, par contre, parfaitement claire. Ici aussi, il y a ségrégation des guerriers non mariés, hommes de moins de trente ans en général, qui cohabitent librement avec les très jeunes filles. Chacun a sa maîtresse favorite qui lui soigne son bétail et lui confectionne des ornements personnels. Cette maîtresse ne se confond jamais avec la jeune fille qui a été fiancée au guerrier dès l'enfance, car celle-ci est obligée de vivre dans le camp d'un autre guerrier. Aussi 
longtemps que le guerrier demeure dans son kraal, sa maîtresse lui reste fidèle, mais, s'il s'absente, ne fût-ce qu'un jour, il perd ses droits à l'exclusivité et elle peut choisir un autre amant. Dans toutes ces relations cependant, les lois tribales sur l'inceste sont strictement observées. Lorsqu'un célibataire est rassasié de l'existence de guerrier, il abandonne la vie commune au kraal et se fixe dans une habitation séparée avec sa fiancée, à la condition toutefois que celle-ci ait réussi à éviter toute grossesse, considérée comme une honte.

Les habitudes des Bororo et des Massaï, de même que la pratique de la prostitution chez nous, ne sont évidemment pas en conflit avec l'institution du mariage individuel, lequel représente, au contraire, l'état normal après la période de licence juvénile. Il existe cependant des tribus où le communisme sexuel nous a été signalé sans aucune de ces limitations.

Le cas le mieux établi est celui des Tchouktche. Il importe de remarquer avant tout que chez ceux-ci le communisme sexuel est une pratique générale, s'étendant en somme à toutes les familles. Des cousins du second ou du troisième degré ou même des hommes sans rapports de parenté, désireux de cimenter fortement des liens d'amitié, formeront un groupe exerçant des droits maritaux sur toutes les femmes des membres du groupe. Les frères ne font pas partie de telles combinaisons, et les célibataires entrent rarement dans ces unions basées essentiellement sur la réciprocité. Parfois le communisme sexuel s'étend jusqu'à dix couples. Lorsque nous étudions de près les faits concrets cités par Bogoras, il devient évident que le terme de mariage par groupe qu'emploient d'autres auteurs n'est pas ici réellement applicable. Les Tchouktche " compagnons de femmes » n'habitent pas avec leurs épouses en un ménage commun. Ils sont membres de campements distincts et le but évident de cette institution est de procurer aux voyageurs des compagnes temporaires. Un Tchouktche a rarement l'occasion de se servir des droits potentiels obtenus par accord mutuel. « Les habitants d'un même campement sont rarement désireux de faire partie de ces mariages par groupes; la raison évidente en est que l'usage réciproque des femmes, qui, dans le mariage par groupe, se pratique rarement, est susceptible de dégénérer en promiscuité complète, si les membres du groupe vivent trop près les uns des autres. » En d'autres termes, cette institution n'a rien à voir avec la licence sexuelle sans restriction, mais elle est fondée sur des notions indigènes d'hospitalité réciproque. Il est vrai que, parfois, un " compagnon » prend la femme d'un autre et vit avec elle pendant quelques mois avant de la rendre, ou qu'un échange de femmes peut devenir permanent. Mais ces faits eux-mêmes, démontrant avec clarté que les notions des Tchouktche sur la fidélité conjugale diffèrent des nôtres, mettent hors de doute la nature individuelle de leur mariage. Bogoras signale des cas isolés de polyandrie, mais n'a jamais trouvé de compagnons se partageant simultanément les faveurs de plusieurs femmes. L'échange des épouses n'implique pas le mariage par groupe, mais plutôt des mariages individuels se succédant l'un à l'autre. Le mariage tchouktche est, en somme, une union individuelle tempérée par une extension temporaire et occasionnelle des droits sexuels du mari proprement dit à ses camarades de contrat. 
Le communisme sexuel, en tant qu'état normal et excluant ainsi le mariage individuel, a été signalé chez les Urabunna et les Dieri, deux tribus australiennes vivant dans le voisinage du lac Eyre. Ne possédant pas assez de détails sur les Urabunna, nous nous en tiendrons aux Dieri.

Chez les Dieri, la forme orthodoxe du mariage pour un homme est l'union avec la fille de la fille du frère de la mère de sa mère ou avec la fille de la fille de la sœur du père de la mère. Lorsqu'un garçon et une fille sont dans ce rapport-là vis-à-vis l'un de l'autre, ils sont époux potentiels et les enfants peuvent être fiancés par leurs mères et leurs oncles maternels; il y aura normalement échanges de filles par les parties contractantes afin que dans chaque famille un garçon reçoive une compagne. Aucune femme n'est la fiancée de plus d'un homme. Cependant, après que le mariage est consommé, il est possible que l'épouse devienne la concubine d'autres hommes, mariés ou célibataires. Nous n'avons malheureusement pas de statistiques précises nous démontrant dans quelle mesure et avec qui de telles relations maritales sont possibles; mais un certain nombre de renseignements fournis par Howitt nous permettent de nous faire une idée de l'état de choses en résultant. Il faut faire observer que, dans chaque cas où un homme et une femme ont des relations sexuelles, ils doivent être l'un vis-à-vis de l'autre dans la parenté orthodoxe définie plus haut. En accord parfait avec cette règle, nous voyons que des frères qui ont épousé des sœurs, peuvent partager leurs femmes et qu'un veuf prend, en échange de présents, la femme de son frère comme concubine. En outre, un visiteur présentant la parenté requise peut recevoir la femme de son hôte comme compagne temporaire. Normalement, cependant, les concubines semblent être réparties officiellement par le conseil des anciens, lequel confère les droits de concubinage aux individus qui sont époux potentiels. En pratique, il semble que seuls les personnages de haut rang soient susceptibles d'avoir plusieurs concubines; les anciens conseillent aux autres de se contenter d'une seule. Un autre obstacle au concubinage excessif réside dans la jalousie mutuelle des conjoints subsidiaires dont chacun désapprouve les nouvelles relations de l'autre et est autorisé à jeter des charbons ardents sur le compagnon ou la compagne coupable de caresser de tels projets. La concubine d'un célibataire est spécialement encline à exercer une surveillance active sur la vie sexuelle de son amant.

De tout cela il ressort surtout : a) que l'épouse prend invariablement le pas sur la concubine lorsque toutes deux occupent le même campement; b) que le mari - celui qui a été dûment fiancé -jouit de privilèges indiscutables sur sa femme. Aucun amant ne peut légalement emmener sa concubine de chez son mari, sauf en des périodes de licence générale; il peut tout au plus exercer ses droits secondaires en l'absence du mari ou avec la permission de celui-ci. Une femme peut, il est vrai, prendre l'initiative et demander à son mari de lui choisir comme concubin un homme présentant les conditions de parenté requises; mais, si le mari refuse, elle doit s'incliner et n'a par ailleurs aucun droit de veto lorsqu'elle est attribuée comme concubine à un autre homme qu'à son mari. 
Ces renseignements soulèvent de nombreuses questions auxquelles il n'est pas possible de répondre; si insuffisants qu'ils soient, il est clair que ce que Howitt décrit sous le nom de " mariage par groupe » ne représente pas un phénomène psychologique ou sociologique uniforme. Pour un homme, céder ses privilèges maritaux à un frère devenu veuf ou se concilier les faveurs d'un hôte de marque en lui livrant son épouse sont deux choses bien différentes; et aucune de ces pratiques n'a la moindre ressemblance, même lointaine, avec l'attribution officielle des époux potentiels par le conseil des anciens. Il pourrait s'agir de " mariage par groupe » dans une communauté où des groupes d'hommes se partageraient des droits maritaux en toute égalité sur des groupes de femmes correspondants. Rien de tel chez les Dieri : le mari est le maître quasi absolu de la vie sexuelle de sa femme et le concubin un simple substitut. Même le terme de " communisme sexuel » peut conduire à des erreurs d'interprétation, car la notion de communisme s'accorde difficilement avec la prépondérance marquée de l'un des partenaires au détriment des autres « communistes ». En outre, tous les renseignements que nous pouvons réunir sur cette matière nous donnent à croire que, alors que le mariage chez les Dieri est permanent, l'état de concubinage véritable est de très courte durée. Enfin la population du groupe local australien est si faible que le nombre des individus qualifiés pour le concubinage par les liens de parenté est extrêmement restreint. Si nous considérons les limitations secondaires qu'apportent au concubinage la jalousie sexuelle et les conseils des anciens (notre auteur insiste sur ces deux facteurs restrictifs), il est évident que dans la vie quotidienne d'un Dieri moyen, le concubinage ne saurait jouer le rôle suggéré par les termes prétentieux de « mariage par groupe » et de « communisme sexuel ». Il existe assurément un certain état de polyandrie mêlé à la polygynie. Les uns cèdent leurs prérogatives maritales par hospitalité et d'autres le font par égard au prestige social. Mais il est évident que la plupart des Dieri observent en général le mariage individuel. Il peut exister un certain communisme à la manière des Toda lorsque deux frères vivent avec deux sœurs; mais, lorsqu'un concubin prend la place d'un mari absent, il ne s'agit que de relations individuelles remplacées par d'autres relations, également individuelles. Du reste, nous avons vu plus haut que le mariage primitif ne saurait être considéré uniquement ou surtout du point de vue sexuel; et il est absurde de conclure du fait que plus d'un homme peut avoir accès auprès d'une femme, à l'existence d'une institution comme le mariage par groupe, ainsi que le fait justement observer Malinowski.

Outre les Dieri et les Tchouktche, on a signalé quelques autres tribus, telles les Ghiliak dans la région de l'Amour, comme pratiquant le communisme sexuel à l'exclusion du mariage individuel. Ces renseignements sont, cependant, d'un caractère si incertain qu'il nous est loisible de les ignorer jusqu'à plus amples informations. En considérant l'extrême rareté des cas de « mariage par groupe » et les résultats de notre analyse du communisme sexuel chez les Tchouktche et les Dieri, nous nous croyons justifiés à conclure qu'à l'heure actuelle, il n'a pas été prouvé qu'aucun peuple du monde ait, à une époque récente, pratiqué le communisme sexuel d'une manière qui exclût la famille individuelle. 
Chapitre 3

\section{d. Communisme sexuel hypothétique}

Retour à la table des matières

Cependant il est possible d'harmoniser ce verdict avec la théorie selon laquelle les tribus primitives, quoique ne pratiquant plus le mariage par groupe, seraient arrivées à l'institution de l'union individuelle après avoir passé par des phases antérieures de communisme sexuel. Telle a été l'opinion prédominante chez les sociologues modernes et son importance exige que nous examinions brièvement les raisons qui ont motivé sa vogue.

Lorsque les théories évolutionnistes, après avoir été généralement admises en biologie, commencèrent à modifier toute la pensée philosophique, on trouva naturel de les étendre à la sphère des phénomènes sociaux. Parmi les premiers à s'embarquer dans cette aventure, il y eut Lewis H. Morgan, dont le traité d'ethnographie sur les Iroquois lui valut la réputation d'un observateur précis et compréhensif des coutumes primitives. Sous l'influence des doctrines évolutionnistes, Morgan trace un schéma complet du développement du mariage humain. Le fait que Morgan suppose, à l'origine, des conditions de promiscuité parfaite où l'instinct sexuel n'était limité par aucune prohibition d'inceste, est nettement caractéristique de l'atmosphère intellectuelle d'alors. Une absence complète de règles dans la vie sexuelle est diamétralement contraire à la monogamie obligatoire, et les évolutionnistes de cette époque tendaient à relier les phénomènes les plus divers par des séries graduées de phases intermédiaires. Chez les penseurs de l'ère victorienne régnait l'opinion toute faite, trop évidente pour qu'il fût besoin de la prouver, que la monogamie était la forme la plus élevée que pût atteindre le mariage dans le meilleur des mondes possible; et il allait également de soi que les premiers hommes vécurent dans des conditions totalement différentes de ce but idéal. Morgan n'essaya donc même pas d'avancer des preuves empiriques d'une promiscuité préexistante, qu'il date d'une période où l'homme hésitait encore aux confins de l'humanité et d'une phase organique antérieure. Il fit intervenir la promiscuité comme un postulat logique, précisément de même que certains philosophes évolutionnistes avancent l'axiome de la génération spontanée, et ce faisant il la plaça par-delà toute discussion scientifique.

Il en va autrement de la seconde phase établie par Morgan, celle de la « famille consanguine », basée sur les mariages entre frères et sœurs, mais excluant ceux entre parents et enfants. Cette phase, quoiqu'on n'ait pu l'observer dans aucune tribu, était inférée par Morgan comme la seule cause possible de certains phénomènes empiriques. En d'autres termes, notre auteur ne se complaisait plus en des axiomes logiques, mais procédait dans l'esprit des physiciens qui, d'après les réactions des substances chimiques, en ont conclu à l'existence de l'atome. La preuve avancée par Morgan que régnait anciennement l'institution du mariage entre frères et sœurs, est le système 
hawaïen de désigner la parenté. Ce système est d'un caractère plus simple que celui qu'on relève d'ordinaire dans les tribus sauvages. Tandis que beaucoup de peuples primitifs distinguent soigneusement entre les parents maternels ou paternels, non seulement les Hawaïens n'introduisent aucune distinction de ce genre, mais encore ils englobent dans une même désignation tous les parents d'une seule génération, quel que soit leur degré de parenté. Par exemple, makua désigne à la fois les parents et les frères et sœurs de ceux-ci, le sexe n'étant indiqué que par des mots qualificatifs signifiant « homme » ou « femme ». Morgan soutient que les oncles maternels étaient appelés comme les pères parce qu'ils étaient effectivement des pères, ayant libre accès auprès de leurs sœurs, et qu'un homme désignait de même tous ses neveux et nièces, de fils et de filles, parce que ses sœurs étaient ses épouses, ainsi que celles de ses frères; ainsi de suite. La thèse de Morgan est que, alors que les coutumes reflétées dans la terminologie ont tendance à disparaître, la terminologie elle-même est Plus conservatrice et fournit ainsi comme une trace paléontologique d'institutions sociales périmées.

Tout d'abord, bien que la supposition de Morgan relative à l'antériorité d'une famille consanguine ait une base empirique, la place qu'il lui assigne dans ses diverses phases n'est pas fondée. Même en admettant qu'il ait réussi à prouver le mariage entre frères et sœurs, rien ne nous oblige à faire remonter cette pratique à quelque période déterminée. Si Morgan date cette institution de temps fort reculés, soit immédiatement après la période de licence absolue, c'est à cause de la conviction tacite, discernable dans toute sa pensée, qu'il nous faut admettre une série unilinéaire de phénomènes franchissant peu à peu la distance qui sépare la promiscuité de la monogamie. C'est uniquement grâce à cette hypothèse que la période supposée de la famille consanguine vient se placer après celle de la promiscuité et avant d'autres types familiaux. Si Morgan n'avait pas été aveuglé par ses obsessions théoriques, il aurait hésité avant d'attribuer aux Polynésiens le rôle qu'il leur assigne dans son système. En effet, la civilisation indigène de la Polynésie, bien loin qu'aucun de ses éléments nous suggère par sa grossièreté une haute antiquité, doit au contraire être rangée parmi les plus avancées des cultures qui furent privées de l'art métallurgique. Lorsque Morgan assigne à cette merveilleuse race, politiquement bien organisée et fort douée esthétiquement, la condition la plus basse parmi les divisions survivantes de l'humanité, il atteint au comble de l'absurde, ce dont il aurait pu se rendre compte même en utilisant les récits d'explorations en Océanie dont on disposait de son temps. Des rapports de missionnaires ont, il est vrai, signalé le mariage entre frères et sœurs dans la couche sociale supérieure d'Hawaï, mais ce phénomène signifie simplement que, dans ces îles, ainsi qu'autrefois en Égypte et au Pérou, de telles unions étaient la résultante d'un orgueil racial qui s'était développé dans une civilisation extraordinairement complexe.

Cependant, Morgan non seulement présuppose la chronologie relative de la famille consanguine et des autres phases sans la moindre garantie empirique, mais encore il s'expose au reproche plus grave d'avoir tiré des conclusions erronées de l'existence du système hawaïen. Quelques-unes des objections qu'on peut faire à ses 
déductions ont été présentées par Cunow dont les critiques sont d'autant plus dignes de considération qu'il accepte en partie et estime l'œuvre de Morgan. Pour commencer par un point précis, Morgan néglige le fait que le système hawaïen ne fait pas qu'englober des parents dans un même terme, mais distingue les diverses relations entre elles : il y a ainsi des désignations différentes pour beau-frère et belle-sœur et il existe même un mot spécial pour indiquer la parenté des parents du mari avec ceux de la femme. Si la nomenclature de Hawaï représentait la phase de la famille consanguine, de tels termes de parenté, ainsi que le fait remarquer finement Cunow, ne sauraient y trouver place. Car, dans les cas de mariages entre frères et sœurs, le frère de ma femme est mon frère, tandis que ses parents à elle sont mes propres parents, ou tout au moins les frères et sœurs de mes parents.

Toutefois, la grande erreur fondamentale réside dans le fait que Morgan tient pour acquis qu'un terme traduit par « père » est synonyme dans l'esprit des indigènes de " procréateur ». Il ne conçoit pas qu'un Hawaïen puisse appeler son oncle maternel « père », si cet oncle n'a pas, à un moment donné, cohabité avec sa sœur et n'est ainsi devenu le procréateur possible de ses enfants. C'est aller contre l'évidence qui nous apprend, non point que le frère de la mère est appelé père, mais que l'oncle maternel et le père sont désignés tous deux par un terme commun qui ne correspond exactement à aucun mot de notre langue. Il est absolument arbitraire de supposer que cette identification linguistique est basée sur des rapports conjugaux réels et cette supposition nous conduit à des conséquences absurdes. Car, ainsi que Mac Lennan l'a observé avant Cunow déjà, la théorie selon laquelle tous les « pères » sont des procréateurs potentiels entraîne la conséquence parallèle que les " mères ", dont chaque Hawaïen possède une bonne douzaine, l'ont toutes conçu et engendré. Morgan, il est vrai, essaie gauchement de tourner la difficulté en affirmant que l'indigène a ici en vue un rapport conjugal plutôt qu'une relation de parenté; le Hawaïen appellerait " mère » la sœur de sa mère parce qu'elle est la femme de son père putatif, donc en quelque sorte sa marâtre. Ce n'est cependant que pur subterfuge. L'extension respective des termes " père » et " mère » est strictement parallèle; ils font partie d'un seul système et exigent une interprétation unique. Si la notion de parenté véritable est sous-jacente au système en un point, elle doit l'être uniformément; et, puisque cette supposition conduit à une conclusion monstrueuse, elle doit être rejetée. L'explication fort simple du système hawaïen se trouve dans la thèse de Cunow pour qui il représente une stratification de la parenté selon les générations. Notre propre nomenclature n'est pas aussi éloignée de ce type qu'il semblerait au premier abord. Nous groupons les frères et sœurs de nos parents sous les termes d' « oncles » et de " tantes »; l'écart réside surtout dans notre différenciation du cercle de famille immédiat par l'emploi de termes distincts pour père et mère. Il est aisé de comprendre comment, dans quelques sociétés, en soulignant le facteur âge ainsi que le fait mainte communauté primitive, des désignations de consanguinité peuvent finir par indiquer seulement la génération au point de confondre les parents les plus proches avec le groupe de leurs contemporains. 
Bref, Morgan ne réussit pas à prouver que la nomenclature hawaïenne a son origine dans les mariages entre frères et sœurs; et, même s'il n'avait pas échoué, rien ne prouverait l'extrême ancienneté soit de cette terminologie, soit de sa cause hypothétique.

Sur ce dernier point, nous pouvons nous avancer un peu plus. Alors que les tribus primitives se conformant au système hawaïen de nomenclature sont en nombre limité, il en est beaucoup plus qui suivent la méthode des Dakota et des Iroquois, laquelle fait bifurquer les relations de parenté selon qu'elles sont maternelles ou paternelles. Les Dakota, de même que les Hawaïens, ont un seul mot pour père et frère du père et un autre pour mère et sœur de la mère; mais le frère de la mère, au lieu d'être classé avec le père, et la sœur du père, au lieu d'être classée avec la mère, sont désignés tous deux par des termes spéciaux. En étudiant de près les nomenclatures qui se rattachent, dans leurs grandes lignes, au système Dakota, nous trouvons des détails nettement hawaïens. On pourrait a priori considérer ces derniers comme des survivances d'un système purement hawaïen plus ancien, mais certaines circonstances nous prouvent d'une façon concluante que l'interprétation opposée est la seule possible. Un Crow, par exemple, s'adresse à la sœur de son père comme à sa mère, ainsi que le fait le Hawaïen. Or, la langue crow est une spécialisation de la branche hidatsa de la famille sioux. Toutes les autres langues sioux, y compris le hidatsa, font la distinction entre la mère et la sœur du père; le Crow lui-même fait aussi cette différence lorsqu'il ne s'adresse pas directement à sa tante paternelle. Il est donc évident que le vocatif crow n'est pas une survivance, mais une innovation. Des changements récents analogues sont signalés chez les Iroquois, les insulaires du détroit de Torrès, les Ghiliak de Sibérie et les Timne de l'Afrique occidentale. On est donc justifié à considérer les caractéristiques hawaïennes comme résultant d'un développement secondaire; et, lorsque ces traits spécifiques se combinent avec le haut niveau culturel des Polynésiens, ils forment un argument de poids contre l'antériorité de la terminologie hawaïenne ou d'autres coutumes sociales s'y rattachant.

Après cette discussion sur la famille consanguine, traitons rapidement des preuves qu'avance Morgan pour sa phase suivante, qui représente ce qu'on appelle communément en ethnographie le mariage par groupe, c'est-à-dire un état où " un groupe d'hommes se marie conjointement à un groupe de femmes ». Morgan considère particulièrement l'institution où plusieurs sœurs cohabitent avec un groupe d'hommes qui ne sont pas nécessairement parents entre eux. Le mariage par groupe pourrait naturellement se concevoir d'une façon quelque peu différente, quoiqu'il implique toujours une combinaison de polyandrie et de polygynie. Ainsi le professeur Kohler cherche l'origine de la nomenclature de parenté dakota dans la coutume selon laquelle les frères AAA épousent les sœurs bbb et les frères BBB les sœurs aaa. C'est encore la terminologie de parenté, en effet, qui fournit à l'ethnographe théoricien le principal argument. Comme dans le cas du système hawaïen, il ignore des possibilités évidentes et associe nos propres concepts de parenté à des termes primitifs comportant une signification toute différente. Nous trouvons dans le lévirat et le sororat des coutumes qui nous indiquent parfaitement pourquoi les termes de père et de frère du père, 
de mère et de sœur de la mère doivent être classés dans la même catégorie. Ces phénomènes nous démontrent que la terminologie de parenté n'exprime pas nécessairement l'état réel des relations sexuelles. Un homme peut ne jamais hériter de la veuve de son frère, soit parce que le frère survit à sa femme, soit parce que la veuve se remarie avec un autre frère. Mais, sans faire état de ce fait, il est appelé père par les enfants de son frère; des considérations analogues sont également valables pour le sororat. Le fait qu'il est théoriquement possible à un certain nombre d'individus d'entretenir des relations conjugales avec la mère d'un autre individu suffit pour que celui-ci les groupe dans une désignation commune. Nous n'avons aucune raison de supposer que les indigènes veulent exprimer plus qu'un statut social semblable en appliquant des termes de parenté identiques.

Morgan, il est vrai, interprète le sororat comme étant un reliquat du mariage par groupe et Frazer a étendu cette explication au lévirat. Mais ce sont des hypothèses dénuées de fondement que nous pouvons négliger. Le sororat et le lévirat sont des institutions réelles, intelligibles par les circonstances concomitantes; on ne contribue en rien à les rendre plus compréhensibles en les concevant comme les survivances d'un état qui n'a jamais été observé.

En résumé, le communisme sexuel, en tant qu'état se substituant à la famille individuelle, ne se retrouve actuellement nulle part et nous devons rejeter comme insuffisants tous les arguments qui tendent à prouver qu'il a existé autrefois. Nous allons voir cette conclusion confirmée par les phénomènes de la vie de famille primitive.

Chapitre 3

\section{e. Références.}

$\underline{\text { Retour à la table des matières }}$

1) THALBITZER : 15, 67. BOAS, $1907:$ 7, 115, 378. CRANZ - 1, 209. ROUTLEDGE : 134. HOLLIS, $1905:$ 303. JUNOD : 1, 97, 125-128, 274. RADLOFF : 484. KEYSSER : 90, 44. JOCHELSON, $1910: 110$ et suiv.; id., $1908:$ 752-755. BOGORAS : 598-602. HEARNE : 124 et passim. MERKER : 27 et suiv.

2) Annual Archaeological Report : 112. ROSCOE, 1907 :105. ROCKHILL : 211 et suiv. TAFEL : II, 124 et suiv. RIVERS, $1906:$ 477-480, 515 et suiv. LOWIE, 1917 (a) : 63; id., $1913: 228$ et suiv. HOLLIS, 1905 : 288. FRAZER, J. 34.

3) VON DEN STEINEN 388. HOLLIS, 1905 : XVI. MERKER : 44, 84. BOGORAS : 602-607. HOWITT : 163-167, 177-187. MALINOWSKI : 100-123.

4) MORGAN, 1877 : Part. III; surtout chap. II, III, VI. RIVERS, 1914 (b) - 1, 275 et suiv. CUNOW, 1894 : 54, 127 et suiv.; Id., $1912: 50$ et suiv. LOWIE, 1917 (a) : 118, 162. KOHLER : 266. FRAZER, J. G., 1910 : IV, 139 et suiv. 


\section{LA FAMILLE}

\section{$\underline{\text { Retour à la table des matières }}$}

Biologiquement chaque communauté a comme point de départ la famille, soit le groupe comprenant un couple marié et ses enfants. Mais les nécessités sociologiques et biologiques ne coïncident pas toujours. Il ne s'ensuit pas que la famille biologique doive exister en tant qu'unité différenciée du reste de l'agrégat social dont elle fait partie. Dans un état comme celui du communisme sexuel décrit par Morgan et son école, la famille serait complètement absorbée par un groupe plus vaste. Il s'agit donc ici non d'un argument a priori mais d'un fait empirique.

Avant d'entreprendre l'enquête imposée par ces considérations, nous commencerons par étudier de près le concept de la famille, tel qu'il se manifeste dans notre civilisation. Le premier point à noter est son caractère bilatéral, impliqué du reste dans sa définition C'est-à-dire que la famille, comme unité sociale, inclut les deux parents, et secondairement la parenté des deux côtés. Cette dualité apparaît nettement dans les devoirs des parents envers les enfants, ainsi que dans les lois d'héritage qui reconnaissent les liens aussi bien du côté maternel que paternel. On verra plus loin combien il est nécessaire de souligner cet aspect. Sur un point important, cependant, le principe bilatéral est abandonné : notre famille est patronymique; la femme et les enfants prennent le nom du père. Ainsi le mari, ses fils et leurs descendants mâles, du côté masculin, ainsi que leurs femmes et filles non mariées, sont différenciés par leur nom du reste de leur parenté. Ceci est à coup sûr un détail d'importance comparative 
plutôt que pratique. Il n'en irait pas de même si tous les porteurs d'un même nom, quoique parents éloignés, formaient une unité sociale définie, se détachant du reste de la communauté et si, par exemple lors de l'héritage des biens, le cousin le plus lointain mais portant le même nom avait le pas sur de très proches parents, tels que des fils de sœurs ou des filles mariées. Puisque nous n'attribuons pas une importance absolue et exclusive à la branche paternelle de la famille, à part la transmission du nom, nous croyons pouvoir définir à bon droit notre famille comme essentiellement bilatérale.

La question qui importe donc avant tout est celle de savoir si les tribus primitives admettent aussi le principe bilatéral dans leur conception de la famille. Si tel est le cas, nous serons fondés à croire qu'elles reconnaissent la famille en tant qu'unité sociale, quelles que soient les autres unités qui peuvent coexister avec elle. Considérons tout d'abord les preuves de l'existence du principe bilatéral et nous passerons ensuite en revue quelques-uns des facteurs les plus importants qui tendent à façonner la vie de famille primitive, la faisant souvent dévier fortement de la norme établie en Europe occidentale.

Chapitre 4

\section{a. La parenté bilatérale.}

\section{$\underline{\text { Retour à la table des matières }}$}

Les systèmes de nomenclature de parenté dont nous avons parlé nous fournissent un témoignage objectif et irréfutable de l'universalité de l'unité familiale. Comme Morgan lui-même l'a fait remarquer dans une controverse avec Mac Lennan, chaque tribu a des termes de parenté, aussi bien pour la lignée maternelle que paternelle, et reconnaît à cet égard la parenté bilatérale. Mais limiter cette notion à des questions de nomenclature serait mal comprendre l'étendue du problème. Dans la grande majorité des tribus primitives, les deux lignées sont prises en considération, non seulement par le vocabulaire, mais aussi par le droit coutumier, certaines fonctions étant communément associées avec certains types de parenté. Ainsi les Hopi, au contraire de nous, sont matronymiques puisque ce qui correspond à notre nom de famille est transmis à l'enfant par la mère; mais le nom personnel est invariablement conféré par une femme de la parenté du père et il évoque symboliquement ce dernier groupe. Chez les Hidatsa, matronymiques également, une série d'usages sociaux témoignent de l'importance de la parenté paternelle. Ainsi les objets sacrés passent de père en fils; les parents du père ont droit en toute occasion à des présents; on donne souvent des surnoms, non à cause des particularités d'un individu, mais à cause de celles d'un parent de son père, et c'est encore un parent du père qui préside au rituel funéraire. D'autre part, les Thonga patronymiques assignent une place importante à l'oncle 
maternel. Ici le frère de la mère a droit à une partie du prix de la fiancée et joue un rôle considérable dans la vie cérémonielle de son neveu, alors que le fils de la sœur peut s'approprier les aliments de son oncle et réclamer sa part d'héritage, recevant parfois l'une des veuves. Pour ne citer qu'un seul autre cas, les insulaires du détroit de Torrès, patronymiques encore, autorisent un homme ou un jeune garçon à prendre les biens les plus précieux de son oncle maternel, et le neveu obéit immédiatement lorsque le frère de la mère lui donne l'ordre de ne pas combattre.

Les règles de parenté sont un sujet immense qui ne saurait être épuisé en un paragraphe. Du point de vue où nous le considérons, il importe simplement de noter que les parentés maternelle et paternelle sont toutes deux reconnues et que le fait de prendre le nom de famille du père ou de la mère n'empêche nullement des relations sociales importantes avec l'autre branche de la parenté.

Toutes ces règles sociales que nous venons d'énumérer impliquent la reconnaissance tacite du père et de la mère. Ceux-ci sont aussi naturellement reconnus en vertu du lien sentimental qui les relie aux enfants et parce que mari et femme, ainsi que leurs plus jeunes enfants tout au moins, forment une unité économique et industrielle. Nous ne saurions répéter avec assez de fréquence et de force que le mariage n'est basé sur des considérations sexuelles que dans une mesure limitée. Son motif essentiel, du moins en ce qui concerne les mâles, est précisément la fondation d'un agrégat économique se suffisant à lui-même. Un Kai ne se marie pas en raison de désirs qu'il pourrait facilement satisfaire en dehors du mariage et sans assumer de responsabilités, mais parce qu'il a besoin d'une femme qui lui fasse sa poterie et lui prépare ses repas, qui lui fabrique ses filets et entretienne ses plantations; en retour de quoi, il subvient aux besoins du ménage en gibier et poisson et construit la maison. Dans le Queensland, le père fournit à sa famille le gros gibier et le poisson, la mère apporte les ignames, les grains, les fruits, les mollusques et les petits poissons. Il existe dans l'Australie centrale une division analogue du travail et, d'après les faits réunis par Malinowski, il est évident qu'à travers tout ce continent la famille individuelle constitue sur cette base une unité nettement différenciée. Ainsi que le remarque Radcliffe-Brown à propos des Kariera de l'Australie occidentale, " l'unité de vie sociale dans la tribu des Kariera était la famille, se composant d'un homme, de sa ou de ses femmes et de leurs enfants. Une telle unité pouvait se déplacer indépendamment des faits et gestes des autres familles du groupe local. Dans le campement, chaque famille avait sa propre hutte ou abri avec son propre feu, ses aliments particuliers, cuits et consommés en commun. L'homme fournissait la viande et la femme les légumes ainsi que les petits mammifères et les lézards ». Les relations économiques et industrielles des conjoints Ewé sont réglées également d'une façon très précise. Il est du devoir du mari d'apporter la viande et le poisson, tandis que la femme doit fournir le sel; tous deux se partagent les travaux d'horticulture; la femme file et l'homme tisse ou raccommode les vêtements.

On pourrait multiplier à l'infini de tels exemples. Nous appuyant donc sur le caractère universel de ce phénomène, nous en pouvons conclure que, quelles que 
soient les autres institutions sociales, la famille individuelle est une unité sociale omniprésente. Il n'importe pas que les relations maritales soient de nature permanente ou temporaire, qu'il s'agisse de polygamie, de polyandrie ou d'un état de licence sexuelle, que les conditions se compliquent du fait de l'adjonction de membres non compris dans notre cercle de famille; il est un fait qui dépasse tous les autres, à savoir que le mari, la femme et les enfants en bas âge constituent une unité séparée du reste de la communauté. Dans la société primitive, il est en général d'usage qu'un individu soit redevable de certains devoirs envers toute une classe d'individus, desquels il attend à son tour d'être traité de telle ou telle manière. Mais, ainsi que RadcliffeBrown le fait admirablement remarquer dans l'article cité, il ne se produit aucune confusion quant à l'intensité de l'obligation qui varie selon le degré de parenté. Quoiqu'on puisse désigner une douzaine d'oncles paternels et autant de cousins du père du même nom que le père, c'est surtout le père réel ou putatif qui fournit aux femmes et aux enfants leur subsistance, tout au moins la part qui en incombe à l'homme dans la société primitive. Ainsi nous avons vu que, quoique le cousin d'un homme au Nième degré puisse être appelé son frère, c'est le véritable frère qui hérite par lévirat de la veuve et c'est seulement en l'absence de frères qu'il s'y substitue un parent plus éloigné.

La seule échappatoire possible aux partisans de la théorie selon laquelle la famille bilatérale serait inconnue des primitifs, est d'abandonner les phénomènes patents des sociétés contemporaines inférieures pour se réfugier dans un passé obscur. Nous avons déjà mentionné l'hypothèse selon laquelle la famille serait partout un produit relativement tardif de l'évolution sociale et nous y reviendrons encore. Qu'il nous suffise pour l'instant d'établir l'universalité actuelle du concept de la famille bilatérale.

Mais cette assertion n'implique pas que la vie de famille doive nécessairement revêtir partout la même forme que chez nous. Du reste les changements fondamentaux que l'évolution économique a introduits depuis un siècle dans notre vie de famille et la modification du statut de la femme rendraient absurde une telle supposition. Des coutumes comme la polygynie et la polyandrie affectent nécessairement le caractère de la famille, ainsi que nous l'avons déjà noté. J'indiquerai brièvement quelques-uns des facteurs qui modifient de façon vitale l'existence familiale primitive et, pour ce faire, je serai obligé de rappeler des faits étudiés dans les chapitres précédents, d'anticiper sur d'autres que je traiterai dans la suite plus à fond et enfin de remettre à plus tard certaines considérations.

Chapitre 4

\section{b. Élasticité du lien familial.}


Même dans les cultures supérieures, la famille individuelle est une unité fort peu stable. Lorsque les filles se marient et vont vivre avec leurs époux, ou lorsque les fils fondent des ménages indépendants, l'intimité du lien qui les unit aux parents est presque inévitablement affaiblie, sinon tout à fait détruite. Parmi les peuples primitifs, qui ne font que bien rarement intervenir des scrupules religieux à l'endroit du divorce, il faut tenir compte de toutes sortes de forces destructrices. Un esprit de pure bravade incitait parfois les Crow à congédier publiquement leurs femmes lors des fêtes, afin de faire montre de force d'âme, et il importait alors apparemment fort peu qu'elles eussent des enfants ou non; ceux-ci naturellement accompagnaient leur mère. Dans cette même tribu, deux organisations militaires rivales se plaisaient au début du printemps à des vols de femmes dont la seule règle était qu'il fallait, pour qu'un homme pût enlever une femme, qu'il eût entretenu avec elle des relations intimes. Dans ce cas, le mari ne pouvait protester et tout essai de recourir à la force eût porté une atteinte irrémédiable à son prestige social.

En dépit de tels usages, il importe de ne pas confondre la fréquence véritable du divorce avec ses possibilités théoriques. Même parmi les Crow, une femme chaste n'y était pas exposée, et d'autres ne pouvaient être enlevées que si elles étaient mariées à un membre des deux sociétés et qu'elles eussent été les maîtresses de membres de l'autre organisation. Ces règles permettaient la répudiation publique et sans conditions des femmes; pourtant, en pratique, un homme y regardait à deux fois avant de se séparer d'une épouse vertueuse et active. Ici comme partout les considérations pratiques interfèrent dans une large mesure avec l'exercice d'une prérogative abstraite. Un Kirghiz qui a payé une somme énorme pour sa femme la renvoie très rarement, en dépit de l'autorisation musulmane. De même, un mari kai est difficilement disposé à se séparer définitivement de sa femme, même lorsqu'elle s'enfuit. Il a acheté ses services et demande que sa personne lui soit restituée ou du moins l'équivalent de la somme payée; si l'amant ne fournit pas les biens requis, la parenté de la femme la retourne à son mari et acheteur. Même lorsque les deux sexes sont également libres de se séparer, il ne s'ensuit pas que le divorce soit plus fréquent. Dans la région de l'Amazone, une Indienne Uitoto n'est jamais blâmée parce qu'elle abandonne son mari; une démarche aussi peu naturelle ne peut être attribuée en effet qu'à de mauvais traitements flagrants puisque, selon les conditions existantes, une femme qu'aucun homme ne protège plus va au-devant de la mort. D'autre part, la réprobation encourue par un mari qui se débarrasse de sa femme, sans motif suffisant, exerce aussi une action préventive.

La présence ou l'absence d'enfants, quoiqu'on n'en tienne parfois pas compte, exerce d'ordinaire une influence profonde sur la stabilité du mariage. La stérilité est presque partout une raison suffisante à la répudiation de l'épouse. Par contre, ainsi que chez nous, les enfants tendent à unir les parents. C'est ce que nous montre clairement le cas des Esquimaux. Avant la naissance des enfants, le divorce est admis par la société groenlandaise sur la moindre provocation; le capitaine Hohm a rencontré une femme d'à peine vingt ans qui venait de quitter son sixième mari. Mais, après la 
naissance des enfants, les relations conjugales deviennent plus stables et, dans les unions de longue durée, il se forme un attachement loyal et même une profonde affection. Cette dernière remarque s'applique aussi aux Tchouktche et s'accorde avec mes propres observations sur les Crow, quoique ces deux tribus fassent preuve d'une tendance marquée à trancher les liens du mariage pour des motifs futiles.

Quoiqu'il soit difficile de généraliser, nous pouvons affirmer que, alors que la famille primitive n'est pas une unité aussi instable que la facilité théorique du divorce pourrait le donner à croire, elle est, cependant, dans l'ensemble beaucoup moins cohérente que la nôtre, bien que son instabilité diminue de façon marquée après toutes les premières années du mariage.

Chapitre 4

\section{d. Résidence patrilocale et matrilocale.}

\section{$\underline{\text { Retour à la table des matières }}$}

Les règles de résidence exercent une influence incalculable sur la vie de famille, car la proximité physique affecte non seulement l'amour sexuel, mais aussi tous les sentiments humains. Un couple de jeunes mariés peut s'établir pour un temps plus ou moins long dans la famille du mari ou de la femme, ou bien ils peuvent fonder un ménage indépendant. Afin de nous faire une idée provisoire des conditions résultant de ces facteurs, commençons par comparer la vie de famille de deux tribus, les Hupa qui sont patrilocaux et les Pueblo matrilocaux.

Chez les Hupa, un homme choisissait sa femme dans un autre village, mais l'emmenait normalement dans son village à lui. Un homme et ses fils ainsi que leurs femmes et filles non mariées étaient donc réunis dans une même localité, tandis que les filles, en se mariant, les quittaient pour suivre leurs maris. Ainsi l'homme naissait, vivait et mourait au même endroit, alors que la femme passait la plus grande partie de sa vie loin de son village natal. Cette règle de résidence fixa un groupement unilatéral de parenté; il y eut ségrégation locale des individus apparentés par le père. Néanmoins, cette lignée paternelle, quoique se distinguant objectivement de l'autre parenté par une résidence commune, n'était pas spécifiquement reconnue par les Hupa comme une unité distincte. il pouvait ainsi arriver qu'un homme, dans l'impossibilité de payer le prix de la fiancée, fût obligé d'aller en service dans le village de son beau-père et les enfants de ce mariage appartenaient alors à la famille de la femme. Dans ce cas, la dérogation aux règles habituelles patriarcales entraînait une association de parenté toute différente de celle qui se fût produite normalement, ce qui aurait été impossible si les Hupa avaient reconnu la ségrégation locale de la famille patrilinéaire, non 
comme une simple coutume, mais comme le reflet d'un principe abstrait soulignant uniformément le côté paternel dans les questions de parenté.

Les règles matrilocales des Indiens Pueblo établissent une autre ligne de parenté, combinée avec la possession de la maison par les femmes. Le noyau de la maison se compose de la grand-mère maternelle, de la mère et des tantes maternelles, des frères célibataires de la mère et de tous les enfants des femmes adultes. Le mari vit dans la maison de sa femme, mais sans droits de résidence bien établis; en cas de divorce, il doit s'en aller et retourner à sa maison natale que possède sa mère ou l'une de ses sœurs. Un homme, même après son mariage, continue donc à regarder la maison de sa mère, plutôt que celle de sa femme, comme la sienne propre. De cette manière, dans toute famille, les enfants sont en rapports constants avec leurs oncles maternels dont le statut est admirablement décrit par Mlle Freire-Marreco : « Ils prennent tout naturellement place au repas, amènent des invités, se comportant comme les seigneurs et maîtres de la maison, et cela quoiqu'ils ne contribuent nullement (s'ils sont mariés) à l'entretien matériel du ménage, car ils ont à fournir en maïs, viande et bois les maisons de leurs propres femmes. " Ils gardent leurs instruments et ornements sous le toit maternel et peuvent adresser des reproches aux enfants de leurs sœurs dont ils sont en droit d'exiger obéissance.

Nous voyons donc que les règles de résidence peuvent mettre l'accent sur l'une des deux branches de la famille et interférer à cet égard avec la symétrie bilatérale des relations de famille. Parmi les Hupa, l'oncle maternel, vivant normalement dans un autre village, n'est pas en mesure d'influer sur l'éducation des enfants qui porteront inévitablement l'empreinte d'influences patrilinéaires. Dans le ménage pueblo, le père continue à former une entité économique avec sa femme et sa progéniture, mais son autorité sur les enfants est déférée, en partie tout au moins, aux hommes appartenant à la maison où lui-même vit en visiteur. Le statut du mari est ainsi profondément modifié. Il est évident qu'un homme occupe une situation toute différente sous son propre toit, en ce qui concerne son statut de père aussi bien que de mari. Dans une communauté matrilocale, il ne peut pas être le maître absolu de la personne de sa femme; lorsqu'il s'élève quelque dispute, il doit tenir compte de sa belle-famille; il peut même être expulsé. En outre la résidence matrilocale limite naturellement la polygynie, sauf sous la forme du sororat.

Néanmoins, nous ne devons pas oublier que la résidence patrilocale ou matrilocale représente les extrêmes d'une série de conditions intermédiaires variables. Les Indiens Pueblo sont matrilocaux au sens plein du terme; il n'en va pas de même de la plupart des peuplades classées sous cette rubrique. Nous trouvons très communément qu'un mari commence sa vie conjugale avec ses beaux-parents, remplissant à tous égards les fonctions d'un domestique, mais qu'il fonde plus tard, souvent après la naissance des enfants, un ménage indépendant. C'est le cas des Hidatsa, des Ovambo de l'Afrique du Sud, des Khasi d'Assam. L'influence de la parenté maternelle est alors moins prononcée que dans les associations matrilocales permanentes. Enfin, il peut ne 
pas exister de règle définie, le jeune couple vivant soit indépendant, soit avec les parents de la femme.

Afin de comprendre ces phénomènes, il nous faut résolument refuser de nous contenter de rubriques classificatoires telles que «matrilocal » et " patrilocal » et étudier les faits en tenant compte aussi bien des usages qui s'y rattachent que des statistiques. Par exemple, dans le nord de la Sibérie, le prétendant Koriak ou Youkaghir fait un temps de service pour gagner sa femme, mais le premier l'emmène dans sa propre famille, alors que le second réside chez ses beaux-parents. Il semblerait donc qu'il existe à cet égard une distinction bien tranchée et infranchissable entre ces tribus. Cependant, la ligne de démarcation n'est pas aussi nette que le simple énoncé des faits le donnerait à croire. Dans 11 mariages sur 181 chez les Koriak, le gendre s'établit chez son beau-père lorsque sa femme n'a pas de frère et que le père de celleci l'invite à prendre la place d'un fils. D'autre part, chez les Youkaghir, il arrive à l'occasion que deux ménages échangent leurs filles en gardant leurs fils. En outre, le père d'une fiancée qui a déjà des fils, peut se désister de ses droits sur la résidence du gendre si celui-ci est fils unique. Finalement il est de coutume chez les Youkaghir que le fils cadet reste avec ses parents. Nos informations sur les Esquimaux sont également fort instructives. Ici nous trouvons une différenciation locale : les Groenlandais sont patrilocaux, les tribus du Labrador et de la terre de Baffin observent la résidence matrilocale, au début du mariage tout au moins. Même chez les Esquimaux du centre, on a observé des variations, quelques communautés suivant la règle patrilocale, d'autres étant matrilocales. Comme c'est la première de ces pratiques qui prédomine en général, c'est elle que nous pouvons considérer comme plus fondamentalement esquimau. On se demandera alors comment se sont produites les déviations et l'on retombera à nouveau sur l'explication suivante : lorsqu'un prétendant n'a pas les moyens de fournir pour sa femme une compensation adéquate, il devient naturellement le serviteur de sa belle-famille. Holm nous apprend plus spécialement que, lorsqu'il y a beaucoup de fils dans un ménage, seuls les aînés emmènent leurs femmes sous le toit paternel, tandis que les cadets vont résider chez les beaux-parents.

En substituant ainsi toute cette série d'observations à des désignations générales pouvant nous induire en erreur, nous pouvons mieux nous faire une idée des contingences qui ont transformé les coutumes matrimoniales d'une tribu. Un appauvrissement général aura pu rendre matrilocaux les Hupa, adeptes autrefois de la patrilocalité, en faisant une règle d'un pis-aller redevenu maintenant une anomalie. Kroeber a démontré aussi que, même dans les communautés généralement matrilocales de la zone Pueblo, des déviations significatives peuvent se développer. Certaines incompatibilités entre un homme et les parents de sa femme peuvent parfois pousser les maris Zuñi à braver la règle traditionnelle et à emmener leur femme chez leur mère à eux. Il arrive que cette migration ait d'étranges conséquences, car, si le mari n'a pas de sœurs ou, s'il en a et que celles-ci n'aient pas d'enfants, la maison, qui est toujours la propriété des femmes, passera ainsi en la possession d'une autre famille. 
En résumé, le mode de résidence exerce toujours une influence profonde sur la vie de famille, à cause de la filiation de parenté, du statut du mari et des relations unissant les enfants à leur parenté maternelle ou paternelle, selon que la règle est matrilocale ou patrilocale. Mais ne perdons pas de vue que la situation peut changer du tout au tout selon que la règle est observée pendant toute la vie conjugale ou pour une période limitée au début du mariage; qu'elle est absolue ou souffre des modifications; enfin selon la nature des motifs opérant dans les cas d'anomalies et susceptibles de devenir habituels sous certaines conditions.

Chapitre 4

\section{d. Division du travail selon les sexes.}

$\underline{\text { Retour à la table des matières }}$

C'est un lieu commun de la sociologie moderne que de répéter qu'une indépendance économique croissante a transformé le statut de la femme et par là le caractère de la famille. Il n'est par suite pas étonnant que la division du travail selon les sexes nuance de colorations diverses la vie de famine des civilisations plus simples. Cette division est, dans une large mesure, conventionnelle, c'est-à-dire qu'elle ne se rattache nullement à des caractéristiques physiologiques des deux sexes, ainsi qu'on peut le prouver en comparant les différentes règles en vigueur chez des tribus parfois voisines. Ainsi les Bantou du sud ne laissent pas approcher les femmes de leurs troupeaux, alors que chez les Hottentots, ce sont elles qui traient régulièrement les vaches.

Contrairement à l'opinion très répandue que la femme primitive est invariablement un souffre-douleur, nous trouvons en général une répartition assez équitable des tâches. Dans les tribus de chasseurs, la femme ajoute au gros gibier tué par le mari l'apport des racines sauvages, des baies et des coquillages. A un niveau de civilisation plus élevé, l'homme reste chasseur, tandis que la femme fait un pas décisif en avant en ne se contentant pas de cueillir, mais en plantant et en récoltant. Dans le langage ethnographique technique, on distingue d'ordinaire entre l'agriculture, ou labour à la charrue, et l'horticulture, ou préparation du sol au moyen d'outils plus rudimentaires. Edouard Hahn, l'auteur de cette classification, a démontré que la charrue était un outil presque uniquement masculin et qu'elle appartenait au même complexe culturel que la houe.

Il a réfuté avec succès l'erreur courante selon laquelle tous les paysans auraient commencé par être des bergers, en avançant comme preuve les nombreux peuples américains ou océaniens qui cultivaient le sol avec des houes ou des bâtons à fouiller et qui ignoraient totalement l'élevage à l'époque de leur découverte. Évidemment ces 
peuples ont passé directement de la chasse à la culture du sol; l'élevage n'a donc précédé nécessairement que l'agriculture à la charrue. Hahn a par malheur quelque peu terni l'éclat de son exposé en insistant sur le fait que les formes primitives de la culture du sol auraient toujours incombé aux femmes. Si cette affirmation est vraie en beaucoup de parties de l'Afrique, de la Mélanésie et de l'Amérique, les exemples contraires sont presque aussi nombreux. La charrue était inconnue de l'Amérique précolombienne et pourtant, du nord de l'Arizona au Pérou, les hommes cultivaient le sol ainsi que tel est le cas dans presque toute la Polynésie. Les exceptions abondent même en Afrique et surtout parmi les tribus du Soudan. Ainsi Nachtigall a observé que, chez les Bornou, les deux sexes se partagent également les travaux à la houe; ailleurs la culture du sol est exclusivement du domaine masculin. Thurnwald a tenté de rectifier les généralisations de Hahn en associant aux femmes non la houe mais le bâton à fouiller. Toutefois, cette version doit, elle aussi, être écartée, car, dans toute la Polynésie, ce sont plutôt les hommes qui se servent de cet instrument, sans parler d'importantes exceptions en Amérique. Voici la seule affirmation que nous puissions nous permettre : selon toute probabilité les femmes furent les premières à cultiver la terre, car, dans les communautés de chasseurs, ce sont toujours elles qui doivent fournir l'alimentation végétale. Toutefois bien des variations locales se sont produites au cours des temps dans la répartition du travail entre les sexes. Il n'est donc pas possible d'adopter à la lettre les théories de Hahn et de Thurnwald.

Alors que les femmes ont créé l'horticulture, la domestication d'animaux tels que le bœuf fut sans aucun doute l'œuvre des hommes. En général les soins des troupeaux sont aux mains des hommes qui ont parfois gardé jalousement leurs prérogatives. Nous avons déjà mentionné que les Bantou interdisent à leurs femmes d'entrer dans les enclos à bétail ; les Toda vont jusqu'à leur défendre de cuire des aliments où le lait entre comme ingrédient. Parallèlement à la domestication du bœuf, les hommes ont aussi développé l'usage de la charrue en agriculture, diminuant ainsi l'importance relative de la contribution féminine à l'alimentation.

Outre les activités économiques, il nous faut considérer les occupations industrielles. Ici, ainsi que nous l'avons déjà dit, nous sommes en présence de variations considérables à l'intérieur d'une même région. Pour la plupart des indigènes de l'Amérique du Nord, par exemple, la préparation des peaux est considérée comme une tâche nettement féminine, mais dans le Sud-Ouest c'est le travail des hommes. Chez les Hopi du nord de l'Arizona, ce sont les hommes qui filent et qui tissent, alors que chez leurs voisins, les Navaho, ce sont là devoirs féminins. En ce qui concerne la céramique primitive, nous sommes redevables à Laufer d'un travail de généralisation comparable à celui de Hahn : partout où la poterie se fait à la main, elle est l'œuvre des femmes; là où on a recours au tour, ce sont les hommes qui la fabriquent. Il est vrai qu'au Ruanda, chez les Pygmées, les hommes confectionnent les récipients en terre et Desplagnes a observé dans des tribus soudanaises que les deux sexes faisaient les pots. Mais de tels cas sont tout à fait exceptionnels. 
La condition de la femme dans la société est un problème si important que nous lui consacrerons un chapitre spécial. Qu'il nous suffise de noter ici que chaque peuple a ses conceptions traditionnelles sur les devoirs masculins et féminins et que des variations à cet égard ne manqueront pas de se répercuter sur la vie de famille. Un Thonga polygyne devient un parasite entretenu par ses épouses-jardinières; une femme Kirghiz vaque aux soins de la maison, tandis que l'homme non seulement s'occupe des troupeaux, mais aussi cherche le bois, travaille la terre et fabrique les ustensiles de ménage; la femme Toda n'a guère d'autre tâche que d'écraser le grain et de le passer au tamis, de nettoyer la hutte et de décorer les vêtements. Le statut théorique de la femme n'est pas ici en jeu puisqu'il atteint sans doute son point le plus bas chez les Kirghiz musulmans et est probablement moins favorable chez les Toda que parmi les Thonga; il s'agit uniquement d'une répartition conventionnelle des travaux selon les sexes. Laufer a insisté à plusieurs reprises sur ce que, à un niveau plus élevé de civilisation, « les formes de la vie de famille chinoise et les relations psychiques de ses membres » diffèrent radicalement des nôtres en ce que la femme n'a rien à diriger à la cuisine et n'en approche même pas; la cuisine est toujours éloignée du centre de la maison et ne sert jamais de point de ralliement.

\section{Chapitre 4}

\section{e. Ségrégation des célibataires.}

\section{$\underline{\text { Retour à la table des matières }}$}

Parmi les coutumes qui donnent à la vie de famille primitive une empreinte toute différente de la nôtre, il y a la ségrégation au sein de la communauté des jeunes gens et des jeunes filles non mariés, se produisant fréquemment lors de l'adolescence, mais parfois aussi plus tôt. Ainsi chez les Dravidiens de l'Inde méridionale, les garçons ne dorment pas avec leurs parents, mais dans un club séparé, et les jeunes filles ont leur propre dortoir surveillé par une patronne. Chaque agglomération kariera est divisée en camp des gens mariés et camp des célibataires, ce dernier incluant aussi les veufs, et le premier les femmes non mariées et les veuves. Nous avons déjà décrit la coutume des Massaï en vertu de laquelle les jeunes guerriers célibataires résident dans un kraal spécial avec les toutes jeunes filles, tandis que les hommes mariés ont leurs propres établissements.

Ces usages nous induisent à introduire un principe de division de la communauté sur la base de l'âge, qu'on y discerne ou non une ségrégation sexuelle. Les problèmes de distribution et d'interprétation qui s'y rattachent seront discutés en détail. Qu'il nous suffise pour l'instant d'attirer l'attention sur la rupture inévitable ou tout au moins sur l'affaiblissement sérieux des liens de famille dans les cas où les adolescents sont séparés de leurs parents par ces institutions établies. 
Chapitre 4

\section{f. Ségrégation sexuelle.}

\section{$\underline{\text { Retour à la table des matières }}$}

Il est un phénomène qui affecte de façon encore plus frappante la famille en tant qu'unité sociale, c'est la séparation du mari et de la femme, soit par la ségrégation des hommes dans leur club particulier, soit par l'exclusion des femmes des formes de l'activité publique qui absorbent spécialement l'attention des hommes. C'est encore là un sujet auquel nous reviendrons dans la suite, mais il nous en faut citer ici par anticipation un ou deux exemples caractéristiques.

Chez les Hupa, les femmes vivaient dans la maison de famille où les maris venaient prendre leurs repas avant et après leurs tâches quotidiennes. Vers le soir, les hommes se retiraient au sudatorium qui ne leur servait pas seulement de bains turcs mais aussi de club et de dortoir. L'isolement où sont tenues les femmes est encore plus marqué en certaines parties de la Mélanésie, aux îles Banks entre autres, où les hommes et les adolescents eux-mêmes non seulement dorment, mais aussi mangent à part, et où on recherche de bonne heure la qualité de membre du club afin de réduire d'autant la période d'ignominie où il faut se nourrir avec les femmes. Mentionnons enfin la coutume presque universelle en Australie de défendre aux femmes la célébration des rites sacrés sur lesquels se concentre la préoccupation des hommes aux heures de gravité.

Chapitre 4

\section{g. Adoption.}

\section{$\underline{\text { Retour à la table des matières }}$}

La constitution réelle de la famille peut être altérée par une fiction légale selon laquelle les parents élèvent les enfants d'un autre couple comme s'il s'agissait de leurs propres. En bien des cas, ces enfants sont apparentés à leurs parents adoptifs, mais ce n'est nullement une condition indispensable. Un motif courant d'adoption est le manque de descendance. Ainsi un couple de Tchouktche sans rejeton adoptera l'enfant, de 
préférence le fils, d'un parent qui deviendra leur héritier principal. Les relations sentimentales se rapprochent alors de très près de celles qui se basent sur les liens naturels. Chez les Indiens Crow, il est fréquent qu'on adopte l'enfant d'un frère ou d'une sœur et on observe alors une certaine exagération des démonstrations d'affection, comme pour compenser par là tout ce que ce lien peut avoir d'artificiel. Mais nulle part probablement l'adoption n'est aussi fréquente que sur lîle Murray, dans le détroit de Torrès oriental, où les enfants, sans raison manifeste, sont adoptés même avant leur naissance et élevés entièrement par leurs parents adoptifs, ignorant souvent l'identité de leurs parents véritables jusqu'à leur maturité ou même jusqu'à la mort.

Chapitre 4

\section{h. Résumé.}

Quoique le caractère de la famille primitive soit notablement modifié par les usages que nous venons d'esquisser, ces altérations n'entament pas le principe bilatéral. Un homme peut passer la majeure partie de ses heures de travail ou de repos loin de sa femme, il n'en restera pas moins lié à elle par l'intérêt commun pour les enfants qui sont réellement ou putativement les siens, et par leur collaboration économique ou industrielle ; des considérations analogues s'appliquent à tous les autres états mentionnés, tout en affectant parfois de façon étrange, du point de vue des Blancs, la dynamique de la vie de famille. Mais la famille bilatérale n'en demeure pas moins une unité absolument universelle de la société humaine.

Chapitre 4

\section{Références.}

$\underline{\text { Retour à la table des matières }}$

1) LOWIE, 1917 (a) : 40 et suiv., 51. JUNOD : 1, 44, 212, 226, 253, 262. Reports, V : 144 et suiv. KEYSSER : 45, 85. ROTH, $1906:$ 6. SPENCER et GILLEN, 1899 : 18. MALINOWSKI : 158167. RADCLIFFE-BROWN : 147. SPIETH : 191.

2) LOWIE, 1912 : 223; id., 1913 : 169. KEYSSER : 86. WHIFFEN : 165. THALBITZER : 65, 72. BOGORAS : 596.

3) GODDARD, 1903 56-58. FREIRE-MARRECO : 269-287. LOWIE, 1917 (a) : 46. SCHINZ 304, 311. GURDON : 78, JOCHELSON, 1908 : 744; id., 1910 : 92. CRANZ : 1, 215 et suiv. BOAS, 1888 : 579. THAL-BITZER :59. MURDOCH :410. KROEBER, 1917 (a) :105.

4) HAHN, RIVERS, 1906 567. LAUVER, 1917 : 148, id., in Amer. Anth., 1918 : 89. RADLOFF 462.

5) BADEN-POWELL 172. BROWN : 147. 
6) GODDARD, 1903 57. RIVERS, 1914 (b) : 1, 63.

7) BOGORAS :556. LOWIE, 1912 :219. Reports, VI : 64,177. 


\section{5 RÈGLES DE PARENTÉ}

Dans ma démonstration du caractère bilatéral de la famille, j'ai insisté sur les relations sociales régnant entre un individu et sa parenté, aussi bien du côté maternel que paternel. En fait, la loi primitive va plus loin et établit des fonctions définies pour chaque degré de parenté, fût-elle de sang ou par alliance. Dans notre société, il n'existe pas de conduite fixe à adopter envers un oncle maternel, le fils d'une sœur ou le mari de la sœur du père. Dans les communautés primitives, au contraire, un comportement spécial peut être rigoureusement déterminé pour chaque forme possible de parenté. Du point de vue de l'individu, cela revient à dire que les membres de sa tribu se rangent en diverses catégories dont chacune implique un certain ensemble de règles sociales à observer. Il est tenu de rendre service à un individu de telle classe; avec un membre d'une autre, il peut plaisanter et prendre des libertés; avec les représentants d'une troisième catégorie, il ne doit avoir affaire qu'à travers des intermédiaires, et ainsi de suite. La proximité de la parenté peut importer ou non; d'ordinaire, ainsi que l'explique Radcliffe-Brown, un Kariera adopte le même type de conduite envers des parents proches ou éloignés, désignés par le même terme, mais l'intensité de l'obligation est plus marquée quand il s'agit du proche parent. Comme cet auteur le fait encore remarquer, un indigène peut être fort embarrassé de savoir comment traiter un étranger qui ne se range sous aucune des rubriques établies. Ce qui se produit alors le plus fréquemment, c'est que, par fiction légale ou par manage avec un membre de 
la communauté, le nouvel arrivé finit par jouir d'un statut défini. Ainsi, dans un mythe des Indiens des Prairies, un adolescent rencontre une jeune fille étrangère qu'il adopte comme sœur ; elle devient automatiquement la sœur de ses frères, à qui il est en conséquence défendu de l'épouser. Dans la vie réelle où ces conséquences sont observées jusqu'au bout, l'étrangère serait devenue la fille des parents de son frère adoptif, la belle-sœur de leurs femmes. Bref, elle aurait été adoptée par le cercle de famille tout entier et ses relations sociales s'en seraient trouvées régularisées.

C'est surtout le caractère de ces règles de parenté qui différencie la vie de famille dans les diverses tribus et qui oppose la vie primitive à la nôtre; ces règles sont si nombreuses et si variées qu'il est nécessaire de leur consacrer un chapitre particulier. Elles impliquent à la fois des devoirs envers les parents et des droits à leur appui et à leurs biens, ainsi que la prohibition stricte de certains rapports ou la sanction de relations extraordinaires. L'étude systématique de cette matière, qui doit beaucoup à l'énergie de Rivers, en est encore à ses débuts; pourtant on peut déjà tirer du vaste amas des faits quelques conclusions valables.

Chapitre 5

\section{A. Parenté maternelle et paternelle.}

\section{$\underline{\text { Retour à la table des matières }}$}

Certaines relations particulières avec la parenté du côté maternel ou paternel affectent profondément les rapports sociaux. Par exemple, lorsque la jeune sœur d'une mère est susceptible, grâce au sororat, de devenir la seconde femme du père, l'attitude initiale des enfants à son égard est nécessairement influencée par cette perspective et vice versa. Similairement, le lévirat crée entre le frère du père et le fils de celui-ci un lien auquel rien dans notre société ne saurait être comparé. Il existe des obligations se rattachant à d'autres Parents maternels ou paternels qui peuvent aussi avoir une portée considérable.

Les ethnologues rangent sous le terme d'avunculat les coutumes réglant d'une manière particulière les relations d'un neveu avec son oncle maternel. Ces relations ont souvent un caractère humoristique auquel nous reviendrons plus tard. Considérées d'un point de vue plus sérieux, elles impliquent une autorité extraordinaire de l'oncle et l'héritage des biens, non par le fils, mais par le fils de la sœur. On a déjà fourni quelques exemples de ce phénomène; le prétendant kai doit obtenir le consentement de l'oncle maternel de la jeune fille et, lors de la transmission des veuves Thonga, nous avons vu que le neveu peut recevoir la femme du frère de sa mère. Des phénomènes de cet ordre sont fréquents. Parmi les Winnebago du Wisconsin, comme nous l'apprend Radin, le neveu sert de domestique au frère de la mère et l'accompagnait autrefois au combat comme une sorte d'écuyer. D'autre part, il lui était permis de 
prendre avec cet oncle des libertés qui lui étaient expressément défendues avec l'oncle paternel, par exemple s'approprier des objets lui appartenant. Chez les Omaha, l'oncle maternel avait un contrôle absolu sur les enfants restés orphelins et, même du vivant des parents, faisait preuve d'un zèle de père pour les défendre ou les venger d'un outrage auquel ils auraient été exposés. Dans les ménages hopi, c'est le frère de la mère qui met les enfants au courant des traditions et de leurs devoirs cérémoniels. Sur la côte de la Colombie britannique, le neveu va vivre avec son oncle maternel, travaille pour lui, épouse sa fille et devient son héritier légal. On signale en Océanie des coutumes analogues. L'insulaire du détroit de Torrès obéissait plus volontiers au frère de sa mère qu'à son père et c'était le frère aîné de sa mère qui le patronnait lors de son initiation à l'état d'homme. Ici, comme chez les Winnebago, un homme pouvait prendre tout ce qui appartenait à son oncle maternel. Ce dernier trait est appliqué rigoureusement chez les Fidjiens. En Afrique, l'avunculat est également assez répandu. Chez les Makondé de l'Afrique orientale, le consentement de l'oncle maternel est indispensable au mariage de la nièce et c'est lui qui reçoit une part du prix de la fiancée, alors que c'est le fils de la sœur qui a droit à hériter des biens d'un défunt. En Guinée supérieure, les Anglo-Ewé reconnaissent à l'oncle maternel une autorité sur les enfants supérieure à celle du père. Le neveu qui est l'héritier présomptif de l'oncle, doit travailler pour celui-ci et l'accompagner dans ses voyages. Chez les Nandi de l'Afrique orientale, probablement hamitiques, le frère de la mère donne son autorisation à la circoncision de son neveu; il a droit à recevoir une vache lorsque celui-ci rentre d'une incursion couronnée de succès, et rien n'est plus terrible que d'encourir sa colère.

Il est évident que quelques-unes de ces ressemblances ne sauraient être attribuées au hasard, mais l'avunculat est si étroitement uni à certains autres phénomènes de la vie primitive que nous n'avons pas encore décrits, que nous remettons à plus tard une discussion sur sa signification. Ce qui nous intéresse ici, c'est le passage inattendu de ce que nous considérons comme l'autorité paternelle sur les épaules de l'oncle maternel, et la tendance également remarquable de celui-ci à faire de son neveu un compagnon et un héritier. Il est superflu de prouver que la vie de famille assume dans de telles conditions un aspect tout différent.

Il est des règles également définies se rattachant à la parenté paternelle. Nous devons à Rivers divers renseignements sur l'importance extraordinaire que revêt, dans les communautés océaniennes, la sœur du père. Dans les îles Banks, non seulement la tante paternelle est entourée d'un respect plus grand même que celui qu'on manifeste à la mère, mais c'est elle aussi qui arrange le mariage des neveux et qui peut opposer son veto à un projet d'alliance. La sœur du père peut s'approprier les biens du neveu pour autant que celui-ci les tient de son frère à elle, et le neveu peut à son tour prendre tout ce qu'elle possède. Le pouvoir de la tante sur les mariages est fréquent en Mélanésie et s'étend jusqu'aux Tonga de Polynésie où cette parente est encore plus vénérée que le père et l'oncle maternel. Chez les Thonga de l'Afrique du Sud, nous ne savons pas grand-chose sur la tante paternelle sinon qu'on la traite avec beaucoup de respect, alors que parmi les Toda c'est elle qui, à la naissance d'une fille, lui choisit un 
nom. Chez les Hopi, cette fonction est régulièrement assumée pour tous les enfants par la grand-mère paternelle. Les Crow ont toute une série d'usages se rapportant aux frères et sœurs du père et à ses parents plus éloignés ou même à des étrangers figurant comme ses frères et sœurs. Tous ces personnages sont entourés de respect; il ne viendrait à personne l'idée de marcher devant eux, quels que soient leur âge et leur sexe. C'étaient eux surtout qui recevaient les cadeaux lorsque le neveu revenait avec le butin pris à l'ennemi et, en retour, le frère du père chantait les louanges du jeune homme par tout le campement. Les titres honorifiques étaient empruntés à un parent paternel et les surnoms avaient également pour origine les exploits de celui-ci plutôt que ceux de l'individu lui-même. Outre ce qui précède, les Hidatsa, apparentés aux Crow, observaient une règle selon laquelle les funérailles d'un défunt devaient être conduites par la parenté paternelle.

Si vitalement que ces règles relatives au comportement des parents unis par le sang affectent les relations sociales, elles sont éclipsées par d'autres préceptes également significatifs et de nature plus frappante encore, se rapportant aux parents par alliance et notamment aux beaux-parents.

Chapitre 5

\section{b. Tabous des beaux-parents.}

\section{$\underline{\text { Retour à la table des matières }}$}

Chez des peuples primitifs en grand nombre, le mari, ou plus rarement la femme, adopte des relations sociales tout à fait particulières vis-à-vis de ses beaux-parents. Il se produit soit une rupture complète de tous les rapports directs avec eux ou l'un d'entre eux, soit une limitation de ces rapports, modifiable parfois après quelques années de mariage ou par l'accomplissement d'un acte spécial. Une série de faits concrets nous feront comprendre de quoi il s'agit.

Une bru Youkaghir ne doit pas regarder en face son beau-père, ni le frère de son mari, pas plus qu'il n'est permis à un gendre de regarder en face son beau-père ou sa belle-mère. En donnant des ordres à son gendre qui, on s'en souvient, vit dans la famille de sa femme, le beau-père lui parle d'une façon impersonnelle ou par allusions. La bru ne doit pas découvrir son corps devant le père de son mari, ni le beaupère devant la bru, et une règle analogue est observée entre la femme et le frère aîné du mari. D'autres tribus sibériennes ont des coutumes presque identiques. Aucune femme mariée Ostiak ne peut apparaître devant son beau-père, ni le mari devant sa belle-mère, avant la naissance des enfants; s'ils se rencontrent par hasard, ils se cachent la face et la femme continue à porter le visage couvert sa vie durant. Avant le paiement intégral du prix de la fiancée, le jeune homme qui vient rendre visite à sa bien-aimée doit tourner le dos à son beau-père, s'il le rencontre accidentellement, ou 
se couvrir la face. L'épouse Bouriate ne s'adresse jamais à ses beaux-parents en les appelant par leurs noms; son visage ne doit jamais être découvert en présence du père de son mari ou d'un parent plus âgé; elle ne doit pas changer de vêtement en leur présence, ni dormir sous la même tente, ni les croiser sur le chemin ou voyager dans le même véhicule. Eux, de leur côté, ne doivent pas non plus s'habiller et se déshabiller devant elle ou employer un langage obscène en sa présence; ils sont tenus de faire connaître leur approche à l'avance afin qu'elle puisse rectifier sa tenue. Les Kalmouk observent des restrictions analogues, ainsi que les Turcs de l'Altaï et les Kirghiz. La femme Kirghiz ne regarde jamais en face le père de son mari ou un parent plus âgé et ne doit pas prononcer leurs noms, même s'ils sont synonymes d'objets courants. Il existe une anecdote à propos d'une femme Kirghiz à qui il était défendu d'employer les mots : agneau, loup, eau et jonc, parce qu'ils faisaient partie de noms d'hommes de sa belle-famille. Une fois qu'il lui fallut avertir son mari qu'un loup emportait un agneau à travers les joncs de l'autre côté de l'eau, elle dut avoir recours à la périphrase suivante : «Regarde là-bas, le hurlant emporte le bêlant à travers les murmurants de l'autre côté de l'étincelante. »

Il est tout à fait compréhensible et explicable parla diffusion qu'un groupe de tribus occupant en Sibérie des territoires adjacents et en relations culturelles intimes aient en commun un même ensemble de tabous. Mais comment nous expliqueronsnous l'existence d'usages analogues dans une région aussi distante que Ceylan? Là, chez les Vedda, le mari ne peut pas approcher, ni à plus forte raison toucher la mère de sa femme. S'il la rencontre dans la jungle, il doit sortir de la piste; il n'entrera pas sous un abri où elle est seule et n'acceptera aucune nourriture de ses mains, si ce n'est par l'entremise d'un tiers; il ne lui parlera pas davantage, sauf en public. Des tabous analogues sont en vigueur entre un homme et la femme de son fils. En outre, tous ces parents par alliance évitent de s'appeler réciproquement par leurs noms qu'ils remplacent par des désignations de parenté.

Ce type de tabou s'étend sur toutes les régions du globe. Dans les îles Banks, en Mélanésie, le gendre ne doit pas prononcer le nom de ses beaux-parents, ni la bru celui de son beau-père; tout mot entrant en composition dans leurs noms est banni du vocabulaire et remplacé par des périphrases. En outre, jamais un homme ne plaisantera avec le père de sa femme; il ne s'adressera pas à lui familièrement et ne touchera à aucun objet placé au-dessus de la tête de celui-ci. Quant à sa belle-mère, si elle est dans le voisinage de la porte, il n'entrera pas dans la maison, et s'il la rencontre dans la brousse, il quittera le sentier et fera un détour pour l'éviter. Elle, de son côté, ne doit pas passer près d'un arbre où il a grimpé, ni boire de l'eau dans un bambou qu'il a porté et, si elle a besoin de son aide, elle doit la demander par l'intermédiaire de sa fille. Les infractions à ces règles s'expient par des paiements en argent. Des prescriptions analogues ont trait aux relations entre bru et beau-père.

Si nous passons à d'autres régions de l'Océanie, nous verrons que les Bukaua du golfe Huon en Nouvelle-Guinée ne permettent pas aux beaux-parents et aux gendres de se toucher, ni de prononcer réciproquement leurs noms. Le beau-père doit se 
couvrir la face s'il mange devant son beau-fils, car, si ce dernier peut apercevoir sa bouche ouverte, le beau-père en ressent de la honte et s'enfuit dans la forêt. S'il désire faire don au jeune homme de bétel, il ne le lui mettra pas dans la main, mais le déposera sur une feuille. A l'ouest du détroit de Torrès, ni l'homme ni la femme ne mentionnent jamais les noms des beaux-parents; l'homme ne s'entretient pas avec eux si ce n'est par l'intermédiaire de la femme ou dans certains cas de nécessité absolue; il parlera alors très peu et à voix basse; une femme ne présente jamais les aliments directement à son beau-père, mais seulement par l'entremise de sa belle-mère.

Dans toute l'Australie, on se heurte à des restrictions semblables. La belle-mère et le gendre doivent s'éviter réciproquement, et, dans certaines localités, elle n'est pas censée entendre le nom de son beau-fils. Un contact accidentel entre eux peut entraîner le divorce du jeune couple, de même qu'une infraction à ce tabou a pour conséquence l'exil du jeune homme; en certaines tribus, on lui applique même la peine de mort. Parmi les Kariera, pour éviter que l'homme ne regarde la mère de sa femme, on interpose entre eux une hutte ou des broussailles, mais le tabou perd de sa virulence à mesure que les années s'écoulent. Dans cette tribu et probablement dans toute l'Australie, les femmes ne sont pas tenues d'éviter leur beau-père.

Le parallélisme n'est pas moins frappant en Afrique. Un Zoulou se couvre le visage de son bouclier s'il croise accidentellement sa belle-mère, crache ce qu'il a dans la bouche si elle passe pendant qu'il mange et ne prononce jamais son nom. Avec le temps cependant, la sévérité de ces règles s'atténue considérablement. Des coutumes analogues sont signalées par Frazer dans diverses tribus bantoues ainsi que chez les Massaï. Elles ne semblent pas répandues dans l'Afrique occidentale, quoique chez les Matsé-Ewé il soit défendu aux beaux-parents de manger dans la maison de leur gendre et vice versa; enfreindre cette règle est considéré comme honteux et de nature à empêcher la naissance des enfants.

En Amérique les exemples abondent. Un Crow ne doit pas parler aux parents de sa femme ni inversement. Le tabou du beau-père est moins strict, mais celui de la belle-mère est rigoureusement observé. Le gendre ne peut parler à sa belle-mère que par l'intermédiaire de sa femme et il ne doit pas prononcer son nom ni aucun mot qui en fasse partie. Ce dernier tabou implique l'usage des circonlocutions, comme chez les Assiniboin où, pour désigner un couteau, on dit « quelque chose de tranchant » et pour le cheval « l'animal sur lequel nous montons ». Prononcer le nom du beau-père passe pour une incorrection. Il était possible autrefois de supprimer le tabou en présentant solennellement aux parents un cadeau d'importance, chez les Hidatsa et les Mandan en leur apportant le scalp d'un ennemi. En outre, une belle-mère Crow peut, à la mort de sa fille, passer par-dessus toutes les restrictions et s'adresser à son gendre comme si c'était son fils. Il n'existe pas de tabou correspondant entre la femme et le beau-père chez les Crow, mais on le trouve par contre chez les Dakota, les Assiniboin, les Kiowa, les Arapaho et les Omaha simultanément avec le tabou entre gendre et belle-mère. 
Contrairement à ce qu'on pourrait supposer, partout où nous recueillons des indices sur l'aspect subjectif de ces tabous, on ne relève aucune marque d'hostilité entre les parents taboués, mais uniquement des démonstrations de respect mutuel. Un interprète Hidatsa avait épousé une femme de la tribu des Arikara où l'on n'observe pas de telles coutumes. Aussi, un jour que ses amis Hidatsa virent sa belle-mère lui parler, ils s'écrièrent avec horreur : «A quoi pense ta belle-mère, Joe? Elle semble n'avoir aucun respect pour toi. »

D'après l'extrême diffusion du tabou des beaux-parents, on pourrait conjecturer l'universalité absolue de ces règles et s'expliquer leur absence en quelques points par le fait qu'elles auraient échappé aux observateurs. Une étude, par ailleurs excellente, peu fort bien ne renfermer nulle indication sur cette coutume, sans que cette omission soit en elle-même décisive. En Amérique du Nord pourtant, où l'on poursuit depuis plusieurs années l'étude systématique de la distribution des tabous, il semble prouvé que des tribus en nombre considérable ignorent totalement cette coutume. Parmi celles-ci, citons les Nutka, les Arikara, les Zuñi, les Hopi, divers Shoshoni des plateaux et certaines peuplades californiennes. Par ailleurs, le tabou fleurit chez les Indiens des Prairies et se rencontre au sud-est, parmi les nomades du sud-ouest, chez quelques tribus californiennes et dans le nord de la Colombie britannique.

Le premier problème dont il faille chercher la solution est celui de la distribution géographique. Comment des coutumes si semblables peuvent-elles être si largement répandues? On ne saurait mettre en doute qu'il ne s'agisse en une certaine mesure de diffusion. Les faits sibériens, par exemple, accusent une telle ressemblance dans le détail qu'on ne peut se l'expliquer que par une origine commune. De même, lorsque, parmi tous les peuples Shoshoni de la zone des plateaux, seuls ceux qui sont en contact avec les tribus des Prairies septentrionales observent le tabou, nous pouvons en conclure à bon droit qu'ils l'ont emprunté à leurs voisins. Mais comment expliquer l'analogie du tabou du nom entre les Kirghiz de l'Asie centrale et les Assiniboin du Montana qui tous font usage de périphrases pour remplacer des mots d'usage quotidien? Pour ma part, j'y vois un exemple intéressant de ressemblances qui se sont développées indépendamment. En effet, alors que le tabou lui-même est identique, il s'applique à des individus différents. Chez les Kirghiz, c'est la bru qui doit user d'une périphrase se substituant au nom du beau-père, alors que chez les Assiniboin c'est au gendre qu'il est défendu de prononcer le nom de son beau-père. Mais jusqu'où vont les possibilités d'emprunt? En vertu de quel mécanisme des coutumes de ce type se sont-elles disséminées? Ce ne peut être un chef conquérant qui les a imposées aux tribus soumises, ainsi que les Chinois furent obligés à porter la tresse. En outre, ces usages sont de nature trop intime et secrète pour qu'un visiteur de hasard les comprenne; celui-ci pourrait tout au plus remarquer que quelques-uns de ses hôtes s'évitent mutuellement, mais sans qu'il connût rien de leur parenté et il lui serait impossible de rapporter chez lui une telle coutume. A coup sûr, s'il observait qu'une femme évite un parent par alliance, ce n'est pas ce spectacle qui le pousserait à créer à son retour un nouveau précepte selon lequel un homme devrait fuir son beau-père ou sa belle-mère, ou faire passer le tabou du nom à un tout autre ordre de parenté. Cette 
notion ne peut pas être comprise sans un contact intime; si elle n'est pas rejetée en tant qu'idiosyncrasie de barbares, elle sera adoptée telle qu'on l'aura trouvée.

Le plus souvent, je n'en doute pas, elle s'est propagée par des mariages intertribaux. Un étranger qui a grandi dans l'idée que la mère de la femme ou pour une femme le mari de sa fille, doivent être tabous et que toute autre conduite est ridicule sinon outrageante, pourra, par son influence personnelle, modifier les usages de la tribu. Par l'imitation de son exemple, le tabou s'introduira dans une tribu qui ne le connaissait pas auparavant, mais cette imitation ne saurait donner naissance à ce qui, dans l'esprit concret du primitif, représente une pratique toute différente. C'est pourquoi je rejette sans hésitation, en dehors de toutes considérations géographiques ou autres se basant sur la signification probable de la coutume sibérienne (page 105), l'hypothèse selon laquelle le tabou des Kirghiz et celui des Indiens des Prairies se rattachent historiquement l'un à l'autre.

La question est quelque peu différente si nous comparons les faits en Afrique et en Australie ou en Mélanésie et en Amérique, car ici les prescriptions s'appliquent aux mêmes individus et présentent réellement des analogies étonnantes. A propos de quelques détails frappants, cependant, il nous faut nous rappeler que tout tabou rigoureux est tenu de prendre certaines formes : par exemple, un homme doit faire un détour s'il rencontre par hasard sa belle-mère. De même, si l'habitant des îles Banks et le Crow ne doivent pas employer certains mots entrant en composition dans le nom de l'un ou l'autre de ses beaux-parents, la périphrase s'impose inévitablement. Les coïncidences particulières ne sont donc pas aussi concluantes qu'il semblerait tout d'abord. En d'autres termes, il s'agit de savoir si le simple fait que des individus unis par le même degré de parenté s'évitent est nécessairement un indice d'origine commune. La réponse dépendra partiellement de l'interprétation donnée à la coutume, car, si celle-ci dérive de conditions se répétant dans diverses localités, l'hypothèse de l'origine indépendante en sera d'autant renforcée. Écartant ce point pour l'instant, je suis d'avis que le mécanisme des emprunts de cette particularité aussi bien que son absence démontrée en certaines régions rendent improbable sa diffusion dans des continents sans rapport les uns avec les autres. Nous avons vu que, pour que cette coutume se transmette, il faut une extraordinaire intimité, telle qu'on ne pourrait en supposer, même s'il y a eu contact de quelque nature entre l'Océanie et l'Afrique ou entre la Mélanésie et l'Amérique.

Il est en vérité bien peu vraisemblable qu'une coutume qui n'a Pas pu traverser la Californie, ni passer des Hidatsa chez leurs voisins les Arikara, et des Omaha aux Pawnie tout proches, aient franchi des océans. J'en conclus donc que la diffusion a joué un certain rôle dans l'histoire du tabou des beaux-parents, mais qu'il convient d'assigner à celui-ci un développement indépendant dans les diverses zones géographiques.

Passant ensuite au problème de l'interprétation, nous trouvons la théorie avancée par Frazer qui, en dépit de toutes ses déficiences, ne saurait être rejetée totalement. 
Frazer fait remarquer avec raison que les règles de tabou ne sont pas limitées aux beaux-parents, mais peuvent aussi s'appliquer aux frères et sœurs, par exemple. Selon lui, toutes les prescriptions de cette nature doivent être classées dans la même catégorie et s'expliquent comme " des précautions destinées à écarter la tentation de rapports sexuels entre individus dont l'union répugnerait pour une raison quelconque au sens moral de la communauté ». Cette explication, il le reconnaît, rencontre une objection sérieuse (quoique pour Frazer non insurmontable) dans le fait de l'existence de tabous restreignant les relations sociales entre individus de même sexe. Ces tabous, selon Frazer, seraient une extension secondaire des règles appliquées primitivement aux individus de sexe différent. 111 nous est impossible de discuter les hypothèses de Frazer sans anticiper sur ce que nous avions à dire à propos d'autres coutumes de parenté. On peut faire cependant une remarque. Même si nous accordons que les tabous imposent des restrictions aux individus dont l'union serait incestueuse, il ne s'ensuit pas que leur but est d'empêcher les relations sexuelles entre lesdits individus. On peut admettre la corrélation empirique mais rejeter l'interprétation causale. Nous reviendrons plus tard à cette question.

Une explication du tabou des beaux-parents dont la tendance est analogue, mais dont les motifs sont tirés de la psychanalyse, a été avancée par Sigmund Freud, dont les opinions méritent d'être prises en considération, non seulement en elles-mêmes, mais parce qu'elles sont typiques de toute interprétation psychologique des faits sociaux. Freud applique aux phénomènes relatifs au tabou de la belle-mère le concept de l'ambivalence, c'est-à-dire qu'il les conçoit comme basés sur un mélange d'affection et d'animosité. Quelques-uns des motifs hostiles sont, à son avis, manifestes : la répugnance de la mère à livrer sa fille, ses soupçons à l'endroit de l'étranger, son désir de conserver la situation prédominante qu'elle s'est créée dans sa propre maison, l'aversion marquée de l'homme envers toute sujétion à une volonté étrangère, sa jalousie à l'égard de tous ceux qui l'ont précédé dans l'affection de sa femme, son refus à voir ses illusions sexuelles troublées par la personnalité d'une femme qui, bien que lui rappelant son épouse par plus d'un côté, est dépourvue cependant du charme de la jeunesse et de la beauté. A tous ces éléments, Freud joint des facteurs latents de nature spécifiquement psychanalytique. La vie psycho-sexuelle de la belle-mère qui avance en âge est prématurément interrompue; elle ne peut plus satisfaire ses besoins émotionnels qu'en s'assimilant à la psyché de ses enfants. Cependant l'identification avec sa fille conduit facilement à l'amour pour l'homme que sa fille aime et, dans le conflit de sentiments qui en résulte, l'élément hostile et sadique de la passion est dirigé vers le gendre afin de supprimer plus certainement l'émotion incestueuse tabou. Le gendre, d'autre part, est mû par des impulsions analogues. Il a vaincu, comme le veut la théorie psychanalytique, sa passion infantile pour sa mère et a substitué à son image une femme étrangère. Avec l'apparition de la belle-mère se produit chez lui une certaine tendance à retomber dans son état juvénile, ce qui choque sa notion de l'inceste, puisque celle-ci exige que l'origine incestueuse du choix de l'épouse reste cantonnée dans son subconscient. Comme la personnalité de la belle-mère, contrairement à celle de sa mère, ne lui est pas familière depuis son enfance et que son image ne dort pas intacte depuis bien des années dans son subconscient, il lui est relative- 
ment facile de vaincre la tentation qui demeure néanmoins une réalité. La raison du tabou serait donc d'empêcher les rapports incestueux.

La première objection qu'il faille présenter à l'explication de Freud est relative à un fait bien courant. Il nous dépeint l'état subjectif de la belle-mère et du gendre avec les couleurs éclatantes qui rehaussent notre vie de famille moderne mais qui font complètement défaut aux relations entre primitifs. Pour en revenir à ce que nous avons dit plus haut, chaque fois que nous avons quelque indice sur les sentiments véritables des individus dans ces questions de mariage, il n'y a jamais trace d'hostilité, le respect est invariablement la note dominante dans les sentiments mutuels, se différenciant ainsi totalement de ceux qu'on attribue au gendre et à la belle-mère dans les caricatures comiques.

Ce que nous savons des autres tabous aura naturellement la même répercussion sur les opinions de Freud que sur celles de Frazer. Mais la recherche des motifs psychologiques de Freud souffre d'un défaut fatal, commun à toutes les interprétations psychologiques des faits culturels. Les phénomènes psychologiques que Freud invoque sont notoirement valables partout; ils doivent donc agir avec une force égale dans les communautés les plus diverses, sauf lorsqu'il y a différence raciale. Or il se trouve que le tabou des beaux-parents a une distribution des plus capricieuses. En Amérique du Nord, les Navaho évitent la belle-mère, tandis que leurs voisins, les Hopi, considèrent cette coutume comme un trait particulier aux Navaho. Les Lemhi Shoshoni tiennent pour fou un homme qui parle à la mère de sa femme; chez les Comanche, qui non seulement sont de la même famille mais qui parlent la même langue, j'ai rencontré une grande difficulté à faire saisir à mes informateurs la notion de ce tabou. Nous faut-il admettre que les sentiments infantiles des Hopi diffèrent considérablement de ceux des Navaho? Ou que les réactions émotionnelles des belles-mères Hopi et Navaho sont de nature divergente? Afin d'ajuster la théorie aux faits, nous devrions supposer deux types psychologiques distincts, l'un pour les habitants des îles Banks, les Australiens, les Zoulou, les Navaho et les Lemhi Shoshoni, l'autre pour les Hopi, les Comanche et les Arikara. Mais ce serait absurde. La théorie psychanalytique est insoutenable parce que c'est une théorie psychologique et parce que les phénomènes psychologiques auxquels nous avons affaire ne sont pas simples, mais déterminés socialement. Si une belle-mère Navaho évite son gendre, ce n'est pas parce qu'elle ressent individuellement telle ou telle émotion à son égard, mais parce qu'elle fait partie d'une société qui prohibe les relations entre certains individus unis par alliance. Tout conflit qui pourrait se produire dans sa situation s'élèverait non entre deux catégories de sentiments opposés à l'endroit de son gendre, mais entre quelque réaction personnelle et la notion d'obéissance aveugle à la norme sociale reconnue. La question ici est de savoir pourquoi les normes sociales diffèrent et l'on n'y peut répondre qu'en comparant ces différences sociales avec d'autres différences d'ordre social également. 
A cet égard, l'interprétation de Tylor respecte beaucoup mieux les exigences scientifiques modernes, car il fait dériver le tabou des beaux-parents des règles de résidence, c'est-à-dire qu'il déduit l'une de l'autre deux particularités sociologiques. Dans le mariage matrilocal, nous explique-t-il, le mari est considéré comme un intrus et n'est tout simplement pas reconnu; un traitement correspondant est réservé à la femme dans les communautés patrilocales, tandis que les deux variétés de tabous se rencontrent dans l'état intermédiaire de résidence matrilocale temporaire. Sur la base de statistiques, Tylor nous démontre que la résidence matrilocale et le tabou de la belle-mère se combinent en fait beaucoup plus fréquemment que tel serait le cas si ces deux phénomènes étaient mutuellement indépendants, et établit ainsi entre eux une relation causale.

L'effort de Tylor pour introduire une méthode rigoureuse dans les enquêtes ethnologiques doit être tenu pour un exploit de tout premier ordre et l'œuvre d'un pionnier héroïque, mais ses résultats statistiques ne sauraient être acceptés sans discrimination. Malheureusement les faits sur lesquels il base ses conclusions n'ont jamais été publiés, et il est par conséquent impossible de les vérifier d'une façon satisfaisante. Il apparaît toutefois clairement que Tylor prend pour des unités statistiques les diverses tribus chez lesquelles on a relevé des phénomènes de cet ordre. Mais c'est là une méthode discutable. Par exemple, il peut exister une douzaine de cas de tabou de la belle-fille parmi les tribus sibériennes. Si, dans chacune d'elles, il s'est produit une association indépendante de résidence patrilocale et de tabou, les probabilités de l'existence d'un lien causal en seront d'autant plus grandes. Mais nous ne pouvons admettre comme prouvée cette indépendance. Il est fort possible que la règle du tabou fût une particularité de certaines tribus ancestrales en Sibérie déjà, en d'autres termes qu'elle n'a été créée qu'une fois, du moins en ce qui concerne chaque famille linguistique, et n'a fait que se conserver dans les diverses branches en lesquelles la tribu ancestrale s'est fractionnée. Il est plus que probable, en outre, que cette hypothèse s'applique à une famille seulement, et que les tribus des autres familles n'ont fait que lui emprunter la coutume avec tout ce qui s'y rapporte. Voilà donc qui modifie complètement la logique des statistiques. Supposons pour simplifier que nous ayons un seul cas contradictoire à opposer aux douze exemples sibériens; prenons quelque tribu américaine, comme les Pieds Noirs patrilocaux chez qui on ne trouve pas la règle sibérienne et qui observent, selon Tylor, un tabou matrilocal. Si les tribus sibériennes représentent douze cas de tabou surgissant indépendamment d'une résidence patrilocale antérieure, il nous faudrait admettre tout de suite qu'elles contrebalancent largement le témoignage négatif des Pieds Noirs. Mais s'il y a eu connexion historique, les douze tribus sibériennes se réduisent, pour ce qui nous intéresse, à une seule unité, et nous avons un cas en harmonie avec la théorie de Tylor et un cas qui la contredit; en d'autres termes, la théorie reste non prouvée.

Tout ceci doit nous montrer que les résultats de Tylor, bien que basés sur des principes mathématiques, peuvent néanmoins être dépourvus de valeur à cause de prémisses erronées, en l'occurrence d'un mauvais choix des unités. En effet, si nous prenons de plus grandes unités, les comparaisons ne sont guère à l'appui de son 
hypothèse. La Sibérie connaît, il est vrai, la résidence patrilocale et le tabou entre bru et beau-père, mais ni en Australie, ni en Afrique du Sud, les faits ne s'harmonisent avec cette théorie. Dans ces deux régions, la femme va habiter dans la famille de son mari et pourtant non seulement on n'y retrouve pas la prohibition en vigueur en Sibérie, mais c'est la règle inverse qui y règne, c'est-à-dire le tabou du gendre qui, d'après l'hypothèse de Tylor, ne devrait se développer que dans une communauté matrilocale. En outre, il est plutôt remarquable que, dans des tribus aussi nettement matrilocales que les Zuñi et les Hopi, le tabou censé y correspondre fasse défaut, alors que des peuplades temporairement matrilocales seulement, comme les Hidatsa, l'observent rigoureusement, ainsi que le faisaient les Pieds Noirs patrilocaux. Chez ces derniers, par contre, on ne retrouve pas le tabou de la belle-fille qui, selon la théorie de Tylor toujours, devrait y être en honneur.

L'interprétation de Tylor présente encore une autre erreur. Elle explique pourquoi les beaux-parents et le gendre s'évitent mutuellement dans les tribus matrilocales et pourquoi les beaux-parents et la bru font de même chez les patrilocaux. Mais, en beaucoup de cas signalés, le tabou est limité à des individus de sexe opposé. Quelques peuplades australiennes ne mettent aucun obstacle aux relations entre beau-père et gendre, pas plus qu'en Sibérie entre belle-mère et bru; et ces cas sont trop fréquents pour qu'on puisse les ignorer. Néanmoins et malgré toutes ces restrictions, je crois que la théorie de Tylor n'est pas dépourvue d'une certaine valeur. La résidence n'est pas, cela va sans dire, la seule cause du tabou des beaux-parents, mais elle peut avoir contribué à le déterminer ou même l'avoir causé en l'absence de facteurs agissant en sens contraire. Une analyse beaucoup plus poussée qu'on ne l'a fait jusqu'ici pourrait, dans une certaine mesure, modifier la conclusion négative à laquelle nous sommes arrivés plus haut. Une simple indication suffira : les tribus sibériennes patrilocales que nous avons énumérées observent le tabou du père du mari ou d'un parent plus âgé que le mari. Mais les Youkaghir, qui sont matrilocaux, non seulement ont aussi le tabou des Sibériens patrilocaux, fait explicable par les effets de la diffusion, mais se différencient nettement de tous les autres en y ajoutant le tabou du gendre. Il est difficile d'échapper à la conclusion qu'il existe une connexion entre le mode de résidence unique et le tabou unique.

Frazer émet l'idée judicieuse que le tabou des beaux-parents ne représente qu'une variété d'un genre plus vaste; nous attendrons donc pour l'interpréter d'avoir passé en revue les prohibitions portant sur d'autres degrés de parenté.

Chapitre 5

\section{c. Autres tabous.}


Dans l'impossibilité de traiter de tous les tabous de parenté qui nous ont été signalés, nous nous contenterons de porter notre attention sur quelques tribus qui ont fait l'objet d'études minutieuses, et sur les restrictions relatives aux rapports sociaux imposées dans chacun de ces cas. On imaginera sans peine les conséquences de ces prescriptions sur la vie de famille quotidienne.

Il est probable qu'aucun peuple n'est allé plus loin à cet égard que les Youkaghir. Outre les prohibitions du gendre et de la bru que nous retrouvons ailleurs, nous relevons chez eux des règles interdisant toute conversation entre le frère aîné ou le cousin et la femme d'un frère cadet ou d'un cousin; entre le frère aîné (cousin) et la femme du fils de son frère cadet (cousin); entre le frère aîné et la femme du fils de sa sœur cadette; entre le frère aîné et le mari d'une jeune sœur. Les Parents du même sang sont aussi soumis à des restrictions : les frères ne peuvent pas parler librement entre eux, ni les frères avec les sœurs, pas davantage que les sœurs entre elles et cette règle s'étend aux cousins. Aucun d'entre eux ne doit découvrir son corps en présence de l'un des autres, même si ce dernier appartient au même sexe, et ils doivent s'abstenir de toute allusion relative à la cohabitation. Ils ne doivent pas s'adresser l'un à l'autre directement, ni se regarder, ni s'appeler réciproquement par leurs noms, mais user d'un terme de parenté.

Dans les îles Andaman, le tabou des beaux-parents n'a pas été mentionné, mais il y est remplacé par une règle analogue. Jusqu'à la maturité, l'homme fait preuve d'une grande timidité en présence de la femme de son frère cadet, ne communiquant jamais avec elle que par intermédiaire. Cette prohibition ne s'étend pas à la femme du fils aîné.

Les Mélanésiens prescrivent une certaine retenue dans la conduite envers certains parents. La prohibition de relations sociales entre frères et sœurs est assez répandue. Dans l'île Lepers, ils ne se voient plus après le tatouage de puberté de la jeune fille, car celle-ci quitte la maison pour aller vivre chez son oncle maternel. Lorsqu'ils se rencontrent par la suite, la sœur s'écarte du chemin et tous deux évitent de se regarder. Ils ne se parlent pas et ne prononcent jamais réciproquement leurs noms. Si le frère désire voir ses neveux, la sœur mariée quittera la maison avant de l'y laisser pénétrer. Les Fidjiens observaient autrefois des règles analogues - les frères et les sœurs ne devaient ni se parler, ni jouer et manger ensemble et ne communiquaient que par des intermédiaires; aucun d'entre eux ne prononçait le nom de l'autre. En NouvelleIrlande où les cousins croisés de sexe opposé sont classés dans la même catégorie que les frères et sœurs, ils sont soumis à une même restriction. Dans les îles Banks, le tabou du frère ou de la sœur s'atténue jusqu'à n'être plus qu'une défense de plaisanter ensemble, mais on signale dans ces régions une multitude d'autres prescriptions. Il est défendu au père et au fils de manger en commun. Un homme ne doit pas toucher à un objet suspendu au-dessus de la tête de son oncle maternel, mais attendre pour le prendre que celui-ci soit parti. La sœur du père est non seulement traitée avec un respect tout particulier, ainsi que nous l'avons dit plus haut, mais on ne prononcera pas son nom, pas plus qu'elle ne dira celui de son neveu. Un mari peut appeler sa 
femme par son nom, mais il est considéré comme fort irrespectueux de sa part à elle de lui rendre la pareille (voir page 109). Les beaux-frères évitent de s'appeler par leurs noms et les belles-sœurs font de même; il est répréhensible pour tous les individus unis par ces liens de parenté d'échanger des plaisanteries.

Prenons un dernier exemple en Amérique. Ainsi qu'il arrive fréquemment dans les tribus primitives, les Crow englobent dans le tabou du gendre non seulement les frères et sœurs du beau-père et de la belle-mère, mais aussi les grand-mères de la femme; et les frères du mari sont également soumis au tabou du mari. Il existe donc une tendance à étendre cette restriction aux individus auxquels la femme s'adresse comme à ses parents ainsi qu'à ceux qui ont le même statut que le mari. En outre, l'homme doit éviter l'épouse du frère de sa femme et vice versa. Les relations entre beaux-frères sont de nature particulière : ils sont grands amis et peuvent se permettre de joyeuses plaisanteries, mais il ne saurait être question entre eux d'allusions obscènes de nature personnelle et, si l'un transgresse cette règle, l'autre peut le frapper. Un homme ne doit pas avoir une conversation obscène en présence de son beau-frère, même s'il s'entretient avec d'autres individus. Il n'est pas absolument défendu de se parler entre frères et sœurs, mais, après la puberté, ils ne doivent plus rester seuls ensemble. Un frère qui entrera dans une tente et n'y trouvera que sa sœur, en sortira immédiatement. Un interprète Crow me reprocha une fois l'indécence des Blancs, assez éhontés pour s'adresser librement à leurs propres sœurs et qui osent trouver la morale des Indiens relâchée. Enfin la femme et le mari évitent généralement de s'appeler par leurs noms sauf après plusieurs années de mariage.

Chapitre 5

\section{d. Privilèges de familiarité.}

\section{$\underline{\text { Retour à la table des matières }}$}

A l'opposé des tabous, nous trouvons une série de coutumes qui permettent et prescrivent presque diverses formes de familiarité, souvent réciproques, consistant à s'adresser des railleries ou des grossièretés entre individus unis par un degré de parenté déterminé. Ces usages, ainsi que bien d'autres se rattachant aux notions de parenté, n'ont été étudiés systématiquement que depuis quelques années, et il n'est guère douteux qu'il reste encore beaucoup d'observations à recueillir dans ce domaine.

A l'heure actuelle, la plupart de nos informations nous viennent de la Mélanésie et de l'Amérique du Nord.

L'avunculat, en dépit de son caractère plus sérieux, demande aussi à être envisagé sous cet angle. Aux îles Fidji, non seulement le fils de la sœur agit avec les biens de 
son oncle comme avec ceux de son père, mais va jusqu'à tuer les cochons de l'oncle et détruire ses plantations dans le seul but de s'amuser. D'autre part, lorsque le neveu s'approprie des objets lors d'un échange entre la tribu de son oncle et un autre groupe, il est rossé par les fils de l'oncle; ceux-ci peuvent le battre, mais non rentrer dans leurs biens. Rappelons que chez les Indiens Winnebago également, le neveu peut prendre ce qui appartient à son oncle maternel. Chez les Thonga le fils de la sœur se nourrit aux frais de son oncle et chez les Hottentots l'oncle peut s'attribuer la propriété de son neveu si elle a souffert quelque dommage, alors que le neveu s'indemnise lui-même sur les biens intacts de son oncle.

L'un des cas les plus nets de privilèges de familiarité est celui entre beau-frère et belle-sœur chez les Pieds Noirs et les Crow; l'obscénité de leurs conversations est illimitée. Quant à leur comportement, j'ai vu un Crow d'un certain âge s'amuser à lutter avec la sœur de sa femme d'une manière qui, à nos yeux, dépasserait les bornes de la décence; et pourtant ils n'étaient nullement gênés par la présence de l'épouse et du fils adulte.

La " parenté de plaisanteries » chez les Crow et les Hidatsa est d'un caractère tout différent. Les participants ne sont pas nécessairement apparentés les uns aux autres, mais simplement enfants de pères appartenant aux mêmes divisions tribales (voir page 117). A l'intérieur de ce groupe, la licence est complète; chacun des membres peut jouer des farces à l'autre en jouissant d'une complète impunité. Mais ces relations ont un but plus sérieux : ces camarades de jeux sont aussi des censeurs éthiques; si un homme a transgressé de quelque manière le code tribal de morale et d'étiquette, un de ses camarades l'accuse tout à coup en public et le tance si vertement que le coupable a envie de disparaître sous terre de honte. Il lui est alors défendu de se défendre et il doit attendre l'occasion d'une revanche.

On signale en Mélanésie des privilèges analogues mais réservés à un certain degré de parenté. Aux îles Fidji un individu peut effrontément s'approprier les biens de son cousin croisé, de même que le neveu ceux de l'oncle maternel; le cousin dépouillé peut réprimander le voleur, mais le simple fait d'essayer de rentrer dans son bien est considéré comme un signe de mauvaise éducation. Même s'ils sont de sexe différent, les cousins croisés peuvent se permettre entre eux des libertés et il était même admis autrefois qu'ils eussent des relations sexuelles. Les propos injurieux entre cousins croisés passent pour naturels et ne créent pas d'inimitié. Dans les îles Banks, il est des localités où le beau-frère et la belle-sœur se plaisent à des jeux brutaux, quoique à un moindre degré que les Indiens des Prairies, tout en évitant cependant de faire intervenir le côté sexuel. Un habitant de ces îles peut traiter n'importe comment le mari de la sœur de son père, le menacer et se moquer de lui continuellement.

Chapitre 5

\section{e. Tabou et licence.}




\section{$\underline{\text { Retour à la table des matières }}$}

Les exemples de tabous et de licence signalés dans les diverses parties du monde justifient l'opinion de Frazer pour qui il est faux de discuter des restrictions relatives aux beaux-parents indépendamment de toutes les autres. Ces restrictions ne représentent évidemment qu'une catégorie spéciale dans une classe plus vaste comprenant les usages qui règlent le comportement social des parents de même sang ou alliés. Il est bien peu probable que tous ces tabous ou tous ces cas de privilèges aient une base psychologique commune. On admettra sans discussion que la coutume Crow permettant la familiarité avec la belle-sœur diffère fondamentalement du phénomène de la " parenté de plaisanteries » avec son mélange d'éléments sérieux et comiques. Il nous faut donc renoncer une fois pour toutes à une théorie qui embrasserait tous les faits signalés. Dans une localité donnée, les prescriptions particulières doivent être considérées dans tout l'ensemble de la culture et les interprétations que l'on déduira d'une telle étude pourront, mais alors seulement, être comparées aux résultats correspondants d'autres régions. Étant donné le caractère insuffisant de nos informations actuelles, il ne faut guère nous attendre à des explications définitives; néanmoins il est quelques résultats auxquels nous pouvons nous tenir en toute sécurité.

Pour avoir un fil conducteur dans le labyrinthe des détails, nous commencerons par un cas simple où nous ne serons pas obligés de lutter contre une multitude de règlements, basés peut-être sur des motifs variés, mais où les tabous sont peu nombreux et peuvent facilement être mis en corrélation avec quelque usage existant. C'est aux îles Andaman que nous trouverons cet exemple. Là, un tabou unique est mentionné; il porte sur les relations sociales entre un individu et la femme de son frère cadet. Nous savons expressément que la femme du frère aîné n'est sujette à aucune restriction de ce genre. Cette règle est donc en connexion directe avec la forme de mariage coexistante, connue sous le nom de lévirat des cadets. Pour cette peuplade tout au moins, nous pouvons énoncer le principe que les restrictions sociales et sexuelles vont de pair, conclusion qui a été adoptée sous une forme plus générale par Goldenweiser, sur la base des travaux encore inédits de Sternberg sur les Ghiliak, ainsi que par Rivers à la suite de ses recherches en Océanie. Qu'il me soit permis de compléter ce postulat par un autre, à savoir que les privilèges de familiarité règnent généralement entre conjoints potentiels.

Examinons maintenant l'exactitude de ces deux propositions en les comparant à ce qui se passe dans d'autres régions. Les Crow restreignent l'intimité entre frères et sœurs, dont l'union est naturellement hors de cause, et permettent par contre des libertés excessives entre un homme et la femme de son frère dont il peut hériter par lévirat, ainsi qu'entre un homme et la sœur de sa femme que, jusqu'à tout récemment, le sororat l'autorisait à prendre comme épouse supplémentaire. En Mélanésie, on a noté une corrélation analogue, positive et négative : les frères évitent les sœurs, alors que le beau-frère est sur un pied de familiarité avec sa belle-sœur. Nous trouvons en 
Afrique du Sud des témoignages corroborants que nous n'avons pas encore cités. Une femme thonga est sans retenue vis-à-vis du jeune frère de son mari qu'elle peut épouser un jour ou l'autre, mais elle observe une réserve distante envers l'aîné dont elle ne sera jamais la femme. C'est toujours le cas des îles Andaman qui se répète. Cependant l'épouse peut aussi être héritée par le fils de la sœur et nous trouvons, en effet, que le neveu prend toutes sortes de libertés avec la femme de son oncle maternel, même du vivant de celui-ci. Enfin un Thonga évite les sœurs aînées de sa femme qui ne sont pas du nombre de ses épouses possibles, alors qu'il traite avec la plus grande familiarité les sœurs cadettes qui peuvent devenir ses femmes.

A ces exemples je joindrai maintenant les tabous sibériens des beaux-parents. La caractéristique de cet ensemble de restrictions réside dans le fait que le frère aîné du mari est assimilé au beau-Père : la femme doit les fuir tous deux ou leur témoigner le même respect. En outre, il y a absence complète (sauf parmi les Youkaghir) de tabou entre belle-mère et gendre, et absence virtuelle de restrictions portant sur les relations entre bru et belle-mère. Ces faits s'éclaireront si nous concevons le tabou des beauxparents comme essentiellement un tabou du beau-frère, basé sur le lévirat des cadets que beaucoup de tribus sibériennes partagent avec les Andaman. L'extension de cette prohibition au beau-père s'explique facilement par une autre caractéristique sibérienne, l'importance attribuée à la supériorité en âge qui va supprimer les différences entre générations. Ainsi les Votiak ont un seul terme pour tout parent plus âgé que celui qui parle et les Youkaghir désignent par le même mot le beau-père et le frère aîné du mari. Mon hypothèse explique donc pourquoi les Sibériens, à la seule exception que nous avons notée, n'ont pas les tabous qu'on trouve si fréquemment ailleurs. La bellemère n'a, en effet, rien à voir dans l'héritage de la bru par les parents de son mari; et le lévirat n'affecte nullement le statut relatif du gendre et du beau-père.

L'exemple sibérien est intéressant à un autre point de vue il démontre la possibilité d'une évolution convergente. Voici quelle est la série causale en Sibérie : lévirat des cadets, d'où tabou entre belle-sœur et frère aîné du mari, identification du père du mari avec le frère aîné du même, d'où extension du tabou au beau-père. Chez les Assiniboin, le tabou de la bru et du beau-père a aussi été signalé, mais, comme cette peuplade ne pratique pas le lévirat des cadets et n'identifie pas le frère aîné du mari au père du même, l'histoire de leur tabou doit être différente de celui du tabou kirghiz par exemple. Ainsi un même résultat peut être atteint au travers de différentes phases de développement.

Revenons-en à notre proposition principale. Les cas cités nous fournissent des preuves évidentes de la corrélation que nous avons avancée plus haut, soit entre les tabous sociaux et sexuels d'une part, la licence sociale et la possibilité de relations sexuelles de l'autre. Mais l'existence de tabous entre individus d'un même sexe nous indique que ce principe n'explique nullement la totalité des phénomènes auxquels nous avons affaire. On peut arguer, il est vrai, qu'une règle a été étendue d'une classe de parenté à une autre lorsque, comme en Sibérie, nous possédons des preuves valables à l'appui de notre assertion. Nous ne pouvons, cependant, comme Frazer, nous 
contenter d'affirmer à la légère que tout tabou entre individus du même sexe est sorti d'un tabou portant sur des individus de sexe différent, hypothèse qui a été d'ailleurs justement réfutée par Parsons. Un véritable historien doit éviter ce genre d'interprétations hâtives en faveur d'une étude intensive de tous les tabous d'une tribu donnée, replacés dans leur milieu culturel normal.

Ici se présente à nouveau un point dont nous avions temporairement remis à plus tard la discussion. S'il existe une corrélation empirique entre les tabous sociaux et sexuels, pourquoi ne pas adopter l'interprétation psychologique qu'en donne Frazer dans les cas où elle est valable? Pourquoi se refuser à admettre que certaines relations sociales sont tabou afin d'empêcher tous rapports sexuels entre les divers intéressés? Je répondrai que d'autres facteurs, plus essentiels que les tabous en question, suffisent amplement à prévenir les relations incestueuses. Nous trouvons en Australie des lois relatives au mariage dont la transgression est punie de mort, même lorsque rien de ce que nous considérons comme incestueux n'entre en ligne de compte. Un homme ne pouvait s'unir qu'avec une femme d'une classe déterminée, par exemple la fille du frère de sa mère. De même, en d'autres parties de la Mélanésie, la tribu est divisée en unités exogames, tous les frères et sœurs appartenant à la même unité. Dans de telles conditions, il est impossible qu'un frère et sa sœur puissent s'unir.

Je dois reconnaître que dans la plupart des cas qui ont été discutés jusqu'ici, c'està-dire dans ceux où interviennent le lévirat des cadets et le sororat, l'interprétation psychologique paraît simple. Les mâles biologiquement désirables ont été dichotomisés par les conventions en ceux qui sont sociologiquement acceptables et ceux qui ne le sont pas; d'où s'ensuit naturellement une différence d'attitude qui, dans un cas, peut dégénérer en licence et, dans l'autre, revêtir la pruderie grotesque du tabou. Lorsqu'il s'agit de certaines relations qui ont été une fois pour toutes définies comme incestueuses, je crois que les tabous ne représentent plus une nécessité pour renforcer la continence, mais qu'ils sont en quelque sorte les résultats spontanés de l'horreur incestueuse prolongée artificiellement. Tout ce qui suggère, même de loin, les relations sexuelles produit un sentiment de répulsion, et, de cet état d'esprit, découle une rupture complète des rapports.

Cependant, je ne suis pas du tout convaincu que les tabous des beaux-parents doivent être toujours ou même souvent considérés comme relevant de la même catégorie que les tabous portant sur les individus de sexe différent mais de même génération. Par exemple, les Crow restreignent les rapports non seulement entre belle-mère et gendre mais aussi entre beau-père et gendre, ainsi qu'entre deux beaux-fils. Il est certainement étonnant que des gens qui sont loin d'être ennemis de la grossièreté dans la conversation quotidienne et dans leur mythologie, fassent preuve d'une telle pudibonderie lorsqu'il s'agit de la conduite imposée à un homme en compagnie du frère de sa femme. Cette particularité a également été observée chez les Pieds Noirs et les Arapaho et pourrait bien être beaucoup plus commune que nos rares informations ne nous l'ont donné a croire jusqu'ici. En ce qui concerne cette région de l'Amérique du Nord, tout au moins, nous voyons en fin de compte que le sentiment essentiel est une 
réserve respectueuse à observer vis-à-vis des membres de la famille de l'épouse, quel que soit leur âge; mais les différences de génération et de sexe ont naturellement intensifié ce sentiment dans le cas du tabou de la belle-mère.

La nécessité de tenir compte de l'ensemble des tabous dans une région donnée est particulièrement évidente en ce qui concerne le tabou du nom. Si l'on considère les superstitions qui s'attachent au nom dans les communautés primitives où il est, dans les rapports quotidiens, remplacé d'ordinaire par la désignation de parenté, la présence de telles prohibitions ne nous étonnera pas; cependant il est essentiel d'observer quels sont les noms taboués. Parmi les Toda, jamais un homme ne prononce le nom de ses beaux-parents, pas plus que des grands-parents, et ce n'est que contre son gré qu'il mentionne celui de sa femme. Ce tabou ne peut donc être rangé parmi les tabous des beaux-parents et ne se rattache certainement pas à des restrictions sexuelles. Cela est également vrai des Crow chez qui les conjoints évitent normalement de s'appeler par leur nom. Dans une peuplade mélanésienne, le mari peut prononcer le nom de sa femme, mais l'inverse est défendu; l'informateur de Rivers l'explique plausiblement comme un signe d'infériorité féminine. Le tabou du nom concernant un parent déterminé peut nous induire à des interprétations erronées, si nous ne faisons que le comparer à un tabou analogue en quelque autre point du globe, au lieu de le rattacher aux restrictions en vigueur dans cette même société sur l'usage des noms d'autres parents. C'est donc uniquement de l'étude approfondie d'une région déterminée et du travail ultérieur de comparaison entre diverses zones que nous pouvons attendre la lumière sur la signification des tabous, et non de parallélismes tracés au hasard.

Ce mode d'enquête intensif sera aussi la seule manière de nous expliquer l'absence de tabous là où on aurait pu s'attendre à en trouver. Pourquoi, par exemple, les Kariera ne connaissent-ils pas le tabou entre gendre et beau-père? Je ne prétends pas avoir découvert une solution, mais il vaut la peine d'indiquer du moins dans quelle direction elle est à chercher. On la trouvera, peut-être, dans le fait que le beau-père d'un individu est normalement son oncle maternel et que la conception kariera des liens avunculaires exclut le tabou. Cette explication serait limitée, il va sans dire, aux Kariera et aux tribus qui partagent les mêmes notions de parenté. En tout cas, il est clair que, si nous voulons connaître l'essence du tabou, il nous faut d'abord pénétrer jusqu'à l'essence de la culture dans la tribu qui l'observe, et, comme ce tabou peut avoir été emprunté, c'est donc toute la civilisation d'une région qu'il nous faut soumettre à une étude attentive. Il n'existe pas de voie royale menant à la compréhension des phénomènes culturels.

Chapitre 5

\section{f. Teknonymie.}


Chez les Ewé, lorsque naît un enfant, on n'appelle plus les parents par leur nom, mais on les désigne comme le père et la mère du nouveau-né, par exemple " père (mère) de Komla ». Cette habitude a été relevée et interprétée pour la première fois par Tylor qui a forgé le terme dérivé du grec de teknonymie pour désigner ce curieux phénomène. Appliquant la méthode statistique dont il a été question plus haut, il suppose que la teknonymie est en relation causale avec l'institution de la résidence matrilocale, ainsi qu'avec le tabou du gendre.

Le gendre, nous dit Tylor, est tout d'abord reçu comme un étranger dans la famille de sa femme, mais, par la suite, il jouit dans la maison d'un statut en tant que père du nouveau-né et c'est d'après celui-ci qu'on le désigne.

Tylor connaissait une trentaine de peuplades où les parents étaient nommés d'après l'enfant, mais il n'en cite que trois, les Bechuana de l'Afrique du Sud, les Khasi d'Assam et les Cri du Canada occidental. On s'est rendu compte depuis que la teknonymie est fort répandue. Les compilations de Frazer nous signalent son existence en Australie, Nouvelle-Guinée, Malaisie, Chine, Sibérie septentrionale, chez diverses tribus Bantou en Afrique, au nord de la Colombie britannique, au Guatémala et en Patagonie. Cette liste, longue pourtant, pèche par des lacunes que peuvent combler divers ouvrages. On a signalé, par exemple, cette coutume chez les Andaman, les Cingalais agriculteurs et les Henebedda Vedda; parmi les Gold de l'Amour, une femme appelle toujours son mari « père d'un tel ». On a aussi trouvé la teknonymie aux îles Fidji, en d'autres points de la Mélanésie ainsi qu'en diverses régions de l'Amérique. Chez les Zuñi et les Hopi, elle est fort en honneur. Une femme Hopi s'adresse à sa belle-mère en l'appelant " grand-mère d'un tel » et désigne de même son beau-père; un homme usera d'appellations analogues pour ses beauxparents. Mon interprète ne parlait jamais de sa femme que comme "mère d'un tel ", mentionnant le nom d'un de ses enfants; elle, de son côté, le désignait de la même manière. Parfois un homme qui n'a pas d'enfant est appelé « oncle d'un tel ».

L'interprétation de Tylor ne nous semble pas en accord avec nos connaissances actuelles. D'une part, elle ne tient pas compte du fait que la mère, aussi bien que le père, peut être désignée d'après les enfants. Quant à la corrélation supposée avec la résidence matrilocale, les Australiens, les Mélanésiens et les Gold, pour ne citer que quelques exemples, sont patrilocaux. D'autre part, parmi les Zuñi et les Hopi matrilocaux, la teknonymie ne peut avoir supplanté le tabou du gendre, puisqu'il n'y existe pas de tabou de cet ordre et que d'autres parents, outre le père, sont nommés d'après l'enfant. Ainsi aucun des deux rapprochements n'est plausible.

Peut-on avoir recours à quelque hypothèse plus vraisemblable? Ici encore nous ferons bien de renoncer à une théorie générale et de nous attacher surtout aux faits en corrélation avec la teknonymie dans ses manifestations particulières. Tout d'abord, il est essentiel de savoir quel est l'individu désignant et l'individu désigné au moyen de 
la teknonymie. Chez les Gold, c'est la femme qui s'adresse ainsi à son mari, tandis que lui l'appelle par son nom; avant la naissance du premier enfant, la femme ne dispose d'aucun terme pour désigner son mari. En corrélation avec ce phénomène, nous trouvons qu'un frère peut appeler sa sœur par son nom, alors que semblable privilège ne lui est pas reconnu à elle; parmi les Gold, le sexe féminin est tenu en très petite estime. Il s'ensuit que, dans cette tribu, la teknonymie est une conséquence du statut inférieur de la femme; le fait que l'épouse peut s'adresser à son mari sans le désigner ni par son nom ni par un terme de parenté est une façon naturelle de résoudre la difficulté. C'est évidemment à une tout autre explication qu'il nous faut avoir recours dans le cas des Hopi. Ici l'usage fréquent de la teknonymie apparaît surtout dans le cas des parents par alliance; et ce ne peut guère être un hasard si le vocabulaire hopi est si pauvre en mots désignant précisément ces parents-là. Ici donc la teknonymie a pris naissance dans la nécessité de désigner des individus auxquels on ne pouvait se référer autrement, et elle peut avoir été étendue secondairement à quelques autres parents. Bref, la teknonymie nous fournit encore un cas d'évolution convergente et ses diverses origines doivent être étudiées en raison des conditions spécifiques. J'en avancerai cependant une autre interprétation se rattachant à un phénomène que je décrirai plus loin.

Chapitre 5

\section{Références.}

$\underline{\text { Retour à la table des matières }}$

1) LOWIE, 1919 (a) : 35-40; id., 1912 : 201; id., 1917 (a) : 40. RIVERS, 1914 (b) : 1, 38, 367; 11, 160; id., 1906 : 332. JUNOD : 1, 223.

2) JOCHELSON, $1910: 75$ et suiv. CZAPLICKA : 120, 122, 127, 128. RADLOFF : 314, 480. SELIGMANN : 68. RIVERS, 1914 (b) : 1, 41. LEHNER : 426. Reports, V : 142. HOWITT : 199, 208, 256, 266. RADCLIFFE-BROWN : 157. JUNOD : 1, 230. FRAZER, 1912 : 77-81, 84, 95. SPIETH : 744. LOWIE, 1912 : 213; id., 1917 (a) : 48, 91. FREUD, 1912 : 30 et suiv. TYLOR, 1889 : 246.

3) JOCHELSON, 1910 : 75. MAN : 68. RIVERS, 1914 (b) : 1, 35-43, 213, 291; Il, 508. LOWIE, 1912 :213 et suiv.; id., 1917 (a) :69, 71, 74.

4) HOCART, 1915 : 641; id., 1913 101. LOWIE, 1912 :204, 215; id., 1917 (a) : 42, 79. RIVERS, 1914 (b) 1, 40, 45 et suiv.

5) GOLDENWEISER, 1910 :251. RIVERS, 1914 (b) :1, 223 et passim; id., 1906 : 494. JUNOD :1, 228-236. PARSONS :282.

6) TYLOR, 1889 : 248. SPIETH : 217. FRAZER, J. G., 1911 : 331-334. MAN : 61. SELIGMAN : 65. LAUFER, 1900 : 3 20. RIVERS, 1914 (b) : 1, 230, 279. LOWIE, 1917 (a) : 92. KROEBER, $1917: 72$. 


\section{6}

\section{LE CLAN}

\section{$\underline{\text { Retour à la table des matières }}$}

A côté du groupe familial omniprésent, nous trouvons fréquemment un type d'unité qui ressemble à la famille en ce qu'il se base sur la parenté, mais qui, à d'autres égards, en diffère fondamentalement.

Le clan («sib » pour quelques ethnologues américains, " clan » pour les Anglais) peut être brièvement défini comme un groupe de parenté unilatérale. La famille est bilatérale; dire qu'un individu appartient à telle famille, c'est affirmer qu'il se reconnaît des relations avec tel homme qui est son père ainsi qu'avec telle femme, sa mère. Le clan institue une parenté soit avec le père, soit avec la mère, mais exclut totalement l'un des deux. Si une tribu est organisée en clans maternels, chaque enfant, quel que soit son sexe, est considéré comme un membre du clan maternel dont il prend le nom, s'il en existe un. Si, au contraire, la tribu se divise en clans paternels (ou consanguins), les enfants suivent le clan du père et en reçoivent le nom. L'autre parent, en ce qui concerne le clan, est tout à fait négligé, de même qu'en Europe, à l'exception de l'Espagne, la mère ne compte pour rien dans la transmission du nom de famille. Si tous les hommes et femmes portant le nom de Durand étaient unis par leur patronyme commun en un groupe social défini, distinct de celui des Dubois et des Dupont, nous pourrions à bon droit affirmer qu'ils forment un clan. Si nous refusons de les désigner ainsi, c'est que, dans notre société, il n'existe pas de lien unissant tous ces Durand apparentés par le sang; s'il s'agit d'héritage, un Dupont 
proche parent passera avant un Durand plus éloigné. Mais, pour plus de clarté dans l'exposition, supposons un clan Durand basé sur une parenté de sang. Ce clan comprendrait les ancêtres Durand avec tous leurs fils et leurs filles non mariées, les fils des fils et les petites-filles non mariées et ainsi de suite ad infinitum. Pour que cette unité devienne un clan primitif typique, il n'y a qu'une modification à y introduire, c'est faire dépendre la filiation (sauf dans les cas d'adoption) uniquement de la naissance et nullement du mariage. Le clan paternel est donc constitué par un ancêtre masculin, ses fils et filles et les enfants des fils. De même, le clan maternel comprend une aïeule avec ses enfants et les enfants de ses filles par descendance féminine.

De tout ceci se dégage dès l'abord une différence importante entre la famille et le clan : celle-là est d'un caractère assez élastique alors que celui-ci est une unité fixe. Le divorce et les migrations dispersent la famille, mais les liens du clan sont permanents. C'est ce que nous démontre clairement l'exemple des Hupa décrit dans un chapitre précédent. Nous y avons vu que, grâce à la résidence patrilocale, les enfants grandissent dans le village paternel, que les filles quitteront plus tard en se mariant. Jusqu'à ce moment, un certain nombre de frères avec leurs enfants formeront le noyau d'un clan patrilinéaire, uni par une résidence commune. Mais, à cause de l'exogamie locale, la même règle qui cimente l'union du groupe amène sa dissolution partielle lors du mariage des jeunes filles. D'autre part, dans les cas exceptionnels où un homme fait une période de service pour gagner sa femme, les enfants seront considérés comme faisant partie de son village à elle, contrairement à ce qui se passe normalement. Or, si les Hupa étaient organisés en clans paternels, jamais les femmes mariées, ni les enfants des hommes travaillant chez leurs beaux-parents, ne perdraient le contact avec le clan du père, et les phénomènes de résidence n'auraient aucune portée. Chaque individu adopterait automatiquement dès sa naissance le nom patronymique, le garderait jusqu'à sa mort et, s'il s'agissait d'un homme, le transmettrait à ses enfants.

D'où suit le corollaire suivant : le clan, éliminant la moitié des parents unis par le sang, est beaucoup plus inclusif que la famille en ce qui a trait aux parents effectivement reconnus tels. Chez nous, un cousin au troisième ou quatrième degré ne fonctionne plus guère comme membre de la famille; cependant, pour les primitifs, grâce à la fixité du lien clanique, même le parent le plus éloigné passe toujours pour un membre de la même unité; celle-ci est le plus souvent désignée par un nom porté en commun par tous ses membres, ce qui ne laisse ainsi subsister aucun doute quant à la filiation clanique. Le sentiment de communauté qui naît alors se reflète dans la terminologie. Les camarades de clan de la même génération s'appellent en général frères et sœurs; de là, étant donné l'attitude des primitifs à l'égard des noms, il n'y a qu'un pas au sentiment d'inceste qu'inspire le mariage entre ressortissants du même clan. C'est pourquoi l'un des traits les plus fréquents du clan est l'exogamie. L'intensité de l'obligation de se marier en dehors du clan varie du reste considérablement. En Australie elle était si prononcée qu'un homme ou une femme coupable d'avoir transgressé cette règle était immédiatement mis à mort; un étranger ne se mariait jamais dans un clan du même nom, même situé à des centaines de milles de sa tribu et bien qu'il ne pût s'agir de parenté véritable. En Amérique du Nord, généralement, les 
réactions des indigènes étaient de nature moins violente. Les Crow se moquaient de ceux qui n'observaient pas la règle, les comparant à des chiens, mais ils ne prenaient aucune mesure pour les punir. De même, les Iroquois ne témoignent d'aucune horreur pour l'inceste clanique et ne châtient les transgressions que par le ridicule, tandis que les Miwok se contentent de faire remarquer combien il est incongru de se marier à l'intérieur de son clan. Mais, quel que soit le caractère de la réprobation qu'inspirent les unions non exogamiques, nous pouvons dire que l'exogamie est une des marques les plus caractéristiques du clan.

Comme nous l'avons déjà dit, le sens de la parenté entre gens du même clan s'associe à un type spécial de nomenclature, les parents les plus éloignés d'une même génération se considérant souvent comme frères et sœurs. La terminologie de parenté des tribus organisées en clans se conforme en effet avec une extraordinaire fréquence à ce que j'ai appelé le type Dakota (page 65). Cette corrélation a été observée par Tylor et plus particulièrement par Rivers qui a démontré combien un tel système de parenté s'accorde bien avec le schéma du clan.

A cause de la loi exogamique, un homme doit épouser une femme d'un autre clan; ses frères à lui et certains de ses cousins sont également des conjoints potentiels pour elle, de même que ses sœurs à elle et certaines de ses cousines pour lui. Mais aux frères et aux sœurs, ainsi qu'à des parents plus éloignés du même clan, l'exogamie clanique défend le mariage. Il est donc tout naturel que le père et celui qui appartient au même clan que le père soient groupés et différenciés de la mère et de son groupe. La filiation de parenté clanique marche donc de pair avec la filiation terminologique. C'est pourquoi il est plausible de convertir le rapport fonctionnel en rapport causal et d'affirmer que c'est bien l'organisation clanique qui produit la terminologie Dakota.

Cependant, avant de tenir ce point pour acquis, il nous faut chercher si d'autres causes n'ont pu peut-être aussi façonner cette classification de parenté. Et nous nous souviendrons à ce propos que la filiation Dakota s'harmonise aussi singulièrement bien avec le lévirat et le sororat (page 43). Il y a donc double hypothèse. Il se peut aussi que l'exogamie et ces deux modes d'union préférentielle soient en rapport causal; nous reviendrons plus tard sur ce point. Mais, quelle qu'en soit l'interprétation, le fait empirique demeure, à savoir que les tribus organisées en clans exogamiques ont un type de nomenclature Dakota, c'est-à-dire une nomenclature où les parents collatéraux ou ceux en ligne directe, soit du côté paternel, soit du côté maternel, sont identifiés, sans égard à leur degré de proximité. Sur l'instigation de Rivers, j'ai étudié ses opinions à la lumière des faits nord-américains et j'ai trouvé que presque toutes les tribus à clans exogamiques ont un système du type Dakota; quelques tribus dépourvues de clans ont aussi adopté cette nomenclature qui leur est parvenue soit par emprunt, soit grâce à d'autres influences. Cependant, beaucoup de tribus sans organisation clanique distinguent les parents collatéraux de ceux en ligne directe. Mes recherches personnelles m'ont également appris que les Hopi, seule peuplade Shoshoni qui connaisse un clan bien défini, sont de même les seuls à avoir une terminologie Dakota. Il nous est donc permis de généraliser et d'affirmer que la nomenclature 
Dakota est en rapport avec l'organisation clanique, quoique sa signification ne puisse être déterminée sans que l'influence du lévirat et du sororat n'ait été étudiée.

Revenons-en à d'autres aspects de l'unité clanique. La règle qui veut que celui qui fait partie d'un clan lui appartienne pour toujours, ne souffre guère d'exceptions par suite de mariages. Je ne connais aucun cas où un homme entre dans le clan de sa femme et, de la possibilité contraire, le seul exemple valable paraît être celui des Toda où la femme adopte le clan de son mari. Les anciens Athéniens, il est vrai, faisaient entrer la femme dans la phratrie du mari, mais les Grecs peuvent difficilement passer pour des primitifs et leurs prescriptions ne font qu'anticiper sur nos lois modernes. L'adoption, par ailleurs, joue un rôle plus important. Un enfant adopté dans une communauté patrilinéaire devient automatiquement du clan de son père adoptif, ou de celui de sa mère dans les cas de descendance utérine, exactement comme s'il s'agissait du véritable rejeton.

Cependant ce ne sont pas ces adoptions individuelles qui ont influencé le plus profondément la constitution du clan. Lorsque nous employons la méthode généalogique pour étudier le clan de type courant, nous trouvons qu'il est en général impossible de faire remonter tous ses membres à un seul ancêtre commun. Nous sommes en présence de lignées indépendantes unies par un lien purement théorique à une origine commune. Il se peut naturellement que les informateurs indigènes aient tout simplement oublié ce qui reliait les ancêtres des générations plus anciennes, mais cette explication ne s'applique pas à tous les cas. Ainsi, en recensant les Hopi, j'ai découvert que quelques très petits clans utérins ne se composaient pas uniquement d'individus unis par les liens du sang, mais qu'ils se divisaient en deux ou trois groupes matrilinéaires distincts qui se considéraient comme unis par une fiction légale seulement. Tout ce que nous savons de la logique primitive nous rend ce phénomène fort compréhensible. Si deux hommes accomplissant ensemble une pratique rituelle s'adoptent réciproquement comme frères, leurs sœurs respectives seront de ce fait également unies par des liens sororaux et leurs descendants seront ipso facto considérés comme les enfants de sœurs véritables, donc rangés théoriquement dans la progéniture d'une même aïeule.

Avant de terminer ces considérations préliminaires, il convient de résoudre ce qui nous apparaît d'abord comme une énigme. Si la famille bilatérale se retrouve partout, comment peut-elle coexister avec le clan? N'est-ce pas contrevenir à la loi de l'identité que de décrire des sociétés qui simultanément reconnaissent le père et la mère tout en ignorant l'un des deux? Il va sans dire que cette reconnaissance et cette ignorance s'exercent dans des aspects distincts de la vie sociale. De même que nous reconnaissons la mère, mais l'ignorons en ce qui a trait au nom de famille, ainsi les tribus primitives admettent le père et la mère à bien des égards et font abstraction de l'un des deux sous d'autres aspects. 
Nous avons considéré jusqu'ici le clan sous un angle morphologique surtout. Il nous faut maintenant voir quelles en sont les fonctions. C'est en décrivant quelques types choisis d'organisation clanique que nous y parviendrons le mieux.

Chapitre 6

\section{a. Types d'organisation clanique.}

\section{$\underline{\text { Retour à la table des matières }}$}

Prenons tout d'abord un cas simple. Les Indiens Crow sont divisés en treize clans utérins exogamiques. Ces unités sont désignées par des sobriquets; l'une s'appelle " Ils rapportent le gibier sans tirer », l'autre " Mauvais honneurs de guerre », une troisième " Mauvaises guêtres » et ainsi de suite. Non seulement les ressortissants du même clan s'interpellent les uns les autres comme s'ils étaient parents, même lorsqu'ils ne sont pas apparentés entre eux, mais ils se comportent véritablement comme tels les uns envers les autres, s'entraidant volontiers lorsque l'occasion s'en présente. Leurs relations, cependant, se limitent à la sphère sociale et n'effleurent jamais le champ de l'activité religieuse. Les Crow se divisaient localement en branche méridionale et branche septentrionale, mais, dans toutes deux, se trouvaient des ressortissants de tous les clans.

Si nous passons des Crow aux Hopi du nord de l'Arizona, nous nous trouvons en présence de phénomènes tout différents. Les Hopi, de même que les Crow, ont des clans utérins exogamiques et il existe un lien social étroit entre les membres du clan. Cependant l'analogie s'arrête là. Les noms de clans sont de caractère fort distinct; ce ne sont jamais des surnoms mais des désignations empruntées en général au règne de la nature; le Serpent, le Sable, le Lézard, le Peuplier en sont des exemples typiques. Les biens immeubles qui, parmi ces peuplades sédentaires, jouent un rôle plus considérable que chez les Crow vagabonds de l'époque de la chasse au bison, sont, dans une certaine mesure, possédés par le clan et transmis en héritage à l'intérieur du clan. Ainsi chaque clan a son district propre pour la chasse à l'aigle; les privilèges les plus importants consistent à remplir certaines fonctions cérémonielles, se transmettent dans le clan, passant à des individus à parenté utérine. Tout ceci peut nous paraître attenter à l'équité des lois de succession. En effet, puisque les clans Hopi sont matronymiques et exogamiques, le père et le fils ne font jamais partie du même clan; un père ne peut donc jamais passer ses privilèges cérémoniels à son propre enfant, mais doit les léguer à ses parents de clan les plus proches, ses frères ou les fils de ses sœurs. C'est de cette manière que, par exemple, la qualité de chef continue à se transmettre dans le clan du Serpent. Le rôle énorme que joue le ritualisme chez les Hopi donne à leur concept du clan une nuance particulière et le différencie nettement de celui des Crow chez lesquels le clan et les cérémonies sont complètement dissociés. 
On trouvera chez les Winnebago du Wisconsin un exemple de la multiplicité des fonctions dont le clan peut être investi et de la complexité dont ce type d'organisation est susceptible. Nous sommes ici en présence de ce qu'on appelle l'organisation bipartite; c'est-à-dire que toute la tribu est divisée en deux clans qui, dans ce cas particulier, sont patronymiques. Ces deux clans consanguins sont exogamiques, de même que les clans plus nombreux des Crow et des Hopi. Ils se rattachent symboliquement au ciel et à la terre, un groupe étant appelé "Ceux d'en haut » et l'autre «Ceux de la terre ». Ces clans, outre leur règlement de mariage exogamique, jouent un certain rôle dans la guerre, les jeux de balle et l'une des fêtes tribales; en toutes ces occasions les ressortissants d'un même clan sont groupés.

Une complication importante résulte cependant du fait que, chez les Winnebago, les deux clans consanguins se subdivisent le premier en quatre, le second en huit groupes plus petits, également consanguins. Comme la nomenclature est de signification secondaire, nous pourrions fort bien appeler ces subdivisions des sous-clans ou sections de clans, ou inventer quelque autre terme. Pour plus de commodité, j'ai appelé ces petites unités clans consanguins (ou paternels) et j'ai donné aux deux grands groupes des Winnebago la désignation qui s'explique par elle-même, de moitié ou phratrie.

Les clans en lesquels se divisent les phratries patronymiques des Winnebago présentent un certain nombre de caractéristiques intéressantes. Ils sont exogamiques, mais dans un sens différent des phratries Winnebago ou des clans utérins des Hopi et des Crow. Ces dernières unités sont exogamiques de leur propre chef, pour ainsi dire; les clans Winnebago ne le sont que par dérivation, c'est-à-dire parce que les plus grandes unités dont ils forment les éléments sont elles-mêmes exogamiques. Leur caractère exogamique ne dépend pas d'eux mais de la phratrie. D'autre part, nous voyons apparaître plusieurs conceptions nouvelles. Non seulement chaque clan porte le nom d'un animal, tel que Serpent, Aigle, Oiseau-tonnerre, Ours, mais encore il prétend descendre de la bête en question; ses ressortissants sculptent, tissent ou gravent des représentations de l'animal clanique, en l'absence de tout indice d'un culte en son honneur. En outre, tout un ensemble particulier de noms propres est associé à chaque clan. A cet égard, le contraste est frappant avec les autres tribus dont nous nous occupons. Parmi les Crow il n'y a aucun rapport entre l'un des clans et le nom propre d'un enfant; celui qui donne le nom, un haut personnage dans la tribu, marque l'enfant d'une désignation évoquant ses propres exploits. S'il a dans un combat frappé trois ennemis du même coup, l'enfant (que ce soit un garçon ou une fille) pourra être appelé « Frappe-trois-ennemis ». De même, chez les Hopi, le nom dépend de la femme qui le donne ainsi que de son clan qui, dans cette tribu matronymique, n'est jamais celui de l'enfant, car elle appartient en règle au clan du père et choisit un nom se référant, d'une façon mystérieuse souvent, à son propre clan. La coutume des Winnebago diffère en ce que chaque individu reçoit l'un des noms tirés d'une série d'appellations appartenant à son clan et qui rappellent souvent le mythe originel. Comme les Hopi, les Winnebago assignent à leur clan des fonctions cérémonielles. Chaque unité possède son " paquet sacré » et accomplit les rites qui s'y associent. Mentionnons en- 
core comme particularité nouvelle l'activité politique de ces unités qui les différencie les unes des autres. Ainsi les gens de l'Ours exercent les fonctions de police tandis que le chef tribal est invariablement choisi parmi les « Oiseaux-tonnerre » et le crieur public chez les « Bisons ».

Voici un dernier exemple américain. Les Miwok de la Californie centrale sont divisés en phratries exogamiques patrilinéaires, associées à l'Eau ou Crapaud-Bœuf, et à la Terre ou Geai bleu. Contrairement aux phratries Winnebago, celles des Miwok ne se subdivisent pas en clans plus petits. Tous les phénomènes naturels sont répartis entre l'Eau et la Terre de façon plutôt arbitraire et cette dichotomie théorique de l'univers trouve sa contrepartie pratique dans l'attribution des noms propres. Ceux-ci sont remarquables en ce qu'ils se réfèrent non directement mais symboliquement à la phratrie du porteur. Au point de vue rituel, les divisions des Miwok sont loin d'être aussi importantes que les clans des Hopi ou des Winnebago. Néanmoins, lors des cérémonies funéraires et de puberté, on observe la division bipartite.

Si nous passons à la Sibérie occidentale, nous trouvons les Ostiak répartis en une quantité considérable de clans paternels exogamiques, se composant chacun d'un certain nombre d'hommes et de leurs descendants. Quoique ces clans comprennent souvent jusqu'à des milliers d'individus et que beaucoup de leurs ressortissants soient incapables d'établir entre eux une parenté quelconque, il existe cependant une croyance marquée en une origine commune. Les membres masculins du clan forment des groupes à part lors des migrations et un certain esprit de fraternité pousse tout naturellement les riches à partager leurs biens avec les pauvres. Le clan est une unité non seulement sociale mais aussi politique; chacun a à sa tête un chef qui transmet ses fonctions à son fils ou à son parent le plus rapproché et dont le devoir principal est d'arbitrer les disputes. Plusieurs clans s'allient en une sorte de ligne présidée par un prince. Au-dessus et au-delà des aspects politiques et sociaux du clan Ostiak, nous trouvons ses fonctions religieuses. Chaque clan a ses idoles distinctes sous la garde d'un devin ou shamane, et les ressortissants du clan s'assemblent pour les sacrifices et autres cérémonies.

En passant encore à une autre région du globe, nous trouvons en Mélanésie un système clanique hautement développé. A Buin, dans les îles Salomon, Thurnwald a découvert huit clans matrilinéaires exogamiques dont chacun est nettement associé à quelque oiseau, tel que la chouette ou le perroquet, qu'aucun des membres du clan ne doit ni tuer ni manger. Les gens de la Chouette seraient même tellement offensés si des hommes appartenant à d'autres clans tuaient une chouette qu'il en résulterait une haine mortelle; c'est pourquoi les membres de la tribu ne chassent aucun des animaux sacrés. Les indigènes ne font pas remonter leur origine à ces oiseaux, mais conçoivent leur parenté mystique d'une façon quelque peu différente. Pour le clan du Perroquet, par exemple, il est question d'une aïeule humaine qui épousa un perroquet et donna naissance à un oiseau de cette espèce. Dans les îles de l'Amirauté, on croit que les obligations entre le clan et l'animal sacré sont réciproques et que les gens du clan de l'Alligator, entre autres, sont à l'abri des attaques de ces reptiles. Contrairement au 
système des Winnebago, celui de Buin est complètement dissocié de l'organisation politique du peuple. Pour ne mentionner qu'un trait, la qualité de chef se transmet de père en fils, en opposition directe au système clanique matrilinéaire observé par la tribu.

Voici enfin un dernier exemple : la tribu des Kariera en Australie occidentale est divisée en phratries et en une vingtaine de clans patrilinéaires sans noms distinctifs, mais possédant chacun un district déterminé par droit inaliénable. En outre, chaque clan est associé à un certain nombre d'animaux, de plantes et de phénomènes naturels. Ainsi, l'un se rattache à l'arc-en-ciel, au cacatoès blanc, à une certaine mouche, à deux espèces de poissons et à un coquillage. Chacun de ces phénomènes ou animaux a son propre terrain cérémoniel à l'intérieur du domaine du clan et certaines cérémonies y sont célébrées à la fois par les hommes et par les femmes pour multiplier les animaux et les plantes en question. La plupart de ceux-ci sont comestibles et il convient de remarquer qu'il n'existe pas la moindre restriction relative à la consommation de ces plantes ou animaux par les ressortissants du clan. Comme d'ordinaire, ces clans sont exogamiques; la résidence étant patrilocale, un jeune garçon grandit sur le terrain du clan paternel et, comme il est censé épouser la fille de son oncle maternel, sa femme est originaire d'un autre clan et d'une autre localité.

Ces exemples suffisent à mettre en lumière la diversité- des fonctions qui peuvent s'associer à un système clanique. Le clan nous apparaît donc comme une unité sociale extrêmement variable. S'il est en général exogamique, il peut ne l'être que par dérivation (Winnebago). Il peut être associé à des animaux et à des plantes envers lesquels une attitude déterminée est prescrite (Buin), mais peut aussi ne présenter aucune connexion de cet ordre (Crow). Ce sera une unité locale (Kariera) ou non locale (Crow); une division politique (Ostiak) ou apolitique (Buin). Ce caractère variable du clan pose un problème important.

Chapitre 6

\section{b. Origine ou origines.}

\section{Retour à la table des matières}

Pour Morgan, l'organisation clanique semble reposer sur un concept si obscur qu'il se voit obligé de supposer une origine commune à tous les clans reconnus. Selon lui, cette institution aurait pris naissance en un point géographique déterminé et de là se serait répandue sur d'immenses zones. Cette hypothèse est d'autant plus étonnante que la théorie de Morgan sur la loi de l'évolution sociale selon laquelle toute l'humanité progresse d'une phase à l'autre, admet la possibilité que des coutumes et institutions analogues puissent êtres créées indépendamment. On peut accorder, en effet, 
que si les primitifs avaient développé un système arbitraire et compliqué pour différencier certains parents à l'exclusion des autres, il serait impossible qu'un tel groupement ait été réinventé à plusieurs reprises. Nous verrons, cependant, plus loin que la classification de la parenté particulière du clan est, dans certaines conditions, fort naturelle, ce qui rend des plus probables les formations claniques parallèles et indépendantes. Écartant ce point pour l'instant, voyons d'abord si les analogies entre clans dans les diverses parties du monde sont suffisamment marquées pour étayer l'hypothèse d'une origine commune. La variabilité que nous avons notée plus haut ne parle guère en sa faveur. Cette hypothèse sera réduite à néant si nous pouvons prouver que, même dans une aire relativement restreinte comme l'Amérique du Nord, il existe des centres indépendants de diffusion clanique; la possibilité d'une origine commune pour le monde sera donc a fortiori écartée.

En Amérique du Nord, l'organisation clanique fonctionne dans quatre, peut-être cinq zones sans relations les unes avec les autres : la région des forêts à l'est du Mississippi, les Prairies du nord-ouest, le sud-ouest et la côte du nord-ouest; à ces aires il faudrait peut-être ajouter comme région distincte la Californie du centre et du sud, quoique d'aucuns ne voient dans l'organisation clanique de cette zone qu'une ramification du système du sud-ouest. Le problème qui se pose est donc le suivant : ces diverses organisations ont-elles évolué indépendamment? Y a-t-il eu à l'intérieur de ces régions des foyers distincts où les clans se seraient développés? ou tous les clans du continent ont-ils une origine unique?

A l'est du Mississippi et même dans le sud des Prairies occidentales, s'étend une immense zone ininterrompue peuplée de tribus appartenant à des familles variées dont les unes sont matronymiques, les autres patronymiques, mais où toutes possèdent en somme linstitution du clan. Que nous passions des Iroquois aux Menomini ou des Omaha aux Choctaw, nous ne pouvons manquer de remarquer que le clan est à peu près toujours du même modèle. Les clans sont d'ordinaire désignés d'après des animaux, la même appellation revenant souvent dans des tribus différentes; chaque clan a une série de noms propres distincts pour ses membres; et presque chaque système de clan se rattache à un groupement par phratries dont la fonction la plus constante est de départager les hommes dans les jeux athlétiques. Même des tribus très éloignées à l'intérieur d'une région déterminée ont en commun des caractéristiques pourtant très particulières. Ainsi les Osage du Missouri ont des phratries se rattachant respectivement aux notions de paix et de guerre, et il en est de même chez les Krik de l'Alabama. L'exogamie est universelle, associée tantôt aux clans restreints, tantôt aux phratries. Les analogies sont trop nombreuses et de nature trop spéciale pour être attribuées au hasard; elles ne sont explicables que par la diffusion. D'autre part, la règle de descendance divise les systèmes claniques de chaque zone en deux catégories qui correspondent au groupement géographique des tribus, si nous adoptons la théorie généralement admise que les Iroquois étaient originairement une peuplade méridionale. Ainsi, on peut concevoir que les Iroquois occupent avec les tribus matrilinéaires des Krik, des Choctaw, des Chickasaw et des Yuchi un territoire ininterrompu, alors que les Algonkin du centre et les Sioux du sud 
représentent une autre zone géographique. A cause des analogies que nous avons mentionnées il ne nous est pas possible de supposer dans chaque région l'existence de plus d'un centre ayant donné naissance à la notion de descendance unilatérale. La seule question qui se pose est celle de savoir si les deux différentes descendances nécessitent deux inventions indépendantes ou une seule pour la zone tout entière. Dans les deux cas, nous aurions à faire la part de la diffusion, ce qui est l'évidence même, si nous présupposons l'existence d'un seul centre. Mais, même si le clan utérin des Iroquois et le clan consanguin des Omaha, par exemple, représentent en fin de compte deux formes indépendantes, il a dû se produire, directement ou non, des emprunts ultérieurs, discernables par le caractère des traits communs. Pour n'en citer qu'un, les séries de noms propres distinctifs de clans ne se retrouvent plus sur des centaines de milles à l'ouest de la zone étudiée; donc leur présence parmi les peuplades consanguines ou utérines à l'est du Mississippi et dans les plaines du sud est une marque certaine d'emprunt. Pour ce que je veux démontrer présentement, il n'est pas essentiel de décider si la zone clanique de l'est correspond à une évolution simple ou double du clan; je laisserai donc la question en suspens.

Cette question peut d'autant mieux être laissée de côté que le point dont il s'agit en théorie est éclairé avec toute la netteté désirable par une comparaison entre les clans orientaux et ceux des Prairies du nord-ouest. Dans cette dernière région, cinq tribus doivent être prises en considération : les Hidatsa et les Mandan du Nord Dakota, les Crow du Montana, les Gros Ventres et les Pieds Noirs du Montana et de l'Alberta. Les Pieds Noirs possèdent une organisation clanique qui est embryonnaire plutôt que parvenue à son plein développement : ils ont donné des surnoms à des groupes localisés à descendance consanguine surtout, mais sans suivre de règle fixe, ce qui fait qu'un individu peut changer de groupe, de même que ses enfants. L'exogamie n'est pas non plus absolue et repose sur la crainte que les ressortissants d'un groupe ne soient unis par des liens de parenté. En hiver, la tribu se fractionne en plusieurs bandes, obligées, pour des motifs d'ordre économique, à se disperser, tandis qu'en été elles se réunissent pour la chasse et la célébration de leurs cérémonies. Cette organisation se répète presque identique chez les Gros Ventres, avec une seule variante significative : il existe chez eux un véritable clan correspondant à cette bande, avec descendance patrilinéaire définie et exogamie stricte. Les trois tribus appartenant à la famille sioux observent la lignée utérine.

Or, si nous présupposons que tous les systèmes de clans orientaux ont une origine commune et qu'il en est de même pour les systèmes des Prairies septentrionales, nous trouvons que les deux images composites fournies par les clans de ces deux zones n'accusent pas la plus petite ressemblance entre elles. Les clans orientaux sont presque toujours désignés d'après des animaux et sont parfois reliés à leurs éponymes par une certaine parenté ou par des obligations religieuses précises. Dans les Prairies du nord, c'est à peine si les nom d'animaux apparaissent et jamais avec la notion d'un lien mystique unissant l'éponyme et les membres du clan. Les appellations de beaucoup les plus communes dans ces clans sont de l'ordre des surnoms : "Les laids », «Ceux qui ne donnent pas sans rendre » ou « Ceux qui apportent le gibier sans le 
tuer ». Il est encore une autre différence à laquelle nous avons fait allusion et qui a son importance : alors que les clans orientaux ont des séries définies de noms propres, on ne trouve rien d'analogue dans les clans des Prairies du nord. Enfin la phratrie qu'on rencontre fréquemment à l'est comme unité exogamique et cérémonielle et à laquelle se rattachent les jeux athlétiques, n'apparaît que chez les Hidatsa et les Mandan et seulement comme un agrégat sans importance de clans dépourvus de fonctions sérieuses bien nettes. Il est donc évident que les systèmes claniques des Prairies de l'est et du nord représentent deux types distincts et qu'il n'y a aucune raison de les faire dériver d'une même source.

Nous pouvons encore nous avancer davantage en utilisant les renseignements apportés par l'ethnographie, grâce à laquelle il nous est possible de retracer avec quelque sécurité l'histoire du système des Gros Ventres. Ces Indiens sont un rameau récent et relativement petit des Arapaho vivant plus au sud et qui ne connaissent pas le clan. Dans leur nouvel habitat, les Gros Ventres ont été en contact étroit avec les Pieds Noirs et l'on retrouve dans leur culture la double marque de leur origine Arapaho et de l'influence des Pieds Noirs. Ainsi que nous l'avons déjà dit, les clans des Gros Ventres, sauf en ce qui concerne leur intégration plus forte, rappellent à un degré étonnant les bandes des Pieds Noirs et, si nous considérons cette analogie en la rattachant à ce que nous savons des relations entre ces deux tribus, il ne saurait subsister de doute que la bande des Pieds Noirs et le clan des Gros Ventres sont historiquement liés. Or les Gros Ventres ignoraient le clan lorsqu'ils se détachèrent de la tribu primitive et, comme ils sont numériquement inférieurs aux Pieds Noirs, il est fort probable que ce furent eux les emprunteurs et qu'en perfectionnant les notions qu'ils trouvèrent chez leurs nouveaux voisins, ils développèrent un système clanique original. En tous les cas, le germe de ce système évolua soit dans l'une des tribus, soit dans toutes les deux conjointement. S'il s'est développé indépendamment des autres systèmes de la région, nous avons là la preuve a fortiori de la diversité d'origine des clans en Amérique du Nord.

Le système Pieds Noirs-Gros Ventres représente donc un développement indépendant. Si, pour des raisons d'ordre géographique, il se rattachait à quelque autre système des Prairies du nord, ce serait à celui des Crow; et, en effet, la grande vogue des surnoms désignant des unités sociales, particularité beaucoup plus accusée dans ces trois tribus que chez les Hidatsa et les Mandan, nous porterait à croire que ce trait caractéristique leur a été emprunté. Mais l'analogie ne va pas plus loin. Les Crow sont utérins, les Gros Ventres et les Pieds Noirs consanguins, et, ce qui est encore plus important, les unités des Crow ne sont pas localisées, mais se retrouvent dans chacune des subdivisions locales de la tribu. Les Gros Ventres et les Pieds Noirs n'ont pas pu faire dériver la base de leur organisation de celle des Crow, car cette base n'était pas la même, et vice versa. Ainsi, dans cette zone restreinte, il existe au moins deux types qui ont évolué séparément, ce qui en porte le total à trois à l'est des Montagnes Rocheuses. La question que nous nous posions sur la diversité d'origine des systèmes claniques dans le monde trouve donc ici une réponse affirmative; il serait bon toutefois de considérer les autres aires de l'Amérique du Nord. 
L'analyse serrée de Kroeber, chef-d'œuvre d'enquête ethnographique, a éclairé d'un jour tout nouveau l'état des faits au sud-ouest; c'est d'après lui que j'en tracerai les lignes essentielles. Les clans Zuñi sont exogamiques et tirent leurs noms d'animaux, de plantes et de phénomènes naturels, mais ils n'ont pas de tabou comme ceux de Buin et ne vénèrent pas leur éponyme. La descendance est utérine. Aux clans se rattachent des cérémonies, non dans le sens que chaque unité célèbre ses rites, mais plutôt que certaines fonctions rituelles déterminées doivent être remplies par des individus appartenant à un clan donné. Ces caractéristiques se retrouvent chez tous les Pueblo qui divergent surtout par l'absence ou la présence de phratries englobant des clans plus petits. Tandis que, sur le Rio Grande, nous rencontrons une organisation bipartite sans exogamie mais avec d'importantes fonctions rituelles et politiques, aucune division de ce genre n'apparaît dans la section occidentale de cette zone. Cette divergence, cependant, devient insignifiante si on la compare à l'extrême complication du système clanique que l'on retrouve dans toutes les tribus Pueblo, ainsi que Kroeber l'a fait ressortir pour la première fois. Les quatre groupes linguistiques de la région répartissent leurs clans en vastes agrégats d'après un plan identique et purement conventionnel. Ainsi les Hopi classent ensemble les clans du Blaireau et de l'Ours, et les Zuñi font de même pour les villages Keresan et Tanoan. De même, les clans du Feu et du Coyote sont toujours accouplés. L'identité est aussi parfaite que possible; là où une variante se produit, il ne s'agit en général que de la substitution d'un clan de la Corne à celui du Cerf, ou d'une espèce d'oiseau à une autre. Comme le dit Kroeber, « un plan unique et déterminé s'étend à toute l'organisation des Pueblo ». On ne trouve rien en dehors de la région qui s'y puisse comparer; le système de cette zone se détache donc comme une entité historique distincte de toutes les organisations claniques considérées jusqu'ici.

Cependant il est possible, quoique nullement certain, que l'organisation clanique de la Californie méridionale soit une dérivation atténuée du système des Pueblo, ainsi que le suppose Gifford. La concentration géographique des clans sur la partie de l'État qui touche plus ou moins à l'Arizona, parle en faveur de cette hypothèse qu'étaie encore un autre fait. Cette même région californienne se rattache, à d'autres égards aussi, à la zone pueblo, surtout en ce qui concerne la céramique. Néanmoins, une difficulté sérieuse s'oppose à l'hypothèse de Gifford : les clans californiens sont uniformément consanguins, alors que ceux des tribus Pueblo sont utérins. Si nous possédions une preuve évidente que les Californiens se conforment à la méthode si spéciale des Pueblo de répartir les clans, nous serions obligés naturellement de présupposer des rapports historiques et une modification dans la règle de descendance. Mais, au point où nous en sommes, nos preuves nous fournissent des indices plutôt que des conclusions et la question doit être pour le moment laissée en suspens.

Tout au nord, allant de l'extrémité méridionale de l'Alaska à la côte septentrionale de la Colombie britannique et aux régions adjacentes, se place le dernier centre de clans, représenté de la façon la plus caractéristique par les Tlingit, les Haïda et les Tsimshian. Les Tlingit sont divisés en deux phratries exogamiques : celle du Corbeau 
et celle du Loup (ou de l'Aigle); les Tsimshian, en quatre clans exogamiques dont deux s'appellent l'Aigle et le Loup, tandis que les deux autres portent des noms ne se référant pas à des animaux, quoiqu'ils se rattachent respectivement à l'ours et au corbeau. La descendance est matrilinéaire. Des séries de privilèges cérémoniels hautement prisés sont associés à chacune de ces divisions tribales; ils sont justement désignés du terme héraldique de " cimier » et incluent entre autres le droit exclusif d'employer des figurations de certains animaux ou objets. L'importance qu'on attache aux « cimiers » donne aux organisations de la côte du Nord-Ouest un caractère tout à fait à Part. On invoque souvent une parenté surnaturelle avec les animaux en question, mais les tabous et la vénération religieuse font complètement défaut. Ce n'est pas toujours de l'éponyme que dérive le principal « cimier » d'une phratrie ou d'un :clan. Ainsi les Corbeaux, chez les Haïda, ont pour cimier principal la Baleine et dans la même tribu, pour les Aigles, le cimier du Castor rivalise en importance avec celui de l'Aigle. Donc, pour ce qui est des relations historiques entre les divisions exogamiques des trois tribus, les cimiers sont plus significatifs que les désignations claniques; les Corbeaux des Tsimshian finissent par être l'équivalent des Aigles chez les Haïda. En outre, tous les ressortissants d'une section ne sont pas tenus d'avoir le même cimier. Il existe des subdivisions matrilinéaires dont chacune possède ses propres cimiers. En fait, ce sont les groupes restreints plutôt que les phratries qu'il nous faut considérer comme des clans dans le sens strict du terme, car ce sont eux surtout qui représentent des ensembles de parents réels ou putatifs tandis que les groupements plus vastes ne dérivent pas d'un ancêtre commun, excepté chez les Haïda. Chaque subdivision ou clan est un groupe localisé, autrefois sans doute une petite communauté matrilinéaire, maintenant dispersée en plusieurs villages.

L'organisation de la côte du nord-ouest ne ressemble à celle d'aucune des autres zones. Elle diffère le plus sensiblement du système des Prairies septentrionales auxquelles, s'il y avait eu diffusion, elle devrait se rattacher à cause de la proximité géographique. Nous avons déjà vu que le système des Prairies est radicalement distinct de celui des tribus toutes proches de l'est et de la région des Pueblo. On ne comprend plus dès lors comment des emprunts auraient pu se produire dans de telles conditions. Il n'est pas non plus possible de supposer que l'idée du clan sous une forme générique se soit répandue à un moment donné sur toute l'Amérique du Nord et se soit différenciée ensuite dans plusieurs zones, car, comme je le prouverai plus loin, le clan n'a jamais existé en dehors des régions mentionnées. Il nous reste donc à en conclure que le clan a évolué quatre fois au moins en Amérique du Nord et que son origine dans le monde est multiple. Je montrerai par la suite que diverses circonstances se rencontrant fréquemment favorisent le développement indépendant de la parenté unilatérale.

Chapitre 6

\section{c. Clans d'un ordre supérieur}


L'étude des systèmes claniques que nous venons de faire nous a révélé l'existence, dans une même tribu, de clans de divers ordres. Nous avons vu que les Winnebago se divisaient en deux phratries consanguines dont chacune se fractionnait en clans plus petits, consanguins aussi. En Colombie britannique, les groupes exogamiques des Tlingit et des Tsimshian comprennent chacun un certain nombre de clans utérins et peuvent eux-mêmes être désignés comme des clans, en étendant quelque peu le sens de ce terme, puisqu'il existe à l'intérieur de chacun un sentiment marqué de parenté, bien que sans croyance distincte en un ancêtre commun. Souvent l'unité la plus vaste a une personnalité plus effacée. Les treize clans des Crow se répartissent en cinq couples et un trio, mais les fonctions de ces groupes anonymes sont dénuées d'importance. Es ne sont pas exogamiques et les clans qui les constituent sont considérés comme unis par des liens, non de parenté, mais simplement d'amitié. De même, ces agrégats chez les Zuñi n'ont pas d'influence sur les mariages et fort peu sur la vie sociale, leur activité rituelle mise à part.

Lorsque coexistent ainsi de grandes et de petites unités, la question de leur parenté se pose inévitablement. On peut concevoir que les groupements plus vastes se soient développés par fractionnement à partir d'un clan originel, les fragments conservant encore la notion de leur unité première et restant cohérents comme les clans d'un agrégat clanique, qu'il s'agisse ou non d'une phratrie. Il est également concevable que des groupes sociaux, autrefois distincts, aient fini, pour certaines raisons, par s'unir, tout en conservant le souvenir de leur séparation première. Enfin il se peut que deux types d'organisation radicalement différents se soient coordonnés de telle manière qu'une unité de l'un des systèmes embrassât plusieurs unités de l'autre. Morgan s'est prononcé exclusivement en faveur de la première hypothèse et il faut reconnaître qu'il y a beaucoup à dire à l'appui de cette théorie. Elle semble a priori fort vraisemblable; rien ne paraît plus simple que la fragmentation d'un clan en cours de croissance en divers segments conservant entre eux une certaine cohésion. En outre, comme Morgan l'a fait remarquer, il existe des cas où les grands agrégats portent le nom d'une de leurs subdivisions et d'autres où plusieurs petits clans représentent diverses espèces d'un même genre désignant la grande division. Par exemple, dans l'organisation tripartite des Mohegan, l'un des tiers s'appelle Dindon et comprend des clans désignés respectivement par Dindon, Grue et Poulet; un autre tiers est appelé Tortue et se subdivise en Petite Tortue, Tortue de Vase, Grande Tortue et Anguille Jaune.

Un, autre argument que rend encore plus convaincante la grande importance que les Indiens de l'Est attachent aux séries de noms propres particulières aux clans, c'est que les Onondaga-Iroquois ont le clan de la Petite Bécasse et celui de la Grande Bécasse, ce qui, non seulement à cause de la similitude de désignation, mais aussi en raison de la possession commune d'un ensemble de noms propres, fait supposer qu'il y a eu scission. 
Néanmoins, si disposés que nous soyons à admettre l'hypothèse de la segmentation, il ne nous faut pas bannir à la légère les deux autres possibilités. Rien n'empêche qu'il ne se soit produit en divers temps et lieux soit la division d'une unité originelle, soit au contraire un amalgame d'éléments distincts. Les preuves de l'amalgame sont, en plus d'un cas, convaincantes et, parfois, tout au moins suggestives. On ne saurait mettre en doute que les clans des Crow ne soient les unités fondamentales de leur organisation sociale, étant donné le manque de signification presque total de leurs agrégats plus vastes. Il est pourtant facilement concevable qu'au cours des temps le lien entre des clans alliés se soit affermi au point que l'une après l'autre les fonctions du clan aient passé aux couples et au trio de clans. Dans le cas analogue des Hopi, mes informateurs m'ont répété à plusieurs reprises que certains clans étaient en rapport les uns avec les autres parce qu'ils s'étaient réunis une fois dans un but cérémoniel. Ici aussi on a pu facilement franchir la distance qui sépare l'agrégat mécanique de la synthèse organique des petits clans en clans d'un ordre supérieur. L'extension au nouveau groupe des termes de parenté employés cérémoniellement a pu contribuer à l'œuvre de consolidation.

Bref, il nous faut renoncer à une solution générale du problème et discuter selon ses mérites chaque cas où de grandes et de petites unités claniques coexistent dans une même tribu.

La troisième possibilité est de grand intérêt théorique surtout. Il est aisé d'appliquer à la comparaison des divers clans d'une seule tribu les principes que nous avons adoptés pour comparer les clans de diverses tribus et de prouver que ce sont des unités d'un caractère tout différent. Prenons l'exogamie, par exemple, l'une des fonctions les plus importantes et les plus répandues. Si la grande unité est exogamique, ses éléments composants doivent l'être aussi par nécessité logique; on peut donc affirmer que la petite unité n'est pas réellement exogamique et diffère dans son essence, par conséquent dans son origine, du groupement plus vaste. La grande unité, au contraire, peut ignorer l'exogamie et sera traitée comme un phénomène distinct du clan exogamique. De tels arguments sont difficilement réfutables, mais ils ne réussissent pas non plus à établir la diversité historique du clan restreint ou étendu. En effet, si le processus de la segmentation, par exemple, se produisait selon l'opinion de Morgan, il s'ensuivrait inévitablement que les petits clans ne seraient jamais exogamiques que par dérivation.

Il nous faut à ce propos envisager un autre facteur qui tend à obscurcir les relations historiques entre le clan restreint et le groupement plus vaste : les fonctions peuvent se transmettre de l'un à l'autre. Ainsi Goldenweiser est d'avis que les phratries des Iroquois étaient autrefois exogamiques, alors qu'actuellement cette particularité se limite au clan restreint. Au contraire, les fonctions de la petite unité peuvent s'étendre à la grande et si ces influences réciproques s'exerçaient pleinement, il serait impossible de reconstruire l'historique d'un cas donné. 
Le type de beaucoup le plus intéressant de grand clan est la phratrie qui se rencontre aussi en unité indivise, comme parmi les Miwok et les Yokuts de la Californie centrale. Il convient de répéter une fois de plus que les phratries ne sont pas nécessairement exogamiques. Celles des Hidatsa n'ont rien à voir avec les mariages et les phratries des Toda sont même positivement endogamiques. Néanmoins, c'est l'organisation bipartite exogamique qui est la plus fréquente et qui, par conséquent, occupera surtout notre attention.

Lorsque nous ne sommes en présence que de deux divisions exogamiques, il en découle logiquement certaines conséquences qui différencient nettement l'organisation par phratrie des systèmes qui se divisent en plus de deux clans exogamiques. Là où le nombre des clans exogamiques est supérieur à deux (à moins que n'interviennent des prescriptions spéciales), l'homme peut épouser une femme de n'importe quel clan sauf du sien. Ainsi un Crow peut se marier avec une ressortissante des douze autres clans; douze treizièmes des femmes nubiles de sa tribu lui sont des épouses potentielles. Mais, avec deux divisions exogamiques, un Winnebago doit limiter son choix à la moitié seulement des femmes de son peuple, ce qui fait une différence considérable. Il existe encore une autre conséquence de portée sociale. Parmi les Crow, un homme a des relations particulières avec les membres de deux clans, du sien propre (c'est-à-dire de celui de sa mère) et de celui de son père; mais le nombre de ces individus relativement à la population totale est faible. Dans le système des phratries, on peut avoir des rapports spéciaux avec tous les membres de sa tribu, puisqu'une moitié appartient à sa propre phratrie, et les autres à la phratrie de celui de ses progéniteurs qui n'intervient pas dans la descendance.

Un trait caractéristique des phratries est le développement des services réciproques. Lors d'un enterrement chez les Iroquois, les rôles ne sont jamais distribués dans la phratrie du défunt, mais dans l'autre, et il en va de même chez les Cahuilla de la Californie méridionale. Sur la côte nord de la Colombie britannique, certaines fêtes ne sont célébrées qu'en l'honneur de la phratrie complémentaire. La question de savoir à quoi tient cette réciprocité est assez embarrassante. S'explique-t-elle par l'essence de la phratrie ou n'en est-il ainsi qu'incidemment, parce que l'une des phratries inclut le père ou la mère? Le cas des Hidatsa est significatif en ce qu'ici, dans un enterrement, ce ne sont pas les phratries non exogamiques qui fonctionnent, mais le clan du père du défunt. Il se peut donc que les phénomènes relevés chez les Iroquois et sur la côte nord-ouest appartiennent à la même catégorie et que, dans ces tribus matrilinéaires, la réciprocité des services signifie simplement la reconnaissance sociale du père. Nous avons cité d'autres fonctions des phratries. Celles des Iroquois sont caractéristiques des Indiens de l'est. A certains jeux, comme à la crosse, les ressortissants des deux phratries s'affrontent les uns les autres. Lors des fêtes et des cérémonies, il se produit un groupement spatial analogue; chacune des phratries, représentée par un porteparole, s'oppose à l'autre.

Nous avons vu plus haut qu'il existe une certaine adaptation de la nomenclature de parenté du type dakota à l'organisation clanique. Cette adaptation est particulièrement 
marquée lorsque les clans apparaissent sous forme de phratries. Certaines particularités de la terminologie dakota pourraient s'harmoniser également avec n'importe quel autre système de clan et quel que fût le nombre des unités. Ainsi le fait que les frères du père et même ses cousins plus éloignés sont tous désignés du même terme que le père, s'ils appartiennent au même clan, pourrait se produire aussi bien dans une tribu de cinquante clans que de deux. Mais d'autres particularités ne peuvent s'expliquer aussi facilement dans une organisation clanique multiple. Prenons par exemple l'identification fréquente du frère de la mère et du mari de la sœur du père. Dans des phratries exogamiques à descendance utérine, par exemple, j'appartiens à $\mathrm{A}$, la phratrie de ma mère, ainsi que mon oncle maternel; mon père, d'autre part, et sa sœur sont de la phratrie B et cette dernière est obligée d'épouser un homme de A. Ainsi le mari de la sœur du père et l'oncle maternel sont forcément de la même phratrie et si la nomenclature reflète à l'origine l'organisation sociale, il est normal que tous deux soient désignés du même terme.

La classification des cousins en cousins parallèles et croisés est encore plus fréquente. Comme Tylor l'a fait remarquer, ce point s'accorde aussi admirablement avec une organisation bipartite. Supposons les mêmes conditions que plus haut, nous verrons que si un homme appartient à la phratrie $\mathrm{A}$, son frère sera aussi du même groupe; tous deux doivent épouser des femmes de B et leurs enfants seront aussi B. De même, deux sœurs de A épouseront des hommes de la phratrie B et leurs enfants seront de A. C'est dire que les cousins parallèles, enfants de plusieurs frères ou de plusieurs sœurs, appartiennent au même groupe social. Il n'en va pas de même pour les cousins croisés. En effet, bien qu'un frère et une sœur de A doivent épouser des membres de la phratrie opposée, les enfants du frère appartiendront, en vertu de la descendance utérine, à la phratrie $\mathrm{B}$, alors que ceux de la sœur resteront dans le même groupe que leur mère, soit $\mathrm{A}$.

Mais la situation change si nous ajoutons un clan seulement. Un homme du clan A peut alors épouser une femme de B ou de C; les femmes et par conséquent les enfants de plusieurs frères appartiendront donc en partie à $\mathrm{B}$ et en partie à $\mathrm{C}$ et il n'y aura aucune raison pour qu'ils soient classés ensemble. Ainsi la présence de plus de deux clans est loin d'expliquer la forme la plus commune de la terminologie dakota aussi bien que le fait la double organisation. C'est pour cette raison, entre autres, que certains sociologues tiennent la phratrie pour le système clanique le plus ancien.

Cette théorie est extrêmement séduisante. Outre qu'elle explique la nomenclature dakota, l'organisation bipartite est certainement la plus simple qui se puisse concevoir. Néanmoins d'importantes objections se présentent. Tout d'abord, la répartition en phratries n'est pas du tout aussi répandue que le type de terminologie de parenté auquel elle semble correspondre. L'absence d'organisation bipartite exogamique dans la plupart et peut-être dans toutes les régions d'Afrique et parmi beaucoup de tribus américaines et asiatiques nous oblige à hésiter avant de donner notre assentiment à l'hypothèse selon laquelle la division bipartite serait la forme première de toutes les organisations claniques. C'est peut-être vrai pour l'Australie et la Mélanésie, mais cela 
ne prouve rien quant au reste du globe. On peut naturellement supposer que les phratries ont existé autrefois là où nous trouvons actuellement des clans en plus grand nombre, mais c'est compliquer arbitrairement la théorie par une hypothèse auxiliaire dénuée de fondement pour préserver l'interprétation la plus simple du contact brutal des faits. Nous pourrions aussi mettre en doute, et avec raison, que l'organisation bipartite soit réellement pour les primitifs la forme la plus simple. Logiquement elle l'est sans doute, et historiquement elle le serait aussi si le développement des clans dans une tribu primitive s'était toujours opéré par scission. Mais si, comme cela paraît vraisemblable en bien des cas, les organisations exogamiques se sont développées par la fusion d'éléments originairement distincts, il est beaucoup plus normal que les relations matrimoniales ne soient pas restreintes à un seul groupe, mais étendues à plusieurs; la réunion de tous ces groupes formerait alors la communauté caractéristique, organisée en un nombre indéfini de clans exogamiques qui pourraient dans la suite se répartir en phratries opposées.

Tout ceci n'est pas un état de choses purement hypothétique. Parmi les Toda, les phratries sont endogamiques, mais subdivisées en clans consanguins exogamiques; chaque phratrie correspond donc à l'ensemble d'une tribu exogamique. Or dans la phratrie Teivaliol qui comprend six clans, celui qui est appelé Kuudr est numériquement prépondérant, à tel point que, pour ne pas enfreindre la loi exogamique, ses ressortissants ont épousé presque tous les membres disponibles des autres clans, ne laissant qu'à très peu la possibilité de se marier entre eux. Rivers signale 161 mariages entre gens de Kuudr et le reste de Teivaliol contre 16 entre membres des cinq autres clans exogamiques. « Grâce au développement énorme de l'un des clans, la division Teivali est presque devenue une communauté à organisation matrimoniale bipartite dans laquelle chaque ressortissant d'un groupe doit épouser un membre de l'autre groupe; cependant, nous n'avons aucune raison de chercher là une autre cause que l'excessif développement numérique de l'un des clans. »

Le phénomène dont se rapprochent les Toda, soit une répartition secondaire en deux unités complémentaires, est discernable, selon Boas, chez les Haïda de la côte nord-ouest de l'Amérique. Boas émet la supposition que les diverses tribus de cette zone se caractérisaient autrefois par une organisation tripartite. Ainsi, en quelques localités encore, les Tlingit ne sont pas strictement organisés en phratries, mais possèdent une troisième unité exogamique dont les membres se marient librement avec ceux des deux autres. B se peut donc que les phratries des Tlingit et des Haïda soient le résultat d'une réduction en nombre des unités originales, ne laissant plus que deux divisions tribales équivalentes.

Citons enfin le cas des Massaï. Dans l'Afrique orientale anglaise, HOLLIS a découvert quatre clans, Aiser, Meñgana, Molelyan et Mokesen; à l'exception du dernier, ils furent aussi signalés par le capitaine Merker sur territoire allemand, où, cependant, Aiser et Molelyan sont alliés en quelque sorte contre les gens de Meñgana qu'ils appellent les "Gloutons » à cause d'une transgression légendaire d'un tabou alimentaire. Ce groupement n'est apparemment pas essentiel et n'a affecté en rien les 
règlements de mariage. Néanmoins il montre bien comment une organisation bipartite peut prendre naissance en tant que phénomène tardif et non originel.

Tous ces faits concrets, ainsi que les considérations générales établies plus haut, nous induisent à rejeter la théorie selon laquelle la phratrie représente fréquemment ou même nécessairement le type le plus ancien d'organisation clanique. Le fait que l'organisation bipartite s'accorde mieux avec la terminologie dakota que toutes les autres formes d'organisation clanique ne doit pas nous faire conclure qu'elle est uniformément la plus ancienne, et négliger ainsi les indices de diffusion, de même que, çà et là, les races d'une origine secondaire des phratries.

Chapitre 6

\section{d. Totémisme.}

$\underline{\text { Retour à la table des matières }}$

Si brève qu'ait été notre étude des organisations claniques, elle a suffi à nous montrer combien fréquemment les clans portent des noms d'animaux et de plantes auxquels se substituent parfois, bien que plus rarement, ceux d'autres phénomènes naturels. Ce mode de désignation s'accompagne souvent de croyances et de pratiques se rattachant aux éponymes. Parfois, comme à Buin, l'animal est tenu pour sacré et il existe dans le clan un sentiment de parenté avec lui fortement enraciné. Ailleurs, comme chez les Kariera, les groupes ne sont pas désignés d'après des plantes et des animaux, mais leur sont néanmoins associés de façon définie, par exemple par la célébration de rites pour la multiplication magique de la faune ou de la flore en question. Le clan croit fréquemment qu'il descend de son éponyme. Toutes ces coutumes et d'autres analogues sont rangées dans la catégorie du totémisme, et l'animal, l'objet ou la plante dont il s'agit est appelé totem. Parmi les Arunta, le groupe se rattachant à un animal ou à une plante n'est pas un clan puisque le fait d'en devenir membre dépend, non de la naissance, mais de la croyance de la mère qui déclare que son enfant est la réincarnation de tel esprit totémique. Des frères et sœurs sont ainsi souvent membres de différents groupes. Pourtant, ces groupes totémiques ressemblent tellement par leur activité à ceux des tribus voisines où ils se confondent avec le clan que nous ne pensons pas devoir exclure les manifestations des Arunta de la catégorie du totémisme.

Le totémisme est très répandu; on le trouve en effet en Amérique, en Australie, en Mélanésie, en Afrique et en quelques régions de l'Asie. Cette vaste diffusion a profondément impressionné les premiers sociologues qui ont étudié la question. Obéissant aux préjugés théoriques de leur temps, ils tinrent pour acquis que tous les phénomènes classés dans la catégorie du totémisme représentaient des processus psychologiques identiques qui, grâce à l'unité psychique de l'humanité, avaient pris nais- 
sance indépendamment dans les diverses aires. Il est devenu de mode actuellement de contester la possibilité de formations culturelles indépendantes; le professeur Elliot Smith soutient même que le totémisme s'est développé dans le nord-est de l'Afrique et de là s'est répandu dans le monde entier.

Si opposées que ces interprétations nous paraissent au premier abord, elles s'appuient sur une base commune, à savoir que le totémisme est partout le même dans son essence. C'est cette croyance naïve qui a conduit à une série d'hypothèses destinées à expliquer l'origine du totémisme en général, les variantes étant traitées en quantité négligeable. Il y avait par exemple une théorie faisant dériver le totémisme de l'habitude de décerner des surnoms d'animaux : ces appellations auraient donné naissance à des légendes explicatives faisant descendre de l'éponyme le porteur du sobriquet, d'où serait résulté le tabou défendant de tuer ou de consommer un membre de l'espèce. Ce furent ces prohibitions qui éveillèrent l'intérêt et qui donnèrent à croire que le totémisme était une forme de religion et même une phase nécessaire dans l'évolution religieuse. D'autre part on souligna le caractère exogamique du clan totémique comme « l'aspect social » du phénomène, qui, cependant, se déduisait directement de la croyance en un ancêtre totémique commun.

Ce fut en 1910 que Goldenweiser s'occupa de la question d'un point de vue tout différent. Boas a démontré à plusieurs reprises que des phénomènes ethnographiques apparemment simples sont en fait le résultat, non d'une unité originaire, mais d'une association secondaire. Ainsi, lorsque des tribus primitives désignent par des noms d'animaux des dessins géométriques, il ne s'ensuit pas que leurs artistes essaient de représenter des animaux et que leurs esquisses se stylisent par la suite en losanges ou en triangles. On peut parfois prouver que ces motifs ont une origine indépendante et n'ont reçu qu'ultérieurement leur interprétation. Boas a prouvé d'autre part que les ressemblances signalées entre tribus éloignées étaient souvent illusoires : elles ne représentent aucune similitude essentielle, comparable aux homologies de l'anatomiste, mais correspondent plutôt à des analogies superficielles. Les ethnologues se sont laissé entraîner dans des erreurs du même ordre que celles qui font classer par les esprits non avertis la baleine parmi les poissons ou la chauve-souris avec les oiseaux.

Par l'application de ces principes à ce qu'on avait considéré jusqu'alors comme un complexe uniforme, Goldenweiser a découvert que le totémisme, loin d'être partout le même, diffère selon les lieux à un degré extraordinaire. Par exemple, le totémisme de l'Australie centrale, avec l'importance qu'il attache aux célébrations rituelles, diverge toto coelo de celui de la Colombie britannique où la représentation artistique du totem et l'idée de l'esprit gardien sont au premier plan de la conscience indigène. Passant en revue les uns après les autres les traits caractéristiques du totémisme, Goldenweiser a démontré qu'aucun n'est universel. L'exogamie peut ou non s'accompagner d'autres particularités; les tabous totémiques peuvent être dissociés du nom désignant le groupe qui les observe; on peut croire ou non en une parenté avec le totem, et ainsi de suite. 
Goldenweiser ne trouve ainsi aucun trait, ou ensemble de traits, qui caractérise le totémisme, aucun donc qui puisse être considéré dans un sens historique ou psychologique comme essentiellement originel. Or, si quelque lien unit les manifestations totémiques des divers peuples, il ne peut consister en la possession de certaines particularités, mais uniquement dans les relations mutuelles de celles-ci. Ici Goldenweiser revient à l'ancienne distinction entre la phase sociale du phénomène et sa phase religieuse. Mais son enquête a démontré que le facteur religieux est souvent des plus atténués. Aussi lui a-t-il substitué dans ses conclusions un terme moins prétentieux et a-t-il défini le totémisme comme " la tendance d'unités sociales déterminées à s'associer avec des objets et des symboles de valeur émotionnelle », ou encore le "processus de socialisation spécifique d'objets et de symboles de valeur émotionnelle ». La socialisation de valeurs émotionnelles à l'intérieur d'un groupe observant telle ou telle descendance, cette définition empêche le totémisme de devenir une dénomination ne correspondant à aucune réalité. Alors que l'unité psychologique du phénomène était ainsi sauvegardée, on soulignait sa diversité historique. A cause du caractère hétérogène du complexe totémique et de la diversité de son évolution historique, Goldenweiser en arriva à la conclusion que les analogies étaient le résultat d'une évolution convergente, c'est-à-dire que, dans des régions différentes, le totémisme avait eu des points de départ distincts et que ce n'est que par la combinaison subséquente de ces éléments que les complexes avaient fini par présenter certaines similitudes. On expliqua facilement la combinaison fréquente de certains éléments particuliers par l'abondance de phénomènes comme les tabous ou les noms dérivés d'animaux qui faisait de leur rencontre une question de probabilité mathématique.

Avant de commenter ce point de vue, il nous faut signaler rapidement la publication de J. G. Frazer qui parut en même temps que l'étude de Goldenweiser, mais qui est de volume beaucoup plus considérable. Une compilation laborieuse de tous les faits se rapportant au sujet y est suivie d'une théorie toute différente du totémisme. En ce qui concerne quelques points spéciaux, il est vrai, ces deux auteurs tombent d'accord dans leurs conclusions. Frazer, comme Goldenweiser, dissocie l'exogamie comme étant une partie non essentielle du complexe totémique. Là où Frazer s'écarte nettement de la méthode de son collègue, c'est lorsqu'il fait dériver le complexe totémique, moins l'exogamie, d'une source psychologique unique : la croyance régnant dans le centre de l'Australie selon laquelle chaque enfant est la réincarnation de l'esprit totémique qui hante les lieux où la mère s'est pour la première fois aperçue de sa grossesse. Alors que cette croyance semble à Frazer une explication suffisante des tabous, de l'ancêtre commun, etc., Goldenweiser considère ces phénomènes résiduels comme non moins indépendants les uns des autres que l'exogamie l'est d'eux tous. Frazer, en d'autres termes, présuppose un lien interne entre les divers symptômes du totémisme (excepté l'exogamie) et envisage l'un d'eux, l'identification du totem et de son descendant, comme essentiel. Goldenweiser ne tient aucun des symptômes pour fondamental, et leur combinaison n'est pour lui qu'une conjonction dans le sens que Hume donne à ce terme, une réunion empirique de Particularités plutôt qu'une synthèse organique. Par l'importance qu'il donne aux simples rapports des éléments les uns avec les autres, il s'oppose diamétralement à l'adhésion que Frazer apporte à un 
système particulier. En outre le choix que fait ce dernier de la croyance australienne comme noyau de tout le totémisme semble à Goldenweiser le comble de l'absurde. Celui-ci concède que la théorie de Frazer s'applique à la rigueur à l'Australie centrale, mais qu'elle ne saurait se soutenir dans d'autres régions où jamais des croyances comparables n'ont été signalées.

Les phénomènes totémiques que nous avons énumérés au hasard, lors de notre étude de l'organisation clanique, suffisent à corroborer les résultats négatifs de l'analyse aiguë de Goldenweiser. Qu'est-ce qui rattache le totémisme des Kariera à celui de Buin ? Les groupes totémiques des Kariera sont des clans locaux sans noms, à totems multiples, lesquels ne sont ni tabou ni vénérés, mais seulement renforcés magiquement. Pour les gens de Buin, au contraire, les animaux de chaque clan sont si sacrés que toute offense d'un autre clan doit être vengée; d'autre part, ils n'ont pas de rites pour la propagation de l'espèce. Dans un cas, il n'y a aucune parenté entre le totem et le clan; dans l'autre, il existe une base légendaire à la notion de parenté, quoiqu'on ne croie pas en un ancêtre commun. Les Winnebago enfin reconnaissent la descendance totémique, possèdent des noms totémiques pour les groupes aussi bien que pour les individus, ainsi que des représentations artistiques des totems, mais ignorent les tabous ou le culte. Nous n'avons pas la moindre raison de supposer que des phénomènes aussi hétérogènes puissent avoir une origine commune historique ou psychologique; et si tous peuvent cependant être traités de totémiques, ce ne sera pas à cause de quelque trait spécifique commun mais en raison de cette ressemblance formelle abstraite qu'a définie Goldenweiser.

Cependant la définition que donne Goldenweiser du contenu totémique, si atténuée qu'elle soit, est-elle suffisante pour s'appliquer à tous les cas? Je n'en suis pas convaincu. Les totems de Buin représentent à coup sûr des valeurs émotionnelles; ceux des Winnebago peut-être aussi; ceux des Kariera certainement pas. Pourquoi donc ne pas nous contenter de noter que quelques groupes sociaux à l'intérieur d'une tribu se différencient fréquemment par des noms souvent empruntés aux règnes organiques, par des emblèmes héraldiques d'origine analogue ou par des tabous distinctifs, etc.? Pourquoi ne pas renoncer à ce vain effort qui consiste à vouloir faire tenir dans une seule définition, ainsi qu'en un lit de Procruste, tout un système d'appellations, d'héraldique, de pratiques religieuses et magiques. Chacun de ces systèmes est à étudier séparément et, lorsque des similitudes se présentent, il faut aussi les analyser rationnellement. La variété de tous ces phénomènes ne doit pas être voilée par la notion captieuse de « valeur émotionnelle ».

Dans une publication ultérieure, Goldenweiser a jeté sur le problème une vive lumière par son analyse serrée du soi-disant totémisme des Iroquois. Les clans iroquois sont désignés d'après des oiseaux et des bêtes et représentent actuellement des unités exogamiques, quoique autrefois, sans doute, ces dernières fussent le caractéristique du clan étendu ou phratrie. Mais aucun tabou ne défend de tuer l'animal clanique et la simple idée d'une telle prohibition paraît absurde à ces Indiens. Il n'existe pas trace non plus de la croyance en une parenté commune avec l'éponyme. L'un des 
traits les plus saillants du système iroquois est l'existence de séries de noms propres, chaque clan possédant sa série particulière; cependant ces noms ne se rattachent nullement à des animaux. Une représentation gravée de l'éponyme était, il est vrai, placée au-dessus de la porte de chaque maison, selon que tel ou tel clan y prédominait; il n'existe cependant aucune preuve que le droit de posséder ces effigies fût restreint aux membres du clan. Les clans des Iroquois doivent-ils donc être tenus pour totémiques? Ces Indiens possèdent-ils un complexe totémique? Goldenweiser répond négativement à ces questions. Les noms d'animaux, dit-il, sont trop courants dans les sociétés primitives pour nous permettre d'en déduire des relations spéciales entre l'espèce et le groupe qui en tire son nom. C'est seulement lorsque le nom implique « une association psychologique avec l'animal dans l'esprit de celui qui donne ou qui reçoit le nom ou de tous les deux », ou lorsque l'exogamie s'associe à cette institution, qu'on peut parler de totémisme. En cas contraire, les clans à désignations animales ne doivent pas plus prétendre à être tenus pour totémiques que les clans à sobriquets, noms locaux ou appellations dérivées d'un ancêtre humain.

Comme Goldenweiser le souligne à juste titre, ce n'est pas une simple question de terminologie. Il s'agit au fond de savoir si le seul fait de désigner des groupes d'après des animaux équivaut à des croyances définies et à des pratiques se rattachant aux éponymes. L'importance du nom dans l'esprit indigène est certaine, mais ce n'est que par l'étude empirique des différents cas qu'on pourra lui associer éventuellement un complexe totémique. La réponse négative de Goldenweiser paraît éminemment raisonnable. Accomplir des rites afin de faire se multiplier une espèce comestible n'est pas du tout la même chose que regarder un animal ou une plante avec une terreur superstitieuse et cette dernière attitude diffère à son tour du simple tatou.

Dans un travail encore plus récent sur ce même sujet, Goldenweiser estime que le totémisme est, après tout, un phénomène spécifique, caractérisé par « l'association du contenu totémique avec un système clanique ». Telle est la conclusion à laquelle aboutit son exposé historico-géographique. Goldenweiser soutient donc à présent que le clan et le complexe totémique sont presque indissolublement liés, les complexes sans clans et les clans sans complexes étant « très rares ».

Je considère ce point de vue comme fort malheureux et en contradiction avec quelques-uns des meilleurs résultats auxquels Goldenweiser était parvenu dans ses travaux antérieurs. Pour moi, il est absolument inconcevable que cet auteur, étant donné les faits, qu'il a cités en partie lui-même dans son étude critique, puisse faire intervenir des arguments historiques et géographiques à l'appui de cette étonnante généralisation. Les Crow, les Hidatsa, les Gros Ventres, les Apache ont tous des clans sans noms totémiques; et, puisque Goldenweiser ne revient pas sur sa conclusion antérieure établissant que le nom en lui-même n'implique pas le totémisme, nous pouvons ajouter à cette liste les Iroquois et quelques tribus Pueblo. Quelle est la tribu sibérienne qui soit organisée en clans totémiques? Et dans quel sens les groupes totémiques des Arunta sont-ils des clans? La conclusion affirmant que le totémisme est « un complément presque universel de l'organisation clanique » n'est obtenue qu'au prix 
de l'ignorance de faits brutalement contradictoires. Il est regrettable qu'un penseur aussi fin et aussi érudit se soit laissé entraîner dans ses derniers travaux hors de la voie droite mais étroite de l'investigation historico-ethnographique.

Pour résumer ma position personnelle sur le sujet du totémisme, je déclare que je ne suis pas convaincu qu'en dépit de la perspicacité et de l'érudition qu'on a dépensées à ce propos, la réalité du phénomène totémique ait été démontrée. Boas a raison assurément de faire remarquer la tendance de quelques groupes à s'associer avec « certains types d'activité ethnique ». Mais c'est simplement énoncer ce qui est évident par soi-même, à savoir que des groupes sociaux déterminés n'existent pas in vacuo, pour ainsi dire, mais doivent être caractérisés par telle ou telle fonction. La question est de savoir si la nature des activités associées au groupe est sans importance, pour autant qu'il y ait une association quelconque, mais c'est là une opinion à laquelle je ne peux souscrire. Pour moi le problème du totémisme se résout en une série de problèmes spécifiques, sans relations les uns avec les autres. L'un de ces problèmes est posé par l'association des noms d'animaux et des clans et, là où elle apparaît, dans une zone continue comme à l'est des États-Unis, les conséquences historiques sont évidentes : il y a eu emprunt du mode de désignation clanique. Ce phénomène n'a absolument rien à voir avec les rites de multiplication des Kariera et des Arunta, mais le fait que cette dernière pratique est commune aux tribus du centre et de l'ouest de l'Australie revêt une grande importance et implique des données historiques également évidentes. La notion de tabou que les clans attribuent souvent à leurs éponymes est un phénomène psychologiquement fort intéressant qui mérite d'être étudié. Mais il ne saurait résulter que confusion de la réunion sous une seule rubrique de matières disparates.

Chapitre 6

\section{Références.}

$\underline{\text { Retour à la table des matières }}$

1) PHILBRICK : 114. RIVERS, 1914 (a). LOWIE, 1915 : 223 et suiv.; id., 1917 (b) : chap. V.

2) LOWIE, 1912 : 182-246. RADIN, 1915. GIFFORD, $1916: 140-148$. CASTRÉN, 1853 : 286 et suiv. THURNWALD, 1912 : 316,327. BROWN : 145,160.

3) MORGAN, 1877 : Il, chap. XV. SKINNER : 8-21. FLETCHER et La FLESCHE : 38, 134-198. DORSEY, J. O. : 252. SWANTON, 1912 : 593 et suiv. LOWIE, 1912 : 186-207; id., 1917 (a) : 722. WISSLER, 1911 :18. KROEBER, 1908 :147 et suiv.; id., 1917 (a) : 91-150. GIFFORD, 1918 :155-218. BOAS, 1916 (a) : 478-530.

4) GOLDENWEISER, 1912 : 464 et suiv. GIFFORD, 1918 : 187. RIVERS, 1906 : 507. BOAS, 1916 (a) :478-530. HOLLIS, 1905 : 260. MERKER : 16-18.

5) SMITH : 33. GOLDENWEISER, 1910; id., 1913 : 370; id., 1918 280. BOAS, 1911 : chap. VIII; id., 1916 (b) : 319. FRAZER, J. G., 1910: IV, 3-71. 


\section{HISTOIRE DU CLAN}

$\underline{\text { Retour à la table des matières }}$

La famille bilatérale est une institution absolument universelle; d'autre part le clan ne connaît qu'une diffusion limitée, quoique relativement vaste. Beaucoup des nations les plus civilisées du monde, il est vrai, comme les Grecs, ont traversé une période d'organisation clanique. Donc, parvenu à un certain degré, le système clanique tend à disparaître et à abandonner le terrain à la famille existant parallèlement, mais sans qu'il soit prouvé par là que le clan est plus ancien. L'étude des faits nous démontre que la famille est présente à toutes les phases de la civilisation, qu'à un niveau culturel un peu élevé elle s'accompagne fréquemment d'une organisation clanique et qu'à un niveau plus élevé encore le clan disparaît.

\section{a. Antériorité de la famille.}

Le simple exposé de ces faits, cependant, s'oppose à l'une des théories les plus généralement admises et les moins autorisées de Morgan. Celui-ci tient la famille pour un produit tardif qui a été presque uniformément précédé du clan. Pour lui, le clan exogamique représente un remarquable mouvement de réforme qui mit des obstacles aux mariages entre Parents, s'implanta grâce aux heureux résultats biologiques remarqués et se répandit en conséquence sur une aire immense. Deux éléments de cette théorie demandent à être examinés : les effets supposés du système clanique et sa prétendue distribution géographique. 
Nous serons bref sur le premier point. Outre le fait que la biologie moderne n'est pas du tout certaine des résultats nocifs des mariages entre parents, d'autres difficultés évidentes s'opposent à l'interprétation de Morgan. Nous n'objecterons pas que l'exogamie clanique n'empêche pas l'union du père et de la fille dans une société consanguine, ni celle de la mère et du fils dans les groupements utérins. Morgan croit, en effet, que les relations incestueuses entre enfants et parents ont déjà été éliminées à une phase antérieure et que le clan n'a fait qu'augmenter les restrictions matrimoniales, les étendant à l'union entre frères et sœurs. Mais ce dont il nous faut faire grief à Morgan, c'est qu'il ne réalise pas que l'exogamie fait bien plus que seulement proscrire l'union entre frères et sœurs : elle empêche les relations sexuelles entre certains cousins fort éloignés et même entre membres du clan non apparentés les uns aux autres, tandis qu'elle ne s'oppose pas au mariage entre cousins du premier degré n'appartenant pas au même clan. L'union entre cousins croisés s'accorde parfaitement avec l'exogamie clanique, ainsi que les mariages entre certains cousins parallèles. Par exemple, dans une tribu matrilinéaire tripartite, deux frères du clan A peuvent épouser respectivement des femmes des clans B et $\mathrm{C}$ et leurs enfants s'uniront sans enfreindre la règle. En réalité le clan interdit l'union de certains proches parents ainsi que celle de parents plus éloignés et putatifs, tout en permettant le mariage de certains autres proches parents.

L'affirmation relative à la présence presque universelle du clan dans les sociétés primitives demande à être analysée plus longuement. J'essaierai d'établir la proposition contraire, à savoir que le clan fait défaut précisément dans les tribus les plus primitives et apparaît en règle générale seulement lorsque l'agriculture ou l'élevage ont totalement ou en partie supplanté la chasse comme base de l'existence économique. A mon avis, c'est la preuve directe de l'antériorité chronologique de la famille. Cependant, afin de donner plus de force à mon argumentation, je crois nécessaire de réfuter d'abord une critique qu'on ne manquera pas de me faire.

Il est notoirement connu que les peuples n'avancent pas uniformément dans les diverses branches de la civilisation. Us Esquimaux suscitent notre admiration par leur ingéniosité mécanique, pourtant leur vie sociale et politique est des plus grossières. Au point de vue architectural, les Maya du Yucatan dépassent, et de beaucoup, les nègres africains, mais leur ignorance de la métallurgie les rabaisse à d'autres égards à un niveau inférieur. Il est donc concevable qu'un peuple très peu développé puisse en distancer d'autres en ce qui a trait à l'organisation sociale et s'élever rapidement aux hauteurs vertigineuses que représente pour Morgan la famille. Ainsi, a-t-on affirmé, l'avancement matériel n'est pas un critère de progrès social et nous ne pouvons pas admettre d'une façon générale que ce sont les tribus dont la culture générale est des moins évoluées qui représentent aussi la forme la plus primitive d'organisation sociale.

Cependant l'application rigoureuse de cette opinion, légitime en elle-même, devient manifestement absurde. Sans doute, nulle phase de culture ne peut être détermi- 
née absolument par telle autre et nous pouvons nous attendre à ce qu'un retard dans une certaine activité soit compensé par une avance ailleurs. Pourtant un moment de réflexion suffira à nous faire voir qu'une corrélation entre les diverses branches existe, bien que non absolue. Il n'y a pas d'exemple qu'une peuplade de chasseurs ait jamais élevé des merveilles architecturales comparables à celles du Pérou et du Yucatan; pour le progrès scientifique et artistique, un certain degré de connaissances techniques, impliquant à son tour une division sociale du travail, est une condition sine qua non, et ainsi de suite. On peut donc admettre que, parfois, une culture très grossière aille de pair avec un appareil social anormalement compliqué. Mais croire que toutes les tribus qui passent pour les plus basses sur l'échelle de la civilisation aient pu atteindre le niveau le plus haut dans la phase culturelle représentée par la famille, alors que d'autres tribus plus avancées seraient uniformément restées en arrière à cet égard, c'est une proposition si monstrueuse que s'en faire une idée nette, c'est reconnaître qu'elle n'a aucun sens. Donc, si nous observons que la famille apparaît et que le clan fait défaut dans les cultures les plus primitives, et cela presque sans exception, nous obéirons à la voix de la raison en concluant que la famille précède le clan. Cette conclusion est d'une telle importance fondamentale que sa base empirique doit être exposée en détail.

Le premier à partir en campagne contre le dogme reçu fut John R. Swanton, le brillant défenseur du principe de l'estimation culturelle. Il se borna à discuter les faits de l'Amérique du Nord, et je crois qu'il n'est personne qui ne se soit sur ce terrain rangé à son opinion. Swanton a démontré de façon décisive qu'à toutes les civilisations indiennes peu développées le système des clans fait virtuellement défaut, alors qu'au contraire il apparaît dans les tribus possédant une plus riche organisation économique, industrielle, rituelle et politique. Ainsi l'immense région sans clans du nord de la Californie, Oregon, Washington, Idaho, Nevada, Utah, ainsi que tout le nord-ouest du Canada, à l'exception de l'étroite bande côtière et de son hinterland immédiat, représentent uniformément la plus basse couche humaine du continent américain. Les Paviotso errant dans les déserts du Nevada en quête de racines comestibles ne sauraient être comparés une minute aux Iroquois, Hopi ou Tlingit sédentaires, sous le rapport des conditions générales de leur civilisation.

On pourrait se demander comment il se fait que l'absence totale du clan sur une aire aussi vaste ait passé si longtemps inaperçue et soit encore ignorée des disciples de Morgan. La raison en est étonnamment simple. Morgan était New-Yorkais et commença ses recherches par les Iroquois, passant ensuite à l'ouest au travers de la zone où prédomine l'organisation clanique. En ce temps, les Indiens du Far West étaient presque complètement inconnus des ethnographes, ce qui explique que Morgan ait pu affirmer de façon générale que toutes les tribus de l'Amérique du Nord connaissaient le clan. S'il avait commencé par l'Oregon ou par l'Idaho, tout son système eût été différent. Mais ce qu'on pouvait excuser en 1877 est impardonnable quarante ans plus tard. Et, alors qu'une conclusion prématurée due à une ignorance partielle n'est qu'une tache vénielle sur l'écusson du maître, elle ternit actuellement la réputation scientifique de ses disciples qui continuent obstinément à s'en faire les champions. 
Mais l'état des faits en Amérique du Nord ne concorde pas nécessairement avec les phénomènes observés ailleurs; il nous faut donc chercher dans d'autres continents des témoignages à l'appui. Nous trouverons que, dans la partie sud du Nouveau Monde, la culture la plus primitive, celle des Fuégiens, se caractérise aussi par le manque d'organisation clanique. Mais cela est surtout vrai de l'Asie. Les clans sont typiques pour les peuples turcs qui possèdent un système très développé d'élevage et sont célèbres comme métallurgistes; mais ils manquent chez les Tchouktche et les Koriak dont la culture marginale a récemment, et en partie seulement, adopté l'élevage du renne et qui étaient auparavant au même niveau que les Esquimaux chasseurs de phoques. Les Khasi d'Assam qui sont horticulteurs et influencés par les conceptions politiques d'une civilisation supérieure, ont des clans, alors que les chasseurs primitifs Sakai et Semang de la péninsule malaise n'en ont pas. L'exemple le plus convaincant est celui des Andaman, appartenant à la famille des Négrito; restés isolés dans le golfe du Bengale, à l'écart des vagues de civilisation qui apportèrent le fer et l'agriculture jusqu'aux lointaines Philippines, ils ignorent l'institution du clan.

Nos connaissances en sociologie africaine sont encore malheureusement fort déficientes, mais, autant que je sache, rien n'y contredit ma thèse. Les clans se retrouvent chez les tribus bantou et soudanaises, s'accompagnant souvent d'organisations politiques complexes, et on les signale parmi des peuples pasteurs comme les Massai qui présentent certains traits hamitiques et occupent à divers égards un niveau relativement élevé. Mais je n'ai trouvé nulle mention du clan dans les ouvrages se rapportant aux chasseurs encore plus primitifs que sont les Bochimans et les Pygmées.

Reste l'Australie. Sur ce continent, le clan est une institution fort répandue et probablement ancienne. Mais cela n'implique nullement qu'il soit antérieur à la famille, car ici, comme ailleurs, nous n'observons jamais le clan sans la famille, mais les deux institutions l'une à côté de l'autre. Tout ce que je pourrais concéder ici, c'est qu'en Australie le problème de l'antériorité reste insoluble. Quand bien même il en serait autrement, les phénomènes australiens ne prouveraient rien sur la succession des phases en d'autres régions, car la civilisation australienne n'est pas plus primitive que celle des Paviotso et des Andaman.

Bref, à la seule exception de l'Australie, les civilisations les plus simples ne connaissent pas le clan, mais possèdent la famille et, même en Australie, nous n'avons pas de preuves que le clan soit plus ancien que l'institution qui l'accompagne invariablement.

Ici, cependant, nous nous trouvons de nouveau face à face avec une objection. Se pourrait-il que les tribus qui ont été énumérées comme dépourvues de clans possédassent réellement une organisation clanique qui aurait échappé à l'observateur? Pour les ethnographes qui ont travaillé sur le terrain, c'est là une objection de savant de bibliothèque. Il n'y a rien de spécialement mystérieux dans une organisation clanique; là où elle existe, elle pénètre la vie sociale de telle manière que l'observateur se heurte 
nécessairement à elle à chaque instant. Un visiteur intelligent ne pourrait pas passer quelques semaines parmi des gens comme les Crow et les Hopi sans noter la présence de divisions héréditaires et unilatérales. C'est pourquoi, lorsqu'une enquête prolongée ne décèle nulle trace d'une telle institution, la seule conclusion à en tirer, c'est qu'elle n'existe pas. Cette conclusion devient une certitude quasi absolue lorsqu'elle est corroborée indépendamment par plusieurs enquêteurs. Ainsi les Athapaskan du nord ont été étudiés par Samuel Hearne, un observateur incomparable, et plus récemment par P. E. Goddard, Frank Russell, J. A. Mason, ainsi que par l'auteur; et pourtant aucun de nous n'a rien constaté qui suggérât un clan.

On peut encore arguer que le fait de ne pas signaler une organisation clanique n'est peut-être pas imputable à l'intelligence de l'observateur, mais que le système a échappé à l'enquête simplement parce qu'il n'existe plus, ayant été anéanti par le choc de la civilisation. Cet argument a lui aussi un fumet de bibliothèque. Il présuppose que le clan a tendance à disparaître au simple contact des Blancs, ce qui est inexact. Les Hopi sont entrés en contact avec les Espagnols en 1540, mais ils sont actuellement encore organisés en clans utérins; c'est aussi le cas du vieux système social des Iroquois qui, au cœur de l'État de New York, a résisté aux influences française, anglaise et américaine; c'est encore celui des quelques Mandan qui survivent dans le Nord Dakota et qui conservent la notion des clans et la vieille tradition matrilinéaire. Les phénomènes californiens sont également significatifs. Les mêmes observateurs ont signalé le clan dans une région sans le trouver dans d'autres, ce qui fait que les préjugés personnels sont, ici tout au moins, hors de cause. En outre il n'est pas du tout exact que des tribus classées comme sans clans aient perdu tout souvenir de leurs anciennes coutumes. Les Hupa conservent encore une curieuse division des sexes, obligeant les maris à ne jamais dormir en hiver dans la maison de leurs femmes, et les Maidu, il y a vingt ans, connaissaient encore bien des particularités sur leurs cérémonies et leur société secrète. En ce qui regarde l'hypothèse selon laquelle toutes les peuplades californiennes possédaient autrefois un système clanique, la limitation actuelle de ce système à certaines régions du centre et du sud de la Californie n'est pas claire du tout. Pourquoi vouloir s'en tenir de si près à la continuité géographique, alors que les conditions modernes sont censées introduire une période de chaos? Cependant, si nous admettons l'alternative contraire, il nous sera facile de concevoir la Californie du sud comme l'avant-poste extrême d'une structure sociale fort complexe, particulière aux tribus indigènes du Nouveau Mexique et de l'Arizona; nous pouvons aussi supposer en Californie un développement local du clan qui ne se serait que relativement peu répandu.

Faisons avancer l'artillerie lourde des nomenclatures de parenté pour renforcer la position et défendre l'hypothèse selon laquelle l'absence de clans n'est pas due à une désintégration récente des coutumes indigènes. Nous avons pu empiriquement déterminer la corrélation existant entre l'organisation clanique et le type Dakota de terminologie de parenté, terminologie qui distingue le côté paternel ou maternel dans la génération des parents, mais qui identifie la parenté, quel qu'en soit le degré de proximité. On a fait remarquer que, dans diverses régions, la terminologie dakota a 
tendance à devenir encore plus inclusive, si bien que les parents ne sont plus groupés que par génération. Ce fait parle en faveur de la théorie que je suis en train d'attaquer. Rivers pense qu'en Océanie les Polynésiens possédaient autrefois l'organisation clanique, ainsi que le système Dakota qu'on trouve encore en quelques points de la Mélanésie, et que ces particularités cédèrent la place à une organisation sans clans à nomenclature hawaïenne. La terminologie hawaïenne, bien qu'incompatible avec une institution clanique, pourrait donc être expliquée par l'hypothèse qu'il aurait existé autrefois dans la tribu en question une organisation clanique; et c'est naturellement l'interprétation qui se présente aussi lorsqu'on trouve une tribu sans clans à nomenclature Dakota. Ce n'est du reste nullement une démonstration irréfutable de l'antériorité du clan, puisqu'il y a alternative : la terminologie peut avoir été empruntée ou être due à d'autres causes sociales (pp. 43-44). Néanmoins l'argument est, dans un certain sens, plausible.

Mais à quoi auront recours les disciples de Morgan lorsque la terminologie, au lieu d'identifier les lignées collatérales et directes, établit une distinction entre les parents et les frères et sœurs de ceux-ci ? C'est précisément le résultat qu'on attendrait d'une organisation familiale où le père et la mère ont une position nettement différenciée de celle des parents plus lointains. Il ne saurait être question ici de la disparition récente d'une ancienne coutume ou d'une innovation due à l'influence des Blancs. En effet, une des principales théories de Morgan veut que les termes de parenté soient plus stables que l'édifice social dans lequel ils ont pris naissance, et qu'ils se maintiennent fort longtemps après qu'il se soit écroulé. Si donc la terminologie de parenté, accompagnant une organisation sans clans, ne fournit aucune preuve de l'existence antérieure de clans, il n'y a pas la moindre raison de supposer que la tribu ait jamais été organisée en clans, puisque ex hypothesi la terminologie aurait survécu à la structure sociale correspondante.

Je ne défends pas ce point afin de remporter un succès de dialectique; car, en cette matière, je suis d'avis que Morgan était sur la bonne voie. Les termes de parenté représentent un phénomène linguistique et le langage est notoirement conservateur. Nous disons encore que le soleil se couche, quoique nous ne croyions plus qu'il tourne autour de la terre. Le choc avec de nouvelles conditions d'existence peut transformer vitalement ou même anéantir la société indigène, sans rien changer à la manière traditionnelle de s'adresser aux parents. Un fonctionnaire colonial pourra abroger les sacrifices humains ou imposer une méthode préférable de travailler la terre, mais il ne s'inquiétera pas de savoir si ses administrés Ont un seul mot ou plusieurs pour désigner le père et l'oncle paternel. En conséquence la distinction entre les lignées directes et collatérales renforce aux yeux des observateurs la Preuve de l'absence d'institution clanique.

Si nous allons maintenant aux faits concrets, nous trouverons fréquemment la réalisation des conditions que, par hypothèse, nous avions d'abord supposées. En Californie, parmi diverses peuplades Salish et Shoshoni, ainsi que sur le territoire des Esquimaux, nous observons des systèmes de parenté différenciant les lignées directes 
et collatérales. C'est aussi ce que font les Andaman, les Tchouktche et les Koriak. Dans tous ces cas, qui pourraient sans doute être multipliés si les informations relatives à des tribus sans clans étaient plus abondantes, l'absence de clans qui nous est signalée prend un caractère définitif. Le dogme de l'universalité du clan dans les communautés primitives est ainsi réduit à néant.

Il est encore un autre ordre d'arguments qu'on peut avancer à l'appui de la théorie que je soutiens ici. Grâce au rôle important joué par le nom dans la pensée primitive, le caractère plus au moins inclusif des termes de parenté est d'ordinaire plus qu'une simple question de terminologie : des désignations semblables impliquent des relations sociales semblables. C'est par une fausse interprétation de ce fait qu'on a souvent admis à tort que la famille et la parenté individuelles n'existent pas là où on trouve des douzaines de " pères » et de " mères », des frères et sœurs par vingtaines. L'erreur consiste à croire que cette similitude signifie l'identité. Mais nous avons vu plus haut qu'en diverses occasions c'est le parent le plus proche de la catégorie requise qui a la priorité. Selon les stipulations du lévirat, c'est le frère qui hérite de la veuve du défunt et les autres parents ne peuvent que prendre sa place à son défaut. Le mariage entre cousins croisés dans les cas les plus nets signifie à l'origine l'union avec la fille du frère de la vraie mère ou avec la fille de la sœur du père. Parmi les Indiens Crow, tous les individus qu'on appelle beaux-frères ont droit au respect, mais, à cet égard, le véritable beau-frère seul jouit d'une prépondérance incontestée. Si l'on tient compte de la fréquence de la notion de clan en Australie, il est d'autant plus satisfaisant de voir que les plus compétents des observateurs australiens aboutissent aux mêmes conclusions. Radcliffe-Brown est aussi d'avis que les fonctions sociales, bien que leur nature soit identique, ainsi qu'il le formule de façon heureuse, diffèrent par le degré; et le degré varie selon la proximité. Bref, les relations entre les membres du cercle de famille restreint sont antérieures, et il y a eu extension secondaire dans la suite sous une forme atténuée à des cercles de plus en plus grands de parents réels ou putatifs. Les phénomènes australiens, dont nous n'avions pas encore déterminé la portée, concordent ainsi avec ceux des autres régions. En Australie, comme ailleurs, la famille est essentielle et plus ancienne, le clan est un développement secondaire relativement peu important. Ce renversement de l'ordre de succession traditionnellement admis est l'une des conclusions les plus certaines de l'ethnologie moderne.

Chapitre 7

\section{d. Origine du clan.}

\section{$\underline{\text { Retour à la table des matières }}$}

Comment le clan a-t-il donc pris naissance sur la base du concept de famille préexistant? Pour répondre à cette question, il faut nous rappeler ce qu'est au fond le clan. Le clan est un groupe de parents choisis et le problème consiste à savoir d'où 
provient le principe de sélection. Pourquoi certains parents, à l'exclusion d'autres, se groupent-ils pour former une unité sociale? Pour trouver la solution, nous devons étudier les conditions sociales relevées dans les tribus sans clans, dans l'espoir de déterminer les facteurs favorables au développement du principe unilatéral; nous aurons aussi à examiner les faits en corrélation avec les organisations claniques, et à rechercher les agents qui auraient pu les produire ou qui auraient contribué à les maintenir en vie. A mon avis, la transmission des droits de propriété et le mode de résidence ont été les moyens les plus efficaces de l'établissement du principe unilatéral, et je vais essayer de démontrer qu'ils ont pu se créer aussi bien dans les communautés patrilinéaires que matrilinéaires.

Rappelons une fois encore l'exemple des Hupa (page 74). Chez ces Indiens, la résidence est patrilocale, mais pas absolument. Dans la majorité des cas, le grand-père paternel, le père, le fils et le fils du fils naissent et vivent dans le même village, prenant leurs femmes ailleurs. En d'autres termes, le système des Hupa unit par la résidence commune les parents mâles qui sont groupés dans un clan consanguin. Nous avons ici le germe favorisant le développement d'un clan consanguin. Deux modifications seulement sont requises. La règle patrilocale doit devenir rigoureuse afin que chaque famille adopte le même principe de ségrégation et, secondement, il doit exister une clause établissant la filiation des membres féminins de la famille aussi bien que celle des hommes. Ce dernier but est facilement atteint par l'emploi d'un nom désignant les individus mâles différenciés : ce nom est donné naturellement lors de la naissance à tous les enfants qui naissent dans le village, qu'ils soient du sexe masculin ou féminin; ainsi filles et garçons portent et conservent une désignation patronymique. Dès lors il importe peu qu'une femme quitte sa localité natale; le nom seul suffit à établir sa filiation. De même, lorsque le principe patrilinéaire est fixé de cette manière, les règles de résidence peuvent tomber en décadence sans modifier le système puisque la qualité de membre est déterminée une fois pour toutes par l'appellation de groupe.

Dans le cas d'une peuplade sud-américaine, nous relevons une influence analogue exercée par la résidence commune.

Dans le bassin nord-ouest de l'Amazone, l'unité sociale est la maison qui peut renfermer jusqu'à deux cents individus. Comme le mariage est toujours patrilocal, le groupement des parents mâles, comme chez les Hupa, se produit sans exception et, pour l'évolution d'un clan typique, il ne manque plus que l'identification permanente des filles avec le groupe habitant la maison où elles sont nées. Un détail est particulièrement significatif. La proximité physique, ici comme ailleurs, a engendré un sentiment de parenté spécialement étroit. Les enfants de frères qui grandissent forcément dans la même maison sont considérés comme trop proches parents pour pouvoir se marier entre eux, mais ces restrictions ne s'appliquent pas aux enfants de sœurs. La communauté résidentielle fonctionne comme une unité exogamique permettant et défendant les mêmes unions entre cousins parallèles que permet ou défend un clan 
patrilinéaire exogamique. Kirchhoff a nettement prouvé la transmission des noms de groupes dans une tribu qui présenterait ainsi une véritable organisation clanique.

Les recherches poussées de Speck parmi les Algonkin du nord-est établissent l'action conjointe de la résidence et des droits de propriété communs. Ici les territoires de chasse se transmettent nettement de père en fils; la femme va habiter chez le mari; et les frères se partagent certains privilèges économiques. Il est évident que de telles conditions ont aussi pour effet une filiation de parenté qui se rapproche d'un clan consanguin caractéristique.

L'explication qui rend compte de la ségrégation de, parenté patrilinéaire peut s'appliquer de même à la parenté matrilinéaire. A cet égard, Tylor a déjà souligné l'importance de la résidence matrilocale. Partout où le mari réside de façon permanente dans la maison de ses beaux-parents, les enfants sont associés tout naturellement à la famille de la femme plutôt qu'à la sienne à lui. Nous avons vu que telle est la conséquence naturelle des cas exceptionnels d'unions matrilocales, même dans une tribu surtout patrilocale comme les Hupa. Mais l'influence de la résidence matrilocale se fait avant tout sentir dans des tribus comme les Hopi et les Zuñi où il y a non seulement résidence matrilocale mais aussi transmission de la maison de mère en fille. Grand-mère, mère et fille forment ainsi le noyau d'une unité sociale et tous les enfants, nés dans la maison, sont naturellement liés à ce groupe permanent.

Toutefois, quoique la résidence matrilocale explique ainsi de façon adéquate l'évolution du groupe de parenté matrilinéaire, deux obstacles sérieux s'opposent à ce que cette interprétation devienne une théorie générale. D'une part, beaucoup de peuples utérins sont patrilocaux. C'est le cas des tribus australiennes matrilinéaires, des Mélanésiens ainsi que de quelques tribus africaines et américaines. On pourrait naturellement supposer que tous les peuples matrilinéaires étaient autrefois matrilocaux, mais ce serait là vaine conjecture. Secondement, la résidence matrilocale est souvent un état transitoire. Si au bout d'un an environ le jeune couple a son propre domicile, pour quelles raisons rattacherait-on ses enfants, ou du moins ceux qui suivent le premier-né, à la famille de la mère plutôt qu'à celle du père?

Il conviendrait donc d'ajouter au facteur de la résidence matrilocale et à celui de la possession par les femmes de la maison quelque autre facteur complémentaire qui pût avoir pour effet une telle filiation de parenté. Voyons donc comment opère la division du travail selon les sexes. Hahn nous a familiarisés avec l'idée que la culture du sol est souvent dans les couches primitives une occupation féminine. Ce fait me semble comporter des conséquences significatives. Les jeunes filles en grandissant apprennent de leur mère le travail de la terre, de même que les autres occupations caractéristiques de leur sexe. Mais, en quelques cas, en s'occupant exclusivement de l'agriculture, elles finissent par établir un titre de propriété se transmettant de mère en fille et, de cette manière, se forme un ensemble matrilinéaire de parenté féminine. Telle était la situation autrefois chez les Hidatsa où l'agriculture était aux mains de la femme, de ses filles et de ses petites-filles, et où les titres se transmettaient entre elles. Des droits 
de propriété communs et la coopération dans l'activité économique unissaient ainsi la descendance féminine des sœurs. S'il avait existé une ségrégation analogue de la parenté masculine, par la transmission, par exemple, des territoires de chasse, ainsi que parmi les Algonkin de Speck, il est possible que le principe patrilinéaire eût survécu ou que le heurt des systèmes eût empêché la naissance de toute règle définie de descendance. Mais, parmi les Hidatsa, il n'existait pas de privilèges de chasse individuels dont pussent hériter les fils, et les règles de résidence n'étaient strictement ni matrilocales ni patrilocales. Il n'y avait donc aucun principe consanguin qui pût contre-balancer le principe utérin actif. Comme les femmes, propriétaires des jardins, formaient le groupe le plus défini, il était naturel que, dès sa naissance, tout enfant eût une filiation le rattachant à ce groupe. Et ce fut ainsi que se développèrent la descendance matrilinéaire et le clan utérin.

On pourrait se demander comment il se fait que des générations successives de femmes puissent s'unir dans un groupe patrilocal. Nous répondrons que la résidence patrilocale ou matrilocale n'entraîne souvent pas le changement de communauté de l'un des conjoints, mais seulement une modification de résidence à l'intérieur de cette communauté. Les villages Pawnie étaient nettement endogamiques; les Kaï admettaient qu'une jeune fille allât vivre chez son mari, à condition qu'elle ne quittât pas le village et en beaucoup d'autres cas la plupart des mariages avaient lieu entre membres du même groupe local. Quoique ces dispositions relatives au domicile ne soient pas sans agir sur la vie de famille, comme nous l'avons dit dans un chapitre précédent, elles n'affectent pas la ségrégation de la parenté consanguine et utérine. Une Kaï, bien que vivant dans une autre maison que ses filles et ses petites-filles, n'éprouve pas de difficulté à les aider dans les travaux quotidiens puisqu'elles résident toutes dans le même village.

La succession des événements, telle que nous l'avons décrite pour les Hidatsa, ne doit naturellement pas être prise pour une description historique des faits, mais seulement pour une interprétation qui, cependant, me paraît des plus probables et digne d'être envisagée dans tous les cas de tribus matrilinéaires où les femmes s'occupent de la culture du sol. Des considérations analogues s'appliquent même en d'autres cas. Les Australiens sont presque toujours patrilocaux, dans le sens que la femme va vivre dans le groupe de son mari. Tandis que le mari parcourt le territoire du groupe en quête de gibier, elle s'occupe à cueillir dans ce même district des plantes comestibles. Or, dans une région du Queensland, on reconnaît à la femme la propriété individuelle de certaines plantations, et elle transmet ses prérogatives à ses filles. $\mathrm{Si}$, ainsi qu'il arrive parfois en Australie, les hommes avaient uniquement des droits communaux sur le territoire et que les femmes établissent leur propriété individuelle sur des sections déterminées, nous nous trouverions en présence de conditions favorisant la descendance utérine au sein d'une peuplade patrilocale.

Du point de vue adopté ici, on comprend pourquoi des tribus en nombre considérable n'ont pas de système clanique; la différenciation unilatérale de la parenté dépend, en effet, de certaines coutumes spéciales, telle une règle définie de résidence, 
qui sont courantes, mais non universelles; il peut se produire, en outre, conflit entre deux principes unilatéraux s'excluant mutuellement et dont aucun ne prédomine. D'autre part, la fréquence de l'institution clanique semble correspondre en gros à la vaste distribution géographique des causes que nous venons d'énumérer. Enfin l'origine multiple du clan, que d'autres motifs nous ont induit à supposer, est rendue encore plus vraisemblable si le clan a comme phénomènes sous-jacents la règle de résidence ou la loi de succession. Car sur ces règles reposent des possibilités de développements indépendants en nombre infini des descendances consanguine ou utérine.

Chapitre 7

\section{c. Le clan et la terminologie dakota}

\section{$\underline{\text { Retour à la table des matières }}$}

Comme nous l'avons déjà vu, on explique parfois causalement la corrélation empirique entre le système clanique exogamique et la terminologie dakota : le clan est alors représenté comme produisant ce groupement de parenté caractéristique. Il n'est pas douteux qu'une fois l'organisation clanique fortement établie, elle réagisse sur la manière de désigner la parenté : tous les individus du même clan et de la même génération passent pour frères et sœurs, tous les hommes du clan paternel sont traités de " pères », et ainsi de suite. Mais ce qui est vrai du système clanique en pleine activité ne l'est peut-être pas encore à sa naissance. Car le clan n'est pas autre chose qu'une filiation particulière de parenté, et, s'il coïncide avec le système dakota, il ne peut y avoir entre eux que des relations d'identité et non de cause à effet. En conséquence, si nous désirons comprendre cette filiation, ne nous contentons pas de prononcer le mot de «clan » comme si C'était quelque terme magique, mais pénétrons jusqu'au concept qu'il représente. J'ai montré dans ce qui précède comment, dans certaines conditions, un père et ses frères ainsi que leurs fils peuvent former un groupe social distinct, et comment, en d'autres conditions, il peut se produire un groupement correspondant de la mère, de ses sœurs et de leurs filles. Néanmoins, un père et ses frères, bien qu'appartenant au même groupe social, pourraient être distingués par des désignations différentes, ce qui arrive fort rarement dans les tribus divisées en clans. Il convient donc de montrer comment cette classification de parenté et d'autres analogues ont pris naissance.

Faisons remarquer tout d'abord que la différenciation des parents maternels et paternels est tout aussi naturelle pour la famille que pour le clan. Dans les deux cas, le frère de la mère, par exemple, est aussi nettement le représentant d'un groupe social distinct que l'oncle paternel. Cette distinction est du reste clairement marquée par beaucoup de tribus sans clans. Où leur nomenclature diffère du type dakota, c'est en ce qu'elle maintient la distinction entre le père et l'oncle maternel, entre la mère et la 
tante maternelle. Si nous réussissons à montrer la disparition de cette distinction, nous verrons par là même comment les ressortissants du clan ne se forment pas simplement en un groupe, mais en viennent à adopter les relations mutuelles si caractéristiques d'une organisation clanique.

Je suis d'avis que le lévirat et le sororat sont des institutions plus anciennes que le clan et que, par leur action conjuguée, ils peuvent produire et ont souvent produit cette classification des parents, particulière au clan et se conformant au modèle dakota. Ces règles, nous l'avons vu, s'appliquent à l'origine au vrai frère ou à la vraie sœur, dont les autres membres du clan ne contestent pas la précédence et qu'ils ne peuvent que remplacer. Secondement le lévirat et le sororat sont beaucoup plus fréquents que le clan; bien plus, ils apparaissent dans plusieurs tribus peu développées et ignorant cette institution. Ce fait n'indique pas seulement leur antériorité relative, mais explique aussi pourquoi certaines tribus sans clans connaissent également la terminologie dakota des peuples claniques. Si cette nomenclature, quoique concordant avec l'institution du clan, n'en est pas le produit, mais constitue le résultat de coutumes matrimoniales plus anciennes, elle peut donc apparaître là où règnent lesdites coutumes, en coexistence avec le clan ou non.

Ma théorie n'implique pas que toutes les terminologies dakota soient un résultat du lévirat et du sororat. En des cas spéciaux, il convient, il s'impose même d'adopter des principes d'interprétation tout différents. Les Hopi, par exemple, ne pratiquent ni le sororat ni le lévirat, Pourtant ils ont à la fois le clan et une intéressante variante de la nomenclature dakota, caractérisée en ce que souvent elle ne fait pas de distinction entre les générations. Ainsi le fils de la sœur du père n'est pas distingué du père, et la fille de la tante paternelle ainsi que la fille de sa fille sont classées avec la sœur du père. Mais ces déviations de la norme dakota et la norme elle-même se déduisent des conditions d'existence antérieures des Hopi qui donnèrent naissance au clan. La résidence matrilocale et la possession des maisons par les femmes suffisent largement à expliquer ces phénomènes. Le fils de la sœur du père sort de la même maison que le père; le père et ses frères ont vécu comme célibataires sous le même toit; (le même, les sœurs habitent ensemble et tel est aussi le cas de la sœur du père, de ses filles et de leurs filles. Les équations terminologiques des Hopi ont donc pour base le domicile commun et sont explicables sans lévirat ni sororat. L'essentiel est que, dans chaque cas, nous puissions retrouver quelque usage en corrélation, que ce soit une règle matrimoniale ou résidentielle, qui aura perfectionné ladite nomenclature dans la mesure où elle dépend de la coutume sociale.

Il est un point important qui s'éclaire à la lumière des principes exposés ci-dessus. On comprend aisément pourquoi le mariage entre cousins parallèles est fréquemment prohibé. Si les pères et leurs frères, les mères et leurs sœurs se classent ensemble, les cousins parallèles peuvent logiquement s'appeler frères et sœurs; et, à cause de l'importance donnée par les primitifs aux noms, ils évitent donc en général de se marier entre eux. Mais, sauf lorsque la société est divisée en deux sections, il n'y a que la moitié des cousins parallèles auxquels la règle du clan défende de se marier, Comme 
Morgan l'a clairement démontré, dans une société matrilinéaire, seuls les enfants de sœurs appartiennent nécessairement à la même unité, et pourtant tous les cousins parallèles sont appelés frères et sœurs. Ce fait nous indique que, après que le lévirat, le sororat ou d'autres conditions eussent fait naître la nomenclature de parenté accompagnant les organisations claniques, il dut se produire une ségrégation des cousins parallèles selon laquelle la moitié seulement de ceux-ci fit partie du clan. Mais ce processus a déjà été exposé lors de la discussion sur l'origine du clan. L'exemple de l'Amazone est typique : la résidence patrilocale amène une dichotomie des cousins parallèles, les enfants de frères sont réunis dans une même maison au contraire de ceux de sœurs. Ici, nous trouvons les règles matrimoniales elles-mêmes profondément affectées par le fait de la proximité physique; les enfants de frères ne peuvent pas s'unir alors que cela est loisible à ceux de sœurs. Cependant, que cet effet se fasse sentir ou non, la séparation des cousins parallèles en ressortissants d'un même clan et ressortissants d'autres groupes est abondamment illustrée par les cas des Hupa, des Algonkin du nord-est et des Pueblo.

Résumons-nous. Le clan prend naissance dans la famille qui existait avant lui, et il grandit grâce à un certain nombre de phénomènes qui aboutissent à la classification caractéristique et à la ségrégation unilatérale de la parenté. La nomenclature dakota est probablement due dans une large mesure à l'action antérieure du lévirat et du sororat, mais celle-ci ne suffit pas à produire les différenciations des cousins parallèles accompagnant une organisation clanique multiple. Cette différenciation résulte naturellement des dispositions réglant la résidence et la transmission des biens, lesquelles finissent par établir des lignées unilatérales de parents.

Chapitre 7

\section{d. Clans utérins et clans consanguins}

$\underline{\text { Retour à la table des matières }}$

Il nous reste à considérer un autre problème historique dont l'importance théorique est de tout premier plan. Quelles sont les relations chronologiques entre descendance patrilinéaire et descendance matrilinéaire? Ici se présentent un certain nombre de possibilités abstraites : les clans utérins peuvent être sortis des clans consanguins ou vice versa; chacun des deux types de clans peut être plus récent que l'autre, sans pour cela représenter une évolution de la forme précédente; ou bien il peut n'avoir existé aucun ordre de succession quelconque.

Cette dernière hypothèse répugne naturellement à ceux qui cherchent à établir des lois sociologiques, comme Morgan et ses disciples, et qui présupposent un processus d'évolution invariable. Selon eux, le clan archaïque était toujours et nécessairement utérin, car le mariage par couples n'existait pas, ce qui rendait la paternité douteuse. 
La filiation était ainsi du côté du groupe maternel et la propriété se transmettait à l'intérieur du clan utérin, que ce fût du frère au frère ou de l'oncle maternel au fils de la sœur, mais jamais du père au fils. Cependant, avec l'accroissement des biens, s'éleva un antagonisme naturel contre cette forme de succession qui excluait de l'héritage les enfants du possesseur et ce motif, joint au fait que la paternité devenait de plus en plus certaine, suffit à renverser la descendance matrilinéaire et à établir le clan consanguin. L'essentiel de cette théorie, c'est que non seulement elle suppose que la descendance matrilinéaire devint par la suite patrilinéaire, mais encore que tous les clans consanguins sont sortis de clans utérins. Par exemple, le genos des Grecs et la gens des Romains étaient patrilinéaires, mais, appliquant le principe de l'uniformité chronologique de l'évolution, Morgan affirme que ces institutions étaient antérieurement des clans utérins.

Chacun des points essentiels de son argumentation est contraire à l'évidence ethnographique. Tout d'abord, le mariage par couples, loin d'être absent, est au contraire fréquent dans les tribus les plus grossières, et rien ne nous autorise à admettre un état antérieur de promiscuité. Il arrive même fréquemment que, dans les couches culturelles les plus basses, on trouve des relations matrimoniales qu'un moraliste de l'époque victorienne n'hésiterait pas à qualifier d'exemplaires. Parmi les habitants des îles Andaman, « la fidélité conjugale jusqu'à la mort n'est pas l'exception, mais la règle ", et un état de choses analogue nous est signalé dans d'autres tribus fort primitives. Même si la paternité ne peut pas être établie, cela ne prouve nullement la nécessité de la descendance matrilinéaire. La paternité biologique et sociologique sont deux choses fort différentes. Les Toda polyandres ne se préoccupent pas de la première, mais établissent la seconde au moyen d'un rite conventionnel (page 53). Lorsque l'adoption joue un certain rôle, comme aux îles Andaman et dans le détroit de Torrès, la paternité biologique ne compte guère et le père adoptif remplit les devoirs d'un véritable procréateur. Le lien entre les rapports sexuels et la conception est, selon des auteurs compétents, inconnu de divers groupes australiens; pourtant, quelques-uns d'entre eux tout au moins sont consanguins, comme les Toda et les insulaires du détroit de Torrès.

D'autre part, il existe assurément une corrélation quelconque entre la loi de succession et la règle de descendance, ainsi qu'il découle du rôle que joue la transmission des biens dans l'établissement d'un groupe de parenté. Mais cette corrélation n'est que partielle. D'une façon générale, il est plus probable qu'on trouvera dans une société utérine la succession matrilinéaire et non filiale, mais que cette dernière apparaîtra dans les cas de descendance consanguine; cependant les exceptions sont trop nombreuses pour être négligées. Il est des tribus matronymiques, comme les Crow et les Hidatsa, où certains biens se transmettent patrilinéairement et d'autres matrilinéairement. Nous voyons aussi que, parmi des peuples patronymiques comme les Warramunga, la propriété passe aux oncles maternels et aux maris des filles du défunt, c'est-à-dire à la phratrie de la mère. On pourrait sans doute harmoniser ces phénomènes avec la norme en supposant que dans le premier cas il s'agit d'un clan consanguin en formation, dans le second d'une survivance d'un état matrilinéaire 
antérieur. Mais les faits gênants peuvent toujours être écartés à l'aide d'un nombre suffisant d'hypothèses auxiliaires. On expliquerait tout aussi bien que les traits consanguins des Crow proviennent d'une ancienne phase patrilinéaire et que les Warramunga sont dans un stade naissant d'organisation matrilinéaire.

Enfin quelles preuves avons-nous que le développement de la propriété puisse non seulement introduire l'établissement de la descendance patrilinéaire se substituant à une organisation sans clan, comme je l'ai soutenu moi-même, mais encore remplacer la descendance matrilinéaire par la filiation consanguine? Cette possibilité existe, sans nul doute, mais certains cas historiquement connus nous démontrent que ce n'est pas une nécessité absolue. Les Navaho du nord de l'Arizona, par exemple, devinrent un peuple pasteur par l'introduction du mouton dans le sud-ouest au XVIIe siècle; et pourtant, en dépit de leurs troupeaux prospères dont les hommes ont la garde, ils sont restés obstinément utérins. De même, l'élevage du cheval a assurément révolutionné la notion de propriété des Crow et des Hidatsa, mais ils observent toujours la descendance par la mère et, lorsque j'ai visité les Crow pour la première fois, c'était au moment où un édit gouvernemental et non une évolution spontanée eut pour effet de modifier la transmission patrilinéaire des biens immobiliers. Parfois, ce que possède une tribu primitive est si peu de chose que Morgan lui-même hésite à attribuer tout changement dans la descendance à la sollicitude du père pour le patrimoine de ses enfants; il invoque alors l'influence récente des Blancs. Cet argument nous parait cependant dénué de valeur si nous nous rappelons que des peuples comme les Hopi et les Iroquois, qui ont été en contact avec les Blancs depuis des siècles, sont au nombre des tribus les plus typiquement matronymiques qui soient, alors que les Pieds Noirs, Plus récemment et moins fortement influencés, sont avant tout patrilinéaires.

Bref, la théorie de Morgan est insoutenable à divers égards. Pour la détruire, il suffit de montrer qu'il est tout à fait possible de transmettre certains biens de père en fils sans altérer en rien la descendance, ainsi que c'est le cas des Crow et des Hidatsa.

Néanmoins réfuter la théorie de Morgan n'équivaut pas à réduire à néant l'ordre de succession qu'elle prétend établir. Malgré la faiblesse de ses preuves, ses conclusions peuvent être justes; mais il faut soumettre à notre analyse d'autres arguments.

Dans une publication qui restera un monument classique de l'ethnologie, Tylor essaie de démontrer entre autres que les tribus consanguines ont passé par une phase matrilinéaire. Avançant ce qu'il appelle lui-même un argument géologique, il soutient que « les institutions de l'homme sont aussi nettement stratifiées que la terre sur laquelle il vit. Elles se succèdent en séries substantiellement uniformes, indépendantes des différences de races et de langues qui semblent relativement Superficielles, et façonnées par la nature humaine identique partout et dont l'action se fait sentir à travers des états successifs qui vont de la sauvagerie et de la barbarie à la vie civilisée ». 
Appliquant ce principe au cas qu'il étudie, Tylor commence Par supposer l'existence de trois couches correspondant respectivement au système maternel, maternelpaternel et paternel. Dans le premier, « la descendance... est maternelle; c'est la mère qui exerce surtout l'autorité et son frère est d'ordinaire le gardien des enfants; la succession aux titres et l'héritage des biens suivent la même voie, passant au frère ou au fils de la sœur ». Dans la phase paternelle, « c'est le père qui a pleins Pouvoirs sur la femme et les enfants, la succession et l'héritage se transmettent à ses enfants ». Entre ces deux états, il existe " une phase de transition où les caractéristiques des deux autres stades se combinent de façon variée ». Tout en reconnaissant avec prudence ce que cette classification a de vague, Tylor continue audacieusement à en tirer des conséquences, comme si elle fournissait des assises suffisamment solides à une construction historique. Avant d'exposer son argumentation, il convient donc d'examiner ce point de plus près.

Du point de vue logique, la classification de Tylor est impeccable. La question est de savoir si la vérité historique correspond à ses catégories logiques et on ne saurait l'admettre qu'en introduisant des restrictions qui enlèvent au système une grande partie de sa valeur. Tylor, remarquons-le bien, substitue à la différence simple, facile et nette entre les descendances patrilinéaire et matrilinéaire, la différence vague, compliquée et variable entre les complexes matrilinéaire et patrilinéaire. Il suppose donc que, conjointement à la règle de descendance, il existe inévitablement, ou du moins en général, certaines coutumes s'y rapportant dont l'ensemble forme un tout organique; et lorsque quelques-unes seulement de ces particularités se présentent, il présuppose l'existence d'un état intermédiaire. On peut admettre, il est vrai, qu'il y a quelques exemples de peuples à système purement paternel, entre autres les anciens Romains et les Chinois. Mais la grande majorité des peuples du monde sont nettement dans ce que Tylor appelle la phase maternelle-paternelle, ce qui n'a rien de surprenant étant donné la coexistence de la famille bilatérale et de l'unité clanique unilatérale. Le père chez les Khasi est respecté même durant sa résidence temporaire dans la maison de sa femme et parvient dans la suite à une autorité incontestée; parmi les Tsimshian, il favorise activement les intérêts de son fils, sans tenir compte de la descendance utérine; les Pueblo le reconnaissent comme le pourvoyeur de la famille et ce sont ses parents à lui qui choisissent à l'enfant un nom marquant leur filiation et non celle du clan de l'enfant. Et pourtant ce sont des cas extrêmes de statut « maternel »; nous en pouvons conclure a fortiori qu'il n'existe pas de tribu utérine à l'état pur.

Une autre question encore plus importante s'impose d'elle-même. D'où Tylor tiret-il son complexe matrilinéaire? Est-ce un fait empirique que les phénomènes cités par lui comme caractéristiques des sociétés utérines sont régulièrement liés ou n'estce là qu'une déduction logique, plausible, mais non basée sur des faits concrets. A la vérité la logique et l'expérience s'y font toutes deux sentir. Il existe quelques tribus où l'avunculat, par exemple, s'associe à la descendance matrilinéaire et, du point de vue de la logique abstraite, il semble que ce soit en accord avec l'éternelle harmonie des choses. Ces tribus seront donc mises à part en tant que tribus utérines par excellence, en tant que norme à laquelle devrait tendre toute tribu utérine digne de ce nom. Mais, 
comme je l'ai démontré ailleurs, on ne peut pas procéder ainsi. Lorsque Bachofen découvrit la descendance matrilinéaire, il paraissait alors vraisemblable que cette notion impliquait la prédominance primitive du sexe féminin, mais cette opinion est maintenant allée rejoindre les vieilles théories ethnographiques périmées. Il ne nous faut donc pas faire trop grand cas des probabilités abstraites. Voulons-nous savoir si et dans quelle mesure l'avunculat et la descendance matrilinéaire sont liés l'un à l'autre, il faut nous servir de l'une de ces coutumes comme d'un pivot et étudier dans quelle mesure l'autre l'accompagne. Une telle analyse portera un coup mortel à la théorie de l'avunculat comme critère certain de la descendance utérine : premièrement parce qu'il est des tribus matrilinéaires sans avunculat, et secondement à cause des tribus patrilinéaires qui, au contraire, le pratiquent.

Les sociologues de l'ancienne école traitaient ordinairement les phénomènes du second groupe comme des survivances d'un état matrilinéaire antérieur et, récemment encore, Hartland s'est fait l'avocat de cette cause avec beaucoup d'habileté et d'érudition. Les partisans de cette théorie convertissent ainsi en argument favorable ce qui semblait d'abord être une objection fatale. Sans doute, diront-ils, les Omaha sont consanguins maintenant, mais leur observance de l'avunculat prouve qu'ils avaient autrefois la descendance utérine, ce qui est la seule manière de l'expliquer. Cette dernière affirmation les expose à l'attaque de leurs adversaires, car on peut concevoir d'autres causes à l'avanculat. Tout d'abord, il faut tenir compte des possibilités de diffusion. Même si l'on concède que l'avunculat découle naturellement de l'organisation matrilinéaire, il peut avoir été adopté ultérieurement par un peuple consanguin, et sa présence n'est pas une preuve de descendance matrilinéaire primitive dans la tribu qui l'a emprunté. En voici un exemple récent : les Pawnie et les Omaha, leurs voisins, ont tous l'avunculat, mais les premiers sont utérins, les seconds patrilinéaires. Nous pouvons supposer avec Tylor et son école que cette coutume a pris naissance chez les Pawnie, mais, selon notre hypothèse, sa présence dans l'autre tribu prouve que les Omaha ont emprunté cette institution aux Pawnie et non point qu'eux-mêmes ont passé par une phase matrilinéaire correspondante. Quant à l'influence des emprunts en général sur les séries uniformes, nous y reviendrons plus loin.

Secondement, l'avunculat s'accompagne parfois du mariage entre cousins croisés de la variété la plus commune. Il n'est en ce cas nullement certain que cette coutume soit attribuable directement à la parenté entre oncle et neveu; elle peut aussi être due à une parenté par alliance. Par exemple, chez les Kariera consanguins, un jeune homme est redevable de certaines obligations au père de sa fiancée et, à un moindre degré, à tous les hommes qui présentent avec lui le même degré de parenté, à savoir celui de frère de la mère. Les termes employés par Radcliffe-Brown ne nous laissent guère de doute à cet égard : c'est le fait du mariage, plutôt que les liens du sang, qui détermine dans ce cas les usages de parenté, et il peut en être de même en beaucoup d'autres cas. L'avunculat finit parfois par n'être plus du tout directement associé à la parenté maternelle. 
Ceci nous amène à un point d'ordre plus général. Dans notre critique des usages de parenté, nous avons vu que les tribus primitives assignent des fonctions définies aux parents, qu'ils soient de la branche paternelle ou maternelle, alliés ou non. Vu d'un angle plus large, l'avunculat est simplement un mode particulier des coutumes de parenté. Il n'est pas plus étonnant que le frère de la mère exerce une certaine autorité sur les enfants de sa sœur que de voir la sœur du père opposer son veto aux plans matrimoniaux de son neveu, ou les ressortissants du clan du père avoir droit à des présents dans une communauté matrilinéaire. Si l'avunculat parmi les Omaha est la survivance d'une phase utérine, la prédominance de la famille du père chez les Mélanésiens et les Crow pourrait aussi représenter un reste d'une ancienne descendance patrilinéaire, antérieure à l'état utérin observé depuis. Ce n'est, il va sans dire, qu'un argument de dialectique pure. En réalité, aucune de ces hypothèses n'est concluante : ces deux ensembles de coutumes sont exactement sur le même plan, nous démontrant la portée des règles de parenté sur les deux branches de la famille et ne se rattachant pas nécessairement à la descendance.

On ne saurait donc admettre qu'on déduise de la présence de coutumes avunculaires dans une société l'existence d'une phase matrilinéaire antérieure, car elles peuvent avoir une tout autre origine. D'autre part, il est avéré que l'avunculat fait assez souvent défaut dans les tribus matrilinéaires. Les Australiens à descendance utérine ne le pratiquent pas sous sa forme typique et on n'en relève aucune trace dans des tribus distinctement matrilinéaires comme les Crow et les Hidatsa. Il se peut en fin de compte que, outre la descendance utérine, d'autres conditions soient requises pour que l'avunculat prenne naissance; son développement le plus haut semble souvent s'accompagner d'une existence sédentaire ainsi que de la résidence matrilocale, et c'est peut-être cette dernière particularité ou tout cet ensemble de conditions favorables qui lui permettent d'apparaître. En tout cas, l'affirmation de Tylor selon laquelle l'autorité avunculaire est la marque d'une société matrilinéaire repose sur une sélection douteuse des faits. Non point que nous contestions toute corrélation, mais elle est certainement de nature plus complexe. La manière la plus sûre de formuler les faits serait de dire que certaines conditions favorables à la naissance de la descendance utérine le sont aussi à la prépondérance de l'oncle, mais la conséquence n'est pas inévitable et le phénomène peut aussi se produire d'autre façon.

Ce qui est vrai de l'avunculat en tant qu'élément du complexe utérin, s'applique également aux règles d'héritage, bien que peut-être à un moindre degré. Celles-ci non plus ne peuvent être considérées comme en corrélation absolue avec les règles de descendance, puisque nous trouvons, d'une part, des tribus patrilinéaires reconnaissant les droits du fils de la sœur et, de l'autre, des tribus utérines où, comme à Buin, les charges se transmettent aux hommes; nous voyons aussi, chez les Crow par exemple, certains biens passer du père au fils. Bref, la classification en système maternel, maternel-paternel et paternel, bien que non dépourvue de fondement empirique, représente bien plus une série logique de possibilités abstraites que l'état normal des faits dans la société primitive. 
Mais revenons-en à l'argument géologique de Tylor. Appliquant sa triple stratification, il compare avec ces couches certains usages et tire de leur absence ou de leur présence la conclusion lourde de conséquences de l'antériorité matrilinéaire. Deux exemples suffiront à exposer sa méthode. Tylor relève le lévirat dans ses trois strates, mais dans le maternel-paternel un nouveau trait s'y ajoute, l'héritage des veuves (sauf de sa propre mère) par le fils. L'héritage filial des veuves accompagnant le lévirat est donc limité à la strate mixte ou purement paternelle. Or, soutient Tylor, la strate maternelle doit avoir précédé les deux autres, sinon on trouverait des vestiges de succession filiale dans la couche maternelle. Il applique le même argument à une autre coutume, la couvade, cette étrange mode, observée en certains pays, de séparer le père plutôt que la mère lors de la naissance d'un enfant et de le soumettre à une série de tabous rigoureux afin de ne pas nuire au nouveau-né. La couvade n'apparaît pas dans la strate maternelle; elle est surtout développée dans la couche mixte et s'affaiblit considérablement dans la troisième. Tylor en tire une conclusion identique à celle qu'il basait sur le cas précédent. « De même que les formes de la vie et même les fossiles actuels de la formation carbonifère peuvent être relevés dans la période permienne, mais que les types et fossiles permiens font défaut dans la strate carbonifère formée avant leur apparition, ainsi l'héritage des veuves et la couvade qui, si le système maternel avait suivi le paternel, s'y seraient prolongés, prouvent par leur absence l'antériorité du maternel. »

Nous pouvons à bon droit objecter que, pour être démontrée de façon adéquate, la proposition de Tylor aurait besoin d'une base plus large. L'héritage des veuves et la couvade ne sont que deux exemples parmi les coutumes en nombre infini qu'on aurait pu choisir pour étudier l'ordre de succession théorique. Quelles garanties avons-nous qu'avec celles qu'il a négligées on n'eût pu parvenir à des résultats contradictoires? Cependant écartons cette considération en faveur d'une autre. Quels sont après tout les faits immédiats sur lesquels construit Tylor? Non point une succession chronologique ni une stratification dans le sens d'une superposition géologique des couches, mais simplement une corrélation négative et positive. Les particularités de la strate paternelle s'accompagnent de l'héritage des veuves par le fils et de la couvade; la strate maternelle les ignore. L'observation directe ne s'étend pas au-delà de la perception d'un rapport de simultanéité. L'affaiblissement de la couvade dans la phase purement paternelle pourrait être pris pour un indice direct du cours de l'évolution sociale, mais ce serait à tort. Si la couvade est plus commune dans l'état intermédiaire, cela signifie simplement que, dans le système paternel, l'influence de certaines particularités favorisant l'évolution de ladite coutume est contrebalancée par d'autres qui lui sont contraires. Il existe entre ces phénomènes des relations fonctionnelles, mais nulle part on n'y discerne un ordre chronologique. Cet ordre ne peut y être introduit que si nous acceptons comme axiome la croyance de Tylor et de Morgan en l'uniformité des lois de l'évolution sociale. C'est alors seulement que toute divergence prendra une signification temporelle. Évidemment, si tous les peuples passent par les mêmes phases, une tribu matrilinéaire doit soit être sortie d'une période patrilinéaire, soit s'y acheminer. L'argumentation de Tylor tend ainsi uniquement à démontrer que, s'il y a eu ordre de succession défini, la phase matrilinéaire est la plus ancienne. 
Or, en ce qui a trait à ce postulat essentiel, nous pouvons faire intervenir l'avertissement de Maitland pour lequel la vaste diffusion par emprunts des traits culturels est forcément fatale à toute tendance hypothétique des sociétés à passer par des phases se succédant en un ordre déterminé. Sous une influence étrangère puissante, une peuplade dont les éléments culturels favoriseraient naturellement l'évolution de la descendance consanguine, peut en venir à adopter la descendance utérine et vice versa. L'ethnographie nous en offre un grand nombre d'exemples frappants. La grande majorité des Athapaskan du nord ignorent le clan, mais, pour ce qui est de l'autorité et de l'héritage, ils se conforment au type paternel de Tylor. Cependant ces mêmes Athapaskan habitant non loin des tribus côtières ont modelé leur organisation sur celle de leurs voisins, adoptant la descendance utérine. Les Hopi appartiennent au même groupe linguistique que les Shoshoni de l'Utah et du Nevada qui tous sont dépourvus de clans et ne présentent nul germe de système matrilinéaire. Leur organisation actuelle a probablement emprunté à leurs prédécesseurs du sud-ouest soit la descendance utérine, soit certaines conditions la favorisant. Les Gros Ventres, branche récente et numériquement faible des Arapaho sans clans, entrèrent en contact avec les Pieds Noirs et adoptèrent le clan consanguin en s'assimilant les concepts des Pieds Noirs et en les développant. Ainsi des influences étrangères peuvent faire passer un état sans clans tantôt à un système utérin, tantôt à un statut patrilinéaire, sans qu'aucun de ces deux états précède l'autre. L'histoire de l'organisation sociale varie selon ses relations intertribales.

Les suppositions émises sur l'origine des clans indiquent de même que, à part l'emprunt de conditions utérines ou consanguines, les clans peuvent évoluer directement à partir d'un état sans clans. Cette hypothèse pare à une sérieuse difficulté : elle explique le passage hypothétique d'une descendance à l'autre. Comme nous l'avons vu, Morgan s'en tire en appliquant le principe de propriété à des tribus parmi lesquelles la propriété est restreinte à très peu de chose. De plus, on ne discerne pas bien pourquoi une règle d'héritage sanctionnée par les traditions donnerait naissance à une opposition subite, et cela d'autant plus qu'un individu peut tout aussi bien bénéficier que pâtir du fait qu'il hérite de l'oncle maternel plutôt que du père. Si, pour certaines tribus d'Algonkin, les clans consanguins se formèrent par la transmission patrilinéaire des prérogatives de chasse, alors que les Zuñi tirèrent leurs clans utérins de la résidence matrilocale et de la possession de la maison par les femmes, la difficulté disparaît du fait qu'aucune des deux règles de descendance ne précède nécessairement l'autre. Je suis convaincu que, dans un grand nombre de cas, les clans consanguins et utérins, soit par emprunt, soit par évolution spontanée, sont sortis directement d'une organisation sans clans.

La Sibérie est si bien connue actuellement qu'elle offre un terrain idéal à l'étude des doctrines sociologiques. Quels indices y allons-nous trouver de l'histoire de l'organisation clanique? Remarquons d'abord que, dans toute cette vaste région, il n'existe pas une seule tribu matrilinéaire. Les Tchouktche et les Koriak, qui y représentent la culture la plus basse, ignorent le clan; les lois de succession, pour autant 
qu'elles sont symptomatiques d'un développement potentiel dans l'une ou l'autre direction, y indiqueraient plutôt le germe d'une organisation patrilinéaire. Il nous est loisible de supposer que l'introduction du renne, animal qui représente un bien des plus précieux, renforça l'importance de la lignée patrilinéaire, mais sans aller jusqu'à la création de clans consanguins distincts. L'histoire de la société des Koriak et des Tchouktche est donc extrêmement simple : c'est un état sans clans à tendance consanguine.

A l'autre extrémité de l'échelle culturelle en Sibérie, nous trouvons des peuples comme les Kirghiz et les Yakut, habiles dans l'élevage des chevaux et du bétail et grands métallurgistes. Ces tribus, comme les Tongouzes, les Ostiak et les Samoyèdes, sont organisées en clans consanguins nettement exogamiques. En outre, nous relevons dans toutes ces populations les coutumes fonctionnellement associées de l'achat de la femme et de la résidence patrilocale, ainsi qu'une extrême uniformité dans la manière dont la femme, considérée partout comme inférieure, est traitée. Il est impossible que ce complexe se soit développé indépendamment un grand nombre de fois dans cette aire ininterrompue. En d'autres termes, ces particularités nettement patrilinéaires ont dû évoluer et se répandre dans toute la zone où on les rencontre actuellement. Nous n'y trouvons aucun indice, même lointain, de tendances matrilinéaires; nous ne sommes sans doute pas loin de la vérité en formulant l'hypothèse que les clans consanguins de ce groupe ont dû naître directement dans un état sans clans, analogue à celui des Tchouktche et des Koriak.

Entre les Tchouktche-Koriak et les Yakut-Tongouzes vivent les Youkaghir qui sont environnés de toutes parts par cette dernière tribu. Les Youkaghir diffèrent de tous les autres peuples de la région par leur matrilocalité. Les Koriak font un temps de service pour gagner leur femme et, en des cas exceptionnels, s'établissent de façon permanente chez celle-ci, pratiques qui semblent avoir abouti chez les Youkaghir à la résidence matrilocale normale. Cette coutume n'a néanmoins pas créé le groupe matrilinéaire; la propriété se transmet de père en fils et l'obligation de satisfaire aux haines de famille incombe à la parenté paternelle. Les Youkaghir, à cause de l'influence des Tongouzes sans doute, ont même des clans consanguins, bien que non exogamiques. Plus intéressante encore est l'assimilation des coutumes des Tongouzes par quelques groupes Youkaghir et vice versa. Les Tongouzes youkaghirisés n'observent plus l'exogamie; les Youkaghir tongoucisés ont adopté l'achat de la femme ainsi que la résidence patrilocale et attribuent au clan une importance plus considérable. Ainsi le développement social, loin de parcourir des phases identiques dans tous les peuples, peut à l'intérieur d'une même région suivre son cours vers des directions opposées. Dans un certain groupe, le contact avec un peuple non exogamique relâchera les liens du clan, alors que dans un autre le voisinage de clans patrilinéaires favorisera la croissance de germes d'organisation paternelle.

Bref, les phénomènes sibériens ne fournissent aucun argument à la doctrine de l'uniformité des lois du progrès social. 
Ils nous montrent, au contraire, la résidence matrilocale n'entraînant pas la descendance utérine, des tribus dépourvues de clans ainsi que de toute trace d'un système clanique antérieur, fût-il consanguin ou utérin, et sans tendance apparente à adopter spontanément un système patrilinéaire défini. Ils sont la preuve surtout de l'extraordinaire influence nivellatrice du contact avec les cultures étrangères.

Ce serait naturellement pur dogmatisme que de se refuser à admettre toute possibilité de modification dans la descendance au sens où l'entendent Tylor et Morgan. Certaines régions de l'Océanie tout au moins nous offrent un bel exemple de l'antériorité relative des clans utérins. On a relevé de façon indiscutable des clans utérins en diverses tribus mélanésiennes. Or, l'hypothèse selon laquelle des peuples apparentés à celles-ci et qui sont actuellement patrilinéaires, ont pu être autrefois matrilinéaires, est fort admissible et le devient d'autant plus si l'on tient compte de certaines conditions d'existence en Mélanésie. Les Mélanésiens, ainsi que beaucoup de groupes matrilinéaires, sont agriculteurs et les travaux des champs incombent souvent aux femmes, situation que nous avons déjà reconnue comme favorable au développement des clans utérins. L'avunculat, bien que n'étant pas décisif en lui-même pour les raisons exposées plus haut, peut faire office, dans ce cas, de preuve à l'appui, car il est possible d'établir une corrélation entre ce phénomène et le système matrilinéaire, et aussi parce que dans certaines tribus océaniennes le pouvoir avunculaire affecte une forme exagérée. Ainsi, parmi les insulaires patrilinéaires du détroit de Torrès occidental, un individu s'arrêtera immédiatement de combattre si son oncle maternel le lui ordonne, alors qu'il ne tiendra pas compte de l'injonction de son propre père. En outre, si nous étudions les tribus matrilinéaires de ladite région, nous trouvons une série de ces conditions qui, précisément, tendent à établir la descendance patrilinéaire dans une communauté sans clans. La résidence patrilocale y est générale; et, tandis que les anciens jardins héréditaires se transmettent au fils de la sœur, le terrain défriché personnellement par un individu passe à ses enfants, ainsi que ses arbres qui représentent une propriété distincte. Le cas des Kaï est spécialement remarquable. Ici les enfants appartiennent de façon non équivoque au groupe de la mère et l'héritage suit la lignée maternelle; pourtant c'est de père en fils que se transmet le titre de chef, et seulement secondairement de l'oncle au fils de la sœur. La résidence est patrilocale dans un certain sens, la femme suit son mari chez lui, mais, comme nous l'avons observé, l'épouse Kaï, bien que vivant dans la maison de son mari, n'est pas autorisée par sa famille à aller habiter un village éloigné afin qu'elle ne la prive pas de ses services. Étant donné ces phénomènes, je ne vois nulle raison de rejeter comme impossible la théorie défendue par Rivers selon laquelle, en certaines parties de l'Océanie, il s'est produit un passage de la descendance matrilinéaire à l'état patrilinéaire. Ce que je maintiens, c'est que cela ne démontre pas l'antériorité de la descendance utérine dans les autres régions du globe, en Australie, en Sibérie ou en Amérique; en Océanie même, l'état matrilinéaire a pu à son tour se surimposer à des conditions patrilinéaires antérieures. 
Nous n'avons pas encore abordé la question de savoir si la descendance matrilinéaire ou patrilinéaire accompagne généralement une forme plus ou moins haute de civilisation. Le point de vue traditionnel assigne naturellement au clan utérin un niveau plus bas. Pour Hobhouse, un auteur plein de bon sens pourtant et qui se refuse à admettre l'universalité de la phase matrilinéaire, la descendance utérine se rencontrerait en général chez les tribus non civilisées et la descendance patrilinéaire parmi les représentants civilisés de l'humanité; à l'intérieur des races non civilisées, la descendance maternelle accompagnerait donc fréquemment les cultures les moins développées. On a, en effet, reconnu que les civilisations les plus hautes, tout en ignorant le clan, sont essentiellement du type paternel, et nous savons que les Grecs et les Romains furent à un certain moment organisés en clans consanguins. Néanmoins, il ne s'ensuit pas que la descendance patrilinéaire soit uniformément un symptôme de haute civilisation. En Australie rien ne nous indique qu'un peuple matrilinéaire comme les Dieri présente une culture plus pauvre que les Arunta patrilinéaires. Les nègres utérins ne le cèdent en rien à leurs congénères consanguins pour ce qui est de l'activité industrielle et de l'organisation politique. En Amérique, au nord du Mexique, ainsi que Swanton l'a fait remarquer, les tribus utérines occupent généralement un niveau plus élevé que les consanguines. Dix ans environ avant Swanton, Cunow, dont la combativité puérile ne doit pas nous faire oublier les mérites de savant consciencieux et de penseur indépendant, avait déjà montré que les indigènes de l'Amérique du Nord qui connaissent la culture intensive du sol sont surtout matrisinéaires, alors que les tribus à descendance consanguine représentent des conditions économiques moins élevées. En outre, si nous tenons compte non seulement des communautés nettement organisées en clans, mais aussi des tribus sans clans dont on a prouvé qu'elles possèdent une culture grossière, nous trouverons que, dans la grande majorité des cas, les quelques germes de descendance unilatérale discernables appartiennent à la variété patrilinéaire. Ce qui revient à dire que les civilisations sans clans les plus basses, si elles présentent quelque tendance à départager la parenté par les procédés exposés plus haut, le feront par ségrégation de la famille paternelle. Ainsi les Bochimans se lèguent de père en fils les terrains habités; les Tchouktche éleveurs de rennes ainsi que la fraction maritime de cette peuplade qui représente son état antérieur, observent l'héritage de la propriété par le fils; les Indiens de Thompson River en Colombie britannique et les Shasta de la Californie transmettent patrilinéairement le droit aux stations de pêche; et nous avons souvent mentionné l'influence de la résidence patrilocale sur l'existence des Hupa et des Algonkin du nord-est. Il se rencontre sans doute des cas de résidence matrilocale parmi les tribus dépourvues de clans; mais, ou bien elle alterne avec la résidence patrilocale, ou bien elle se limite au début du mariage et n'arrive pas ainsi à créer une base suffisante à la formation d'un clan unilatéral, d'où la rareté des clans utérins dans les tribus de chasseurs.

Bref, les généralisations de Hobhouse ne s'accordent nullement avec la réalité. Les plus hautes civilisations connues, telles celles des Chinois, des Grecs et la nôtre, sont, il est vrai, essentiellement patrilinéaires, mais c'est aussi le cas des cultures les moins développées pour autant qu'elles présentent une filiation unilatérale, alors que 
les peuples matrilinéaires occupent une position intermédiaire. Cette affirmation, contrairement à mon intention et à ce que j'ai déclaré plus haut, pourrait être interprétée, mais à tort, comme fixant un ordre de succession déterminé dans les phases culturelles. C'est pourquoi je m'empresse de parer à la possibilité de telles erreurs en esquissant ce qui me semble être une ligne possible d'évolution dans un cas hypothétique concret et en démontrant que la corrélation reconnue entre certains phénomènes économiques et sociologiques s'accorde parfaitement avec mon refus d'admettre que la descendance utérine représente une phase universelle dans l'histoire de l'humanité. Imaginons les habitants des îles Andaman, peuple dépourvu de clans et sans penchant discernable vers l'une ou l'autre branche de la famille, s'élevant par des emprunts successifs à une phase culturelle plus haute sans nécessairement s'organiser en clans consanguins ou utérins. La question de savoir si, dans ces circonstances, il se formerait de telles institutions, dépend pour une large part de la nature, de l'intensité et de la durée des influences étrangères. Je n'affirme pas par là que le progrès industriel que nous supposons ne produise aucun effet sur l'organisation sociale, mais simplement qu'il ne donne pas naissance à des groupes de parenté unilatéraux. Si, par exemple, ces insulaires apprenaient à travailler le fer, ce progrès industriel impliquerait ou, du moins, favoriserait l'évolution sociale d'une corporation professionnelle de forgerons. Celle-ci pourrait se développer en une caste héréditaire, comme c'est arrivé chez les Massaï, sans que ce processus porte un caractère de nécessité. Il pourrait se produire simplement une ségrégation des individus qui présentent des aptitudes spéciales dans ce sens, donnant naissance à une corporation commerciale et à la complication sociale qui en résulte, mais non à un clan héréditaire. En outre, même si le métier devenait héréditaire, cela n'entraînerait pas la création de clans, aussi longtemps que le reste de la population ne s'organiserait pas sur un modèle analogue. En d'autres termes, les Andaman ne pourraient franchir le pas qui sépare l'âge de la pierre de l'âge du fer, sans opérer quelque réajustement social, mais ils n'adopteraient pas pour tout autant le principe de la parenté unilatérale.

En dépeignant comme un tout l'histoire sociale de l'humanité, il convient de ne jamais perdre de vue les phénomènes se rattachant à la division du travail entre les sexes. J'ai déjà démontré comment la descendance matrilinéaire peut se développer dans une communauté sédentaire où les femmes travaillent le sol à la houe. Notons cependant que là où l'agriculture se pratique de façon plus intensive, bien que toujours avec des outils primitifs, ainsi que dans les civilisations supérieures américaines, c'est à l'homme que revient d'ordinaire la part essentielle de ces travaux. Il est donc fort compréhensible que, dans de telles conditions, les clans consanguins se soient développés. La descendance matrilinéaire des Pueblo ne peut s'expliquer par les mêmes motifs que celle des Hidatsa, parce que chez les Pueblo ce sont les hommes qui s'occupent du mais; elle se base sur le facteur tout différent de la résidence matrilocale et de la propriété du sol par les femmes. Sans cet élément, les Hopi n'auraient peut-être pas connu de groupes unilatéraux de parenté ou se seraient organisés en clans consanguins. Cela signifie que la phase de la petite agriculture n'implique pas nécessairement la descendance utérine, car, puisque les hommes prennent parfois part aux travaux de culture ou les assument en totalité (ainsi qu'ils le font 
en certaines parties de l'Amérique et de l'Océanie), les conditions requises à la ségrégation de la parenté matrilinéaire peuvent faire défaut.

Or, ainsi que Hahn nous l'a enseigné, l'agriculture dans les civilisations avancées repose sur l'emploi de la charrue et du bœuf domestiqué (ou d'un équivalent), et ces conquêtes culturelles sont certainement associées au sexe masculin. La domestication est, sans aucun doute, un exploit des hommes; il est intéressant de noter les tabous sévères qui maintiennent parfois les femmes à l'écart des troupeaux, ainsi que le fait encore plus important que la femme, dans les tribus pastorales les plus développées, occupe un rang nettement inférieur. Il est hors de doute que l'agriculture et la domestication ont fait leur apparition plus tard que l'horticulture; à supposer qu'elles se soient développées à partir de cette dernière, nous aurions de nouveau des phases en ordre de succession déterminé, correspondant à des stades parallèles de descendance utérine et consanguine, selon les vieilles théories longtemps en honneur. Mais la question n'est pas essentielle. En dehors du fait que l'horticulture intensive, ainsi que nous l'avons vu plus haut, incombe parfois à l'homme, nous n'avons aucune preuve que l'agriculture, si différente par ses méthodes de l'horticulture, ait son origine dans celle-ci. Il est fort possible que le complexe agricole tel qu'il existe en Asie occidentale ait évolué grâce à l'effort masculin indépendamment du complexe horticole féminin ; et il n'y a, en conséquence, nulle raison de supposer que les représentants des grandes civilisations historiques aient jamais traversé une phase maternelle. Il va sans dire que si les femmes furent les premières à cultiver le millet et le blé, les hommes ne furent pas tenus de découvrir à nouveau la culture de ces céréales. Mais lorsque les circonstances permirent le développement de la charrue, l'usage de la houe ou du bâton à fouiller revint probablement aux hommes dans les civilisations du Proche Orient et du nord-est des Indes, ainsi que la chose est certaine au Mexique et au Pérou. Il ne faut pas voir là un refus dogmatique d'admettre qu'aucune de ces civilisations ait connu la descendance utérine qui, en quelques cas, aurait pu résulter d'influences étrangères, mais simplement le rejet du dogme selon lequel ces cultures doivent autrefois avoir observé la descendance utérine. Je ne vois pas, par exemple, pourquoi les anciens Grecs n'auraient pas passé directement d'un état sans clans, mais à tendance paternelle, à des conditions nettement patrilinéaires représentées par le genos et la phratrie.

Passant en revue les phénomènes de toutes les parties du monde, nous ne pouvons manquer d'observer que, alors que la descendance consanguine nettement marquée fait souvent défaut, il est extrêmement courant de trouver une certaine accentuation asymétrique de l'influence du père. L'hypertrophie des facteurs matrilinéaires nous apparaît en comparaison comme un phénomène hautement spécialisé qui s'ajoute aux caractéristiques paternelles plutôt qu'il ne s'y substitue. C'est pourquoi, même dans des sociétés nettement matrilinéaires, le père et les siens jouent un rôle relativement plus important que la mère et son groupe dans les sociétés fortement paternelles comme les Chinois et les nomades turcs. C'est ce qui nous explique aussi l'instabilité des institutions matrilinéaires en certains cas spéciaux, par exemple en Océanie. Non point que les facteurs maternels aient tendance à disparaître en faveur des paternels; 
ce sont plutôt les facteurs paternels qui, jamais supprimés mais suspendus temporairement dans certaines conditions, reprennent toute leur force dès que ces conditions disparaissent. Cette évolution, comme toutes les autres, n'est pas inévitable, mais sujette à l'influence de cultures voisines.

En résumé, il n'existe pas d'ordre de succession déterminé des descendances utérine et consanguine; les tribus sans clans peuvent passer directement à l'état matrilinéaire ou patrilinéaire; si les civilisations les plus avancées soulignent le côté paternel de la famille, beaucoup des moins évoluées le font aussi; et l'histoire sociale d'un peuple donné ne peut être reconstruite selon un système d'évolution valable pour la généralité des cas, mais seulement sous l'angle des relations culturelles certaines ou probables qu'il a entretenues avec ses voisins.

Chapitre 7

\section{Références}

$\underline{\text { Retour à la table des matières }}$

1) MORGAN, 1871 :484,490; id, 1877. SWANTON, 1905 (b) :663; id., 1906 : 166. MARTIN : 861. SKEAT et BLAGDEN : 1, 65; 11, 62, 258. MAN : 58 et suiv., 202. SCHULTZE : 305. RIVERS, 1914 (a) : 67-70. LOWIE, $1915:$ 231. BOGORAS : 538. JOCHELSON, 1908 : 759. RADCLIFFE-BROWN : 157.

2) WHIFFEN : 63, 66. SPECK, 1918 : 143; id., 1915 (a) et (b). TYLOR, 1889 :258; id., 1896 :81. WILSON 9 et suiv., 113 et suiv.

3) LOWIE, 1919 (b) :28. MORGAN, $1871: 475$.

4) MORGAN, 1877 : part. II, chap. II et XIV. MAN : 67 et suiv. SPENCER et GILLEN, $1904: 524$. TYLOR, 1889 : 245. LOWIE, 1919 (a) : 29. HARTLAND : 1 et suiv. Reports, V : 144. RIVERS, 1914 (b) : 1, 55; 11, 126. KEYSSER : 42, 85, 100. HOBHOUSE : 162. CUNOW, $1894: 138$ et suiv. BLEEK et LLOYD : 305. BOGOR As : 679. TEIT, 1900 : 293. DIXON, $1907: 452$. 
8

\section{CONDITION DE LA FEMME}

$\underline{\text { Retour à la table des matières }}$

Des opinions diamétralement opposées ont cours dans le publie instruit au sujet du sort de la femme dans les sociétés primitives. On se la représente d'une part comme une esclave, une bête de charge ou guère plus, condamnée aux corvées les plus dures, achetée comme un objet et livrée sans recours aux brutalités de son maître. Ceux, au contraire, qui ont entendu parler de tribus à descendance maternelle et qui se sont assimilé quelques bribes de doctrines sociologiques périmées d'il y a un demisiècle, considèrent la femme comme le chef incontesté de la famille sinon de la communauté elle-même. Ces deux conceptions sont, dans la grande majorité des cas, également éloignées de la réalité. Cependant, telle est la variabilité des relations entre la femme et la société que toute affirmation d'ordre général doit être sujette à caution. Il convient d'approcher notre sujet par plusieurs voies différentes avant d'émettre un jugement qui ait quelque valeur.

\section{a. Théorie et pratique}

Tout d'abord, il convient de noter que le traitement de la femme, son statut légal, les occasions qui lui sont offertes de prendre part à l'activité publique et enfin la nature et l'étendue de ses travaux appartiennent à quatre catégories distinctes. S'il 
existe une corrélation entre deux d'entre elles, elle est purement empirique. Du point de vue conceptuel, elles ne sont pas les mêmes et l'ignorance de ces distinctions ne peut qu'aboutir à la confusion. Nous le comprendrons immédiatement en considérant certains phénomènes bien connus. La favorite de harem n'est pas astreinte aux tâches d'une servante et pourtant le sort qui lui est échu n'est pas compatible avec notre idéal de dignité humaine; la même remarque s'applique, à un moindre degré seulement, à la dame noble du temps des chevaliers. Dans un milieu tout différent, les femmes Toda, bien traitées pourtant, passent pour des êtres inférieurs et sont exclues des pratiques rituelles qui jouent un rôle de tout premier plan dans la civilisation Toda; on ne leur laisse même que fort peu d'occupations puisqu'on leur défend aussi de préparer les repas, du moins les aliments où il entre du lait. D'autre part, la femme des îles Andaman est virtuellement sur un pied d'égalité avec son mari, bien que des tâches en plus grand nombre retombent sur ses épaules. En Asie centrale, il est spécialement instructif de comparer les Kirghiz et les Altaïens. Ces deux tribus assignent à la femme une position nettement inférieure, mais les Kirghiz, peut-être sous l'influence de l'Islam, traitent leurs femmes avec beaucoup plus de sévérité que les Turcs Altaïens. Une lecture superficielle des travaux de Radloff pourrait nous faire accroire que le statut légal de la femme est, à peu de choses près, le même dans ces deux tribus, avec une amélioration sensible toutefois de son sort chez les Altaïens. Mais un examen plus attentif nous révélera qu'en dépit des conceptions légales renforcées par des sanctions religieuses, la femme Kirghiz est décidément en meilleure posture. Chez les Altaïens, elle est à l'œuvre dès l'aube jusque tard dans la nuit; outre les soins du ménage, elle doit s'occuper du bétail, chercher le combustible, traire les vaches, les brebis et les chèvres, fabriquer tous les ustensiles et même cultiver les champs d'orge. Les hommes ne font guère plus que couper le bois, traire les juments et apporter leur aide à la fabrication des ustensiles de ménage. Chez les Kirghiz, la division du travail est beaucoup plus équitable, car ce sont les hommes qui soignent le bétail, apportent le combustible, font les récipients et travaillent la terre. En conséquence, les femmes Kirghiz jouissent d'une liberté bien plus grande que leurs sœurs de l'Altaï : elles participent aux fêtes, font des visites à leur gré, assistent aux jeux et aux assemblées publiques et prennent part aux concours de chant. Bref, elles sont amplement dédommagées des mauvais traitements signalés.

Cet exemple nous est une preuve de la prudence à observer lorsqu'il s'agit de formuler le statut des femmes dans une société déterminée. Les relations entre hommes et femmes sont multiples et il est dangereux d'en souligner uniquement un aspect. C'est à la théorie qu'il faut attacher le moins d'importance. La théorie influence la pratique, mais souvent modérément. En théorie, le Kirghiz musulman peut divorcer d'avec sa femme à son gré; en pratique il ne le fait que fort rarement. La métaphysique chinoise assimile au mal le principe féminin universel et, dans ce peuple, le statut légal de la femme est d'une abjecte infériorité. Cela n'a pas empêché beaucoup de Chinoises de régner sur leur maison par la force de leur personnalité, ni de jouer un rôle appréciable dans les affaires et en littérature. $\mathrm{Si}$, au lieu de prendre un cas extrême, nous revenons aux périodes récentes de notre civilisation, nous verrons que les Américaines n'étaient nullement maltraitées avant qu'elles obtinssent le droit de 
vote; leur incapacité à posséder ou à administrer des biens n'impliquait en aucune façon qu'elles fussent soumises à la volonté du mari; enfin, en un temps où il était peu question d'émancipation féminine, les mondaines des salons parisiens exerçaient une influence qu'on ne saurait surestimer. En d'autres termes, il est important de connaître ce que le droit coutumier et la philosophie théorique statuent sur les obligations et les droits féminins, mais il l'est encore bien plus de se rendre compte si la pratique se conforme à la théorie ou la dépasse, ainsi qu'il arrive parfois. Le rôle exagéré qui est fréquemment assigné aux propositions abstraites et aux décrets légaux relève de ce rationalisme pervers qui a si souvent obscurci la compréhension des institutions des hommes et de leur psychologie.

Chapitre 8

\section{b. Matriarcat}

\section{$\underline{\text { Retour à la table des matières }}$}

On interprétait autrefois la descendance utérine comme un signe de suprématie féminine non seulement dans la famille, mais aussi sur ce qui équivalait primitivement à l'État. Il n'est probablement pas un seul problème théorique sur lequel les sociologues soient en aussi parfait accord maintenant que sur la non-valeur absolue de cette déduction. Le témoignage apporté par l'ethnographie est trop net pour qu'il puisse être écarté par des spéculations a priori. Parmi les Australiens, quelques tribus sont utérines, d'autres consanguines, mais la condition de la femme dans les premières n'est en rien meilleure que dans les autres. Il en va de même pour la Mélanésie. En Colombie britannique, les Tlingit et leurs voisins sont matrilinéaires, cependant l'autorité sur les enfants est exercée par les oncles maternels et non par la mère elle-même. Certains biens particulièrement estimés ne sont pas possédés par les femmes, mais transmis avec une régularité automatique de l'oncle maternel au neveu. On nous signale en Afrique des femmes-chefs, mais leur présence semble n'avoir aucun rapport avec la descendance et n'affecte pas plus le statut des négresses moyennes que le règne de Catherine ne modifia le sort des paysannes russes.

Il existe quelques exemples, si rares qu'on pourrait les compter sur les doigts d'une seule main, de peuples où les femmes jouissent de droits de propriété inusités ou jouent un rôle extraordinaire dans la vie publique. Les cas les plus connus sont les Khasi d'Assam, les Iroquois et les Pueblo.

Chez les Khasi il existe une combinaison pour ainsi dire unique de prérogatives féminines. Ici la maison, les biens immobiliers et les beaux bijoux de famille ne se transmettent pas seulement en ligne maternelle, comme c'est le cas en Colombie britannique, mais sont détenus par les femmes de la lignée maternelle, c'est-à-dire qu'ils passent de la mère à la fille. Dans une certaine localité même, la dignité de grand prêtre est occupée par une femme, et celle qui lui succède est choisie parmi ses 
parentes. Ce serait, cependant, une grave erreur que d'en inférer que l'homme ne compte pour rien dans les communautés Khasi. Dans le ménage, bien que la femme soit propriétaire, c'est son frère aîné qui règne et, lorsque le mari, après la résidence matrilocale du début, s'établit pour son compte, c'est lui le maître incontesté. En outre, le droit coutumier reconnaît au mari la possibilité de tuer la femme adultère surprise en flagrant délit. La souveraineté politique se transmet en ligne féminine, mais d'un homme à l'autre; c'est seulement en l'absence d'héritiers mâles que la femme est appelée à succéder; dans la suite elle passe sa charge à son fils et non à sa fille. Ce n'est, à n'en pas douter, que par courtoisie que ce système est appelé matriarcat.

Les Iroquois sont ceux qui se rapprochent probablement le plus de l'état matriarcal. Chez ces Indiens, c'étaient les femmes qui arrangeaient les mariages et qui, semble-t-il, possédaient maisons et terres. Quelques-unes des plus importantes organisations cérémonielles étaient en bonne partie constituées et dirigées par les femmes et c'était dans leurs rangs qu'on choisissait trois sur six des fonctionnaires rituels de chaque clan. Les femmes nommaient leur candidat lors d'une vacance au conseil des chefs et avaient le droit de désapprouver et même d'empêcher l'élection d'un chef qu'elles jugeaient indigne. Néanmoins, c'est un fait certain que, même parmi les Iroquois, les femmes n'entraient pas dans le conseil suprême de la Ligue.

Dans les villages pueblo, la situation de la femme est nettement moins importante que chez les Iroquois. Ainsi que l'a formulé Kroeber avec justesse: " c'est sur la propriété de la maison par la femme que repose le soi-disant matriarcat des Zuñi ». Les femmes n'ont pas voix aux affaires gouvernementales; dans les rites elles jouent un certain rôle, il est vrai, mais secondaire; et, même dans la maison, l'homme, pour autant qu'il y habite, est le maître véritable. Ces observations coïncident en grande partie avec celles que j'ai faites chez les Hopi.

Tous ces exemples fournissent une base a fortiori à la conclusion que le matriarcat pur n'existe nulle part, quoique en quelques localités les privilèges féminins aient atteint, dans certaines conditions, un développement notable. Le fait que ces privilèges se rattachent parfois à la descendance utérine ne garantit nullement que celle-ci en soit la cause efficiente; les phénomènes relevés en Australie, en Mélanésie et en Colombie britannique sont significatifs à cet égard. Nous avons reconnu que la descendance utérine résulte de conditions plus fondamentales, telles que le mode de résidence et l'activité économique; il convient donc d'étudier si ce sont aussi ces mêmes causes qui ont influé sur la situation de la femme. En d'autres termes, s'il y a relation causale, l'ordre probable ne sera pas : descendance utérine, d'où résidence matrilocale, d'où amélioration du statut juridique de la femme, mais plutôt : résidence matrilocale, d'où amélioration du statut et peut-être aussi descendance utérine. Il en va de même pour les autres phénomènes essentiels.

Chapitre 8 


\section{c. Résidence matrilocale}

$\underline{\text { Retour à la table des matières }}$

Les effets de la résidence matrilocale sur la condition de la femme ont déjà été brièvement exposés. Ici comme ailleurs, nous ne devons pas nous laisser éblouir par les mots ni tirer des conclusions extrêmes d'une mince base de réalité. Le résultat immédiat de la résidence matrilocale n'est pas la prépondérance de la femme, mais celle de sa famille à elle. Il existe une grande différence entre les ménages des Esquimaux du centre où la femme s'établit dans la famille du mari et ceux dans lesquels l'époux va vivre chez ses beaux-parents; mais cette différence n'affecte pas le statut de la femme en tant que femme. Dans le premier cas, elle est subordonnée à sa bellemère, dans le second à sa propre mère; dans l'un comme dans l'autre, le chef suprême, c'est l'homme, que ce soit son père ou son beau-père. Naturellement, lorsqu'elle n'est pas entourée de ses parents, la femme est moins protégée que lorsque le mari est obligé de tenir compte de ses beaux-parents avec qui il vit. Chez les Kaï, la résidence matrilocale n'est pas incompatible avec une infériorité féminine bien marquée.

Bien qu'en apparence patrilocaux, puisque la femme va vivre chez le mari, nous avons vu qu'ils sont au fond matrilocaux, les parents de la femme s'opposant à ce qu'elle sorte de leur sphère d'influence. C'est vis-à-vis d'eux que l'homme est responsable s'il maltraite sa femme ou s'il détruit ce qui lui appartient. Mais, quoi qu'il en soit, elle reste sous la tutelle d'un homme, que ce soit son frère, son oncle maternel ou son grand-père. En outre, il est évident que la résidence matrilocale, en limitant les unions polygamiques, tend aussi à éliminer les difficultés auxquelles une femme est parfois exposée du fait d'un favoritisme conjugal dans les cas de polygynie. En somme, il est clair que la femme a avantage à résider de façon continue sous le toit de ses parents, mais elle ne s'émancipe pas nécessairement par là même de l'asservissement à une volonté étrangère; et lorsque, ainsi que parmi les Youkaghir, le mari finit par être le maître de la maison, elle sera exactement dans la même situation que s'il l'avait été dès le début.

Il en va différemment lorsque les femmes sont propriétaires de la maison. La résidence matrilocale donne alors véritablement l'avantage à la femme, puisqu'elle peut expulser son mari de chez elle, ainsi que cela arrive chez les Pueblo. C'est assurément une supériorité dans les relations conjugales, mais la situation de l'homme n'en est pas aussi sérieusement affectée qu'il semblerait au premier abord, puisqu'il lui est toujours loisible de se réfugier chez sa mère ou ses sœurs; il trouve toujours un abri, en raison d'un droit reconnu à se domicilier dans sa propre parenté féminine.

Chapitre 8 


\section{d. Interprétation économique}

$\underline{\text { Retour à la table des matières }}$

Les facteurs économiques ont considérablement transformé plusieurs aspects des civilisations actuelles et en sont venus à jouer un rôle exagéré dans plusieurs systèmes sociologiques. Il est un problème d'une complication extrême, c'est celui de savoir comment et dans quelle mesure ils ont contribué à la formation du statut de la femme, dans la part de travail qui lui incombe tout au moins. Pourtant, les pêcheurs de l'Amour, les cultivateurs chinois, les cavaliers et éleveurs turcs ainsi que les Ostiak nomades et éleveurs de rennes, tous ont une même conception du sexe féminin. On peut nous objecter que, chez ces peuples si divers par leur mode d'existence, on retrouve un trait commun : le fait que la femme ne contribue pas matériellement à l'alimentation; cependant, dans des régions comme le sud de l'Afrique ou l'Amérique du Sud, nous voyons que les femmes plantent et récoltent, et occupent pourtant une situation humiliante, du moins nettement subordonnée. D'autre part, il existe des tribus de chasseurs, comme les Vedda et les Andaman, où la femme ne contribue que modérément à fournir des vivres et est néanmoins considérée socialement comme l'égale de l'homme.

Cependant, il est un rapprochement qu'on peut faire, qu'on a fait souvent, et que la réalité rend fort vraisemblable. Dans les populations qui se vouent à l'élevage, le statut féminin est uniformément et d'une façon indiscutable défavorable. Ainsi, selon Hobhouse, le pourcentage des cas où la femme occupe un niveau peu élevé dans l'échelle sociale est de 73 parmi les agriculteurs et de 87,5 dans les tribus pastorales. La chose s'explique aisément par des raisons d'ordre économique. La domestication des animaux fut, sans aucun doute, le fait des hommes et le soin des troupeaux est resté à peu près partout une occupation masculine. Cette dissociation complète opérée entre la femme et le travail productif aboutit, selon cette hypothèse, à l'abaissement social du sexe féminin. A mon avis, cette remarque doit s'étendre aux peuples agricoles, distingués ici des horticoles. En effet, ainsi que Hahn l'a montré, ce n'est pas seulement la domestication, mais aussi le labour à la charrue qui sont, dans l'histoire de la civilisation, liés à l'effort masculin. Nous avons ainsi une explication fort plausible des relations entre sexes dans tous les centres culturels de l'Ancien Monde qui ont fourni la base de notre civilisation. Nous comprenons pourquoi la femme en Chine, en Asie centrale et aux Indes, qui est économiquement dépendante, occupe un niveau nettement inférieur à celui de l'homme.

Je ne crois pas, néanmoins, que la relation causale soit aussi étroite qu'il semblerait tout d'abord, Personne ne conteste que la culture de l'orge, du millet et du blé, la domestication du bœuf et du cheval, l'usage de la charrue et de la roue ont eu une même origine et se sont ensuite répandus sur de vastes régions. On peut donc supposer que le corrélat sociologique du développement économique, c'est-à-dire la condi- 
tion inférieure de la femme, ne fut qu'un accident historique. En une certaine région et à un moment donné, il résulta de la dépendance économique de la femme qu'on lui assigna un statut inférieur; ce n'en fut, cependant, pas une conséquence nécessaire et on aurait pu l'éviter par un ensemble approprié de conceptions éthiques et idéologiques. Celles-ci n'existant pas, l'association, tout empirique, d'un statut inférieur avec la non-participation à un système économique avancé put se produire à un certain moment; elle se répandit ensuite et s'est maintenue par pur conservatisme.

Je crois, pour ma part, que la force de suggestion et l'inertie mentale sont, ainsi que l'ethnologie et la sociologie nous l'ont prouvé, si puissantes qu'elles rendent fort vraisemblables la propagation et la conservation d'un complexe accidentel. On pourra m'objecter, et à juste titre, que le seul fait de la diffusion et de la durée d'une coutume présuppose qu'elle répond à certains besoins dans le milieu qui l'adopte. Nous voyons, d'autre part, chez les Hottentots la vie pastorale marcher de pair avec l'égalité des sexes, alors que parmi leurs voisins, les Bantou, les femmes, bien que travaillant aux champs, ont une condition inférieure. Ces faits sont nettement contraires à la doctrine selon laquelle l'activité économique élève automatiquement le statut de la femme, ainsi qu'à la théorie de la vie pastorale préjudiciable au statut féminin. Le facteur économique est peut-être une cause agissante, mais il ne saurait représenter au plus qu'une déterminante parmi d'autres, et ses effets peuvent être réduits ou même rendus imperceptibles par d'autres. Par exemple, une croyance religieuse bien définie a pu retarder et même empêcher que les Hottentots acceptassent l'infériorité sociale de la femme, alors qu'ils adoptèrent volontiers les aspects les plus utiles du complexe de l'élevage.

Nous aboutissons ici à un principe important : une peuplade peut n'adopter que partiellement un complexe en diffusion. Il arrive que ce soit l'élément sociologique ou utilitaire qui se répande indépendamment du résidu, et je suis fortement convaincu que c'est ce qui nous empêche de saisir nettement le lien causal entre la vie économique et le statut féminin. Si bien que s'ajustent par la suite les phénomènes les uns aux autres, la raison immédiate du point de vue adopté vis-à-vis de la femme dans une grande partie de l'Asie réside dans le fait qu'il y a eu contact entre les diverses peuplades partageant cette manière de voir. La chose nous deviendra évidente si nous observons que nous n'avons pas affaire à une notion vague mais, au contraire, à une conception parfaitement définie de l'infériorité féminine. Comme le montre l'exemple des Kirghiz, cette notion n'implique pas la réclusion, ainsi qu'en d'autres régions. Elle signifie que la femme est un bien dépendant qui peut être vendu au plus offrant, hérité par les parents du mari et qu'étant une propriété elle-même, elle est incapable de posséder des biens. Lorsque ces mêmes notions se retrouvent parmi les Syryan, les Ostiak, les Altaïens, les Kirghiz et d'autres tribus occupant une zone ininterrompue, il est manifeste qu'il s'agit d'une caractéristique ethnologique définie qui s'est répandue dans le territoire en question. Le fait devient encore plus certain lorsque nous voyons que, dans les tribus paléo-asiatiques de la Sibérie orientale, la femme, quoique toujours inférieure à l'homme, occupe une situation bien moins humble que sa sœur de l'ouest sibérien. Ainsi la femme tchouktche n'est pas achetée par son mari, elle le 
quitte si elle est provoquée et elle peut posséder des biens. L'introduction du renne a modifié quelque peu son sort, comme on le verra plus loin, mais sans la faire descendre au niveau de la femme ostiak, car l'ancienne culture des Tchouktche et de leurs voisins avait déterminé son mode d'existence, et celui-ci n'a pu être transformé simplement par l'action d'un facteur économique nouveau.

La condition de la femme dans une tribu donnée est en fonction des relations historiques de cette tribu; cela est encore plus évident en Océanie et en Australie. Les particularités saillantes de l'infériorité féminine dans cette région sont toutes différentes de celles qui nous ont frappés en Sibérie. Dans presque toute l'Australie ainsi qu'en Mélanésie et en Nouvelle-Guinée, nous trouvons une forte tendance à séparer les sexes, quelquefois même pendant les repas et surtout lors des cérémonies. L'exclusion des femmes de la vie publique forme un contraste marqué avec leur participation aux fêtes chez les Kirghiz et nous montre combien de nuances peut renfermer le terme d' " infériorité ». Mais ce ne peut être par hasard qu'une notion aussi bien définie soit répandue dans toutes les Mers du Sud; la présence de ce phénomène dans toute localité de cette aire est attribuable, sans aucun doute, au fait que ladite localité fait partie d'une zone géographique sur laquelle cette particularité s'est répandue. Quelle que soit l'origine de la coutume, sa cause seconde, par conséquent son explication scientifique, ne repose pas sur quelque facteur économique mais sur des relations historiques et géographiques.

L'Amérique du Nord nous fournit des témoignages à l'appui. Dans la plus grande partie de ce continent, les Indiens, quelle que soit la condition de la femme, n'observent pas cette séparation minutieuse des sexes si frappante dans les Mers du Sud. L'importance rituelle des femmes iroquois a déjà été notée. Même dans les tribus où leur statut est beaucoup moins élevé, les femmes prennent part aux cérémonies et, parmi certains Indiens des Prairies, la conception de l'union de l'époux et de l'épouse au point de vue cérémoniel apparaît clairement dans le transfert et les soins des objets sacrés. Ce ne saurait être par accident que tout ce qui rappelle une dichotomie sexuelle de la société nous est signalé dans le voisinage de la côte du Pacifique. C'est seulement dans l'Alaska que les Esquimaux ont une maison dont les femmes sont exclues; c'est seulement parmi les Athapaskan du Nord que les filles sont séparées des garçons et qu'il est défendu aux femmes de prendre part aux danses; c'est seulement en Californie que nous rencontrons des sociétés masculines comparables par l'exclusion jalouse du sexe féminin aux organisations mélanésiennes. Si, chez les Hupa, les hommes ne dorment pas avec les femmes, c'est qu'ils ont eu probablement des relations culturelles avec d'autres populations californiennes qui observaient cette coutume. Pour toutes les tribus, à l'exception de celle dans laquelle s'est développé le phénomène diffusé par la suite, la cause agissante n'est pas tel ou tel facteur économique mais simplement l'emprunt. Dans le lieu d'origine, qu'on ignore généralement, l'activité des facteurs économiques a dû se faire sentir; partout ailleurs leur action a été purement sélective, c'est-à-dire qu'ils ont favorisé ou empêché la diffusion, sans agir comme force créatrice. Ceci nous explique pourquoi des tribus totalement diver- 
gentes par leur mode d'alimentation en sont venues à partager des opinions identiques sur la femme, ce qui, pour la théorie économique, reste un mystère complet.

Un examen hâtif des faits économiques ne suffit donc pas à établir une corrélation particulièrement intime entre eux et la condition de la femme. Répétons quelles sont les divergences les plus significatives : dans les communautés horticoles en Mélanésie et en Amérique du Sud où les femmes travaillent le sol à la houe, leur prestige est moindre que dans les tribus de chasseurs comme les Vedda et les habitants des îles Andaman. La vie pastorale n'a pas dégradé la femme chez les Hottentots. Nous voyons que partout l'influence des relations culturelles intertribales a été énorme.

Néanmoins, une étude plus précise de l'influence possible des faits économiques s'impose. Plusieurs problèmes importants se présentent à l'ethnographe, mais en général les informations concrètes nécessaires à leur solution font défaut. Ainsi, parmi tous les Bantou, les Herero sont les seuls à ne faire que de l'élevage. Il serait donc intéressant de comparer le statut de leurs femmes avec celui de leurs voisins, les Ovambo, où l'élevage coexiste avec le jardinage. Mais je ne connaît pas de travaux qui puissent nous renseigner à ce sujet. Les Navaho sont devenus depuis l'arrivée des Espagnols une peuplade de pasteurs prospère, mais nous ignorons tout de la condition sociale de leurs femmes au XVIe Siècle. Les Indiens des Prairies nous fournissent des exemples de tribus adonnées uniquement à la chasse et d'autres, proches parentes, où la culture du maïs se combinait avec la chasse; mais on pourra sensément objecter que, puisque ces tribus dépendaient toutes du bison surtout, l'absence ou la présence de champs cultivés ne pouvait produire de modification sociale profonde. Il n'existe certainement aucune différence sensible entre le statut de la femme chez les Hidatsa semi sédentaires et chez les nomades Crow, leurs parents. On a signalé en Mélanésie des divergences locales relatives à la division sexuelle des travaux horticoles, mais sans mettre en corrélation les faits qui nous intéressent avec les coutumes sociologiques correspondantes.

L'exemple qui peut le mieux nous satisfaire ici est celui des Tchouktche; la description détaillée que Bogoras nous en donne est un chef-d'œuvre de l'ethnographie. Les Tchouktche étaient à l'origine un peuple de chasseurs maritimes ressemblant aux Esquimaux par leur mode d'existence. Une partie de la tribu adopta l'élevage du renne, ce qui eut pour conséquence de la fractionner en deux branches vivant maintenant l'une à côté de l'autre avec des traditions culturelles identiques et différant seulement par leur vie économique. C'est ce qui nous permet d'isoler ici presque aussi complètement qu'il est possible le facteur économique. Quel sera donc le statut de la femme chez les Tchouktche de la mer et chez ceux du renne? Si nous ne faisons qu'appliquer un terme général à toute une série de faits, nous ne décèlerons aucune différence, car, dans les deux groupes, la femme est subordonnée à l'homme. Les divergences sont néanmoins intéressantes. Les expéditions en mer des chasseurs de la côte sont difficiles et pleines de dangers; les femmes ne se lancent naturellement dans ces entreprises masculines que poussées par la nécessité. Parmi les Tchouktche éleveurs de rennes, il arrive fréquemment que les jeunes filles se substituent aux 
hommes pour garder les troupeaux et mènent ainsi une existence indépendante; les femmes aident normalement à soigner les rennes et font tout le travail d'écorchage et de boucherie. Pour le berger nomade, le mariage est une nécessité. " L'homme ne peut avoir une vie supportable s'il ne possède sa propre maison et une femme qui en prenne soin. » Il a besoin de quelqu'un qui lui raccommode ses vêtements, les lui fasse sécher, qui l'aide à soigner les troupeaux; au contraire de son frère sédentaire, il lui faut une femme pour s'occuper de sa tente de nomade. Il n'est donc pas étonnant que le célibat soit un peu plus fréquent parmi les chasseurs de la côte. D'un autre côté, le caractère indispensable de la femme dans les bandes nomades s'accompagne d'un accroissement considérable de travail. Ses occupations ménagères sont multiples et d'autres tâches encore viennent s'y ajouter. Le chasseur de mer ne peut guère entretenir plus d'une femme; parmi les nomades, on rencontre assez fréquemment des familles polygynes, car un riche éleveur aspire à avoir une épouse pour chacun de ses troupeaux. Chez les tribus de la côte, la femme est forcément dépendante; chez les autres, la veuve administre les troupeaux de son mari défunt durant la minorité des enfants et, en l'absence de progéniture, elle peut même, en vertu de cet héritage, dominer son second mari.

Les modifications que produisirent chez les Tchouktche l'introduction du renne ne sont donc pas moins significatives du fait qu'elles ne peuvent être étiquetées comme améliorant ou nuisant à la condition de la femme. Celle-ci, dans une communauté maritime, ne produit rien et, entretenue uniformément par le mari, mène une vie assez facile; chez les autres Tchouktche, elle est active économiquement parlant, sa vie est en général plus dure, mais il lui est aussi plus aisé d'acquérir l'indépendance. Cet exemple nous prouve donc que les phénomènes économiques peuvent être des causes actives dans le développement du statut féminin.

Toutefois, étant donné la tendance actuelle à surestimer les motifs économiques, il convient de souligner qu'ils ne constituent qu'une déterminante parmi d'autres. Si nous comparons les Tchouktche du renne avec les Ostiak, nous trouverons des différences qui ne s'expliquent pas par les conditions économiques, puisque celles-ci sont les mêmes. Le Tchouktche nomade n'achète pas sa femme et n'acquiert aucun des droits absolus du mari ostiak; sa femme peut le quitter ou être emmenée par sa famille, en dépit du temps de service qu'il a rempli avant son mariage. Chez les Ostiak, par contre, la veuve ne peut hériter d'un troupeau de rennes, puisque les femmes sont inaptes à posséder; elles restent donc dépendantes toute leur vie. En d'autres termes, c'est la culture préexistante qui détermine pour une bonne part dans quelle mesure un nouveau facteur économique affectera le statut de la femme. Comme les Tchouktche n'avaient aucune idée préconçue relative à l'aptitude de la femme à posséder, le nouveau facteur put avoir chez eux des effets qu'interdisait l'idéologie des Ostiak. Si les Tchouktche avaient conçu la femme comme un bien qui pût être acheté, ils auraient substitué les présents de rennes au temps de service; mais leurs traditions culturelles les en empêchaient. On pourrait objecter que l'élément de temps doit être considéré : si les Tchouktche avaient possédé le renne durant une période suffisamment longue, les résultats dus à l'élevage seraient beaucoup plus sensibles. Mais c'est 
là pure supposition que n'étaie aucune preuve. S'il arrive que les femmes tchouktche se trouvent jamais réduites au niveau de leurs sœurs ostiak, on pourra se demander s'il en faut chercher la cause dans un facteur économique commun ou plutôt, comme je le pense, dans l'extension graduelle et due à des emprunts de notions sociologiques provenant de l'ouest de la Sibérie.

En résumé, le facteur économique semble être actif, mais d'une activité restreinte, sujette à être contrebalancée et même supprimée par d'autres déterminantes.

Chapitre 8

\section{e. Corrélation avec les diverses phases de la civilisation.}

Retour à la table des matières

Nous n'avons pas encore traité explicitement des possibilités de corrélation entre un statut féminin élevé et une phase culturelle avancée. Cependant, d'après les faits que nous avons cités, on devine quelle attitude l'ethnographe devra adopter relativement à l'opinion courante selon laquelle la condition de la femme est un indice certain du développement culturel. Cette affirmation va à l'encontre des phénomènes ethnographiques. Dans les communautés de chasseurs les moins évoluées, comme les Vedda et les Andaman, la femme est à tous égards l'égale de l'homme. Ce n'est pas le cas des couches primitives plus hautes, d'un village Bantou moyen par exemple, où la femme, sans devenir pourtant une esclave, est loin d'être sur le même plan que l'homme. A un niveau plus élevé encore, en Chine et en Asie centrale, elle devient un être nettement inférieur. Enfin, pour considérer la question d'un autre angle, George Eliot et Mme Récamier, en dépit de leur influence sociale, n'ont jamais occupé, il s'en faut de beaucoup, la position légale dont jouit la mère de famille chez les Iroquois.

Nous revenons ainsi à notre proposition du début selon laquelle le droit coutumier codifié ou tout au moins rationalisé n'est pas un critère toujours sûr des phénomènes sociaux. Reconnaissons pourtant que, dans la grande majorité des cultures primitives ou plus complexes, une condition moins favorable est assignée à la femme qu'à l'homme, si nous la comparons à notre idéal éthique le plus avancé. Mais croire, ainsi qu'on le fait généralement, que relativement à sa sœur blanche, la femme primitive est une esclave en butte aux mauvais traitements, c'est travestir les faits. En certaines régions, parmi les Athapaskan du nord, par exemple, les femmes devaient accomplir les travaux les plus durs et étaient en outre traitées brutalement par leurs maîtres. Cependant, on ne saurait répéter assez souvent que les cas non douteux de cette nature sont d'une extrême rareté. La description de Spencer et Gillen se rapportant à 
l'Australie centrale se rapproche beaucoup plus de ce qu'on peut considérer comme l'état primitif moyen : « Si l'on tient compte de tout,... la vie d'une de ces sauvages est loin d'être aussi misérable qu'on l'a souvent dépeinte; pour la juger, il faut envisager ce qui est nécessaire à la femme pour lui rendre la vie plus ou moins agréable. » D'ordinaire la division du travail est équitable. Souvent, il est vrai, la femme parait constamment occupée, alors que le mari se repose pendant de longues heures; mais, en compensation, sa tâche à lui exige plus de courage et, comme chez les Esquimaux, elle le met souvent en péril de mort. Pour ce qui est des droits de propriété, même chez les tribus où l'on achète l'épouse, les conséquences logiques de l'achat ont rarement été poussées à l'extrême. Sauf en quelques rares cas, la femme possède des biens et en dispose à son gré. Lorsque j'achetais des objets ethnographiques en Amérique du Nord, je n'ai jamais rencontré d'Indien qui consentît à se dessaisir d'une chose appartenant à sa femme ou qui en fixât le prix avant de l'avoir consultée; les voyageurs ont fait en Amérique du Sud des expériences identiques. Toute théorie mise à part, la femme primitive peut, par la force de sa personnalité, devenir aussi souvent que chez nous maîtresse au logis et, ainsi que nous l'avons remarqué plus haut, il en va de même en Chine, en dépit des affirmations abstraites sur l'indignité de la femme que soutiennent les philosophes chinois.

Pour ce qui a trait aux interdictions imposées aux femmes, il convient de faire allusion à certain trait de la psychologie primitive : l'horreur si répandue de la menstruation. C'est ce qui a souvent abouti à la ségrégation des femmes dans des huttes séparées pendant la durée de leurs règles, coutume si persistante que j'ai pu l'observer dans toute sa vigueur en 1906 encore chez les Shoshoni de l'Idaho. Je ne doute pas que ce ne soit en partie pour cette raison et par crainte de souillure que les femmes sont si souvent tenues à l'écart des activités investies d'un caractère de sainteté. C'est du reste le motif qu'on invoque ouvertement pour les empêcher d'approcher de certains objets sacrés. On a connu des Indiens instruits qui sont toujours demeurés sous l'emprise de ce sentiment; l'influence de celui-ci sur la formation des conceptions primitives relatives au sexe féminin ne saurait être surestimée. La réclusion mensuelle de la femme a été interprétée comme une marque de son infériorité dans les communautés primitives; il est cependant probable que l'ordre de succession causal doit être renversé et que son exclusion de certaines activités et, par conséquent, sa moindre liberté sont les effets de l'horreur inspirée par le phénomène de la menstruation.

Les conclusions générales auxquelles nous a conduits une étude impartiale des faits sont donc les suivantes : ni l'horreur superstitieuse dont il vient d'être question, ni la supériorité physique de l'homme n'ont beaucoup contribué à l'abaissement de la femme primitive; celle-ci est en général bien traitée et peut exercer une certaine influence sur l'homme, en dépit de son infériorité et de son impureté théoriques; c'est précisément parmi quelques-uns des peuples les moins évolués qu'elle jouit pratiquement de l'égalité avec son compagnon. Si des faits contraires ont été relevés et soulignés avec force, il s'agit soit de tribus exceptionnelles comme les Chipeway, soit d'une certaine incompréhension de la part des observateurs, explicable dans la plupart 
des cas par le fait que l'homme, chez les primitifs, ignore cette galanterie sentimentale que nous a léguée le Moyen Age et que beaucoup de femmes modernes désavouent comme contraire à leur dignité d'êtres humains.

Chapitre 8

\section{Références}

$\underline{\text { Retour à la table des matières }}$

1) RADLOFF :295,313,462,484.

2) GURDON : 66 et suiv., 76 et suiv., 82, 93. GOLDENWEISER, 1912 468. KROEBER, 1917 (a) : 89.

3) SELIGMAN : 88. MAN : 107. HOBHOUSE : 177. THEAL : 1, 49, 117. CASTRÉN : 297 et suiv.

4) SPENCER et GILLEN, $1904: 33$. 


\section{9 \\ LA PROPRIÉTÉ}

$\underline{\text { Retour à la table des matières }}$

Le concept de propriété pénètre toutes les phases de la vie sociale. Le mariage est constitué en partie par un transfert de biens et la femme, devenue épouse, peut ellemême être considérée comme un effet mobilier et voir de ce fait se modifier son statut au sein de la famille. Nous avons vu que la polygynie dépend de la fortune du mari; et chez les Wahuma, la polyandrie temporaire découle de l'impossibilité de se procurer une épouse, faute de biens. D'autre part, si notre, théorie s'avère conforme aux faits, la transmission de la propriété a été un facteur qui a puissamment contribué à la création de l'organisation clanique. Nous verrons dans un chapitre suivant l'influence exercée par la richesse sur le développement des castes. Il ne serait donc pas trop de tout un volume pour décrire exclusivement les fonctions de la propriété dans la société. Dans ce chapitre nous nous occuperons plus particulièrement de la manière dont elle est transmise et des formes qu'elle revêt parmi les primitifs. Il nous faudra donc tout d'abord nous attaquer à un problème épineux qui a fait l'objet de longues discussions : celui de savoir si et dans quelle mesure les tribus primitives reconnaissent la propriété individuelle ou si elles se bornent à pratiquer le communisme.

\section{a. Communisme primitif.}

Les partisans du dogme évolutionniste soutiennent que toute condition sociale actuelle est l'aboutissant d'un long développement, partant de quelque état fort éloigné 
et passant par des séries de phases intermédiaires; ils émettent donc l'hypothèse que notre sens de la propriété, maintenant si fortement développé, faisait complètement ou partiellement défaut à la société primitive; il serait donc sorti lentement de son antithèse directe, la communauté des biens de toutes sortes. Or cette affirmation est visiblement erronée. Néanmoins, on peut avancer plus d'un argument à l'appui de ceux qui refusent au sauvage et à l'homme primitif la notion de la propriété privée.

En premier lieu, alors que le communisme proprement dit, supprimant tous les droits personnels, ne se rencontre probablement jamais, nous trouvons fréquemment la propriété collective, non pas nécessairement de toute la communauté, mais d'un certain groupe. De même que le mariage est, comme nous l'avons vu, un arrangement entre des groupes de parents, ainsi la propriété concerne souvent un groupe plutôt qu'un individu. Sir Henry Maine, penseur profond, doué au plus haut point du sens historique, fut si impressionné par divers phénomènes observés aux Indes qu'il lança la théorie de la propriété collective représentant l'état ancien qui aurait précédé les droits personnels de propriété. "Il est plus que probable », écrit-il dans Ancient Law, " que la propriété multiple et non individuelle est la vraie institution archaïque et que les formes de propriété qui pourront le mieux nous renseigner seront celles qui seront associées aux droits de familles et de groupes de parents. » La copropriété ne doit pas, répétons-le, se confondre nécessairement avec la propriété commune. Les copropriétaires peuvent être un couple d'amis, un ménage individuel, un club, une association religieuse, un clan ou une fraction de clan ne comprenant que de proches parents du côté du père ou de la mère. Cependant, il est des cas où le village entier est habité par les hommes d'un seul clan paternel qui possèdent conjointement les champs ou la brousse; il se produit alors une assimilation de la communauté et de la corporation à qui appartiennent les terres, et nous sommes en présence d'un véritable communisme dans les limites du village.

Secondement, nous voyons des phénomènes relevant d'une catégorie toute différente de la pensée sociale affecter parfois l'aspect d'un communisme légal. Même dans notre civilisation si complexe, il arrive que l'autorité de la loi soit contrebalancée par les conceptions éthiques des jurés, conceptions que l'opinion publique élève plus haut que les décrets de la jurisprudence. Dans les tribus où la loi n'est pas codifiée et où la plupart des individus se rattachent les uns aux autres par des liens personnels, les conséquences pratiques de tels sentiments sont d'autant plus fortes. Néanmoins, bien que l'élément juridique puisse parfois s'affaiblir, il s'efface rarement; et, en bien des cas, la ligne de démarcation est tracée avec une netteté qui ne laisse aucun doute. Ainsi l'hospitalité est un élément essentiel de l'étiquette chez les Indiens des Prairies. Un hôte qui ne mettrait pas à la disposition de son visiteur toutes les provisions qu'il possède à n'importe quelle heure du jour ou de la nuit, passerait pour une brute et perdrait sa situation dans la communauté. Mais il y a loin de cette générosité imposée par la morale et le savoir-vivre à la théorie communiste qui autoriserait le visiteur à tout s'approprier sans y être invité. Une telle théorie n'est ni énoncée ni pratiquée; donc les droits du propriétaire individuel ne sont pas mis en question. Plusieurs sociétés primitives envisagent, il est vrai, de façon spéciale les choses indispensables 
à la vie, les rangeant dans une catégorie de biens à part; il est d'usage, en effet, que dans les périodes de disette personne ne souffre de la faim tant qu'un membre de la communauté possède encore des réserves alimentaires. Mais ce point de vue s'explique par l'existence précaire que mènent beaucoup de ces peuples et que nous comprenons d'autant mieux que la guerre mondiale nous a familiarisés avec l'usage des cartes de rationnement.

Voici un autre exemple destiné à illustrer la différence qui sépare la morale de la loi à l'intérieur d'un même groupe d'Indiens. Un Crow, qui avait organisé une expédition guerrière et qui rentrait couvert de gloire, était théoriquement le seul maître du butin capturé, de même qu'il était entièrement responsable de toute perte humaine. Cependant un homme qui aurait exercé ses privilèges légaux jusqu'à tout garder pour son propre usage, aurait certainement été traité d'avare et aurait eu de la peine à recruter des partisans pour une expédition ultérieure. Thésauriser son butin de façon si mesquine répugne tellement au sentiment des Crow qu'il est probable que jamais un capitaine ne s'est exposé ainsi à la réprobation générale. Toutefois, il ressort avec évidence des dires des indigènes que, s'il l'avait fait, ses soldats n'auraient pas pu se plaindre. Ces hommes avaient risqué leur vie sous les ordres de leur chef et pourtant ils étaient obligés de mettre à la disposition du capitaine tous les biens que leurs efforts réunis avaient accumulés.

L'existence de la propriété privée parmi les primitifs est un fait beaucoup trop important pour qu'il suffise d'un ou de deux exemples choisis au hasard pour l'établir. C'est même à cette question que nous consacrerons la plus grande partie de ce chapitre. Voici tout d'abord quelques faits utiles à une orientation préliminaire.

Un missionnaire, excellent observateur, cite les Kaï de la Nouvelle Guinée comme une peuplade communiste. Cependant, à la page suivante, il écrit qu'un voleur pris sur le fait dans le champ d'autrui peut être mis à mort immédiatement, sans crainte que les parents du coupable cherchent à venger sa mort; des individus qui ont volé des biens tels que des défenses de sanglier ou des dents de chiens, ne peuvent sauver leur vie qu'en prenant la fuite; enfin chaque arbre fruitier a son propriétaire. Un indigène peut, il est vrai, planter un arbre sur le terrain d'un autre, mais il n'a pas le droit d'y construire une hutte sans en demander l'autorisation. Les droits de propriétaire sur le gibier reviennent à celui qui le voit le premier, de même l'homme qui découvre un nid d'oiseau passe pour son propriétaire légitime. Lorsqu'un Kaï a commencé à abattre un arbre et se trouve interrompu dans sa tâche, il rend manifeste son droit par une marque de propriété qui empêche tout autre d'y toucher. Ajoutons à tout cela les exemples de biens immatériels que nous décrivons plus loin; il est donc évident que les Kaï ont des notions bien définies de propriété privée. Leur prétendu communisme se réduit simplement à un certain sens de la solidarité, non point communale, mais familiale, grâce auquel les parents se prêtent assistance mutuelle lors de l'achat d'épouses et coopèrent dans les travaux qui dépassent leurs capacités individuelles. 
Les populations arctiques fournissent un exemple encore meilleur à notre enquête critique précisément parce que beaucoup de leurs institutions ont un certain parfum de communisme. Au Groenland, une grande baleine n'est pas considérée comme la propriété exclusive de celui qui l'a harponnée; les spectateurs passifs en ont aussi leur part, même si leur nombre s'élève à une centaine. Un homme est autorisé à se servir d'une trappe dont son propriétaire n'a pas fait usage pendant quelque temps et ce dernier n'a aucun droit sur la bête prise au piège. Dans la terre de Baffin, lorsque les aliments se font rares, celui qui a tué un phoque en distribue la chair et l'huile aux habitants du campement. Cette indifférence à l'égard des droits de propriété individuels, nous la retrouvons chez les Esquimaux du détroit de Béring : « ... Si un homme emprunte quelque objet à un autre, et ne le lui rend pas, il n'est pas tenu d'en rendre compte. Selon l'opinion généralement acceptée, si quelqu'un a suffisamment de biens pour pouvoir en prêter, c'est qu'il en possède au-delà de ses besoins. Celui qui prête, dans de telles circonstances, ne se sent pas justifié à exiger le retour de l'objet et attend qu'il lui soit rendu volontairement. »

Les conceptions qui sont à la base de ces usages se retrouvent sous une forme presque identique chez les tribus asiatiques dont le mode de vie se rapproche étroitement de celui des Esquimaux. Le chasseur idéal des Koriak d'autrefois « entasse le produit de sa chasse sur le rivage et prie les habitants du village de le répartir entre eux, ne gardant pour lui que ce qu'ils lui laissent ». Chez les Tchouktche, un homme qui a tué un morse ne s'approprie pas le produit de sa chasse, mais le partage avec les spectateurs passifs.

Toutefois, une lecture plus attentive de nos sources nous montrera que, même dans ces sociétés communistes, les éléments individualistes, bien qu'à demi effacés, ne font pas complètement défaut; elle nous fournira aussi une explication de la prédominance du communisme, Pour des raisons superstitieuses ou autres, les droits individuels sont parfois reconnus sans conteste. Chez les Tchouktche, quand le courant a fait échouer une baleine sur le rivage, tous les assistants se partagent effectivement sa chair, mais les fanons appartiennent intégralement à l'individu, enfant ou adulte, qui l'a aperçue le premier; tout autre qui se les approprierait commettrait un péché et courrait risque de mort. On a signalé des notions analogues chez les Esquimaux du centre et chez les Groenlandais méridionaux. A l'ouest de la baie d'Hudson, le chasseur qui frappe le premier un morse reçoit ses défenses et l'un des quartiers de devant. Les Koriak et les Youkaghir admettent la propriété privée des vêtements et des ornements tout au moins, tandis que d'autres objets ne sont possédés ni par l'individu ni par la communauté, mais font partie du ménage. Une certaine coutume Tchouktche nous met au cœur de la question : l'individu qui possède un bateau supplémentaire autorise ses voisins à s'en servir. « il est contraire au sentiment de la justice indigène de laisser un bon bateau inactif sur le rivage, alors qu'il y a tout près des chasseurs qui en auraient besoin. » On ne paie rien pour l'utilisation de ce bateau, même dans les coups de chance extraordinaire. En d'autres termes, la société arctique reconnaît deux axiomes : le partage altruiste des produits alimentaires et la nécessité de l'emploi effectif des moyens existants de production économique. Ce communisme est donc 
centré sur des considérations purement économiques, en dehors desquelles il y a place pour des éléments individualistes.

De tout ce qui précède, il découle que nous ne pouvons nous satisfaire de l'alternative facile : communisme ou individualisme. Une peuplade sera communiste en ce qui concerne certains biens et reconnaîtra pourtant, à d'autres égards, la propriété individuelle. En outre, le principe communiste peut être appliqué non point par l'unité politique tout entière, de quelque ordre qu'elle soit, mais seulement à l'intérieur d'une classe d'individus plus restreinte, ou différemment constituée; dans ce cas, nous pourrons parler non de communisme au sens propre du terme, mais de collectivisme. Ce sont ces divers points qu'il nous faut avoir présents à l'esprit en passant successivement en revue au regard du droit de propriété les biens immobiliers et mobiliers, les richesses immatérielles et les règles de succession.

Chapitre 9

\section{b. Propriété du sol.}

$\underline{\text { Retour à la table des matières }}$

La loi primitive concernant les biens immobiliers est affectée par diverses circonstances; elle est souvent en corrélation avec les conditions politiques d'une peuplade et avec la manière dont elle envisage la notion de chef. Le statut économique est naturellement fort important et les facteurs géographiques, inefficaces à eux seuls, peuvent, en se combinant avec les aspects culturels, exercer une influence considérable. Il convient de grouper les tribus selon leurs moyens de subsistance et d'examiner brièvement les règles sur la propriété foncière parmi les représentants typiques des peuples chasseurs, éleveurs et agriculteurs.

On affirme fréquemment que, lorsqu'une tribu vit de la chasse, la propriété communale des terrains de chasse s'impose. Cette opinion a été non seulement sérieusement ébranlée, mais même dépouillée de toute valeur par des témoignages émanant de diverses régions. Elle n'est pas vraie de façon générale mais s'applique uniquement à des zones telles que les Prairies du Nord, ainsi qu'à des tribus comme les Maidu de Californie et les Indiens de Thompson River en Colombie britannique. Ces deux derniers exemples sont instructifs en ce que le communisme virtuel des membres de la tribu s'accompagnait, chez ces peuplades, de l'exclusion jalouse de tous les étrangers, c'est-à-dire que la tribu considérait une aire donnée comme son terrain héréditaire, exploitable par tous ses ressortissants, mais n'admettait aucun empiétement du dehors. Quiconque pénétrait dans le territoire de Thompson River payait son intrusion de sa mort. Les Maidu protégeaient leurs frontières par un système compliqué de sentinelles. Le socialisme tribal n'était restreint qu'à l'égard de certaines améliorations apportées au sol; si un Indien de Thompson River ou un Maidu avait 
construit une palissade à cerfs ou une station de pêche, il avait droit à l'usage exclusif du produit de son effort individuel et transmettait ce droit à ses héritiers. Grâce aux investigations importantes de Speck parmi certains groupes Algonkin du nord-est, il nous faut considérer maintenant comme un fait établi que, dans une partie de l'Amérique du Nord, non seulement ces améliorations mais les terrains de chasse eux-mêmes étaient la propriété individuelle des familles. « Tout le territoire appartenant à la tribu se subdivisait en districts possédés depuis des temps immémoriaux par les mêmes familles et transmis d'une génération à l'autre. Les limites approximatives de ces districts étaient connues et observées par tous et, si on les franchissait, ce qui ne se produisait que rarement, le coupable était sommairement exécuté. »

Parmi les Timiskaming, la peine encourue était la mort, mais on recourait plus communément à la sorcellerie. Pour faire montre de courtoisie, le privilège de chasser dans certain district pouvait être accordé à une autre famille, mais à titre temporaire seulement. Si la nécessité était cause de la violation de la propriété, le braconnier se sentait obligé d'offrir les peaux au propriétaire lésé. C'était seulement lorsque tous les ayants droit mâles d'un district s'étaient éteints que celui-ci était divisé entre les familles survivantes. Les limites étaient si nettement établies que Speck put dresser le cadastre des districts possédés par les diverses familles. Ce même auteur interprète avec raison dans le même sens des citations d'anciens voyageurs et y trouve que les privilèges de chasse individuels étaient fréquemment admis par les Indiens à l'est et au centre du Canada. Nous rencontrons des preuves analogues sur la côte de la Colombie britannique où chaque groupe de maisons possédait sa propre crique à saumons ou une fraction de la crique, et où chaque famille individuelle avait son banc de helbuts et de mollusques, et disposait d'une zone où elle récoltait baies et racines.

Il convient de donner ici une indication. En parlant de familles individuelles, nos sources omettent souvent de nous préciser le sens exact de ce terme. Une famille individuelle, dans le sens où je l'ai entendu jusqu'ici, inclurait le père et la mère; mais Speck nous montre clairement que les droits dont il parle ne se rattachent pas à l'unité bilatérale mais à son chef mâle et à ses descendants de sexe masculin. La chose est toute naturelle puisque la chasse est une occupation masculine, les sociétés primitives tendant à associer l'utilisation effective à la propriété, ce qui dans ce cas exclut automatiquement les femmes. Une autre question se pose relative à la possession simultanée d'un district par plusieurs frères. Dans queue mesure ce partage des droits entre-t-il en conflit avec la propriété individuelle? Bien que nos informations ne soient pas concluantes sur ce point, un fait est certain : les droits territoriaux étaient dévolus tout au plus à un groupe de proches parents du côté paternel, jamais à un ensemble plus inclusif de parents réels ou putatifs et jamais non plus à un groupement politique plus vaste. La propriété communale, au sens légal du terme, était donc inconnue. Le seul point qui reste en suspens est de savoir si la terre héréditaire appartenait à un homme individuellement ou s'il la partageait avec ses fils ou ses frères. Mon interprétation personnelle des faits avancés par Speck est que les deux se produisaient à des époques différentes, que la propriété multiple était simplement due à un héritage en commun des biens du père, mais qu'il existait tout au moins une 
tendance à régler les choses sur une base purement individuelle et à assigner à l'occasion son domaine propre à chaque fils.

L'attitude des indigènes australiens à l'égard du sol est extrêmement intéressante. Un groupe local, non pas nécessairement la tribu tout entière, mais peut-être la fraction mâle localisée de chaque clan paternel, comme parmi les Kariera, occupait un certain district auquel il était indissolublement lié. En cas de guerre, jamais l'idée d'exproprier le vaincu ne se présentait au conquérant. Rien de surprenant donc à ce que toute naturalisation dans un autre groupe fût également inconcevable. " De même que le pays appartenait au Kariera, de même ce dernier appartenait au pays. » Les violations étaient extrêmement rares et les colons blancs de l'Ouest australien rencontrèrent des difficultés à obtenir que les bergers indigènes allassent mener paître leurs troupeaux de moutons ailleurs que sur leurs territoires ancestraux. Les informations obtenues sur quelques-unes de ces tribus donnent à croire que cet attachement à un district particulier peut avoir été dû à des raisons mystiques, plus particulièrement à la localisation des ancêtres totémiques de l'individu; le changement de territoire aurait rompu le contact avec ces êtres mythiques. Hors de l'association de groupes définis avec certaines localités, il existe d'importantes différences régionales. Les Kaï exploitaient chaque district en commun et chaque membre du clan paternel pouvait n'importe quand chasser sur le terrain commun ou faire usage de ses produits sans être arrêté par des droits de propriété spéciaux. Il n'en va pas de même dans le Queensland où les familles individuelles, au sens de Speck, se réservent le droit de récolter racines et baies, ainsi que de chasser dans des zones particulières. Le braconnage est rare, bien qu'il ne soit pas considéré comme un tort grave s'il est commis par un membre de la tribu. En général, les privilèges mentionnés se transmettent aux frères et aux fils, mais, dans un certain district, les terrains où poussent les plantes comestibles sont répartis parmi les femmes qui les lèguent à leurs filles.

Enfin les Vedda de Ceylan peuvent être cités comme un peuple de chasseurs doué d'un sens remarquablement aigu de la propriété, ce qui a attiré l'attention des observateurs blancs au XVIIe siècle déjà. Chaque groupe, ainsi que les Maidu, faisait garder soigneusement ses frontières par des archers et toute violation s'expiait par le sang. Mais, au contraire des Maidu, les Vedda reconnaissaient aussi des fiefs plus restreints et Seligman a pu dresser la carte des territoires appartenant aux diverses familles des Henebedda. Personne ne chassait sur le terrain d'autrui, même pas de son frère, sans permission et si le gibier poursuivi s'enfuyait sur le territoire d'autrui, le propriétaire du sol avait droit à une portion de la chair. Les conditions dans lesquelles la tom se transmettait nous donnent un aperçu de la véritable nature de la propriété chez des Vedda. Le transfert des collines ou des étangs avait normalement lieu au bénéfice des enfants et des gendres, mais non sans le consentement de tous les mâles adultes de la famille. On ne sait pas s'il s'agissait simplement de tous les fils adultes ou aussi des frères auxquels le droit héréditaire de chasser sur le territoire en question était refusé. En tous cas, nous avons ici affaire à un droit non communal bien que collectif sur des biens immobiliers, exercé par de proches parents. Afin de symboliser ou de ratifier le transfert, celui qui donnait la terre faisait présent au nouveau 
propriétaire d'une ou deux pierres auxquelles il ajoutait parfois une de ses dents. D'ordinaire la limite d'un district était fixée par des frontières naturelles, sinon une figure représentant un homme avec un arc tendu était gravée sur les troncs des arbres, le long de la ligne de démarcation.

Nous avons ainsi des preuves incontestables que certaines tribus de chasseurs reconnaissent fréquemment des droits non communaux et héréditaires sur certaines fractions du territoire tribal.

Parmi les peuples pasteurs, nous trouvons d'ordinaire un sens hautement développé de la propriété individuelle en ce qui regarde le bétail, mais, quant à la terre qui nous occupe plus spécialement pour l'instant, il règne souvent un communisme intégral ou presque. Un Massai partage les pâturages avec tous les autres habitants de son district et quand l'herbe s'épuise, il se produit un exode général. Dans cette région, la steppe se prête admirablement à la pâture, les bergers ont donc à leur disposition une grande étendue de terrain. De même parmi les Toda, le groupe local, ici assimilé en clan, possède collectivement les pâturages. Les Hottentots pratiquaient le communisme tribal en ce qui avait trait aux prairies; cependant les guerres intertribales d'autrefois avaient souvent Pour motif le désir de posséder de beaux pâturages. il était néanmoins une catégorie de biens immobiliers qui se rattachaient à la famille individuelle : les arbustes dont on obtenait la calebasse nara. Lorsqu'il y avait eu violation des terrains de nara, on allait se plaindre auprès du chef si le délinquant était un ressortissant de la même tribu, alors que les étrangers coupables de ce forfait étaient impitoyablement tués.

Parmi les Kirghiz qui possèdent d'immenses troupeaux de moutons, de chèvres, de bétail et de chevaux, les règlements sont beaucoup plus compliqués. Ils n'élèvent les chameaux qu'en petit nombre et pour ces pâturages ils observent en général le principe de la communauté. Mais les nécessités naturelles requises par les autres troupeaux dans le cadre géographique donné, exigent une répartition minutieuse des terrains disponibles. En été, il faut aux troupeaux des plaines bien irriguées qui ne soient pas infestées d'insectes; en hiver, les pâturages doivent être pourvus d'abris contre les rigueurs du climat, offrir de l'eau et du bois en abondance et ne pas être exposés aux trop grandes chutes de neige. Comme les conditions requises pour de bons quartiers d'hiver sont plus difficiles à remplir, l'histoire primitive des hordes kirghizes se compose en grande partie de querelles au sujet des meilleurs terrains d'hivernage. Ces escarmouches ont cessé depuis longtemps et, dans la seconde moitié du siècle passé, chaque famille était en possession de son propre pâturage d'hiver. Étant donné que les exigences territoriales varient en proportion de l'importance des troupeaux, ce système s'accompagne inévitablement d'un transfert de territoire imposé par les besoins. Un éleveur dont le bétail s'accroît doit se procurer un supplément de pâturages; si c'est le contraire qui se produit, une partie du terrain reste sans emploi et de ce fait sera vendue. Les quartiers d'hiver sont d'ordinaire délimités par des frontières naturelles, telles que cours d'eau, collines ou lacs; à leur défaut des pieux ou des rochers indiquent leurs limites. Les confins de ces réserves sont bien connus 
de tous et chaque individu reçoit l'aide de son clan pour se défendre contre les violations. En contraste marqué avec cette répartition des terrains d'hivernage, les pâturages d'été sont possédés non individuellement, mais par toute la communauté $(a u l)$. Voici donc une nouvelle preuve qu'il est vain de se servir -de ces grands mots d'usage facile mais dépourvus de signification. Les Kirghiz ne sont ni communistes ni individualistes au sens absolu de ces termes, en ce qui regarde la propriété du sol. Ils sont communistes une partie de l'année et individualistes le reste du temps.

Pour ce qui est de la loi sur les biens immobiliers chez les tribus vivant de l'agriculture, nos informations sont souvent déficientes et, bien pis, viciées par les préjugés qui ont longtemps eu cours. Cette remarque s'applique avec une force toute spéciale à l'Amérique du Nord où notre ignorance est à déplorer. Elle est due partiellement à la disparition sur des aires étendues des usages anciens, mais bien davantage au facteur de confusion qu'est le dogme du clan. Toute affirmation de nos auteurs relative au clan en tant qu'unité possédante, doit être soumise à l'examen le plus attentif, car il s'agit souvent, non point d'une observation, mais d'une inférence de faits observés que rien ne justifie. Ainsi la détention de la propriété à l'intérieur du clan ne doit pas être confondue avec la possession par le clan. Lorsqu'une Indienne Hopi meurt, sa maison passe à ses filles, ressortissantes de son clan à elle par descendance matrilinéaire; mais elle ne devient pas et n'est jamais devenue la propriété du clan. Tout d'abord, la loi des Hopi interdisant aux hommes de posséder des maisons, une moitié environ de la tribu ne peut se classer parmi les propriétaires. Secondement, il s'agit de la possession collective d'une maison non point par toutes les femmes du clan, mais tout au plus par les descendantes en lignée féminine de la défunte. La loi de succession peut, comme je l'ai moi-même indiqué, conduire à la notion de clan, mais il y a loin de là à affirmer que le clan en plein développement, comprenant des parents non seulement éloignés mais simplement putatifs, possède en corps des biens.

Pour ce qui est des Zuñi, nous avons la preuve irréfutable que le sol n'a jamais appartenu au clan. La propriété communale existe, du moins dans le sens que la terre inutilisée, les chemins et les puits sont à la disposition de tous les Zuñi. Mais les champs, les enclos à bétail, les maisons et les biens mobiliers sont la possession des individus et des groupes de parents. Ce que nous en dit Kroeber ne fait que corroborer les témoignages antérieurs de Mrs. Stevenson : « Les champs n'appartiennent pas aux clans et les Zuñi affirment que tel ne fut jamais le cas. » Une simple appropriation et le travail du sol donnent droit au titre de propriété et, chose importante à noter, la terre est inaliénable. Les petits jardins dont s'occupent les femmes se transmettent, il est vrai, de mère en fille; toutefois, ce fait n'a évidemment rien à voir avec la propriété par le clan, mais découle du même principe qui fait passer les terrains de chasse de père en fils.

Là où la condition primitive des indigènes a été profondément modifiée par la civilisation, il n'est guère possible d'établir avec sécurité l'état des faits. Ainsi Speck ne nous dit pas clairement si, parmi les Yuchi, les fractions arables du territoire appartenaient aux individus ou aux clans. Cependant, lorsqu'il parle de l'occupation et 
de l'utilisation de la terre établissant le titre de propriété, ainsi que des pierres angulaires à dessins variables servant de marques de possession, il semble bien qu'il s'agisse de propriété privée.

C'est aux recherches de G. Wilson parmi les Hidatsa que nous devons les travaux de beaucoup les plus précis sur la forme de propriété admise chez les peuplades américaines horticoles au nord du Mexique. Nous les avons déjà utilisés pour montrer comment la notion de clan matriarcal sort naturellement de l'attribution des jardins et des travaux d'horticulture aux femmes. Il est hors de doute que les sœurs et les descendantes qui cultivent un champ conjointement, s'en partagent également les produits et, dans ce sens, il est possible de parler de propriété collective chez ces Indiens. Mais les informations de Wilson précisent que c'étaient invariablement les proches parentes de la mère, et non le clan maternel plus vaste, qui possédaient et cultivaient la terre. Dans les riches plaines basses du Missouri, les femmes de la famille, c'est-àdire la grand mère, ses filles et les filles de celles-ci, défrichaient un champ et le délimitaient ensuite par des pieux, des pierres et des monticules. Des conflits de revendications donnaient lieu parfois à des difficultés mais, dans la tribu, un certain sentiment s'opposait fortement aux querelles relatives à la terre et, d'ordinaire, en offrant une compensation pour le terrain cédé, on arrivait à s'arranger à l'amiable. Cependant il est probable que les jardins ne se vendaient pas intégralement. Lorsqu'une femme mourait et que ses parentes ne s'appropriaient pas ses terrains, quelque autre femme pouvait les utiliser, mais non sans en avoir auparavant demandé l'autorisation à la famille de la propriétaire défunte.

En ce qui concerne l'ancien Mexique, Bandelier a tenté, avec toutes les ressources de sa vaste érudition, d'interpréter les documents historiques dans un sens favorable au système clanique de Morgan. Rejetant complètement la notion d'une monarchie féodale qui avait été répandue parmi les vieux chroniqueurs espagnols, il se représente le Mexique central comme une confédération de tribus indépendantes qui avait soumis d'autres populations, mais sans jamais les réduire à l'état de vassaux. Au lieu de s'emparer du territoire des vaincus, les conquérants se seraient contentés d'exiger un tribut, en stipulant que la récolte de certains champs leur serait réservée.

Pour ce qui est des territoires héréditaires appartenant aux tribus dominatrices ou même à toutes les autres peuplades indigènes, le clan paternel (car Bandelier donne au terme aztèque de calpulli le sens de clan) constituait l'unité possédante, complètement indépendante de toute autre subdivision tribale. L'aliénation était impossible. Si un clan s'éteignait, son territoire s'ajoutait à celui d'un calpulli mal loti ou était réparti entre les autres clans. Les terres du clan étaient distribuées à ses ressortissants mâles, qui étaient obligés de cultiver leur lot ou du moins de fournir une compensation si d'autres devoirs les empêchaient de se consacrer aux travaux des champs. S'ils ne s'en occupaient pas, leurs champs revenaient au clan à la fin d'une période de deux ans et étaient distribués à nouveau. Le chef du clan, assisté du conseil des anciens, veillait à la répartition des terres. Bandelier insiste avec force sur le fait que les chefs tribaux eux-mêmes n'avaient pas de domaine à la manière des seigneurs 
féodaux, mais possédaient certains champs en leur qualité de membres du clan tandis que d'autres champs étaient réservés aux besoins de leurs charges, sans qu'ils en fussent le moins du monde propriétaires. Bref, Bandelier est d'avis que la notion abstraite de possession par le chef ou par la nation était étrangère aux Mexicains; pour lui le clan avait un droit de propriété inaliénable sur son territoire et les familles individuelles se contentaient de jouir de l'usufruit des champs dans la zone du clan.

Malheureusement, aucun des successeurs de Bandelier n'a étudié les chroniques espagnoles avec autant de conscience que lui; la discussion ne saurait donc être considérée comme close. L'affirmation de Bandelier selon laquelle les seigneurs féodaux étaient inconnus au Mexique s'avérera probablement exacte; de même la propriété collective du sol par les calpulli, l'usufruit seul étant réservé aux familles, se trouve confirmée par les travaux les plus récents sur ce sujet. Par contre, on a tout lieu de douter que les calpulli aient été de véritables clans patrilinéaires; pour Spinden, ce sont plutôt des « organisations militaires se recrutant parmi tous les hommes de la tribu ». Pour pouvoir connaître véritablement comment les anciens Mexicains possédaient le sol, il est indispensable de réviser à nouveau nos anciennes sources en nous dégageant de toute théorie préconçue.

Nous n'avons que peu de renseignements précis sur l'état des choses en Amérique du Sud. On ne saurait guère s'attendre à trouver la propriété individuelle dans un pays pratiquant une sorte de socialisme d'État comme l'ancien Pérou. Les terres y étaient réparties entre le gouvernement, le clergé et le reste de la population. Le territoire était aux mains du clan paternel (ayllu) qui répartissait aux différentes familles des lots à cultiver en proportion de leurs besoins. En ce qui concerne les Chibcha, nous avons un témoignage des plus sommaires sur la propriété individuelle des biens immobiliers : " La propriedad individual de las tierras existia entre los Chibchas, y los bienes raices se transmitian por herencia á las mujeres y á los hijos del difunto. » Nous accueillerions avec plaisir des informations plus détaillées. Parmi les tribus plus primitives, les deux formes de propriété nous sont signalées. Au nord-ouest de l'Amazone, les plantations tribales appartiennent au chef, apparemment non point en vertu d'une souveraineté abstraite, mais pour la raison pratique suivante : puisque toutes les femmes non mariées de la communauté sont à lui, c'est lui qui peut le mieux faire cultiver les champs. Cela n'empêche nullement les autres Indiens qui ont leur habitat individuel dans la brousse de posséder leurs champs particuliers de manioc. Les frontières tribales sont souvent minutieusement observées. A l'intérieur de celles-ci, les Bakaïri ont des plantations communales, quoique Schmidt fasse observer que le travail communal créait un droit d'usufruit et de possession à l'individu en lui débroussaillant un champ.

Ainsi, dans la mesure où la rareté de nos informations nous permet de généraliser, il semble que les indigènes du Nouveau Monde aient adopté des systèmes variés de propriété du sol. La propriété communale se rencontre surtout dans le sud; l'existence de la possession non communale mais collective et clanique a été sinon prouvée du moins vigoureusement défendue; enfin la propriété par de proches parents du côté 
maternel est observée chez les Hidatsa, alors que les Zuñi connaissent la propriété individuelle.

En dehors de quelques exceptions relativement rares, la société américaine était organisée sur une base démocratique. Si nous passons aux autres continents, l'influence qu'exercent les autres régimes politiques sur la propriété du sol nous apparaît souvent avec clarté, surtout là où, comme en Afrique et en Océanie, des formes plus ou moins accusées de pouvoir monarchique se rencontrent les unes à côté des autres.

Par suite de leurs conceptions politiques, les indigènes africains considèrent souvent le sol comme appartenant au roi ou au chef. Es ne peuvent donc pas acquérir de terres. Néanmoins, à part l'aliénation rendue impossible, l'individu qui a reçu un territoire en devient parfois le maître absolu. Voici de quelle manière s'y prennent les Thonga : un chef qui a obtenu une étendue de terres considérable, la divise entre les hommes de son village et ceux-ci commencent alors à travailler les parties les plus fertiles de leur lot. Lorsqu'un nouveau venu désire s'établir sur le territoire, on le mène à un terrain non cultivé dont les limites sont déterminées par des obstacles naturels, tels que lac, fourmilière ou arbre. Il en est dorénavant l'occupant légal, mais, s'il s'en va, soit parce qu'il est mécontent de la terre, soit par suite de difficultés personnelles avec ses voisins, la propriété ne peut être vendue, mais revient au donateur. Sinon, les droits de possesseur passent normalement aux héritiers du cessionnaire. De même, le titre de chef perdrait toute valeur si ce dernier quittait le pays, quelle qu'ait été la durée de son occupation. Aussi longtemps que le tenant reste en possession de la terre, son pouvoir est incontesté et il peut, à son tour, donner des fractions de son territoire à ses parents. Chose étrange, le donateur se dépouille de ses droits au point qu'il est obligé de demander l'autorisation du cessionnaire pour ramasser des fruits tombés sur le district cédé. Sur ces points de détail ainsi que sur d'autres, il y a naturellement des variations locales parmi les Bantou et le pouvoir personnel des différents chefs et souverains jouait sans doute un rôle considérable; cependant, en général, on a l'impression que la propriété du sol était relativement assurée. Ainsi, parmi quelques-unes des tribus méridionales, un chef pouvait, il est vrai, chasser de ses biens un homme du commun et se saisir de la récolte sur pied au profit d'un autre chef, mais il était illégal de déposséder un cultivateur de sa terre afin d'en faire bénéficier un autre individu. Une intéressante déviation de la coutume Thonga a aussi été notée parmi leurs congénères du sud dans les cas d'abandon. Celui-ci n'annulait pas le titre de propriété; le premier occupant pouvait recouvrer les terres qu'il avait autrefois cultivées, mais n'avait aucun droit sur les terrains défrichés et mis en valeur par le nouvel arrivant.

Chez les Bantou du sud, le cultivateur reste tenancier quoique occupant le terrain en général avec sécurité; l'usage effectif du sol par le cessionnaire prive le cédant de tout droit d'intervention, sauf parfois en cas de délit politique. Dans la mesure où l'on peut s'en rendre compte, les droits de possession sont détenus individuellement. 
Parmi les Ewé du Togo, les chefs ont moins d'importance que chez les Zoulou et tribus apparentées. D'autre part, l'individu semble s'effacer devant le groupe de parents patrilinéaires. Ces deux ordres de faits nuancent de façon différente la loi des Ewé sur les biens fonciers. Chaque tribu et chaque village a son domaine distinct, soigneusement délimité des unités voisines par une sorte d'arbrisseau réservé à cet usage. A l'intérieur de la zone du village, chaque famille a sa propre terre dont la limite est aussi dûment marquée. En théorie, le titre de propriété se base sur une appropriation ancestrale et sur la prescription. Pour tout ce qui a trait aux terres de la tribu et du village, la propriété est publique. Les fractions en lesquelles se divise la zone du village sont aux mains de groupes de parents patrilinéaires dont les chefs de famille font office d'administrateurs. Celui qui est à la tête du groupe est tenu d'assister les autres membres dans les temps de disette, et tout parent est autorisé à cultiver une partie du territoire héréditaire. Une famille qui va s'éteignant peut ne mettre en valeur qu'une partie seulement des terrains qu'elle possède, et pourtant ses droits ne sont pas frappés de caducité du fait qu'il n'en est pas fait usage. Dans de tels cas, d'autres familles sont autorisées à occuper ces champs sans obligation de payer une location, mais elles doivent observer les coutumes locales relatives aux jours de repos et planter des graines de palmier, de manière que le possesseur puisse plus tard avoir une palmeraie sur ses terres. A l'intérieur du groupe familial patrilinéaire, il n'existe guère de propriété individuelle. Le fait qu'un membre occupe un certain champ ne porte aucun préjudice aux intérêts des autres membres sur le sol qu'il travaille et ne modifie pas davantage les droits du cultivateur sur le reste du territoire héréditaire. Pour autant que la propriété individuelle est reconnue, elle semble ne concerner que les plantations de palmiers.

Puisque l'existence même des Ewé a pour base la possession de leurs champs, la vente intégrale d'une propriété ancestrale est donc hors de cause; autrefois, plutôt que d'en venir à cette extrémité, on préférait, en cas de dettes, vendre ou livrer en gage des membres de la famille qui avait contracté l'obligation. Il arrive toutefois que certains champs soient vendus avec le consentement de l'administrateur-chef de groupe. En de telles occasions, il est des plus vraisemblables que les ressortissants du groupe feront tendre tous leurs efforts à récupérer leurs biens en les achetant à nouveau. S'ils n'y réussissent pas, la propriété vendue passera aux héritiers de l'acheteur, selon les lois de succession habituelles. Le transfert ne va pas sans formalités. L'acheteur paie le prix convenu, puis le vendeur apporte une quantité stipulée de coquillages cauris qui est divisée entre l'acquéreur et lui. Ensuite une rangée de ces coquillages est partagée en deux, coupée par la moitié et chacune des parties lie la moitié qui lui revient à son siège ou à son panier, comme preuve évidente de la légalité de l'achat. Finalement acquéreur et vendeur, escortés de témoins, se rendent sur le terrain transféré où trois coups de feu doivent être tirés qui sont comme une ratification publique de la vente; en cas de litige ultérieur, les juges s'informeront en premier lieu si cette formalité a été remplie. Si l'on peut prouver que les coups réglementaires ont bien été tirés sur le champ, le titre de propriétaire est dûment établi. Les dissensions relatives aux biens fonciers ont été de tous temps fréquentes et étaient autrefois tranchées par le chef et par une commission de deux experts devant qui chaque plaignant devait réciter les 
noms de tous les propriétaires précédents et indiquer la ligne de démarcation du terrain litigieux. S'il n'y réussissait pas, on se prononçait contre lui. Parfois en avait recours à une ordalie et le plaideur qui succombait le premier était considéré comme mal fondé dans sa plainte.

La loi foncière, telle que nous venons de l'étudier, ne s'applique pas au royaume du Dahomey où le souverain autocrate était en théorie le chef de tout le pays, permettant ou accordant par faveur spéciale la jouissance de l'usufruit aux cultivateurs. Il avait donc, entre autres prérogatives, bien qu'il n'en fît pas normalement usage, celle de chasser les occupants à son gré et de les remplacer par qui bon lui semblait.

Dans l'État d'Ouganda, hautement organisé, le mode de propriété se rapproche, ainsi qu'au Dahomey, du système féodal. C'est le roi qui possède tout le pays et qui en dispose à son gré, à l'exception des lieux de sépulture claniques d'ancienne date qui peuvent être taxés, mais qui ne changent pas de propriétaire. Le souverain accorde des terres aux chefs et ceux-ci en distribuent aux paysans, lesquels en retour sont obligés de travailler pour eux et de remplir une période de service militaire. Il n'est pas possible de vendre des terres, car ce serait empiéter par là sur les droits du souverain; les lieux de sépulture eux-mêmes sont inaliénables.

Nous voyons donc qu'en Afrique toute la question de la propriété du sol prend un aspect différent. Elle est inextricablement liée à la nature et à l'étendue du pouvoir royal qui réduit parfois le cultivateur à n'être qu'un simple fermier à la merci de son maître. D'autres facteurs se font aussi sentir, sans doute, ainsi que dans le cas des Ewé où la solidarité entre en conflit avec la possession individuelle des terres. Étant donné que les travaux horticoles sont si souvent dévolus aux femmes, nous nous attendrions à voir le sexe féminin plus fréquemment associé aux droits territoriaux. En fait, la femme Kikouyou possède le champ qu'elle cultive, et, quoique parmi les Bakouba la terre soit au chef, ses produits appartiennent aux planteuses. Mais de tels exemples sont rares; le fait que la femme africaine est souvent considérée elle-même comme un bien a porté atteinte à son aptitude à posséder.

En Océanie, les Mélanésiens, qui n'ont pas été influencés par des exemples polynésiens, se rapprochent de l'état démocratique; les Polynésiens, eux, estimaient la noblesse de race, mais, à part certains groupes, se constituaient plutôt en républiques de nobles qu'en États autocratiques; à Hawaï, cependant, et dans une partie de la Micronésie, une fraction de la population rampait dans la poussière devant la caste des patriciens. Ces différences se répercutent inévitablement sur la loi foncière.

En Mélanésie, on ne trouve généralement guère de trace du système féodal; Codrington cite même un intéressant exemple de chefs ne possédant pas de terres. Même aux Fidji où, sous l'influence polynésienne, les chefs occupaient une situation élevée, il semble que ce n'ait été que dans certaines conditions qu'on se soit senti à cet égard redevable envers le souverain. Selon Thomson, le chef, en sa qualité de représentant tribal, assignait des champs aux fugitifs qui demandaient sa protection, et le 
tribut qu'il recevait était immédiatement divisé entre ses sujets. Mais, usurpant des prérogatives dont il n'avait pas été investi à l'origine, il lui arrivait de lever des taxes spéciales et même de modifier à tel point la forme première de la propriété qu'il faisait figure de seigneur régnant sur des sujets qui lui étaient personnellement soumis.

D'une façon générale, les terres en friche étaient propriété tribale dans ce sens que tout membre de la tribu pouvait s'en approprier les fractions vacantes, les défricher et les cultiver. Les indigènes des îles Banks et archipels voisins distinguent nettement entre les vieilles terres héréditaires et le sol récemment acquis. Les premières appartenaient aux clans utérins ou tout au moins aux membres de la famille maternelle; à la mort d'un homme, ses jardins devenaient la propriété des fils de sa sœur et ceux-ci possédaient en commun le terrain, en choisissant chacun une partie à cultiver; par ailleurs les champs qu'un individu par son effort personnel avait récemment défrichés lui appartenaient en propre et passaient à ses fils; ceux-ci ne les possédaient pas conjointement, mais s'en réservaient chacun une part distincte.

Une particularité fort intéressante des droits sur les biens-fonds en Océanie est la distinction qu'on établit entre les arbres fruitiers et le sol sur lequel ils poussent, particularité qui a occasionné bien des erreurs de la part des administrateurs coloniaux. Aux Fidji, aux îles Banks et dans une partie de la Nouvelle-Guinée, le planteur a un droit inattaquable sur ses cocotiers et autres arbres de valeur, même s'il les a plantés sur le terrain d'autrui sans autorisation, quoique en général la permission soit demandée et accordée. Les colons européens sont donc obligés d'indemniser non seulement le propriétaire du terrain, mais encore chaque indigène qui possède un arbre sur le terrain qu'ils désirent acheter.

En Mélanésie, on n'aliénait pas les terres. Dans l'État fidjien de Rewa, celles-ci se transféraient de neuf manières différentes, mais six d'entre elles prévoyaient des dispositions spéciales pour le rachat à une cérémonie particulière, la propriété ne revenant pas automatiquement à la lignée des premiers propriétaires. Ainsi une famille pouvait donner en dot à sa fille une terre dont son mari et ses fils feraient usage. En l'absence d'héritiers mâles, le donateur de la terre rentrait en sa possession par un présent approprié, mais, jusqu'à ce que cette formalité eût été remplie, le mari ou ses représentants pouvaient à leur gré cultiver le sol ou l'affermer. Il n'existait pas de dispositions spéciales limitant ces mesures, mais, si la famille du donateur laissait dormir l'affaire pendant trois ou quatre générations, les descendants du cessionnaire, en rejetant l'offre tardive de rachat, avaient pour eux l'assentiment public.

La propriété du sol variait énormément chez les Fidjiens selon les localités et, en ce qui a trait au communisme, les informations de notre auteur sont parfois contradictoires. Cependant il est clair que, dans certaines régions tout au moins, la propriété individuelle était reconnue. C'était le cas à Rewa surtout où chaque champ devait être gagné sur la mer ou la rivière grâce à l'effort personnel qui établissait des droits de propriété. A la vérité, il semble que le prétendu communisme des îles Fidji s'efface devant la prédominance de la morale sur les concepts légaux : la terre qu'un homme 
cultivait et les arbres qu'il avait plantés lui appartenaient légalement, mais, en pratique, les revendications de sa parenté finissaient par égaliser à peu près les profits retirés par le possesseur et ceux qui revenaient à ses parents.

Il n'est probablement aucune région de l'Océanie où les droits de propriété individuels tels que nous les concevons aient été plus développés qu'aux archipels du détroit de Torrès. Chaque rocher et chaque anse avait son propriétaire; il n'y avait que la rue du village qu'on possédât en commun. Là où on affermait les jardins, on recevait comme loyer les

prémices. Contrairement à la coutume si répandue parmi les primitifs, il était permis d'aliéner ses terres et d'en disposer par testament. Un père irrité pouvait déshériter ses enfants ou répartir ses biens entre eux à son gré, ce qui prouve bien qu'il n'existait pas par naissance de droit inaliénable sur la terre, ni des droits de copropriété, même exercés par des parents.

Le système de castes en vigueur aux îles Marshall forme un contraste marqué avec les gouvernements démocratiques de la Mélanésie. Ici la haute et la basse noblesse prédominent sans conteste, considérant avec un mépris parfait leurs serfs plébéiens et n'accordant qu'à la bourgeoisie des " artisans » des sortes de fiefs à la manière féodale pour les remercier de leurs loyaux services. Ce n'est donc que la caste limitée des patriciens qui possède le sol et son pouvoir est absolu. Le titre de propriété d'un noble a pour origine l'héritage ou la conquête; le noble peut à son gré donner ou vendre son territoire, mais les chefs peu importants paient tribut à ceux d'un rang plus élevé. Les terres sont cultivées par des serfs complètement soumis aux caprices de leur maître. Quelques-unes de ces familles plébéiennes peuvent rester pendant plusieurs générations à la même place, mais sans acquérir par là un droit de propriété quelconque. Les serfs peinent pour leur maître qui vit intégralement à leurs dépens et qui exige en outre un tribut annuel.

En Nouvelle-Zélande, d'autre part, nous voyons une égalité politique générale coexister avec un profond respect envers la noblesse. La grande masse de la population se compose de ce qu'on pourrait appeler des gentilshommes terriens dont la position au sein de la tribu ne saurait descendre à l'état de dégradation qui caractérise les serfs micronésiens. Nous ne sommes pas très au clair sur les prérogatives précises des chefs et des autres individus. Les droits collectifs et individuels existaient parallèlement d'une manière parfois surprenante. Il semble en général que les droits de la communauté se rapportaient à la zone tribale qui n'avait pas encore été définitivement occupée; mais, dès qu'un homme marquait comme sien un arbre dont il voulait faire un bateau, son titre de propriété était incontesté. Au contraire des Australiens, les Maori appliquaient le principe selon lequel le territoire du vaincu appartient au vainqueur; ce territoire était en fait considéré comme une sorte d'indemnité pour les pertes humaines du conquérant. Les terres étaient-elles possédées à la manière féodale? Nous ne pouvons le dire. L'estime en laquelle on tenait les chefs et le fait que les régions qui n'appartenaient à personne étaient morcelées à leur gré, semblent 
indiquer que le pays conquis était également réparti selon ce système. Nous n'ignorons pas d'autre part que n'importe qui pouvait acquérir des privilèges de propriétaire sur les terrains pris à l'ennemi en les mettant en valeur et posséder tout le territoire qu'il avait traversé avant de rencontrer un colon rival. Il lui suffisait d'enfoncer sa lance dans le sol pour marquer qu'il lui appartenait. Il se peut naturellement que ces excursions de prospection aient été au nombre des privilèges des nobles.

Outre la conquête et l'héritage, on pouvait devenir propriétaire de diverses manières. Les revendications se basaient sur le fait que les ancêtres du plaignant avaient été enterrés dans la terre contestée, que son propre cordon ombilical avait été coupé là, qu'il y avait été blessé ou maudit et ainsi de suite. Ainsi des notions religieuses ou même des allégations fantaisistes pouvaient influencer la loi foncière de cette civilisation si complexe. Quoique les Maori fussent surtout cultivateurs, la terre représentait pour eux toute une série de valeurs économiques; les individus et les familles possédaient divers privilèges spéciaux. L'un avait le droit de chercher en tel endroit des coquillages ou des baies; un autre avait l'exclusivité de la chasse aux oiseaux en certaines localités. Il est particulièrement intéressant de noter la présence de la saisine multiple; parfois, à l'intérieur d'une même aire, une famille avait le droit de déterrer des racines de fougères tandis qu'une autre se livrait à la chasse aux rats.

A Samoa, les conditions politiques rappellent en substance celles de la NouvelleZélande. Le peu que nous connaissons de la manière dont on possédait le sol ne laisse pas de doute sur la question. Chaque district protégeait ses frontières contre les empiétements des colons étrangers. A l'intérieur du district, les familles individuelles, représentées chacune par leur chef, possédaient séparément la terre. Le chef de famille, en dépit de sa position dominante, ne pouvait pas aliéner le sol sans consulter ses parents ; s'il y manquait, la parenté aurait déposé l'administrateur arbitraire de ce qu'on considérait évidemment comme une propriété de famille.

Pour citer un dernier exemple des formes de propriété foncière chez les primitifs, la co-possession par la famille caractérise aussi nettement la loi sur les liens immobiliers chez les Ifugao du nord de l'île Luçon. C'est à eux, plus qu'à toute autre peuplade, qu'on peut appliquer l'axiome de Sir Henry Maine, selon lequel l'individualité chez les peuples primitifs est engloutie par la famille; la famille, dans ce cas, comprend les parents du côté maternel et du côté paternel. Les champs de riz et, dans une certaine mesure, les forêts sont aux mains d'administrateurs Plutôt que de véritables propriétaires. Selon Barton, « les propriétaires possèdent ou plutôt occupent la terre d'une façon fugace, éphémère et de durée insignifiante en comparaison des décades et peut-être des siècles qui se sont écoulés depuis que le champ ou l'objet en question est devenu la possession de la famille ». Lorsqu'il n'y a qu'un champ pour un grand nombre d'héritiers, c'est l'aîné qui le prend, car, outre les difficultés d'ordre pratique, on juge préférable que la famille soit représentée par un individu unique mais puissant qui soutienne les autres membres du groupe, plutôt que de diviser les terres en petits lopins occupés par des hommes sans influence dans la communauté. En sa qualité d'administrateur, le titulaire de la terre ne peut pas en disposer à son gré, 
mais est tenu de consulter auparavant sa parenté. Les transferts n'ont jamais lieu que sous le coup d'une nécessité urgente, telle que l'obligation de faire un sacrifice afin que quelque membre de la famille, dangereusement malade, recouvre la santé; et le transfert des biens est alors célébré avec une solennité qu'on ne trouve à aucun degré lors de la vente de biens personnels.

Les terres peuvent sortir de la famille de deux manières par la vente ou par l'affermage. Si un propriétaire foncier a besoin d'un prêt, par exemple pour couvrir les frais de funérailles, il peut donner son champ de riz en garantie au prêteur; celui-ci y sème et moissonne jusqu'à remboursement de la somme avancée. D'ordinaire, lorsque le prêt est restitué (en général, il s'agit de la moitié de la valeur du champ), la terre revient à l'emprunteur avec la réserve que le créancier l'occupera jusqu'à la fin des récoltes. Le prêteur peut à son tour affermer la terre, mais jamais pour une somme supérieure à celle qu'il a avancée lui-même, de manière à ce que, par le paiement de la dette, le champ revienne sans contestation possible à son propriétaire originel. La mise en gage a lieu devant un témoin qui est aussi l'agent ayant négocié l'affaire; la commission de celui-ci est avancée par le créditeur, mais doit être restituée en même temps que la dette.

Lorsqu'il y a vente, la cérémonie est beaucoup plus solennelle. Le prix est divisé en dix parties dont chacune est représentée par une encoche dans un bâton ou par un nœud à une ficelle. Les deux premiers paiements sont les plus considérables et doivent être effectués au terme fixé, tandis que l'époque où les autres paiements peuvent se faire n'est pas déterminée, La livraison des biens a lieu dès la réception de la somme initiale. Les parents éloignés du vendeur servent souvent de témoins ainsi que les indispensables intermédiaires qui ont négocié la transaction et qui ont de ce fait droit à une rétribution. Le transfert de la propriété ne peut intervenir sans la célébration d'une fête; d'autre part, comme le début du banquet délie l'acheteur de toute obligation relative aux paiements ultérieurs, celui-ci a parfois recours à la ruse pour que le vendeur se laisse entraîner à un festin prématuré.

Si le propriétaire foncier abandonne un champ et que celui-ci soit repris et cultivé par un autre homme sans que l'occupant héréditaire intervienne, ce dernier perd tous ses droits sur le champ pendant une durée égale à celle où il ne l'a pas utilisé. Au terme de cette période, le premier possesseur recouvre son champ ; s'il veut rentrer auparavant On sa Possession, il est obligé de le racheter.

L'étude des diverses formes de possession du sol décrites au cours des pages précédentes, établit de manière indiscutable l'existence chez les primitifs de cette copropriété qui fit tant d'impression sur Sir Henry Maine. Mais il est faux que les copropriétaires constituent toujours une unité sociale de type invariable. Outre la zone tribale en général, nous n'avons trouvé la propriété communale que dans le cas très spécialisé où un clan consanguin est localisé et se confond ainsi avec la commune. Bien plus souvent, les prérogatives de propriétaire sont réparties entre des corporations de type différent, des groupes de proches parents, unilatéraux chez les Ewé par 
exemple, ou bilatéraux comme cela semble être le cas parmi les Ifugao. Ceci revient à dire que le communisme foncier n'existe pas en ce qui concerne le groupe territorial, mais seulement à l'intérieur d'un groupe strictement limité de parents véritables. En outre, la co-propriété, bien que fréquente, n'est pas universelle. Nous trouvons aussi des droits de propriété individuels, comme à Rewa et au détroit de Torrès ; bien plus, il arrive parfois, notamment dans le cas des pâturages Kirghiz, que communisme et individualisme coexistent. C'est à ceux qui croient à un état universel de propriété communale, antérieur à la propriété foncière individuelle, à faire la preuve de leurs assertions. C'est à eux de nous montrer que la terre était autrefois possédée communalement au détroit de Torrès, que les Algonkin, à certain moment de leur histoire, ignoraient les terrains de chasse individuels, ou que, durant une période donnée, les Vedda ne connurent pas la propriété individuelle.

Par bonheur, il n'est pas besoin de s'en tenir à l'attitude passive du sceptique. Les études de Baden-Powell aux Indes nous permettent de prendre l'offensive et de démontrer que, selon toute probabilité, cette région a été témoin d'une évolution de la loi foncière diamétralement opposée à celle que supposent inconsidérément les théoriciens de la sociologie. Baden-Powell fait remarquer que la zone attribuée en commun aux villages n'atteint pas à la moitié de celle où la propriété individuelle prédomine. De plus, cette dernière forme se rencontre surtout précisément parmi les populations dont la civilisation est plus primitive. Les Kandh d'Orissa nous en offrent une preuve qui n'est pas à négliger. Ici, c'est le chef de famille qui possède la maison et la terre qui en dépend. Us fils continuent à vivre avec lui après leur mariage, mais sans rien Posséder jusqu'à la mort du père; à ce moment les biens sont répartis également entre eux. On ne relève aucune trace de propriété communale ni de répartition par lots de la zone du village aux diverses familles. Chacune de celles-ci défriche et occupe une fraction de la vaste étendue de brousse, selon ses propres besoins. Une fois occupée de cette manière, la terre devient une propriété transmissible qui peut être achetée et vendue. A ce type de propriété individuelle des indigènes agriculteurs, est venu s'ajouter, en certaines localités, le principe de la possession multiple par le village ; il arrive alors souvent qu'un groupe conquérant non agriculteur s'empare du soi et rabaisse les premiers propriétaires au rang de simples tenanciers. Dans de tels cas, les propriétaires terriens forment une confrérie à laquelle appartient la zone du village et la brousse qui en dépend, le tout formant une unité foncière. Les parts sont assignées soit au prorata des individus, chaque ménage en recevant un nombre proportionné à celui de ses membres; ou bien les parts ancestrales sont évaluées selon le pedigree, si bien que l'unique héritier du mandataire originel possède un territoire plus vaste que celui qui est attribué à chacun de trois héritiers par exemple. Baden-Powell déclare qu'il n'existe pas de propriété en commun du sol, au sens strict du terme, par quelque groupe important, quoiqu'on décèle un certain sens de la parenté et de l'obligation de s'aider mutuellement; la co-possession ne dépasse jamais un certain degré de parenté. Sa conclusion est également importante : dans les cas où prédomine la co-possession, elle n'est pas « originaire mais consécutive à un titre de propriété unique et antérieur, détenu par le fondateur ou le 
cessionnaire, etc. du village; la co-possession n'est que le résultat de la succession de plusieurs (par parts ancestrales) à un fondateur unique ».

Ainsi l'étude intensive d'une zone unique mais vaste conduit à une reconstruction historique qui est en contradiction directe avec le dogme de la propriété primitive commune. Ce dernier état ne semble pas être universel, mais représenter plutôt un cas très spécialisé, un développement tardif plutôt que primitif.

Chapitre 9

\section{c. Biens mobiliers.}

\section{$\underline{\text { Retour à la table des matières }}$}

La loi primitive relative à la propriété mobilière est beaucoup plus simple que celle qui régit les biens fonciers, et peut donc être traitée plus sommairement. D'une façon générale, le caractère purement personnel des titres de propriété est plus manifeste que dans le cas de la terre. Le communisme, en ce qui a trait aux plantations, peut fort bien s'accompagner d'un individualisme complet pour ce qui est des effets mobiliers. Cette remarque s'applique, entre autres, aux Bakaïri, où chaque homme et chaque femme a ses biens propres. Il est particulièrement digne d'attention que le droit des femmes à posséder n'est pas contesté, même lorsqu'elles occupent une condition nettement inférieure. Une Ewé est achetée par son mari et est jugée inapte à hériter du terrain, mais elle peut posséder des effets mobiliers tels que chèvres ou volailles et elle ne livre à son mari que contre compensation le coton qu'elle obtient par son travail. Il n'est pas moins remarquable que parfois même les droits de propriété individuels de l'enfant sont considérés comme inviolables. Dans une réserve de Paviotso du Nevada, il m'est arrivé une fois de demander à acheter une couverture appartenant à un petit garçon. Ses parents non seulement allèrent le consulter en tant que propriétaire légitime de l'objet, mais ils s'obstinèrent à s'en tenir au prix ridiculement bas que leur fils avait fixé et qu'en toute équité je m'étais senti obligé d'élever. Schmidt a fait au Brésil une expérience analogue en essayant de se procurer la natte d'un garçon de onze ans que le père se refusait à vendre sans le consentement de son fils.

Le titre de propriété sur les biens mobiliers a fréquemment pour base l'effort individuel; c'est ce qui explique que les femmes possèdent si souvent la poterie qu'elles fabriquent. Un autre principe a trait à ce qu'on pourrait dénommer l'utilisation effective. Chaque Youkaghir est propriétaire de ses vêtements, le chasseur de son fusil, la femme de ses objets de couture. Néanmoins, cette notion elle-même peut conduire à une conception de la propriété collective plutôt que personnelle : les 
Youkaghir considèrent les bateaux, les maisons et les filets comme la possession de toute la famine. Mais il arrive que ces principes si courants ne correspondent plus du tout à la structure de la société politique. Lorsque le système des castes atteint la rigidité caractéristique des îles Marshall, le travailleur est complètement à la merci du chef qui peut s'approprier non seulement le produit de ses travaux horticoles, mais aussi ses effets mobiliers. Dans ce cas, l'organisation sociale introduit un type particulier de propriété : les serfs ou esclaves dont nous étudierons plus loin le statut. Sur la côte africaine occidentale, la servitude peut n'être que temporaire, l'esclave faisant office de gage ou de garantie pour le règlement des dettes.

Le bétail représente une autre catégorie de biens mobiliers. Parmi les peuples pasteurs, les troupeaux constituent la seule ou tout au moins la principale forme de la richesse, le moyen de se marier ou d'acquérir du prestige social. Le sens des droits de propriété individuels sera donc chez eux fortement accentué, ce que vient attester l'habitude de marquer le bétail qu'observent les Tchouktche, les Kirghiz ou les Massai. Parmi les peuples qui se consacrent surtout à l'élevage, la propriété individuelle s'affirme hautement, parfois même à l'encontre des revendications familiales. Un vieillard massai peut diviser une partie de son bétail entre ses femmes ; en retour de leurs soins, elles jouissent de l'usufruit, mais les bêtes restent la propriété du mari. Ce n'est que lorsque l'épouse a un fils de dix à douze ans qui n'est pas tenu de s'occuper des troupeaux paternels, que celui-ci devient propriétaire du bétail ; toutefois, mère et fils doivent alors quitter le kraal et s'en construire un autre plus petit à plusieurs kilomètres de distance afin que les troupeaux du père et du fils ne se mélangent pas au détriment du premier. Le Tchouktche que la perte d'animaux domestiques a appauvri, peut, il est vrai, demander aide à son frère ou à son cousin afin d'être en mesure de continuer à élever des rennes, mais cette revendication reste toute morale et non légale ; un vieux berger gardera jalousement intacts ses droits de propriété, même contre ses fils.

En résumé, pour ce qui a trait aux biens mobiliers, la propriété individuelle prédomine.

Chapitre 9

\section{d. Propriété immatérielle.}

Retour à la table des matières

Contrairement à ce qu'on supposerait, la notion de patente ou de droit d'auteur est très développée dans les couches basses de la civilisation, et sa force, parmi certains peuples, rend manifestement absurde le dogme d'un communisme primitif universel. Si les anciens auteurs n'ont pas apprécié à sa juste valeur l'importance de ce phénomène, c'est à cause du préjugé rationaliste qui est la perte de toute enquête historique. 
Pour des esprits imprégnés de l'atmosphère d'une ère industrielle, il est difficile de concevoir que des privilèges, ne rapportant apparemment aucun bénéfice utilitaire, puissent être hautement estimés et parfois même assimilés nettement à des richesses. Même dans un milieu aussi humble que celui des îles Andaman, nous trouvons des droits de disposition exclusive dépourvus d'objet matériel. Ce fait est d'autant plus remarquable que, en ce qui a trait aux ustensiles de cuisine, par exemple, ces insulaires font preuve, par contre, d'une largeur d'esprit qui touche au communisme : « Les droits de propriété privée se limitent à l'interdiction de s'approprier sans permission ou d'emporter à une certaine distance tout objet appartenant à un ami ou à un voisin. » Cependant cette générosité ne s'étend pas aux chants composés à l'occasion d'une assemblée publique. Un chant qui a eu du succès peut être répété lors de réunions de moindre importance, mais, quelle que soit sa popularité, personne n'a le droit de le chanter en dehors de son compositeur.

Les Koriak croient que le cours des événements peut être modifié par des formules magiques qui ont pour effet de bannir les maladies, d'exorciser les mauvais esprits, de donner la chance aux joueurs et de rendre efficaces les charmes. L'origine de toutes ces incantations remonte au Créateur. Elles sont connues maintenant par des vieilles femmes qui en gardent jalousement le secret ; on croit, en effet, que ce serait détruire la force agissante de la formule que de la divulguer. Quand une de ces propriétaires de formules a prononcé une incantation, elle reçoit en récompense des sortes de galettes de thé compressé, plusieurs paquets de tabac ou un renne. "Lorsqu'une femme vend une incantation, elle doit promettre qu'elle la vend tout entière et que l'acquéreur devient le seul possesseur de son pouvoir mystérieux. »

Dans l'une des îles du détroit de Torrès oriental, Haddon a découvert des notions de propriété distinctes, se rapportant aux légendes locales :les informateurs n'y racontaient qu'à contrecœur les histoires ayant trait à une autre localité. Beaucoup d'ethnographes ont fait cette expérience parmi les Indiens de l'Amérique du Nord. Les Kat nous en fournissent encore d'autres exemples. Chez ces derniers, comme parmi les Andaman, le poète est le maître absolu de sa composition. Personne ne peut la chanter sans son consentement et ce n'est pas gratuitement qu'il accorde cette faveur. Ils sont de même propriétaires de formules magiques ; celui qui en enseigne une reçoit de son élève une compensation. Il est également certains dessins gravés qui ne peuvent être reproduits sans autorisation spéciale. Les noms personnels eux-mêmes sont, dans un certain sens, un aspect de la propriété patentée : un jeune homme qui adopte un nom qu'un autre porte déjà offre en manière de conciliation un présent à son homonyme plus âgé.

Parmi les indigènes de la Colombie britannique, les Nutka se font remarquer par le nombre et la variété de leurs biens immatériels. D'après les informations dont Sapir a bien voulu nous faire part, il semble que chez ces gens les droits de patente se divisent en deux catégories : ceux qu'on nomme topati et qui passent obligatoirement du père à son fils aîné, et ceux qu'un père peut ou non transmettre à son fils. Ainsi un privilège tel que la connaissance de la légende familiale ne peut être refusé au fils 
aîné puisque sa naissance lui y donne droit. D'autre part, il arrive qu'un père garde par devers lui le secret du rituel pour harponner le poisson ; s'il n'en juge pas son fils digne, il refuse de lui donner les instructions nécessaires. Les topati sont extrêmement nombreux. En voici quelques-uns : des désignations comprenant non seulement le nom du possesseur lui-même, mais aussi ceux qu'il applique en vertu d'un droit exclusif à ses esclaves, ses maisons, ses canots ou ses harpons; l'exclusivité de certains motifs gravés sur les poteaux funéraires ou totémiques; le privilège de chanter certains chants, même des berceuses, et d'exécuter certaines danses ; et bien d'autres encore. Quelques-unes de ces exclusivités sont d'un caractère étonnamment spécial. Par exemple, dans le rituel du Loup, la coloration et la décoration particulière de tels exécutants constituent la patente héréditaire de l'individu qui organise la fête ; le droit de poser une trappe afin d'y prendre les personnificateurs du Loup s'associe à telle famille ; de même l'acteur qui boite et hurle de certaine façon y est autorisé en vertu d'un privilège qu'il a hérité. Cette remarque s'applique aussi aux chants solennels qu'on fait entendre en des occasions spéciales, à la cérémonie du lasso pour les novices, aux battements de tambour sonores et rapides qui marquent certains points du drame, au port de peaux d'ours à un autre moment, au droit de noircir les visages de tous les spectateurs. D'autres cérémonies sont marquées par des exhibitions analogues de topati ; ainsi, lors de la fête de puberté des filles, le droit de recevoir une torche cérémonielle est jalousement gardé et transmis comme un privilège héréditaire.

Alors que les Nutka soulignent le caractère héréditaire des diverses formes de propriété immatérielle, ce qui équivaut à faire de ces privilèges la possession multiple d'un groupe, le caractère individualiste des biens immatériels est en général beaucoup plus accusé parmi les Indiens des Prairies. Afin de bien comprendre les phénomènes relevés dans cette région, il nous faut quitter momentanément la sphère des faits purement sociologiques et entrer dans le domaine des croyances religieuses. Le pivot de la religion chez les Indiens des Prairies est constitué par des concepts et des pratiques se rattachant à des visions. Parfois celles-ci visitent sans qu'il l'ait voulu l'heureux mortel favorisé des esprits surnaturels; mais bien plus fréquemment, la venue du rêve est facilitée par un jeûne de plusieurs jours sur le sommet d'une colline solitaire. Le contenu de la révélation déterminait souvent toute la carrière de l'heureux visionnaire. Si son rêve lui montrait un bison lui recommandant l'usage de certain mélange de racines pour soigner les blessures, il s'établissait guérisseur et le succès lui apportait honneurs et richesses. S'il lui était enseigné comment organiser une nouvelle société de danses avec certains chants et insignes, il devenait dans la suite le fondateur d'une telle organisation, en retirait un prestige considérable et peut-être aussi d'autres bénéfices. S'il voyait un cavalier portant un bouclier décoré de certaine manière et sortant indemne d'un combat, il était plein dès lors de confiance en lui et se faisait une réputation de bravoure et de témérité.

Cependant, les visions pouvaient étendre leurs bienfaisantes révélations à d'autres individus qui ne s'étaient jamais hasardés à rechercher une révélation ou qui, l'ayant tenté, n'avaient pas réussi à obtenir les faveurs surnaturelles. Il arrivait donc qu'ils en partageassent les bienfaits, non seulement de façon indirecte, en tant que patients gué- 
ris par un visionnaire ou que participants à une nouvelle danse, mais intégralement et comme s'ils avaient reçu eux-mêmes la bénédiction spirituelle. La chose était rendue possible par le fait que les privilèges conférés par un esprit passaient pour transmissibles ; et ce concept, joint à la notion qui s'y ajoutait de l'aliénation de la vision par vente, devint une source de gain pour le visionnaire.

Pourquoi certains de ces droits jouissent-ils d'une estime variable selon les tribus? Pas plus que dans le cas des Nutka, l'explication n'est aisée. L'important est que ces droits sont en général hautement prisés et accroissent la situation sociale de leur possesseur. Personne ne se hasarde à les lui contester ; et celui qui désire partager un de ces droits ou l'acheter tout entier sacrifie dans ce but des biens représentant une valeur que nous considérerions comme absurdement exagérée. Le transfert par don n'est pas possible, même quand les deux parties sont unies par les liens de parenté les plus étroits. Je connais un Crow qui a acheté à sa propre mère le droit d'user d'une certaine espèce de peinture cérémonielle, et les " paquets sacrés » des Hidatsa qui dérivent toujours de visions ancestrales, héréditaires dans certaines familles, doivent, néanmoins, faire l'objet d'une transaction entre pères et fils. En beaucoup de cas, ainsi que dans le dernier cité, les objets eux-mêmes, tels que pipe, plume ou sonnaille, peuvent être transférés, mais ils ne sont pas l'essentiel invariablement constitué par le privilège immatériel et les instructions s'y rattachant, relatives aux chants, à la façon de manier l'objet sacré et aux tabous inséparables des prérogatives du visionnaire. En raison du peu de signification de l'objet visible, il est possible de substituer à celui-ci une réplique que l'acquéreur fournit d'ordinaire. Il importe peu que ce dernier prenne au vendeur sa patte de lapin, sa peau d'hermine ou sa plume d'aigle, ou qu'il se les procure personnellement ; ce qu'il achète, c'est l'autorisation d'utiliser la combinaison de certains objets avec tels chants ou telle activité. Toutefois, il s'engage aussi par là à observer les devoirs et les règles de conduite qui en découlent.

Les dispositions relatives aux divers privilèges cérémoniels varient naturellement. Tantôt le vendeur n'aliène pas complètement sa propriété, mais permet simplement à l'acquéreur d'en partager les bénéfices, avec la réserve parfois que lui-même ne pourra plus user que quatre fois de ses droits, la quatrième fois y mettant fin; tantôt l'acheteur acquiert intégralement les privilèges en question, un seul transfert suffisant au changement de possesseur.

C'est par des exemples concrets surtout que ces principes généraux deviendront compréhensibles. Je choisirai deux cas : le droit de planter le Tabac sacré parmi les Crow, et l'achat du titre de membre d'une organisation militaire chez les Hidatsa.

Afin de s'assurer le droit de pouvoir participer à la plantation du Tabac sacré, il est nécessaire de devenir un membre initié de la Société du Tabac, d'être adopté comme son " fils ». Ce trait rend sensible le caractère individuel de la cérémonie. Personne ne peut entrer par lui-même dans la société; le novice est patronné par un parrain qui lui-même a été présenté par quelque garant, de même que le fondateur de la société fut dirigé par l'être surnaturel qui lui ordonna de planter le Tabac, pour le 
plus grand bien de sa tribu. Le fait que tout un groupe se partage ce privilège est, du point de vue des Crow, purement accidentel; c'est par hasard que, dans le cas de cet ordre par exemple, ceux qui se procuraient la « médecine » du Tabac ont fait preuve de plus de solidarité que ceux qui achetaient la même « médecine » de guerre et qu'ils en sont venus par la suite à former une société, au lieu de rester des individus non associés, jouissant de privilèges identiques. Outre le droit de planter le Tabac, le novice acquiert certains remèdes dont il peut faire un choix parmi ceux que lui présente la section adoptrice de la société. De plus, il existe de nombreux privilèges spéciaux, rappelant ceux des Nutka, mais en différant par le fait qu'ils ne sont pas héréditaires. Chaque objet médicinal et chaque privilège se paient à part. Ainsi une femme nommée « Coupe-la-mule-attachée » livra un cheval pour un sac de tabac, un autre cheval pour des ornements de poitrine en peau de loutre et encore un cheval pour le privilège d'être assise près de la porte. Ces paiements, n'incluant pas la rétribution initiale au « père », s'effectuaient aux membres de la section qui détenaient le privilège. L'aide que reçoit le novice de ses parents et amis et le fait que le privilège recherché est possédé conjointement, donnent à toute l'affaire certaine apparence d'achat collectif qui est au fond étrangère à l'essence de la transaction. Mettant un point d'honneur à se montrer généreux lors de l'initiation, le candidat est plus ou moins libéralement assisté par sa parenté, ses amis et son club. Par ailleurs, le " père » qui reçoit, par exemple, cinquante chevaux, serait traité d'avare s'il les gardait tous pour lui et n'en distribuait une partie aux membres de sa section. Ceci n'est, toutefois, que l'aspect personnel ou éthique que peut revêtir un processus strictement légal par lequel l'individu $\mathrm{A}$ se dessaisit d'une partie de ses privilèges au profit de l'individu B. lequel est obligé de fournir compensation.

Dans les clubs guerriers des Hidatsa apparaît un curieux mélange d'éléments collectifs et individualistes. Nous étudierons dans un chapitre ultérieur d'autres particularités de ces organisations. Ce qui nous occupe ici, c'est le concept du titre de membre en tant que propriété. Selon la théorie des Hidatsa, ces sociétés, avec leurs chants, leurs insignes et leurs fonctions, furent révélées à des visionnaires qui en devinrent les fondateurs pour obéir à leurs maîtres surnaturels, Lorsqu'un Hidatsa ne connaît pas la vision légendaire sur laquelle une société est basée, il suppose immédiatement qu'elle est d'origine étrangère. En d'autres termes, nous retrouvons ici la notion d'une révélation individuelle, dotant son bénéficiaire de prérogatives transmissibles.

Puisqu'il a été révélé au visionnaire comment fonder une société, l'élément collectif apparaît inévitablement. En principe, pourtant, nous avons affaire à un droit de propriété qu'un groupe peut se partager, mais qui est aussi détenu par un seul individu. C'est ce que nous prouve le fait que, à un moment donné, lorsque tous les membres d'une société, à l'exception d'un seul, eurent été emportés par la maladie, le survivant resta seul propriétaire et vendit le titre de membre à un groupe de jeunes gens, désireux de se le procurer. Si on la compare au privilège de planter le Tabac sacré, la qualité de membre dans un club militaire diffère en ce qu'elle est vendue intégralement, le " père » abdiquant en faveur de son « fils ». 
Normalement, l'élément collectif se manifeste par le fait qu'un groupe d'acquéreurs achète conjointement le titre de membre à un groupe de vendeurs. Nous indiquons plus loin une des raisons qui peuvent expliquer cet achat collectif. Ce qui nous importe ici, c'est que le transfert de la propriété était essentiellement individuel. Les « fils » en corps commençaient par accumuler des biens, puis, forts de ces richesses, ils cherchaient à faire accepter aux membres de la société l'idée de vendre leur titre. Mais, dès le début de la vente proprement dite, chaque acheteur choisissait un vendeur, appartenant au clan de son père, qui devenait son " père » individuel. En entrant dans certaines de ces sociétés, on livrait sa femme; il ne se produisait pas alors la remise en groupe aux vendeurs des femmes des acquéreurs, mais chaque individu amenait son épouse à son " père ". De même, si celui-ci possédait quelque fonction ou insigne particulier au sein de la société, ils étaient automatiquement transférés à son « fils ». Le pivot de toute cette transaction, c'était non le transfert de droits de propriétaire d'un groupe à un autre, mais une série de transferts multiples et simultanés entre individus.

Ainsi s'affirme le caractère individuel des droits de propriété basés sur des visions, quand bien même ces droits sont partagés par une association d'individus. Ce caractère devra donc être a fortiori incontestablement reconnu lorsque, ainsi que dans le cas des " médecines » de guerres et des " paquets sacrés ", il y a négociation entre deux individus seulement, l'acquéreur et le vendeur. Certaines formes de propriété immatérielle confirment indubitablement l'existence de la possession individuelle dans les premières phases de la société. Aucun doute n'est plus possible quand nous voyons qu'un père ne peut pas transmettre à son propre fils ses peintures faciales ou un ensemble rituel héréditaire sans recevoir en échange une compensation. Naturellement, il ne nous faut pas perdre de vue que les biens immatériels se transmettent aussi automatiquement, comme parmi les Nutka, et représentent dans ce sens une possession multiple. Mais ce qui importe, c'est que parmi les Andaman, les Kaï, les Koriak et les Indiens des Prairies, quelles que soient les lois relatives à la possession matérielle, il existe des patentes et des droits réservés qui sont détenus individuellement et dont seuls les titulaires peuvent se servir.

La propriété immatérielle, cependant, n'est pas à considérer uniquement sous cet angle. Le simple fait de son existence parmi les primitifs est du plus haut intérêt, et la variété des formes qu'elle assume en certaines circonstances favorables est également curieuse.

Chapitre 9

\section{e. Héritage.}


Rien ne fait mieux ressortir la différence entre les régimes de propriété individuel et collectif que les divers degrés de liberté accordée à l'individu dans ses dispositions testamentaires par différentes sociétés. Le contraste est frappant entre un insulaire du détroit de Torrès qui peut priver tous ses enfants de leur part d'héritage, et le Kaï dont l'avoir se transmet automatiquement en vertu du droit coutumier, dont les cochons sont abattus pour la cérémonie funéraire, dont les sacs en défenses de sanglier et en dents de chiens passent aux mains de ses frères ou oncles maternels et dont les fils héritent des arbres fruitiers qu'il a plantés personnellement.

Ce dernier cas illustre un principe important qu'avait déjà reconnu l'intelligence pénétrante de Maine. La loi archaïque distingue souvent entre la possession héréditaire et la possession acquise; nous l'avons déjà fait remarquer à propos de la Mélanésie. Lorsque se produit cette différenciation, il y a tendance à ce que les biens acquis soient doués de plus de liberté, l'individu disposant en maître de ce que ses efforts lui ont valu. Ces considérations peuvent naturellement s'effacer devant des facteurs d'ordre différent. Un Indien des Prairies ne peut léguer sans autre ses droits sur une vision obtenue à force de jeûnes, en vertu du principe qui veut que de tels droits ne soient accessibles que par le songe lui-même ou par l'achat; dans le cas des « paquets sacrés " des Hidatsa, ce sentiment peut prendre une telle force qu'un privilège, même héréditaire, n'a de valeur que s'il est formellement racheté au père.

Les règles de succession se trouvent parfois considérablement simplifiées là où la coutume exige qu'on brûle ou qu'on détruise de quelque autre manière tout ce qui a appartenu au défunt. Les Maidu, par exemple, brûlent presque tous les effets du mort; le peu qui reste est réparti entre le fils aîné, héritier principal, et les autres enfants ou parents; l'exclusivité sur certains trous de pêche ou sur les battues de cerfs se transmet par les mâles en ligne directe. Parmi les Assiniboin, les armes, les vêtements et les ustensiles du défunt étaient déposés à côté du cadavre, ainsi que les boucliers sacrés et les pipes. Là et dans d'autres régions des Prairies, ses meilleurs chevaux étaient parfois relâchés ou tués, seuls quelques-uns d'entre eux étant distribués aux survivants. En outre, nous voyons parfois que l'habitation qu'il a occupée de son vivant est abandonnée ou détruite, coutume qui a pour origine une crainte morbide de la mort. Ainsi les Pima de l'Arizona non seulement abattent et mangent le bétail d'un mort, mais détruisent ses effets personnels et vont jusqu'à brûler sa hutte.

Certains principes, que nous avons déjà décrits à propos des titres de propriété, s'appliquent forcément aussi à l'héritage. Ainsi les vêtements d'une femme et les objets qui sont son oeuvre, la poterie par exemple, passent généralement à ses filles ou à d'autres parentes, car elles sont seules à pouvoir en faire usage. D'un autre côté, c'est un homme qui héritera des armes du mort. C'est ce principe, joint à d'autres causes sans doute, qui explique l'une des règles de succession les plus fréquentes : le fait que la veuve est exclue du nombre des héritiers. Cette incapacité ne saurait s'expliquer par son statut inférieur, car elle se retrouve aussi dans des régions où la femme occupe dans la société une situation élevée; elle correspond d'autre part à 
l'incapacité du mari à hériter de sa femme. C'est pourquoi lorsqu'on nous représente la veuve ostiak comme inapte à posséder les troupeaux de rennes de son mari défunt, il nous faut envisager la chose de la manière suivante : les hommes, en domestiquant les rennes et en les soignant, ont établi une association empirique entre leur sexe et cette catégorie de biens; il apparaît donc anormal qu'une femme puisse en posséder, de même qu'il ne convient pas qu'un homme hérite d'objets féminins. Les troupeaux passent aux parents mâles les plus rapprochés et les femmes n'en reçoivent aucune part. Cette notion n'affecte pas en elle-même leur statut puisqu'elles peuvent a priori posséder des droits de propriété équilibrant leur incapacité. En pratique cependant, dans les tribus pastorales, les femmes n'ont pas en compensation de tels privilèges, d'où leur relative dégradation.

Un autre facteur qu'il nous faut considérer à ce sujet est le concept du mariage en tant que contrat entre divers groupes de parents : le mari et la femme sont des alliés dont l'individualité reste distincte, mais qui se confond avec le groupe qu'ils représentent et auquel reviendront leurs biens. Ainsi, alors que les héritiers d'un Kaï sont ses frères et ses oncles maternels, les biens de sa femme deviennent la propriété de ses frères à elle et des frères de sa mère. Ce facteur est particulièrement manifeste lorsque ces groupes sont des clans, en d'autres termes lorsque le groupe de parenté se définit avec une précision parfaite. Chez les Turcs, ce sont les fils qui héritent ou, en l'absence d'héritiers mâles, les frères du père ou des parents du sexe masculin. Ce n'est qu'à défaut de tous ceux-ci qu'une fille peut devenir l'héritière.

Toutefois, en reconnaissant la puissance du facteur clanique sur les formes de succession, nous devons veiller à ne pas en exagérer l'importance. En premier lieu, alors qu'un système clanique, une fois qu'il est fortement établi, réagit sur la loi de propriété, nous avons vu que fondamentalement c'est souvent sur les règles de succession que repose la notion de clan. Ce qui constitue l'organisation clanique, c'est de faire passer un parent éloigné mais du même clan que le défunt avant un proche parent d'un autre clan; cependant, on ne peut jamais, à proprement parler, appeler le clan l'unité possédante. Le clan, en effet, comprend des hommes et des femmes et, étant donné la répartition universelle des tâches selon les sexes, en général les droits de propriété, quels qu'ils soient, ne sont pas détenus à la fois par les hommes et par les femmes, mais par l'un des sexes seulement. Les Hopi nous en fournissent un exemple instructif. Parmi ces Indiens, les maisons appartiennent invariablement aux femmes et se transmettent en conséquence de mère en fille. La dignité de Prêtre Serpent, de même, reste dans les limites du clan, mais passe d'un frère à l'autre ou de l'oncle maternel au neveu. Il n'est donc pas exact d'affirmer que le clan possède les maisons ou telle charge cérémonielle; les véritables co-propriétaires sont, dans un cas, les membres féminins, dans l'autre les ressortissants masculins du clan. En outre, il est souvent faux de dire que tous les hommes ou toutes les femmes du clan soient copropriétaires puisque les possesseurs véritables sont un groupe de parents à l'intérieur du clan. Dans le cas des droits de propriété, exactement comme dans celui du lévirat, la transmission s'opère au bénéfice du parent le plus proche dans le même clan, mais non de celui d'un membre quelconque. Bref, l'organisation clanique exclut de l'hérita- 
ge certains proches parents tout en admettant d'autres plus éloignés ou putatifs, mais son action s'accompagne de l'influence des notions relatives à la division fonctionnelle des sexes et de la présence des parents les plus proches.

Il est une autre particularité également importante : dans les communautés organisées en clans, la propriété ne se transmet pas nécessairement à l'intérieur du clan. Us Crow qui ont dû récemment s'adapter à un nouveau type de propriété, les biens fonciers, appliquèrent leur système matriarcal aux nouvelles lois relatives à la transmission des terres; mais c'est un autre principe qui prévalait d'ordinaire en ce qui avait trait aux possessions sacrées et héréditaires, lesquelles passaient du père au fils et d'un frère à l'autre. A cet égard, les Crow rappellent les Hidatsa qui héritaient des jardins matriarcalement mais des " paquets sacrés » patrilinéairement. On peut naturellement arguer que de telles particularités sont la marque d'une phase de transition, du passage d'une loi de succession à une autre. Mais c'est vouloir que toutes les règles d'héritage adoptent pour toutes les formes de la propriété une norme immuable. Par malheur, les processus historiques ne se conforment pas à cette belle régularité logique. Supposons qu'une peuplade observe une loi de descendance déterminée en ce qui concerne telle forme de propriété, l'adoption d'une nouvelle catégorie de biens peut entraîner un ensemble de règles toutes différentes relatives à la transmission. Ce nouveau code a alors une origine spontanée ou est simplement emprunté en même temps que la propriété elle-même. En conséquence, à défaut de faits concrets, je ne saurais appuyer la théorie qui veut que les lois d'héritage mixtes soient un signe de transition entre deux descendances différentes, du système consanguin au système utérin ou vice versa.

Par exemple, la coutume mélanésienne d'hériter des arbres fruitiers patrilinéairement et de la terre matrilinéairement peut être due à l'histoire différente de ces formes de propriété; et, s'il ne s'est pas produit d'unification, c'est pour la simple raison que l'esprit concret du juriste primitif ignore le concept abstrait de la propriété foncière, confondant dans une même notion les arbres et la terre, alors qu'il conçoit les arbres comme une chose, le sol comme une autre. Cette manière de penser a pris pour moi toute sa netteté le jour où j'ai tenté de raconter un conte de nourrice en Crow classique : je parlais d'une petite fille qui ne possédait que le pain qu'elle tenait à la main et les habits qu'elle avait sur le dos, lorsqu'on m'arrêta pour me dire que les deux choses ne pouvaient aller ensemble; dans l'esprit des Crow, il n'existait pas de lien entre la nourriture et le vêtement. Il n'était donc pas possible d'exprimer ma pensée en une seule phrase; il fallait la diviser en deux affirmations séparées, la première relative à la rareté des aliments, la seconde concernant cette catégorie de biens mobiliers que représente l'habillement.

En d'autres termes, si, au point de vue logique reconnu inapplicable, nous substituons le point de vue psychologique et historique, la coexistence de lois de succession distinctes pour des catégories de biens différentes devient parfaitement compréhensible. Les règles d'héritage sur lesquelles repose le concept du clan seront naturellement toujours en vigueur lors du plein développement du système clanique; mais 
d'autres règles, aussi anciennes ou davantage encore, continueront à s'appliquer à des types de biens spéciaux; enfin, avec l'introduction de nouvelles possessions, de nouvelles lois viendront s'ajouter aux autres.

Passons maintenant à l'étude plus poussée de certaines règles de succession, nous contentant de mentionner l'avunculat dont nous avons déjà vu plus haut les particularités.

Le droit d'aînesse existe, mais est relativement rare dans les sociétés primitives, même lorsque le fils aîné jouit d'un certain ascendant sur ses frères et sœurs. Parfois la différence d'âge ne joue aucun rôle; les Vedda répartissent les biens également entre les enfants adultes, la part des filles étant souvent attribuée nominalement à leurs maris. Les Kandh d'Orissa observent aussi une division équitable du sol entre les fils, quoique la charge de chef passe à l'aîné. Les Ifugao attribuent au premier-né la portion de terrain la plus importante, mais nous avons vu plus haut qu'il n'en est au fond que l'administrateur, pour le plus grand bénéfice de tout le groupe de ses parents. Parmi les Chukchi de la côte, le fils aîné reçoit la plupart des armes et des instruments de son père, mais les autres frères ont aussi leur part et la maison elle-même est fréquemment divisée. Dans les communautés polygynes, le droit d'ainesse est encore restreint du fait du statut supérieur de l'une des femmes, d'ordinaire mais non toujours la première épouse, et ceci quel que soit l'âge du fils. Chez les Massaï, c'est le fils aîné de la femme principale qui reçoit la plus grande partie du troupeau paternel et qui commande aux filles de sa famille. Cependant, tous les autres fils héritent de la part de bétail dont on a attribué à leurs mères respectives l'usufruit.

En pratique, toutes sortes de complications peuvent survenir. Les pouvoirs testamentaires dont un individu peut faire usage modifient la répartition habituelle des biens. Un Kikouyou qui sent sa fin approcher lègue plus ou moins de bétail à ses fils selon la place qu'ils occupent dans son affection. Quand le fils aîné adulte prend possession du legs, il n'est au fond qu'un exécuteur administrant les biens selon la volonté du testateur. S'il n'y a que des enfants en bas âge, la propriété passe à un ou à plusieurs des frères cadets du défunt, suivant les désirs de celui-ci. Mais ces derniers aussi ne sont que des administrateurs, jalousement surveillés par les veuves dont les fils réclameront leur legs en atteignant l'âge d'homme. Parmi quelques-unes des tribus Cafres de l'Afrique du Sud, deux des femmes étaient spécialement désignées comme passant avant les autres, « la grande » et « celle de la main droite ». Le fils aîné de la " grande » héritait du rang de son père et de tous les biens qui n'avaient pas été expressément attribués au fils de « celle de la main droite ». Le père pouvait mettre à part certains biens pour les premiers-nés de ses autres femmes, mais, s'il montrait par trop ouvertement sa préférence pour l'un de ces derniers, ses dispositions testamentaires étaient annulées après sa mort. En l'absence de progéniture, c'étaient les frères qui héritaient; à défaut de frères, le chef s'appropriait les biens, les filles n'y ayant aucun droit. Chez les tribus Cafres et Kikouyou apparaît donc la loi de succession collatérale qui, dans certaines parties du monde, a beaucoup plus d'importance que l'autre; ce sont alors les frères qui représentent les principaux héritiers, tout au 
moins au sens de la possession, à l'exclusion ou au détriment des fils. Cette coutume est bien mise en relief par la loi des Thonga qui règle la transmission de la charge; elle cherche, en effet, à concilier deux principes disparates, la préséance d'une certaine branche de la famille et la possession collective des biens par les frères. Lorsque meurt un chef de famille ou de tribu, le fils aimé de l'épouse principale est considéré comme son héritier légitime, mais il ne peut lui succéder qu'après que tous les frères cadets du défunt ont successivement occupé sa fonction et sont morts à leur tour. C'est ce même système qui réglait la succession des chefs militaires à Tenochtitlan, chez les Aztèques. Le cas des Maori est également intéressant. En ce qui a trait au rang, le droit d'aînesse prédominait; le grand-prêtre était le fils aîné du fils aîné... etc., de la lignée qui prétendait descendre des dieux. Mais, pour ce qui était de l'héritage du sol, le droit d'aînesse coexistait avec des principes collatéraux : " Si un père avait des fils appelés A, B, C, D, à sa mort, les biens passaient à A; mais si A mourait, ce n'était pas son fils qui héritait mais $B$, puis $C$, puis $D$ et, à la mort de ce dernier, ils revenaient enfin au fils de A. Quelquefois les prétentions des héritiers collatéraux allaient encore plus loin. Parmi les Indiens Arapaho, l'essentiel des biens qui, jusqu'à récemment, se composait surtout de chevaux, devenait la propriété des frères et sœurs du défunt, les fils adultes étant à tous égards privés d'héritage. Les Crow observaient des pratiques analogues en ce qui concernait la terre et les chevaux.

Lorsqu'une organisation matriarcale a des répercussions sur les lois de succession, les terres et la charge peuvent passer toutes deux à un frère et secondairement à un fils de la sœur, les deux méthodes ayant du reste le même effet, celui de garder les biens au sein du clan. Partant de ce fait, quelques auteurs en ont conclu que l'héritage fraternel dans une tribu donnée constitue une survivance d'un ancien système matrilinéaire. Les Arapaho qui n'ont pas de clan devraient donc être classés parmi les tribus utérines. Il est difficile d'avancer plus faible argument à l'appui d'une cause perdue d'avance. Sans doute, les frères sont membres du même clan maternel, si la descendance est matriarcale, mais, en cas contraire, ils appartiennent aussi tous au même clan consanguin. Le simple fait de l'héritage par les frères n'a donc pas la moindre portée sur la descendance, quelle qu'elle soit. Par ailleurs, si nous nous plaçons sur le terrain de l'équité, la règle collatérale est bien supérieure au droit d'aînesse. Si un titre de propriété s'établit sur la base de l'utilisation effective, le frère adulte doit certainement avoir le pas sur le fils adolescent ou enfant. De même, si un père laisse à ses quatre fils une propriété qu'il a fondée et que le partage est impossible, la manière de beaucoup la plus juste de procéder est d'en transmettre le contrôle au fils aîné, puis successivement à chacun des autres. La succession collatérale, même dans une société patriarcale, ne présente donc aucun mystère.

A l'opposé du droit d'aînesse, nous trouvons une coutume qui fut en vigueur dans certaines régions de la Grande-Bretagne sous le nom de " bourg anglais » et qu'on désigne en ethnologie par droit du cadet. C'est en effet le plus jeune des enfants qui devient alors l'héritier principal ou tout au moins préférentiel. Les Indes constituent l'un des centres de cette coutume. Chez les Badaga, voisins des Toda, les fils doivent en se mariant quitter le toit paternel et fonder leur propre ménage; seul le plus jeune 
reste avec ses parents, les entretient lorsqu'ils sont devenus vieux et, à leur mort, devient automatiquement le maître de la maison. Ce droit du cadet est aussi observé chez les Toda eux-mêmes, bien qu'à un moindre degré. Les buffles du père sont souvent la propriété de tous les fils conjointement; toutefois, si le besoin de partage se fait sentir, chacun en emporte une part égale; mais, lorsqu'il y a des animaux en surplus, l'aîné et le cadet en reçoivent chacun un. S'il n'y a que deux fils, tous deux héritent de la moitié. Si quatre fils se partagent seize buffles, l'aîné et le cadet en prennent chacun quatre, le deuxième et le troisième trois et les deux animaux qui restent sont soit vendus et leur valeur répartie également, soit attribués à l'un des frères qui indemnise les autres en divisant entre eux les trois quarts de ce qu'ont rapporté les buffles.

Les Naga de Manipur, bien qu'observant en général un droit d'ânesse limité, pratiquent le droit du cadet en certaines localités; le plus jeune des fils hérite alors à la fois de la maison du père et des plus précieux de ses biens mobiliers. Les règles de succession des Khasi, avec leur mélange de prérogatives féminines et de droit du cadet, sont des plus intéressantes. Ici la fille cadette célèbre le rituel de famille destiné à rendre les ancêtres propices; elle hérite de la maison ainsi que de son contenu et reçoit la part du lion des bijoux de famille; cependant, elle ne peut disposer de la maison sans le consentement unanime de ses sœurs. A sa mort, c'est la plus jeune après elle qui lui succède, et ainsi de suite. En l'absence de filles, l'héritage passe à la fille cadette de la sœur, puis à la fille cadette de cette nièce, etc. S'il n'y a pas non plus de nièces de cette catégorie, les biens reviennent aux sœurs de la mère. Toutefois, la loi réglant la transmission de la charge repose sur un principe différent. A la mort d'un chef, ce sont les fils de sa sœur ainée utérine qui lui succèdent par ordre d'ancienneté, ou à leur défaut les fils de la sœur qui vient ensuite. En un certain district où c'est une femme qui exerce les fonctions de grand-prêtre, sa fille aînée la remplace. On a également décelé des traces du droit des cadets dans les couches supérieures de la civilisation hindoue; les Lois de Manou, en effet, tout en assignant une part plus importante au fils aîné, stipulent également que le cadet reçoit une portion spéciale.

Nous rencontrons encore plus loin vers le nord cette coutume si contraire aux notions légales courantes. Chez les Kirghiz, un père cherche de son vivant à faire de ses fils des éleveurs indépendants. Il attribue à l'aîné une grande partie de ses troupeaux; en cas de nécessité, il ira jusqu'à lui acheter de nouveaux terrains d'hivernage, sinon il lui réservera une section des siens. Il dote de façon analogue ses autres fils, mais le cadet reste l'héritier des pâturages paternels et des autres biens. Si plusieurs fils demeurent à pourvoir, les troupeaux sont répartis équitablement entre eux, et les quartiers d'hiver sont utilisés conjointement ou divisés selon entente; cette division, cependant, se produit rarement, car eue serait au désavantage du cadet. Selon la loi kirghiz, c'est le devoir du fils aîné d'acquérir de nouveaux terrains d'hivernage aussitôt que les troupeaux, en se multipliant, dépassent la capacité du territoire hérité ou assigné, et si, après son départ, le bétail continue à s'accroître, le plus âgé des autres fils se trouve à son tour dans l'obligation de chercher de nouveaux 
pâturages, jusqu'à ce que finalement le cadet reste l'unique possesseur des terres de son père.

Parmi les Youkaghir, une cause différente a un effet identique. L'usage de la résidence matrilocale entraîne le départ des frères aînés hors de la maison paternelle; cette dernière, ainsi que d'autres biens, sont alors hérités par le cadet. C'est sans doute par extension de cette importante coutume que le \&oit du cadet est appliqué à certains objets féminins qui se transmettent de la mère à la fille cadette. Ceux des Youkaghir qui vivent sur la toundra et qui ont adopté des Tongouzes la coutume d'acheter la fiancée, sont naturellement patrilocaux, et tous les frères se partagent l'essentiel des biens, leur mère administrant la maison et le fils aîné ayant la haute main sur les troupeaux de rennes. Mais l'ancien usage a encore suffisamment de force pour que s'ajoutent à la part du fils cadet le fusil et les vêtements du père.

Les lois de succession chez les Esquimaux sont significatives, à la fois en ce qui touche au \&oit du cadet et aux notions de propriété en général. Au premier abord, une profonde différence de principe paraît régner entre la coutume observée dans l'Alaska, autour du détroit de Béring, selon laquelle le fusil paternel et la plupart des objets de valeur passent au fils cadet, et le fait que chez les Groenlandais c'est à l'aîné que se transmet la propriété. Mais une lecture plus attentive de nos sources nous éclairera. En réalité, les Groenlandais modifient la règle que nous venons de citer en introduisant le principe de la possession basé sur l'utilisation effective. Or, étant donné le mode d'existence dans les zones arctiques et, d'autre part, personne ne pouvant avoir l'usage simultané de deux tentes ou de deux bateaux, le fils aîné, s'il possède déjà de tels biens, ne s'appropriera pas un legs dont il n'a que faire. Si ses frères sont encore mineurs, il l'abandonnera à un étranger, et ses fils à lui, en approchant de la majorité, n'y auront aucun \&oit. En conséquence, le droit d'aînesse au Groenland, dans la mesure où il existe, n'implique nulle préséance mystique du premier-né, et c'est sans surprise que nous entendrons préciser que, parmi les Esquimaux du Centre, c'est le plus âgé des fils vivant avec les parents qui figure comme héritier principal, alors que les fils et filles qui possèdent déjà leur propre maison sont exclus de l'héritage. Nous n'aurons, en effet, qu'à en induire que les peuplades de l'Alaska, voisines du détroit de Béring, observaient la coutume des Youkaghir ou des Kirghiz relative à la séparation des fils adultes et à l'attribution au cadet des biens paternels qui en découle naturellement. Le droit du cadet, ainsi que tant d'autres éléments culturels, nous ramène une fois de plus au problème de la diffusion ou de l'origine indépendante. $B$ ne nous est pas possible d'ignorer que des emprunts ont dû se produire en une certaine mesure. La présence sporadique du droit du cadet chez les Naga, habitant une région où cette coutume atteint son plein développement, indique que nous n'avons affaire chez eux qu'au pâle reflet d'une institution caractéristique des tribus voisines. De même la double préférence pour l'aîné et pour le cadet que nous trouvons dans les Lois de Manou et dans les règles de succession des Toda, nous incline à croire qu'il y a eu connexion historique. Par ailleurs, il n'existe pas la moindre preuve que la coutume asiatique soit à rattacher à l'usage anglais. Puisque le droit du cadet s'accompagne si fréquemment du départ de l'aîné, les circonstances qui favorisent une telle séparation 
tendront régulièrement à établir ce mode d'héritage. Ceci revient à dire qu'il n'est nullement nécessaire de faire dériver la coutume de l'Alaska de celle des Youkaghir, ou cette dernière des Kirghiz.

Ces faits sont théoriquement intéressants d'un autre point de vue encore. Le départ des aînés peut avoir dans des régions fort distantes les unes des autres une même conséquence, le droit du cadet. Dans ce sens, il y a évolution parallèle. Mais ce parallélisme est strictement limité. Nous n'avons qu'à comparer l'évolution du droit du cadet chez les Kirghiz et les Youkaghir pour rencontrer deux ordres de phénomènes tout différents. A la base de la scission de la famille parmi les Youkaghir et la conditionnant, nous trouvons la résidence matrilocale, dont l'action ne se fait pas sentir chez les Kirghiz. Ainsi, par-delà le parallélisme partiel, la ligne d'une évolution remonte à des déterminantes distinctes; en d'autres termes, il s'agit une fois de plus d'un cas de convergence.

Dans cette étude brièvement esquissée des lois de succession primitives, il a été impossible de traiter plus que par allusion certaines des ramifications et des complexités de la question. En concluant, il me reste à rappeler au lecteur que les effets du droit coutumier peuvent être encore plus variés et complexes que ne l'indique cette rapide étude. Chez les Thonga, un administrateur de biens, dépourvu de scrupules, peut chercher à faire dériver de son côté l'héritage de son frère aîné. Ailleurs, l'absence d'héritiers et l'extinction de toute la lignée en présence d'une succession importante suggèrent au juriste indigène de nouvelles dispositions, créant une nouvelle jurisprudence. Les exemples cités suffisent à démontrer que l'héritage des diverses catégories de biens peut obéir à des principes différents : les objets sacrés se transmettront de père en fils tandis que les chevaux deviendront la Propriété des frères du défunt; la qualité de chef peut être léguée en ligne paternelle et les biens fonciers passer au fils de la sœur; le fils aîné hérite de telle charge alors que la transmission des biens fonciers ou mobiliers obéit au droit du cadet. Ici comme partout en ethnologie, ce qui nous a empêchés trop longtemps de saisir nettement la réalité des faits, c'est la simplicité tentante des grands mots.

Chapitre 9

\section{Références.}

$\underline{\text { Retour à la table des matières }}$

1) KEYSSER : 92 et suiv. CRANZ : 1, 234. BOAS, 1888 : 582. NELSON : 294. JOCHELSON, 1908 : 769. BOGORAS, : 633.

2) TEIT, 1900 :293. DIXON, 1905 :224. SPECK, 1915 (a); id., 1915 (b) : 289. SWANTON, 1908 : 425. MALINOWSKI : 150. SPENCER et GILLEN, 1899 : 590; id., 1904 : 13. FRAZER, J. : 36. 
RADCLIFFEBROWN : 146. ROTH, 1906 : 8 et suiv. SELIGMAN : 106-115. MERKER 28, 176, 212. RIVERS, 1906 : 557. SCHULTZE : 197, 318. Radloff 414-420, 452.

3) STEVENSON : 290. KROEBER, 1917 :178. SPECK, 1909 :18. WILSON : 9, 10, 108-114. BANDELIER, 1878 : 385. SPINDEN, $1917:$ 184. MARKHAM : 35, RESTREPO : 121. VON DEN STEINEN 285. SCHMIDT : 439. WHIFFEN : 103.

4) JUNOD : Il, 6 et suiv. MACLEAN : 149. SPIETH Il 1-115. ELLIS, A. B. : 162, 216. ROSCOE : 14, 133, 238, 268, 424. ROUTLEDGE : 121. TORDAY et JOYCE : 91.

5) CODRINGTON : 60. THOMSON : 70, 354-386. Reports, V : 284-291 ; VI : 162-168. ERDLAND : 99-113. TREGEAR : 127-136. TURNER : 177. STAIR :83. BARTON : 39-60.

6) MAINE chap. VIII. BADEN-POWELL : 1-37, 171 et suiv., 398-423.

7) SPIETH 116, 191. VON DEN STEINEN : 285. SCHMIDT : 316. MERKER : 28. BOGORAS : 677.

8) MAN : 120, 169. JOCHELSON, 1908 : 59. Reports, VI 167. KEYSSER : 100. SAPIR, 1911 : 15 ; id., 1913 : 67; id., 1915 355. LOWIE, 1913 : 225 et suiv.

9) DIXON, 1905 : 226. RUSSELL : 194. MERKER :200. SELIGMAN : 118. BADEN-POWELL : 172, 305. ROUTLEDGE : 143. MacLEAN : 11, 116. JUNOD : 1, 303, 383. SPINDEN, 1917 : 185. TREGEAR : 122-129. KROEBER, $1904: 11$. LOWIE, 1912 :188. RIVERS, $1906: 559$ et suiv. HODSON : 103. GURDON : 68, 83. Radloff : 416. JOCHELSON, 1910 109. CRANZ, 1, 247. BOAS, $1888: 580$. NELSON : 307. 


\section{LES ASSOCIATIONS}

$\underline{\text { Retour à la table des matières }}$

Morgan et ses disciples nous décrivent la société primitive comme un agrégat atomique. La tribu se composerait d'unités façonnées sur un modèle unique : la notion de clan, tous les clans étant généralement identiques par leurs fonctions et leur importance; à l'intérieur de chaque clan, les membres occuperaient démocratiquement le même niveau. En d'autres termes, si les vues de Morgan étaient exactes, les individus des cultures primitives ne différeraient socialement que par le clan auquel ils appartiennent. Nous avons déjà vu que ce système est faux parce qu'il fait abstraction de la famille bilatérale. Mais il présente encore un autre défaut; il ne tient pas compte de principes de classification qui ne dépendent pas de la parenté, unilatérale ou bilatérale. Les tribus primitives sont pour ainsi dire stratifiées par les différences d'âge, de sexe ou de statut matrimonial, et le fait que tel individu est affilié à l'un de ces groupes peut affecter son existence de façon bien plus puissante que sa qualité de membre de tel clan. Cunow fut peut-être le premier théoricien à observer le rôle que joue l'âge dans la société primitive, mais c'est surtout à Schurtz que nous devons l'étude systématique de toutes les associations. (Je propose qu'on désigne de ce terme toutes les unités sociales qui n'ont pas pour base le facteur de parenté.) Quelque temps après, Hutton Webster décrivit, dans une compilation de valeur, à peu près le même 
ordre de faits. Cependant, c'est surtout à Schurtz que revient le mérite d'avoir distrait les ethnologues de l'étude exclusive de l'organisation clanique et de les avoir incités à s'occuper de phénomènes qui semblaient devoir échapper à leur myopie. Quoique, comme bien d'autres pionniers, il se soit trompé et même gravement en plusieurs de ses interprétations, son insistance sur la signification théorique des associations doit être tenue pour l'un des points de départ les plus importants dans l'étude de la sociologie primitive.

Afin de donner une idée des divers types d'associations, le plus facile serait de grouper les faits en compartiments séparés. Mais ces catégories nuiraient plutôt à la compréhension synthétique de l'organisation sociale. Par exemple, nous pourrions dans un sous-chapitre traiter de la dichotomie sexuelle et dans un autre des classes d'âge. Une telle division serait néanmoins artificielle. En Australie, il est vrai, les sexes sont strictement séparés pendant les cérémonies, mais les jeunes garçons sont soumis comme les femmes à une même interdiction d'y prendre part, et, dans un certain sens et à un moment donné, la scission véritable s'opère entre les individus mâles initiés et le reste de la tribu. Ailleurs nous rencontrons une division tripartite en couples mariés, hommes célibataires et femmes non mariées, la dichotomie sexuelle ne s'appliquant donc qu'aux célibataires. De même, il est probable que souvent les dortoirs des hommes non mariés et les clubs masculins se rattachent génétiquement les uns aux autres : après leur mariage, les hommes continuent à venir se distraire ou travailler au lieu qu'ils occupaient du temps de leur célibat. Une classification logique entraînerait donc facilement la séparation d'institutions pourtant apparentées. C'est pourquoi, dans ce chapitre, je préfère choisir quelques tribus appartenant à diverses zones géographiques et décrire leur organisation sociale en laissant de côté le clan et la famille. Dans ce choix, je me laisserai guider en partie par la qualité des sources disponibles et en partie aussi par le désir de présenter toutes les variétés principales des unités associationnelles. Ce sera donc une étude limitée à quelques points particulièrement intéressants.

Chapitre 10

\section{a. Îles Andaman.}

\section{Retour à la table des matières}

Selon la théorie de Morgan, puisque les Andaman ignorent le clan, chacune de leurs communautés devrait se réduire à une horde désordonnée d'individus. Cependant ils s'obstinent à se diviser en groupes indépendants de toute notion de clan ou de parenté quelconque. Chaque campement présente une triple disposition des huttes par couples mariés, hommes célibataires et femmes non mariées, le premier groupe occupant une place intermédiaire entre les célibataires des deux sexes. Et à l'intérieur de l'habitation ils ont également soin de séparer ces derniers par des couples mariés. 
Cette classification selon le statut matrimonial ou l'état civil et le sexe n'équivaut pas à une simple répartition selon l'âge, comme nous la trouvons ailleurs, puisque même des veuves âgées occupent le côté de la hutte réservé aux femmes sans mari. Néanmoins, le facteur âge joue pourtant un certain rôle, le mariage dans les tribus primitives suivant généralement de près la puberté physiologique des filles et puisque aux îles Andaman aucun obstacle économique ne semble s'opposer à ce que les hommes se marient de bonne heure. En fait, l'importance que revêt dans la conscience de ces indigènes l'âge et ses corrélats, les statuts conjugal et paternel, appert clairement de l'abondance des termes qui s'y rapportent, ce qui permet de définir nettement tout individu de l'un ou l'autre sexe en mentionnant son âge et son état civil. Ainsi un enfant est désigné par telle appellation durant sa première année, par telles autres durant sa seconde, sa troisième ou sa quatrième année, alors que des termes spéciaux définissent la période qui va de quatre à dix ans et de onze à douze ans. Un homme qui est marié depuis plusieurs mois est distingué de celui qui n'a que quelques jours de mariage et celui qui deviendra père est différencié de celui qui l'est déjà; une nomenclature également subtile désigne les épouses et les mères. Cependant, parmi tous ces termes, les plus significatifs, socialement parlant, sont ceux qui se rapportent à l'initiation des garçons et des filles à la qualité de membres véritables de la tribu, et les cérémonies qui se célèbrent alors demandent à être spécialement étudiées.

Vers la onzième année, à peu près, garçons et filles sont soumis à une période de jeûnes durant laquelle il leur est défendu de manger de la tortue, du miel, du porc et d'autres plats savoureux. L'abstention de ces mets de luxe est considérée comme une preuve de renoncement de la part du néophyte. Cette période, qui ne prend fin que sur la décision du chef, est divisée en trois parties; chacune de ces divisions se termine par une cérémonie après laquelle est levée l'une des interdictions; les tabous de la tortue, du miel et du foie gras de porc sont donc successivement supprimés.

Lorsqu'un jeune garçon va être autorisé à rompre le tabou de la tortue, le chef fait cuire un grand morceau de graisse de tortue, la laisse refroidir et la verse sur la tête du novice, cependant que les assistants en frottent le corps de ce dernier; il ne lui est permis de se laver qu'à la fin du jour suivant. On lui donne alors à manger de la chair de tortue, puis on le conduit à sa hutte où on le fait rester assis, les jambes croisées et silencieux; un groupe d'amis lui apporte ce dont il a besoin et l'empêche par des chants de s'endormir. Il va entrer, croit-on, dans une période importante de sa vie où il aura des épreuves à surmonter; aussi sa mère et les femmes de sa famille font-elles entendre de grandes lamentations. Elles lui peignent le corps et ornent toute sa personne, après quoi le novice, tenant à la main des bouquets de feuilles semblables à des balais, se met à danser avec force, tandis que les femmes marquent la mesure et que les hommes regardent ou prennent part à sa danse. Quand il est épuisé, il s'arrête et se mêle à ses amis, ayant atteint ce qu'on pourrait appeler le premier degré de la majorité: Une cérémonie analogue élève les filles au même rang social.

Après cette cérémonie, les tabous secondaires, comme l'interdiction de manger du foie gras de porc, peuvent être supprimés par le chef sans autre formalité que le 
silence obligatoire du néophyte lorsqu'il rompt le jeûne pour la première fois. Il n'en est pas de même avec le miel dont on ne peut recommencer à manger qu'après une cérémonie particulière. Le chef présente au novice une large portion de ce mets, jusqu'alors tabou, et l'en oint; pour qu'il ne soit pas attaqué par les fourmis, il lui est cependant permis de se laver peu après. Il n'a pas d'autres prohibitions à observer que le silence. Le matin suivant, le jeune garçon, décoré de feuilles, entre dans la mer et éclabousse l'eau sur lui et sur les spectateurs, plongeant aussi, la tête en avant. Toute l'opération passe pour protéger magiquement contre les serpents. Les jeunes femmes y sont également soumises, mais seulement après la naissance de leur premier-né.

En général, un an après qu'a pris fin le tabou de la tortue, se place la cérémonie finale, supprimant l'interdiction relative au foie gras de porc. Les amis du novice organisent une chasse au cochon; pour un garçon on tue un verrat et pour une jeune femme, une truie. Le candidat reçoit à manger de ce mets jusqu'alors défendu, on verse sur lui la graisse fondue du cochon, puis il doit rester assis en silence et sans dormir. Le matin suivant, il exécute une danse comme la première fois.

Pour pouvoir établir des comparaisons, il nous faut étudier avec soin certains faits relatifs à ces cérémonies. Tout d'abord, il est évident que le commencement de l'initiation coïncide en substance avec la puberté. La maturité physiologique de la jeune fille est indiquée plus nettement par le fait qu'elle prend alors un « nom de fleur ", c'est-à-dire celui d'une fleur épanouie au moment de sa première menstruation; elle conserve cette appellation jusqu'à la naissance du premier enfant. Secondement, bien qu'il ne soit pas obligatoire pour les jeunes gens de passer par ces trois cérémonies avant d'être mariés, beaucoup retardent le mariage jusqu'à ce qu'ils aient traversé les trois phases, alors que d'autres se font un point d'honneur de célébrer leur initiation peu après leur union.

Si nous considérons la société andamane comme un tout, nous trouverons que ses membres se classent de différentes manières. Le groupement le plus apparent est celui en gens mariés et célibataires des deux sexes, puisqu'il correspond à une répartition spatiale du campement. A part cette particularité, il serait exagéré de parler d'une dichotomie sexuelle de la tribu. Bien que certains travaux tels que raser, chercher l'eau et le bois, incombent nettement aux femmes, alors que ce sont les hommes qui chassent, pêchent et construisent les bateaux, cette division du travail n'implique pas la ségrégation en castes distinctes qu'on rencontre en d'autres régions. D'autre part, les noms de fleurs que prennent les jeunes filles créent une démarcation bien nette entre celles qui ont atteint la puberté et les autres. L'abondance de désignations relatives au statut est l'indice de toute une classification de la communauté qui n'est pas moins réelle du fait qu'hommes ou femmes d'une même catégorie ne sont pas nécessairement unis par un but commun, mais ne font que porter une même appellation plus ou moins honorifique. 
La minutie dont font preuve à cet égard les Andaman nous fournit rétrospectivement une explication partielle plausible de la teknonymie, ainsi que l'a déjà suggéré Elsie Clews Parsons. Lorsque le statut d'un individu est estimé de façon différente selon qu'il a des enfants ou non, la désignation teknonymique est toute naturelle. La période de jeûne est sans doute en corrélation avec la désignation selon le statut. Nous avons vu qu'en substance elle coïncide avec l'adolescence, mais les subdivisions de la période d'initiation ne correspondent plus à un état physiologique.

En général, les Andaman sont moins rigoureusement organisés que beaucoup d'autres peuples; néanmoins, la segmentation de leurs communautés selon des principes définis qui n'ont rien à voir avec la parenté, est un fait indéniable.

Chapitre 10

\section{b. Australie.}

Retour à la table des matières

Alors que les Andaman présentent des groupes associationnels dans une société sans clans, l'Australie offre de multiples exemples de la coexistence des clans et des associations, ces dernières jouant un rôle pour le moins aussi important que les premiers. Le mode d'existence des Australiens a été décrit bien des fois dans des livres populaires et semi-populaires, nous n'en parlerons donc ici que sommairement. Plusieurs des particularités présentées par les Andaman caractérisent également les Australiens et, en s'ajoutant à certains autres facteurs tels que la gérontocratie et la petite estime en laquelle sont tenues les femmes, elles ont produit une division encore plus tranchée de la société en associations.

Tout d'abord la question des sexes prédomine nettement dans l'activité sociale des tribus australiennes. Le fait d'appartenir au sexe féminin ne détermine pas seulement l'activité économique et industrielle; il ne suffit pas non plus de dire que la condition de la femme en Australie est basse. Les femmes kirghiz, ainsi que nous l'avons déjà démontré, sont en pratique et en théorie subordonnées aux hommes; cependant elles n'en participent pas moins aux fêtes tribales sur un pied presque absolu d'égalité. Pour les Australiennes, l'activité publique masculine est un mystère défendu, voilé par des récits fabuleux ad mulierem. Il est vrai qu'elles partagent cette exclusion avec les garçons non initiés, mais, puisque chaque individu mâle a droit à l'initiation, nous avons affaire en somme à une véritable dichotomie sexuelle : les hommes se constituent en une société secrète dans laquelle aucune femme n'est admise. Ce fait doit être rapproché des particularités de l'affiliation clanique. La dichotomie sexuelle rompt nécessairement le lien unissant les membres des deux sexes d'un même clan; en groupant tous les hommes en association tribale, elle remplace la solidarité clanique par ce que Webster appelle « la solidarité sexuelle ». Un membre du clan du Casoar 
qui se retrouve constamment dans les cérémonies rituelles et dans les assemblées politiques avec les hommes de l'Aigle-Vautour, de la Chauve-Souris, de la Corneille et de la Grenouille, développe à leur endroit une solidarité de classe qui ne peut être ressentie dans de telles conditions par les hommes et les femmes appartenant au même clan du Casoar. Tous ces derniers doivent, il est vrai, s'abstenir totalement ou presque de consommer la chair de leur totem, mais les femmes en tant que femmes observent des restrictions alimentaires analogues. Selon Howitt, les Yualaroi défendent aux femmes de manger le casoar ou ses oeufs et, parmi les tribus du centre, aucune femme, de quelque clan qu'elle soit, ne peut toucher au vautour brun. Ainsi, même en matière alimentaire, le facteur sexuel s'oppose à celui du clan. Il n'est pas moins significatif d'appartenir à tel sexe que de faire partie de tel groupe totémique.

Cependant, alors que le sexe est socialement bien plus important en Australie que parmi les Andaman, l'âge et l'état civil jouent aussi leur rôle. Les Kariera et les Kurnai opèrent une ségrégation typique des hommes non mariés et du reste du campement, sans prendre de mesures analogues à l'égard des femmes célibataires. En général, on distingue aussi minutieusement les différentes phases sociales. Les Kurnai usent d'appellations différentes pour l'enfant en bas âge, le garçon de huit ou neuf ans, le jeune homme non initié vivant avec ses parents, le novice, le célibataire et le pater familias adulte. Ailleurs, les divers degrés de l'initiation sont différenciés avec soin. Notons encore une particularité significative : la suppression des tabous alimentaires pour les vieilles femmes.

Les cérémonies d'initiation des Australiens ressemblent à celles des Andaman en ce qu'elles sont célébrées à l'époque de la puberté; à tous autres égards, elles en diffèrent notablement. Chez les habitants des îles Andaman, chacun se soumet à l'épreuve des jeûnes partiels impliquant la disparition successive des tabous alimentaires, mais ces cérémonies ne sont pas des préliminaires indispensables au mariage. En Australie, par contre, personne ne doit prendre femme qui n'a pas passé par les rites prescrits; et, dans le centre du continent, il existe même pour les jeunes filles un rite de nubilité analogue. Parmi les Andaman, les cérémonies d'initiation ne sont pas conçues comme des mystères dont les femmes ne doivent rien savoir, ainsi que le font la plupart des tribus australiennes. En d'autres termes, l'initiation des Australiens est nettement un rite préparant le mariage et proclamant la suprématie de l'homme; la cérémonie de nubilité elle-même, dans les endroits où elle est observée, est dirigée par des hommes, en présence d'hommes, alors que les femmes sont presque toujours exclues des rites les plus importants de l'initiation des garçons. Enfin, l'initiation ellemême est de caractère tout différent. Il est des tribus comme celle des Kariera qui se contentent de marquer le néophyte de signes conventionnels, par exemple en lui enserrant les biceps d'un cordon. Mais, bien plus fréquemment, les Australiens admettent les garçons à la dignité d'hommes après les avoir soumis à une épreuve infiniment plus sévère; dans telle aire, on leur arrache une dent; dans telle autre, on pratique la circoncision et la subincision (opération supplémentaire de l'organe génital). Ailleurs encore les jeunes gens qui se préparent à être initiés doivent se retirer en un lieu solitaire où ils ne reçoivent qu'une nourriture insuffisante et supportent sans 
broncher les brimades des vieillards; en outre, on les oblige à couper et à transporter de gros troncs d'arbres, ou bien on les soumet à quelque autre travail pénible. Lorsqu'il a passé son examen avec succès, chaque novice reçoit des vieillards officiants un bâton sacré ( « bull-roarer ») qu'il lui faut cacher en un lieu secret et sûr. Bref, si, en ce qui regarde la forme, l'initiation australienne met durement à l'épreuve la maîtrise de soi de l'adolescent, dans son essence elle est aussi beaucoup plus solennelle que chez les Andaman. C'est un véritable cours propédeutique en sciences et en éthique tribales : on exhorte le néophyte à obéir implicitement aux enseignements des anciens et à observer le secret en présence des non-initiés sur les arcanes qui lui sont révélées; on lui apprend quels tabous alimentaires il doit observer, enfin il s'entraîne à gagner sa vie par lui-même.

Il est un détail de l'initiation australienne qui mérite une mention spéciale. Entre autres secrets, on révèle au néophyte l'origine de certain bourdonnement bizarre qui l'avait inquiété avant l'initiation et que les femmes et les enfants considèrent comme la voix de l'esprit présidant au rituel d'initiation. L'initié apprend alors que le bruit est simplement produit par le tournoiement en l'air du bull-roarer, bâton plat ou dalle de pierre attaché à une ficelle. Chose curieuse, on insiste surtout sur la nécessité de garder la véritable nature de l'objet ignorée des femmes; celles-ci ne doivent jamais le voir. Autrefois, il y avait même dans certaines tribus une règle stipulant que si un homme montrait un bull-roarer à une femme, tous deux devaient être punis de mort. On verra dans la suite pourquoi nous soulignons cette particularité. En quelques tribus qui restent une exception, le bull-roarer intervient dans l'initiation, mais on ne cherche pas à le cacher aux femmes.

L'initiation, tout en permettant au jeune homme d'accéder au mariage et à d'autres fonctions sociales, ne le met nullement sur un pied d'égalité avec les vieillards. Pendant bien des années encore, sa place dans la société restera modeste; il n'a pas à émettre son avis en politique et, même en matière alimentaire, il se passera encore longtemps avant qu'il soit libéré des dernières restrictions. C'est sur ces faits que Schurtz et avant lui Cunow ont insisté à bon droit comme démontrant que, outre l'organisation clanique, les Australiens sont divisés en unités sociales qui jouent un rôle tout aussi important dans la vie de l'individu. Chacun fait partie, de par sa naissance, de la phratrie masculine ou féminine, soit de la caste des privilégiés ou des incapables; chacun, au cours de son existence, passe par une série de degrés sociaux, exigeant un comportement spécial et fixé par les conventions. Chez les Warramunga, un homme faisant partie du clan du Chat sauvage est, dans la mesure où il s'agit des règlements du clan, libre de manger de la viande de casoar; cependant, il doit s'abstenir d'y toucher jusqu'à ce qu'il ait atteint la quarantaine. Quels que soient la phratrie, la famille et le clan dont il est membre, s'il est initié et célibataire, il vivra séparé de sa famille, de son clan ou de sa phratrie avec tous les autres hommes non mariés du campement. Ainsi, dans la vie quotidienne, l'action conjuguée du sexe et de l'âge s'avère plus importante que celle des groupes de parenté. 
Il est un trait de l'organisation australienne que j'ai à dessein omis de mentionner jusqu'ici. Sur une grande partie de ce continent règne ce qu'on appelle le système des classes (« sections » de Radcliffe-Brown), comprenant la variété de huit classes et celle de quatre classes. Il nous suffira d'étudier cette dernière. J'ai déjà parlé de l'existence de l'organisation bipartite en Australie. Les phratries se divisent fréquemment chacune en deux classes, ce qui porte à quatre le nombre de ces dernières dans la tribu. Jusqu'à récemment, les auteurs nous représentaient ces quatre classes comme des groupes matrimoniaux, réglant les mariages sur le principe suivant : choisissant pour exemple les Kariera patrilinéaires, nous désignons par A et B les phratries exogames, la première se subdivisant en classe 1 et 2 , la seconde en 3 et 4 , ces chiffres étant substitués à des noms indigènes phonétiquement compliqués. Selon la loi des Kariera, un homme de 1 ne peut pas épouser une femme de sa propre classe non plus que de 2, puisque ce sont des subdivisions de la même phratrie; mais il ne peut pas davantage se marier en 3. Il est obligé par les lois du système à, chercher sa femme dans une seule classe, qui est 4 . On se demandera, cependant, selon quelle méthode est déterminée la classe de chaque individu; c'est ici qu'intervient la partie la plus intéressante du système.

L'enfant d'un homme de la classe 1 doit appartenir à la phratrie de son père, la descendance étant, en effet, patriarcale chez les Kariera. Néanmoins, il ne peut pas faire partie de cette classe 1, mais entre dans la classe complémentaire 2. De même, lorsqu'un individu de 4 épouse une femme de 1, l'enfant sera de 3, puisque la phratrie mais non la classe doit être la même que celle du père. Si, au lieu des Kariera, nous avions choisi comme exemple quelque tribu matriarcale, le rapport aurait été le même; chaque enfant appartient alors à la même phratrie que sa mère, mais à la classe complémentaire. Particularité intéressante du système, le grand-père et le petit-fils se trouvent réunis dans la même classe. Chez les Kariera, un homme de 1 a dans la classe 2 non seulement ses enfants, mais aussi son père. Ainsi le père du père et le fils du fils appartiennent à une même unité.

J'ai dit plus haut que, jusqu'à une époque récente, les ethnologues s'en sont tenus à ce système matrimonial; à l'heure actuelle en effet, les enquêtes de Radcliffe-Brown nous indiquent que, alors que la division en quatre classes est formellement correcte, elle ne pénètre pas jusqu'à l'essence du Phénomène. Ainsi que les anciens ouvrages le démontraient déjà, quoiqu'un membre d'une classe donnée ne puisse se marier que dans l'une seulement des trois autres classes, il n'est pas permis à n'importe qui de 1 d'épouser un membre quelconque de 4; il existe encore d'autres restrictions. Radcliffe-Brown nous prouve qu'au cœur même du système nous trouvons simplement l'obligation pour un homme d'épouser la fille de son oncle maternel (ou une femme que la nomenclature indigène désigne du même terme), à l'exclusion de toute autre femme. Le système des quatre classes ne semble correspondre aux faits que parce que les conjoints possibles doivent appartenir à des phratries opposées et à une autre classe que leur père ou leur mère, selon la descendance. En réalité, le mariage n'obéit qu'à la consanguinité et n'est admis que pour des cousins croisés d'un type 
spécial. C'est pourquoi j'ai rangé les faits s'y rapportant sous la rubrique de l'union préférentielle.

Ce même auteur a expliqué la division encore plus complexe qu'observent certaines tribus australiennes : la société se divise en huit sections, chaque classe se scindant en deux. Il a prouvé que la plupart des peuplades de ce continent se conforment soit au modèle de quatre sections, soit à celui de huit et que, alors que le premier s'associe au mariage entre cousins croisés, les huit subdivisions impliquent toujours la prohibition de mariage entre cousins croisés du premier degré, tout en préconisant l'union entre les enfants de tels cousins, soit entre cousins croisés du second degré. Ici aussi cette règle matrimoniale est parfaitement compatible avec le système des huit sections : les conjoints possibles, relativement aux sections, sont précisément les enfants de cousins croisés dont nous avons défini la parenté à la fin de notre second chapitre.

L'interprétation de Radcliffe-Brown explique admirablement pourquoi les prétendues classes matrimoniales ne rendent pas compte de toutes les unions individuelles; celles-ci dépendent, en effet, du degré de parenté des cousins dit croisés. Cependant, si on leur enlève leurs fonctions matrimoniales, il n'est pas facile de comprendre quelles peuvent bien être les attributions de ces classes. Qu'elles représentent un type quelconque d'unité sociale, le fait est attesté par une douzaine d'observateurs. Elles ont souvent des appellations définies, alors que les phratries en sont dépourvues. Quelques savants ont émis la supposition que ce ne sont que des moitiés de phratries, mais cette hypothèse n'est guère défendable. Si elle était conforme à la réalité, ces classes observeraient la même règle de descendance au lieu de prescrire cette curieuse voie indirecte.

Quoique je ne sache pas de théorie expliquant de façon satisfaisante les classes australiennes, la contribution de Cunow doit être citée comme un apport intéressant au nombre bien restreint d'idées originales relatives à la théorie de l'organisation sociale. Cunow suppose que ces classes sont, pour ainsi dire, des strates d'âge; la règle stipule que seuls les membres d'une même strate sont conjoints potentiels et les phratries auraient été à l'origine deux groupes locaux distincts, mais se mariant entre eux. Or, puisqu'un enfant ne peut pas appartenir au même groupe d'âge que ses parents, on comprend que la règle de descendance par clan présente ce caractère d'anomalie que nous avons relevé : l'enfant est de la même phratrie que son père ou sa mère, mais non de la même classe, car le système de classes est au fond précisément une méthode différenciant les âges.

La théorie de Cunow se heurte à plusieurs obstacles. Si ces classes étaient bien des degrés d'âge, l'affiliation de classe devrait naturellement varier avec l'âge; cependant, en fait, chaque indigène australien fait partie de la même classe de sa naissance jusqu'à sa mort. Cunow suppose que cette affiliation a été rendue immuable afin d'empêcher qu'un homme épouse deux femmes de différents degrés. Selon lui, il y aurait trois degrés : celui de l'enfant, de l'initié et de l'homme âgé, c'est-à-dire celui 
qui a un enfant dans le groupe des initiés. Lorsque Cunow insiste sur le fait que seuls des individus du même âge ont le droit de se marier entre eux, il veut dire que le mari et la femme doivent appartenir tous deux soit au degré des initiés, soit à celui des adultes. Supposons alors qu'après dix ou quinze ans de mariage, un individu prenne une seconde épouse qui est du même degré que lui et sa première femme, mais qui continue à rester dans le même degré, alors que le couple aura déjà passé à la subdivision supérieure. Elle sera donc du même degré que le fils aîné de la première femme. Afin d'éviter cet état de choses, il était nécessaire, selon Cunow, de rendre les affiliations de classe permanentes, ainsi que les désignations claniques. On ne saurait nier que le raisonnement est ingénieux, mais rien ne nous indique que ce soit là plus qu'une simple conjecture.

En outre, puisqu'il n'y a que quatre désignations de classes au lieu des six demandées par l'organisation tripartite de chaque phratrie, Cunow se croit fondé à supposer que les membres d'une génération sur deux qui portent un même nom de classe, n'appartiennent pas réellement à la même classe, mais à des classes distinctes, que, pour plus de commodité, on désigne d'une appellation identique. Encore une fois, l'interprétation nous semble arbitraire. Cunow lui-même attire notre attention sur le fait que, dans beaucoup de nomenclatures de parenté australiennes, le grand-père et le petit-fils sont désignés par un seul terme qui s'emploie réciproquement. C'est pour lui un phénomène incompréhensible qui pourtant s'expliquerait par le fait que grand-père et petit-fils portent le nom de la même classe. Cependant, il est possible de recourir à une autre interprétation. Grands-parents et petits-enfants peuvent être désignés de la même appellation parce que, précisément, selon le système des quatre classes, ils sont en fait ce qu'ils paraissent être : membres d'une seule et même classe; en ce cas, la classe ne coïnciderait pas avec le degré d'âge. Néanmoins, en dépit de ses déficiences, la théorie de Cunow fournit une explication si simple des modalités énigmatiques de l'affiliation de classe que nous nous en tenons à ses lignes essentielles, espérant que les difficultés qu'elle soulève s'aplaniront quelque jour.

Résumons, en dehors de toute hypothèse, l'état des choses en Australie. Nous avons trouvé que, dans la plupart des tribus, chaque individu est simultanément membre de plusieurs unités sociales. Il se rattache à un sexe, à une phratrie, à un clan totémique et à une classe auxquels il est affilié de façon permanente. Il reçoit aussi un état civil qu'il modifie plus tard lorsque, après une cérémonie spéciale, il passe à l'état d'adulte puis, par des degrés moins perceptibles, à celui d'ancien de la tribu. A toutes ces périodes de sa vie, ses devoirs et ses privilèges dépendent, tout autant que de sa parenté, des associations auxquelles il se rattache.

Chapitre 10

\section{c. Massaii.}


Alors que chez les Australiens, clans et associations sont d'importance à peu près égale, chez les Massaï les premiers s'effacent indiscutablement, sans qu'il soit possible, toutefois, de les ignorer. Autrefois c'était d'après les clans que se concluaient les mariages et maintenant encore les fonctions religieuses et les marques de bétail se rattachent aux groupes de parenté restreints ou étendus. En outre, les chefs et les hommes-médecine se recrutent exclusivement dans la section supérieure d'un certain clan. Néanmoins, les attributions du clan sont peu nombreuses en comparaison de celles que présente la qualité de membre d'autres unités.

Tout d'abord, il existe une ségrégation spéciale des hommes non mariés et de leurs maîtresses, les filles impubères, du reste de la communauté, ségrégation qui n'offre aucune analogie avec celle des îles Andaman où les célibataires des deux sexes sont séparés par des couples mariés.

Secondement, hommes et femmes sont nettement groupés d'après leur statut. Ainsi que chez les Australiens, chaque garçon est soumis à une épreuve de puberté (circoncision) après laquelle il atteint au grade de guerrier. Durant la période d'initiation et jusqu'à ce que la plaie soit cicatrisée, il ne passe plus pour un garçon mais pour un néophyte (littéralement " cloîtré ») et, pendant les deux années qui suivent, il est appelé apprenti (« le rasé »). Jusqu'à 28 ou 30 ans, c'est un véritable guerrier; après quoi, il se marie, quitte le kraal des célibataires et revêt pour le restant de ses jours la dignité d'ancien. L'initiation et l'apprentissage n'étant évidemment que des périodes préparatoires, la vie des hommes se divise donc en trois phases : l'enfant, le guerrier et l'homme mûr. Dans cette peuplade belliqueuse, c'est la seconde de ces phases qui joue le premier rôle. Les filles, après leur première menstruation, sont soumises à une opération analogue à la circoncision des garçons, la clitoridectomie ou excision, et sont également traitées de novices jusqu'à cicatrisation de la plaie. Elles occupent ensuite un rang à part jusqu'à la ménopause, après quoi leur statut est désigné par un nouveau terme qu'elles échangeront contre un autre quand leurs cheveux blanchiront. Les individus non apparentés des deux sexes s'adressent les uns aux autres par des désignations conformes à leur statut.

Ces phases s'accompagnent de coutumes bien définies. Les femmes mariées sont distinguées des jeunes filles par des colliers et des anneaux de fer. Dépouillées de ceux-ci, jamais les épouses n'oseraient se présenter devant leurs maris. Elles sont aussi reconnaissables à leurs longs vêtements. Les garçons et les filles se mettent dans les oreilles des tampons de bois de plus en plus grands, tandis que les guerriers et les anciens portent des anneaux et des bracelets faits de chaînes. Après la circoncision, les jeunes gens s'arment de l'épée, de la lance, de la massue et du bouclier qui marquent leur rang, arborant aussi un bonnet particulier, une coiffure en plumes d'autruche, une cape, des anneaux de chevilles, des ornements de bras, une peau de veau et une peau de chèvre autour de la taille. Ils se tressent les cheveux, se nourrissent uniquement de viande, de lait et de sang, et s'abstiennent de tous intoxicants. 
Avant que la plaie causée par la circoncision ne soit guérie, le novice doit imiter les femmes en portant leurs vêtements et leurs boucles d'oreilles. S'il a soutenu l'épreuve sans broncher, il est autorisé à tirer sur de petits oiseaux et à porter leurs dépouilles sur sa tête.

outre le fractionnement en habitants du kraal des guerriers et autres individus, et la triple division de la population masculine selon le statut, il existe encore d'autres groupements. L'organisation tripartite des hommes réunit dans un seul degré le nouveau marié de trente ans et l'octogénaire. Il s'est donc produit dans cette phase une division importante : les pères d'enfants circoncis ont en privilège le port de certaines boucles d'oreilles. Cependant, il ne semble pas que les anciens de cette catégorie soient unis par un sentiment quelconque de solidarité. Quelques-uns d'entre eux se distinguent de manière encore plus spéciale : les propriétaires de grands troupeaux qui ont beaucoup d'enfants exhibent un anneau de bras en ivoire ou une corne de buffle, insigne de leur opulence. Toutefois, ce critère mal défini n'a pas réussi à produire un groupe social déterminé et n'est intéressant que comme preuve de la variété des types classificatoires auxquels les indigènes ont recours. D'autre part, il existe chez les Massaï une division basée sur la période d'initiation qui est de toute importance. Le rite de la circoncision ne fait pas que séparer la catégorie des garçons de celle des guerriers; elle produit une classification encore plus subtile de la société.

Tous les garçons qui ont été circoncis pendant telle période de quatre ans, appartiennent au même « âge ». Il y succède un laps de temps de trois ans et demi au cours duquel aucune initiation n'a lieu. Puis revient un nouveau quadrennium. où l'on recommence à circoncire les garçons. Partant de quelque moment arbitrairement choisi ou du moins inconnu, les Massaï désignent les individus circoncis durant tel quadrennium comme appartenant à la « circoncision de la main droite », les suivants faisant partie de celle « de la main gauche ». Ces classes d'âge ne relèvent donc point de la gauche ou de la droite relativement les unes aux autres, mais de façon absolue et par rapport à un certain point de départ. Deux âges, l'un de gauche, l'autre de droite, constituent ce qu'ils appellent une " génération ». Il convient de décrire en détail la manière dont une de ces classes d'âge remplace celle qui la précède en tant que représentante de la catégorie des guerriers et comment les deux « âges » complémentaires sont soudés en une seule génération.

Dès que prennent fin initiation et convalescence, les novices se trouvent occuper une situation particulière par rapport aux véritables guerriers. Afin de faire figure de vrais braves, ils doivent tout d'abord trouver un nom spécial pour leur classe, une décoration nouvelle sur leurs boucliers et construire un kraal séparé. La première condition n'offre pas de difficulté puisque c'est le chef de la tribu qui, contre compensation d'un troupeau de bétail, choisit la désignation. Mais l'ornement des boucliers n'est pas aussi facile à découvrir. Les principaux des anciens font choix dans ce but de figures noires; néanmoins, c'est surtout la coloration rouge du bouclier qui caractérise le grade de guerrier. Aussitôt que les nouvelles recrues ont essayé de peindre leurs boucliers en rouge et se sont mis à élever leur kraal, les guerriers fondent sur eux, 
attaquent leur installation et, si possible, font disparaître les peintures rouges. Si les aînés sont victorieux, les novices doivent attendre que revienne une occasion favorable et s'entraîner dans l'intervalle par des expéditions contre les tribus hostiles. S'ils font preuve de bravoure au cours de ces dernières, les guerriers condescendent à les reconnaître comme leurs pairs; en cas contraire, les novices en sont réduits à soumettre leurs adversaires par la force. Une fois qu'ils y ont enfin réussi, il y a donc deux kraals distincts pour les célibataires, bien qu'ils se réunissent pour les entreprises guerrières. Mais, du point de vue indigène, c'est là un état d'anomalie transitoire. Bientôt, les plus âgés des guerriers se décident à partir; ils s'en vont successivement après avoir fêté leur départ, se marient et fondent un ménage individuel. Lorsque tous les membres d'une même classe d'âge se sont mariés et ont quitté le kraal, ils reçoivent collectivement le titre d'anciens et laissent leurs successeurs seuls occupants du campement des guerriers.

Ce qu'il y a de plus significatif dans tous ces phénomènes, c'est que l'initiation simultanée crée des liens plus forts que ceux du statut identique. Quoique deux classes d'âge puissent temporairement se partager la qualité de célibataire, elles restent des unités distinctes. De même, le groupe des hommes récemment mariés ne se confond nullement avec celui des anciens. Le seul lien étroit, qui se forme du reste beaucoup plus tard, est celui qui unit les classes de « gauche » et de « droite » dont l'ensemble, formant une génération, reçoit une désignation commune et adopte une marque de flèche distinctive. Mais, avant et après cette réunion, la classe d'âge garde son nom et son individualité et règle l'activité de ses membres. C'est surtout un agent régulateur des relations sexuelles. Les jeunes filles qui ont été initiées durant telle période de quatre ans sont considérées comme appartenant à la même classe d'âge que les garçons circoncis en même temps. Aucun Massaï ne peut avoir de relations avec une femme de la classe de son père; par contre, il n'est pas défendu d'avoir des rapports avec une femme de sa propre classe; et les veuves ou les divorcées peuvent vivre avec les camarades de leur mari. Un visiteur d'un autre district est admis d'emblée chez un membre de la même classe; celui-ci quitte alors sa hutte et y laisse sa femme à son visiteur. Il y est du reste forcé, car un hôte peu obligeant encourt la malédiction de ses camarades. Une femme que son mari bat, ira chercher refuge auprès des contemporains de celui-ci; elle jouira ensuite de la tranquillité domestique, son époux ne récidivant pas de peur d'être maudit par sa classe.

Nous appuyant sur tous ces faits, nous pouvons affirmer que le groupe des anciens ne constitue pas une unité nettement formée, mais qu'à l'intérieur de ce groupe, tous les sentiments de solidarité sociale se rattachent aux associations plus petites que nous venons de voir, la " génération » et plus particulièrement la classe d'âge. Comparons en manière de conclusion les associations des Massai avec leur organisation clanique. Notre affirmation du début sur la prépondérance relative des premières paraît démontrée. Même une particularité aussi typiquement clanique que le règlement des mariages est ici une fonction se rattachant surtout à la classe d'âge. Si on éliminait des communautés Massaï la division en clans, nous nous trouverions encore en présence d'un vaste ensemble de relations sociales; mais, privée du kraal des célibataires, des 
grades initiatoires et des classes d'âge, la société des Massaï prendrait un aspect dénudé et tristement monotone.

Chapitre 10

\section{d. Îles Banks.}

$\underline{\text { Retour à la table des matières }}$

Aux îles Banks, comme parmi les Massaï, la structure clanique disparaît devant les unités associationnelles, mais ces dernières sont de nature toute différente dans les deux régions. Les Mélanésiens nous font même connaître des principes d'association tout nouveaux. Néanmoins, une des particularités qui ont attiré notre attention en Australie, reparaît aux îles Banks de manière encore plus marquée. La scission de la société en phratries sexuelles atteint ici son maximum d'intensité. Non seulement les femmes sont exclues des solennités publiques, mais même dans la vie quotidienne il se produit une dichotomie presque absolue. Les hommes ne mangent ni ne dorment avec leurs femmes mais dans un lieu à part où les jeunes garçons essaient de se faire admettre le plus tôt possible. Comme en Australie, les hommes constituent donc une sorte d'association tribale secrète. Il existe pourtant une différence fort importante : l'admission n'a rien à voir avec la puberté, mais a lieu à tout âge; elle ne dépend pas d'une épreuve impliquant quelque mutilation personnelle ou une mortification physique, mais du paiement d'un droit d'entrée. En outre, la société des hommes ne forme pas un groupe compact où les vieillards ne se différencient des initiés que par des degrés plus ou moins imperceptibles; au îles Banks elle est constituée par une série de divisions hiérarchiquement étagées.

La manifestation de cette exclusivité masculine est le club du village, un lieu où les hommes mangent et se reposent de jour, et viennent dormir la nuit. Cette maison est divisée en un certain nombre de compartiments d'importance variable dont chacun a son foyer autour duquel se réunissent les membres d'une même catégorie. Ne pas se joindre à son groupe équivaut à ne compter pour rien dans l'estime sociale; c'est pourquoi, bien que l'admission puisse être retardée, elle a lieu tôt ou tard. "Si un homme n'est pas admis au Sukwe (club), il doit manger avec les femmes et excite par là à tel point la pitié d'un ami que celui-ci se décide à se faire son introducteur, bien qu'il lui en coûte de ce fait une forte somme d'argent. » Parmi les gens du commun, l'introducteur, qui est souvent l'oncle maternel du candidat, prend sur lui tous les frais; dans les couches sociales plus élevées, les dépenses sont à la charge du novice, de ses parents et de ses amis. L'acte d'initiation est constitué par un repas cérémoniel auquel prennent part les nouveaux condisciples du candidat, mais tous les membres du club, quel que soit leur rang, semblent tirer profit de ces paiements. 
Puisqu'il est impossible d'être respecté par la communauté si l'on n'est affilié au club, l'accroissement de prestige sera donc en proportion directe de l'avancement dans la société des hommes, ce qui exige des sacrifices pécuniaires de ]plus en plus considérables. Il est donc tout à fait exceptionnel qu'un individu se hisse à l'échelon le plus élevé où il devient à la fois chef et héros de légende. Il est alors en mesure de récupérer, et au-delà, la fortune qu'il a consacrée à parvenir à cette dignité suprême, en exigeant des contributions exorbitantes de ceux qui après lui aspirent aux honneurs. La plupart ne rêvent pas pareille distinction. Bien qu'ils soient rentrés enfants dans le club, ils s'estiment heureux d'avoir atteint les degrés moyens de l'organisation. Toutefois, il est vrai, bien que les divisions soient nettement localisées à l'intérieur du club où chacun occupe un rang bien défini, elles offrent en théorie la possibilité de s'élever à un niveau élevé dans ladite société. Mais, en pratique, les sommes à payer sont beaucoup trop considérables pour que la théorie puisse devenir réalité. Tout ce qu'on peut essayer, c'est d'entrer gratuitement dans quelques-uns des degrés les plus bas; ces derniers sont par suite tombés en désuétude dans quelques villages. En outre, même en théorie, cette possibilité est limitée : pour pouvoir continuer à progresser après avoir dépassé un certain point, un homme doit appartenir à une autre organisation de type apparenté bien que distinct, que nous étudierons plus loin.

Nous voyons donc chez les habitants des îles Banks le club s'associer à des droits de propriété. Seul l'individu fortuné peut atteindre les degrés supérieurs et acquérir par là du prestige. Cependant, dans la conception des indigènes, il ne s'agit pas d'accumuler en avare ses richesses, mais au contraire de faire preuve de sa noblesse en affectant de mépriser ses biens. Ainsi les plus hauts dignitaires du club peuvent encore accroître leur renom en déployant leur générosité et en dépensant sans compter à l'occasion de certaines fêtes; s'ils se montraient tant soit peu chiches en de telles circonstances, toute leur influence s'évanouirait.

Les divers degrés du club étaient autrefois différenciés avec une telle rigueur que c'était encourir une mort certaine que de pénétrer dans un compartiment plus élevé que le sien. Pourtant ces phases sont douées de peu d'individualité. Elles ont des masques ou des chapeaux distinctifs; en quelques-unes on fait usage de certains couteaux et c'est dans les degrés supérieurs seulement qu'il est permis de boire le stimulant indigène, le kava. Mais il est difficile de spécifier en quoi diffèrent les fonctions et les privilèges de degrés voisins. L'idée essentielle semble avoir été tout simplement la suivante : en faisant un sacrifice supplémentaire en vue d'une nouvelle initiation, l'individu avait droit à de plus grands honneurs, droit qui se manifestait visiblement par l'accès à un compartiment spatialement distinct.

Les particularités significatives du système des îles Banks prennent un relief plus accusé si nous les comparons à celui des Massaï. En Afrique orientale, le principe est purement démocratique; en Mélanésie, il devient ploutocratique. Tout Massaï est tenu de passer de l'état de garçon à celui de célibataire, puis d'ancien, la différenciation des classes d'âge parmi les anciens n'entraînant nulle infériorité ou supériorité de caste. Si les hommes d'âge, riches et prolifiques, arborent un signe extérieur, les conséquences 
pratiques de cette distinction sont négligeables. Dans ce système démocratique, c'est collectivement que les contemporains passent d'un statut à l'autre. Aux îles Banks, l'accroissement de la finance d'entrée, qui devient bientôt prohibitive, empêche forcément que les divers degrés soient franchis avec une égale rapidité par des individus du même âge. A chaque phase, sauf à la plus basse peut-être, nous rencontrons des âges et des situations fort variés. Autour du même foyer et traités momentanément de pairs par l'opinion publique, se réunissent des individus d'âge mûr et peu entreprenants que la gêne empêchera de dépasser le niveau où ils viennent à grandpeine de se hisser et des jeunes gens pleins d'espoir qui, grâce à leurs relations de famille, jouissent d'un traitement de faveur et offrent des chances sérieuses d'atteindre plus tard au pinacle des aspirations indigènes.

Jusqu'ici nous n'avons décrit qu'une seule phase des associations compliquées des îles Banks. Elles sont complétées par tout un ensemble de sociétés secrètes, « sociétés d'esprits » comme les appellent les insulaires; la petite île de Motta n'en a pas moins de soixante-dix-sept. Au contraire du club, le lieu de réunion de ces " sociétés d'esprits », que ce soit une hutte ou simplement une clairière, n'est jamais situé dans le village, mais isolé dans la brousse, et le sentier qui y conduit reste tabou à tous les non-membres. En dépit des contributions précieuses de Codrington et de Rivers, il n'est pas encore possible de se faire une idée nette de ces organisations si complexes, et nous ne ferons dans ce chapitre qu'esquisser leurs particularités les plus certaines.

Les organisations secrètes sont de caractère fort varié, mais, en général, elles peuvent passer pour des compléments de clubs. Elles présentent un trait commun entre elles et avec le club des hommes : l'admission dépend de l'initiation et du paiement du droit d'entrée. Quelques-unes de ces sociétés admettent les garçons qui ne font pas encore partie du club, alors qu'en d'autres la chose serait impossible. Dans certaines organisations, plusieurs individus entrent à la fois et paient pour leur initiation une somme globale, mais les sociétés les plus importantes ne prévoient pas de telles dispositions.

Presque toutes ces sociétés ont des masques distinctifs ou des objets qu'on porte à la main. On ne les montre que de loin au non-initiés lorsque, en certaines occasions solennelles, les membres font une procession dans l'île. Le reste du temps, il n'est permis sous aucun prétexte à un non-membre, et surtout à une femme, de voir et d'approcher ces objets sacrés. La plupart des sociétés possèdent d'autres emblèmes, de nature totalement différente, qui rendent la qualité de membre fort désirable : ce sont des feuilles de croton. Lorsqu'un membre les plante dans le sol, elles protègent ses biens contre les entreprises de ceux de sa tribu qui n'appartiennent pas à la confrérie. Quiconque transgresse ce tabou doit livrer un cochon à la société qui a subi l'outrage, et une amende identique est exigée de qui coupe ou utilise l'espèce particulière de croton qui sert d'emblème à l'organisation. Puisque les femmes ne sont pas admises dans les confréries, elles ne doivent toucher à aucune de ces plantes. Entrer dans ces organisations équivaut donc, dans une certaine mesure, à prendre une police d'assurance contre le vol. Étant donné qu'on n'est protégé que contre les non-membres, les plus 
petites organisations sont, de ce point de vue, les plus efficaces, ce qui entraîne des conséquences amusantes. Si l'une d'elles devient populaire par sa valeur protectrice, à mesure que le nombre de ses membres s'accroît, elle perd automatiquement de son efficacité, baisse par suite dans l'opinion publique, voit diminuer ses effectifs et redevient bientôt haut cotée. Certaines confréries ne sont pas sujettes à de telles fluctuations parce qu'on en recherche l'admission pour des raisons étrangères à la protection des biens. Par exemple, il est indispensable de faire partie de l'organisation Tamate liwoa pour pouvoir accéder aux degrés supérieurs du club.

Toutes ces sociétés secrètes présentent un trait typique : le désir de terroriser les non-initiés. Voici, par exemple, un homme qui se noircit la face et le corps jusqu'à devenir méconnaissable et qui sort, un bâton dans une main, une massue dans l'autre. Tout en faisant tournoyer continuellement le premier de façon qu'on ne le voie pas distinctement, il frappe de sa massue ceux qui ne s'écartent pas de son chemin. A l'occasion aussi, une bande organisée de membres de la même confrérie détruira sans motif valable les biens de quelque individu malchanceux ou l'en dépouillera. Lorsqu'un candidat est admis à entrer dans la Tamate liwoa, il est nécessaire qu'on renverse une maison choisie par le père du novice. Les membres de la société, partiellement déguisés, se précipitent au dehors, effraient les femmes et les non-initiés et détruisent l'habitation désignée. Lors du passage à un degré supérieur, selon le rituel d'admission, non seulement le candidat, mais encore tout individu que rencontre la confrérie et qui ne jouit pas d'un rang élevé dans le club, doit être battu.

Certaines formalités relatives à l'entrée dans la Tamate liwoa rappellent l'initiation tribale en Australie. L'un des secrets révélés à celui qui est admis dans une confrérie mélanésienne est la manière de produire un son particulier en frottant un bâton sur une pierre. Quoique les détails ne soient pas les mêmes, nous pensons involontairement à l'attitude des Australiens à l'égard du bull-roarer. Bien qu'aucune mutilation ne soit infligée, le novice doit se soumettre à toute une série d'épreuves durant une période de cent jours. On vient jeter sa nourriture dans le feu, lui briser ses couteaux et on le met à l'amende s'il émet une plainte; des tâches difficiles lui sont imposées et on le reçoit mal lorsqu'il vient présenter le fruit de son labeur. Ici encore, les analogies sont générales plutôt que spécifiques, mais elles nous offrent un exemple significatif des notions communément sous-jacentes à l'admission rituelle dans une association, dans les basses couches de la civilisation.

Si nous voulons maintenant récapituler la situation d'un habitant des îles Banks, en nous en tenant à ses diverses relations sociales, nous serons surpris de l'abondance de ces dernières. Nous avons déjà fait suffisamment ressortir l'importance du sexe. L'existence de la double organisation implique une nouvelle affiliation. Tout individu appartient, de par sa naissance, à une phratrie exogame et, les phratries étant subdivisées en sections observant des tabous spéciaux il fait partie automatiquement d'un de ces derniers groupes. Tôt ou tard, il entre dans le club des hommes, passant successivement par neuf ou dix peut-être de ses degrés. En même temps, il est probablement membre d'une demi-douzaine de confréries secrètes, soit afin de sauvegarder ses 
biens, soit pour accroître son prestige social. Il devient dès lors superflu de prouver que réduire leur organisation sociale à des divisions claniques serait faire disparaître ce qui constitue aux îles Banks le cachet particulier de la société.

Chapitre 10

\section{e. Indiens Pueblo.}

\section{$\underline{\text { Retour à la table des matières }}$}

L'abondance des organisations cérémonielles chez les Indiens Pueblo fait de leur société un véritable labyrinthe. par bonheur, depuis la publication du guide de Kroeber, après les études approfondies d'Elsie Clews Parsons sur toute la région et le travail intensif de Bunzel chez les Zuñi, il y a quelque espoir que, après bien des courses vaines dans ce dédale, on revienne à la lumière du jour.

Les corporations religieuses des Zuñi peuvent se ranger sous deux rubriques. D'une part, il existe une société du Danseur masqué comprenant obligatoirement tous les hommes de la tribu, à l'exclusion des femmes; ensuite, nous trouvons une série de confréries entre lesquelles les Zuñi ont le choix et qui sont ouvertes aux deux sexes. Le but principal de la société du Danseur masqué est la production de la Pluie par les prêtres de la pluie. Quant aux confréries, elles guérissent les maladies et font des démonstrations de puissance magique.

Il semble, à en juger d'après la constitution de la société du Danseur masqué, que nous devions la considérer comme l'équivalent du club tribal en d'autres régions. Mais, quoiqu'elle ait à sa tête un chef unique, ce n'est pas à proprement parier une organisation d'individus isolés, comme en Australie, mais une série de six unités, chacune se rattachant à un local cérémoniel distinct. Ces six subdivisions symbolisent les quatre points cardinaux, plus le zénith et le nadir. Ces locaux séparés nous rappelleraient les groupements mélanésiens si le système des degrés ne faisait défaut aux Indiens du sud-ouest. Les groupes des Zuñi sont des unités sociales coordonnées. C'est à la naissance que se décide de quelle unité l'enfant fera partie; celui-ci se rattache, en effet, à l'unité du mari de la sage-femme qui est la première à le toucher. C'est cet homme qui fait office de parrain à la première initiation involontaire de l'enfant, puis lors de la véritable qui a lieu vers douze ou treize ans. A la seconde initiation, l'enfant est rudement fouetté par des membres de la société personnifiant des dieux. Chaque novice, car on en admet plusieurs à la fois, reçoit un masque de faiseur de pluie qui devient sa propriété personnelle et qu'à sa mort en enterrera avec

lui. C'est alors seulement que les garçons apprennent que les prétendus dieux, une fois leurs masques déposés, ne sont que des hommes. Ces derniers remettent aux néo- 
phytes leurs propres masques et fouets et les jeunes garçons fustigent à leur tour ceux qui les avaient châtiés. Puis les aînés se recoiffent à nouveau de leurs masques et on menace les novices, s'ils divulguent les mystères de l'initiation, de leur couper le cou avec un couteau de pierre. Il arrive parfois, mais très rarement, qu'un homme préfère plus tard s'affilier à une autre unité, ce qui ne l'empêchera pas de retourner à son gré au local de son enfance. En outre, un individu convaincu d'adultère avec la femme d'un membre du même groupe peut être expulsé et obligé de se faire admettre par quelque autre division; cependant, sauf dans les cas extrêmes, on ne se résout qu'à contrecœur à l'expulsion.

Étant donné le degré de développement atteint par le système clanique des Indiens Pueblo, on peut se demander si et dans quelle mesure l'affiliation à tel clan affecte la qualité de membre des diverses unités cérémonielles. A en juger par la manière dont on entre dans l'une de celles-ci, il est évident qu'elles n'ont en général rien à voir avec le clan. Cependant, certaines charges dans le rituel du Danseur masqué se rattachent plus ou moins étroitement au clan. Ainsi le directeur du club appartient invariablement au clan du Cerf; certains masques sont confiés à la garde d'un homme de l'Aigle et, à la fin de la cérémonie célébrée tous les quatre ans, c'est un homme de la Grenouille qui fait entendre les chants de pluie. Toutefois cette règle souffre de nombreuses exceptions : il arrive souvent que la fonction cérémonielle alterne entre un membre de tel clan et son fils, lequel, en vertu de la descendance matriarcale et de la loi exogamique, fait partie de n'importe quel clan sauf celui de son père. Les prêtres de la pluie du club tribal se rattachent en théorie à des clans déterminés; en pratique., il se produit dans le clergé les mêmes dérogations à la règle. Un prêtre de haut rang peut avoir pour successeur et adjoints ses fils qui appartiennent obligatoirement à un autre clan. Quelquefois ce sont ses frères ou les fils de sa sœur, donc des ressortissants du même clan; d'autres fois encore, ce ne sont nullement des parents. Par ailleurs, il est des fétiches que seule une certaine catégorie de prêtres peut manipuler; on les conserve dans des maisons spéciales et ils se rattachent nettement aux unités claniques.

En général donc, les membres du club ne sont pas groupés selon l'affiliation clanique; les relations de cette institution avec le principe du clan n'apparaissent que lorsqu'il s'agit des charges cérémonielles du club et surtout en ce qui concerne les prêtres et leurs fétiches.

Les liens entre la confrérie et l'organisation clanique sont bien moins apparents, ainsi que nous allons tout de suite nous en rendre compte d'après le mode d'entrée. C'est d'ordinaire sur la base d'une guérison effectuée par la confrérie qu'on est admis dans celle-ci; le patient n'est pas obligé d'y entrer s'il paie une compensation adéquate au guérisseur, mais en général c'est la solution qu'il préfère. Dans une confrérie typique, trente-quatre membres sur quarante-deux sont entrés de cette manière. Il existe une autre méthode, c'est l'initiation forcée qu'on impose à l'individu qui s'est trouvé par hasard assister à une session exécutive de la confrérie. A part une ou deux 
exceptions, il est possible à tout membre d'une confrérie de passer dans n'importe quelle autre; dans ce cas, une cérémonie de pure formalité remplace la véritable initiation. L'indépendance vis-à-vis du clan, discernable au mode d'admission, se retrouve également dans les charges rituelles; dans une importante confrérie, Kroeber cite une seule fonction qui ne peut être remplie que par les membres de tel club.

Par bonheur, Kroeber n'a pas limité ses études aux Zuñi; il nous présente également une analyse des confréries chez les Hopi. Celles-ci présentent une importance théorique toute spéciale. On considérait, en effet, il y a quelque temps, ces confréries comme des prolongations naturelles du clan, comme représentant dans leur essence l'aspect rituel du concept clanique. Cette opinion semble s'appuyer sur le fait que les confréries des Hopi portent fréquemment le même nom que les clans et que les philosophes indigènes ont attribué la fondation de la confrérie à son homonyme ou à quelque autre clan déterminé. En dressant un tableau de toutes les informations disponibles relatives à l'affiliation clanique des confréries Hopi, Kroeber prouve de façon concluante que, dans la plupart des cas, le prétendu lien est inexistant. Sur trente-cinq membres de la confrérie du Serpent, sept seulement appartiennent au clan du même nom; sur dix membres de la confrérie de l'Antilope, trois font partie du clan de la Corne; on ne trouve pas plus de six ressortissants du clan du Lapin dans la confrérie prétendue y correspondre. Mes observations personnelles sur le terrain corroborent les conclusions de Kroeber avec la réserve suivante : la charge rituelle se transmet en général à l'intérieur du clan. Ainsi, dans le village de Mishongnovi, la danse du Serpent est exécutée par des ressortissants des clans du Maïs, du Perroquet, de l'Ours, du Blaireau, de l'Aigle, du Nuage et du Jeune Vautour, mais le clan du Lézard, qui représente les clans du Serpent d'autres villages, assume la direction de la danse. Le mode d'admission dans la confrérie du Serpent est identique à celui qui est de règle parmi les Zuñi pour faire partie de ces confréries; un individu qui a été guéri par un homme du Serpent entre dans l'association et se trouve dispensé par là de payer des honoraires au guérisseur. Il est évident qu'on n'attache que peu d'importance au fait que les noms coïncident. Dans le village de Shipaulovi, il n'existe pas de clan du Serpent; la direction de la confrérie du Serpent revient à un homme de l'Ours qui la transmet à un fils de sa sœur qui appartient au même clan. Sur ce point, Kroeber a peut-être exagéré. Il semble bien qu'une ou deux sociétés reconnaissent aux membres d'un certain clan un droit inné à entrer dans la confrérie. Néanmoins, de façon générale, les affirmations de Kroeber sont bien conformes aux faits.

En d'autres termes, la société des Pueblo, pas plus que celle d'autres régions, ne peut être considérée comme une structure atomique d'unités claniques. Une division selon le sexe est créée par l'initiation obligatoire de tous les hommes à un club, puis, à l'intérieur de cette organisation tribale, nous trouvons l'affiliation à une association indépendante du clan, quelles que soient les règles de succession relatives aux charges rituelles. Les confréries de guérisseurs des Zuñi sont encore plus dégagées de l'influence du clan, et c'est aussi le cas des sociétés analogues chez les Hopi. Ainsi l'importance du phénomène associationnel, partout où il coexiste avec les groupes de 
parenté, est manifeste. Comme Kroeber le fait remarquer, un individu du clan A peut appartenir à la famille $\mathrm{B}$, avoir son père dans le clan $\mathrm{C}$, entrer dans la confrérie $\mathrm{D}$ ainsi que dans le clergé $\mathrm{A}$ et se rattacher au local $\mathrm{B}$. Rien ne nous autorise à supposer que le lien particulier que représente le clan doive à tout prix passer avant les autres rapports sociaux. C'est comme si nous prétendions que celui qui vote pour le parti radical se comporte en radical dans les assemblées littéraires, aux réunions de son syndicat et dans l'intimité du foyer. Pourquoi vouloir attribuer à l'une de ces attaches sociales la préséance? L'individu agit normalement en tant que membre du groupe avec lequel il se trouve momentanément associé et ces divers groupes n'entrent pas nécessairement en contact ou en conflit les uns avec les autres.

Chapitre 10

\section{f. Crow.}

$\underline{\text { Retour à la table des matières }}$

Dans le chapitre concernant la propriété, il a été fait mention de la Société du Tabac des Crow, dans laquelle hommes et femmes pénètrent en payant une certaine somme à l'adoptant. Le novice subit un entrainement approprié durant une période préliminaire; il apprend les chants qu'a choisis pour lui son « père » ou parrain. C'est en acquérant le droit de planter le Tabac sacré au cours d'une cérémonie publique qu'il devient un membre véritable de l'association. L'Ordre du Tabac est divisé en plusieurs sections. Le novice entre naturellement dans celle de son " père ». Mais toutes ces subdivisions font preuve d'un esprit de corps très fort; bien que seuls les membres du groupe adoptif aient normalement le droit de se partager la finance d'entrée et de donner au novice le choix entre leurs différents objets sacrés, toutes les sections y participent. L'initiation du Tabac ne présente nul de ces phénomènes de castigation ou de mutilation du candidat; elle prescrit surtout une instruction appropriée, des chants cérémoniels, des insignes et des prérogatives en compensation du droit d'entrée qui est considérable. Autrefois, il était d'usage de présenter une demande d'admission, mais actuellement l'association, qui se pique de compter le Plus grand nombre de membres possible, use de persuasion en cas de besoin. Il est aussi fréquent qu'un individu qui passe par un moment difficile de son existence, par exemple lorsque sa vie ou celle d'un parent chéri est en danger, fasse le vœu d'entrer dans la société si le cours des événements répond à ses désirs. Il est un autre point sur lequel diffèrent l'ancien et le nouvel usage. Autrefois la qualité de membre était limitée à un nombre restreint de couples d'un certain âge et les éléments religieux prédominaient; mais dernièrement, appartenir à l'ordre est devenu une marque de prestige social; aussi presque tous les adultes en font-ils partie et on a même adopté de jeunes 
enfants. Néanmoins, le caractère cérémoniel de la société n'a pas disparu le moins du monde. On continue à croire que l'existence de la tribu dépend du Tabac sacré, lequel symboliquement représente les étoiles. Du point de vue strictement social, les organisations du Tabac sont importantes par le fait qu'un lien tout à fait particulier unit les individus dits " père » et « fils » qui font preuve d'autant d'affection mutuelle que de véritables parents. Une autre particularité qui mérite d'être signalée, c'est qu'on a tendance à initier en même temps mari et femme. Tous ces phénomènes se sont maintenus chez les Crow jusqu'en 1915.

Actuellement quatre clubs laïques jouent un rôle important dans la vie tribale : les Danseurs ardents de la Nuit, les Grands Trous d'Oreilles, les Derniers Danseurs ardents et les Danseurs Sioux. Ils célèbrent tous la même Danse ardente qui a été introduite par les Hidatsa dans la seconde moitié du siècle passé. Presque tous les hommes ont appartenu jusqu'à il y a vingt ans à l'un de ces clubs. Ces derniers, au contraire de l'Ordre du Tabac, n'exigent pas de droit d'entrée, pas davantage qu'une initiation formelle, de quelque nature qu'elle soit. Chaque club aspire à avoir beaucoup de membres; aussi, bien loin d'extorquer des paiements, les clubs offrent des cadeaux de valeur aux candidats éventuels afin de les engager à devenir membres; on sollicite ainsi surtout les individus dont la générosité est connue et qui sont susceptibles de traiter largement leurs camarades. Il arrive même que ceux qui jouissent d'un grand renom à cet égard, sont incités à changer de club par l'offre tentatrice d'une récompense en biens. Il nous faut considérer ces clubs comme étant dans une large mesure des coopératives. Si un homme des Grands Trous d'Oreilles doit exécuter certains travaux, tous ses camarades de club l'assistent. S'il cherche à être initié dans l'Ordre du Tabac, les autres l'aident à réunir la somme que nécessite une admission dans toutes les règles. En de telles occasions, le club du candidat se comporte exactement comme le clan. De temps à autre, le club célèbre des fêtes non dans un local spécial mais sous la tente de l'un de ses membres.

Ces clubs, néanmoins, ne sont que l'édition atténuée d'une ancienne forme d'association qui florissait encore dans la seconde moitié du XIXe siècle. En 1833, le prince Maximilien de Wied-Neuwied avait trouvé jusqu'à huit de ces organisations; un peu plus tard, elles n'étaient plus que quatre : les Mains boueuses, les Grands Chiens, les Renards et les Bois renflés; vers 1870, seules les deux dernières étaient encore en pleine vigueur. Ces clubs étaient en partie des associations militaires cherchant à se faire connaître sur le champ de bataille et n'étaient par suite accessibles qu'aux hommes, sans toutefois exclure absolument les femmes; celles-ci participaient aux fêtes et aux excursions des clubs. Le chef tribal attribuait chaque année à l'un des clans une fonction publique : le maintien de l'ordre durant la chasse communale au bison. En outre, chaque société avait sa danse et son chant, mais tout caractère religieux en était absent, ce qui surprend chez des Indiens des Prairies dont on connaît la tendance à répandre sur des phénomènes de toutes sortes un parfum de religion.

Comme dans les clubs actuels, on entrait dans une association militaire sans aucune formalité; il n'y avait ni adoption, ni achat, ni solennité d'aucune sorte. A la 
mort d'un membre, ses camarades cherchaient à le remplacer par un frère ou par un parent proche, offrant à ce dernier des présents afin d'obtenir son adhésion. Cependant les différents frères d'une même famille n'étaient pas obligés d'appartenir à la même société et, dans ces clubs, on trouvait des représentants de toutes les familles et de tous les clans. Même lorsqu'il ne se produisait pas de vacance, les membres pouvaient inviter par des dons quelque guerrier illustre à prendre place parmi eux, qu'il appartînt déjà ou non à un autre club. Bien que des griefs personnels pussent causer un changement de club, en général on était membre perpétuel. Quelques individus entraient dans tel club simplement parce qu'ils en aimaient les chants et les danses.

De même que les clubs modernes, les associations militaires étaient des unités coordonnées. Chacune se composait d'individus de tout âge et remplissait à tour de rôle les devoirs de corps de police. Chacune avait son ensemble de chants et de cérémonies, et toutes modelaient leur organisation interne sur un seul patron. Évidemment cette égalité n'allait pas jusqu'à s'étendre à un club pour jeunes garçons imité de ceux des adultes qui, sous le nom de société du Marteau, reproduisait les activités des grands.

Bien qu'il soit impossible de décrire dans ce chapitre tout le système des associations militaires chez les Crow, on ne saurait s'en faire une idée vivante sans traiter l'une d'elles quelque peu en détail. Mon choix se porte dans ce dessein sur l'organisation du Renard.

Les Renards étaient divisés selon l'âge en plusieurs petits groupes qui ne présentaient aucune différence sensible à l'égard du vêtement, des chants et de l'éligibilité aux charges honorifiques. Les membres ne portaient guère d'emblèmes distinctifs, à l'exception d'une cape de renard. Quelques-uns des officiers, cependant, arboraient des signes particuliers. Leur dignité, il importe de le noter, ne rappelait en rien les fonctions de président ou de secrétaire d'une de nos organisations; ces dernières incombaient à des membres âgés qui les remplissaient sans aucune solennité. Pour ce qui est des officiers crow, c'était sur des prouesses militaires, passées ou à venir, que se basait leur préséance. Chaque année, au printemps, les plus vieux des membres convoquaient les autres dans une même tente et désignaient les huit ou dix braves à qui incombait de porter haut l'honneur de l'association. Parmi ceux-ci, les quatre porte-insignes avaient les devoirs les mieux définis; deux d'entre eux recevaient des lances incurvées et les deux autres des lances droites; lorsque l'ennemi était en vue, ils devaient ficher en terre ces emblèmes et les défendre sans s'en éloigner, même au risque de leur vie. S'ils y réussissaient, qu'ils fussent tués ou non, ils devenaient célèbres dans la société et dans la tribu; s'ils battaient en retraite, ils perdaient leur rang et étaient traités avec mépris de " femmes en règles ». A cause du danger qu'on courait en acceptant cette charge, il arrivait souvent qu'on déclinât ce périlleux honneur, mais, en cas de nécessité, les anciens pouvaient l'imposer en touchant à la dérobée avec une pipe les lèvres d'un guerrier. Même de tout proches parents pouvaient ainsi forcer un jeune homme à devenir officier, non qu'ils lui voulussent du 
mal, mais parce qu'ils étaient impatients de le voir se distinguer. Ce lourd devoir prenait fin avec la première chute de neige; au printemps suivant, un nouveau titulaire recevait la lance fatale.

L'un des phénomènes les plus remarquables que présentât cette société était l'esprit de rivalité qui mettait aux prises les Renards et les Bois renflés, c'est-à-dire les deux clubs qui finirent par avoir le pas sur les autres organisations militaires des Crow. Dans cette hostilité bien définie, il n'y avait nulle trace d'animosité personnelle. A certains jeux, les Renards et leurs femmes jouaient contre les Bois renflés et leurs femmes; à la guerre, chaque club essayait de s'attribuer le premier coup frappé sur l'ennemi; et au début du printemps, au cours d'une brève période, un Renard pouvait enlever la femme d'un Bois renflé et vice versa, si elle avait été auparavant sa maîtresse.

Cette dernière coutume est remarquable à divers égards. Aussitôt que le temps de l'enlèvement avait été proclamé par des marques de défiance mutuelle dans chaque club, les hommes sortaient pour se saisir de leur ancienne amante, devenue plus tard l'épouse d'un membre de la société rivale. Toute résistance était vaine, car, en cas de nécessité, ils avaient recours à la force. Le mari, moins que tout autre, ne devait manifester aucun signe de ressentiment sous peine de perdre l'estime publique et de devenir l'objet de la risée générale. La seule manière dont une femme pût éviter l'enlèvement était de s'en remettre à la générosité de son ancien amant. Pour le mari, la meilleure attitude à adopter était de le prendre de haut et même d'encourager le séducteur dans son dessein. Aussitôt que les Renards avaient enlevé une femme des Bois renflés, ils l'emmenaient dans leur campement où elle recevait de la parenté de son amant tous les ornements dont on fait don d'ordinaire à une mariée. Le lendemain, les membres du clan exhibaient leur captive en procession solennelle. La femme, cependant, ne pouvait monter que sur le cheval d'un Renard qui s'était couvert de gloire à la guerre, sinon les Bois renflés les faisaient tomber, elle et son nouveau compagnon, de l'animal qu'ils chevauchaient. A cette exception près, les Bois renflés se comportaient en spectateurs à peine intéressés, faisant ostensiblement montre d'une indifférence feinte. En général, la femme enlevée était bientôt abandonnée par son amant, mais elle ne pouvait en aucun cas retourner auprès de son mari. Un homme qui reprenait sa femme après rapt, non seulement se dégradait aux yeux de tous, mais attirait l'ignominie ur sa société et donnait au club rival le droit de déchiqueter les couvertures de tous ses camarades.

La coutume relative à la compétition militaire est presque aussi curieuse. Si c'était un Renard qui, durant la bataille, frappait le premier coup de la saison, les Bois renflés perdaient de ce fait le droit de chanter leurs chants, ces derniers devenant pour une année la propriété de leurs rivaux. Toute cette émulation se limitait à la période comprise entre le début du printemps et la première chute de neige; puis elle cessait automatiquement et les deux organisations passaient l'hiver dans la plus parfaite amitié. 
En résumé, la classification par clans ne constituait qu'un type particulier de groupement social, s'entre-pénétrant avec d'autres divisions d'importance égale et peut-être même supérieure.

Chapitre 10

\section{g. Hidatsa.}

$\underline{\text { Retour à la table des matières }}$

Les Hidatsa ont, eux aussi, une série d'organisations militaires dont beaucoup sont semblables à celles des Crow, ce qu'indique déjà l'identité des noms qu'elles portent. Ainsi les deux tribus ont en commun les sociétés du Renard, du Bois renflé, du Marteau et du Chien. Souvent les analogies se manifestent jusque dans les plus petits détails. Les officiers aux lances incurvées des Renards Crow réapparaissent chez les Hidatsa avec les mêmes devoirs; dans les deux tribus, les gens du Chien portent des sonnailles particulières en sabots de cerfs ou de bisons liés à un bâton et certains officiers ont des écharpes fendues. Toutefois, alors que ces clubs étaient identiques en leur essence et correspondaient sans doute aux mêmes besoins sociaux de leurs membres, le mode d'admission différait totalement. Comme nous l'avons déjà dit (voir p. 228), les Hidatsa n'étaient pas invités individuellement et gratuitement à entrer et bien moins encore incités par des présents de valeur; ils devaient acheter collectivement une qualité de membre à laquelle avaient renoncé les titulaires antérieurs, si bien que toute la transaction équivalait à un transfert de droits de propriété.

Toutefois, outre ces différentes manières d'entrer dans l'organisation, ces deux systèmes présentent une divergence essentielle. Chez les Hidatsa, les sociétés militaires formaient une série étagée, les hommes passant au cours de leur existence d'un degré à l'autre. Du fait que l'achat jouait un rôle considérable dans l'opération, on pourrait conclure que nous sommes simplement en présence d'une réplique du système des îles Banks. Mais il n'en est rien. Chez les Mélanésiens, la promotion est individuelle et le jeune homme aisé peut facilement dépasser l'indigent. Parmi les Hidatsa, l'avancement s'opère toujours collectivement et les acquéreurs sont tous du même âge. En d'autres termes, les Hidatsa établissent la qualité de membre sur une double base, l'achat et l'âge. Aucun groupe d'individus ne peut, à partir d'un certain âge, faire partie automatiquement des Renards ou des Chiens; ils ne deviennent membres qu'une fois la finance d'entrée payée. D'autre part, personne ne peut isolément entrer dans les Chiens ou dans les Renards, mais seulement avec tout un groupe de candidats à peu près contemporains.

On trouvera au chapitre suivant une explication de ce dualisme. Ce qui nous importe pour l'instant, c'est la stratification de la société qu'opère le système des 
Hidatsa. Puisque les détails ont varié selon les époques, je choisirai en exemple un moment précis de l'histoire des Hidatsa. En 1833, le principe Maximilien trouva toute la population masculine, à part les très petits garçons, groupée en dix sociétés. Les plus jeunes étaient les Marteaux de Pierre, âgés de dix à onze ans; les garçons de quatorze à quinze ans constituaient les Bois renflés; puis venaient les jeunes gens de seize à dix-sept ans et ainsi de suite jusqu'au club du Corbeau qui groupait les vieillards de la tribu.

A proprement parler, les hommes de la tribu étaient répartis en dix classes d'âge, plutôt qu'en dix sociétés. Ces sociétés correspondaient réellement à des degrés d'âge et, selon le système des Hidatsa qui excluait la promotion automatique, il devait arriver que certains degrés devinssent vacants, lorsque les membres d'une classe d'âge avaient vendu leurs titres et qu'un certain temps s'écoulait avant qu'ils ne fussent en mesure d'entrer dans le degré supérieur. En d'autres termes, chaque classe d'âge était une unité permanente, mais elle n'occupait pas toujours une place définie dans la série. En employant la désignation de classe d'âge, il faut nous souvenir que ces Indiens n'estiment jamais leur âge par années et que ce groupement était assez approximatif. A cet égard ainsi qu'à d'autres, il sera instructif d'en rapprocher les classes d'âge des Massaï. Les deux tribus n'appliquent pas strictement le critère de l'âge. Certains Massaï sont initiés plus tôt que d'autres pour des raisons économiques ou parce que la circoncision n'a lieu qu'en telle année; la classe d'âge est ainsi formée d'individus qui sont à peu près contemporains, tout en incluant ou en excluant d'autres qui pourraient se classer différemment. Parmi les Hidatsa, la classe d'âge est également fondée sur des principes assez larges : un groupe de compagnons de jeux décide de s'acheter leur premier titre de membre d'une société; aussitôt que l'achat collectif est effectué, ils se constituent par là même en une unité sociale définie. Dans la suite, chez les Massaï comme chez les Hidatsa, l'affiliation de l'individu à une classe d'âge est immuable comme celle qui le rattache à son clan.

Il existait une alliance curieuse parmi les Hidatsa entre les classes d'âge alternées. Lorsqu'une classe 2 essayait d'accéder à la classe 3, elle pouvait compter sur l'aide de la classe 4, ainsi que des autres classes à nombre pair, tandis qu'en vertu d'un lien analogue les classes impaires s'assistaient mutuellement. La chose s'explique par les relations des degrés mitoyens. Entre ces derniers, il se produisait nécessairement « une lutte des classes ", les vendeurs tâchant d'extorquer les sommes les plus élevées possible; aussi les classes alternées étaient-elles unies par leur commun antagonisme contre la classe intermédiaire qui allait bientôt dépouiller les plus jeunes, tandis qu'elle deviendrait elle-même la victime des aînés.

Alors que les Indiennes Crow n'avaient pas de sociétés propres, leurs sœurs Hidatsa se constituaient en une série plus restreinte mais parallèle à celle des hommes, quelque peu élargie par des emprunts à leurs voisins, les Mandan. Le mode d'achat était le même que pour les clubs d'hommes ; il existait même certains rapports entre les clubs masculins et féminins, tels groupes de femmes venant en aide aux séries paires ou impaires respectivement. Naturellement ces organisations féminines 
n'étaient pas à proprement parler militaires, mais associées indirectement à des prouesses guerrières. Us femmes du Putois célébraient la mort d'un adversaire, tandis que celles de l'Ennemi organisaient une danse en l'honneur des braves tombés au cours d'un combat récent. Dans les unités supérieures de la série, surtout dans les organisations de l'Oie et de la Blanche Femelle de Bison, l'aspect laïque disparaît presque tout à fait, les activités les plus importantes étant de nature magicoreligieuses. Les femmes de l'Oie étaient censées aider à la croissance du maïs, tandis qu'à celles de la Blanche Femelle de Bison incombait le devoir d'attirer les troupeaux de bisons près des villages, en temps de disette. Ces sociétés étaient par suite investies d'une auréole de sainteté que ne possédaient pas les unités inférieures de la série, ni la plupart des clubs militaires des hommes. Dans les sociétés féminines, l'âge jouait aussi un rôle bien moins marqué. D'une part, l'usage voulait qu'on admît une ou deux petites filles dans les groupes de l'Oie et de la Blanche Femelle de Bison, composés surtout de vieilles femmes. Secondement, il n'est pas certain que toutes les femmes fissent partie de ces sociétés; d'après les faits, il semble que, contrairement au groupement par âge des associations masculines, beaucoup de femmes n'appartinssent à aucune classe d'âge déterminée et que le sexe féminin ne fût donc pas stratifié selon ce principe.

Outre les classes d'âge auxquelles il nous faudra revenir encore une fois dans la suite afin de les étudier d'un autre angle, les Hidatsa avaient des corporations sacrosaintes, unies par des " paquets " d'objets sacrés et célébrant les rites qui s'y rattachaient. Chaque Hidatsa avait droit au " paquet sacré » de son père et à ses prérogatives cérémonielles; mais, pour que les privilèges potentiels pussent devenir une réalité, il fallait qu'il fût racheté au père; les frères et sœurs s'unissaient généralement dans ce but. Cette dernière particularité, sans aller jusqu'à créer une association, aurait pu contribuer a renforcer le groupe de parenté si un même " paquet sacré » avec son rituel n'avait appartenu à plusieurs familles à la fois. Des individus qui ne se rattachaient les uns aux autres par aucun lien de parenté étaient donc réunis par des rites et des privilèges cérémoniels communs. Ainsi, en dépit de leur caractère héréditaire, les « paquets » des Hidatsa entraînèrent la formation d'un type différent d'unité sociale. Les femmes jouaient aussi leur rôle dans ces associations rituelles; c'était à leur garde qu'étaient commis les « paquets » achetés par leurs maris.

Ainsi un Hidatsa typique appartenait, du fait de sa naissance, à tel clan; à quelque moment de son enfance, il contribuait à la formation d'une classe d'âge, passant avec ses camarades par des achats successifs d'un degré à l'autre; indépendamment de ces affiliations, il devenait, par l'acquisition du « paquet » patrimonial, membre d'une des associations rituelles de son village. Une femme, bien que ne se rattachant pas nécessairement à une classe d'âge, en faisait souvent partie et avait également des devoirs précis à l'égard du « paquet » de son mari. 


\section{h. Résumé.}

Si brève que soit cette étude, elle suffit à prouver l'importance et la variété des associations, aussi bien dans les communautés à clans que dans celles qui n'en ont pas. Divisions fondées sur le sexe ou l'état civil, clubs sociaux, confréries secrètes, tous ces phénomènes s'entremêlent à ceux de la famille et du clan, créant de nouvelles unités dont l'influence sur l'existence sociale de l'individu est immense. A cet égard donc, l'opinion de Schurtz sur la société primitive est entièrement justifiée, alors que les théories de l'école de Morgan doivent être reconnues comme inexactes.

\section{Références.}

Retour à la table des matières

1) SCHURTZ : 1-82. WEBSTER.

2) MAN : 40, 60 et suiv., 108, 207. PARSONS : 282.

3) CUNOW, $1894: 25$ et suiv., 144 et suiv. SPENCER et GILLEN, 1904 : 257, 498, 611. 328, HOWITT : 509 et suiv. RADCLIFFE-BROWN, 1931.

4) HOLLIS, 1905 : XVI, 260 et suiv., 280 et suiv. MERKER : 16, 67 et suiv.

5) RIVERS, 1914 (b) :1, 60-143. CODRINGTON : 101 et suiv.

6) KROEBER, 1917 (a) : 150-188. STEVENSON : 62-107. BUNZEL, 1932.

7) LOWIE, $1913: 147-211$.

8) LOWIE, $1913: 225-354$. 


\section{1}

\section{THÉORIE DES ASSOCIATIONS}

\section{$\underline{\text { Retour à la table des matières }}$}

Dans le chapitre qui précède, je n'ai fait qu'indiquer la place importante qu'occupent les groupes associationnels dans l'organisation sociale primitive, sans essayer d'élucider les problèmes d'ordre historique ou sociologique que soulèvent ces phénomènes. Avant de les examiner, il convient de procéder tout d'abord à un exposé du système de Schurtz qui nous fournira toute une série de points de repère pour des considérations théoriques.

\section{a. Système de Schurtz.}

Selon cet auteur, la distinction entre les groupes de parenté et les groupes associationnels correspond à une différence profonde dans la psychologie des sexes. Contrairement à l'opinion courante, les femmes sont des êtres fort peu sociables, ne formant pas d'union sur la base des intérêts communs et restant centrées sur le groupe de parenté qui repose sur les relations sexuelles et sur les fonctions reproductrices. Les associations créées par les femmes et même celles où les femmes se trouvent sur un pied d'égalité avec les hommes, sont rares et doivent être envisagées comme de pâles imitations des associations exclusivement masculines. L'homme, d'autre part, n'attache qu'une importance épisodique aux relations sexuelles et estime que «qui se 
ressemble s'assemble ». Ainsi les différences physiologiques entre hommes et femmes entraînent une ségrégation sociologique.

Il se forme encore dans le cercle de famille une autre scission qui détruit quelques liens sociaux tout en en créant d'autres. L'antagonisme entre l'ancienne et la nouvelle génération qui sépare enfants et parents, contient le germe d'une classification selon l'âge que Schurtz considère comme le type le plus ancien d'association.

Schurtz ne prétend du reste pas que le type de groupement selon l'âge, qu'on trouve chez les primitifs, ait une base purement naturelle, mais plutôt qu'il est dû à un mélange de facteurs psychologiques et conventionnels. L'état typique, c'est la division tripartite de la société en enfants, adolescents nubiles et couples mariés. La démarcation entre les deux premiers groupes a pour base une différence naturelle, tandis que celle qui sépare le second du troisième est artificielle. Pour Schurtz, cette division est censée régler les relations sexuelles à la manière des Massaï qui admettent l'union libre entre jeunes gens et établissent plus tard des liens conjugaux sérieux. Dans une telle société, les hommes non mariés forment le groupe le plus net et le mieux organisé; les jeunes filles de condition égale, à cause de l'inaptitude féminine à la camaraderie, n'en présentent qu'un reflet affaibli. L'admission dans le groupe important des célibataires mâles est dûment marquée au moment de la puberté par des cérémonies compliquées, tandis que l'initiation des filles à l'état de femmes est d'un caractère bien moins significatif, correspondant à l'organisation moins définie de la classe des jeunes filles non mariées.

Schurtz est d'avis que partout où nous rencontrons un nombre plus élevé de classes d'âge, c'est en vertu d'une modification ultérieure de la division tripartite. Ainsi, tout en admettant la possibilité qu'une initiation simultanée puisse créer une union, il soutient que les classes résultant de celle-ci sont venues s'ajouter à un schéma primitif plus simple. Dans le cas des Hidatsa et des systèmes apparentés dont il traite plus particulièrement, il déduit leur origine secondaire non seulement du grand nombre des classes d'âge, mais aussi des modalités d'admission. C'est, en effet, un de ses axiomes favoris que, puisque le groupement selon l'âge physiologique et l'état civil a précédé tous les autres, l'achat, en tant que facteur d'admission dans une unité sociale, représente une phase d'évolution ultérieure.

Le développement du club masculin est en connexion intime avec les classes d'âge et plus particulièrement avec le rôle prédominant que joue l'organisation des jeunes gens non mariés. Ce club est un local où les hommes adultes mais célibataires préparent leurs repas, travaillent, jouent et dorment, tandis que les hommes mariés demeurent à part avec leur famille. Les femmes et les enfants sont en général exclus du club, mais les jeunes filles pubères y viennent vivre librement avec ses occupants. Bien que considérant cet état comme le prototype de l'institution, Schurtz reconnaît que les déviations s'écartent beaucoup de la norme. Il interprète invariablement de telles déviations comme des différenciations secondaires et admet que plusieurs lignes d'évolution peuvent être relevées. D'une part, l'habitation des célibataires peut 
conserver son attrait sur les hommes mariés et se transformer ainsi en un club général et même en un dortoir pour tous les mâles adultes. Ce même local, perdant son caractère premier, devient parfois un lieu de réunion lors des assemblées, des fêtes et des danses où les femmes sont quelquefois admises. Il peut encore servir de sudatorium, de salle du Conseil, d'arsenal, de taverne ou d'atelier, pour ne mentionner que quelques-unes des possibilités. Toutefois, l'habitation des hommes n'est caractéristique que pour certaines branches apparentées de l'humanité et sa distribution géographique pourrait être expliquée comme les effets de relations historiques plutôt que par une loi sociologique. Schurtz n'ignore pas cette objection; il va même jusqu'à admettre que les formes les plus typiques du club masculin sont limitées à la famille malaise. Néanmoins, l'ensemble des preuves ethnographiques l'induit à conclure que les sociétés ont une tendance inhérente à donner naissance au club masculin, qui n'est que « la manifestation extérieure d'une division simple et des plus évidentes (nahe liegenden) en classes d'âge et, dans ce sens, un état intermédiaire presque inévitable dans l'évolution de structures sociales plus hautes ».

Mais le sexe et l'âge ne sont pas les seuls agents sociaux. Dans les phases plus développées de la société, la tendance à l'exclusivisme commence à se faire sentir et les clubs se forment, supplantant les classes d'âge antérieures. Cette tendance est favorisée par les différences de rang et de fortune. Les esclaves et les pauvres sont exclus des classes d'âge qui prennent ainsi le caractère de clubs. Là où une finance est exigée, le facteur âge et avec lui la stratification de la société selon ce principe disparaît et nous voyons surgir à sa place un système hiérarchique, tel celui des îles Banks. Les clubs peuvent se développer dans l'une ou l'autre des directions suivantes : ils deviennent des associations cérémonielles, représentant des ordres nettement religieux ; ou bien c'est surtout le facteur sociabilité, particulier aux groupements masculins, qui l'emporte ; nous sommes alors en présence de joyeux convives dont les festins n'ont d'autre but que l'entretien d'une bonne camaraderie.

Enfin les sociétés secrètes représentent la dernière forme d'association qui se fonde sur l'instinct grégaire des hommes. Elles ne sont pas une phase nécessaire dans l'évolution sociale des associations ; on ne peut pas non plus faire remonter directement toutes les sociétés secrètes aux classes d'âge, quoiqu'il soit possible de déceler une parenté indirecte. Leurs activités sont variées. L'une de leurs particularités les plus évidentes est le secret à observer vis-à-vis des femmes et des esclaves, ce qu'explique assez bien l'interprétation que donne Schurtz des associations. Quant au côté formel des organisations secrètes, il découle également des phénomènes relatifs aux anciennes divisions par âge avec leurs épreuves et leurs cérémonies initiatoires. Il est également possible de découvrir dans ces rites tribaux d'initiation, tels que beaucoup de sociétés les pratiquent, le germe d'un culte des ancêtres et souvent les notions qui y correspondent. Les sociétés secrètes ont développé ce germe, de même que quelques éléments spécifiques, tels que le port des masques, le culte des crânes et l'usage de tabous pour sauvegarder la propriété. S'appuyant sur leurs pouvoirs prétendus mystiques, ces organisations dégénèrent parfois en bandes terrorisant et dépouillant les non-initiés. Mais elles peuvent aussi revêtir l'aspect d'un corps rendant la 
justice. Elles punissent les membres qui ont divulgué le secret ou qui se sont rendus coupables d'autres transgressions et infligent des amendes aux non-membres qui se sont opposés à leurs intérêts. Elles peuvent aussi dispenser le pouvoir politique et, comme telles, elles ont souvent été utilisées par les administrateurs des colonies anglaises. Quoiqu'il y ait toujours potentialité d'organisations secrètes, les unités les plus typiques sont éminemment instables. Selon Schurtz, il ne serait pas possible à la longue de garder secrets les mots d'ordre et les autres mystères.

Schurtz considère comme typiques les conditions observées dans le Brésil central où telle peuplade exclut les femmes de toutes les danses, alors qu'une autre tribu les admet à des cérémonies d'importance moindre ; où, dans certaines régions, les bullroarers ne doivent jamais être vus par le sexe féminin et sont soigneusement cachés dans la maison des hommes, alors que dans une zone voisine ils sont exposés aux regards du public. Ailleurs l'atmosphère de mystère disparaît et la société n'est plus qu'un simple club. Dans d'autres parties du globe enfin, les organisations secrètes se transforment en un corps de police au service du chef ou en ordres religieux qui admettent jusqu'à des femmes.

Cette brève esquisse suffira à indiquer la nature du raisonnement de Schurtz. En comparant son système avec celui de Morgan et en les étudiant de près, nous leur trouverons une analogie fondamentale. Schurtz tient compte de phénomènes dont Morgan ignorait totalement l'existence, mais, comme lui, il impose aux faits un système d'évolution qui se prétend universellement applicable et cherche ainsi à formuler des lois sociologiques. Schurtz possède, il est vrai, un sens plus fin de la complexité des phénomènes sociaux et admet qu'un même état-type se développe de façon multiple, comme nous l'avons vu dans le cas du club. Mais cela ne l'empêche pas de s'en tenir strictement à son ordre de succession chronologique, dans la mesure où il s'agit des grandes phases de l'évolution. Il est aussi sûr de l'antériorité universelle des classes d'âge relativement aux clubs et aux sociétés secrètes que l'est Morgan de l'ancienneté nécessaire de la descendance matriarcale. Schurtz fait, il est vrai, de fréquentes concessions au principe de la diffusion, mais sans grande conviction. Il va même jusqu'à affirmer que, dans les enquêtes sociologiques le problème des emprunts est dépourvu d'importance.

Cependant, ainsi que nous l'avons déjà noté, la question de la diffusion n'est jamais insignifiante. On peut accorder sans doute que le seul fait de savoir que telle particularité a été empruntée par un peuple à un autre n'en fait pas comprendre l'essence ; mais l'importance des preuves d'emprunt provient de ce qu'elles indiquent l'origine étrangère dans la société empruntante de la particularité en question, dont la présence en ce point n'est donc pas le résultat d'une loi sociologique. On pourrait soutenir qu'elle aurait de toute façon évolué indépendamment, mais c'est là une allégation toute gratuite. Rien n'empêcherait, dans ce cas, d'affirmer que les anciens Brites auraient pu inventer, l'art d'écrire ou que les Esquimaux de l'Alaska auraient fini par savoir à eux seuls domestiquer le renne. De telles affirmations sont faciles à lancer, moins faciles à démontrer. Pour appliquer ce principe au cas qui nous occupe, 
il n'est nullement secondaire de savoir si les clubs masculins ont évolué indépendamment en Assam et aux îles Banks, ou s'ils sont originaires du même point que les institutions indonésiennes analogues.

A l'appui de la première hypothèse, on se saurait nier que les sociétés humaines ont une certaine tendance à séparer la population mâle dans un édifice à part. D'après la seconde, la force de cette tendance est considérablement diminuée et on en arrive inévitablement à se demander si un phénomène qui a couvert déjà de si grandes distances, n'aurait pas pu aller encore plus loin, bref si tous les cas de clubs masculins, en quelque lieu qu'ils se rencontrent, ne remontent pas à une même source. Si cette hypothèse pouvait être prouvée, ce serait enlever tout fondement à la supposition d'une force sociologique poussant à la création de clubs masculins ; tout ce qu'on pourrait avancer en sa faveur serait de reconnaître qu'il existe une tendance à imiter cette institution quand l'occasion s'en présente.

Schurtz essaie d'éluder ces faits évidents. Ses remarques marginales sur les relations historiques, tout en indiquant qu'il n'ignore pas que la diffusion est un principe actif de l'histoire culturelle, dénotent qu'il n'en a pas fait lui-même un usage effectif; elles ne permettent donc pas de distinguer logiquement: sa méthode de celle de Morgan. Cela est d'autant plus vrai que les fantaisies occasionnelles de Morgan à l'endroit des connexions historiques dépassent en audace les diffusionnistes récents les plus téméraires. Selon Morgan, non seulement le clan a une origine unique, mais encore, pour ce qui est des systèmes de parenté, la diffusion elle-même ne lui suffit pas, et il ne lui faut pas moins que des affinités raciales pour expliquer une analogie de classification chez les Zoulou et les Hawaïens. Tout cela n'entame nullement l'évolutionnisme essentiellement unilinéaire de son système.

En substance donc, Morgan et Schurtz vivent dans la même sphère et se servent des concepts de manière identique. Le mérite de Schurtz est d'avoir étendu matériellement le champ de ses enquêtes sociologiques plutôt que d'avoir inventé de nouvelles méthodes de le cultiver. Les pionniers de la science, pour employer la comparaison de Tourguenieff, commencent par effleurer légèrement le sol vierge avec la houe et c'est plus tard seulement que la charrue y pénètre profondément.

Après ces commentaires en manière d'introduction, je passe aux problèmes généraux les plus importants que soulèvent les associations.

Chapitre 11

\section{b. Dichotomie sexuelle.}


Schurtz insiste à plusieurs reprises de façon fastidieuse sur la différence psychologique essentielle qui conduit les hommes à se grouper en associations, alors que les femelles peu sociables de l'espèce se bornent tout au plus à copier timidement leurs prototypes masculins ou entrent dans quelque société mixte. Il n'est pas indispensable d'être un féministe ardent pour réfuter ce raisonnement. Pour pouvoir entrer dans une société, il faut davantage que le seul désir d'en devenir membre. Étant donné que les Australiennes qui s'aventuraient près du lieu où l'on procédait à l'initiation étaient impitoyablement mises à mort, il n'est pas surprenant que bien peu s'exposassent à se faire blackbouler de façon aussi irrémédiable. En outre, il n'est nullement certain que les hommes eussent admis partout la formation de sociétés féminines indépendantes. En certaines régions tout au moins, l'absence totale de clubs de femmes est due surtout à l'attitude peu encourageante des hommes. Mais faisons abstraction de ce facteur. Les faits que nous avons déjà signalés dans un autre chapitre à propos des Kirghiz et des Turcs Altaïques sont particulièrement révélateurs. Parmi ces derniers, la femme absorbée par les besognes serviles du ménage n'a matériellement pas le temps d'être sociable, que son mari soit d'accord ou non. Grâce à une répartition différente des tâches, sa sœur kirghiz, se moquant des préceptes théoriques de l'Islam, se mêle librement aux hommes et aux autres femmes et prend même part avec l'autre sexe à des compétitions de chants animées. Quelles que soient les différences psychologiques qui séparent les sexes - et ce n'est pas moi qui les nierai -, le caractère moins grégaire des femmes ne pourra guère être tenu pour une déficience féminine aussi longtemps qu'on pourra expliquer d'autre façon pourquoi son activité associationnelle est restée limitée.

L'une des plus convaincantes de ces explications a été suggérée par Karl von den Steinen et par Paul Radin. Pour le premier, les mystères des Bakaïri sont des fêtes de chasseurs, dont les femmes sont naturellement exclues. De même, Radin fait remarquer qu'il serait peu concevable qu'un cercle militaire admit des femmes, pas davantage qu'une société de couture, des hommes. L'exclusion est, dans ce cas, presque automatique. Nous pourrions ajouter que l'activité féminine n'exige pas fréquemment un effort concerté, ainsi qu'une entreprise militaire. Une potière peut fabriquer ses vases sans pour cela s'entourer d'aides. D'autre part, Mooney a trouvé que chez les Cheyenne les femmes étaient organisées en une série de corporations groupant les ouvrières des métiers les plus difficiles, tels que la confection des tentes, la broderie en piquants de porc-épic, la décoration des mocassins et la peinture des peaux. L'admission n'avait lieu que contre paiement d'une finance élevée; d'une façon générale ces guildes rappellent à celui qui les a découvertes les trade unions. Ici la nécessité de demander des conseils et de se faire connaître dans un champ d'activité limité a occasionné l'essor de toute une quantité de sociétés, réduisant ainsi à néant le dogme de l'insociabilité féminine.

A la vérité, les faits nord-américains nous prouvent amplement à quel point ce principe cardinal de Schurtz est dépourvu de base. Parmi les Crow, les femmes jouent un rôle aussi important que les hommes dans la Société du Tabac; elles y sont généralement admises avec leur mari, un couple marié représentant presque toujours 
une unité fixe. Il en va de même chez les hidatsa : les "paquets sacrés » sont d'ordinaire transmis à l'acquéreur par l'intermédiaire de sa femme qui est la première à toucher de son corps les fétiches. La présence dans cette tribu d'organisations nettement féminines a déjà été signalée et si elles sont moins nombreuses que les sociétés analogues des hommes, elles ont, par contre, un caractère plus sacré. Ainsi que le fait remarquer Radin, la grande société secrète des Algonkin du Centre, les Midewiwin, admet comme shamanes des hommes ou des femmes. En plusieurs des organisations Omaha, il était indispensable, pour acquérir la qualité de membre, de recevoir un message de tel être surnaturel, comme le Tonnerre ou le Bison, et les femmes que visitaient ces visions étaient éligibles au même titre que les hommes. Dans le sud-ouest des États-Unis, les hommes ont certainement une activité cérémonielle plus forte que les femmes; cependant ces dernières ont leurs sociétés et danses propres. Les Shoshoni du Grand Bassin n'ont rien qu'on puisse à proprement parler désigner du terme de société, mais, à l'occasion de plusieurs fêtes et danses, les femmes sont les égales des hommes.

Tout aussi intéressantes que les nombreux exemples de phénomènes grégaires féminins en Amérique du Nord sont certaines analogies qui nous ont été signalées dans d'autres continents avec les maisons d'hommes et les sociétés tribales. Ces analogies, ainsi que nous l'avons déjà vu auparavant, se limitent aux régions voisines des rivages du Pacifique, circonstance qui rend probable leur diffusion à partir d'un certain centre commun. Ce qui nous intéresse plus spécialement pour le moment, c'est que les conséquences des mêmes institutions sont en général toute différentes en Amérique du Nord ou en Australie et en Mélanésie. Dans les îles Banks, il existe une véritable organisation bipartite sur la base du sexe, les hommes initiés mangeant, vivant et dormant à l'écart des femmes. Les Australiens, sans observer jusque là la ségrégation, la font intervenir pourtant dans la vie publique et religieuse. Dans ces deux régions, tous les hommes sont initiés, mais jamais les femmes. Or, précisément, cette manière de considérer le statut de la femme, ses fonctions et ses incapacités, paraît à l'américaniste totalement étrangère à la notion indienne et à tous les phénomènes nord-américains.

Dans la partie septentrionale de la Californie par exemple, il existe un club purement masculin et une société secrète des hommes, mais les conceptions sousjacentes sont fort différentes de celles qu'on observe en d'autres continents. Parmi les Hupa, les hommes ne dorment pas en hiver avec les femmes, mais dans le sudatorium du village; cependant ils mangent toute l'année avec leurs épouses et vivent avec elles durant l'été dans des abris en branchages. La ségrégation des hommes n'est donc que temporaire. En outre, elle n'implique pas l'exclusion des femmes des activités cérémonielles; il existe des femmes shamanes et, au temps du séjour de Goddard, la danse des Branches était dirigée par une vieille femme, la seule qui pût s'entretenir avec la « médecine » requise. A peu près dans la même région, les Shasta ont pour les hommes des dortoirs d'hiver et des clubs où ils se rendent pour jouer, flâner ou prendre leur bain de vapeur. Le rituel des Shasta se réduit presque entièrement à des cérémonies shamanistes et nous voyons avec surprise que ce sont seulement les fem- 
mes qui assument la charge de shamane. C'est dire que, en dépit du club des hommes, l'activité cérémonielle revient pour une large part aux femmes, ce qui semblerait certainement à un Océanien le monde renversé.

Si nous passons aux Maidu, nous nous trouvons devant des conditions quelque peu différentes. Les grands édifices qui rappellent extérieurement les clubs des Shasta, ne remplissent pas tout à fait les mêmes fonctions; tout en servant d'étuves, ce sont, bien plus que des clubs, des locaux cérémoniels. En outre, les femmes ne sont pas toujours exclues de ces lieux de danse, mais elles prennent une part active à plusieurs cérémonies; les femmes shamanes, moins nombreuses que parmi les Shasta, célèbrent dans le sudatorium la fête shamaniste annuelle.

Jusqu'ici nous ne trouvons pas en Californie une séparation des sexes aussi rigide qu'en Océanie. La ségrégation des hommes durant une partie de l'année coexiste avec la commensalité et, dans une certaine mesure, avec les cérémonies communes. Cependant les Yuki, les Pomo et les Maidu ont une organisation secrète qui, au premier abord, rappelle fortement les sociétés tribales en d'autres continents, puisqu'elle exclut absolument les femmes. Powers, dans son enquête (qui tient quelque peu du reportage mais qui se trouve confirmée par Loeb et par d'autres) sur les deux premières tribus, par sa description des danseurs masqués qui personnifient de mauvais esprits et qui, tout en terrorisant les femmes, les obligent à observer soumission et chasteté, communique à ces sociétés l'atmosphère particulière à l'Afrique occidentale et à la Mélanésie.

Mais, pour pouvoir juger des phénomènes californiens, il ne nous faut pas oublier un fait de toute importance. La société secrète n'était pas un groupe comprenant tous les hommes, ni l'initiation une phase indispensable dans la vie d'un individu et préludant nécessairement au mariage. Par cette seule particularité, elle se distingue de la société tribale des Australiens. On peut la comparer aux sociétés d'esprits des îles Banks, mais les points de parallélisme ne concernent pas ce qui nous occupe ici, c'està-dire la présence de phratries sexuelles. On ne peut même pas relever ces dernières chez les Pomo, puisque, ainsi que le démontre Barrett, un nombre indéterminé d'hommes et de femmes prennent part à la plupart des danses; pour deux autres danses, le nombre des participants de chaque sexe était fixé. Il y avait en outre cinq danses où n'entraient que des hommes, mais à deux de celles-ci les femmes pouvaient assister; plus deux danses des femmes dont les hommes n'étaient, cependant, pas exclus. Ainsi le fait que les femmes et les hommes non initiés étaient tenus à l'écart d'une cérémonie ésotérique, ne peut pas être rangé parmi les phénomènes relatifs à la dichotomie sexuelle.

Des considérations analogues s'appliquent au cas des Maidu. La situation dans cette tribu des femmes shamanes est spécialement intéressante si nous la rapprochons de celle des guérisseuses analogues au Queensland. Ces dernières observent certaines cérémonies, mais elles ne doivent ni toucher ni regarder les charmes qui constituent 
l'inventaire du vrai guérisseur et « en aucun cas elles ne peuvent se joindre ostensiblement aux délibérations secrètes des autres médecins ».

Ce qui est vrai de la Californie s'applique aussi à la région des Pueblo. Nous savons que les salles de cérémonies souterraines, utilisées d'ordinaire par les hommes comme atelier ou lieu de repos, faisaient autrefois office de dortoir et d'étuve pour hommes; mais ces salles ne jouent aucun rôle dans la vie quotidienne en ce qui a trait à la libre association des sexes. L'organisation tribale masculine elle-même n'est pas parvenue à ce résultat et n'empêche pas les femmes de participer aux cérémonies. Dans l'extrême nord, les peuplades de l'Alaska diffèrent des autres Esquimaux en ce qu'elles possèdent pour les hommes, et plus particulièrement pour les célibataires, un dortoir qui est à la fois club, maison communale, salle de danses et taverne. Les femmes en sont exclues à certaines époques mais, d'une façon générale on ne refuse pas de les y admettre. Au contraire, elles apportent deux ou trois fois par jour la nourriture au club, restent assises près de leurs parents mâles durant le repas et souvent ne se contentent pas d'assister aux cérémonies, mais y prennent une part active. Étant donné tous ces phénomènes, les observations de Hearne parmi les Chipewyan de la région du Mackenzie représentent une exception; ces Indiens excluent les femmes de toutes les danses et veillent avec autant de vigilance qu'une gouvernante anglaise à ce que jeunes gens et jeunes filles ne se mêlent pas. Ce point de vue représente une anomalie non seulement de la norme des Indiens nord-américains en général, mais aussi de celle des Athapaskan du nord, puisque d'autres branches de cette famille, tels les Flancs de Chiens, admettent que les deux sexes participent aux danses.

En résumé, non seulement les Indiens de l'Amérique du Nord ne présentent pas cet exclusivisme masculin qui divise une tribu en deux phratries sexuelles, mais encore leurs femmes font preuve d'un certain instinct grégaire, puisqu'elles se joignent aux hommes dans les manifestations sociales lorsqu'elles y sont autorisées et vont même fréquemment jusqu'à fonder leurs associations propres. Si ces dernières sont moins nombreuses que celles des hommes, la chose s'explique fort bien comme nous l'avons indiqué plus haut, sans qu'il soit besoin de recourir à quelque absence d'instinct de sociabilité.

Les phénomènes africains concordent en général avec ceux de l'Amérique du Nord. En dépit du statut souvent inférieur de la femme, on observe d'ordinaire que les deux sexes se mêlent librement dans leurs rapports sociaux. Il existe, il est vrai, sur la côte nord-ouest surtout, des sociétés secrètes où les femmes ne sont pas admises, mais, ainsi que Radin l'a fait remarquer, le nombre de sociétés féminines parallèles est considérable. A quelques organisations masculines incombent des fonctions militaires et juridiques, d'autres se spécialisent dans le châtiment des épouses adultères; il est donc tout naturel que les femmes en soient exclues. Le fait que les sociétés féminines sont non seulement assez nombreuses, mais socialement importantes, est beaucoup plus significatif. Ainsi, à la société masculine des Poro chez les Mendi de Sierra Leone s'oppose le Bundu des femmes; bien qu'il soit exagéré de prétendre que cette dernière est de rang égal, les hommes reconnaissent son caractère sacré. " Aucun 
homme, sous quelque prétexte que ce fût, ne se hasarderait à approcher du Buisson Bundu, car on sait que les effets de la médecine Bundu sur le délinquant sont très violents; et cette croyance est si profondément enracinée dans l'esprit de tous les hommes que les jeunes filles Bundu, lorsqu'elles sont sous la protection de cette médecine, peuvent se promener seules, sachant qu'elles sont parfaitement à l'abri de toute poursuite. " Miss Kingsley raconte que, dans une région située un peu plus au sud, un homme qui s'introduirait dans les mystères féminins serait mis à mort, de même qu'on tuerait une femme qui essaierait de violer le secret des réunions d'hommes. Les organisations féminines sont souvent en relation tout au moins avec l'initiation des filles pubères, et il convient de noter qu'en Afrique l'admission des filles à la condition de femme s'entoure d'autant de solennité que les rites équivalents pour les garçons. Tel n'est certainement pas le cas en Australie ni, pour autant que je sache, en Mélanésie.

En général, les sexes ne sont pas strictement séparés en Afrique. Là où se produit une ségrégation cérémonielle, elle diffère toto cœlo de celle qui a été signalée en Australie et en Mélanésie, puisque les femmes ne forment pas régulièrement une masse amorphe d'individus, mais se constituent souvent en sociétés tribales ou de caractère plus particulier. Enfin, si nous considérons que, dans une très grande partie de l'Asie, Schurtz lui-même n'a pas pu découvrir de société tribale masculine, ni d'autre association, nous rejetterons comme dépourvue de fondement la doctrine qui veut que l'insociabilité virtuellement absolue soit un caractère sexuel secondaire de la femme. Schurtz a pris à tort un phénomène à distribution géographique limitée pour un trait universel; et, même dans la région propice à ses vues, il a ignoré la différence entre le résultat d'une institution et l'incapacité organique. C'est seulement lorsqu'on aura démontré que l'influence de la division du travail combinée avec celle de la gérontocratie masculine en Australie ou du club des hommes aux îles Banks, est favorable ou tout au moins ne s'oppose pas à la création d'associations féminines, c'est alors mais alors seulement qu'il sera possible d'expliquer psychologiquement la rareté des confréries féminines par la moindre sociabilité des femmes.

Avant de quitter la question du sexe par rapport aux associations, il me faut d'abord traiter un sujet apparemment banal, mais du plus haut intérêt ethnologique. En esquissant les rites d'initiation australiens, j'ai fait mention du bull-roarer, instrument de musique défendu aux femmes. Le soin qu'on prend pour empêcher les noninitiés de savoir que c'est ce simple appareil qui émet certains sons sinistres est extrêmement comique. Il semble que l'essence de tous les mystères soit la production de ce bourdonnement et que toutes les souffrances, tous les tourments d'un long rituel atteignent, du point de vue indigène, leur apogée lorsqu'on révèle aux garçons comment faire siffler dans l'air une petite pièce plate. Il est assez curieux que la peine de mort soit infligée à la femme qui découvre ce secret ou à l'homme qui le divulgue. Toutefois, nous retrouvons en différentes régions du globe cette même association d'idées. En voici comme preuve quelques exemples: 
Parmi les Urabunna de l'Australie centrale, on enseigne aux non-initiés que ce bruit est la voix d'un esprit « qui s'empare du garçon, lui enlève toutes ses entrailles, les remplace par d'autres et le ramène initié. On avertit le jeune garçon de ne jamais laisser voir le bâton à des femmes, sinon sa mère et ses sœurs tomberaient mortes comme des pierres ». Plus au nord, les Anula, du Golfe de Carpentaria, racontent à leurs femmes que le sifflement du bull-roarer est produit par un esprit qui avale le garçon puis le vomit, devenu initié. Chez les Bukaua qui vivent en Nouvelle-Guinée, près du golfe de Huon, lors de l'initiation, on dit aux mères des novices que le bruit des plaques en forme de feuilles est la voix d'un ogre insatiable qui engloutit puis rejette les petits garçons. Aux Salomon et dans les îles françaises, le bull-roarer est également dissimulé aux femmes qui croient que ce bruit étrange est la voix d'un esprit, et les Sulka de Nouvelle-Bretagne ajoutent encore que cet être dévore à l'occasion les non-initiés. Les exemples qui précèdent sont choisis entre beaucoup d'autres dans les ouvrages traitant de l'Australie et de l'Océanie. Des conceptions identiques apparaissent en diverses régions de l'Afrique. Les Ekoi du Sud-Nigéria ne permettent pas aux femmes de voir le bull-roarer ou de connaître l'origine des sons produits et on signale, dans une zone très distante, chez les Nandi de l'Afrique orientale, des règles analogues. Une fois que Frobenius fit, par manière de plaisanterie, semblant de faire tourner un bull-roarer, toutes les femmes manifestèrent une violente terreur, et on raconte qu'autrefois les femmes qui se trouvaient sur le passage de la procession des hommes, lorsqu'ils faisaient vibrer ces instruments, étaient impitoyablement mises à mort. Citons enfin un exemple tiré de l'Amérique du Sud. Dans les rites funéraires des Bororo du Brésil central, on fait tournoyer des bullroarers et, à ce bruit, les femmes s'enfuient dans les bois ou vont se cacher dans leur hutte afin d'éviter la mort. Les hommes croient également que le simple fait de voir un bull-roarer causerait la mort des femmes; ils recommandèrent donc à von den Steinen à plusieurs reprises de cacher aux femmes et aux enfants les spécimens du bull-roarers qu'il se procurait, afin de ne pas causer de catastrophe.

Ces analogies ne sauraient passer inaperçues. Elles éveillèrent l'intérêt d'Andrew Lang qui les expliqua comme les effets " d'une mentalité similaire, cherchant à atteindre par des moyens simples des buts identiques », et qui répudia expressément " la nécessité d'avoir recours à l'hypothèse d'une origine commune ou à celle d'emprunts pour rendre compte de la présence si fréquente de cet objet sacré ». Von den Steinen est du même avis que lui; il fait remarquer qu'un appareil aussi simple qu'une planchette attachée à une ficelle ne peut avoir coûté un tel effort au génie humain qu'il faille faire intervenir l'hypothèse d'une invention unique dans toute l'histoire de la civilisation. Mais c'est mal saisir le problème. Il n'importe pas de savoir si le bull-roarer a été inventé une seule fois ou douze, et pas davantage que ce simple jouet soit fréquemment ou non associé à des cérémonies. J'ai vu moi-même chez les Hopi les prêtres de la confrérie de la Flûte faire tournoyer des bull-roarers en des occasions solennelles, mais il ne m'est pas venu à l'idée de rattacher ce fait aux mystères australiens ou africains, car rien n'indiquait que chez les Hopi cet instrument fût défendu aux femmes. C'est ici, en effet, que se trouve le nœud du problème. Pourquoi les Brésiliens et les Australiens du centre jugent-ils dangereusement mortel 
que les femmes voient le buil-roarer ? Pourquoi cette insistance à leur cacher la nature de cet objet en Afrique occidentale ou orientale ainsi qu'en Océanie? Je ne connais pas de loi psychologique qui oblige l'esprit des Ekoi et celui des Bororo à maintenir les femmes dans l'ignorance sur la question du bull-roarer et, on attendant qu'on la découvre, je n'hésite pas à admettre, comme l'hypothèse la plus probable, l'existence d'un centre unique de diffusion. Cette hypothèse établit des relations historiques entre les divers rituels d'initiation à la société tribale masculine en Australie, en NouvelleGuinée, en Mélanésie et en Afrique; en outre, elle étaie notre conclusion, à savoir que la dichotomie sexuelle n'est pas un phénomène universel, jaillissant spontanément des besoins de la nature humaine, mais un élément ethnographique ayant un seul centre d'origine et transmis ensuite à d'autres régions. Loeb a émis une intéressante hypothèse qui mérite d'être étudiée de près : le bull-roarer serait originaire d'une époque très ancienne, du paléolithique peut-être, et se serait répandu avec d'autres éléments cérémoniels, tels les rituels de mort et de résurrection.

Chapitre 11

\section{c. Classes d'age.}

\section{$\underline{\text { Retour à la table des matières }}$}

Avec la doctrine d'une différence sexuelle conduisant les hommes à former des associations et les femmes à s'en tenir aux liens de parenté, Schurtz, dans son système, établit un autre principe : les associations créées par la solidarité masculine dérivent toutes des classes d'âge. C'est de façon très différente qu'il nous faut estimer la valeur de ces deux affirmations. Nous avons vu que la première n'est que la généralisation d'un ensemble de faits ethnographiques dans lequel ce théoricien se trouvait plongé. Par contre, sa façon de concevoir la société comme un corps fractionné en couches d'âge nous livre sur le dynamisme social un aperçu profond, bien que Schurtz en fasse étroitement l'application à un type particulier de stratification selon l'âge. Si, négligeant dès l'abord sa formule spéciale du facteur âge, nous ne nous attachons qu'au principe général, nous verrons qu'en effet l'importance de ce facteur est surprenante.

Dans la famille elle-même, ainsi que le répète Schurtz, nous trouvons cette opposition entre l'ancienne et la nouvelle génération à laquelle Tourguenieff a donné son expression classique dans Pères et Fils. S'élever au-dessus des limitations imposées par l'âge demande un effort d'imagination dont la plupart des esprits sont incapables. Les gens âgés, se targuant de leurs expériences, n'apprennent jamais à s'abstenir sagement de donner des conseils qui ne seront pas suivis et ne pourront pas l'être; ils ne se rendent pas non plus compte de l'ennui profond que cause à leur jeune et impatient auditoire le récit interminable et vain de leurs souvenirs. Les jeunes, de leur côté, sont tentés de traiter les anciens de radoteurs bien intentionnés tout au plus, 
et de n'en faire aucun cas, mais ils n'ont pas le don prophétique qui leur ferait distinguer la sénilité pure de l'expérience de la vie. Souvent la rupture peut être évitée, mais il subsiste toujours la possibilité latente de discorde et une incompatibilité manifeste de pensée, de goûts et de mode d'existence. Il va de soi que cet isolement des générations ne se limite pas à la famille, puisque c'est en son essence non un combat personnel, mais une lutte de classes. Ainsi toute assemblée mêlée révèle la même scission, les mêmes différences de tempérament qui séparent pères et fils, mères et filles. Est-il une réunion qu'on pourrait croire plus exempte des imperfections de l'esprit humain qu'un congrès scientifique? Pourtant, en de telles occasions, point n'est besoin de beaucoup de pénétration pour deviner le mépris mal contenu que ressent le jeune chevalier défenseur de la vérité à l'égard du rabâchage de ses aînés, alors que le cynisme protecteur avec lequel ces derniers persifflent ses envolées, n'est surpassé que par l'indifférence suprême dont les jeunes font montre à l'égard de tout ce qui ne sort pas de leurs rangs. Ce groupement et cette différenciation sont si profondément enracinés dans l'esprit humain qu'ils ne peuvent manquer d'avoir leur répercussion sur le flot des variations culturelles, bien que la classe dominante ne soit pas partout la même et que l'idéal des diverses classes puisse varier. En Australie, l'emprise absolue qu'exercent les vieillards sur la vie publique est un phénomène évident; parmi les belliqueux Massaï, c'est aux jeunes guerriers célibataires que revient la préséance sociale; et dans quelques communautés des Indiens des Prairies, il y avait antagonisme constant entre les jeunes gens impatients de se distinguer par des incursions chez les tribus hostiles et les chefs qui cherchaient prudemment à éviter toute entreprise hasardeuse.

Jusqu'ici, donc, Schurtz est sur la bonne piste. Mais là où il se trompe, c'est lorsqu'il prétend que cette tendance invétérée doit toujours être formellement organisée et que là où elle est reconnue, elle entraîne invariablement une organisation tripartite de la société en garçons, célibataires et hommes mariés, ce système représentant la forme la plus ancienne d'association. C'est de nouveau là une généralisation sans fondement, basée sans doute sur les aspects pittoresques de l'existence chez les Massaï et les Bororo. Si nous étudions sans idée préconçue le club à degrés mélanésien, nous n'y relevons pas la moindre trace de discrimination entre les célibataires et les hommes mariés; une telle distinction serait même étrangère à l'esprit du club. En d'autres régions de l'Océanie, il est vrai, la division entre mâles s'opère en grande partie sur ce critère, puisque les célibataires ont leur dortoir à part et que les individus mariés dorment avec leurs épouses. Mais cette division est des plus imparfaites, car les hommes mariés passent souvent presque toute leur journée dans le dortoir des célibataires et y dorment même, ainsi qu'aux Fidji, en certaines occasions. Schurtz, comme nous l'avons déjà dit, est d'avis que le dortoir était à l'origine le local des célibataires et que ce ne fut que plus tard qu'il prit le caractère secondaire de club pour tous les hommes en général. Mais comment parvient-il à cette conclusion? Si nous nous limitons à une zone étroitement circonscrite de la Nouvelle-Guinée, nous relevons les variations suivantes : les Bukaua ont des salles de Conseil servant de locaux pour les célibataires et les visiteurs, mais les hommes mariés du village y dorment aussi à l'occasion, alors que les délibérations de tous les membres du sexe 
masculin ont lieu sur les plates-formes de ces édifices. Les Kaï ont des locaux publics pour le rituel de la circoncision seulement; les hôtes sont reçus par le chef et chaque famille a sa hutte à part. Plus à l'intérieur, les sexes sont complètement séparés, les hommes vivant ensemble dans des maisons particulières. Sur quel critère se baser pour déterminer l'antériorité relative de ces diverses conditions? En tenant compte de la tendance incontestable à la dichotomie sexuelle que présente cette région, ne pourrait-on soutenir que l'état mentionné en dernier lieu est chronologiquement le plus ancien et que les hommes en sont venus plus tard à dormir avec leurs femmes, laissant aux seuls célibataires la possession du dortoir qui autrefois avait abrité tous les hommes ? Selon cette supposition, il se serait produit d'abord une division de la tribu en initiés et non-initiés et ce n'est que sur le point particulier du local nocturne qu'il y aurait eu ensuite une segmentation secondaire des initiés. Cette hypothèse n'est nullement plus arbitraire que celle de Schurtz et paraît même concorder mieux avec les observations relevées.

En Afrique, il est des peuplades qui établissent une classification sur la base de l'état civil. Nous avons déjà décrit les coutumes des Massaï. Les Zoulou, sous le roi Chaka, nous en fournissent un autre exemple : ce chef choisissait ses guerriers en séparant les célibataires des hommes mariés. Mais, en beaucoup d'autres tribus, cette distinction ne produit aucune division dans la société; les initiés forment une classe à part, quel que soit leur état civil.

Schurtz reconnaît que l'Asie présente peu de systèmes d'organisation tripartite, sauf à l'extrême sud, dans le rayon de l'influence malaise. Les Andaman, bien que de race négrito, n'ont évidemment pas échappé à ce courant culturel. Chose significative, ils ne séparent pas seulement les célibataires mâles, mais aussi les femmes non mariées. Or cette double ségrégation a dans le monde une distribution géographique limitée. Elle se rencontre parmi quelques tribus des Philippines comme les Bontoc Igorot, à Sumatra, parmi les Naga d'Assam et les Dravidiens de l'Inde méridionale. On ne peut tenir pour accidentel le fait que les Négrito andaman ainsi que des tribus voisines présentent cette variante caractéristique de ladite institution ; il est donc à supposer qu'ils ont emprunté cette coutume ainsi que beaucoup d'autres éléments de leur culture, tel le bateau à balancier, aux peuplades plus avancées avec lesquelles ils sont entrés en contact. Ce point est important, car l'évolution spontanée parmi les Andaman d'un groupe de célibataires non seulement confirmerait la thèse de Schurtz selon laquelle le système tripartite serait un produit social naturel, mais étaierait aussi dans une large mesure sa chronologie; il serait, en effet, vraisemblable qu'un peuple aussi primitif que les Andaman ait conservé un système d'organisation fort ancien.

Cependant, c'est en Amérique du Nord que nous trouvons une réfutation écrasante de la théorie de Schurtz. A part les faibles indices d'un groupement des célibataires dans le sud-ouest des États-Unis et parmi les Esquimaux de l'Alaska, les différences entre individus mariés et célibataires n'ont pas influencé la structure sociale. Même là où on nous signale des dortoirs de célibataires, rien ne nous prouve que ce groupement soit fondamental. Chez les Zuñi et les Hopi, il est certain que tous les hommes 
initiés, quel que fût leur état civil, faisaient partie de la société tribale, et une quantité d'autres liens n'avaient rien à voir avec ce facteur. Schurtz suppose que le sudatorium du nord de la Californie n'était autre que le dortoir des célibataires, mais les faits ne correspondent pas à cette opinion.

Si peu nombreuses que soient en Amérique du Nord les preuves d'une ségrégation des hommes mariés et des célibataires, il est encore plus difficile d'en trouver démontrant une différenciation formelle entre les jeunes gens initiés et ceux qui ne le sont pas. A part le cas des Pueblo déjà cité, il n'y en a pour ainsi dire pas. Dans son désir d'établir des processus d'évolution semblables dans les diverses régions, Schurtz identifie les jeûnes de puberté en Amérique et les fêtes d'initiation en Australie et en Afrique. Mais c'est là, certes, la moins défendable de ses opinions, bien que Webster l'ait malheureusement adoptée à son tour. Le rituel d'initiation des Arunta et des Massaï, par exemple, est une affaire tribale et tout individu doit passer par là avant de se marier. Cependant, lorsqu'un jeune Hidatsa ou un Crow se retire sur quelque colline solitaire et dénudée et mortifie sa chair pour se faire entendre des êtres surnaturels, cela ne concerne nullement la communauté, c'est une question personnelle ou tout au plus familiale. S'il réussit à avoir une vision, il en retirera un avantage personnel; sinon, personne ne peut lui reprocher d'avoir échoué. En fait, les Indiens des Prairies ne bénéficiaient pas tous de visions, loin de là. En général, la vision ne modifiait pas la position sociale des Indiens et n'avait rien à voir avec leur avenir matrimonial. En outre, la recherche de la vision ne coïncidait pas nécessairement avec l'époque approximative de la puberté. Parmi les Arapaho, c'étaient d'ordinaire les hommes adultes qui essayaient d'avoir des esprits gardiens; le cas est exceptionnel, sans doute, mais il est facilement explicable que ce fût plutôt l'adolescent qui recherchât la faveur divine : le jeune Indien des Prairies avait l'ambition de se distinguer sur le champ de bataille; il était obsédé par l'exemple des héros qui avaient jeûné, qui s'étaient peut-être torturés, mais qui avaient été récompensés par une vision et s'étaient par là acquis un grand renom. Rien n'était donc plus naturel pour celui qui aspirait à une telle gloire que de suivre l'exemple laissé par ces hommes, et cela dès que l'occasion s'en présenterait, mais en fait souvent après l'âge de la maturité physiologique. Il se soumettait volontiers aux mortifications qu'il s'infligeait lui-même dans le seul espoir d'apitoyer les puissances universelles. Rien ne ressemble donc moins aux tortures que devait endurer un jeune garçon chez les Arunta ou les Massai. Bref, le jeûne de puberté en Amérique du Nord n'était pas une cérémonie tribale d'initiation, n'entraînait pas la formation d'un groupe de célibataires différencié des garçons non initiés et n'était souvent même pas un rite de puberté.

Il semble que les phénomènes nord-américains aient voulu donner un démenti formel au système de Schurtz. En effet, ce continent, qui est le plus dépourvu de tous en cérémonies de jeunes garçons, est aussi celui où se détachent avec le plus d'évidence les fêtes de puberté des jeunes filles, ou tout au moins les rites qu'on observe à l'époque de la première menstruation. Diverses tribus, telles les Apache, les Dakota et les Shasta, célébraient alors des cérémonies fort complexes. Celles-ci n'entraînaient pas, il est vrai, l'organisation d'une unité sociale distincte. Il est toutefois notable que, 
d'une part, alors que l'existence de telles formalités fournissait une base à une classification éventuelle des femmes, de l'autre, l'absence presque totale de rites de puberté pour les garçons était loin de favoriser un groupement analogue chez les membres du sexe masculin.

Ainsi, alors que le facteur âge est une véritable déterminante de la vie sociale, comme nous le prouverons encore plus loin, la conception particulière d'une triple classification des mâles selon l'âge, modifiée par les conventions relatives à l'initiation et au mariage, doit être tenue pour erronée et écartée.

Chapitre 11

\section{d. Types d'associations.}

$\underline{\text { Retour à la table des matières }}$

Il serait possible de continuer de la même manière l'analyse du système de Schurtz, mais il nous paraît préférable d'y revenir par une autre voie. Cessons donc d'analyser l'application de ses principes aux diverses régions du globe et considérons les modalités associationnelles à l'intérieur d'une zone culturelle limitée. Dans ce dessein je choisirai la région des Prairies en Amérique du Nord. Après avoir énuméré les variétés les plus importantes d'associations qu'on y relève, je procéderai à l'étude historique des phénomènes se rangeant sous la rubrique des sociétés d'âge.

On peut citer comme un embryon d'association d'un caractère tout à fait particulier l'union de deux amis non apparentés qui se sont juré aide réciproque et camaraderie éternelle. C'était surtout parmi les Dakota et leurs congénères que florissait cette amitié à la Damon et Pythias. Les obligations morales qu'elle imposait apparaissent clairement dans un conte des Assiniboin : un père renie son fils qui s'est montré un camarade déloyal et l'ami trompé se sent si honteux qu'il se retire volontairement en exil. Dans la formation de telles amitiés, l'âge jouait indubitablement un rôle essentiel, mais l'exclusivisme de ce lien créait un type tout différent de celui que suppose Schurtz. Il se formait un nombre infini de paires d'amis, représentant autant d'amitiés indépendantes, mais celles-ci n'allaient pas jusqu'à opérer une coupe transversale de la société à la manière des classes d'âge.

Les associations basées sur une expérience surnaturelle commune sont d'un caractère tout différent. On pourrait s'attendre à trouver ce type de société dans toute cette zone puisque les indiens des Prairies recherchent tous des visions; il serait bien naturel, d'autre part, que des individus, protégés par les mêmes esprits gardiens, se sentissent unis par un sentiment de solidarité sociale. Pratiquement, ce résultat n'a été atteint que dans le sud et seulement parmi des tribus intermédiaires, comme les Dakota. Une abondance extraordinaire de telles associations a été signalée chez les Omaha où les individus qui ont eu la vision du Bison ou du Tonnerre se réunissent en 
organisations shamanistes, douées parfois de vertus médicales et présentant toujours un caractère nettement religieux. Ces groupes se composent d'individus d'âges divers et, en principe, n'excluent pas les femmes. Ils s'écartent donc autant qu'il est possible du type premier d'association tel que Schurtz l'imagine. Cependant, le rôle qu'ils jouent dans l'existence des Omaha indique qu'ils représentent une très ancienne acquisition culturelle de la tribu. D'un autre côté, certaines sociétés de fête parmi ces mêmes Omaha, représentant en substance les classes d'âge, sont d'importance très accessoire. Ces dernières sont au nombre de trois : celle des hommes mûrs, celle des jeunes gens et celle des garçons, formant chacune un groupe à part de commensaux. Schurtz ne manque pas d'avancer ce fait à l'appui de sa théorie et prétend que c'est là le résidu d'un ancien système à trois classes qui, cela va sans dire, aurait précédé toute autre forme d'association. Il ne daigne, du reste, pas nous faire part de ce qui, dans l'histoire des Omaha, le porte à affirmer que cette camaraderie de table est la plus ancienne. Privée de toute autre fonction sérieuse, elle jouait un rôle si secondaire qu'elle n'est même pas mentionnée dans la longue monographie de Miss Fletcher et de la Flesche. Pour nous, cet exemple démontre surtout l'aspect varié et parfois banal que revêt le facteur âge, lequel n'introduit pas nécessairement une scission primordiale de toute la société. Étant donné la faible diffusion et le peu d'importance des camaraderies de table chez les Omaha, il semble à peu près certain qu'elles représentent un développement accidentel, ultérieur aux corporations religieuses qui reposent sur l'un des traits essentiels de leur civilisation.

L'exemple de deux organisations de danse chez les Omaha correspondant aux Chiens et aux Renards des tribus du nord: est encore plus révélateur. La première ne comprenait des vieillards et des hommes mûrs; la seconde était constituée par des garçons. Voici donc une stratification par âge bien marquée. Une seule question se pose : à quelle date remonte-t-elle ? Par bonheur, nous connaissons l'histoire de ces sociétés. Les Omaha les ont empruntées toutes deux assez récemment aux Ponka qui, à leur tour, les tenaient des Dakota. Or les Ponka n'introduisent nulle gradation dans leurs associations; celles-ci, que pour plus de commodité j'appellerai les Chiens et les Renards, comprennent des hommes adultes et des jeunes garçons; mais les Chiens sont en général plus âgés, quoique les jeunes gens d'avenir y soient aussi admis. Les conséquences théoriques de ces faits sont fort intéressantes. Puisque les organisations des Ponka n'étaient pas différenciées selon l'âge et gardaient tout au plus de leur prototype dakota un embryon presque imperceptible de classification par âge, l'application du critère de l'âge représente donc une addition indépendante des Omaha. Cela revient à dire que l'âge n'intervient que tardivement dans l'histoire de ces sociétés; les Omaha reconnaissent eux-mêmes que c'est un élément récent. En outre, ce facteur ne modifie pas les organisations les plus anciennes et les plus caractéristiques, mais seulement celles qui sont nettement d'origine étrangère. C'est là une belle preuve de la vigueur du facteur âge et nous ne saurions nier qu'il a pu se manifester sporadiquement à des époques antérieures aussi. Cependant rien ne nous le prouve. Tout ce que nous savons, c'est que, chez les Omaha, il ne forme pas la base des anciennes sociétés et ne s'est que récemment imposé dans des organisations étrangères. 
Les Renards et les Chiens nous introduisent dans la catégorie de ce qu'on appelle les sociétés militaires. Mais puisque, pour ce qui nous occupe actuellement, le mode d'admission est plus significatif que la fonction, nous devons les subdiviser en deux classes tout au moins : celles qui se conforment au type Crow et celles qui suivent le modèle des Hidatsa. Rappelons que les premières sont sans degrés, les individus étant invités à en faire partie ou y entrant à leur gré; les autres sont graduées selon l'âge et on n'y est admis que contre une finance d'entrée. L'un de ces types a pour base à la fois l'âge et l'achat, l'autre est indépendant de l'âge. Mais, comme il est possible que ces sociétés aient perdu leurs degrés au cours de leur évolution, nous reviendrons plus loin à ce cas.

Cependant il existe des sociétés aux fonctions variées où la qualité de membre ne dépend pas de l'âge, mais d'un paiement. L'Ordre du Tabac chez les Crow rentre dans cette catégorie, ainsi que les corporations féminines des Cheyenne que nous avons déjà décrites à d'autres égards. Parmi les Hidatsa, dans les confréries des " paquets " qui sont en corrélation avec les cérémonies les plus sacrées de la tribu, le titre de membre est héréditaire, mais doit être racheté à un taux prescrit.

Il n'est pas nécessaire d'énumérer les associations des Indiens des Prairies. Nous en avons dit assez pour montrer que les modalités d'admission varient, que l'âge dans quelques-unes des sociétés militaires n'est qu'un des facteurs déterminants et que, si nous nous en tenons aux faits observés, il n'est pas certain que ce soit le facteur prédominant. Le système tripartite n'est, en outre, appliqué que dans les sociétés de fêtes des Omaha, d'importance secondaire. Ce serait donc le comble de l'arbitraire que de décréter qu'une particularité relativement rare dans cette zone et virtuellement absente sous ce que Schurtz considère comme sa forme typique, doive être la plus ancienne, celle à laquelle toutes les autres seraient venues se surajouter. On se demande comment une notion comme celle de la vision qui a donné naissance à tel type d'association, aurait pu sortir de la conception toute différente d'un groupement selon l'âge et surtout d'une congrégation de célibataires ou d'hommes mariés. Et si cette première catégorie n'a pas évolué, mais a une origine indépendante, pourquoi ne serait-elle pas plus ancienne que le critère d'âge en tant que mode de classification? A toutes ces questions, il serait bien difficile à un disciple de Schurtz de répondre.

Si nous élargissons notre enquête et l'étendons au monde entier, il va de soi que nous rencontrerons encore bien d'autres principes de classification, rendant encore moins possible une réduction moniste de toute la série des associations. Mais il vaut mieux prendre le taureau par les cornes et examiner avec attention l'histoire de tel groupement d'âge afin de déterminer par là dans quelle mesure la théorie de Schurtz est admissible ou de quelle manière peuvent s'éclairer les problèmes généraux ou théoriques en question. La quantité d'études consacrées aux sociétés d'âge parmi les Indiens des Prairies nous incitera une fois encore à choisir cette région comme présentant le terrain le plus favorable à une enquête critique. 
Chapitre 11

\section{e. Les sociétés d'age chez les indiens des prairies.}

$\underline{\text { Retour à la table des matières }}$

Nous avons vu que, d'après le système des Hidatsa, la population masculine est divisée en dix classes environ, d'importance croissante; chacune possède sa danse, ses chants, ses accessoires et ses privilèges. Ces classes n'étaient pas accessibles automatiquement aux individus qui avaient atteint un âge déterminé; tout un groupe de contemporains devait acheter conjointement le droit d'entrée. Ce système n'est pas restreint aux Hidatsa, mais s'étend à leurs voisins immédiats, les Mandan et à trois autres tribus, les Pieds Noirs, les Arapaho et les Gros Ventres. Ces variantes doivent donc être étudiées historiquement comme un tout. Mais ce n'est pas encore suffisant. Quoique ce système d'organisation qui présente l'action conjointe des facteurs âge et achat soit limité aux cinq tribus mentionnées, les complexes et les éléments qui caractérisent ces degrés ont une distribution géographique beaucoup plus vaste.

Par exemple le degré du Chien chez les Hidatsa, accessible aux hommes d'âge et même aux vieillards, a entre autres insignes une certaine ceinture fendue, une sonnaille en sabots de daim ou de bison et une coiffure en plumes de hibou. La société des Chiens chez les Crow, constituée par des hommes de tout âge, arbore les mêmes emblèmes. Il est incontestable que ces deux complexes ont une seule origine, mais laquelle des deux tribus l'a emprunté à l'autre? Le cas se complique encore du fait que ces éléments se retrouvent aussi dans les quatre autres tribus à degrés et chez d'autres peuplades classées en séries non graduées, comme les Cheyenne et les Dakota. Ainsi, sur la seule base des faits avancés jusqu'ici, la danse du Chien pourrait s'être développée dans l'une quelconque de ces tribus et avoir erré çà et là par bien des voies différentes. Des problèmes analogues se posent à propos de complexes hidatsa se rattachant à d'autres degrés, et d'autres problèmes particuliers se présentent.

Ce ne sont pas, cela va sans dire, les détails historiques qui nous intéressent surtout. Nous désirons savoir si telle danse, commune aux systèmes à degrés et à ceux non gradués, s'associe a il origine avec un groupe d'âge ou non. Si la réponse est négative, une fois de plus la succession des faits, telle que l'établit Schurtz, se trouvera renversée, puisque le groupement se sera à l'origine formé sur une autre base et n'aura adopté que dans la suite le critère de l'âge. Mais si, au contraire, la société du Chien, du Renard ou du Bois renflé était à l'origine une organisation de contemporains, la théorie de Schurtz serait, dans cette mesure tout au moins, corroborée. 
Cependant il surgirait alors une autre question : que signifie la corrélation entre telle danse et le facteur âge? Cela veut-il dire que la danse est associée aux hommes d'un âge déterminé, par exemple aux hommes mariés, aux jeunes gens ou aux vieillards de soixante ou soixante-dix ans? ou cela implique-t-il simplement que l'essentiel pour tous les membres est d'être contemporains? Et finalement, puisque nous nous occupons de classes d'âge, pourquoi retrouvons-nous si souvent le facteur achat?

Cette dernière particularité va nous permettre d'entrer dans le problème. Ce n'est pas simplement le rapport entre l'âge et l'achat qui retient notre attention, mais aussi le rapport également important entre l'achat et les sociétés militaires non graduées. Dans une véritable stratification par âges, la promotion devrait être automatique. Schurtz affirme que tel était bien le cas à l'origine et que la notion de paiement est venue s'ajouter dans la suite. L'ordre de succession serait donc : tout d'abord avancement automatique avec l'âge, puis introduction de quelque autre condition. Fort bien. L'absence du facteur d'âge et la présence du facteur achat relèveraient donc d'une époque ultérieure. Mais, s'il en est ainsi, pourquoi, dans les sociétés militaires, l'élément prétendu postérieur de l'achat s'agrippe-t-il tenacement à celui qui nous est représenté comme le plus ancien de toutes les associations? Par contre, on ne le trouve jamais associé à des notions plus ou moins contemporaines, selon la théorie de Schurtz. Tout ceci est bien mystérieux et donne à penser que cette chronologie hypothétique est fausse et que l'affirmation selon laquelle les sociétés d'âge des Prairies sont au fond des classes d'âge authentiques, pourrait bien être dépourvue de fondement.

Attaquons-nous donc tout d'abord au problème suivant les complexes ou degrés dans les séries d'âge représentent-ils vraiment un âge déterminé ou bien un rang précis dans la série? Comparons des complexes équivalents dans différentes tribus, ou le même complexe au sein de la même tribu mais à des époques diverses. Le complexe du Chien, très fréquent et s'associant presque toujours à une société importante, nous fournira un exemple favorable. En 1833, le prince Maximilien observa que les Chiens des Pieds Noirs étaient surtout des jeunes gens, alors que ceux des Hidatsa et des Mandan étaient des hommes mûrs, ainsi que chez les Arapaho et les Gros Ventres, comme l'a prouvé Kroeber. Si, sans nous en tenir à la statistique pure, nous évaluons l'importance de cette organisation dans la zone des Prairies, nous considérerons certainement comme atypique la phase ancienne des Pied

Noirs. Il est cependant impossible d'affirmer catégoriquement que la danse du Chien appartient aux hommes mûrs ou s'associe à un âge quelconque, pour la simple raison que nous n'ignorons pas que cette relation avec l'âge a varié selon les époques dans une même tribu. Les Pieds Noirs d'il y a quarante ans assignaient aux Chiens un rang beaucoup plus élevé que ce n'était le cas vers 1830, et parmi les Hidatsa ils représentaient en même temps la catégorie des vieillards.

D'autres complexes suggèrent la même conclusion. La société du Renard, également commune, était un groupement de jeunes gens parmi les Hidatsa, d'hommes 
d'un certain âge chez les Gros Ventres, alors que parmi les Pieds Noirs elle était en 1833 supérieure à celle du Chien et plus récemment atteignait presque la place la plus élevée dans la série. Les Corbeaux des Pieds Noirs étaient des hommes mûrs à l'époque du prince Maximilien, mais, en la même année, c'était parmi les vieillards qu'ils se recrutaient chez les Hidatsa. Il est donc évident qu'il n'existait pas de relation essentielle entre tel complexe et tel âge dans une peuplade déterminée. Comme un complexe faisait partie d'une série hiérarchique, il s'ensuivait inévitablement qu'à un moment donné il devait occuper un rang défini, impliquant un rapport d'âge plus ou moins déterminé puisque les contemporains achetaient en groupe le droit d'entrée. Mais c'est tout. Un changement dans la situation, quelle qu'en fût la cause, ne passait évidemment pas pour un outrage à l'ordre immuable des choses. Tant que les possesseurs d'un complexe appartenaient à la même classe d'âge il importait peu qu'ils eussent quinze ou soixante-dix ans.

C'est ce que nous démontrent encore plus nettement les rapports autobiographiques d'informateurs indiens. Lorsque les conditions de vie modernes firent disparaître les anciennes coutumes chez les Mandan et les Hidatsa, on cessa d'acheter l'admission aux degrés. Les individus qui seraient normalement devenus des Taureaux ou des Corbeaux ne purent plus faire cette dépense, et il en alla de même sur toute l'échelle. Il fut impossible aux hommes âgés non seulement d'acheter leur avancement mais aussi de se défaire des prérogatives qu'ils possédaient, puisque aucun groupe plus jeune n'en était acquéreur. Chose bizarre, il arriva alors qu'un homme conservât jusqu'à sa mort des privilèges acquis dans sa jeunesse, ce qui n'aurait pas pu se produire si c'était le facteur âge qui avait prédominé. Pour prendre un exemple concret, un individu nommé Pauvre-Loup se trouvait à quatre-vingt-dix ans le possesseur d'un complexe acheté à sept ans, d'un autre qu'il s'était procuré à vingt ans, d'un troisième qu'il possédait depuis vingt-sept ans et d'un quatrième obtenu vers quarante-cinq ans. Le principe qui apparaît alors manifestement et que formulent les indigènes euxmêmes est simple : un homme possède tous les complexes qu'il a achetés s'il ne les a jamais revendus par la suite. Or on ne peut pas à quatre-vingt-dix ans être classé comme le contemporain d'enfants de sept ans et c'est monstrueusement absurde d'être simultanément membre de trois ou quatre classes d'âge différentes; mais cet individu peut très bien posséder tous les titres qu'il a acquis au cours de son existence. En d'autres termes, la notion qui forme la base d'un degré dans une série est la possession de droits de propriété dûment acquis, l'élément âge étant secondaire.

C'est aussi à cette conclusion que tendent certaines particularités du système des Gros Ventres. Ici les contemporains ne se substituent pas à un groupe homogène situé au-dessus du leur, mais achètent les privilèges cérémoniels désirés à une assemblée hétérogène comprenant des individus de tous les groupes qui ont acquis en même temps ces droits. L'origine immédiate d'une telle acquisition était toujours le vœu fait par un membre du groupe d'opérer cette transaction s'il se remettait de telle maladie ou si telle difficulté s'aplanissait. Dans ces conditions, rien n'empêchait plusieurs classes d'âge de détenir simultanément le même complexe cérémoniel et, en fait, les Gros Ventres avaient un nombre bien plus élevé de classes d'âge que de danses, 
chaque danse étant la propriété de plusieurs classes. C'étaient ces classes qui formaient les unités sociales. Aucun lien ne réunissait les trois ou quatre classes qui possédaient le même complexe. Chacune exerçait ses privilèges à part et leur caractère distinct était souligné par des appellations différentes qui, contrairement au nom de leurs danses, ne changeaient pas avec le temps, mais duraient toute leur vie. Ainsi, par exemple, un homme partageait avec les hommes de sa classe, mais avec eux seulement, la désignation permanente et exclusive de " Qui-se-tient-à-la-queue-duChien ». Mais il pouvait devenir pour une période limitée un Chien ou un Renard et posséder ce titre en commun avec des individus d'autres classes également. En d'autres tribus, certains complexes de biens immatériels étaient détenus exclusivement par une seule corporation; les Gros Ventres, eux, admettaient que plusieurs corporations possédassent simultanément un même complexe, mais sans impliquer par là qu'elles perdissent leur individualité distincte. Cette situation était analogue à celle, par exemple, de plusieurs maisons d'édition en Angleterre, en France et en Amérique qui auraient le droit de publier un même livre.

En d'autres termes, alors que les classes des Hidatsa pouvaient posséder en même temps des complexes relevant des degrés les plus variés, chez les Gros Ventres le même degré ou complexe était détenu à la fois par plusieurs classes distinctes. Ces complexes étaient donc des biens négociables pouvant être associés a priori avec différents âges et différents degrés. Un seul problème se pose : comment ces complexes en sont-ils venus à être gradués en une série associée à des différences d'âge? C'est la question de l'origine des degrés d'où découle tout un ensemble de problèmes relatifs à la manière dont certains complexes se sont associés à certains degrés. Schurtz ne se préoccupe guère du développement ultérieur du système et le considère en fait comme un aspect plus compliqué de la division tripartite des tribus. Pour nous, au contraire, la connaissance historique positive des modifications récentes est la base de toute spéculation sur des phénomènes plus anciens. C'est donc à la manière dont les complexes ont été gradués durant la période historique que nous attacherons le plus d'importance.

Le facteur qui a évidemment exercé l'influence la plus profonde sur le développement ultérieur du système des degrés, est l'imitation des sociétés étrangères ou les achats qui leur ont été faits. Nous savons qu'en 1833 les Mandan n'avaient pas de société du Renard, qu'ils l'empruntèrent plus tard aux Hidatsa et l'incorporèrent à leur propre système. De même, les Hidatsa adoptèrent des Mandan la société du Chien enragé et les Mandan empruntèrent aux Hidatsa l'organisation du Petit Chien. A l'époque de Maximilien, les Marteaux de pierre des Hidatsa avaient acquis la Danse ardente des Arikara, ce qui eut pour effet qu'un complexe non gradué fut associé à un degré défini. Il devint par la suite possible aux Marteaux de pierre soit d'assimiler complètement le nouvel élément à l'ancien, soit de conserver dissociés les deux complexes et de les vendre indépendamment. Quoiqu'on ne sache pas exactement pour quelle solution ils optèrent, il semble que ce fut pour la seconde, qui impliquait l'addition à la série d'un nouveau degré. Lorsque les possesseurs furent sollicités par la classe cadette, ils lui vendirent soit la Danse ardente, soit le complexe des 
Marteaux de pierre. Dans le premier cas, la danse récemment acquise aura constitué le degré le plus bas, dans le second cas l'avant-dernier.

Il ne fait aucun doute que cette manière de procéder ait été en vigueur bien longtemps avant que nous n'en possédions de preuves. Par exemple, les Arapaho et les Gros Ventres sont des tribus étroitement apparentées avec des systèmes de degrés très semblables. Les Gros Ventres, après leur séparation d'avec les Arapaho, vécurent avec les Pieds Noirs. L'un des points sur lesquels les Gros Ventres s'écartent du système des Arapaho est la Danse de la Mouche, tout au bas de la série. Cette danse se rencontre aussi parmi les Pieds Noirs chez qui elle a été signalée pour la première fois ainsi que dans l'une des tribus voisines des Pieds Noirs, mais nulle part ailleurs. La seule conclusion possible est que les Gros Ventres l'ont empruntée aux Pieds Noirs et l'ont incorporée à leur système. Or cette incorporation dut forcément affecter tout le reste de la série, les différents degrés perdant leur place respective. Partout où un système a ainsi reçu l'apport d'éléments étrangers, il s'en est suivi une modification complète et manifeste de toute la série. Tout semble indiquer que c'est précisément ce qui s'est produit et, vues de cet angle, les anomalies étranges, relatives au rang de telle société dans les différentes tribus, deviennent compréhensibles. Si les Pieds Noirs ont adopté le complexe du Chien plus tard que celui du Renard, l'amour caractéristique des primitifs pour tout ce qui est ancien leur aura fait placer leur nouvelle acquisition au bas de l'échelle. En d'autres cas, le caractère sacré d'un complexe récemment acheté a pu le faire passer avant tous les autres, dont la position, par suite, se trouva modifiée. Puisqu'il est démontré que chacun des cinq systèmes s'est développé peu à peu par de tels apports, il s'ensuit naturellement que les divers complexes n'ont qu'un rapport de hasard avec un rang ou un âge donnés.

Toutefois, l'élaboration des séries graduées n'a pas eu pour seul agent la diffusion. Tout système, une fois formé, présentait une tendance naturelle à entrer en relations avec d'autres complexes, d'origine indigène peut-être. C'est ce qui est arrivé parmi les Hidatsa pour le rituel du Bâton à Encoches.

Pour autant qu'on en puisse juger, c'était une cérémonie indigène, n'ayant rien à voir avec les classes d'âge. Mais l'un des villages hidatsa l'intégra dans son système où elle constitua le degré le plus bas. Les conséquences qui en résultèrent Pour les autres degrés sont naturellement identiques à celles qu'aurait entraînées l'adoption d'un complexe étranger.

Jusqu'ici, un disciple de Schurtz serait d'accord avec nous et interpréterait les faits historiques à l'appui de ses théories. "Fort bien, dirait-il, l'amplification des séries doit être relativement récente, car, à l'origine, il n'a pu exister que les trois classes fondamentales des garçons, des célibataires et des anciens. Celles-ci ne sont pas dues à des emprunts, ni à une évolution interne ultérieure, mais représentent un groupement premier, résultant d'une loi sociale immuable. » Cependant, nous avons quelque difficulté à admettre que les principes dont nous avons observé l'action pendant toute la période historiquement connue, aient pris corps soudainement alors qu'ils auraient 
été inopérants durant la période qui précéda immédiatement. Et, en fait, la réalité nous prouve indubitablement que le système des sociétés graduées dérive en tant que système d'une source unique.

En comparant les séries des Pieds Noirs et celles des Hidatsa, par exemple, nous trouvons une conception identique de l'achat, jointe à un autre élément caractéristique, la remise cérémonielle de la femme au vendeur, ainsi que la présence de quatre complexes communs : le Renard, le Chien, le Corbeau et le Taureau. Ce serait folie dans ce cas d'envisager une évolution indépendante. L'une des deux tribus a certainement emprunté son système à l'autre ou à une source commune; et si la comparaison s'étend aux trois autres peuplades possédant des sociétés à degrés, les analogies observées nous renforcent dans notre conviction que ce ne sont pas là cinq évolutions spontanées d'un système gradué, mais qu'un seul système de base a subi des modifications locales dans les différentes tribus. Même en accordant que les degrés ou danses représentent à l'origine la division tripartite, cette hypothèse ne pourrait s'appliquer qu'à la seule tribu qui a transmis le système aux autres. Ces dernières présentent cette particularité non point en vertu d'une loi de progrès inhérente, mais parce qu'elles sont entrées en contact avec la peuplade qui a produit ce phénomène. Pour autant qu'elles sont en cause, rien ne prouve que, dans ces tribus, les hommes se groupent automatiquement en trois classes d'âge ou davantage. D'autres faits s'élèvent encore à l'encontre de la théorie de Schurtz. Celui-ci n'est, en effet, nullement autorisé à prétendre que la tribu qui la première a présenté les sociétés à degrés, celle des Hidatsa, par exemple, a créé à la fois la notion de degré et les sociétés elles-mêmes. Alors que cinq tribus des Prairies présentent des sociétés d'âge, d'autres peuplades en nombre considérable ont les mêmes complexes, mais sans relation avec l'âge. Il est donc possible et probable que, même parmi les Hidatsa, la graduation est un phénomène secondaire ; ils ont copié chez leurs voisins quelque organisation non graduée et d'une façon ou d'une autre en sont venus à disposer les degrés hiérarchiquement. L'importance de ce processus est manifeste; il détruit dans sa racine le raisonnement de Schurtz. Ce n'est point une classification selon l'âge mais quelque autre modalité sociale qui est à la base des organisations guerrières des Prairies, le facteur âge n'apparaissant qu'à une époque relativement tardive et dans une variante spécialisée de ces sociétés.

Nous avons déjà vu, à propos des variétés de types associationnels, quels ont pu être ces agents sociaux originels. L'influence des visions, l'élément culturel fondamental de la région, se fait sentir de différentes manières. Il peut y avoir groupement des individus selon leurs visions, comme chez les Omaha; ou bien le visionnaire peut en initier d'autres et organiser avec eux une société distincte, telle la Danse du Tabac chez les Crow; ou encore il peut apprendre à un groupe à célébrer la cérémonie dont il a rêvé, l'union d'abord temporaire des participants finissant alors par se stabiliser, ainsi que tel a été le cas probablement chez les Dakota de l'est. Une particularité culturelle également importante de cette région est l'expédition guerrière, et il semble bien que parmi les Dakota les compagnons d'armes aient formé une association permanente. Plusieurs causes ont donc contribué à produire ces associations masculines, 
bien avant que prît naissance ce phénomène hautement localisé et spécialisé qu'est l'association graduée selon l'âge.

Il convient de récapituler les points essentiels de cette argumentation qui a été forcément quelque peu complexe. En présence d'un système chronologique qui faisait dériver les clubs, les sociétés secrètes et toutes les autres associations des classes d'âge, nous avons étudié, en nous appuyant sur les phénomènes d'une zone donnée, leur succession hypothétique. D'une rapide étude des associations chez les Indiens des Prairies, il est ressorti que les classes d'âge ne forment nullement le groupement prédominant, et que dans certains cas il est possible de démontrer que le groupement selon l'âge est un élément secondaire. Restreignant la discussion aux systèmes sociaux qui indiquent clairement une stratification par âges, nous avons découvert plusieurs faits significatifs. Les prétendus degrés avaient pour les indigènes si peu de fixité qu'une même société, représentant dans telle tribu un club de jeunes gens, se composait dans une autre d'hommes mûrs et même de vieillards. Les Indiens nous prouvent donc par ce traitement des degrés qu'ils ne se divisaient pas essentiellement selon l'âge, mais achetaient surtout certaines prérogatives cérémonielles estimées; un individu pouvait atteindre simultanément plusieurs degrés, ce qui aurait été parfaitement impossible si ces degrés avaient représenté des différences d'âge ou d'état civil. Restait le problème du développement historique des séries graduées. Préférant raisonner du connu à l'inconnu, nous avons trouvé que, dans la période sur laquelle nous sommes directement renseignés, le facteur principal a été la diffusion et que la complexité des systèmes observés est due à des apports successifs par emprunts. Il serait cependant concevable qu'à une époque antérieure un groupement tripartite plus simple ait été à la base de ces systèmes de sociétés, en harmonie donc avec la grande loi d'évolution sociale que préconise Schurtz. Mais puisque les cinq tribus ont indubitablement tiré leurs séries graduées d'une seule source, cette loi n'aurait pu agir que dans le cas d'une seule de ces tribus; pour les quatre autres, le système tripartite hypothétique n'eût pas été l'effet de quelque force sociale inhérente, mais d'emprunts, et il n'aurait pu être question de " loi ». Même en ne prenant que la peuplade qui la première divisa ses sociétés selon l'âge, il serait téméraire de prétendre que ce fut elle aussi qui créa les premières sociétés guerrières puisqu'on trouve bien plus fréquemment ces dernières dépourvues de degrés. Enfin les phénomènes essentiels de l'existence chez les Indiens des Prairies présentaient diverses possibilités d'associations dont il a été effectivement fait usage.

Ainsi le système unilinéaire de Schurtz, reposant sur la théorie d'une division tripartite, ne peut même pas s'appliquer à un type de société qui présente des marques ostensibles d'un groupement selon l'âge. Dans une zone fertile en associations, les classes d'âge apparaissent comme un phénomène local et tardif qui, de plus, ne correspond pas à la division tripartite.

Cependant, même après qu'ait été sapée dans sa base la chronologie de Schurtz, un fait reste certain, c'est que l'âge a joué son rôle dans l'histoire des organisations militaires, bien que de façon différente de celle qu'imagine cet auteur. Parmi les 
Crow, les Dakota et les Kiowa qui avaient tous des sociétés coordonnées, la vraie classe d'âge se formait de la manière la plus naturelle du monde, lorsque les garçons imitaient les organisations de leurs aînés. Ce fait, apparemment banal, nous fournit une indication sur l'origine possible des degrés. Supposons que, parmi ceux qui les ont créés, les Hidatsa par exemple, ce mimétisme juvénile fût en vogue. Supposons encore qu'il existât une seule société à laquelle une partie ou la totalité des adultes avaient accès sur paiement d'un droit d'entrée, cette assemblée rehaussant le prestige social de ses membres, ce qui est un motif constant d'association chez les primitifs. Il ne manquait alors plus aux jeunes garçons, dans leur impatience de posséder un vrai complexe de danses et de privilèges, que d'acheter conjointement la qualité de membre. Le caractère collectif de la transaction constitue son élément révolutionnaire, puisque par là le groupe amorphe des compagnons de jeux devient une classe d'âge aussi nettement définie que celle des jeunes Massaï se soumettant ensemble à la circoncision. Les vendeurs dépossédés perdaient leur danse, mais leurs liens antérieurs les maintenaient vraisemblablement unis et, à la première occasion, ils voyaient en songe, c'est-à-dire inventaient, une nouvelle danse, ou en achetaient une à quelque tribu étrangère. Cette nouvelle danse devenait le but prochain de l'ambition des jeunes garçons et constituait donc un second degré.

Il convient de noter que le groupe original des adultes n'était pas nécessairement une classe d'âge distincte. D'une part, elle pouvait être composée d'individus entre vingt et quatre-vingts ans; de l'autre, soixante pour cent des adultes seulement en faisaient peut-être partie. Mais les jeunes imitateurs s'étaient formés approximativement en une classe d'âge et lorsqu'ils eurent en corps acheté une société plus élevée, ils avaient mis en branle les rouages nécessaires à la création d'un système tel que celui qui caractérisait les Hidatsa et les quatre autres tribus à degrés. Une nouvelle génération achetait ensuite le degré le plus bas à ceux qui l'avaient inventé; ces derniers avançaient collectivement, et graduellement la masse amorphe des adultes disparaissait, laissant derrière elle des groupes successifs d'individus plus jeunes, gradués approximativement selon l'âge. Le fait de la réunion des jeunes garçons, en luimême un exemple de la puissance associationnelle de l'âge en général, peut ainsi avoir entraîné cette association fortuite des différences d'âge avec les sociétés décrites plus haut. Le facteur âge est donc une réalité, bien que ce ne soit pas le seul élément, ni celui qui a nécessairement prédominé dans l'histoire des associations. Dans la zone des Prairies, ce n'est pas le plus ancien.

Chapitre 11

\section{f. Conclusions générales.}

$\underline{\text { Retour à la table des matières }}$ 
On peut tirer plusieurs conclusions générales de l'histoire des associations chez les Indiens des Prairies. Tout d'abord nous avons noté une fois de plus l'effet funeste des grands mots. Nous nous trouvons selon les cas devant des phénomènes distincts : division des mâles en groupes de non-initiés, célibataires et anciens; division en classes d'âge, comme celle des Hidatsa; division des Renards chez les Crow en jeunes gens, adultes et vieillards, ce qui est encore un phénomène différent, puisqu'il n'implique pas de répartition tribale mais n'est qu'un groupement à l'intérieur d'une même association choisie parmi plusieurs. Il nous faut donc être sur nos gardes lorsque, pour désigner des phénomènes dans des zones distantes, on fait usage de termes également larges et également vagues. Il est alors fort probable que l'identité de nomenclature suggère, mais à tort, l'identité des phénomènes. Si, par exemple, nous comparons les sociétés secrètes mélanésiennes avec celles des Indiens Pueblo, nous ne trouvons aucune analogie ni de constitution ni de fonctions; leur seul point commun est l'exclusion des non-membres. C'est par achat qu'on entre dans les sociétés secrètes des îles Banks; dans le sud-ouest des États-Unis, c'est après avoir été guéri par un membre de l'association, ou par hérédité, ou encore parce qu'on a été reçu à sa naissance par la femme d'un membre, mais jamais par suite d'un paiement. Les habitants des îles Banks n'admettent pas les femmes; chez les Pueblo, quelques-unes des sociétés le font et d'autres sont composées uniquement de femmes.

Les sociétés mélanésiennes concentrent leur activité autour de la production d'un bruit étrange et de la protection des biens de leurs membres, tout en terrorisant à l'occasion les non-initiés ou en détruisant ce qu'ils possèdent. Nous ne trouvons rien d'analogue dans les confréries médicales des Zuñi, ni dans les associations de faiseurs de pluie chez les Hopi. Il n'existe donc pas entre ces organisations d'affinités historiques ou psychologiques.

Ces considérations ont une portée énorme sur le problème de l'évolution unilinéaire. L'étude intensive des sociétés chez les Indiens des Prairies tend à nous prouver que, en ce qui a trait à cet ordre de phénomènes, l'histoire ne se répète pas, sauf dans ses grandes lignes ou pour une durée très limitée. Dans toutes les communautés, l'instinct grégaire se manifeste d'une façon ou d'une autre et le facteur âge produit parfois, je l'admets moi-même, des sous-groupes ou même un groupement premier des individus. Cependant, ce sont là des généralisations sociologiques plutôt qu'historiques; elles ne nous livrent pas la formule d'un ordre de succession fixe des événements.

Voyons maintenant quels sont les différents principes dont la conjonction est requise pour que se créent des sociétés d'âge telles que celles des Hidatsa : concepts de la danse, des insignes qui s'y rattachent, de la bonne camaraderie régnant entre participants, de certaines obligations militaires et enfin la possibilité d'achat de tels complexes, ce dernier élément reposant sur la théorie indigène des visions. Il n'est donc pas surprenant qu'avec une telle abondance de facteurs essentiels, ce type d'association ne se rencontre pas à double. Les Massai sont aussi belliqueux que les 
Hidatsa, mais cette ressemblance générale ne va pas jusqu'à produire des analogies spécifiques, puisque ces deux peuplades n'ont pas les mêmes traditions culturelles. Les Massai qui ont une conception tout autre du cérémonialisme et qui ne possèdent pas, comme les Hidatsa, la théorie des visions individuelles, doivent forcément présenter un système tout différent. Puisqu'il en est ainsi, la recherche de lois d'évolution embrassant la totalité des phénomènes, à la manière des théories de Morgan ou de Schurtz, est une entreprise chimérique. Seule une étude ethnographique intensive de chaque zone culturelle peut établir la succession véritable des diverses phases.

Chapitre 11

\section{Références.}

$\underline{\text { Retour à la table des matières }}$

1) SCHURTZ : 83-109, 125-128, 202-213, 318-333, 347-367

2) VON DEN STEINEN : 268. RADIN, $1911:$ 198-207. LOEB : 249-288. MOONEY : 415. GODDARD, 1903 : 15, 50, 67. Dixon, 1905 : 269, 272; id. 1907, 420, 471. BARRETT : 397. POWERS : 141,158, 305. ROTH, 1903 : 31. NELSON : 285, 347. HAWKES, 1913. Annual Archaeological Report : 213. ALLDRIDGE : 220. KINGSLEY : 376. SPENCER et GILLEN, 1904 : 498-501. LEHNER : 404 et suiv. PARKINSON : 636, 640, 658. HOLLIS, 1909 : 40, 56. TALBOT : 284. FROBENIUS : 170 . VON DEN STEINEN : 384. LANG : 29-44.

3) LOWIE, 1916. 


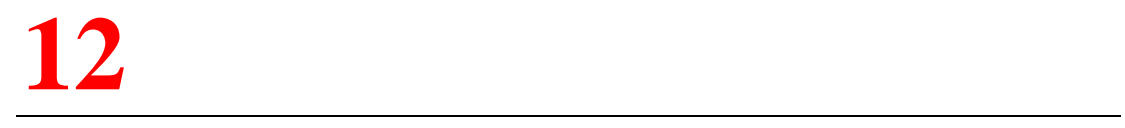

\section{LE RANG}

$\underline{\text { Retour à la table des matières }}$

Ainsi que je l'ai déjà fait remarquer, Morgan avait de la société primitive une conception atomiste. C'était peut-être la tendance américaine aux institutions démocratiques qui le rendait aveugle aux signes évidents de discrimination sociale dans les civilisations primitives. Il n'accorde que peu d'attention aux différences qui séparent les clans d'une même tribu ou les individus d'une même communauté et il date d'une époque beaucoup plus récente les ordres à privilèges. Et pourtant, même en restant en Amérique du Nord, il aurait pu découvrir des systèmes d'organisation sociale basés sur une différenciation des classes supérieures et inférieures; et, chose plus importante encore, il aurait trouvé que l'absence de castes héréditaires n'exclut nullement les distinctions vitales reposant sur le mérite personnel. Le primitif n'est pas un imbécile; il est prompt à percevoir et à apprécier ces différences individuelles qui, ainsi qu'un phénomène biologique inévitable, apparaissent dans tous les groupes, même dans les plus primitifs, comme l'a, à juste titre, fait remarquer Marett. Le primitif n'ignore pas que $X$, quoique maladroit pour tordre un fil, est de première force quand il s'agit de tirer à l'arc; que $\mathrm{Y}$, en dépit de toute son éloquence à la réunion du Conseil, s'est montré poltron devant l'ennemi ; et que $\mathrm{Z}$ est un aimable médiocre. Imperceptiblement il les situe; imperceptiblement leur influence sur ses propres actions et sur ses pensées dépend de l'estime qu'il a d'eux. Cette attitude ne le concerne pas seulement à titre individuel, mais constitue une question sociale, affectée par l'idéal tribal. Celui qui, dans tel milieu, passe pour un héros, sera considéré dans tel autre comme une brute grossière; l'habileté mécanique peut être fort prisée d'une peuplade et ne compter pour rien dans une autre. Il incombe à l'ethnologue précisément de montrer que, dans les 
diverses sociétés, l'appréciation personnelle varie selon les préjugés sociaux. Les indigènes de l'Amérique du Nord présentent un terrain exceptionnellement favorable à qui veut rendre sensible la puissance des différences individu elles. En effet, à part quelques cas isolés auxquels nous reviendrons plus tard, ce continent était en grande partie occupé par des tribus démocratiques d'esprit. Commençons par étudier quelques groupes des Prairies.

Chapitre 12

\section{a. Bravoure.}

$\underline{\text { Retour à la table des matières }}$

Parmi les Indiens des Prairies, la recherche de la renommée guerrière était aussi hypertrophiée que chez nous la soif de l'or dans les milieux les plus typiques de la haute finance. C'était afin d'obtenir une vision qui lui permettrait de se distinguer dans la mêlée que le jeune brave jeûnait et qu'il traînait des crânes de bisons fixés aux muscles perforés de ses épaules. Et, pour atteindre à la gloire ardemment souhaitée, il rejetait toute prudence et risquait sa vie dans les audaces les plus folles. Ces dernières, cependant, devaient se conformer à un certain modèle conventionnel afin d'être considérées comme héroïques et ces modèles différaient sensiblement d'une tribu à l'autre.

En temps normal, parmi les Crow, quatre exploits passaient pour honorables et procuraient conjointement à celui qui s'était ainsi signalé le titre de chef, généralement dépourvu de signification politique. Le guerrier devait détacher et emmener un cheval du camp adverse, prendre l'arc ou le fusil d'un ennemi dans un corps à corps, frapper un " coup », c'est-à-dire toucher l'adversaire avec une arme ou simplement avec sa main, et diriger une expédition victorieuse. Naturellement, seuls quelques rares individus parvenaient à remplir ces quatre conditions; mais, même sans atteindre au titre de chef, le brave qui avait accompli un ou plusieurs de ces exploits jouissait de la considération de la tribu; sa situation était en fonction directe de ses hauts faits militaires. A toutes les assemblées tribales, il avait le privilège de réciter la liste de ses exploits; il pouvait les peindre sur ses vêtements et sur la porte de sa tente; les parents d'un nouveau-né venaient lui demander d'imposer un nom à leur enfant; les jeunes gens ambitieux lui achetaient de sa " médecine » de guerre; à chaque manifestation publique, on lui assignait un poste honorifique, celui de héraut par exemple; les compagnons de clan de son père chantaient ses louanges par tout le camp; et même dans les cérémonies, on accordait la préséance au guerrier victorieux en raison de ses hauts faits.

On trouve une expression, parfois exagérée, de cette mentalité dans les adages et les chants populaires. " Il convient de mourir jeune », tel était le dulce et décorum des 
sages parmi les Crow et les Hidatsa. Voici le refrain d'un chant crow : «Éternels sont les cieux et la terre; les vieilles gens ne valent rien; n'aie pas peur. » Ainsi, dans une société militaire, un frère aîné pouvait forcer son cadet à accepter les obligations dangereuses associées à telle charge, non point par mauvaise intention, mais afin que le jeune homme se couvrît de gloire. Et nous voyons les guerriers non seulement rivaliser d'intrépidité, mais encore se jeter délibérément au-devant de la mort dans des entreprises insensées, par exemple se précipiter seul contre une troupe d'ennemis.

Naturellement le lâche était l'objet d'un mépris suprême; ses " parents de plaisanterie » se moquaient de lui et le traitaient de « femme en règles ». Ce mérite exclusif attribué à la valeur guerrière faisait passer inaperçus des individus qui, dans des communautés dont l'idéal eût été différent, auraient joui d'un prestige considérable. Ainsi, chez les Crow, l'un de mes meilleurs informateurs était un homme étonnamment au courant du folklore tribal; aux yeux de ses concitoyens, c'était une nullité. J'appris qu'il ne s'était jamais distingué sur le champ de bataille et avait encore aggravé sa situation en s'attribuant des exploits qu'il n'avait pas accomplis. En d'autres termes, il arrive qu'un individu, pourtant fort bien doué, ne soit pas estimé par son entourage social, simplement parce que la rigidité des préjugés indigènes empêche que ses mérites soient reconnus.

Les Crow prisaient encore d'autres qualités, non qu'elles pussent se substituer à la valeur guerrière, mais elles achevaient de la parfaire. C'était tout d'abord la libéralité, entraînant par contre-coup le mépris de l'avarice. Les femmes étaient également jugées selon leur mérite individuel, quoique l'échelle des valeurs différât. En dépit de la morale sexuelle généralement relâchée, les femmes chastes étaient tenues en haute estime et, pour remplir certaines charges cérémonielles, on exigeait d'elles une vertu sans tache. L'habileté dans les travaux manuels et l'amabilité rehaussaient aussi le prestige féminin. On voit donc que, dans une civilisation relativement simple et dans une communauté nettement démocratique, les variations individuelles entraînaient des différences considérables dans l'estime sociale.

Nous ne serons donc pas surpris de trouver chez d'autres peuples guerriers des systèmes analogues, rappelant une aristocratie basée sur le mérite individuel. Les Maori de la Nouvelle Zélande, il est vrai, nous fournissent l'exemple d'une peuplade belliqueuse où l'esprit de caste est trop profondément enraciné pour pouvoir s'accommoder de la phrase de Napoléon sur le bâton de maréchal. Mais, en général, l'existence militaire chez les primitifs s'accompagne de notions du type de celles des Crow. Parmi les Massaï, dans le kraal des célibataires, règne une certaine égalité de statut; ceux qui se sont distingués par leur courage ou par leur munificence sont qualifiés de Taureaux et d'Hommes généreux et autorisés à porter des ornements spéciaux. On se moque des lâches devant les jeunes filles, et un homme qui ne prend pas part à une expédition, décidée en séance solennelle, peut être impunément tué. De même que chez les Indiens des Prairies, le brave se pique de témérité; ainsi les Massaï plantent un pompon au bout de leur lance et jurent de ne pas l'enlever avant qu'elle n'ait traversé le corps d'un ennemi. 
Dans une tout autre région, les Bagobo de Mindanao font preuve d'une mentalité analogue. Leur but principal dans l'existence est de porter les attributs dont peut se targuer celui qui a tué au moins deux êtres humains après son second meurtre il est autorisé à exhiber un bandeau frontal couleur chocolat; le quatrième lui donne le droit de porter des pantalons rouge sang et quand il en est à son sixième, il a tout le costume de cette couleur, plus un sac rouge. Tout meurtre supplémentaire, bien que n'apportant pas de modification à son vêtement, rehausse encore son prestige. Ceux qui n'ont jamais tué personne sont des nullités; les braves reconnus, au contraire, remplissent les charges importantes et passent pour jouir de la protection particulière de deux esprits puissants auxquels ils servent d'intermédiaires. Ni le statut du brave, ni ses vêtements ne sont transmissibles; ces derniers doivent être brûlés avec leur possesseur.

Chapitre 12

\section{b. Shamanisme : richesse.}

\section{$\underline{\text { Retour à la table des matières }}$}

En d'autres parties du monde, nous trouvons des notions radicalement différentes. Les Maidu septentrionaux nous en donnent l'exemple. Ici nous sommes en présence d'un chef électif dont l'autorité se basait sur la richesse et la générosité; en réalité, le shamane, surtout s'il est à la tête d'une société secrète, supplantait complètement ce chef. C'était en effet par le shamane, chargé de révéler la volonté des esprits, que le chef était élu et c'était une communication surnaturelle analogue qui entraînait sa dégradation. La charge de shamane n'était pas héréditaire. Celui qui se destinait à cette profession l'embrassait après avoir été mystérieusement visité et avoir passé avec succès l'examen imposé par de vieux shamanes. Autrement dit, l'aptitude aux expériences religieuses était le moyen de parvenir à la prééminence sociale. Le shamane qui se trouvait à la tête de l'organisation secrète était à tous égards l'individu le plus influent de la communauté. Il réglait la vie cérémonielle de la tribu, jugeait les contestations, assurait une bonne récolte de glands et, grâce au pouvoir magique, infligeait à l'ennemi une juste punition; parfois il dirigeait lui-même les expéditions guerrières. Il était par-dessus tout l'autorité suprême en mythologie tribale et en folklore, et son devoir l'obligeait à enseigner ces nobles sciences aux gens de sa tribu.

Dans le nord de la Californie, un motif qu'on discerne déjà faiblement chez les Maidu, devient le thème principal : c'est sur la richesse que repose la prééminence. Le chef des Hupa était l'homme le plus opulent de la tribu; les autres s'adressaient à lui en temps de disette afin qu'il les entretînt et ils lui empruntaient de l'argent en cas de dispute. Il léguait son pouvoir à son fils à condition qu'il lui transmît aussi ses biens, 
mais si quelque rival, extraordinairement capable et actif, le surpassait en richesse, c'était à ce dernier que passait la dignité de chef. Dans la société des Shasta, prédominait la même conception.

Le rôle que joue la richesse dans le système politique des tribus primitives offre en général un grand intérêt comparatif. Dans la phase pastorale, quand une nouvelle forme de propriété s'introduit, elle pourrait entraîner des différences considérables de statut si la force nivellatrice des conditions naturelles ne se faisait sentir, déshéritant brusquement le nabab d'hier. La mentalité reste donc démocratique. Chez les Turcs Altaïques, le gueux fait montre de la fierté d'un noble râpé en traitant avec les gens aisés de sa tribu; il se comporte dans la maison d'un riche éleveur comme un membre de la famille, ne tolérant en aucune façon d'être considéré comme un serviteur et préférant la faim plutôt que de se soumettre à un ordre trop impératif. Chez les Chukchi à rennes, le statut du pauvre est moins favorable, puisque son maître peut l'injurier et même le frapper. Cependant la morale indigène, en exigeant un traitement généreux, empêche qu'il soit fait usage de ces privilèges. Le courage relatif des individus s'y oppose également; en effet, un valet vigoureux que son maître frappe peut lui rendre la pareille.

On trouve en Mélanésie une conception particulière de la richesse. Ainsi que je l'ai expliqué en décrivant le club des habitants des îles Banks, l'avancement dans l'organisation et, par conséquent, dans l'échelle sociale, dépendait de la richesse, non cependant d'une accumulation de biens ou d'argent, mais du fait qu'on dépensait sans compter. Cette même notion caractérise également les Indiens de la Colombie britannique, quoique ici la situation se complique par la coexistence des castes héréditaires, comme nous allons bientôt le voir. " La richesse », dit Boas en décrivant le point de vue des Kwakiutl, « passe pour honorable et chaque Indien s'efforce d'y parvenir; ce n'est point tant la possession de biens que la possibilité de donner de grandes fêtes qui la lui rend désirable. » Plus un homme distribue de biens à l'occasion de ces fêtes ou potlatchs, comme on les appelle, plus il jouit de la considération générale. De jeunes garçons, des chefs, des communautés entières rivalisent d'extravagance. Nous avons affaire ici à un concept primitif très répandu dont Mauss a souligné à juste titre l'importance : l'individu auquel un présent est offert, doit l'accepter et assume par là certaines obligations. Celui qui lance le défi commence par donner un grand nombre de couvertures à son adversaire; celui-ci ne peut pas les refuser et doit en rendre dans la suite le double s'il ne veut pas consentir à l'humiliation de passer pour insolvable. Parfois, afin de prouver combien il méprise les richesses, un chef détruit sans nécessité des biens de valeur et autrefois il faisait tuer ses esclaves par bravade pure. L'importance attribuée à cet élément modifie même, en certaines occasions exceptionnelles, l'armature rigide des castes. Selon Sapir, "il est des cas où des individus de basse extraction se sont, à force de potlatchs et de prodigalités, élevés au-dessus de leurs supérieurs, accaparant plusieurs des privilèges qui reviennent aux hautes castes. Parmi les Indiens de la côte du Pacifique ainsi qu'en Europe, un parvenu peut par son activité modifier les normes habituelles ». 
Ainsi la valeur guerrière, la tendance aux expériences mystiques et, d'une façon ou d'une autre, la richesse, toutes ces causes entraînent dans les communautés primitives la différenciation d'individus égaux par ailleurs. Ajoutons-y encore une qualité en honneur dans les couches les plus basses : l'habileté à se procurer des aliments. Parmi les Tchouktche de la côte, c'est la famille la plus ancienne du village, possédant donc une connaissance profonde des conditions économiques de la région, qui passe au premier rang. L'organisateur d'une chasse au phoque occupe une situation prééminente. Chez les Youkaghir, il existe un poste spécial de chef des chasseurs, charge assez lourde, du reste, puisque le titulaire doit procurer des vivres à toute la communauté et que cet honneur n'est, pour ainsi dire, pas rétribué. Beaucoup d'Indiens, tels les Chipeway et les Shoshoni des plateaux, témoignent du respect aux chasseurs habiles, bien que de façon moins formelle.

Chapitre 12

\section{c. Castes.}

$\underline{\text { Retour à la table des matières }}$

Les facteurs que nous avons étudiés jusqu'ici sont basés sur des différences individuelles qui n'ont rien à voir avec le rang dû à la naissance. Toutefois, il est de nombreux cas où la distinction sociale est avant tout une question d'hérédité, bien qu'elle soit modifiable plus tard par la compétence personnelle. Là où fonctionne un véritable système de castes, son action se fait généralement sentir sur les conditions politiques; ce qui nous importe pour l'instant, c'est son influence sur le rang social.

En Polynésie, l'orgueil de famille des nobles indigènes est aussi pointilleux que celui d'un seigneur castillan. Ce sentiment dérive d'une croyance en l'origine divine de la noblesse. Les chefs sont des descendants, des représentants et, en un certain sens, des incarnations des dieux, ainsi que l'a montré Hocart. Chez les Maori typiques, la prééminence sociale dépendait de la descendance en ligne directe par droit d'aînesse des grands dieux. Chaque individu de rang élevé était obligé de connaître par cœur son arbre généalogique, mi-historique mi-légendaire, afin de pouvoir, lorsqu'on l'y provoquait, prouver son ancienneté. Ainsi un célèbre guerrier Maori faisait remonter sa famille au Ciel et à la Terre à travers soixante-cinq générations. L'héraldique indigène était aussi enchevêtrée qu'un labyrinthe, car les deux branches entraient en ligne de compte et, pour régler les contestations entre rivaux, il ne fallait manquer ni de précision ni de discernement. Les enfants d'un chef occupaient naturellement un rang variable selon l'extraction de leur mère. Un enfant né de mère noble passait avant ses demi-frères, puisque en lui coulait le sang de deux hautes lignées. Si le premier-né d'un chef et d'une esclave pouvait exercer bien des privilèges, il n'était pas considéré comme un véritable chef et jamais ses mérites 
personnels n'effaçaient sa tare originelle. Rares étaient ceux qui possédaient un blason immaculé et leur noblesse pouvait leur devenir à charge lorsqu'il ne se trouvait pas de jeunes filles de sang assez pur auxquelles ils pussent s'allier. Parfois le grand-prêtre légitime qui avait hérité de sa dignité par droit d'aînesse, passait pour un personnage trop considérable pour pouvoir remplir les obligations de sa fonction; il les déléguait donc au frère qui venait après lui. L'une des prérogatives du futur pontife était d'être admis dans le collège sacré où il apprenait l'histoire légendaire de son peuple et la science des incantations redoutables. Cette dernière n'était pas enseignée aux femmes. Si, dans la lignée sainte, c'était une fille qui naissait la première, elle jouissait de privilèges extraordinaires; elle était la seule femme à pouvoir goûter à la chair humaine et aux offrandes sacrées; on lui enseignait quelques-unes des anciennes traditions et personne n'avait le droit de manger en sa compagnie. En traitant des régimes politiques, nous énumérerons encore d'autres caractéristiques de la haute noblesse. Après les grands-prêtres venaient les chefs des subdivisions tribales et leurs parents, selon le degré de proximité; puis suivait la classe des professionnels, c'est-àdire des sorciers et des artisans de métier; le gros de la population était formé de hobereaux plus ou moins apparentés avec la famille du chef et possédant quelques biens. Tout en bas de l'échelle sociale, nous trouvons les esclaves qui se recrutaient surtout parmi les prisonniers de guerre. En général, leur situation matérielle n'était pas trop mauvaise. Grâce à la crainte superstitieuse des nobles de se souiller en accomplissant certaines tâches indispensables, l'un des piliers de la civilisation Maori reposait sur le groupe des esclaves. Les besognes serviles, comme de faire la cuisine ou de porter des fardeaux, pouvaient contaminer un guerrier mais non un esclave dont le statut spirituel et temporel n'importait pas. Parfois des liens d'amitié se nouaient entre le maître et son esclave et le code moral des classes supérieures défendait les mauvais traitements. On autorisait quelquefois un esclave à travailler pour un autre que son maître et à garder son gain pour lui. Bref, sa position était supportable. L'esclave n'essayait jamais de s'enfuir, car il aurait été renié chez lui, le fait d'avoir été fait prisonnier étant une preuve de la défaveur divine. Un danger cependant le menaçait constamment - être exécuté à l'occasion d'un sacrifice cérémoniel, par exemple lors de l'érection d'un édifice ou même par suite de quelque caprice du maître. Les hommes les moins fortunés de la tribu épousaient souvent des esclaves et leur progéniture se confondait bientôt avec celle des gens libres; toutefois leur basse extraction les exposait souvent à des commentaires méprisants. Parfois, mais rarement, un homme dans les veines duquel coulait du sang d'esclave pouvait, grâce à son courage, occuper une situation éminente, mais il était toujours considéré comme un parvenu incapable de rivaliser avec les aristocrates. Peu importait qu'avant de devenir captif l'esclave eût occupé le rang le plus haut; le simple fait d'être prisonnier effaçait toute trace de sang bleu et souillait de façon indélébile l'écusson de sa lignée.

Le système social des habitants de Samoa présente une ressemblance générique avec celui de la Nouvelle-Zélande, si nous ne tenons pas compte naturellement des variations locales. Stair distingue cinq classes d'hommes libres : les chefs, les prêtres, la noblesse terrienne, les grands propriétaires et les gens du commun. Cependant les différences de rang étaient bien plus nombreuses que ne l'indique cette liste. Les chefs 
n'étaient nullement égaux entre eux et le terme de respect dont il fallait user pour s'adresser à chacun d'eux était précisé dans le plus petit détail. Puis les grands chefs avaient leurs assistants qui, tout en faisant partie d'une des cinq divisions mentionnées, tiraient leur titre de gloire particulier de leur profession: barbiers, échansons, trompettes ou bouffons.

Les artisans de métier, tels que constructeurs de bateaux, architectes et tatoueurs, correspondaient à nos classes professionnelles. Du fait qu'ils exerçaient des industries importantes et grâce à leur organisation, ils pouvaient imposer leur volonté à toute la communauté, ainsi que Stair l'a décrit en des termes frappants. Lorsqu'un homme haut placé désirait se faire construire un bateau, il commençait, avec l'aide de ses voisins, par accumuler autant de biens que possible, puis il se rendait auprès des spécialistes, demandant formellement dans un petit discours flatteur les services du charpentier en chef, et offrant, pour convaincre celui-ci, une natte de valeur ou une hache. Sa demande n'était pas toujours exaucée, car ces constructeurs étaient très occupés et ne pouvaient pas constamment accepter de nouvelles commandes. S'il était disposé à entreprendre le travail, le maître charpentier répondait par un discours dont les termes étaient fixés; il recevait un paiement initial et prenait ses dispositions pour qu'on se mit à l'ouvrage. Au jour dit, le constructeur en chef, tous ses ouvriers et les familles de ceux-ci se présentaient et il allait de soi que c'était au client à les entretenir tous pendant les trois mois peut-être que durait le travail, même si tout son avoir y passait. Tout était mis en œuvre afin de maintenir dans de bonnes dispositions les nombreux visiteurs, et quelque important représentant de la maison veillait quotidiennement à ce que les travailleurs ne manquassent de rien; ceux-ci étaient protégés des passants par un hangar spécial. On ne convenait pas à l'avance d'un prix déterminé; il était d'usage de faire cinq paiements cérémoniels, séparés par des intervalles plus ou moins longs. Si les deux premiers semblaient un peu modestes, les ouvriers se mettaient froidement en grève jusqu'à ce que leur employeur fût venu s'excuser ou offrir un supplément. C'était la seule manière de mettre fin à ce dilemme, car aucune autre équipe n'aurait repris le travail commencé, sous peine d'être chassée de la corporation et d'être privée de ses outils, donc de tous moyens de subsistance, par les autres membres de ce tyrannique syndicat.

Des scènes analogues se produisaient durant la construction d'une maison, car les architectes étaient également constitués en une puissante organisation. En 1927 encore, Buck fut frappé de l'orgueil dont faisait preuve cette corporation; ses membres menacèrent de se mettre en grève parce que leur employeur avait enfreint une règle sans importance sur la manière de préparer la volaille de leur dîner. Ces deux corporations pourraient naturellement être considérées comme des associations, mais il paraît préférable de les étudier par rapport aux strates de la société dont ils faisaient partie et où ils occupaient une place nettement déterminée.

Chez les Samoans, nous trouvons une importante différence: ils ignoraient le droit d'aînesse dans la transmission des charges élevées. Ces dernières ne passaient pas automatiquement au fils aîné du chef et il n'était pas possible à celui-ci de désigner 
son successeur. S'il avait pourtant entre autres privilèges celui de nommer quelqu'un, son choix devait être ratifié par les hommes influents de la localité qui pouvaient s'arrêter à une tout autre désignation. Les choses se passaient plus simplement lorsqu'il s'agissait de transmettre le titre d'un gentilhomme terrien qui le léguait à sa mort non point nécessairement à son fils aîné, mais souvent à celui de ses enfants qui avait meilleur air ou plus grande valeur, ou même à un favori adoptif.

Cette brève description des conditions polynésiennes suffit à indiquer l'importance des titres héréditaires et des autres distinctions de classes. Ajoutons encore que, dans l'au-delà, patriciens et plébéiens étaient séparés et on se rendra compte du rôle que jouaient ces différences de castes dans la conscience indigène.

L'Afrique est, comme la Polynésie, un continent où les distinctions sociales sont très marquées, mais elles y ont un caractère tout différent. On y trouve souvent des potentats auxquels leurs sujets témoignent la révérence la plus servile et qui sont entourés d'un essaim de fonctionnaires dont les diverses charges forment une échelle hiérarchique qui aurait fait honneur à une cour du Moyen Age. Ces dignitaires ne reçoivent pas leur titre en vertu d'une haute lignée; ce ne sont pas des nobles dont les arbres généalogiques remontent jusqu'à quelque personnage légendaire mais des hommes politiques et, comme tels, d'ordinaire les créatures du roi. Le souverain et les membres de sa famille sont à part; tout le reste de la population est au même niveau.

L'exemple de l'Ouganda est typique: le roi faisait descendre sa famille d'un héros légendaire; les autres dignitaires de l'État devaient leur situation à leur compétence ou à leur fidélité au service royal et toute charge était accessible à n'importe qui.

Rien n'est aussi éloigné de la mentalité africaine que la notion d'une caste patricienne dont les membres rivalisent d'ancienneté. Une curieuse particularité qu'il convient de noter ici, c'est la prééminence de la reine douairière qui a souvent sa propre cour. Parmi les Bakuba, quoique le roi soit une incarnation de la divinité suprême, il laisse la parole à sa mère; elle a le privilège de s'adresser à lui en premier, ce qui, selon l'étiquette indigène, est une marque de supériorité.

Les esclaves occupent, cela va sans dire, une condition inférieure, mais ici il ne faut pas négliger quelques nuances. Les prisonniers de guerre pouvaient, en effet, être vendus comme du bétail et exécutés au gré de leur maître, mais il existait une autre classe d'esclaves indigènes qui avaient été privés de leur liberté pour dettes. Ceux-ci jouissaient d'un traitement beaucoup plus doux et ne perdaient pas leur prestige social puisque leur servitude avait souvent pour motif de sauver quelque parent ruiné. Dans l'Ouganda, une esclave qui avait des enfants d'un homme libre devenait libre ellemême ainsi que sa progéniture et quelquefois, mais rarement, il arrivait que ses fils héritassent des biens de leur père.

Les remarques qui précèdent s'appliquent aux territoires des nègres. Là où les races se mélangent, les choses se compliquent. Ainsi la situation des éleveurs de 
bétail wahuma en Afrique orientale varie selon les localités. Au Ruanda, ils constituent la classe gouvernante, traitant avec mépris les cultivateurs Bantou, alors qu'ailleurs ce sont simplement des bergers de métier dans un pays agricole. Frobenius, Arcin et Desplagnes nous décrivent de façon à peu près identique la société soudanaise, elle est constituée par une série de classes graduées : nobles, troubadours faisant également office de tanneurs, serfs nés de femmes indigènes et de seigneurs, forgerons et esclaves. Selon Frobenius, cette complexité résulterait en grande partie du contact entre les éleveurs conquérants et la population agricole indigène.

On constate chez les Massaï et leurs voisins un curieux phénomène. Quoique démocratiques en général, ces tribus tiennent à l'écart les corporations ou les clans des forgerons, comme d'impurs parias. Ce sont pourtant eux qui fabriquent les armes et on imaginerait a priori qu'ils jouissent d'un rang élevé dans cette communauté guerrière. Rien n'indique que ces forgerons soient de race différente et les motifs de cette attitude restent énigmatiques.

L'Amérique du Nord, ainsi que nous l'avons déjà remarqué, est la région des démocraties sociales et politiques. La chose apparaît nettement lorsque nous comparons les récits des anciens explorateurs sur ce continent et ceux des voyageurs en Afrique ou en Océanie. Il est assez compréhensible que Morgan ait salué « la liberté, l'égalité et la fraternité » comme les " principes cardinaux » de l'organisation clanique en Amérique et de la société indienne tout entière et qu'il en ait déduit que « le sens de l'indépendance et la dignité personnelle sont des attributs du caractère indien». Mais, en ethnographie, il est bien peu d'affirmations qui aient une valeur absolue. Citons deux notables exceptions: les Natchez du Mississippi et les indigènes qui vivent sur la côte de la Colombie britannique.

Les Natchez avaient évidemment un système de castes fort intéressant. Par malheur nous ne le connaissons qu'à travers les récits des voyageurs du XVIIIe siècle qui ont été réunis avec soin par Swanton. Les gens du commun, désignés du terme peu flatteur de Puants, étaient tenus à l'écart par les nobles; ceux-ci se divisaient en trois catégories: les Honorables, les Nobles et les Soleils, le chef appelé Grand Soleil occupant la place suprême. Le statut se transmettait à l'origine matriarcalement. Les titres de noblesse n'étaient pas sans affecter la condition des descendants, mais leur importance allait en s'affaiblissant avec les générations. Les enfants d'un Soleil n'étaient que des Nobles, ses petits-enfants des Honorables et ses arrière-petitsenfants devenaient des gens du commun. Ce système était, en une certaine mesure, mitigé par des conceptions démocratiques puisqu'un Puant pouvait, grâce à son courage, être admis dans la catégorie la moins élevée de la noblesse et qu'un Honorable passait de la même manière dans la caste supérieure.

L'élément le plus curieux peut-être des coutumes des Natchez était la loi réglant les mariages. Alors que là où règnent des distinctions de classes rigides nous trouvons presque toujours des règles endogamiques, les Natchez non seulement autorisaient 
mais encore prescrivaient l'union des Soleils avec les gens du peuple, et cette loi s'appliquait aux deux sexes. Lorsqu'une femme Soleil épousait un Puant, il se produisait une dérogation aux coutumes patriarcales de la tribu ; le mari occupait alors la situation d'un domestique, ne pouvait pas manger en compagnie de son épouse et, en cas d'infidélité, était exécuté. Voici comment, selon Swanton, le système fonctionnait : les Soleils comprenaient des enfants de mères Soleils et de pères Puants ; les Nobles étaient les enfants de mères Nobles et de Puants ou de mères Puantes et de Soleils ; les Honorables incluaient les enfants de mères Honorables et de Puants, ainsi que de mères Puantes et de Nobles ; enfin la vaste classe des gens du commun était constituée par des mariages entre plébéiens ou par les unions entre mères Puantes et Honorables.

Les manifestations extérieures de ce système de castes étaient plus étonnantes encore pour qui connaît la mentalité démocratique des Indiens de l'Amérique du Nord. Nous n'y trouvons plus trace, en effet, de cette dignité personnelle que Morgan admirait chez les Peaux-Rouges. La description que les chroniqueurs français nous font de l'étiquette de cour chez les Natchez rappelle l'atmosphère environnant un potentat oriental plutôt que la situation d'un chef indien. "La vénération que ces sauvages ont pour leur grand chef et pour sa famille va si loin que chaque fois qu'il parle, en bien ou en mal, ils le remercient par des génuflexions et par des grognements qui marquent leur respect. » Cette attitude était d'origine religieuse puisque les Soleils étaient considérés comme des descendants de cet astre, leur divinité suprême, et comme capables par leur intercession de prévenir les maux. Le Grand Soleil s'élevait de toute sa hauteur au-dessus des autres membres de sa caste et était protégé par des prohibitions spéciales. Seule son épouse avait le droit de manger avec lui, et lorsqu'il abandonnait à ses frères les restes de ses repas, il repoussait les plats de leur côté avec les pieds. Les Puants n'étaient pour lui que poussière et à sa mort ses serviteurs étaient sacrifiés. Les degrés intermédiaires avaient aussi leur importance ; il y avait surtout un conseil de vieux guerriers qui servait à enrayer l'autorité du souverain.

Grâce aux travaux de Boas, Swanton, Sapir, Barbeau et d'autres, notre connaissance des phénomènes relevés sur la côte nord-ouest suffit à nous donner une idée de ce système de castes. Aucun doute n'est possible ni sur son existence ni sur l'intensité des sentiments qui s'y rapportaient. L'endogamie était strictement observée. Il y a quelques années encore, Barbeau a pu noter l'abjecte servilité que de petits plébéiens témoignaient dans une école du gouvernement à l'égard de rejetons royaux. Les tribus se divisaient en trois classes : les nobles, le peuple et les esclaves. Nous passerons rapidement sur ces derniers ; c'étaient des captifs qui, comme chez les Maori, n'étaient pas maltraités mais qui pouvaient être mis à mort n'importe quand, soit lors d'un sacrifice rituel, soit pour obéir à un caprice de leur maître. Ainsi, à l'occasion de ces fêtes où chaque grand seigneur cherchait à surpasser ses pairs par son dédain superbe des biens matériels, le maître faisait tuer un esclave afin de rehausser encore l'étalage de sa munificence ; et un affront, même s'il avait des causes naturelles, ne pouvait être complètement lavé que par la mise à mort d'un esclave. Les plébéiens étaient des 
groupes d'hommes libres, de basse condition ou apparentés de loin avec les nobles dont ils formaient la suite, sans jouir d'aucun privilège.

Parmi les esclaves et les plébéiens, il n'y a pas d'autres différences de rang. Il n'en va pas de même dans la noblesse où les questions de préséance ont autant d'importance que chez les Polynésiens. Le statut dépend surtout de ces formes immatérielles de la propriété que nous avons décrites dans un chapitre précédent. La manifestation extérieure la plus nette est la disposition des sièges lors de manifestations publiques, chacun étant assis selon son rang. Mais grâce à la variété des privilèges, il n'est aucun noble qui puisse jouir d'une prééminence absolue. L'un sera mieux placé que les autres à telle cérémonie, mais ne sera pas invité le premier à telle autre, et ainsi de suite. Cependant, en général, ces privilèges s'enchaînent les uns aux autres et il est fréquent qu'on en possède plusieurs à la fois.

L'indépendance théorique des divers privilèges est due en partie, selon Sapir, au sentiment très fort que chaque privilège est originaire d'une localité déterminée, et le souvenir s'en conserve longtemps après que le lieu et le privilège se sont dissociés. Ainsi, il peut arriver qu'un individu ait hérité de droits se rattachant à plusieurs villages et, en ce cas, il peut fractionner son patrimoine, en transmettant une partie à son fils aîné et l'autre à un cadet qui va s'établir dans la communauté en question sous la tutelle du père. Cette forme de succession n'a cours naturellement que parmi les tribus patrilinéaires; chez les peuplades septentrionales de la côte qui sont utérines et observent l'avunculat, c'est au fils de la sœur que se lèguent les privilèges.

Sapir insiste avec force sur le fait qu'en dépit de l'importance du rang, c'est la lignée, le groupe plutôt que l'individu qui compte. "Chez les Indiens Nutka, par exemple, un vieillard, son fils aîné, le fils aîné de son fils et enfin l'enfant de ce dernier, qui peut être une fille, constituent à tous égards une personne sociale unique. Nominalement, parmi les Nutka, c'est au plus jeune que revient le rang le plus élevé, car c'est toujours la dernière génération qui, en théorie, jouit de la plus grande considération. Il va sans dire qu'en pratique ce sont les membres les plus âgés du groupe qui ont le plus de crédit et qui administrent, en quelque sorte, le patrimoine dont ils ont hérité. Mais il serait difficile de préciser dans un tel cas où s'arrêtent les privilèges de l'arrière-grand-père et où commencent ceux du fils, du petit-fils ou de l'arrière-petite-fille. " Nous retrouvons ici un phénomène que nous avons déjà étudié sous d'autres aspects, dans les règles matrimoniales par exemple, la tendance du primitif à faire disparaître l'individu dans le groupe.

On ne saurait nier que cette organisation en castes des Indiens du nord-ouest ne rappelle sur certains points la Polynésie. Le droit d'aînesse des Noutka est identique à la coutume des Maori; la mise à mort d'un esclave lors de l'érection d'un édifice dans les deux tribus représente une ressemblance assez spécialisée ; enfin le caractère exceptionnel en Amérique de tout système de castes, joint à la localisation de cette anomalie sur la côte du Pacifique, pourrait faire croire à son origine océanienne. Cependant ces indices ne sauraient être tenus pour décisifs. Les Natchez nous 
fournissent l'exemple d'une tribu de l'intérieur chez qui s'est développé un système de classes héréditaires radicalement différent de tout ce qui l'entoure, prouvant ainsi que de telles divisions peuvent se produire spontanément.

En outre, les systèmes de la Polynésie et de la Colombie britannique présentent quelques divergences : les Indiens sont dépourvus de toute passion pour la généalogie et les Polynésiens n'estiment nullement ces privilèges immatériels qui différencient les nobles sur la côte du nord-ouest. Nous pouvons donc conclure à une évolution indépendante des castes dans ces deux régions.

Chapitre 12

\section{d. Conclusion.}

$\underline{\text { Retour à la table des matières }}$

Cette énumération des phénomènes américains, africains et océaniens réfute de manière décisive l'opinion qui veut que les sociétés primitives soient uniformément hostiles au principe aristocratique. Ce n'est pas, comme Morgan se l'imaginait, dans les communautés qui travaillent le fer, qui ont des bêtes domestiques ou qui connaissent l'irrigation et la construction en pierre que l'esclavage a débuté. On le trouve dans une phase plus primitive, représentée par les Polynésiens néolithiques et par les Nutka non agriculteurs. Il en va de même pour la segmentation de la société en castes et en degrés. Toutefois, même dans les communautés vraiment démocratiques, où les enfants naissent socialement égaux, les différences psychologiques qui les séparent impliquent des variations inévitables dans la façon dont ils sont publiquement estimés. C'est ce qui fait de la société primitive un agrégat d'individus plutôt qu'une agglomération d'automates indifférenciés.

Un autre point encore attire notre attention. Dans quelle mesure les faits cités s'harmonisent-ils avec l'interprétation économique de l'histoire que nous avons eu l'occasion d'esquisser plus haut? Il faut reconnaître qu'il n'existe aucun rapport. Quand un chef tsimshian tue un esclave pour relever l'honneur de sa fille blessée ou lorsqu'un Kwakiutl, dans un paroxysme de vanité, écrase son rival en détruisant un bateau ou en brisant une plaque de cuivre de la valeur de mille couvertures, les mobiles de ces actions ne sont évidemment Pas économiques, loin de là. De même, les Indiens des Prairies n'avaient pas en vue dans leurs combats une extension territoriale ou le butin du vainqueur, mais la guerre était pour eux un jeu qu'ils aimaient à cause de l'estime sociale dont était récompensé celui qui jouait selon les règles. 
Il est vrai que l'un des aspects essentiels de la guerre était le vol des chevaux. Mais pourquoi un Crow risquait-il sa peau en allant détacher un cheval en plein camp ennemi lorsqu'il aurait pu facilement en emmener tout un troupeau qui paissait aux abords du camp? Et pourquoi décernait-on une distinction non point au guerrier qui avait tué ou blessé un ennemi, mais à celui qui avait touché son corps, même légèrement? Pourquoi enfin les Polynésiens étaient-ils si pointilleux en matière de préséance? S'il se fût agi de différencier le souverain de ses sujets, on pourrait invoquer quelque raison utilitaire. Mais tel n'était pas le cas. Entre le noble et le plébéien, aucune compétition n'était concevable; il ne s'agissait que de la supériorité des patriciens, et cette supériorité n'impliquait: pas d'avantages temporels mais avait sa fin en elle-même. Ainsi la doctrine de l'utilitarisme s'avère incapable d'expliquer la mentalité indigène. Le primitif n'est ni un avare, ni un sage, ni une bête de proie mais, selon le mot heureux de Tarde, un paon.

Chapitre 12

\section{Références.}

$\underline{\text { Retour à la table des matières }}$

1) MARETT.

2) LOWIE, 1912 : 230 ; id., 1917 (a) : 82. MERKER : 92, 216. HOLLIS, 1905 : 289, 298, 353 et suiv. COLE : 96.

3) DIXON, $1905:$ 223, 267, 323; id., $1907:$ 451. GODDARD, 1903: 58. RADLOFF : 1, 312. BOGORAS : 614, 639. MAUSS. BOAS, $1897: 341$ et suiv. SAPIR, $1915: 355$ et suiv.

4) TREGEAR : 123, 146,163, 383. STAiR : 65 et suiv., 147. TURNER: 173. BUCK : 84-95. KRAEMER : 1, 31. ROSCOE, $1911: 14,187,269$. TORDAY et JOYCE : 60. MERKER : 111. FROBENIUS, $1910: 1-44$. ARCIN : 257-276. DESPLAGNES : 167 et suiv. SWANTON, 1911 : 93, 100 et suiv. SAPIR, $1915: 355$ et suiv. 


\section{3}

\section{LE GOUVERNEMENT}

\section{$\underline{\text { Retour à la table des matières }}$}

En comparaison des efforts qu'on a dépensés pour élucider les problèmes de la famille et de l'organisation clanique, les ouvrages théoriques consacrés aux institutions politiques des tribus primitives sont en nombre infime. C'est que, ainsi que nous le verrons plus loin, on a essayé à diverses reprises de mer jusqu'à l'existence de véritables organisations politiques dans les basses civilisations. On comprend que c'est là un point important auquel nous reviendrons. Pour l'instant toutefois, il est préférable de passer en revue les phénomènes observés sans aucune idée préconçue, en cherchant quels sont les gouvernements existants dans les diverses zones et quels rapports ils entretiennent avec les autres éléments de la vie indigène que nous avons déjà esquissés dans les chapitres précédents. Je n'emploie pour l'instant le mot « politique » que pour plus de commodité et afin de désigner l'ensemble des fonctions législatives, exécutives et judiciaires; mais son emploi n'indique nulle solution anticipée du problème que nous étudierons après avoir énuméré les faits concrets.

Les trois pouvoirs, ainsi qu'on l'a déjà remarqué, se confondent souvent. En Australie, le conseil promulgue les décrets, en surveille l'exécution et juge les délits criminels, et c'est le cas des gouvernements primitifs un peu partout. Il convient, cependant, de noter que le pouvoir législatif semble particulièrement atrophié dans la plupart des communautés primitives lorsqu'on en rapproche l'extension qu'il prend 
dans les civilisations plus complexes. Le droit coutumier répond à toutes les exigences des relations sociales normales et la tâche de ce qui tient lieu de gouvernement est d'exiger l'obéissance à l'usage traditionnel plutôt que de créer une nouvelle jurisprudence. Sous l'autorité despotique d'autocrates africains et océaniens, ce principe souffre quelques exceptions. Un monarque zoulou abrogea par un édit royal la coutume vénérable de la circoncision, et à Hawaï les décrets du souverain pouvaient délivrer ses sujets de leur obéissance aux lois établies. Néanmoins il est probable que, même dans ces cas extrêmes, les rapports sociaux étaient réglés bien plus par les anciens usages que par les oukases de ces autocrates.

Dans le présent chapitre, nous traiterons surtout de l'existence et du caractère du gouvernement central. Pour des raisons pratiques, nous remettons au chapitre suivant l'administration de la justice.

Chapitre 13

\section{a. Australie.}

\section{$\underline{\text { Retour à la table des matières }}$}

Le trait saillant de la vie publique en Australie est la prééminence des vieillards. Il existe des variations locales quant aux pouvoirs des anciens, mais presque partout les femmes sont sévèrement exclues des manifestations politiques et partout les jeunes gens jouent dans les délibérations tribales un rôle insignifiant ou même nul. Néanmoins on peut constater d'intéressantes différences à l'intérieur des limites de cette gérontocratie.

Les Dieri nous fournissent l'exemple d'une de ces variations. L'assemblée des hommes véritables, c'est-à-dire de ceux qui, avaient passé par les cérémonies d'initiation conférant officiellement le statut d'adulte, constituait en somme une société secrète, car c'était risquer la mort que de divulguer les actes de ce parlement. Il discutait, entre autres, des cas de meurtre par magie et de transgression des lois sur l'inceste, ainsi que de l'organisation des cérémonies. C'était cette assemblée qui envoyait un groupe armé tuer un sorcier coupable de manœuvres criminelles et qui procédait lors des fêtes à la nouvelle distribution des concubines.

Apparemment on ne votait pas. « Si tous étaient d'accord sur telle mesure, le conseil se séparait; en cas contraire, il s'ajournait à une date ultérieure. » Jusqu'ici nous n'avons affaire aux anciens qu'en tant que corps. Le président des sessions dirigeait le conseil et menait la discussion. Vers la moitié du siècle passé, cette charge était remplie par un certain Jalina-piramurana qui l'avait héritée de son père. On nous le dépeint comme « un homme d'une éloquence persuasive, un guerrier habile et brave 
et un puissant homme-médecine ». Il était vénéré par tous et recevait des divers groupes locaux des présents qu'il distribuait généreusement à ses amis. Si forte était son emprise qu'il pouvait empêcher une guerre. Aucun membre du conseil, pas même ses propres frères, n'osaient s'opposer à ses décrets. Bref, nous sommes ici en présence d'un cas d'autorité extraordinaire, exercée par une personnalité exceptionnelle. Jalina-piramurana éclipsa son père, et son successeur ne fut sans doute pas à sa mesure. Il convient cependant de noter que, dans cette région de l'Australie, on remarque souvent une tendance vers l'accaparement du pouvoir par des chefs éminents qui se transmettent leurs fonctions de père en fils.

Les conditions sont toutes différentes dans la zone du centre qu'habitent des peuplades comme les Arunta et les Warramunga. Ici il n'est pas de personnalité qui puisse être considérée comme le chef tribal, mais rien non plus ne rappelle une assemblée générale des anciens. Chaque groupe totémique local a son président, dignité se transmettant de père en fils, et c'est parmi ces présidents dont les fonctions sont surtout sinon uniquement cérémonielles qu'on choisit les membres du conseil gouvernant. C'est une corporation fermée, constituée par quelques vieillards particulièrement influents. Leur nombre, sans être fixé, est généralement de cinq; on cite des cas exceptionnels où il s'est élevé à huit ou dix. Ce conseil tient ses sessions sans aucune solennité et on n'y prononce pas de véritables discours. Tous ses membres sont des chefs et si quelque autre chef a attiré leur attention par ses capacités ou son intérêt pour la chose publique, ils le récompensent quelque jour en l'invitant à se joindre à leurs délibérations. Toute la tribu obéit aveuglément à ces oligarques qui exercent un contrôle absolu sur les affaires publiques. Par ailleurs, les hommes plus jeunes, quoique déjà mûrs, ne jouent aucun rôle quelconque et manifestent comme le reste de la tribu le respect le plus absolu envers les vieillards dirigeants.

Chez les indigènes du Queensland méridional, le conseil du campement ne répète exactement ni le type des Dieri, ni celui des Arunta. C'est un corps plus vaste qu'en Australie centrale puisqu'il comprend tous les vieillards de quelque influence, mais aucun d'entre eux ne s'élève au-dessus des autres. Les nuances du pouvoir s'établissent d'après des facteurs personnels; un individu qui a traversé toutes les phases conduisant à la qualité d'ancien, qui s'est distingué comme guerrier ou qui, par suite de plusieurs mariages, se trouve à la tête d'une grande famille, est susceptible d'exercer une autorité exceptionnelle. A peu près comme chez les Dieri, les questions de paix et de guerre, la réception des étrangers, les déplacements du camp, la punition des incestes et les tentatives de réconciliation, tout cela est du ressort des anciens, alors que l'infanticide ou la mutilation d'une épouse adultère passent pour des affaires privées dans lesquelles le conseil n'a pas à s'immiscer.

Chapitre 13

\section{b. Polynésie et Micronésie.}


L'importance extrême attachée aux différences de naissance, qui est un trait si frappant des civilisations polynésiennes, n'implique pas nécessairement l'ascendance politique de la caste patricienne. C'est ce que démontre fort clairement l'exemple de la Nouvelle-Zélande et de Samoa, quoiqu'il semble que dans ce dernier cas nous ayons affaire à un développement ultérieur, comme on le verra plus loin.

Il n'est pas facile de définir avec précision et brièveté le régime politique des NéoZélandais. Évidemment, il n'y a eu nulle part en Nouvelle-Zélande de vaste royaume gouverné par un suzerain. Mais il est malaisé de déterminer les pouvoirs constitutionnels des chefs de haute lignée qui, tout en régnant sur un territoire exigu, étaient à la tête de nombreuses tribus constamment en guerre les unes contre les autres. D'une part, nous apprenons que, bien que le droit d'aînesse pût conférer la pourpre et la mitre, celle-ci seule était l'apanage inaliénable de la naissance. La petite noblesse terrienne, qui formait l'ossature de la nation, pouvait déposer un prince héritier qui, par sa couardise, son avarice ou son incapacité, s'était aliéné les sympathies de son peuple; on élisait à sa place un représentant plus digne de sa lignée, un oncle ou un frère. Dans un certain sens donc, le prince n'était que le premier parmi les nobles. D'autre part, ses attributions religieuses, s'il ne s'était pas rendu coupable de graves fautes, l'entouraient d'une atmosphère de crainte et de vénération qui contribuait dans une large mesure à rehausser son autorité dans toutes les phases de son existence. Non seulement il agissait en intercesseur auprès des dieux, consacrant les guerriers avant la bataille, bénissant les moissons et réglant le rituel, mais il faisait aussi office de juge dans les contestations à propos de terres, dirigeait les travaux horticoles et avait droit aux prémices, aux bateaux qui avaient fait naufrage et aux cétacés pris sur le rivage.

Créer un tabou était un privilège indissolublement lié au pouvoir du roi ou des nobles. Cette prérogative, typiquement polynésienne, atteint en Nouvelle-Zélande son plus haut point de développement. Il n'est pas possible de suivre cette notion indigène dans toutes ses ramifications enchevêtrées et du reste nous ne nous occupons ici que de son aspect politique. Celui-ci était assez simple en principe, mais les applications pratiques s'étendaient plus loin qu'on ne l'aurait cru tout d'abord. Le noble ou le chef, participant à l'essence divine dont il était l'incarnation, pouvait communiquer autour de lui sa sainteté, si bien que certains objets ne devaient plus être possédés que par des individus de rang élevé. Il pouvait arrêter le trafic d'une rivière et créer de grands embarras à ses sujets en tabouant une forêt. On dressait alors un poteau portant un bouquet de feuilles ou de chiffons, ou bien on élevait quelque " écriteau " analogue. L'infraction au tabou n'était pas seulement un crime, mais aussi une iniquité spirituelle, éveillant la colère des dieux qui envoyaient à l'offenseur les maladies ou la mort. 
Même en dehors de la Polynésie, les Océaniens observent la coutume du tabou, bien que sous une forme moins pure. La méthode adoptée par les sociétés d'esprits mélanésiennes pour sauvegarder leurs biens personnels fait partie de cet ordre de phénomènes, quoique dans les îles Banks ce soit l'organisation lésée qui inflige le châtiment mérité. Rivers nous montre que, dans les îles Salomon, des transgressions analogues sont censées être automatiquement punies par les esprits, croyance qui forme un chaînon intermédiaire entre les notions de tabou en Mélanésie et en Polynésie. Il est superflu d'insister sur la puissance énorme que le privilège du tabou conférait à celui qui l'exerçait lorsqu'il s'alliait au pouvoir du chef. Il est donc étonnant que, malgré le développement qu'elle atteignit en Nouvelle-Guinée, la dignité de chef ait vu parfois décroître son autorité laïque.

Nos informations sont plus claires en ce qui a trait aux grandes lignes de la constitution dans les îles Samoa. Il y avait pour tout le groupe un chef nominal, un seigneur qui avait réuni les cinq titres royaux dont disposaient les districts électoraux privilégiés. Mais chacun de ces districts était, dans une mesure considérable, autonome; les nombreuses guerres d'extermination et la défense jalouse des frontières locales sont les signes manifestes de cette autonomie. Chaque district avait un corps gouvernant qui convoquait le parlement local. Celui-ci siégeait en séance exécutive sur une place publique qui devenait alors tabou pour ceux qui n'étaient pas admis; toute intrusion était punie de mort. Le chef de la province, l'aristocratie rurale et les petits propriétaires avaient accès à cette assemblée qui combinait les pouvoirs législatif et judiciaire. Les chefs de villages pouvaient prendre la parole, mais d'ordinaire ils étaient représentés par un noble qui faisait office de porte-parole. Le droit de s'adresser à l'assemblée était fort prisé et, en conséquence, tous ceux qui étaient autorisés à parler se levaient en même temps et luttaient à qui ferait le premier discours; beaucoup de temps se perdait ainsi jusqu'à ce que la question de préséance fût réglée. L'orateur qui avait la permission de s'exprimer devait remplir une formalité préliminaire, laquelle consistait à réciter les diverses désignations honorifiques de tous les districts de Samoa; une seule omission était considérée comme un affront. Tandis qu'il parlait, ses partisans le soutenaient, lui rafraîchissant la mémoire ou lui présentant un choix de sujets; si son éloquence les ennuyait, ils avaient le droit de lui dire de s'asseoir. Les autres groupes ne l'interrompaient pas jusqu'à ce qu'il effleurât une question épineuse; alors l'orateur d'un autre parti pouvait prendre la parole et se lancer dans le débat. Pendant les discours peu importants, on passait des rafraîchissements fournis par les villages environnants et durant toute la journée les membres qui ne s'adressaient pas à l'assemblée s'occupaient à tresser des fibres de noix de coco.

Lorsqu'on traitait de questions intéressant plusieurs provinces, le village principal de celle qui prenait l'initiative envoyait des messagers pour faire part aux autres districts du sujet qui allait être discuté. Chaque province avait son assemblée à part qui se rendait en ces occasions à ce qu'on pourrait appeler le parlement provincial. Parfois les personnalités du village principal allaient faire une tournée dans les autres zones, sollicitant les membres afin d'obtenir leur appui à l'assemblée générale. En 
l'absence de tout gouvernement centralisé, il n'y avait guère de coopération et aucun pouvoir, en dehors des petits groupes, qui pût appliquer les décisions prises.

Dans l'assemblée des nobles, c'étaient les porte-parole qui prédominaient. Grâce à leur influence, ils pouvaient nommer chef de district un simple particulier, le faisant passer avant ceux que désignaient leurs qualités ou leur naissance. Bien que les chefs extraordinairement doués jouissent d'une grande autorité, ils étaient incapables de dominer leurs orateurs lorsque ceux-ci s'appuyaient sur les autres chefs de famille. En conséquence, les chefs impopulaires n'étaient pas seulement bannis mais encore battus et parfois même tués par leurs sujets. Leur sainteté héréditaire, qui les rendait tabou au même titre que les chefs-prêtres des Maori, constituait bien un obstacle à un traitement aussi sommaire, mais l'ingéniosité des Samoans se montrait à la hauteur des circonstances. En faisant gicler sur le corps du chef de l'eau de noix de coco, ils le rendaient profane, le dépouillaient formellement du titre qu'ils lui avaient jadis conféré et pouvaient à leur gré le mettre en pièces, si tel était leur bon plaisir.

Le régime politique des Maori et des habitants de Samoa peut être comparé à un État de barons, accordant la préséance au chef de leur choix et se réservant de décider en dernière instance dans les questions gouvernementales. C'est donc un régime tout différent de celui de Hawaï et des îles Marshall où le gros de la population ne se composait pas de propriétaires fonciers, mais d'esclaves rampant servilement devant la classe des nobles. A Hawaï, on discerne même une certaine tendance vers le despotisme monarchique, quoique la caste patricienne ne fût évidemment pas sans influer sur les décisions importantes. A propos des droits de propriété, nous avons déjà traité de la stratification sociale des îles Marshall. Nous avons vu que l'aristocratie y est nettement séparée de la classe intermédiaire et du peuple. La bourgeoisie comprend des navigateurs experts et d'autres gens de métier dont les services sont estimés dans cette région, quoique leurs avis n'aient aucun poids; ils jouissent du respect des nobles et peuvent avoir des terres en fief. La noblesse se divise en caste supérieure et caste inférieure, mais toutes deux possèdent de façon absolue leurs terres héréditaires. Quoique le chef secondaire soit tributaire du plus grand, sa soumission est volontaire et, dans les cas de désaccord sérieux, il reprend toute son indépendance. Par rapport aux deux castes patriciennes, les plébéiens sont un corps de parias, cultivant les terres des chefs et exécutant les besognes viles, Les chefs sont non seulement entretenus par leurs sujets, mais ils peuvent se servir de leurs femmes et de leurs filles et avaient autrefois droit de vie et de mort sur eux. Ces rapports, qui durent sans doute depuis des âges, ont engendré dans la caste opprimée une mentalité servile qui a survécu à la pression de la nécessité. En 1905 encore, le Père Erdland observa des plébéiens qui risquaient volontairement leur vie pour assujettir les murs d'une hutte princière, secouée par l'ouragan. La même année, un vieux pêcheur qu'un cataclysme avait réduit à l'indigence, vint emprunter de l'argent à ce missionnaire afin de pouvoir apporter aux coffres bien garnis de son maître son impôt annuel. Quoique l'administration coloniale l'eût sans doute protégé et qu'Erdland lui offrît de plaider sa cause auprès de son chef et de le faire dispenser du paiement, le pauvre homme n'eut de cesse qu'il n'eût obtenu ce prêt, en retour duquel il offrit à son créancier de le 
fournir en poisson sa vie durant. Il n'est pas facile à l'homme de s'adapter à des conditions sociales nouvelles. La mentalité de cet indigène ne diffère pas beaucoup de celle du gentleman appauvri qui conserve dans notre propre civilisation une anachronique notion de classe au lieu d'accepter son sort avec ses nouveaux compagnons.

Les conceptions sociales et politiques, avec toutes leurs variations locales, qui sont en vigueur en Polynésie et en Micronésie ont une histoire fort compliquée. Seuls les travaux de spécialistes pourraient l'élucider dans ses détails. Mais les grandes lignes de leur évolution ont été retracées de façon plausible par Waitz et Gerland. Les observations des anciens voyageurs et les légendes indigènes sur la société d'autrefois indiquent que les faits relevés plus récemment à Hawaï existaient depuis bien des siècles dans toutes ces régions. Cette conclusion se déduit du reste logiquement de l'origine divine de la noblesse, qui impliquait naturellement un gouvernement de droit divin. Vue sous cet angle, la passion des Maori et des Samoans en matière d'étiquette et de généalogie est le résidu d'une phase antérieure où les différences de rang n'étaient pas des questions d'intérêt théorique, mais entraînaient une autorité politique plus ou moins considérable. Il est évident qu'une importance aussi minutieuse attachée aux distinctions de castes, un développement aussi hypertrophié de la théorie du tabou ont dû nécessiter une longue évolution. Mais aucune enquête historique ne nous a encore révélé ce qui a bien pu précéder la conception théocratique et aristocratique des philosophes et des politiciens polynésiens. Nous en sommes réduits à conjecturer que l'état antérieur participait à la simplicité qu'on observe dans tout le reste de l'Océanie.

Chapitre 13

\section{c. Mélanésie et Nouvelle-Guinée.}

\section{$\underline{\text { Retour à la table des matières }}$}

Les régions de la Mélanésie qui ont été exposées aux influences polynésiennes, surtout la Nouvelle-Calédonie et certaines parties des Fidji, partagent la conception des princes divins; ailleurs le statut du chef est beaucoup plus modeste. Parmi les Baining de la péninsule de la Gazelle, il n'y a même pas de chef du tout; un guerrier qui se distingue par sa bravoure gagne le titre de " héros » et forme le noyau du groupe aussi longtemps que sa valeur le rend populaire. Aux îles Banks, il n'y avait pas non plus de chef à proprement parler; celui qu'on pourrait à la rigueur considérer comme tel devait obligatoirement être membre du degré le plus élevé dans le club des hommes. L'accès à ce degré dépendant de la richesse, nous serions donc en présence d'un gouvernement ploutocratique. Mais il est évident qu'il n'y avait pas de véritable corps gouvernant, que les membres du degré supérieur étaient non point tant des 
potentats que des individus hautement estimés par la communauté et que, d'autre part, les diverses sociétés d'esprits détenaient un pouvoir considérable.

Les choses se compliquent à Buin, dans les îles Salomon. Ici les chefs se divisent en trois catégories : les petits chefs, les " chefs de cent » et les grands chefs. Les premiers ne sont guère plus que des pères de famille influents. Quiconque est assez fortuné pour construire une halle contenant plusieurs tambours de bois et pour y donner des fêtes, devient un petit chef. Les « chefs de cent », qui s'allient en général les uns aux autres, ne sont pas nécessairement à la tête d'un groupe de cent partisans; ce chiffre tombe parfois jusqu'à soixante, y compris les serviteurs. Les grands chefs se recrutent dans certaines familles influentes et se liguent toujours avec les " chefs de cent » et les petits chefs de plusieurs districts. C'est sur ces alliances que repose en fait le pouvoir. Ces trois grades sont héréditaires, mais ils n'ont de valeur que lorsque l'ayant droit a construit une halle de réunion et y a reçu ses voisins; le fait que ceux-ci acceptent son invitation signifie qu'ils reconnaissent sa supériorité. Ainsi un élément nettement ploutocratique s'associe ici au principe de l'hérédité. La halle de réunion sert à la fois de club pour les hommes, de salle du conseil et de chapelle cérémonielle; les femmes en sont d'ordinaire exclues.

Les alliances sont ratifiées par une cérémonie qui coïncide avec les rites d'initiation des garçons. Le père du jeune initié promet alors son appui à tel chef ou se vante d'exploits passés qu'il a accomplis au service de ce chef. En retour, il lui demande d'accorder sa protection au novice. Un échange de cadeaux s'ensuit. Il est possible d'étendre le cercle de ses alliés en envoyant des présents à d'autres chefs. Toux ceux qui forment une telle alliance se soumettent à l'obligation de la vendetta. Ce lien est purement personnel; il ne peut pas être hérité par la nouvelle génération, qui doit renouveler la cérémonie afin de perpétuer ces relations. Quoiqu'un grand chef puisse, grâce à ses nombreux alliés, jouer un rôle important à la guerre, il n'a aucune autorité en dehors de celle que lui vaut sa personnalité. Il ne s'immisce pas dans les affaires privées et ne peut pas obliger ses partisans à obéir à un ordre. Si l'un d'eux se fâche et quitte la halle, le chef essaie de se le concilier par des présents, car le guerrier vexé pourrait abandonner le district et suivre un autre chef, affaiblissant par là son campement natal.

En Nouvelle-Guinée, le rôle du chef est également modeste. Parmi les Kaï, c'est lui qui a le champ le plus vaste, mais il doit, par contre, nourrir tous ses invités et ses propres gens par surcroît. Comme c'est lui l'individu le plus riche du village, ses partisans s'attendent à ce qu'il soit généreux en tabac et en noix de bétel, à ce qu'il tue des cochons pour eux et les régale d'autres friandises. A part le droit qu'il a à leur aide, il ne jouit que de peu d'autorité. Chaque membre de la tribu agit comme bon lui semble et le chef ne dispose d'aucun moyen de coercition. La situation est la même chez les Jabim. Chacun aide le chef à construire sa maison et à planter son champ, mais il est entendu que les travailleurs seront en retour généreusement traités. Il n'y a pas d'assemblée populaire, mais, en certaines occasions importantes, quelques individus éminents tiennent une session et leurs délibérations ont lieu à voix basse. Le 
débat contradictoire est inconnu et on ne vote pas, ainsi qu'il est fréquent parmi les primitifs. La formule qu'on retrouve souvent et par laquelle on se soumet à la volonté des autres est : «Tu as parlé, ainsi soit-il. »

Chapitre 13

\section{d. Afrique.}

\section{$\underline{\text { Retour à la table des matières }}$}

Les institutions politiques sont particulièrement intéressantes en Afrique. Nous avons remarqué dans un chapitre précédent certaines différences entre le caractère des distinctions sociales en Afrique et en Polynésie. Dans le domaine politique également, des variations sont à noter, bien que dans les deux continents on rencontre des monarques despotiques. D'une part, les chefs noirs étendaient souvent leur domination sur une région immense, si bien qu'en comparaison les grands chefs de Tahiti ou de Hawaï n'apparaissent plus que comme de petits princes locaux. Cette divergence ne saurait s'expliquer par des considérations géographiques, car les Polynésiens étaient d'admirables navigateurs et auraient pu facilement fonder une sorte d'empire maritime. Pourtant la force des tendances centrifuges les empêcha d'y parvenir. C'est ce dont on peut se rendre compte en Nouvelle-Zélande surtout, où aucune barrière géographique ne se serait opposée à ce qu'un souverain puissant réunît sous son sceptre une douzaine de tribus.

Secondement, le cours des événements politiques paraît avoir été beaucoup plus variable et capricieux en Afrique qu'en Océanie. En Polynésie, si l'on admet la théorie de Waitz et de Gerland, il y avait généralement tendance à ce qu'un vaste corps de propriétaires terriens s'emparât de plus en plus du pouvoir politique. Parmi les nègres, une sympathie invétérée pour la monarchie, limitée toutefois par l'influence des anciens et d'autres fonctionnaires, a toujours offert aux personnalités violentes la possibilité de devenir des tyrans absolus. Mais l'édifice que ceux-ci avaient élevé grâce à leurs propres efforts s'écroulait complètement lorsqu'il passait à des chefs de caractère moindre ou dégénérait en un simulacre d'autocratie, le vrai pouvoir étant exercé par divers fonctionnaires.

Les Thonga et les Zoulou nous présentent deux exemples typiques de l'histoire politique africaine; nous leur accorderons donc une place d'honneur dans notre description.

Le territoire des Thonga est fractionné en un nombre considérable de petites principautés politiquement indépendantes les unes des autres, bien qu'unies par une communauté de langage et de coutumes. Dans chacun de ces minuscules royaumes 
règne un monarque héréditaire, le fils aîné de la reine-mère; celle-ci n'est pas nécessairement la première épouse du monarque défunt, mais la première qu'il ait prise après son avènement au pouvoir. La dignité de roi est investie d'une atmosphère de sainteté. Lui seul a droit à la salutation honorifique de "Bayete »; son nom est tabou, sauf dans les serments, dans le rite des prémices, il a sa part immédiatement après l'offrande aux esprits; et surtout il possède un charme puissant qui rend le pays magiquement invincible. Le roi lève des impôts en nature; il reçoit de chaque kraal un panier d'aliments au moment de la récolte, ainsi qu'une partie du gibier tué à la chasse. Les sujets doivent travailler aux champs du roi, nettoyer sa place publique, construire et réparer ses huttes. Enfin le roi s'approprie une grande partie des amendes qu'il a lui-même imposées, car les pouvoirs législatif, exécutif et judiciaire sont réunis en sa personne, et son jugement est sans appel.

Quoique le roi jouisse donc de grands privilèges et d'une autorité considérable, il ne saurait être qualifié d'autocrate absolu. S'il contrevient aux notions courantes sur la propriété, il est sévèrement critiqué par son peuple et peut même être déposé. Secondement, il partage son pouvoir avec certains membres de sa famille. En effet, les parents du roi, fils ou frères, sont à la tête des diverses provinces de l'État. Lors de son avènement au trône, il arrive que la parenté du nouveau monarque refuse de reconnaître -sa suzeraineté et on a vu à plusieurs reprises se former des principautés indépendantes. Enfin le roi est entouré d'un groupe de conseillers composé surtout d'hommes adultes de sa propre famille; ils peuvent gagner de l'influence sur lui et aller jusqu'à lui imposer leurs décisions. Cette assemblée peut décider sur bien des points, car le roi, qui préside la réunion, ne prend fréquemment aucune part au débat et se contente d'approuver de la tête.

Les disputes de famille ont pour origine non seulement ce système de provinces semi-autonomes, mais aussi les lois de succession qui tentent de concilier deux principes distincts : le droit d'aînesse et l'héritage par les frères. Le prince héritier est théoriquement le fils aîné de la reine, mais il ne peut accéder au trône qu'après la régence successive de tous les frères cadets du roi, son père. Ceux-ci sont en somme des rois, eux aussi; ils se montrent peu enclins à remettre la souveraineté au descendant de leur aîné mais cherchent à la léguer à leur propre fils. C'est ainsi que prennent naissance de violentes haines de famille et certains témoignent à se débarrasser de leurs rivaux et parents d'aussi peu de scrupules que les Borgia. Cet état de choses peut avoir pour résultat qu'une partie de l'autorité passe aux mains de la populace, les rivaux ayant en effet besoin de l'aide des guerriers pour parvenir à leurs fins.

En comparaison des autres souverains africains, les rois thonga déploient peu de faste; ils ne diffèrent pas de leurs sujets par la mise; parfois leurs kraals ne sont pas plus grands que ceux des autres et il leur arrive de se livrer à une occupation aussi modeste que d'éloigner les moineaux d'une plantation en les effarouchant. Néanmoins, chaque souverain est entouré d'une cour de dignitaires qui doivent observer tout un ensemble de formalités typiquement africaines. 
Nous avons déjà parlé des conseillers. A côté du cabinet composé des parents du roi, il y a les chefs militaires, puis le corps diplomatique dont chaque membre a comme rayon d'action l'une des principautés voisines, et enfin les magistrats de district secondaires. Un autre groupe de courtisans se compose des amis personnels et des camarades du roi, compagnons de ses fêtes et de ses chasses, qui vivent habituellement dans un club de célibataires près du bâtiment royal. Alors que ces favoris n'ont pas de fonctions spéciales, le héraut, lui, remplit une charge particulière. Son devoir est de se présenter chaque matin devant la porte du roi et de vanter les mérites des ancêtres du souverain, puis de critiquer vigoureusement le maître actuel. Il semble que l'éloquence soit la seule qualité requise pour ce poste; on se rendra compte de quel genre de rhétorique il s'agit d'après ces quelques phrases choisies parmi les plus typiques d'un long discours prononcé par un héraut distingué :

"Muhlaba Shiluvane (le père du roi), tu es comme un rhinocéros qui saisit un homme, le mord de part en part, le roule et le coupe en deux. Tu es comme le crocodile qui vit dans l'eau; il mord les hommes. Tu es comme ses griffes; il saisit un homme par les bras et par les jambes et l'entraîne dans la mare profonde pour le manger au coucher du soleil; il en défend l'entrée afin d'empêcher les autres crocodiles de prendre sa proie...

" Et toi, pourquoi gouvernes-tu avec tant de douceur? Regarde les autres avec leurs yeux terribles. Tu es un lâche... Agis avec courage et défends-toi. »

Tout aussi remarquable est le " vitupérateur public » ou bouffon de cour; ce personnage jette à la tête de n'importe qui, y compris le roi, les insultes les plus graves. Il peut accuser à la légère ses concitoyens d'inceste et enlever les aliments des mains mêmes du roi.

La précision de l'étiquette de cour apparaît surtout lors de la visite d'un étranger. Celui-ci s'assied hors de la place centrale de la capitale et déclare à un passant qu'il désire voir le roi. Aussitôt le diplomate chargé des relations avec la principauté de l'étranger vient le saluer et annonce son arrivée au roi, qui prend ses mesures pour le recevoir. Si le sujet qui l'amène est d'importance, le visiteur l'expose au diplomate chargé de la catégorie des affaires étrangères dont la question relève. Ce fonctionnaire répète son discours, phrase après phrase, à l'un des conseillers du roi qui le redit encore une fois à son maître, comme si celui-ci ne l'avait pas déjà entendu. Ainsi que Junod le fait remarquer, cette manière de procéder se justifie chez des gens qui ignorent les rapports écrits.

Les Zoulou sont une peuplade étroitement apparentée aux Thonga qui vit au sud de cette tribu. D'après l'ensemble considérable du matériel ethnographique, il semble qu'il y a cent cinquante ans environ leur existence était à peu près la même que celle des autres Bantou plus au nord. Au début du XIXe siècle, ils furent gouvernés par un souverain voisin, Dingiswayo, qui avait organisé une armée permanente grâce à 
laquelle non seulement il soumit diverses tribus indigènes mais encore devint un autocrate dont le pouvoir dépassait celui de ses prédécesseurs. Il fut cependant défait par un chef zoulou, Chaka. S'inspirant des principes militaires de Dingiswayo, Chaka les développa, organisa un militarisme puissant et, par ses conquêtes lointaines, mérita le titre de « Napoléon de l'Afrique du sud ». Les troubles que son règne suscita s'étendirent au nord jusqu'au lac Tanganyika. Chaka introduisit deux innovations essentielles : tout d'abord il remplaça la javeline de roseau jusqu'alors en usage par une lance à jet court, et les résultats de la tactique de choc qu'il inaugura furent irrésistibles. Secondement, il organisa les tribus vaincues de manière à accroître ses propres forces; en particulier, lorsque la peuplade soumise n'était pas immédiatement voisine, à l'exception des adolescents nubiles, tous étaient mis à mort, mais les survivants étaient traités comme ses propres sujets, les filles entrant dans son sérail, les garçons dans l'armée.

De cette façon, Chaka réussit à avoir une armée permanente de douze à quinze mille guerriers. Chaque régiment, comptant de six cents à mille hommes, occupait un kraal spécial. Les jeunes garçons s'enrôlaient volontairement et il fallait construire chaque année de nouveaux kraals. Les novices se remarquaient à leurs boucliers noirs; ceux des vétérans étaient blancs. Aussitôt que les jeunes guerriers s'étaient distingués dans la bataille, on leur rasait la tête sur l'ordre du roi et ils jouissaient du titre officiel d' « hommes ». Mais ils n'avaient pas encore le droit de se marier et de s'établir. Les soldats observaient le célibat tout au moins en ce qui a trait au mariage légitime, mais rien ne les empêchait de nouer des intrigues avec les femmes des kraals voisins. Pour se marier, il fallait une dispense spéciale du roi; celle-ci était accordée individuellement ou à un régiment tout entier en récompense de services particuliers ou lorsque l'âge avait diminué l'ardeur des guerriers. Les hommes mariés passaient pour socialement inférieurs et constituaient les réserves de l'armée royale. Les soldats étaient entretenus aux frais du roi. Pour une seule caserne, on abattait quotidiennement jusqu'à douze têtes de bétail. La sécurité économique, la participation au butin et le prestige social dont jouissait cette profession, telles étaient les compensations aux rigueurs d'un système qui avait été moulé par une main de fer. La vie du soldat zoulou n'était pas facile; il devait obéir aux ordres les plus extravagants et supporter la douleur sans broncher. On punissait de mort ceux qui battaient en retraite devant l'ennemi et, en vertu d'un terrorisme systématique, les officiers devaient même après chaque bataille désigner les « lâches » qui allaient être mis à mort, qu'il y en ait eu ou non.

Disposant d'une telle force, Chaka gouverna avec un despotisme auprès duquel celui de Dingiswayo ne comptait plus. Ses sujets étaient à tous égards des esclaves qu'il pouvait faire mourir sur un seul signe de tête. Es n'étaient pas autorisés à travailler pour un autre et lui-même se réservait le monopole du commerce avec les Blancs. Chaka avait comme conseillers deux grands ministres qui surveillaient son propre kraal, et une vingtaine de conseillers secondaires, mais il paraît certain que, parvenu à l'apogée de sa puissance, le monarque ne faisait aucun cas de leur opposition. Cependant, ce type d'autocratie, tel que l'avait fondé Chaka, porte en lui-même 
un germe de désintégration. Afin de réussir, il nécessite une personnalité virile et même brutale. Cette forme de gouvernement ne comporte en soi aucune stabilité et de même qu'elle a varié selon que ce fut un Pierre le Grand ou un Nicolas qui exercèrent le pouvoir en Russie, de même elle changea du tout au tout lorsque Dingaan succéda à Chaka. Sous un souverain plus faible, la puissance royale s'écroula. Alors que Chaka avait dominé son armée, ce furent ensuite les guerriers qui gouvernèrent Dingaan et celui-ci garda l'apparence plutôt que la réalité de l'autorité souveraine.

L'histoire du gouvernement zoulou pendant une brève période de moins d'un siècle nous offre un raccourci typique des phénomènes africains. Dans le territoire limité d'un petit État, dirigé en général, non sans obstacles et heurts, par un monarque bénévole, apparaît un chef impérieux qui organise l'armée, terrorise et soumet les populations avoisinantes et devient un despote absolu. Mais le royaume que son énergie a créé tombe après lui en décadence et se fractionne en petites unités gouvernées à nouveau par des souverains du type patriarcal.

Si nous comparons le régime politique des Ewé avec celui des tribus parentes du Dahomey, nous voyons que le cours des événements s'est déroulé parmi les Noirs soudanais selon le même rythme que parmi les Bantou du sud-est.

Les chefs des Ho-Ewé sont encore plus éloignés de tout autocratisme que les rois Thonga. Quoique l'hérédité joue aussi son rôle dans les questions de succession, le roi est choisi parmi un certain nombre de candidats éventuels. L'aspirant au trône essaie de se concilier par des cadeaux les électeurs influents, mais ces présents ne sont pas tout-puissants, on tient compte dans une large mesure du caractère du candidat. Les chefs nomment un roi de leur choix et la populace ratifie leur nomination. Puis le couronnement a lieu en présence des chefs et d'un groupe limité de représentants du peuple; en général les gens du commun ne doivent jamais voir le trône ni les autres attributs royaux. Le roi peut être déposé par ceux qui l'ont élu, mais cette mesure est exceptionnelle; on n'y a recours que lorsqu'il a porté une sérieuse atteinte à la prospérité de la tribu. Le roi est affable et traite ses sujets avec hospitalité; lorsqu'il rend la justice, il tempère d'une phrase aimable la dureté du verdict; dans la vie privée, il se montre un tisserand et un cultivateur industrieux.

Le noyau du gouvernement se compose du roi et de deux chefs locaux, assistés par un orateur et ses adjoints, plus un groupe de conseillers recrutés parmi les chefs des familles les plus nombreuses. En face de ce corps plus ou moins aristocratique s'oppose le gros de la communauté avec son président et le porte-parole de celui-ci. Ce président est le chef de l'armée; il joue le rôle d'intermédiaire entre le peuple et les chefs. Le peuple a le droit de protester contre les décrets du gouvernement et ses objections sont transmises par le président au conseil dont les membres considèrent dûment les vœux des sujets. Dans les moments de crise, le roi convoque le peuple en assemblée. 
Quoique le souverain exerce donc un pouvoir très limité, il possède du moins les attributs extérieurs de la royauté. Nous avons déjà mentionné le trône, caché à la vue du vulgaire. Plus importante est l'institution des serviteurs personnels dont le nombre atteint parfois vingt-quatre. Quelques-uns sont des huissiers qui convoquent le peuple, d'autres portent le roi sur un cadre spécial en vannerie. Lorsqu'ils sont en fonction, ils ont le privilège de fouetter tous ceux qu'ils rencontrent, ainsi que de tuer ou de manger les chèvres ou les moutons qui leur tombent sous la main. Ils ne reçoivent pas de gages fixes mais ils s'approprient en manière de pourboire une grande partie des revenus de la cour de justice. Si on inflige de fortes amendes, ils sont les premiers à en profiter et cet état de choses conduit inévitablement à des abus.

Les lois qu'on respecte le plus et dont la transgression est le plus sévèrement punie, ne sont pas les décrets spéciaux du conseil du roi, mais les règles de droit coutumier qui sont en vigueur depuis des temps immémoriaux, les lois contre le meurtre et le vol par exemple. En général, le conseil législatif ne fait que promulguer des ordonnances d'importance secondaire. Ces décrets sont d'abord discutés par le conseil des chefs, puis soumis aux assemblées de villages; les membres de celles-ci agitent la question entre eux avant d'en délibérer avec les chefs. Ce n'est que lorsque les deux groupes sont parvenus à s'entendre que les lois sont ratifiées et proclamées au peuple.

Les Ewé de la côte diffèrent de leurs congénères, les Ho-Ewé, en ce que chez eux le peuple n'a pas voix au gouvernement; mais, en limitant le pouvoir royal par un conseil de chefs, ils se conforment de près au modèle des Ho. Par contre, on trouve des conditions totalement différentes parmi la population du Dahomey dont la langue et la culture s'apparentent pourtant à celle des Ewé. Ici le souverain nous apparaît comme un monarque presque absolu. Théoriquement, il était le seul propriétaire de la terre et de tous les autres biens et ne faisait que tolérer qu'en pratique d'autres les partageassent avec lui. Les fonctionnaires les plus élevés n'étaient guère plus à ses yeux que des esclaves, obligés à ramper honteusement devant lui. Pas d'aristocratie héréditaire naturellement. Le roi désignait les chefs provinciaux et les grands ministres d'État et parmi ceux-ci un seul, qui cumulait les charges de chef de police et de grand exécuteur, ne pouvait pas être décapité au gré de son maître. Le pouvoir absolu de vie et de mort était l'attribut distinctif de la royauté. Les chefs provinciaux ne pouvaient qu'emprisonner et frapper d'amende, mais il leur était naturellement possible de mettre à mort leurs sujets par des méthodes indirectes, en les affamant dans des cachots, par exemple.

Les grands fonctionnaires étaient au nombre de sept. Tout d'abord venaient le grand exécuteur et l'organisateur des fêtes publiques à qui incombaient également les charges de collecteur d'impôts et d'intermédiaire entre le souverain et le peuple. C'étaient ces deux personnages qui désignaient parmi les fils du roi le prince héritier; quoique le droit d'ainesse fût en faveur, ils avaient le droit de déroger à la coutume. Ils jugeaient aussi les affaires criminelles et donnaient surtout des conseils au roi. Après eux se plaçait le gouverneur de la ville maritime de Ouidah, puis le maître du 
palais, un eunuque qu'on exécutait à la mort du souverain. En cinquième et en sixième rang venaient le commandant de l'armée et son adjoint, tous deux résidant près de la porte principale d'Abomey, la capitale. Enfin le surintendant des plantations fournissait les vivres à la maison royale et servait en outre d'aide au grand exécuteur. Ces sept hauts fonctionnaires étaient salués par tous les inférieurs avec les marques d'un respect profond. A cet organisme correspondait une série de femmes, occupant des dignités analogues à l'intérieur du palais; elles passaient en somme avant les fonctionnaires masculins, grâce à la fiction légale selon laquelle toutes les femmes attachées à la cour étaient les épouses du roi.

Au-dessous des dignitaires de l'État venaient les hommes de loi, les serviteurs royaux, puis les officiers de l'armée, les chefs de province ainsi que leurs adjoints et enfin les commerçants. Tous les autres sujets ne valaient guère plus que des esclaves.

Les grands fonctionnaires arboraient les insignes de leur rang : un siège de bois, un dais, une pipe et une blague à tabac, ainsi qu'un sceptre d'ivoire. Le messager du roi ou d'un chef portait invariablement le bâton de sa fonction qui lui servait de saufconduit et grâce auquel il était reçu avec autant de respect qu'on en eût témoigné à celui qui l'envoyait. Tout affront à cet emblème était considéré comme une offense grave.

Ellis nous dépeint en couleurs vives l'existence au Dahomey. Les gens du peuple étaient dépouillés et traités brutalement par les autorités locales; les chefs et les ministres faisaient montre d'une servilité totale devant le despote; un système d'espionnage mettait en danger la vie de chacun; on célébrait pour honorer les monarques défunts des sacrifices cérémoniels qui coûtaient l'existence à des centaines de victimes, chargées d'aller porter le message du souverain à ses ancêtres. Il régnait, certes, au Dahomey une tyrannie extrême, mais la nature humaine est telle qu'elle ne saurait supporter un despotisme total et, si nous étudions les faits de près, nous discernerons que le monarque du Dahomey n'obéissait pas qu'à ses caprices.

Théoriquement tout-puissant, en pratique le roi était souvent obligé d'agir avec quelque circonspection. Ainsi, au début du XIXe siècle, un souverain qui se vautrait dans le sang et qui organisait lui-même des expéditions de pillage contre ses propres sujets, souleva finalement une révolte qui eut pour conséquence son détrônement et sa mort. Et quoique le roi pût condamner des chefs et des officiers à être exécutés, il avait en général soin de ne pas défier simultanément les prêtres, les chefs et l'opinion populaire. Sans doute, il exerçait l'autorité de la manière la plus arbitraire, mais en confisquant des biens et en infligeant la peine de mort, il était moins guidé par la puissance dont il jouissait théoriquement que par le souci de donner une apparence de justice à des procédés oppressifs. Dans tel cas concret, le paysan ou le chef n'était pas dépouillé en vertu de quelque droit royal abstrait, mais parce qu'il avait désobéi à une ordonnance arbitraire ou qu'il était accusé de comploter contre son maître. En tout ceci, l'autocratie du Dahomey ne diffère que bien peu des gouvernements despotiques de nations plus civilisées. 
Une étude comparative des autres tribus de la famille Ewé nous révélera quelle a été l'évolution en matière politique dans cette région. A la base des systèmes de tous ces peuples, nous avons ces différences de rang qui sont si développées parmi les Bantou et les nègres du Soudan. Dans le territoire des Ho, elles sont parfaitement compatibles avec l'influence non seulement des fonctionnaires, mais aussi du gros de la population. Au Dahomey, d'autre part, l'organisation d'une armée permanente relativement considérable, entretenue par le roi, donna à celui-ci sur le reste de la société une prépondérance qui ne rencontrait d'obstacle qu'en cas d'abus criant. Néanmoins l'étendue exacte du pouvoir du souverain dépend inévitablement de ses qualités personnelles.

Ce sont des conditions quelque peu différentes qui caractérisent d'autres populations nègres, par exemple les Bakuba dans le sud-ouest du Congo. Ici, les institutions politiques semblent avoir joui d'une certaine stabilité. Le souverain, incarnation de la divinité, est en théorie un monarque absolu, entouré d'une cour magnifique comprenant des dignitaires, hommes et femmes, en grand nombre. Lors des manifestations publiques, on le traite avec un grand respect; ses ennemis eux-mêmes n'admettent pas qu'on lui manque d'égards. Cela n'empêche pas ses six ministres d'assumer presque toute l'autorité et de le rendre complètement esclave des formalités fastidieuses de l'étiquette traditionnelle de cour. Quoiqu'il ne soit pas absolument impossible à un roi doué de qualités extraordinaires de faire usage de ses prérogatives théoriques, la chose est moins faisable parmi les Bakuba que dans la plupart des autres régions de l'Afrique. Les dignitaires, jouissant de fonctions stables, traitent le roi avec beaucoup d'indépendance et, alors que le souverain peut en théorie pourvoir une vacance, son choix est au fond déterminé par la pression de l'opinion publique. En face d'une opposition résolue, il n'osera pas nommer ses favoris et sera parfois obligé d'offrir une situation élevée à un individu qu'il déteste du fond du cœur.

$\mathrm{Au}$ Yoroubaland également, nous ne trouvons guère plus que l'illusion de la royauté. Le roi, qui tient son titre de son origine divine, est traité avec toutes sortes de marques de respect apparent, mais ce n'est qu'une marionnette entre les mains de son cabinet. Hors de sa capitale règne une autonomie locale complète. Le roi doit se contenter de ce que sa souveraineté soit reconnue nominalement par une multitude de cités-États. Le système gouvernemental de ces communautés, dont certaines comptent jusqu'à cent mille âmes, est fort intéressant, car il nous prouve une fois de plus combien il est nécessaire de ne pas séparer les uns des autres les divers aspects d'une culture. L'Afrique occidentale est la région des organisations secrètes et au Yoroubaland c'est une association de vieillards, nommés les Ogboni, qui a usurpé le pouvoir suprême. Ils constituent un collège électoral qui élève un homme influent de la tribu à la dignité de gouverneur de la ville; celui-ci s'entoure d'une cohorte impressionnante de fonctionnaires dont les uns sont désignés par lui et les autres choisis par le peuple. Pendant la durée de sa charge, le gouverneur a droit à l'aide de ses sujets et peut accroître ses terres par des expéditions punitives contre les rebelles. Mais il dépend complètement des Ogboni. Aussitôt qu'il agit contrairement aux intérêts de cette 
association, les vieillards déclarent, après avoir tiré au sort, que la divinité s'oppose à ce qu'il reste en fonctions et ils le suppriment en lui administrant clandestinement un breuvage empoisonné. On dit qu'en moyenne le règne du gouverneur ne dépasse pas deux ans. C'est grâce à leurs procédés divinatoires que les Ogboni terrorisent toute la population. Il arrive fréquemment qu'un parvenu qui a peu de partisans et dont la richesse excite la cupidité des membres de cette association, soit accusé de quelque crime et soumis à une ordalie. Le sort est naturellement contre lui, il est reconnu coupable et sommairement exécuté; les Ogboni et le gouverneur se répartissent ses biens.

Si nous faisons abstraction de quelques variations locales ou historiques, nous pouvons affirmer que les nègres font preuve d'une sympathie invétérée pour le gouvernement monarchique ou pour son apparence tout au moins. Celui-ci représente probablement un vieil héritage culturel des Bantou et des Soudanais. Ce fait ne saurait servir à l'appui de ce mysticisme géographique qu'on a remis en vogue dernièrement. En effet, il est des peuplades qui ont un ensemble de traditions très différentes de celles des nègres en général, quoiqu'elles vivent au milieu de tribus nègres, c'est-àdire dans un milieu géographique identique. C'est ce que nous démontre l'exemple des Massaï qui est aussi instructif d'un autre point de vue. On serait tenté a priori de supposer que le chef d'une peuplade aussi belliqueuse doit être quelque guerrier célèbre, mais cette supposition, si logique soit-elle, est bien loin de la réalité. Ce qu'on pourrait appeler le pouvoir central est exercé par un devin héréditaire qui n'a même pas l'autorisation d'accompagner les expéditions militaires, mais qui doit préparer aux guerriers la médecine qui leur assurera la victoire; il prédit aussi l'avenir et désigne par divination les chefs des différents districts; mais, quoiqu'il jouisse du respect dû à sa sainteté, il est très éloigné de l'autocrate nègre typique. Il tient davantage du saint national que du chef, n'a pas pouvoir de vie et de mort, fait rarement office de juge et ne s'entoure jamais de la splendeur d'une cour. Naturellement une personnalité très forte, telle celle de Mbatian qui mourut en 1890, peut exercer une grande autorité, mais même alors nous sommes encore très loin des despotes apparents ou réels si communs dans les autres régions du continent.

Pour terminer cette description des phénomènes africains, nous nous sentons obligé par souci d'exactitude de modifier ce tableau d'ensemble. Il est bien exact qu'en comparaison d'autres populations illettrées, les nègres d'Afrique sont les seuls à avoir formé non point une seule fois, mais à diverses reprises de grands États monarchiques avec des souverains autocrates. Mais le développement de formes sociales aussi complexes implique une longue histoire. En fait, plusieurs sociétés africaines ont conservé jusqu'à maintenant leur simple état démocratique, à partir duquel a dû à l'origine se développer le despotisme typiquement africain. Ainsi les Lobi de la Haute-Volta, que Labouret nous a décrits, ne présentent aucun des éléments si bien marqués au Dahomey, dans l'ancien Bénin ou chez les Zoulou du temps de Chaka. Ils n'ont ni chefs suprêmes, ni conseils réguliers; et, quoiqu'il y eût des procès légaux, aucun fonctionnaire ne veillait à l'exécution des décisions du tribunal; on obéissait surtout par crainte des puissances surnaturelles. Nous ne nous tromperons pas de 
beaucoup en nous imaginant les groupes africains les plus anciens comme se conformant dans l'ensemble au modèle des Lobi. Certes les sociétés les plus primitives de ce continent, Pygmées et Bochimans, sont beaucoup plus près de cette société égalitaire.

Chapitre 13

\section{e. Amérique du Nord.}

$\underline{\text { Retour à la table des matières }}$

Nous avons vu que les Indiens de l'Amérique du Nord ont en général des tendances démocratiques et qu'ils contrastent ainsi fortement avec les nègres africains. On s'attendrait à trouver des exceptions à cette règle parmi les tribus qui ont un système de castes bien défini, mais ce n'est guère que chez les Natchez que nous sommes en présence d'une centralisation de l'autorité comparable à la puissance d'un despote africain. Le chef des Natchez était obéi avec respect, bien plus avec humilité. Ses moindres souhaits étaient exécutés aveuglément, si bien que lorsque les Français avaient besoin de rameurs ou de chasseurs, ils n'avaient qu'à payer le chef afin qu'il réquisitionnât les services de ses sujets dans la mesure nécessaire, ceux-ci ne recevant aucune récompense de leur travail. Il avait sur eux pouvoir absolu et, à sa mort, ses serviteurs et d'autres même considéraient comme un honneur de l'accompagner dans l'au-delà. Il est superflu d'ajouter que les meilleurs produits de la chasse, de la pêche et de l'horticulture lui étaient livrés en tribut. Le souverain qui partageait, dans une certaine mesure, ses prérogatives avec ses parents les plus proches, désignait ses ministres, plus particulièrement deux chefs militaires, deux prêtres et les fonctionnaires chargés de régler les traités de paix et d'organiser les fêtes. Quoique les ouvrages contemporains cités par Swanton soient d'accord pour faire du chef un autocrate absolu, certaines allusions indiquent que parfois les membres d'un conseil ou les chefs des villages les plus influents, probablement tous de caste noble, pouvaient restreindre matériellement sa liberté d'action. Néanmoins les institutions des Natchez restent le plus curieux exemple de monarchie au nord du Mexique.

Sur la côte du nord-ouest, malgré la rigidité des distinctions de classe, le pouvoir strictement politique du chef était faible relativement à son rang social. Parmi les Tlingit, il ne faisait guère que présider les délibérations collectives. En général, chaque chef de famille pouvait agir à son gré pour autant qu'il n'enfreignait pas le droit coutumier. Le pouvoir d'un chef Tsimshian était peut-être plus grand : il décidait quand la tribu devait se déplacer ou fixait le début de la pêche, il avait droit à certain tribut. Il avait la préséance à toutes les danses et commandait aux messagers et aux serviteurs. Cependant il portait certaines responsabilités. En sa qualité de chef militaire, il devait répondre des pertes subies et fournir des compensations aux familles des défunts. Les questions d'importance étaient tranchées par le conseil et, sans le 
consentement de celui-ci, aucun chef ne pouvait proclamer la célébration d'un potlatch.

L'individualisme démocratique, à demi supprimé seulement dans les sociétés de la côte du nord-ouest, retrouve toute sa vigueur dans le reste de l'immense territoire nord-américain. Ce n'est certes pas que le contact des Blancs ait causé la ruine des anciens chefs. Au contraire, tout concourt à démontrer que la colonisation blanche tend à renforcer le pouvoir des chefs indigènes. Les anciens voyageurs, dont les observations se rapportent aux régions les plus éloignées, nous fournissent de ce fait des preuves indiscutables. Le grand chef des Chipeway, Matonabbee, que Hearne exalte dans un amusant panégyrique qui s'appliquerait flatteusement à Lincoln ou à Pitt eux-mêmes, n'avait pas suffisamment d'autorité pour empêcher un Indien bien musclé d'enlever une de ses femmes. A propos des Algonkin du Canada oriental, un père jésuite écrit en 1612 : « ... Chacun est son propre maître et son propre défenseur. Ils ont bien des Sagamores, c'est-à-dire des chefs militaires, mais leur autorité est fort précaire et même on ne peut guère appeler autorité ce à quoi nul n'est tenu d'obéir. » Et James Adair, qui connaissait bien les Indiens Muskoghi du sud-est des États-Unis, déclare au début du XVIIIe siècle : " Le pouvoir de leurs chefs est un mot creux. Ils ne peuvent que persuader ou dissuader leur peuple, soit par la force de leur caractère, soit par la logique, soit en présentant les choses de manière à émouvoir les passions populaires. " Nous avons déjà expliqué que les " chefs » crow étaient surtout des guerriers qui avaient accompli certains exploits reconnus par les conventions.

Quelques réserves sont à faire, mais en général l'absence d'autorité centrale est l'un des traits les plus caractéristiques des sociétés nord-américaines. On pourrait en conclure que le chaos était l'inévitable conséquence de cet état de choses. Mais ce serait ignorer la force énorme, pour ne pas dire terrible, qu'exercent les coutumes établies et l'opinion Publique. S'exposer à la réprobation générale des voisins, voir ses méfaits tournés en dérision dans des chants satiriques, s'entendre reprocher publiquement sa conduite par ses " parents de plaisanterie ", c'étaient là des sanctions auxquelles les Indiens préféraient se soustraire. C'est ainsi qu'ils pouvaient se passer d'une autorité exécutive puissante et d'institutions pénales. Le droit coutumier rendait inutile toute législation nouvelle.

Toutefois, cette affirmation, bien que conforme à la plupart des phénomènes, ne s'applique pas à tous. En certaines circonstances affectant les intérêts de la tribu, il était besoin d'un pouvoir plus concentré que celui dont disposait communément une personnalité plus ou moins honorée. C'est ce que nous démontre l'organisation de la police chez les Indiens des Prairies, lors des grandes chasses au bison, alors qu'un seul faux pas pouvait effaroucher le troupeau entier et aventurer les réserves alimentaires de tout le campement. C'est pourquoi la rigueur la plus absolue remplaçait momentanément l'extrême individualisme qui régnait en temps normal. Les femmes ne devaient pas abattre d'arbres et il était défendu aux hommes d'aller chasser pour leur propre compte afin de ne pas diminuer les chances de succès de l'entreprise collective. Non seulement la police confisquait le gibier du délinquant, mais battait 
celui-ci, lui brisait ses armes et détruisait sa tente. S'il résistait, il courait le risque d'être tué sur place. Ces agents de police avaient encore d'autres fonctions, bien que moins apparentes. Ils empêchaient les expéditions de guerre de partir au moment inopportun et il était de leur devoir de tenter de réconcilier les individus dont l'hostilité personnelle pouvait déclencher une querelle grave. La constitution de ce corps de police variait selon les tribus. Parmi les Crow, l'un des chefs en titre faisait office de chef de campement aussi longtemps qu'il plaisait à la tribu, c'est-à-dire qu'elle prospérait sous son égide. Il avait pour fonction de diriger les déplacements du camp et de déléguer l'autorité de police à l'une des organisations militaires; celles-ci se relayaient sans ordre fixe pour faire la police du campement pendant une saison. De même les Hidatsa avaient un chef de village, responsable de la prospérité de la tribu, mais les forces de police se rattachaient de façon permanente à la société des Bouches noires qui occupait dans la série des organisations à degrés une place élevée, mais non la plus haute.

Étant donné le pou d'autorité dont disposait ordinairement le chef, il est naturel que ce ne fût pas lui personnellement qui prît les décisions importantes, par exemple celles qui avaient trait à la paix et à la guerre, mais qu'il consultât un conseil d'adultes. Ce sénat ou cabinet était parfois de nature assez vague, ainsi que parmi les Maidu septentrionaux où cette assemblée se recrutait en général parmi les membres âgés de la société secrète. Ailleurs il y avait un nombre fixe de conseillers. Les Cheyenne avaient un conseil de vingt-quatre chefs électeurs; quatre d'entre eux occupaient un rang supérieur et choisissaient parmi eux le représentant suprême de la tribu. Chez les Omaha également, il y avait un conseil fixe de sept chefs nommés à vie et grâce à leurs hauts faits. Deux d'entre eux passaient avant les autres en vertu de leurs exploits particuliers et le reste du conseil était gradué selon ce même critère. Il n'y avait pas d'assemblée populaire et ce sénat omaha aurait rappelé une oligarchie si, comme nous l'avons déjà dit, la qualité de membre n'eût point dépendu uniquement du mérite individuel; d'autre part le champ de leurs délibérations était si restreint que ce conseil ne portait pas atteinte à la liberté personnelle dans les questions de la vie quotidienne. Il concluait la paix, fixait la date de la chasse annuelle et choisissait celui qui se mettrait à la tête de cette expédition. Durant la chasse, les Omaha se subordonnaient à l'homme qu'ils avaient choisi. Un trait particulier qui caractérise les discussions non seulement des Omaha, mais apparemment de toutes les assemblées analogues chez les Indiens est l'absence de vote majoritaire; chaque décision exigeait l'approbation unanime de ceux qui avaient pris part au débat.

L'individualisme indien a comme corollaire sur une échelle plus grande un séparatisme très développé. Les deux mille Hopi sont répartis en sept ou huit villages complètement autonomes; même le petit hameau de Shipaulovi, avec ses cent cinquante habitants, a son chef cérémoniel et célèbre tout le rituel hopi indépendamment de ses voisins. Cette attitude s'hypertrophie encore sur la côte du Pacifique, ainsi que l'a démontré Kroeber. Le terme de " tribu » perd là toute signification politique, aucune unité n'étant reconnue en dehors du campement. Les villages maidu s'unissaient parfois pour attaquer un ennemi commun, mais cette alliance était d'un caractè- 
re éminemment fragile et éphémère, et, si l'on veillait sur le territoire communal avec le soin jaloux que nous avons décrit ailleurs, c'était aussi bien par crainte du prochain village maidu que des intrusions étrangères. Les Shasta étaient un peu moins particularistes, car ils groupaient leurs campements en quatre catégories dont chacune avait à sa tête un seul chef dirigeant donc plusieurs communautés. Cependant ces unités étaient peu considérables puisque la population des Shasta tout entière ne dépassait pas deux mille âmes à l'époque où on les découvrit. En allant vers l'est, la tendance au fractionnement en petits groupes, pour être moins apparente, reste tout de même assez prononcée. Les Dakota sont loin de représenter un agglomérat politique unique. Les Crow sont non seulement demeurés distincts des Hidatsa, étroitement apparentés, mais sont aussi divisés en deux ou peut-être trois groupes locaux indépendants. Et tout tend à prouver que plusieurs des villages Hidatsa et Mandan étaient des unités complètement indépendantes.

Néanmoins, il se nouait des alliances entre plusieurs tribus, c'est-à-dire entre groupes de langues différentes, mais sans aucune tentative d'intégration. Seuls les Krik dans le sud-est et les Iroquois dans l'État de New York fondèrent des unions de plus grande envergure et la Ligue des Iroquois mérite tout particulièrement d'être étudiée. Elle comprenait à l'origine cinq tribus parlant des langues distinctes, mais se comprenant mutuellement. Chacune conservait toute sa souveraineté dans les affaires locales et était gouvernée par un conseil de chefs. A la tête de la ligue, il n'y avait pas une autorité suprême unique, mais un conseil fédéral de quarante-huit sachems; les Mohawk et les Oneida envoyaient neuf délégués, les Onondaga quatorze, les Kayuga dix et les Seneka huit. Cette répartition inégale n'impliquait nulle hégémonie tribale puisque le scrutin individuel n'existait pas; grâce au vote à l'unanimité, chaque tribu était représentée par une seule opinion. En outre, pour qu'une décision fût valable, il était indispensable que tous les représentants tribaux fussent d'accord; si cela n'était pas possible, la question était écartée, comme n'étant pas susceptible d'être réglée. Le conseil fédéral était convoqué sur l'initiative de l'un des conseils tribaux. Tout membre de la ligue pouvait prononcer un discours sur le forum, mais seuls les quarante-huit sénateurs avaient le droit de décider.

Les conseils représentaient leurs clans respectifs, quoique chaque clan n'eût pas son délégué. Chacun était choisi dans une certaine section du clan et nommé par les femmes du clan, mais cette nomination devait être ratifiée d'abord par le conseil tribal, puis par le sénat fédéral. Un député indigne de sa charge pouvait être déposé. Nous sommes donc en présence d'un mélange du principe héréditaire et du principe électif. L'investiture d'un nouveau sachem, en cas de mort ou de démission de son prédécesseur, était l'un des principaux motifs justifiant la convocation du conseil. Il incombait, en outre, à celui-ci de recevoir les ambassades d'autres tribus, de ratifier la paix ou de déclarer la guerre. Le développement que prit cette confédération contribua sans nul doute à l'ascendant qu'exercèrent les Iroquois sur les autres tribus de la côte septentrionale de l'Atlantique. 
Chapitre 13

\section{f. La démocratie et les organisations primitives.}

Retour à la table des matières

Après cette brève étude des faits ethnographiques les plus saillants, nous sommes prêts à nous attaquer aux problèmes généraux qu'ils soulèvent. Il nous faut avant tout analyser l'affirmation de Morgan, selon laquelle les institutions primitives s'associent invariablement à un gouvernement démocratique. Pour lui, la monarchie est incompatible avec le clan qu'il considère, rappelons-le, comme un élément presque universel. « Il est impossible qu'un royaume se forme de façon naturelle en quelque région de la terre pendant les phases de barbarie inférieure, intermédiaire et supérieure » en même temps qu'une organisation clanique. Une telle évolution se produirait plus tard, à l'époque de l'écriture phonétique et de la notation littéraire. Les différences de castes elles-mêmes, y compris l'esclavage, n'ont pas eu lieu avant la phase supérieure de la barbarie, c'est-à-dire avant la fabrication du fer.

Il faut avouer carrément que Morgan, même dans ses plus mauvais moments, n'a jamais prononcé incohérence pareille, et c'est beaucoup dire. Les nègres africains qui savent travailler le fer, mais qui ignorent l'alphabet phonétique, seraient à classer, selon Morgan, dans la phase supérieure de la barbarie, et pourtant il est peu d'éléments qui se retrouvent aussi constamment chez eux que la constitution monarchique. Sur la côte de la Colombie britannique, les indigènes ne connaissent pas la céramique et rentrent donc dans la catégorie des sauvages supérieurs, selon Morgan, ce qui ne les empêche pas d'établir des castes fixes de nobles, de plébéiens et d'esclaves.

On a trouvé une classification analogue chez les Polynésiens qui se rangeraient encore plus bas dans cet étrange système. Quant à la prétendue incompatibilité des clans avec l'aristocratie ou la monarchie, l'on observe que les Indiens de la côte du nord-ouest et beaucoup de tribus nègres sont organisés en clans.

A propos de cette théorie atomiste de la société, il est encore un autre point qui mérite d'être mentionné. Morgan prétend que tous les clans d'une tribu sont sur un même pied d'égalité. Mais le contraire arrive souvent. Dans l'Ouganda, par exemple, certains clans passaient pour inférieurs aux autres; on ne pouvait pas aller chercher dans tels clans le prince héritier du trône, ce qui revient à dire que les fils que le roi avait eus de femmes appartenant à ces clans n'héritaient pas de la royauté. De telles discriminations sont naturelles, sans doute, dans un royaume puissant; toutefois, elles apparaissent aussi chez les Massaï, généralement démocratiques, où les clans des forgerons sont considérés avec une bizarre horreur et ne doivent pas s'unir à d'autres clans. Même dans la zone typiquement démocratique de l'Amérique du Nord, quelques clans passent avant les autres et, en plus d'une tribu, le chef, ou celui qui en tient 
lieu, sort toujours d'un clan déterminé. Bref, prétendre que l'organisation clanique et les distinctions de rang s'excluent mutuellement, c'est avancer une proposition insoutenable qui ne peut s'appuyer que sur quelques rares constatations.

Chapitre 13

\section{g. Organisation tribale et territoriale.}

$\underline{\text { Retour à la table des matières }}$

Selon la théorie atomiste de Morgan, la société primitive diffère fondamentalement de la société civilisée en ce qu'elle est dépourvue d'organisation politique basée sur la contiguïté territoriale. Les tribus primitives, prétend-il, traitent l'individu comme le membre d'un clan, c'est-à-dire d'un groupe de parenté, et ne tiennent compte par suite que de ses relations personnelles; l'État civilisé ne néglige pas, au contraire, les rapports de l'individu avec le territoire en tant que membre d'une ville, d'un canton ou de quelque autre unité spatiale plus grande. Cette organisation politique, dans un sens restreint du terme, représente pour Morgan une évolution relativement récente, se produisant à un niveau culturel très élevé. Il nie que ce point ait jamais été atteint par les Aztèques au Mexique, et son disciple CUNOW soutient une théorie analogue pour le Pérou. Les tribus primitives pouvaient combiner plusieurs clans en un clan plus vaste ou organiser des confédérations sur le type des Iroquois, mais les devoirs de l'individu ne dépassaient pas le statut de la parenté. Tel était l'état de la Grèce antique, y compris Athènes, jusqu'à ce que Cleisthènes, vers 509 avant J.-C., divisât l'Attique en une centaine de dèmes ou municipalités. A partir de ce moment, tout citoyen fut enregistré en tant que membre d'une unité locale; il votait et était taxé non point comme membre de tel ou tel clan, mais en sa qualité d'habitant de telle ville; et conjointement avec ceux du même dème et non du même clan, il élisait ses représentants. Les unités plus grandes étaient basées sur un principe identique; dix dèmes formaient un district et dix districts composaient l'État athénien.

Seize ans avant que Morgan ne mêlât les différenciations conceptuelles que nous avons décrites plus haut, à son système d'évolution sociale, Sir Henry Maine avait émis une opinion analogue : «... L'histoire des idées politiques s'ouvre par l'affirmation initiale que la parenté de sang est la seule base possible de communauté dans les fonctions politiques. Il n'est aucun de ces bouleversements sentimentaux que nous appelons avec emphase révolutions, qui soient aussi surprenants et aussi complets que le changement qui s'est accompli lorsqu'on a passé à un autre principe, par exemple à celui de la contiguïté locale, celle-ci devenant pour la première fois la base d'une action politique commune. On peut dire des communautés primitives que leurs citoyens considéraient tous les groupes dont ils étaient membres comme fondés sur une parenté commune. » 
Il est hors de doute que Morgan et Maine ont raison de différencier nettement l'organisation basée sur la parenté (tribale) et l'organisation territoriale (politique). Tout le problème est de savoir dans quelle mesure cette distinction est parallèle à celle entre civilisations primitives et avancées. Reconnaissons tout de suite qu'il est des tribus primitives qui se conforment admirablement à la théorie selon laquelle la parenté est l'unique facteur de toutes les relations gouvernementales. Les Ifugao du nord de l'île Luçon nous en fournissent un exemple parfait. Ici tout le droit coutumier tourne autour du pivot que constitue le groupe de parenté, et il n'existe absolument aucune autorité centrale qui prenne des décisions engageant les diverses familles. Le groupe est collectivement responsable vis-à-vis d'un autre groupe des méfaits d'un de ses membres; chaque ressortissant du groupe apporte aux autres membres une aide proportionnée au degré de proximité de la parenté, et il est impossible que les parents s'élèvent les uns contre les autres. La terre et les biens précieux sont détenus en commun par les individus qui ne peuvent en disposer qu'avec l'assentiment de la famille. Aucun lien n'unit entre eux les habitants d'un territoire donné, sauf la force d'une opinion publique intangible. " Le fait que deux individus habitent le même village ou le même district n'entraîne nul comportement obligatoire. » En cas de dispute entre groupes, on prend, il est vrai, un intermédiaire pour régler la difficulté, mais il est choisi ad hoc et son autorité est nulle.

Il convient de remarquer que chez les Ifugao l'unité n'est pas le clan, mais le groupe familial bilatéral; toutefois elle correspond en substance au concept général de groupe de parenté en tant qu'organisme de gouvernement. Ce qu'il importe de savoir, c'est dans quelle mesure les systèmes excluant le facteur territorial de ce qu'on considère généralement comme les relations politiques sont fréquents. On peut fort bien concevoir que la société humaine, même dans ses manifestations les plus grossières, est de façon générale plus complexe que ne le supposent Maine et Morgan et qu'elle n'est pas forcément basée soit sur un rapport personnel, soit sur un rapport spatial, mais sur tous les deux à la fois. Il n'est pas nécessaire de se ranger d'un côté ou de J'autre; la parenté peut régler tel ensemble d'obligations, les relations territoriales tel autre, de la même façon que chez nous l'Église et l'État. On ne saurait méconnaître, il est vrai, la possibilité de heurts entre ces deux catégories d'obligations, mais les juridictions peuvent être si bien séparées que tout conflit est écarté quand les événements suivent leur cours normal.

Avant toute autre chose, il nous faut chercher s'il existe dans plusieurs cas un groupement territorial d'individus s'élevant au-dessus d'une classification de parenté coexistante. Il nous suffira de récapituler des faits déjà cités dans d'autres chapitres pour répondre par l'affirmative, toutefois non sans discrimination. En Australie, les groupes des Kariera sont indissolublement rattachés par des liens mystiques à une localité déterminée, et c'est ce groupe local qui fait la guerre à d'autres groupes locaux de la même tribu. Toutefois nous aurions tort de voir là un exemple d'organisation territoriale, car les habitants et co-propriétaires d'un district sont tous parents en 
lignée paternelle. Leur cohésion peut donc s'expliquer de façon plausible comme le résultat de la consanguinité plutôt que de la contiguïté spatiale. Mais en Australie nous trouvons des tribus entières qui sont aussi étroitement unies à leur habitat que les clans kariera à leurs localités respectives, à tel point même que la possibilité de dépouiller une tribu vaincue de son sol ancestral ne se peut concevoir. Ici donc la tribu entière, réunissant plusieurs groupes de parenté, fait fonction d'unité territoriale. C'est le cas des Arunta et de leurs congénères ; toutefois les Dieri nous en fournissent J'exemple le plus favorable. Chez eux la descendance est matriarcale, mais le mariage patrilocal, en conséquence les hommes d'une même localité ne sont pas membres du même clan totémique. Alors que chaque clan a à sa tête le vieillard le plus âgé, le groupe local a aussi son chef qui peut être en même temps ou non le chef d'un groupe totémique. Celui qui dirige le groupe totémique n'occupera la charge de chef de la localité que s'il fait preuve de qualités spéciales. Nous voyons donc l'unité territoriale coexister avec l'unité de parenté, mais en rester indépendante. Nous avons vu, en outre, que les Dieri possèdent ce dont les Kariera sont absolument dépourvus : un chef suprême pour toute la tribu, réunissant sous son autorité tous les habitants du territoire.

Citons comme exemple parallèle à celui des Dieri le caractère disparate que présentent en Mélanésie les règles de descendance et l'organisation politique. Les indigènes patrilocaux de Buin sont affiliés au clan de leur mère ; le village comprend donc des membres des divers groupes de parenté utérine. Mais, du point de vue du gouvernement, c'est ici le groupe territorial qui importe ; c'est lui, par exemple, qui entreprend les guerres ; et, chose significative, la fonction de chef ne se transmet pas à l'intérieur du clan, mais de père en fils, c'est-à-dire sans sortir de l'unité locale fixée par la règle de résidence.

Nous avons déjà observé qu'une extrême jalousie à l'endroit de leurs droits territoriaux caractérise diverses tribus, dont quelques-unes représentent un état culturel fort primitif, tels les Vedda, les Maidu, les Shasta et les Indiens de Thompson River. A un degré de civilisation plus élevé, nous trouvons ce sentiment parmi les habitants de Samoa. Dans tous ces cas, le groupe local est une unité indépendante des liens de parenté.

De même, parmi les Tchouktche de la côte, le village est fondé " non sur les relations de famille, mais sur la contiguïté territoriale » et c'est le village aussi bien que le groupe de parenté qui intervient dans les cas de vendetta.

Même lorsque le sentiment qui rattache les individus à telle section de territoire est moins prononcé que dans les cas qui viennent d'être cités, la localisation géographique peut influencer le statut politique. Les deux divisions principales des Crow parlent la même langue, s'unissent librement et sont formées de membres de mêmes clans ; mais lorsqu'un individu s'était fixé dans telle division, son sort était à 
certains égards identifié à celui de ce groupe, quelles que fussent ses autres affiliations.

La question n'est pas ici de trancher lequel, du groupe de parenté ou du groupe local, exerce la plus grande influence, ou si l'un dérive de l'autre. Ce qui importe, c'est que, même dans les couches culturelles les plus basses, la contiguïté locale est l'un des facteurs qui déterminent la solidarité sociale, indépendamment de la parenté. Or j'ai désigné du terme d'association les unités sociales qui ne se basent pas sur la parenté, et le groupe territorial peut être véritablement conçu comme une forme spécialisée d'association. Les membres, ou beaucoup d'entre eux, sont en plusieurs cas plus passifs que dans les clubs ou les sociétés secrètes, mais ils n'en sont pas moins unis par une communauté d'intérêts.

C'est l'un des mérites les plus remarquables de Schurtz que d'avoir expliqué l'origine première de la société politique, au sens que Morgan donne à ce terme, sans avoir fait intervenir les décrets légaux. A Buin, un chef qui construit une halle de conseil et qui rassemble autour de lui en un club tous les hommes du village, détruit ce faisant les liens de la famille et du clan ou plutôt il crée une nouvelle attache qui, du fait de son existence, diminue l'emprise de la parenté. Cette attache est de nature territoriale puisqu'elle unit des hommes de la même localité et de familles différentes ; et elle revêt une signification politique dès que les hommes du même village ne se contentent plus de célébrer des fêtes en commun, mais entreprennent des expéditions contre les villages voisins. Comme il y a déjà été fait allusion, il n'est pas nécessaire que tous les habitants du village participent activement à l'association. Les femmes en sont naturellement souvent exclues et, sous le régime de la gérontocratie australienne, seuls quelques vieillards, ou tout au plus la classe des vieillards, font office de conseil exécutif.

Néanmoins, les femmes et les jeunes gens du district, quoique spectateurs passifs, sont souvent associés à leurs aînés par des rapports qui diffèrent totalement de ceux qu'ils peuvent avoir avec les anciens d'une autre localité. Dans ce sens, ils sont membres, bien qu'inefficaces, d'une association territoriale. La même remarque s'applique aussi aux résultats de l'association secrète des Ogboni dans le Yoroubaland. Ceci revient à dire que plusieurs des types d'associations étudiés par Schurtz sont au fond des agents politiques potentiels, en faisant de la population habitant une zone donnée un agrégat qui fonctionne comme une unité distincte, quelles que soient les autres affiliations sociales des individus qui la composent. Un historien philosophe, Teggart, se plaint de ce que Maine et Morgan ont défini la différence entre les organisations politiques et de parenté sans avoir expliqué les phases de transition ; et il a raison en ce qui touche à ces deux érudits, mais il ne tient pas compte de la précieuse contribution de Schurtz. Même à une époque très ancienne et dans un milieu très primitif, il n'était pas nécessaire de rompre les liens de parenté pour fonder un État politique. En effet, en même temps que la famille et le clan, il a existé pendant un nombre de siècles incalculable des associations, telles les clubs masculins, les classes d'âge ou les organisations secrètes, indépendantes de la parenté, évoluant, pour ainsi dire, dans 
une sphère toute différente de celle du groupe de parenté et capables de revêtir facilement un caractère politique, si elles ne le présentaient pas dès le début.

Toutefois un avertissement est ici de mise. Il arrive que les associations opèrent la fusion d'une société en une unité politique stable, mais elles n'atteignent pas toujours à ce résultat. Les Chinois ont eu à diverses périodes et ont encore une multitude d'associations, sans pouvoir parvenir à l'unité nationale. Quelques-unes de leurs trade unions ont parfois lutté avec succès contre les agents du gouvernement central. Comme le dit Courant : « Pour le Chinois, la patrie n'est pas l'Empire mais la province, plutôt encore le district d'où il est originaire. » Dans des conditions plus primitives, les guildes de Samoa représentent une force centrifuge analogue. En outre, il est évident que des associations rivales telles que celles qui caractérisent quelques tribus indiennes des Plaines, tendent tout autant que des clans rivaux à disloquer un État en formation. Enfin nous ne pouvons manquer de noter que les pasteurs nomades de l'Asie, qui sont des constructeurs d'États célèbres, ne présentent aucun développement associationnel. Bref les associations sont un facteur important pour la création d'une solidarité politique, mais non le seul ; même là où elles se rencontrent, tout dépend encore du type d'union qu'elles créent

Chapitre 13

\section{Références.}

$\underline{\text { Retour à la table des matières }}$

1) HOWITT : 295-326, surtout 297 et suiv., 320 et suiv. SPENCER et GILLEN, $1904:$ 20-30. ROTH, 1906 : 5 et suiv.

2) RIVERS, 1914 (b) : Il, 409. TREGEAR : 123, 150, 192. STAIR: 65-91, 128. ERDLAND : 99113. WAITZ et GERLAND : 165-222, 343 et suiv.

3) THURNWALD, 1912 : 314-326; id., 1910 : 9-15. KEYSSER : 100. ZAHN : 308.

4) JUNOD 1, 355-409. FERGUSON : 197 et suiv. SPIETH : 98-110. ELLIS, A. B. 161-181. TORDAY et JOYCE $: 53$ et suiv. FROBENIUS : 172 et suiv. MERKER : 18. LABOURET $: 215$, 383 et suiv. 
5) KRAUSE 122. BOAS, 1916 (a) : 429, 496. Relations des Jésuites : II, 73. ADAIR 428. FLETCHER et LA FLESCHE : 206. LOWIE, 1912 : 228 ; 1913 : 274. KROEBER, 1917 (b) : 396. MORGAN, 1877 : Part. II, chap. 5. GOLDENWEISER, 1912 : 468.

6) MORGAN, 1877 : part. II, chap. 2, 8, 10. MAINE : chap. V.

7) RADCLIFFE-BROWN : 144. SPENCER et GILLEN, $1904: 13$. HOWITT : 47. BOGORAS : 50, 628, 668. LOWIE, 1912 : 245. COURANT : 68. 
14

\section{LA JUSTICE}

$\underline{\text { Retour à la table des matières }}$

L'administration primitive de la justice fournit des exemples particulièrement significatifs des relations du facteur de parenté avec d'autres éléments. Lorsque l'autorité centrale fait complètement défaut, ainsi que parmi les Ifugao, le groupe de parenté devient un corps judiciaire qui s'oppose aux autres corps analogues, de même qu'un État souverain s'oppose aux autres États. Mais c'est là un cas extrême ; bien plus fréquemment, c'est le pouvoir central qui intervient, non point dans tous les cas qui exigeraient chez nous une action gouvernementale, mais dans les circonstances qui, du point de vue indigène, présentent un intérêt collectif.

Citons à ce propos la comparaison de Maine entre la jurisprudence primitive et celle des civilisations plus évoluées. La première, dit-il, est caractérisée par une étrange prépondérance du droit pénal sur le droit civil. « Plus le code est archaïque, plus sa législation pénale est complète et circonstanciée. » Les anciens auteurs ont expliqué ce phénomène par la prétendue brutalité de la vie primitive. Avec sa pénétration habituelle, Maine ne se contente pas de cette interprétation facile, mais démontre que le volume relativement réduit du code civil à l'époque primitive est dû au fait que cette partie de la jurisprudence n'avait guère alors l'occasion de se manifester. Le règlement des affaires personnelles par le statut des individus, l'administration et 
l'héritage des biens qui, selon le droit coutumier, ne sortaient pas de la famille, enfin l'absence de contrats entre individus, tout cela explique que la jurisprudence civile jouât un faible rôle en comparaison des lois pénales ; selon Maine, " il est téméraire d'affirmer que l'enfance des nations est toujours une période de violence et de désordre ».

Cette dernière affirmation est à dessein trop faible. On ne saurait répéter assez souvent que l'individualisme extrême qu'on trouve souvent dans les sociétés primitives est loin de favoriser l'anarchie universelle ou tout autre état s'en rapprochant. De façon générale, on peut dire que les indigènes observent plus volontiers les lois non écrites du droit coutumier que nous les articles de nos codes. Malinowski a critiqué à juste titre un passage d'une édition précédente du présent ouvrage où il était question de l'obéissance spontanée du primitif au droit coutumier. De même que dans nos civilisations avancées, la théorie et la pratique ne vont pas toujours de pair, ainsi, ditil, les règles de conduite ne sont pas toujours suivies. C'est là une conséquence directe de l'existence des différences individuelles qui ont été suffisamment soulignées dans le chapitre relatif au rang. De même qu'il se trouve des lâches dans les sociétés qui exaltent l'héroïsme, ainsi il est des libertins parmi les puritains et des foudres de guerre dans la population pacifique des vinages chinois. Pourtant, à part ces concessions, il reste certain que, dans la plupart des groupes primitifs, la force de l'opinion publique stimule ou prohibe de façon plus effective que dans notre civilisation moderne.

Chez les Crow, on considère avec mépris les querelles personnelles, et un homme hésitera à risquer sa position sociale et à s'exposer aux moqueries publiques de ses " parents de plaisanterie » en en venant aux mains avec un membre de sa tribu. Devenir par suite de quelque écart de conduite un objet de risée pour ses compagnons d'existence ou se voir infliger un ostracisme complet en châtiment d'un tort grave, ce sont là de terribles punitions pour l'indigène ; elles exercent sur lui une action préventive dont nos sanctions sont souvent totalement dépourvues. Il faut y ajouter encore le facteur religieux. Certains crimes sont considérés comme des péchés ; ce sont des offenses envers les puissances invisibles de l'univers et elles méritent un châtiment approprié en dehors de toute sanction laïque. C'est cette conception qui était à la base, par exemple, de l'observance polynésienne des règles sur les tabous. Bref, même dans les sociétés primitives les plus individualistes, il existe des motifs suffisamment puissants pour maintenir l'ordre, bien que la manière dont ce dernier est conçu varie naturellement selon les localités et s'écarte parfois beaucoup de notre propre conception.

Après avoir expliqué pourquoi les lois pénales prédominent dans la société primitive, Maine limite sa définition en ajoutant qu'au fond « la loi pénale des anciennes communautés ne statue pas sur les crimes, mais sur les torts ou dommages ». Cette remarque nous met au centre du problème. Maine veut dire par là que, pour la jurisprudence archaïque, ce n'est pas l'État qui est considéré comme la partie lésée, mais l'individu qui a souffert le tort et sa parenté. En d'autres termes, nous retrouvons 
ici le problème des relations personnelles contre les relations territoriales. J'ai déjà indiqué que, dans la plupart des cas, le primitif reconnaît à la fois les crimes et les torts, mais, avant de citer des exemples, je dois mentionner certains principes très répandus de la législation primitive. Nous ne nous y attarderons pas, ces questions ayant été exposées avec clarté par Hobhouse dans un ouvrage qu'il est facile de se procurer.

Chapitre 14

\section{a. Responsabilité collective.}

$\underline{\text { Retour à la table des matières }}$

Si l'individu se confond avec son groupe, il s'ensuit logiquement que les autres membres sont collectivement responsables de ses méfaits. Bien que ce soit là une notion archaïque, eue a survécu jusqu'à maintenant dans la manière dont les nations civilisées envisagent la guerre, faisant sommairement abstraction de toute faute ou innocence individuelle. Il convient de noter que les phénomènes qui sont à ranger dans cette catégorie ne peuvent pas servir à l'appui du dogme clanique, car le groupe dont il s'agit n'est souvent pas le clan mais la famille, l'association ou la tribu. Les Hupa sans clans punissaient les meurtres en tuant quelque membre de la famille de l'assassin. Parmi les Crow, si un Renard s'était couvert de honte, lui et sa société, en reprenant une femme séduite, les Bois renflés, leurs rivaux, avaient le droit de déchiqueter les couvertures de tous les Renards ; et dans la même tribu, la douleur que les parents manifestaient à la mort d'un fils tué par les Dakota, s'apaisait immédiatement lorsqu'on s'était vengé sur quelque membre de la peuplade hostile.

En raison de ce principe, toute offense d'un groupe à un autre ressemble à une atteinte qu'un État porterait à la souveraineté d'un autre État. Par ailleurs, un crime commis sur un individu par un membre du même groupe ne concerne personne en dehors dudit groupe. C'est ce que nous démontrent constamment les Ifugao ; ainsi deux cas de parricide restèrent impunis, parce que c'était une affaire de famille, ne regardant personne d'autre que les parents ; ceux-ci jugèrent que ce meurtre était justifié puisque le vieillard avait à tort mis en gage le champ de son fils et par là porté atteinte aux moyens de subsistance de sa famille. Les lfugao ne prennent aucune mesure contre un membre du même groupe, quand bien même il commet les crimes les plus monstrueux. Ainsi, si un père a des relations incestueuses avec sa fille, il peut être châtié par les parents de la mère de la jeune fille qui se prétendront offensés, mais sa propre famille ne fera rien contre lui.

Étant donné cette loi suprême de la solidarité du groupe, il s'ensuit que, lorsqu'un individu a lésé un membre d'un autre groupe, son propre groupe le défend alors que le 
groupe opposé soutient les prétentions de l'offensé à une compensation ou à la vengeance. C'est là l'origine des vendettas et des guerres civiles. Le degré d'opiniâtreté qu'on y apporte varie selon les régions. Les Tchouktche font en général la paix après le premier acte de rétribution, mais parmi les Ifugao la lutte peut se poursuivre indéfiniment jusqu'à ce qu'enfin une alliance matrimoniale rétablisse des relations pacifiques. Ces deux tribus nous démontrent de façon intéressante comment un même principe peut être à l'origine de coutumes toutes différentes : alors que les lfugao tendent à protéger un parent en n'importe quelles circonstances, les Tchouktche évitent souvent une querelle en tuant un parent qui, par son irritabilité, est susceptible de les brouiller avec les autres familles.

Nous trouvons en Australie une étrange variante de ce principe - les Dieri infligent la peine de mort au frère aîné du criminel plutôt qu'au coupable lui-même.

Les haines de famille se sont maintenues jusqu'à nos jours dans les régions éloignées de l'Europe et des États-Unis. Il y a dix ans environ, le baron Nopcsa a publié des statistiques démontrant que, dans certains districts d'Albanie, vingt-cinq ou trente pour cent des morts sont dues à des vendettas.

Chapitre 14

\section{b. Intention criminelle.}

\section{$\underline{\text { Retour à la table des matières }}$}

Du fait que la justice se contente qu'un membre du groupe de l'offenseur soit puni, il est facile de déduire que l'intention criminelle ne joue pas dans les lois primitives un rôle aussi considérable que dans notre propre jurisprudence. Goddard raconte un incident survenu chez les Hupa et qui sert d'exemple classique: « Un enfant est mort brûlé par un feu qu'une femme avait allumé dehors pour faire chauffer l'eau de sa lessive. Quoique la femme ne fût pas coupable, on exigea en compensation la vie de son fils. » Cependant, ce postulat n'est pas universellement reconnu. Les Ifugao se font spécialement remarquer par le soin qu'ils apportent à discriminer les actes volontaires des involontaires, ceux qui sont accidentels ou qui résultent d'une négligence. Si le couteau d'un individu lui échappe de la main et va se planter dans l'œil d'un voisin, on ne réclame aucune indemnité. Dans les escarmouches à propos des buffles de sacrifice, beaucoup d'hommes sont blessés, quelques-uns tués; pourtant, même dans ce dernier cas, la famille ne perçoit rien. Au contraire, si un homme tue un enfant qui s'est trouvé sur son passage, alors qu'il s'exerçait à atteindre une cible avec sa lance, on juge qu'il n'a pas pris suffisamment de précaution et il doit payer une amende équivalant à la moitié de ce qu'on paie pour le meurtre d'un homme. Une amende encore plus forte est infligée à celui qui tue un voisin en le prenant pour un 
ennemi, car ici l'intention de tuer s'aggrave du délit de négligence. Les lfugao font une exception à cette règle: lors des grandes fêtes, l'hôte et le prêtre officiant sont tous deux responsables des accidents qui peuvent survenir, le premier parce que, s'il n'avait pas organisé cette fête, il ne se serait pas produit de querelle, le prêtre parce qu'on en conclut qu'il ne s'était pas acquitté avec conscience de ses devoirs religieux.

Les Bantou du sud-est font une distinction très intéressante entre un homicide accidentel et une atteinte fortuite à la propriété. Tout homicide est criminel puisqu'il prive le chef d'un de ses sujets; même s'il n'y a pas eu intention criminelle, le meurtre doit être expié par une compensation fournie au chef. Mais quand on porte atteinte aux champs d'un individu ou à ses autres biens, on ne commet qu'un dommage et, s'il n'y a pas eu préméditation, aucune indemnité n'est à payer.

Ces trois exemples doivent nous mettre en garde contre le danger des généralisations prématurées. Toutefois, en tenant compte de ces réserves, il reste certain que le motif éthique d'un acte est plus souvent dénué d'importance dans les cultures primitives que dans nos cours de justice.

Chapitre 14

\section{c. Wehrgeld (Composition).}

\section{$\underline{\text { Retour à la table des matières }}$}

Les démêlés entre le groupe de l'offenseur et celui de l'offensé Prennent souvent fin par un accord mutuel, c'est-à-dire par le paiement d'un Wehrgeld compensant le tort qui a été fait. Il existe souvent un tarif des sommes à payer pour tous les dommages possibles. Il convient, cependant, de ne pas oublier que les sociétés divergent quant à la nature des offenses pour lesquelles il peut être offert un paiement en compensation. Les Ifugao ont un système compliqué d'amendes pour le règlement de toutes sortes de difficultés, mais le meurtre volontaire ne peut s'expier que par le sang.

L'usage est moins rigoureux chez les Tchouktche; cependant ils sont beaucoup plus enclins à accepter le Wehrgeld dans les méfaits véniels, à la place de châtiment personnel, que dans les cas de meurtre. En beaucoup de régions, la trahison ellemême se règle à l'amiable dans l'intérêt de la paix publique. Quelques exemples concrets nous feront mieux pénétrer le sens de ces tarifs conventionnels.

Le droit coutumier des Ifugao établit une division tripartite de la société sur la base de la fortune, l'amende variant selon la situation financière des parties intéressées. Ainsi, pour l'adultère consommé après la seconde cérémonie de mariage mais 
avant le rite final, un homme riche paie une amende de dix objets totalisant une valeur de quarante-sept pesos; un individu de classe moyenne livre un nombre d'objets égal, mais pour une valeur de vingt-quatre pesos vingt, et à un indigent on ne demande que six objets valant en tout douze pesos. Dans les cas d'homicide qui n'exigent pas que le sang soit répandu en retour, le meurtrier fortuné doit organiser de grandes fêtes et livrer toutes sortes d'objets que se répartissent les héritiers du défunt; la dépense globale s'élève parfois à neuf cent soixante-quinze pesos. Cette somme dépend toutefois du rang de l'individu qui a été tué; elle sera moins considérable s'il s'agit d'un membre de la classe moyenne et, à plus forte raison, d'un pauvre. Par contre, si le meurtrier appartient à la classe la plus basse, l'amende ne sera pas diminuée; il devra passer le reste de son existence à la payer et après sa mort ses enfants en devront encore le solde.

Les Kirghiz d'autrefois établissaient aussi des distinctions de classes dans la taxation des amendes. Pour le meurtre d'un homme libre, l'assassin devait payer un kun, c'est-à-dire cent chevaux ou mille moutons; la famille d'un noble avait droit à une somme sept fois plus élevée; pour le meurtre d'une femme ou d'un esclave, on s'en tirait avec un demi-kun plus neuf animaux domestiques variés et pour un enfant on payait un tiers de kun. Un bras brisé, la perte de la main gauche ou d'un pied étaient compensés par trois fois neuf têtes de bétail; pour un pouce cassé, le prix était de neuf animaux domestiques. En cas de dent et de doigt cassés ou de blessure à la tête, l'individu qui avait souffert le dommage recevait du coupable un cheval et un vêtement. Le vol était puni par une amende de trois fois neuf bêtes, un chameau équivalant à trois chevaux et à trente moutons. Si une femme enceinte était renversée et accouchait d'un enfant mort-né, l'assaillant devait payer un cheval par mois de grossesse si la femme était dans son cinquième mois, mais si elle était plus près du terme, il fallait livrer un chameau pour chaque mois.

Ces règles sont évidemment très détaillées. En beaucoup de cas, on admettait le principe de composition sans qu'on stipulât de façon aussi précise la somme à payer. Ainsi chez les Indiens des Prairies, il semble qu'on livrât à la famille du mort un nombre variable de chevaux, alors que d'autres offenses étaient effacées par des offrandes ou des cadeaux de valeur indéterminée. Dans cette région, une coutume curieuse et très en honneur se substituait à la composition. Au lieu d'attendre des dédommagements, l'individu lésé pouvait détruire un ou plusieurs chevaux appartenant à son ennemi ou d'autres objets précieux. Cet usage était particulièrement commun dans les cas d'adultère.

Chez les Shasta, le système des compensations s'écarte quelque peu de la norme. Le prix du sang doit toujours être accepté si on l'offre; cependant, quand on se venge sur quelqu'un qui n'est pas le meurtrier lui-même, la compensation habituelle doit être fournie aux parents du second mort, la dette de ceux-ci consécutive au premier meurtre étant ainsi supprimée en grande partie ou totalement. Les accords mutuels sont très faciles dans cette tribu, car chaque individu a déjà sa valeur fixe, déterminée 
par le prix qu'on a payé pour sa mère lorsqu'elle s'est mariée. Dans tout ceci, l'élément le plus curieux est peut-être le rôle joué par le chef qui non seulement essaie d'arriver à un arrangement entre les parties adverses, mais avance ou fournit même la valeur exigée si l'agresseur s'avère insolvable. Citons enfin la manière dont les Samoans envisageaient le meurtre et l'adultère. Fréquemment le criminel ne se contentait pas de livrer des nattes précieuses ou d'autres biens, mais faisait preuve d'humilité en apportant également à la partie lésée du bois, des pierres et des feuilles, indiquant par là symboliquement qu'on pouvait le tuer, le faire cuire et le manger et qu'il s'en remettait à la générosité de ses adversaires. En général, cette combinaison de cadeaux et de marques d'humilité ne manquait pas d'apaiser la colère des offensés. Les gens de basse classe ne manifestaient pas cette mortification symbolique et n'offraient qu'un paiement qui pouvait être refusé.

Chapitre 14

\section{d. Preuves.}

\section{$\underline{\text { Retour à la table des matières }}$}

Ainsi que Hobhouse l'a souligné, la législation archaïque tend moins à déterminer exactement la faute ou l'innocence qu'à éviter des luttes d'extermination. Néanmoins, même dans les civilisations primitives, on fait usage de certaines méthodes pour découvrir si telle accusation est fondée ou si telle dispute a sa raison d'être. D'ordinaire ces moyens sont pénétrés des notions magico-religieuses qui ont cours dans la tribu. A cet égard, deux ensembles de coutumes requièrent notre attention: les serments et les ordalies.

Parmi les Indiens des Prairies, les serments servaient à l'origine à établir la validité d'un titre contesté à des honneurs de guerre. Il arrivait ainsi chez les Crow que deux hommes prétendissent chacun être le premier à avoir touché le corps d'un ennemi; en ce cas, on prononçait des serments solennels devant l'assemblée des guerriers. Deux méthodes étaient en vigueur.

Selon l'une chacun des plaignants prenait à son tour un couteau, l'introduisait dans sa bouche, le dirigeait ensuite vers le soleil et récitait une formule par laquelle il prenait le soleil à témoin en souhaitant la mort au faux plaignant. Selon l'autre, une flèche traversant une tranche de viande était posée sur le crâne desséché d'un bison, puis les deux parties prenaient la flèche, goûtaient à la viande et récitaient une même formule. Naturellement le verdict ne pouvait pas être rendu sur l'heure, mais s'il survenait peu après à l'un des rivaux un accident grave ou qu'il fût éprouvé de quelque 
manière, la tribu considérait qu'il s'était parjuré et que c'était l'autre qui avait droit à la distinction, motif du litige.

En d'autres parties du monde, le serment a pour but de déterminer la culpabilité. Chez les Samoyèdes et les Ostiak, on fait jurer le plaignant sur une gueule d'ours. Tandis qu'il y enfonce un couteau, il prononce: «Que l'ours me dévore si je ne dis la vérité ». Le parjure sera, croit-on, châtié; aussi quiconque prononce ce serment est-il tenu pour innocent. Si, dans la suite, l'individu est tué par un ours ou meurt accidentellement, on attribue sa fin au fait qu'il a prononcé un faux serment. Les Kirghiz observent une coutume curieuse; ce n'est pas le plaignant qui prononce le serment, mais un homme connu pour sa probité qui par là prend sur lui le péché du criminel. Rappelons aussi l'ancienne coutume des Arabes et du Moyen Age germanique selon laquelle chaque plaignant était secondé par autant d'amis et de parents possible qui renforçaient par leurs serments ses affirmations. D'une façon générale, les serments sont caractéristiques de l'Ancien Monde, quoique, ainsi que nous venons de le voir, ils apparaissent sous une forme limitée parmi les indigènes américains.

L'ordalie est également une institution de l'Ancien Monde, assumant les formes les plus variées. Parmi les Tchouktche, les différends se règlent parfois par une séance de lutte; les assistants sont fermement convaincus que l'individu lésé doit vaincre. Les Ifugao font usage des mêmes méthodes pour fixer des limites contestées entre des champs de riz, alors qu'ils emploient d'autres moyens pour mettre fin à des litiges d'ordres différents. Ainsi, dans les cas d'adultère, les adversaires se jettent réciproquement des œufs. D'autres fois, chacun des plaignants doit enfoncer lentement sa main dans un récipient d'eau bouillante pour en retirer un caillou; une hâte exagérée ou une sérieuse brûlure sont interprétées comme des preuves de culpabilité. En traitant de l'Afrique, nous citerons encore d'autres exemples de cet ordre.

On emploie aussi naturellement des moyens magico-religieux servant de diverses manières à déterminer la faute, mais ne portant pas toujours un caractère judiciaire. Ainsi lorsque les lfugao désignent un voleur par divination en faisant tenir un œuf en équilibre sur une lame de lance, l'œuf se dressant sur sa pointe lorsqu'on prononce le nom du coupable, le processus intellectuel est le même que dans le cas des ordalies, mais légalement les deux phénomènes sont différents. L'acte divinatoire a ici une signification purement personnelle; il ne fait qu'inciter l'individu dépouillé à intenter contre le criminel une action qui, elle, a une portée juridique.

Après avoir rapidement analysé quelques-uns des traits les plus caractéristiques de la jurisprudence primitive, passons à l'étude des phénomènes juridiques dans diverses peuplades, surtout afin de nous rendre compte si et de quelle façon la société punit les transgressions au droit coutumier. 


\section{e. Australie.}

$\underline{\text { Retour à la table des matières }}$

Les Australiens nous fournissent l'exemple admirable d'un peuple situé très bas dans l'échelle du progrès matériel, mais possédant une autorité centrale déterminée pour punir les crimes. Il est vrai que certains actes que nos lois tiennent pour criminels, ne concernent personne en dehors du cercle de famille immédiatement intéressé. Un habitant du Queensland peut brutaliser ou même tuer sa femme; la tribu ne s'en inquiétera pas, mais la parenté de la femme pourra lui demander des comptes. Une mère peut légalement tuer son enfant quelques heures après la naissance. Pour certaines transgressions du code indigène, il n'y a pas d'autre châtiment que le ridicule et la réprobation générale, qui sont infiniment plus pénibles aux primitifs que nous ne saurions l'imaginer. Enfin d'autres méfaits sont, croit-on, punis automatiquement par des moyens magiques; ainsi les cheveux d'un homme blanchissent prématurément s'il parle à sa belle-mère. Cependant, à part toutes ces restrictions, il existe un ensemble de délits qui ne peuvent être réglés par un accord mutuel, ni trouver leur châtiment simplement dans des moqueries ou par une punition impersonnelle, mais qui nécessitent l'intervention de l'État sous la forme du conseil de la tribu.

Considérons d'abord quelques exemples typiques d'administration privée de la justice. Le Queenslandais, nous venons de le voir, a un pouvoir absolu sur sa femme; si elle lui est infidèle, il peut la frapper avec un boomerang, lui percer la jambe d'une lance ou lui mettre des braises sur l'estomac. De même, le père châtie à son gré un fils non initié sans que quiconque ait le droit de s'en mêler. Lorsqu'on empiète sur un terrain possédé individuellement, le propriétaire peut se contenter d'injurier le braconnier, mais aussi lui percer la jambe d'une flèche si c'est un membre de la tribu; un étranger peut être tué. Puisqu'on reconnaît la responsabilité collective, il arrive que le meurtre d'un homme soit la cause d'une vendetta, mais, grâce à l'une des plus curieuses des institutions indigènes, on l'évite en y substituant une rencontre légale où le criminel, armé d'un bouclier, tient tête aux parents ou au groupe local du mort. Ceuxci lui jettent des lances qu'il évite de son mieux jusqu'à ce que son sang coule, ce qui met fin aux hostilités. Ces combats expiatoires ont été désignés du terme d'ordalies, mais ce sont évidemment des phénomènes tout différents puisqu'ils ne déterminent pas la culpabilité du criminel, patente dès le début. De tels combats se produisent aussi à l'occasion de fautes moindres; l'accusé peut alors être activement soutenu par ses parents; il est autorisé non plus seulement à se défendre, mais à jeter des projectiles sur ses adversaires.

L'expiation formelle est appliquée pour certains méfaits, mais semble être d'une fréquence limitée. Elle est très populaire dans le sud-est ainsi qu'au Queensland; là, en effet, un voleur exprime son repentir et présente sa tête aux coups, tandis qu'un diffamateur se laisse frapper sur la bouche par la personne calomniée. Dans le centre 
de l'Australie, les réparations de cet ordre ne semblent pas être aussi fréquentes, bien qu'après un enlèvement le séducteur puisse avoir à se soumettre aux brutalités du mari offensé afin d'éviter qu'il y ait lutte entre les groupes locaux intéressés. Dans cette région, lorsqu'un décès est attribué à la magie noire, le conseil tribal charge une bande organisée de venger le crime.

S'il existe des variations locales quant à la conception du crime envers la société, dans tout le continent, cependant, on s'accorde à considérer comme telle l'infraction aux lois sur l'inceste. Ainsi, chez les Dieri, un jeune homme fut accusé devant le conseil tribal d'avoir pris femme dans une catégorie défendue. Les anciens examinèrent la question, maintinrent l'accusation et tuèrent presque le coupable qui n'échappa à la mort qu'en faisant plaider l'imbécillité par un homme influent de la tribu. Toute différente était la sanction punissant l'enlèvement d'une fille promise en mariage à un autre homme. C'était alors à sa famille à elle de régler l'affaire. Les frères lésés se battaient contre le séducteur jusqu'à ce que le sang de celui-ci coulât; quant à la jeune fille, elle était violemment fustigée par sa mère et ses sœurs. Cependant, lorsque le jeune homme et la jeune fille qui s'étaient enfuis ensemble étaient l'un vis-à-vis de l'autre dans le rapport de conjoints potentiels, l'enlèvement était en dehors de la compétence du sénat des anciens.

Ce corps gouvernemental punissait encore d'autres crimes, tels que le meurtre par magie noire et la divulgation des secrets révélés lors de l'initiation. En ce qui a trait aux autres formes de l'homicide, l'usage variait probablement selon les localités. Dans le Queensland septentrional, le règlement des disputes ordinaires était affaire privée, mais tout tort grave infligé à un membre du groupe local était vengé par le conseil tribal, à moins que le délit ne fût considéré comme justifié par quelque provocation flagrante.

Bref, ce qui distingue la jurisprudence australienne de celle de peuples plus civilisés, ce n'est pas qu'elle reconnaisse exclusivement les torts ou dommages, mais qu'elle considère comme dommages ce que nous tenons pour des crimes, réservant l'inculpation de crimes à un nombre relativement faible de délits.

Chapitre 14

\section{f. Ifugao.}

\section{$\underline{\text { Retour à la table des matières }}$}

Les lois des Ifugao présentent une extraordinaire combinaison d'éléments. Il serait difficile à une société de porter plus loin les tendances séparatistes des diverses familles que ne l'ont fait ces indigènes de l'île de Luçon. L'individu doit obéir à sa 
parenté et celle-ci accorde sa protection à ses membres contre les autres groupes. Il n'existe pas d'obligations vis-à-vis des autres familles du même village ou du même district. Aucun fonctionnaire ne fait donc office d'arbitre en vertu de quelque autorité dont il aurait été investi. Lorsque deux familles se dressent l'une contre l'autre, on choisit d'un commun accord un intermédiaire non apparenté, mais tout le pouvoir de celui-ci réside dans ses dons de persuasion personnels. En théorie donc, cette désintégration atteint presque le point où la communauté se fractionnerait en familles complètement dissociées les unes des autres.

Par ailleurs, il serait difficile de trouver une tribu primitive où le droit coutumier fixe avec une plus grande abondance de détails la conduite à adopter dans toute une série de circonstances possibles. Donc, ce qui manque en cohésion formelle est partiellement compensé par la force de l'opinion publique qui statue sur les plus petits incidents des rapports sociaux. Ainsi, lorsqu'un amant surpris in delicto est tué par le mari furieux, la famille peut bien chercher à tirer vengeance du meurtre, niais ce faisant elle n'excuse pas l'adultère même, elle affirme simplement que l'époux offensé aurait dû exiger l'amende habituelle et que « si l'amende n'avait pas été immédiatement payée par l'amant, même la famille de celui-ci eût admis que le mari avait le droit de le tuer ». En d'autres termes, les délits ne sont pas uniquement considérés du point de vue de la parenté, 8 jugés selon des canons reconnus par la communauté.

Il n'en demeure pas moins qu'il est étrange de voir les lfugao pour qui le facteur de parenté a une telle importance, déterminer avec tant de minutie ce qu'on pourrait comparer à une législation internationale. Il est vrai qu'il se produit des infractions à la norme, de même que dans les relations entre nations; la famille puissante peut molester le groupe plus pauvre ou moins nombreux. Néanmoins, le mépris de la justice semble ne pas dépasser certaines limites. Un amant fortuné peut refuser de livrer la forte indemnité qu'exige un plaignant indigent, mais il fait une concession à la moralité en daignant payer l'amende la plus faible, celle qui est exigible en tel cas lorsque les rivaux sont tous deux de la classe pauvre. Par ailleurs, il arrive que le lésé indigent, rendu téméraire par le sentiment qu'il n'a rien à perdre, se montre si menaçant que le coupable, sur le conseil de sa propre parenté terrifiée qui l'engage à être prudent, consente à payer une amende exorbitante. Us lois sur la saisie des biens prouvent également de façon irréfutable que certains comportements sont reconnus par tous. Lorsqu'un débiteur refuse de payer l'amende punissant tel délit, le créancier peut à la dérobée ou par la ruse aller enlever dans sa maison un gong ou quelque autre objet de valeur. Son acte est légalement reconnu et tenu pour juste s'il a eu soin de laisser sur les lieux son couteau, par exemple, permettant de l'identifier, sinon il est inculpé de larcin.

Nous voyons donc que des concepts identiques, observés en substance par la communauté tout entière, peuvent pallier à l'anarchie qui découlerait logiquement de la coexistence dans une même localité de toute une série de groupes indépendants. On peut aller jusqu'à affirmer que la société des lfugao, malgré son caractère centrifuge, contient certains germes dont pourrait sortir, dans des conditions favorables, une 
organisation politique. L'existence de l'intermédiaire en est un. Il est vrai qu'il n'a pas d'autorité au sens strict du terme, mais il peut se faire une réputation de pacificateur qui devient pour lui une source de revenus ainsi que de prestige social. Afin de soutenir sa renommée, il se rend à des distances considérables; il poursuit le plaignant irréconciliable ou le coupable obstiné, le couteau à la main, et les oblige à l'écouter. En second lieu, malgré la neutralité de toutes les familles qui ne sont pas directement intéressées, l'opinion publique n'est pas indifférente à la querelle. « Les voisins et les gens du village n'aiment pas voir leur entourage déchiré par des dissensions intestines et se trouver ainsi affaibli lorsqu'il s'agira de lutter contre les ennemis du dehors. » Donc le principe territorial existe, bien qu'à l'état d'embryon et s'effaçant complètement devant les liens de parenté. A l'occasion d'une dispute à propos de terres, les spectateurs s'écrièrent: " En voilà une manière pour des gens du même village de régler une querelle. Rentrez chez vous, faites des enfants, mariez-les entre eux et donnez-leur les champs contestés; la limite n'aura alors plus d'importance ».

Diverses remarques isolées çà et là dans l'ouvrage de Barton indiquent que ce sentiment se fait jour de différentes manières. On nous donne à entendre qu'un Ifugao doit se comporter de façon à éviter à ses voisins des difficultés avec les indigènes des districts ennemis ou semi-hostiles. Il est également important de noter que la responsabilité collective, bien que s'appliquant surtout à la parenté, peut aussi s'étendre au district. Le créancier se saisira de biens appartenant au parent fortuné d'un débiteur lent à s'acquitter, mais, lorsque l'occasion se présente, il peut aussi confisquer le buffle d'un individu du même village que son débiteur. Citons enfin la distinction qu'on établit en matière de châtiment entre un individu du village et un étranger : lorsque ce dernier est surpris en flagrant délit de vol, il est presque toujours tué, tandis que le voisin s'en tire en payant l'amende ordinaire. On ne saurait donc nier qu'il existe parmi les Ifugao un sentiment basé sur la contiguïté locale, bien qu'il soit faible en comparaison des liens de parenté.

Il a déjà été fait dans les chapitres précédents une brève allusion aux éléments essentiels de la jurisprudence des Ifugao: la solidarité du groupe, la charge d'intermédiaire, l'influence de la caste et l'institution des ordalies. Il reste encore à signaler deux particularités: l'une a trait à la nature des sanctions, l'autre à la procédure. Toute punition entre dans l'une des deux catégories suivantes: infliction d'une amende ou peine de mort; les Ifugao ne connaissent pas la fustigation ou d'autres châtiments corporels d'usage courant en Australie, pas davantage que l'emprisonnement. En matière de procédure, il est à noter que le plaignant et l'accusé ne sont jamais confrontés. Aussitôt que le débat est formellement ouvert, il y a rupture complète des relations diplomatiques; toute l'affaire est menée par l'intermédiaire qui écoute séparément les dépositions des deux parties et les communique aux intéressés. Naturellement, lorsqu'on a recours aux ordalies, cette méthode n'est pas appliquée. 


\section{g. Esquimaux.}

$\underline{\text { Retour à la table des matières }}$

Les Esquimaux sont une peuplade nettement différenciée, tant au point de vue racial que linguistique, et pourtant ils ne présentent pas la moindre cohésion politique, même dans des districts voisins. " Les habitants du village, dit Holm, forment souvent une société à part et diffèrent fréquemment de ceux qui vivent dans d'autres campements. Ainsi les habitants de la partie supérieure du fjord d'Angmagsalik et ceux de la partie inférieure s'insultaient copieusement. Ils entretenaient des relations tout aussi amènes avec les habitants des trois fjords. » Parmi les Esquimaux du centre, il règne une si profonde méfiance à l'égard des tribus voisines que les rapports sont forcément espacés. On défie souvent un étranger à une épreuve de force ou d'endurance, mais il expie sa défaite de sa vie. Au Groenland, personne ne peut s'établir dans un village d'hiver sans le consentement unanime des habitants.

Ces faits tendent, sans doute, davantage à prouver que le sentiment de communauté territoriale a pour base une zone très limitée plutôt que l'absence totale de celui-ci. Comme il n'y a pas dans un village esquimau d'autorité gouvernementale, le règlement des délits incombe aux individus et aux familles plutôt qu'à la communauté. A cet égard, les Esquimaux rappellent donc les Ifugao, mais ils représentent un état social beaucoup plus primitif et sont dépourvus en matière judiciaire de cette précision si caractéristique des Ifugao. Les tendances communistes des Esquimaux suffiraient à elles seules à simplifier la jurisprudence, réduisant la loi sur les biens à un strict minimum et n'aboutissant pas à un tarif compliqué d'amendes. Celles-ci sont, en effet, inconnues au Groenland.

Dans l'ensemble, les Esquimaux sont gens pacifiques et la manière dont se règlent les différends d'ordre personnel est un symptôme typique de leur mentalité. Un Groenlandais qui a souffert quelque dommage, soit du fait d'un vol, de destruction de sa propriété ou de l'enlèvement de sa femme, compose un chant satirique tournant en dérision le coupable et invite ce dernier à un tournoi public. En chantant et en s'accompagnant du tambour, il jette à la tête de l'autre ses méfaits, tout en les exagérant et en les rendant ridicules, ressortant parfois des secrets de famille désagréables. L'accusé reçoit ces moqueries avec une indifférence feinte et lorsque l'autre a terminé, il riposte à son tour. A part les chants alternés, on ne déploie aucune hostilité. Les spectateurs écoutent les productions avec grand intérêt, excitant les acteurs à donner toute leur mesure. De tels tournois ne prennent pas nécessairement fin en une seule soirée mais peuvent se continuer pendant des années, les plaignants s'invitant à tour de rôle à la riposte.

Les cas de meurtre sont naturellement traités tout différemment, mais sans apparence non plus d'une administration publique de la justice. C'est le parent le plus 
proche de la victime qui se venge sur le meurtrier ou sur un membre de sa famille. En vertu du principe de la responsabilité collective, la querelle peut se transmettre à la génération suivante et beaucoup d'innocents trouveront la mort avant qu'une réconciliation formelle ne s'opère. A propos de cette prolongation de la querelle, on signale chez les Esquimaux de curieuses coutumes dans des régions très éloignées les unes des autres. Plusieurs années peuvent s'écouler avant que l'offensé ne cherche à se venger d'un méfait et, dans l'intervalle, le meurtrier vit en paix et rend visite à la famille de sa victime où il est bien reçu et bien traité. Puis, brusquement, ses compagnons le massacrent au cours d'une partie de chasse ou bien il est défié à un match de lutte et, s'il est vaincu, on le met à mort.

Toutefois, il est des cas où un meurtrier ou tout autre délinquant s'est rendu odieux à toute la communauté. Il peut alors être tué par un individu quelconque qui fait fonction de justicier. Celui qui s'en charge doit d'abord demander à chacun de ses concitoyens en particulier s'il est d'avis que le coupable mérite la peine capitale. Si tous répondent affirmativement, le condamné est alors mis à mort et personne n'est autorisé à le venger. Un châtiment sommaire est également réservé à ceux qu'on accuse d'avoir par sorcellerie causé la mort d'un parent; cependant le meurtre d'un sorcier peut déclencher une vendetta du type habituel.

D'autres délits rentrent dans la catégorie des péchés. C'est un article de foi parmi les Esquimaux que le fait de la transgression de l'un des innombrables tabous met en danger l'approvisionnement de la tribu: on tente alors en vain d'attraper des phoques et le village est menacé de la faim. C'est seulement lorsque le transgresseur confesse sa faute que les puissances surnaturelles suspendent leur châtiment. Pour découvrir la cause de la calamité, on a donc recours à un shamane; si le criminel avoue, tout va bien, " mais s'il s'obstine à soutenir qu'il est innocent, seule sa mort apaisera la divinité offensée ».

Il semble donc que la communauté n'est pas uniformément indifférente aux actions de ses membres, mais, comme le font d'autres sociétés, elle fixe à sa manière les circonstances dans lesquelles il est désirable que l'unité territoriale intervienne collectivement.

Chapitre 14

\section{h. Indiens des Prairies.}


Comme on pouvait s'y attendre étant donné le caractère nettement individualiste de la civilisation de ces Indiens, la plupart des difficultés étaient réglées entre individus ou entre familles. Ainsi l'adultère était une question privée et si un mari irrité battait brutalement sa femme infidèle ou même l'estropiait, il n'avait pas à en rendre compte devant l'autorité communale. Même dans les cas d'homicide, à l'origine seuls les clans ou les familles du meurtrier et de la victime étaient impliqués, mais en de telles occasions les chefs ou d'autres fonctionnaires tâchaient d'éviter la vendetta. Es y parvenaient souvent en introduisant à l'improviste une pipe dans la bouche du deuilleur principal, ce qui l'obligeait moralement à accepter la composition que le meurtrier et ses parents étaient trop contents de lui offrir.

L'officier de police, chargé de surveiller la chasse au bison, avait aussi généralement pour devoir de maintenir la paix. Nous avons déjà vu que les attributions de ces agents de police étaient fort étendues lors des chasses collectives. En effet, le seul crime contre la société que reconnussent les Indiens des Prairies était de désobéir alors à leurs ordres et par conséquent de mettre en danger l'approvisionnement du village. Nous avons déjà décrit le châtiment sévère réservé au coupable. Le corps de police défendait aussi aux individus les expéditions intempestives, maintenait l'ordre dans les marches ou lors des cérémonies et, d'une façon générale, exerçait l'autorité lorsque le succès d'une entreprise collective était en jeu. L'administration de la justice chez les Omaha présente les caractéristiques des Prairies avec en plus quelques particularités. Le Conseil des Sept avait pouvoir de tuer un membre de la tribu qui s'était montré indocile et rebelle. Le décret était exécuté par un homme de confiance qui se servait pour cela d'un bâton empoisonné. D'ordinaire, on donnait un avertissement au coupable en lui tuant ses chevaux; s'il ne s'amendait pas, c'était à son tour de mourir. Les conseillers jugeaient aussi les meurtres prémédités qui étaient punis par quatre ans de relégation. Durant cette période, le meurtrier était obligé de résider sur les confins du campement et de n'avoir de relations qu'avec sa famille immédiate; celle-ci allait le voir et lui apportait des vivres. La durée du châtiment dépendait dans une certaine mesure des sentiments des parents en deuil, car sitôt que leur douleur diminuait, l'exilé était autorisé à revenir.

Donc l'homicide, en dépit de l'intervention tribale, passait pour un dommage personnel; ce n'était pas la tribu qui exigeait la punition, mais la famille lésée, et le conseil n'intervenait pas pour infliger une sanction méritée, mais pour satisfaire au sentiment individuel de vengeance et pour empêcher que des dissensions intestines affaiblissent la communauté. Selon le code des Omaha, il y avait donc deux crimes seulement: mépriser l'autorité des sept chefs et partir avant les autres à la chasse lors des expéditions collectives.

Chapitre 14 


\section{i. Polynésie.}

$\underline{\text { Retour à la table des matières }}$

Le corps parlementaire qui gouvernait villages et districts à Samoa combinait les trois pouvoirs exécutif, législatif et judiciaire, mais il est difficile de savoir dans quelle mesure exacte les délits personnels étaient poursuivis par le conseil. Nous avons déjà vu que dans quelques cas on recourait à un accord mutuel; mais on ne discerne pas bien comment s'opérait la démarcation et quelle catégorie de délits était réservée à la vengeance publique. Ainsi il est évident que parfois l'homicide se réglait à l'amiable, mais, dans le cas cité par Stair, d'un meurtre multiple particulièrement atroce, l'assassin fut formellement jugé et exécuté.

Le droit coutumier des Samoans présentait une particularité bizarre; c'était l'organisation par l'assemblée locale d'une expédition de pillage contre le coupable. Une fois la décision prise, les chefs de la communauté se rendaient à la demeure de l'accusé, prononçaient la sentence et marquaient d'un cercle un des arbres à pain de la propriété; à ce signal, les autres saccageaient les champs de taro, tuaient le bétail, mettaient le feu à l'habitation et obligeaient la famille à se réfugier en exil. Ce n'est pas à Samoa seulement qu'on trouve ce genre d'entreprises; elles florissaient en d'autres parties de la Polynésie. Ainsi, si un Maori avait accidentellement détruit une propriété collective en y mettant le feu, ou avait par sa négligence privé sa tribu de nourriture, ses voisins arrivaient en corps, s'appropriaient ses biens et le rossaient par surcroît.

D'autres châtiments font preuve d'une cruauté raffinée. En cas de vol ou d'insultes à des voyageurs et pour quelques autres délits, il arrivait que l'assemblée ordonnât au coupable de se frapper la tête et la poitrine avec une pierre jusqu'à ce que son sang coulât. Ou bien on le forçait à mordre dans une racine vénéneuse qui lui faisait enfler la bouche et lui infligeait pendant quelque temps une douleur intense. Une autre torture réservée aux voleurs était de leur lier pieds et mains et de les exposer aux ardeurs du soleil.

La jurisprudence hawaïenne reconnaissait la suprématie absolue du roi. La volonté de celui-ci avait force de loi et il pouvait autoriser quelques privilégiés à enfreindre la tradition. Quoique les chefs secondaires jouissent d'une puissance analogue sur les habitants de leur district, il était possible pour un individu d'en appeler de la décision de son chef à la cour suprême du roi. En dépit des institutions monarchiques et féodales, quelques coutumes typiquement polynésiennes continuaient à être en vigueur dans la vie courante. Par exemple, en représailles d'un vol, il était légal d'organiser une expédition de pillage à laquelle le délinquant se soumettait, même s'il était à la tête d'une force considérable, de peur que tous les hommes du district ne se liguassent contre lui. A Hawaï, une épreuve curieuse était en honneur: plaignant et accusé 
devaient tenir leurs mains au-dessus d'une calebasse pleine d'eau; cette eau était censée trembler et dénoncer ainsi le coupable.

Les Polynésiens doivent évidemment être rangés parmi les peuples qui, quelle que fût leur législation sur les dommages, punissaient comme des crimes les délits envers la communauté ou le souverain.

Chapitre 14

\section{j. Afrique.}

$\underline{\text { Retour à la table des matières }}$

C'est parmi les nègres africains que la jurisprudence primitive atteint son développement le plus haut. Leur code rivalise en précision et en étendue avec celui des lfugao, mais, au contraire de ces derniers, les nègres observent presque partout une certaine méthode de procédure devant les tribunaux constitués. Ils font preuve de beaucoup de goût pour la casuistique judiciaire et l'éloquence oratoire. On trouve aussi chez eux la notion de responsabilité collective et, par conséquent, déléguable, mais l'autorité des cours de justice est telle que les vendettas sont rares, probablement même inconnues, dans le sens plein du terme. Lorsqu'un Ewé a commis un meurtre, les parents de la victime enlèvent parfois un membre de la famille du criminel ou détruisent ses maisons et ses champs, mais la chose semble être plutôt exceptionnelle. En l'absence de toute étude sérieuse sur la jurisprudence africaine, il nous faut résumer les phénomènes des diverses régions dont l'ensemble nous donnera un tableau complet de la justice sur ce continent.

En étudiant les institutions légales des Ewé, comme en somme de toutes les peuplades nègres, nous retrouvons le lien intime unissant chez les primitifs les diverses catégories culturelles qui sont chez nous séparées dans une large mesure sinon complètement. La jurisprudence Ewé reste incompréhensible à qui ne connaît pas leur philosophie de l'univers. Nous avons surtout affaire ici à deux conceptions essentielles: la croyance à la sorcellerie et la conviction que les ordalies sont efficaces pour déterminer la faute. En face du magicien bienveillant qui guérit les maladies se trouve le méchant sorcier qui jette du poison à la dérobée dans les aliments de sa victime, causant ainsi ses souffrances et sa mort. Lorsqu'un homme est accusé d'avoir ensorcelé un membre de sa tribu, il essaie de prouver son innocence en se soumettant à l'une des épreuves prescrites. Ces épreuves sont dirigées par une corporation spéciale; chacun de ses membres a acquis sa science au moyen d'un achat et en se faisant adopter dans la confrérie. 
Tout d'abord le propriétaire de l'ordalie soumet l'accusé à un interrogatoire contradictoire, s'enquérant s'il n'a pas déjà ensorcelé quelqu'un ou s'il a été auparavant condamné par une ordalie. Naturellement il lui est répondu par une dénégation énergique. Vient ensuite le choix de l'épreuve à appliquer. On jette, par exemple, un grain de sel dans un bol plein d'huile de palme bouillante; s'il se fend en deux, c'est signe de culpabilité; s'il reste entier, l'accusé est innocent. Cette épreuve est complétée par une autre: on verse de l'huile bouillante dans la main de l'accusé; s'il la garde sans manifester de douleur, son innocence est prouvée; sinon on maintient l'accusation. Tout dépend naturellement de celui qui dirige l'épreuve. S'il est favorable à l'accusé, il feindra de verser de l'huile bouillante, mais y substituera une huile tempérée. Dans une autre épreuve, on frotte les yeux du coupable avec un liquide vénéneux; s'il est innocent, sa vue reste intacte.

Telles sont les épreuves typiques permettant de déterminer le délit de sorcellerie. Bien que reconnu coupable, le sorcier peut échapper à la sanction suprême, si sa prétendue victime vit encore. On place alors devant lui une houe ou une pioche et un panier, et on lui donne à choisir lui-même son destin. La pioche et la houe symbolisent la tombe qu'on creusera pour lui, à moins qu'il ne prenne le panier qui ne représente qu'une forte amende. Mais, si la victime est morte, le sorcier est lié avec une corde et conduit hors de la ville par plusieurs bourreaux qui l'enterrent vif ou le fustigent à mort.

Il nous est difficile, à nous autres modernes, de considérer autrement qu'avec horreur ce qui nous apparaît comme un mélange d'ignorance et de sauvage brutalité. Toutefois, ces cruautés découlent de la conviction que le sorcier est réellement ou intentionnellement un meurtrier nuisible; et, pour ce qui est de l'ignorance, souvenons-nous que les sorciers ont été exécutés légalement jusqu'en 1782 dans l'ouest de l'Europe.

L'ordalie n'est pas appliquée uniquement dans les cas de sorcellerie; on y a recours aussi pour découvrir un voleur. Le condamné peut faire appel du verdict, mais si les chefs refusent de l'éprouver à nouveau, rien ne le sauvera. Si, par contre, le chef l'y autorise, il peut s'en remettre pour une nouvelle épreuve à un autre propriétaire d'ordalie. Mais comme tous ces derniers forment une corporation et se tiennent réciproquement au courant de leurs affaires, ce privilège est assez illusoire. C'est le coupable et sa parenté qui paient les honoraires du propriétaire de l'ordalie; ces honoraires sont en général extrêmement élevés et ne peuvent être réduits qu'après due supplication du malheureux condamné. En voici la composition ordinaire: une certaine quantité de cauris, quatre poulets, une chèvre et quatre bouteilles de whisky.

Il est évident que toute action légale né requiert ni n'admet nécessairement l'ordalie. Dans des circonstances ordinaires, les procès sont simplement jugés par le conseil des chefs, que les indigènes appellent « la vieille femme ». Le plaignant et l'accusé prennent la parole à tour de rôle, puis leurs témoins sont entendus, enfin les juges se retirent et font proclamer la sentence par le porte-parole. Ce dernier, tandis 
qu'il fait connaître la décision, frotte avec de la terre blanche le bras de celui qui a gagné le procès. Le perdant est condamné aux frais et doit en outre souvent fournir une compensation à son adversaire. Les juges sont indemnisés de leurs peines sur les honoraires de la cour; cependant les vieux chefs reçoivent une part énorme des profits d'un procès.

Il est difficile de donner une idée de ce que sont ces audiences si on ne les décrit pas en détail. Le porte-parole officiel répète les dépositions de chaque témoin et les juges doivent passer par son intermédiaire pour prendre connaissance des témoignages. Comme dans le cas des ordalies, les jugements ne sont pas sans appel; une amende peut, sur demande, être réduite à la moitié de la somme initiale. Dans les discours des témoins et des plaideurs, les dictons, les comparaisons interminables et les paraboles abondent. "Écoutez », dit le porte-parole pour exhorter l'auditoire à l'attention, « il n'est pas nécessaire que nous nous querellions dans cette assemblée. Si nous discutons calmement l'affaire point par point, nous découvrirons qui est à blâmer et nous saurons ce que nous avons à faire. Si on jette une pierre dans une volée de petits oiseaux, d'ordinaire nul n'est atteint; mais si l'on en vise un particulièrement, on est sûr de le frapper... " Voici en quels termes se plaint un chef de ce que son adversaire l'entraîne en des procès fréquents : « La souris a donné un soufflet au chat, mais lorsque le chat a voulu rendre le soufflet à la souris, la souris a dit que le chat cherchait querelle. » Parfois même, on raconte tout un récit populaire pour illustrer tel point de l'argumentation.

Une particularité de la jurisprudence des Ewé est le caractère qu'y revêtent les serments. Il y en a de privés, de tribaux, de royaux et de religieux. En les prononçant, l'accusé affirme son innocence et oblige à ce qu'on enquête officiellement sur l'affaire.

Chose curieuse, la formule évoque souvent quelque événement malheureux. Ainsi le serment tribal " Je le jure par le soir des Ho » fait allusion à une sorte de SaintBarthélemy qui mit fin à l'attaque hostile des Asante. Lorsque le roi a désigné comme objet de serment quelque catastrophe, celle-ci ne doit dès lors plus être mentionnée qu'en cette occasion. Les serments privés font allusion de façon analogue à des difficultés éprouvées par l'individu.

Si nous passons du pays Togo à la région du Limpopo, nous y rencontrerons les mêmes éléments essentiels de la jurisprudence africaine. La notion de responsabilité collective se retrouve dans les deux zones. De même que chez les Ho on peut détruire les champs d'un individu dont le frère a commis un meurtre, ainsi un Thonga est responsable des dettes de ses parents. Dans les deux tribus, un tribunal puissant rend les décisions, et la sorcellerie ainsi que les ordalies jouent un rôle important. Ce n'est que dans les détails que nous relevons des variations intéressantes. Ainsi il existe parmi les Thonga une croyance bien ancrée en la divination par les os et les coquillages magiques; le devin commence donc par tirer au sort lorsqu'il procède à ce qu'on pourrait appeler l'enquête préliminaire sur un décès par magie noire. Si le sort 
désigne par deux fois le même sorcier, celui-ci est accusé officiellement. On l'amène devant le tribunal, sur quoi le devin procède à une nouvelle enquête par questions, se mettant lui-même en état de transe. Si les premiers résultats sont corroborés, mais que l'accusé maintienne son innocence, ce dernier est soumis à une épreuve; on l'oblige à avaler une boisson intoxicante. S'il cède à l'influence de celle-ci, sa culpabilité est avérée et la loi indigène le condamne à la mort par la pendaison, le pal ou la noyade.

Nous ne trouvons donc ici aucun principe nouveau, et il en est de même dans les affaires civiles. Seuls les détails varient selon les différents éléments culturels. Comme la vie sociale des Thonga est centrée autour de la conception du contrat de mariage, sur dix procès civils, neuf ont pour origine le prix de la fiancée; ils sont jugés selon la façon dont la tradition applique cette coutume. Lorsqu'une femme quitte définitivement son mari, sa parenté doit rendre la somme que le mari a payée pour elle, et les enfants reviennent à la mère.

Pour ce qui a trait aux lois en vigueur chez les tribus cafres, au sud des Thonga, on a relevé des observations fort intéressantes.

Les Amaxosa et leurs voisins font une distinction fondamentale entre les affaires pénales et civiles. Les premières comprennent les délits politiques, la sorcellerie et les crimes commis sur les membres de la tribu. Ces délits sont jugés par les chefs auxquels l'amende payée appartient par droit inaliénable. Tous les autres sont poursuivis par les plaignants eux-mêmes et les chefs n'ont aucune part à la compensation. Cependant les plaignants doivent livrer un tiers de l'amende aux huissiers afin de rendre la sentence exécutive. Si toute l'affaire s'est déroulée en dehors de la cour, il n'y a aucun frais à payer. Les procès civils se règlent à l'amiable par devant un conseiller, mais les deux parties peuvent en appeler à la décision du chef. Parfois le conseiller met à l'amende un individu pour voies de fait et garde pour lui la somme versée, mais c'est là un acte illégal et le chef peut réclamer quand il lui plait l'amende empochée par son subordonné.

La loi cafre a pour base essentielle que les personnes des individus appartiennent au chef. C'est donc celui-ci qui doit être dédommagé lorsqu'il perd un de ses sujets. L'amende est de sept têtes de bétail pour un homme et de dix pour une femme; cette différence en faveur des femmes est due à leur valeur matrimoniale. Dans tous les cas d'homicide et quelles que soient les circonstances, on exige un dédommagement. Si on torture un sorcier à mort ou si on le tue sans l'autorisation formelle du chef, celuici a droit à une compensation, bien qu'il renonce souvent à cette prérogative. Dans le cas d'une querelle générale, l'amende pour chaque individu tué est exigible de tous ceux qui ont pris part à la dispute. Avant 1820, un mari pouvait impunément tuer un amant pris en flagrant délit, mais le chef Gaika abrogea cette loi et assimila ce cas aux autres formes d'homicide. Pour voies de fait, l'amende varie entre une et cinq têtes de bétail; en général les deux parties sont punies d'amende, car on considère que rien n'autorise un homme à en frapper un autre, même lorsqu'il se défend. Pour un avortement, la femme et ses complices doivent payer quatre à cinq têtes de bétail et 
on imposa cette même amende au seul cas de sodomie dont Warner entendit parler durant le séjour de vingt-cinq ans qu'il fit dans le pays.

L'adultère se classe parmi les affaires civiles. L'amende est d'une à cinq têtes de bétail, selon la situation sociale du mari; elle peut s'élever à sept ou dix têtes s'il en résulte une grossesse. L'enfant adultérin appartient au mari qui doit l'entretenir. Une femme ne peut pas poursuivre son mari ou ses amants pour adultère. De même, on ne frappe d'aucune amende la séduction des vierges, mais, si la jeune fille est enceinte, le séducteur doit livrer un animal domestique; il peut, dans la suite, revendiquer son rejeton en payant encore deux ou trois têtes de bétail pour dédommager la parenté de la mère des frais que lui a occasionnés l'enfant. Celui-ci reste à la charge de la famille de sa mère si le père ne s'acquitte pas de cette indemnité. Le vol n'est guère commis que sous la forme de vol de bétail. Autrefois, lorsqu'on pouvait rentrer dans ses biens, aucune amende n'était imposable; sinon on exigeait une compensation s'élevant à dix fois le montant du vol. Ainsi que nous l'avons déjà dit ailleurs, l'atteinte volontaire à la propriété est passible d'une indemnisation intégrale, mais les dégâts accidentels n'entraînent aucun dédommagement.

L'amende constitue donc la sanction normale que reconnaît la loi cafre. C'est seulement lorsqu'un sujet porte un défi à l'autorité du chef que celui-ci rassemble clandestinement une troupe armée, fait une descente chez le rebelle, se saisit de son bétail et, s'il rencontre de la résistance, fait tuer sans cérémonie les révoltés.

Le royaume de l'Ouganda était le mieux organisé des États indigènes; aussi trouverons-nous dans ses institutions légales quelques éléments nouveaux. Il y existait une série hiérarchique de cours. Les chefs d'importance secondaire rendaient la justice, mais leurs sujets avaient le droit d'en appeler successivement aux cours supérieures, jusqu'à ce qu'ils parvinssent au dignitaire qui réunissait les fonctions de premier ministre et de magistrat suprême. C'était devant lui que se terminaient la plupart des procès, mais certaines causes exceptionnelles étaient déférées au roi luimême. Le juge suprême avait un assesseur qui examinait les affaires de moindre importance, mais celui-ci devait présenter à son supérieur un rapport sur la base duquel sentence était rendue.

Dans chacun des tribunaux inférieurs le plaignant devait payer en honoraires vingt cauris lorsqu'il introduisait la demande, plus un supplément d'une chèvre et d'un vêtement en écorce avant que l'accusé fût convoqué. Si l'accusé était condamné, il livrait au demandeur, en sus de la sentence, deux chèvres et un vêtement en écorce. Si le plaignant en appelait au juge suprême, il fournissait comme paiement initial dix chèvres et cinq vêtements d'écorce. A part ces honoraires, ce magistrat recevait un quart de l'amende imposée et le perdant était en outre condamné aux frais.

En plus des éléments communs aux diverses tribus africaines, tels que l'ordalie et la composition collective du crime, les lois de l'Ouganda admettaient qu'on fît usage de la torture pour extorquer des aveux. Comme sanction, elles appliquaient aussi la 
peine du pilori: on introduisait les pieds du condamné dans des trous creusés à travers une grosse pièce de bois; une corde fixée à la jambe lui permettait de la lever, mais le frottement constant du bois sur son pied et les gardes qui l'accompagnaient rendaient toute fuite impossible. Parfois on emprisonnait ainsi deux bras et une jambe. Ces coutumes, fort en honneur dans l'Ouganda, nous prouvent une fois de plus qu'il existe bien peu de rapports entre la complexité de la vie sociale et son raffinement véritable.

Il est intéressant de noter que, bien que les monarchies abondent en Afrique, la tendance à obéir aux formalités légales s'y fait sentir encore plus fortement. Ainsi les Lobi n'ont pas de chefs; pourtant un prêtre dirige les ordalies et les anciens discutent selon les formes sur le bien-fondé d'une plainte, recueillant les témoignages du plaignant et de l'accusé. Même des indigènes qui ne sont ni Soudanais, ni Bantou, tels les Massai et les Hottentots, font preuve du même amour pour la procédure légale. C'est probablement un trait caractéristique de toutes les sociétés africaines, sauf des plus primitives.

Chapitre 14

\section{k. Conclusion.}

\section{$\underline{\text { Retour à la table des matières }}$}

Nous croyons avoir suffisamment démontré que la majorité des communautés primitives non seulement reconnaissent les dommages causés aux individus et susceptibles d'occasionner des querelles entre les familles intéressées, mais qu'audessus de la législation sur les dommages elles établissent une loi criminelle, relative aux délits intéressant non plus seulement un groupe restreint de parents mais la communauté tout entière ou son chef. Cette conclusion a sa répercussion sur celle du chapitre précédent qu'elle vient renforcer; elle prouve, en effet, la réalité de l'unité territoriale dans certaines activités sociales. Naturellement l'influence relative de la parenté et du sentiment territorial varie selon la tribu; ou, plus exactement, les sphères d'action de ces deux forces diffèrent selon les régions du globe. Toutefois, même dans un cas aussi net de particularisme familial que celui des Ifugao, nous discernons une vague solidarité entre voisins et nous voyons aux relations mutuelles des habitants d'un même village s'opposer les réactions soulevées par un étranger. Le sentiment territorial doit donc être considéré comme l'un des nombreux liens sociaux dont l'action se fait sentir dans les phases primitives de la civilisation.

Chapitre 14

\section{Références.}


1) MAINE - chap. X. HOBHOUSE : chap. 111. MALINOWSKI, 1926. 2) BARTON : passim. BOGORAS : 662. GODDARD, 1903 : 59, MACLEAN : 60, 67. RADLOFF : 523. STAIR : 96. DIXON, $1907:$ 452. LOWIE, 1912 : 238. NOPCSA : 52. THURNWALD, $1934: 178$.

3) ROTH, 1906. SPENCER et GILLEN, 1904 : 25, 556. HOWITT : 183, 254, 296, 326 et suiv. 4) BARTON.

5) THALBITZER : 59, 127. BOAS, 1888 : 465, 561, 582, 609; id., 1907 :115-121, 467. CRANZ :1, 231, 249. HAWKES, $1916: 109$.

6) FLETCHER et LA FLESCHE : 213.

7) STAIR : 91 et suiv. TREGEAR : 139. ELLIS, Wm. : IV, 419-423.

8) SPIETH : 123-181, 278-283, 535-543. JUNOD : 1, 410-421. MACLEAN : 57-75. ROSCOE : 260267. 


\section{5}

\section{CONCLUSION}

\section{$\underline{\text { Retour à la table des matières }}$}

La société primitive présente donc en réalité un caractère tout différent de celui que l'école de Morgan a rendu populaire. Au lieu d'une monotone uniformité, nous trouvons une diversité nuancée; à la place d'un seul système clanique, multiplié fastidieusement à l'infini, nous découvrons tout un ensemble d'unités sociales qui tantôt accompagnent le clan, tantôt prennent sa place. Afin de rendre plus compréhensibles les conditions d'existence dans une tribu primitive, prenons un exemple concret en le choisissant dans un milieu social qui ne soit pas particulièrement compliqué.

Il y a quelque quatre-vingts ans, parmi les Crow de la Montagne, une femme du clan des Tentes épaisses donne le jour à un garçon. Son mari fait appeler un guerrier célèbre de son clan, les Mauvaises Jambières, qui baptise le nouveau-né Frappe-troishommes, en souvenir d'un de ses propres exploits. En grandissant, Frappe-troishommes apprend à se comporter envers ses parents maternels ou paternels et quelle conduite il doit attendre de leur part. Les femmes des Tentes épaisses font pour lui des chemises et des mocassins brodés de perles; les hommes lui fournissent leur appui en cas de difficulté. L'enfant considère son père comme le protecteur naturel du cercle de famille immédiat et comme celui qui pourvoit à ses besoins. Quand il peut, il offre des cadeaux à tous les autres hommes du clan des Mauvaises Jambières et les traite 
avec respect. De leur côté, ils se font ses panégyristes officiels dès qu'il se distingue par son habileté à la chasse ou par sa bravoure dans le combat; et le lien qui l'unit à ces hommes est si étroit que, lorsque l'un d'eux se rend coupable de quelque manquement à, l'étiquette tribale, il reçoit lui aussi un sobriquet approprié. Entre lui et les enfants de ses " pères " s'établissent de curieuses relations. Ils sont les mentors les uns des autres et se jettent réciproquement à la face leurs faiblesses et leurs erreurs.

A ces diverses relations, basées sur la famille et le clan, viennent bientôt s'ajouter les liens de l'association. Il entre dans une société que ses compagnons de jeux ont constituée à l'instar des sociétés de guerriers, et il essaie de se couvrir de gloire en chassant des cerfs et des bisons, de même que les vrais braves collectionnent les dépouilles des ennemis Dakota ou Cheyenne qu'ils ont tués. Bientôt l'un de ses compagnons, Taureau albinos, devient son meilleur ami. Ils vont ensemble courtiser les filles et se prêtent leurs maîtresses; ensemble ils prennent part aux expéditions militaires, chacun risquant sa vie pour protéger l'autre; ils entrent ensemble dans la société des Renards où Taureau albinos a été invité et ils passent tous les deux à celle des Bois renflés, lorsque ces derniers, favorablement impressionnés par les exploits guerriers des jeunes gens, les pressent de faire partie de leur association. Ce sont alors de nouvelles relations qui se créent pour eux. Frappe-trois-hommes assiste les Bois renflés, ainsi qu'il le fait pour les autres membres de son clan; lui et son ami participent aux fêtes et aux danses de la société; et ils passent leurs heures de loisir à flâner et à fumer dans les tentes de leurs nouveaux camarades, en chantant les chants des Bois renflés. Lorsque Frappe-trois-hommes s'achète une femme, une unité nouvelle vient s'ajouter aux autres groupes sociaux; elle s'ajoute plus qu'elle ne se substitue au groupe de famille primitif, car les liens qui unissent le jeune homme à ses frères et sœurs, loin de se relâcher, conservent toute leur force.

Supposons qu'à cette époque la fantaisie le prenne de partager la fortune de ceux qui chassent vers le confluent du Yellowstone. Dorénavant les relations politiques de ces derniers deviennent aussi les siennes. Avec ses nouveaux compagnons, il va rendre visite aux tribus amies des Village, sur le haut Missouri, puis il se lance à la poursuite d'une bande Dakota; et si les Crow des Montagnes refusent de se joindre à ceux de la Rivière pour une expédition punitive contre ces ennemis héréditaires, Frappe-trois-hommes est le premier à dénoncer la pusillanimité de la bande dont il faisait autrefois partie. Du reste, il ne s'est jamais senti étranger dans la nouvelle région; sur le Yellowstone, il a retrouvé des Tentes épaisses qui l'ont accueilli comme un frère et il s'est mêlé sans formalité aux Bois renflés de la contrée.

L'un de ses enfants tombe malade et il fait le vœu de se faire admettre dans l'Ordre du Tabac si l'enfant recouvre la santé. Quatre Ours, de la section des Belettes, consent à l'initier, et c'est ainsi que Frappe-trois-hommes et sa femme deviennent membres de cette société, jouissant du privilège de planter chaque année la plante sacrée et de participer aux autres cérémonies de leur section. Un lien particulièrement intime les unit dès lors à leur parrain, Quatre Ours; celui-ci, en signe d'affection paternelle, leur fait à l'occasion don d'un cheval. 
Ainsi notre Crow est devenu membre d'une demi-douzaine environ de groupes distincts. De par sa naissance, il appartient à une famille, un clan et une bande. Plus tard, il se lie d'amitié éternelle avec Taureau albinos; il entre dans l'organisation des Renards, puis des Bois renflés et est finalement admis dans l'ordre religieux du Tabac. Arrivé à la maturité il est simultanément membre des Tentes épaisses, des Bois renflés, des Crow de la Rivière, des Belettes; il est en outre l'ami de Taureau albinos et le centre de la famille qu'il a fondée. Ses affiliations, pour variées qu'elles soient, ne dépassent pas en nombre celles de la moyenne de ses concitoyens. En certaines conditions spéciales, il peut s'en ajouter bien d'autres. S'il se fait remarquer par sa bravoure, il devient chef; l'achat d'une médecine crée entre lui et le vendeur un lien de caractère cérémoniel; s'il se procure une autre médecine, il entre dans une organisation distincte, les Danseurs du Cheval.

Dans l'ensemble, il se produit étonnamment peu de heurts d'intérêts du fait de ces obligations variées. Les liens émotifs qui unissent l'individu aux divers groupes, bien loin d'entrer en conflit les uns avec les autres, prennent de plus en plus d'extension. Sans doute, certaines obligations sont traitées plus à la légère que d'autres. Si de deux camarades l'un est insulté par les membres de leur société militaire, ils la quittent tous deux et se font admettre dans une autre. Il est probable, par contre, que l'attachement à l'épouse s'efface devant la parenté de sang ou de club. Selon le code d'honneur quelque peu bravache auquel le Crow a soin de rendre publiquement hommage, une femme reste une femme et il serait malséant de montrer trop de sollicitude à son endroit. Peut-être en va-t-il différemment dans la vie privée du bourgeois crow. Toutefois les occasions où ces divers sentiments peuvent se manifester ne sont pas très nombreuses et les membres de la tribu ne pâtissent pas en général de la multiplicité de leurs affiliations.

Nous aurions pu tout aussi bien choisir d'autres exemples afin de rendre sensible l'abondance des relations sociales. Chez les Andaman qui ignorent le clan, nous aurions eu à tenir compte du statut déterminé par les restrictions alimentaires, de l'état civil et des degrés de parenté. Parmi les insulaires des îles Banks, nous aurions trouvé que chaque indigène appartient simultanément à un clan, à un degré du club et à une demi-douzaine de sociétés d'esprits. Chez les Vedda, le groupement territorial aurait figuré au premier plan alors qu'il serait resté en arrière dans les distinctions de castes polynésiennes. Dans chacun de ces cas, nous aurions donc dû envisager diverses unités coexistantes.

Cette multiplicité n'est pas incompatible avec un système unilinéaire d'évolution sociale. En effet, on peut concevoir logiquement que, à une certaine phase de l'histoire de l'organisation clanique, les groupements selon le statut se forment, puis à une autre les classes d'âge, et ainsi de suite. Mais il se trouve qu'empiriquement les divers types d'unités sociales se combinent de la façon la plus capricieuse. Dans une zone, nous trouvons des sociétés secrètes avec clans; dans une autre, des clans mais pas de société secrète; dans une troisième, une société secrète sans clans; une qua- 
trième tribu a l'un de ces éléments ou tous les deux en combinaison avec d'autres types d'associations; enfin une cinquième n'en présente aucun. Sur quels principes pourrait-on baser l'ordre chronologique des combinaisons observées? Dirons-nous que l'absence de clans des Andaman, jointe au groupement selon le statut, est antérieure aux conditions observées chez les Maidu où il n'y a ni clans ni groupement selon le statut mais une organisation secrète? La réunion chez les Mélanésiens des clans utérins et de la dichotomie sexuelle avec les clubs gradués et les sociétés d'esprits est-elle antérieure ou non au complexe des Hidatsa, composé de clans utérins, d'organisations militaires et de sociétés sacrées? Il semble bien vain de vouloir réduire des phénomènes si divers à un seul ordre de succession chronologique. Les partisans de l'évolution unilinéaire eux-mêmes admettraient sans doute que toutes les manifestations sociales ne peuvent s'expliquer de cette manière et ils se contenteraient de soutenir simplement que chaque type distinct d'unité sociale ou chaque phénomène pris en lui-même tend à passer par une série de phases déterminées.

Toutefois, cette affirmation, même sous cette forme limitée, s'avère inexacte dans presque toutes les catégories de l'organisation sociale. L'erreur apparaît immédiatement si nous comparons les institutions de tribus dont les habitants sont distants mais appartiennent en général au même niveau culturel. Les indigènes australiens ont leur existence économique basée sur la chasse et la cueillette des fruits, et tel était aussi le cas des Paviotso du Nevada. Ces deux tribus ignorent l'agriculture et l'élevage de l'Europe néolithique, mais présentent certains éléments du complexe néolithique; les Australiens, par exemple, se font des haches là où la pierre peut être travaillée. Pourtant, si nous voulons mettre en parallèle les divers aspects de leur vie sociale, nous nous heurtons à des dissemblances profondes. Us Australiens ont des clans, des phratries, des classes d'âge et le totémisme; parmi les Paviotso, on ne relèverait pas la moindre trace de ces institutions. Rien donc ne nous autorise à croire que l'apparition ou la décadence de ces dernières coïncide avec telle forme d'organisation. Les phénomènes politiques non plus ne présentent pas la moindre analogie; il n'existe pas chez les Paviotso de corps gouvernemental jouissant des mêmes pouvoirs que la gérontocratie australienne. D'autre part, rien en Australie ne saurait se comparer au chef d'une chasse au lapin, seule forme d'autorité centrale qui existe dans le Nevada. Donc, non seulement les Australiens et les Shoshoni des plateaux sont différents, mais il est impossible de les comparer; ils ne représentent pas une seule évolution, mais deux lignes bien séparées. Et si on nous répond que ce sont là deux cas arbitrairement choisis, voyons-en d'autres. Les Andaman représentent la même phase culturelle que les Paviotso et ne connaissent pas non plus le clan. Mais leur division en couples mariés et célibataires des deux sexes ne peut être rapprochée de rien d'analogue dans le Nevada. Et quoique la ségrégation des célibataires se retrouve en Australie, nous avons vu que cette ressemblance partielle est bien plus probablement le résultat de rapports historiques avec les mêmes peuples que celui d'une évolution indépendante et spontanée.

Non, il n'est aucune base pour l'argumentation spécieuse qui soutient que le progrès culturel et le progrès social peuvent marcher indépendamment l'un de l'autre. 
Nous avons déjà analysé plus haut cette manière de voir et constaté son absence de fondement, précisément lorsqu'on groupe les peuples selon la complexité de leurs institutions sociales. Vus de cet angle, les nègres et les Polynésiens qui occupent technologiquement des catégories toutes différentes, pourraient être considérés comme à peu près équivalents. Pourtant, comparer l'Ouganda et Hawaï, c'est passer d'un univers culturel dans un autre; les Africains n'ont pas le système polynésien de castes, fondé sur l'origine divine, et dans toute la Polynésie il n'y a pas trace de cette jurisprudence compliquée qui est un trait si typique de l'Afrique nègre. Or, si les prétendues lois d'évolution sociale n'opèrent ni parmi les peuples présentant en général les mêmes conditions d'existence, ni chez ceux dont l'organisation sociale est également complexe, où pourrait-on bien déceler leur action?

Comment expliquerons-nous donc les analogies que nous retrouvons dans des zones fort distantes? N'est-ce pas la même loi interne qui produit la polyandrie dans les communautés esquimaux et dans celles des Toda, ou les clans parmi les Pueblo et les Indiens Gros Ventres? Parvenus à ce point, il convient que nous distinguions de manière plus précise que nous ne l'avons encore fait jusqu'ici la théorie de l'évolution indépendante, dont je me suis fait à plusieurs reprises le défenseur, de la croyance en des lois donnant naissance à des répétitions indépendantes des mêmes séries de phases, croyance contre laquelle je m'inscris formellement en faux. Il existe sans nul doute certaines conditions qui peuvent se reproduire dans des zones distantes et avoir des résultats identiques. Ainsi, nous avons vu que la rareté des femmes et la polyandrie se rattachent causalement; cependant, ainsi que je l'ai démontré en son temps, ce parallélisme est strictement limité. La cause la plus fréquente de la polyandrie est l'infanticide des filles, mais l'origine de l'infanticide varie et les effets de la polyandrie, après une brève période de ressemblance, marquent des divergences dans les deux régions en question.

Généralement parlant, des conditions identiques peuvent produire deux séries identiques, mais l'analogie s'arrête là. Le cours de l'évolution culturelle ne dépend pas, en effet, d'un seul élément, mais de tout le complexe des éléments associés, et comme ces derniers ne sont pas les mêmes et ne sauraient l'être étant donné la différence des traditions culturelles, si quelque évolution se produit, il s'ensuit presque inévitablement des divergences.

Enfin il convient de noter que souvent on ne remarque aucun progrès. Le développement se termine en cul-de-sac et rend impossible tout parallélisme. Nous avons dit comment un même point de vue social peut donner naissance à un terme d'insulte identique, comme c'est le cas des Australiens et des Indiens Crow (page 21), mais notre constatation ne va pas plus loin. Aucune conséquence sociale ne découle de l'usage d'épithètes injurieuses analogues et, s'il en existait, on se représente malaisément de quelle nature elle serait. Rappelons à cet égard l'analogie linguistique à laquelle il a été fait allusion dans notre introduction. Si les Shoshoni et les Grecs ont les uns comme les autres le duel, c'est sans doute là le résultat de processus classificatoires identiques, mais quelle importance générale présente cette ressemblance isolée? 
Absolument aucune. Elle n'a pas marqué le début d'une série de modifications morphologiques qui auraient ramené les deux langues à un même modèle linguistique. On pourrait, à la vérité, concevoir qu'une classification de cet ordre puisse être rapprochée de certains éléments extérieurement distincts mais apparentés psychologiquement. Néanmoins, la ressemblance de structure resterait dans l'ensemble très vague. Or, ce cas illustre assez bien la manière dont j'envisage l'évolution indépendante des éléments sociologiques ou culturels. L'évolution indépendante existe, mais ses résultats n'ont que bien peu d'influence sur l'ensemble des événements dans leurs séries respectives; celles-ci restent essentiellement distinctes.

L'existence de l'évolution convergente (résultats identiques obtenus par des moyens différents) pourrait être avancée comme prouvant la réalité de lois aboutissant à des fins prédéterminées. Mais, dans la grande majorité des cas, l'analogie ne résiste pas à une analyse serrée et se réduit à une ressemblance superficielle ou partielle. Ainsi avons-nous vu que la teknonymie peut être le résultat d'un système particulier de désignation du statut, de l'infériorité féminine ou de la rareté des termes de parenté. Évidemment, la signification de cette coutume n'est pas la même dans ces différents cas; il s'agit bien plutôt de trois coutumes qu'il convient parfois d'appeler du même nom. De la même façon, nous groupons sous le même terme de démocratie les régimes politiques de l'antique Athènes et des États-Unis. Cette désignation commune les différencie de certaines autres constitutions, mais n'implique nullement qu'on reconnaisse les affinités psychologiques ou génétiques de ces deux systèmes.

Même là où il s'agit d'une identité véritable, la divergence succède rapidement à la convergence, et tel est le cas de la polyandrie.

Ainsi, ni les cas d'évolution indépendante à partir de causes semblables, ni ceux d'évolution convergente découlant de causes dissemblables ne peuvent établir une loi innée de progrès social. Il est un phénomène cependant qu'on rencontre dans toutes les phases sociales et qui est fatal à toute théorie des lois historiques, c'est la diffusion. Bien que ne créant rien de nouveau, ce facteur fait passer tous les autres au second plan par l'action qu'il exerce sur le développement de la civilisation humaine. Une explication du clan des Omaha n'éluciderait qu'une seule organisation clanique, alors que la diffusion rend compte de celle d'une douzaine de tribus ou davantage. La diffusion ne fait pas que répandre tel ou tel élément. Elle peut aller jusqu'à niveler ces différences de races, de milieu géographique et de statut économique qui passent auprès du public pour de puissants facteurs d'évolution culturelle. C'est grâce à la diffusion que les Chinois ont adopté les notions politiques de l'Occident; c'est grâce à la diffusion que les Indiens des Prairies méridionales partagent avec les Iroquois des forêts un certain type de clan qui les distingue des autres Sioux vivant dans les mêmes conditions géographiques; c'est à cause de la diffusion que des pêcheurs, des nomades et des agriculteurs conçoivent de façon identique l'incapacité féminine. Toute tendance hypothétique de la société humaine vers des séries de phases fixes doit s'effacer complètement devant le penchant constant à l'emprunt et rester une entité inconnue, donc dépourvue de valeur scientifique. 
Chose curieuse, ce fut un juriste qui s'en rendit compte le premier, à une époque où les ethnologues en étaient encore à courir après les feux follets des lois historiques. Il convient de citer intégralement les paroles de Maitland, dans son Livre du Jugement dernier et de l'Au-delà: « Même si nos ethnologues avaient à leur disposition un matériel qui les autorisât à affirmer que chaque fraction indépendante de l'humanité doit, si elle veut avancer, passer par une série de phases immuables qu'on pourrait désigner par phase $A$, phase $B$, phase $C$ et ainsi de suite, même si cela était, il est un fait que nous devrions envisager: les groupes progressant rapidement ne sont précisément pas ceux qui sont indépendants, mais, au contraire, ceux qui se sont approprié des idées étrangères et qui ont ainsi pu, à l'encontre de toute classification, sauter de la phase A à la phase X, sans passer par les états intermédiaires. Nos ancêtres anglosaxons n'ont pas adopté l'alphabet ou le symbole de Nicée après avoir traversé une longue série de " phases » mais en procédant par bonds successifs. »

Nos connaissances actuelles en ethnographie confirment la manière de voir de Maitland. Nous savons maintenant que les peuplades relativement stationnaires tout autant que les peuples relativement progressistes se sont développées culturellement par le contact avec des idées étrangères. Par conséquent, nous ne trouvons nulle part les conditions requises pour que les lois sociales puissent opérer parmi des peuples indépendants. Certes il est indispensable de prendre note des séries parallèles qu'on peut relever chez des peuples éloignés, mais ne décorons pas du titre prétentieux de lois historiques des rapports strictement limités et parfois banaux, tels ceux qui relient la polyandrie à la rareté des femmes.

Nous avons reconnu que la complexité et la particularité des phénomènes culturels sont dues surtout à la diffusion. Nous abandonnons par là cette recherche des formules brèves que préconisait Pearson, non point parce que nous sommes mus par quelque inutile mépris pour la simplification en elle-même, mais parce que nous préférons les faits dans toute leur complexité à un énoncé plus simple, mais qui ne correspond pas exactement à la réalité. Les théories évolutionnistes qui avaient cours récemment encore parmi les sociologues appartiennent à la catégorie de ces « lois » que dénonçait Sir Henry Maine lorsqu'il écrivait en 1861: « On préfère d'ordinaire des théories plausibles et compréhensives mais nullement vérifiées à la recherche sérieuse de l'histoire primitive des sociétés et des lois. » Il est temps maintenant que nous fuyions les théories universelles et sans fondement à la mode d'antan pour nous mettre à ces recherches historiques sérieuses qui préconisent l'étude intensive des diverses régions.

Nous faut-il donc renoncer à tout espoir d'atteindre jamais, par-delà l'étude des séries isolées de phénomènes, à une interprétation générale? Nullement. Tour d'abord, en renonçant aux lois historiques, nous n'avons pas renoncé aux identités indépendantes du facteur temps et véritablement inhérentes à l'existence sociale dans son essence. L'universalité de l'emprunt est en elle-même une généralisation de cet ordre, 
de même que l'aversion innée pour l'effort créateur ou l'incapacité à créer qui sont à leur tour en corrélation avec la persistance des éléments culturels une fois établis.

Secondement, c'est précisément la combinaison particulière des éléments formant l'histoire actuelle ou passée d'un trait culturel donné qui, rapprochée des grands principes sociologiques tels que ceux-ci, peut nous éclairer sur sa signification mieux que tout autre procédé. Afin de rendre la chose plus claire, empruntons un exemple à Maine, ce défenseur des saines méthodes historiques. Il étudiait le fait que, vers la fin de la République romaine, la peine de mort fut supprimée, suppression qui avait été jusque-là expliquée par quelque particularité psychologique des Romains. Mais Maine découvrit qu'à cette époque-là les corps judiciaires permanents tenaient leur autorité de l'assemblée législative; or cette dernière n'ayant pas pouvoir pour infliger la peine capitale, pouvait encore bien moins déléguer cette autorité. Cette explication éclaire donc parfaitement le problème, convainc du premier coup et rend en même temps sensible le caractère spécieux de toute explication qui ne repose pas sur des principes analogues. Si nous voulons comprendre les classes d'âge des Massaï ou les sociétés des Hidatsa, nous ferons sagement de ne nous guider ni d'après Morgan, ni d'après Schurtz, mais de suivre Maine. Il faut nous saturer de l'esprit et du passé des civilisations Massai et Hidatsa ainsi que de celles de leurs voisins, plutôt que d'attendre notre secours de quelque loi chimérique d'évolution sociale.

Les principes qui déterminent la croissance de l'organisme social ne diffèrent pas de ceux qui président à l'évolution de la culture en général. On croyait autrefois que toute l'humanité avait nécessairement traversé les périodes établies en Europe occidentale par les recherches archéologiques. Mais l'exemple de la technologie africaine suffit à prouver que tel n'a pas été le cas : les Africains n'ont pas passé successivement de l'âge de la pierre à l'âge du cuivre et du bronze, puis à celui du fer. Soit en raison d'une évolution autochtone, soit par suite d'emprunts à des sources asiatiques, ils passèrent directement de la fabrication des outils de pierre à celle des instruments de fer. Dans une autre phase de la civilisation matérielle, les indigènes américains, le Pérou excepté, ne connurent jamais les animaux domestiques, ce qui est une preuve évidente qu'un édifice culturel aussi complexe que celui des Mexicains ou des Maya ne repose pas nécessairement entre autres bases sur la domestication. En l'absence d'une loi innée d'évolution, l'histoire sociale se conforme simplement aux faits de l'histoire culturelle en général.

Néanmoins, une différence importante sépare l'histoire de la culture matérielle de celle de l'organisation sociale, non pas tellement d'un point de vue objectif que de celui de l'observateur. Quand il s'agit de la culture matérielle, on se trouve en présence de périodes de régression et de stagnation alternant avec des ères de progrès. Or l'emploi même de ces termes implique des jugements de valeur dont il n'est pas difficile d'établir l'origine. Les outils sont destinés à des buts pratiques précis; si tel type d'outil y parvient plus rapidement et avec plus d'efficacité, ce type est préférable. C'est donc émettre un jugement purement objectif que de dire que les haches de métal sont préférables aux haches en pierre. L'activité économique a pour but d'entretenir la 
vie humaine. Si on observe des possibilités d'existence plus larges à la suite d'une découverte agricole ou autre, nous avons le droit de parler d'un progrès.

Cependant, dans le domaine de la vie sociale, il n'est pas de critère objectif permettant d'évaluer les phénomènes culturels. Les plus grands philosophes n'ont jamais pu se mettre d'accord sur l'idéal ultime vers lequel doit tendre l'existence sociale. En l'espace d'un siècle, la pensée et l'action en Occident ont sauté d'un pôle à l'autre, passant de l'individualisme manchestérien le plus accusé à un socialisme d'État extrême. La manière dont un sociologue étudiera les penchants communistes des Esquimaux, par exemple, variera selon qu'il se rattache aux idées de Spencer ou à celles de Kropotkine. La démocratie est le refrain constant de l'époque moderne, et pourtant elle a soulevé les protestations passionnées d'hommes de génie et de biologistes réactionnaires dont quelques-uns jettent des regards d'envie du côté de la Micronésie, se lamentant qu'ait disparu ce sentiment de respect envers les supérieurs dont les îles Marshall sont un si noble exemple. De même l'émancipation sans réserve de la femme est peut-être le seul but qui soit conciliable avec un individualisme strict, mais qu'arrivera-t-il si ces aspirations individualistes sont subordonnées à d'autres, à la conservation des traditions de famille par exemple ou à des buts eugéniques? Ici encore la manière dont seront jugées les conceptions primitives dépendra de la réaction subjective de l'observateur devant les problèmes épineux de la pensée moderne. Même lorsque le verdict de la société actuelle est quasi unanime, l'enquêteur critique ne doit pas attacher une valeur absolue à son opinion. Il n'est pas certain, par exemple, que la monogamie obligatoire soit absolument préférable à toutes les autres formes matrimoniales, surtout lorsqu'elle est tempérée comme chez nous par un système de libertinage qui ne diffère guère dans l'ensemble de celui des Massaï.

Bref, l'appréciation des éléments sociologiques est fort différente de celle des éléments technologiques de la civilisation. Ces derniers peuvent être jugés selon qu'ils remplissent plus ou moins bien leurs fonctions; les autres tendent vers des buts inconnus ou plutôt vers des buts sur la valeur desquels le doute règne. Ils ne peuvent donc pas être gradués selon un critère subjectif et doivent du point de vue scientifique être considérés comme incommensurables.

Sans doute, les diverses organisations sociales ne sont pas toutes également complexes, mais le degré de complexité ne nous fournit pas un critère de progrès. Quand les Andaman ont développé la ségrégation des hommes et des femmes non mariés entre eux, d'avec les couples mariés, leur culture sociale est devenue plus complexe, mais il serait malaisé de prouver qu'elle s'est améliorée ou qu'elle a reculé. Si nos civilisations éclairées résolvaient le problème du maintien de l'ordre, par exemple, avec autant de succès que les peuples primitifs dans un milieu plus simple, on pourrait accorder que toute notre machine administrative représente réellement une avance intellectuelle. Mais il n'en est rien; notre manière incommode de maintenir la paix et la solution plus élégante qui a été donnée à ce problème par des peuplades primitives restent deux phénomènes sans commune mesure. 
Si nous quittons les coutumes et les institutions elles-mêmes pour passer à l'élément dynamique de l'histoire sociale, nous nous heurtons de nouveau à l'impossibilité d'évaluer les diverses cultures, mais pour une tout autre raison. Les institutions sont généralement différentes et ne peuvent pas être comparées; les processus, par contre, sont non seulement comparables mais identiques dans les civilisations inférieures et supérieures. Ainsi nous trouvons chez les primitifs le thème de la coopération et le besoin de se réunir entre compagnons sous de nombreuses formes différentes qui prennent parfois l'aspect d'institutions tout à fait modernes, telles les trade unions de Samoa. Plus loin, nous rencontrons cet élément omniprésent de l'existence primitive: l'éternelle recherche du prestige qui est évidemment une caractéristique de tous les agrégats sociaux. Ainsi la vantardise, ce trait de caractère que nous avons retrouvé chez tous les primitifs, réfute définitivement un lieu commun périmé selon lequel, dans les sociétés primitives, l'individu disparaît dans le groupe. Il est vrai que l'individualité n'y est guère prisée ; les primitifs n'acceptent les variations personnelles que dans une certaine direction et, pour pouvoir admettre une personnalité, ils exigent qu'elle soit conforme à leur idéal. Mais, à cet égard, la société civilisée et la société primitive se rencontrent en principe, si différentes soient-elles par les détails. Avec une ténacité qui ne s'est jamais démentie, chaque société, de la plus simple à la plus complexe, a adhéré au principe proclamant que le seul péché impardonnable consiste à élever son propre jugement individuel contre l'autorité sociale reconnue et à enfreindre par là les tabous de la tribu. C'est pourquoi, lorsque Sir Henry Maine insiste sur l'importance croissante des relations contractuelles dans la société moderne au détriment de celles qui découlent du statut, son argumentation présente une portée de forme plutôt que de substance pour l'histoire de la liberté individuelle. S'il s'agit de disposer de ses biens, un Ewé jouit de moins de liberté qu'un Américain, mais il en a davantage à d'autres égards. Tous deux sont entourés par une barrière de conventions et s'exposeraient, s'ils la franchissaient, au mépris, à l'ostracisme ou même à la mort. Donc, ni du point de vue morphologique, ni du point de vue dynamique, on ne peut prétendre que la vie sociale ait passé d'une phase de barbarie à une époque de progrès.

La croyance au progrès social accompagnait naturellement la foi en des lois historiques, surtout lorsque ces dernières étaient teintées de l'optimisme évolutionniste qui régnait autour de 1870. Si une nécessité interne fait avancer toutes les sociétés le long d'une voie prédéterminée, les métaphysiciens peuvent continuer à discuter sur le caractère divin ou diabolique de la force qui les pousse, mais on ne saurait mettre en doute que telle communauté reste en arrière, alors que telle autre est plus rapprochée du but. Mais l'étude de l'histoire culturelle ne nous révèle nulle nécessité de cet ordre. Les civilisations se développent surtout par des emprunts dus à des contacts accidentels. Notre propre civilisation est, plus que toute autre, un mélange d'éléments empruntés. Le cours des événements qui lui ont donné naissance n'ouvre nulle voie à d'autres cultures. L'argumentation spécieuse selon laquelle un peuple donné doit passer par telle ou telle phase de notre histoire avant d'atteindre tel ou tel but, ne saurait donc être soutenue plus longtemps. Celui qui s'est pénétré de la pensée de Maitland, reconnaîtra immédiatement que c'est là une absurdité historique et ethnologique. Si nous voulons prescrire à d'autres peuples un programme social, 
nous ne le ferons que mus par des motifs subjectifs. Tout au moins nous est-il maintenant possible d'agir sans être retenu par la crainte de transgresser une prétendue loi d'évolution sociale.

Enfin, les phénomènes de l'histoire culturelle ne sont pas sans influer sur notre propre avenir. L'historien ne peut actuellement plus considérer avec un respect superstitieux ce mélange incohérent, fait de pièces rapportées, que nous appelons civilisation. Mieux que tout autre, il se rend compte des obstacles que rencontreront ceux qui voudront imposer un plan à cette masse amorphe. Toutefois, en pensée tout au moins, il ne se contentera pas d'une soumission humble et fataliste à ce qui est, mais il rêvera d'un système rationnel qui viendra quelque jour mettre fin au chaos. 


\section{APPENDICE}

\section{LA FAMILLE, UNITÉ SOCIALE}

\section{$\underline{\text { Retour à la table des matières }}$}

La famille est « un groupe se composant des parents et des enfants, qu'ils vivent ensemble ou non »- (dictionnaire Murray). Cette conception peut être élargie et comprendre « ceux qui sont apparentés par alliance ou par consanguinité », mais elle devient ainsi assez vague. Adoptons la définition la plus restreinte et voyons si la société humaine doit a priori être constituée par des unités de familles. La réponse sera négative. Il existe des espèces se reproduisant sexuellement qui ne présentent aucune trace de vie de famille. La ségrégation du mari, de la femme et de l'enfant en un groupe distinct doit donc faire l'objet d'une démonstration empirique. En fait, l'existence de cette unité chez l'homme préhistorique a été catégoriquement niée par beaucoup d'auteurs. Au commencement, nous dit-on, était la promiscuité, la licence sexuelle que ne limitait aucune restriction. Les prohibitions les plus anciennes interdisent les mariages entre parents et enfants; elles furent suivies d'interdits sur les unions entre frères et sœurs, et c'est ainsi que par des mouvements de réforme successifs l'humanité finit par atteindre les hauteurs vertigineuses de la monogamie à l'époque victorienne, en théorie du moins.

Par malheur, nous ne savons rien directement quant à la vie sexuelle du précurseur immédiat de l'homme, et une étude comparative du comportement des primates, bien que réfutant définitivement certaines affirmations, apporte à la reconstitution des 
coutumes préhistoriques un minimum de faits positifs. Un zoologue, M. Gerrit S. Miller, a rassemblé toutes nos informations à ce sujet. Il a prouvé que, contrairement à une notion très répandue mais fausse, chez tous les primates observés il n'y a pas au fond d'époque de rut. Il est donc improbable au plus haut degré que le prédécesseur immédiat de l'homme connût un accouplement saisonnier. De même que les primates, il avait des rapports sexuels chaque fois que l'occasion s'en présentait. Il semble, en outre, que de récentes aberrations humaines eussent leur contrepartie chez les primates et soient donc l'héritage d'un passé fort lointain ${ }^{1}$.

Cependant, parmi les informations dont nous disposons, nous ne trouvons rien qui démontre la théorie de la promiscuité humaine primitive. Les observations faites sur les anthropoïdes les plus proches, les chimpanzés et les gorilles, sont indécises et parfois contradictoires lorsqu'elles se rapportent à une même espèce. Reichenow, par exemple, attribue au gorille des habitudes monogamiques, alors qu'Akeley émet la supposition qu'il est polygame. "A la vérité, ajoute-t-il sagement, nous ne savons que peu de chose sur les habitudes du gorille ${ }^{2}$. »

Yerkes, avec une prudence exemplaire, nous déclare ce qui suit: " Nous concluons par l'hypothèse que la monogamie et la polygamie coexistent chez quelques types anthropoïdes ou chez tous et qu'elles se retrouvent selon toute probabilité parmi tous les singes plus ou moins proches de l'homme. Nous proposons, sous toutes réserves, la liste suivante indiquant en ordre croissant les probabilités de relations monogamiques: gibon et siamang, gorille, orang-outang, chimpanzé. Pour découvrir la vérité, il faudrait une étude beaucoup plus systématique, approfondie et critique que celles qu'on a faites jusqu'ici. Comme point de divergence entre les trois types de grands singes, nous indiquons que chez l'orang-outang nous trouvons des relations temporairement monogamiques ou polygamiques, chez le chimpanzé des relations monogamiques relativement permanentes, mais peut-être aussi polygamiques, et chez le gorille la famille patriarcale avec probablement la polygamie dans l'espèce des montagnes et peut-être la monogamie dans celle des plaines ${ }^{3}$. »

Si nous ne connaissons rien de plus positif sur les espèces existantes, il sera vain et prématuré d'en tirer des conclusions dogmatiques sur le comportement hypothétique d'un type ancien disparu.

MILLER, G. S., « Some Elements of Sexual Behavior in Primates and Their Possible Influence on the Beginnings of Human Social Development », Journal of Mammalogy, 9 (1928) : 273-292; idem, " The Primate Basis of Human Sexual Behavior ", The Quarterly Review of Biology, 6 (1931) : 379-410. Après avoir terminé cet article, je vois que les conclusions de Miller sont contestées par S. ZUCKERMAN dans Social Life of Monkeys and Apes (New York, 1932). C'est aux zoologues de décider.

2 AKELEY, Carl E., In Brightest Africa (Garden City, 1925), p. 247.

3 YERKES, Robert M. et Ada W., The Great Apes, a Study of Anthropoid Life (New Haven et Londres, 1929), pp. 542 et suiv. 
Il est un point, cependant, dont nous pouvons être certain. Quelles qu'aient été les habitudes sexuelles du précurseur de l'Homo sapiens, tous ceux qui croient à l'évolution doivent admettre l'existence à un moment quelconque d'une phase de promiscuité, au sens technique de plaisir sexuel non limité socialement. Il n'existe pas anthropologiquement d' " index de promiscuité », obtenu en divisant le nombre des individus mâles et femelles, s'accouplant sans égard à la parenté, par la somme de ceux auxquels il est physiquement possible de s'accoupler. Vue sous cet angle, la question ne comporte que l'alternative suivante: le désir charnel est-il limité dans l'une quelconque de ses manifestations par la désapprobation du groupe? si oui, il n'y a pas de promiscuité; sinon, elle existe. Prenons le cas d'un gorille mâle qu'Akeley a trouvé avec trois femelles. Il n'importe pas de savoir si le mâle cohabitait avec les trois femelles. Il s'agit plutôt de ceci: à supposer que deux d'entre elles soient ses filles, l'attitude des gorilles sera-t-elle indifférente ou non? On peut même concevoir cette situation sur le plan humain. Un conte très répandu parmi les Indiens du Grand Bassin et des Prairies occidentales se déroule autour de ce thème : un fourbe possède au moyen d'artifices ses propres filles. Dans l'histoire, toutefois, cette conduite soulève une réprobation morale intense. Pour ma part, je ne trouve nul indice d'une telle conscience sociale dans l'ouvrage de Koehler ${ }^{4}$, non plus que dans d'autres informations se rapportant au plan infra-humain. Si cette interprétation est exacte, les anthropoïdes pratiquent la promiscuité. D'autre part, il n'est aucun groupement connu d'Homo sapiens qui reste indifférent au comportement sexuel des membres qui le constituent. Partout où nos renseignements sont valables, partout les unions sont jugées, mises hors la loi, réprouvées, excusées, admises Ou nettement sanctionnées. Sous cette dernière forme, l'accouplement peut être qualifié de " mariage " et c'est à partir de ce point que la famille évolue. Nulle part la fornication et le mariage ne sont confondus en une catégorie indifférenciée de « copulation animale ».

Un abîme sépare ainsi l'Homo sapiens du chimpanzé et du gorille. A quelle phase de l'évolution le saut entre ces deux conceptions a-t-il donc eu lieu? Je hasarde l'hypothèse que l'homme de Neandertal faisait preuve de quelque discrimination quant au comportement sexuel et je la fonde sur le fait qu'on décèle chez lui des indices de tradition sociale dans l'artisanat; il me semble donc probable qu'il avait de même développé des normes de conduite sociale. Je me refuse, par contre, à toute supposition relative à l'attitude adoptée à cet égard par l'homme de Heidelberg, l'Eoanthropus, l'homme de Pékin et le Pithécanthrope. Je me contente de croire qu'il exista une phase de licence non contrôlée à quelque époque entre l'anthropoïde le plus reculé et l'ancêtre hominidé plus proche dont les descendants constituent l'humanité géologiquement récente.

Je ne suis pas certain d'être d'accord avec M. Miller sur l'éloignement de cette phase. Il argue que les échantillons humains existants sont des survivants spécialisés et que beaucoup de races se sont éteintes. Il en déduit « qu'on ne peut guère espérer réussir à trouver parmi ces peuples actuels spécialisés une race ou une tribu vivant

4 The mentality of Apes (New York, 1925). 
dans des conditions sociales qui représenteraient le reflet le plus fidèle de ce que fut la mentalité de l'homme primitif ». Tout dépend ici de la signification qu'on attache aux termes de " homme ", " plus fidèle » et " mentalité primitive ». Non seulement j'admets, mais encore je soutiens que les habitants des îles Andaman, les Fuégiens, les Australiens et les Tchouktche ne nous apportent aucune précision sur la mentalité de l'homme de Piltdown ou de Pékin. J'insiste avec force sur le fait qu'aucun groupement humain ne représente la mentalité des premiers hominidés sous sa forme pure. Mais puisque des groupes hautement spécialisés comme les Andaman, les Australiens et d'autres encore exercent sans exception un contrôle social sur leur vie sexuelle, ce contrôle n'est donc pas d'hier, ni de l'an 4000 avant J.-C. par exemple, mais remonte probablement à une période comprenant les tout premiers échantillons d'Homo sapiens, bien que plusieurs des races de cette espèce soient irrémédiablement soustraites à notre observation.

Faute d'espace, il ne nous est pas permis d'étudier en détail plus d'une société humaine récente. Je choisis donc les Australiens qui, à cause de leur infériorité anatomique et de la grossièreté de leur le mode d'existence, sont devenus le point de départ favori des historiens spéculant, entre autres questions, sur la famille ou la religion. En outre, on leur a attribué une forme de vie sexuelle qu'on pourrait considérer comme intermédiaire entre la promiscuité et la monogamie obligatoire, c'est le « mariage par groupes ». Cette institution a été définie comme l'union non préférentielle d'un groupe d'hommes avec un groupe de femmes. Ce n'est pourtant pas de la promiscuité pure, car jamais les Australiens ne toléreraient les unions entre frères et sœurs. Mais les partisans de l'existence de la promiscuité aux époques de l'aurignacien et du moustérien regardent le mélange de polyandrie et de polygynie qu'implique le mariage par groupe comme un début de contrôle sur les accouplements. Par ailleurs, aussi longtemps qu'un groupe d'hommes s'unit sans discrimination à un groupe de femmes, la famille n'existe pas en tant qu'unité sociale.

Afin que mon exposé soit aussi concret que possible, je baserai mes dires sur l'ouvrage que le professeur Radcliffe-Brown tient pour la meilleure description des Australiens, celui de Warner sur les Murngin vivant à l'ouest du golfe de Carpentaria ${ }^{5}$. Je compléterai ce tableau par des informations sur d'autres peuplades australiennes et je terminerai par une sélection de traits saillants relevés dans divers groupes. Les questions dont nous aurons à nous occuper seront les suivantes : existet-il une forme de mariage qui se différencie de la cohabitation? Si oui, quelles sont les relations sociales entre mari et femme? entre frères et sœurs? entre parents et enfants?

Commençons par les Australiens. Aucun Murngin ne peut s'unir à son gré ; quand il veut se marier, il est censé épouser la fille de son oncle maternel. A son défaut, on lui substituera une femme présentant le même statut de parenté, par exemple la fille d'un cousin de la mère. Les conjoints potentiels seront fiancés avant la naissance.

5 WARNER, Wm. Lloyd, « Morphology and Functions of the Australian Murngin Type of Kinship », American Anthropologist, 32 (1930) : pp. 207-256. 
Tout homme, il est vrai, désire avoir le maximum de femmes accessibles sans danger, mais elles ne sont jamais choisies au hasard. Afin de rendre possibles leurs relations sociales, les Australiens rangent les individus en catégories de parenté. Ainsi, même lorsque les Murngin font une incursion dans un campement hostile, les femmes qu'ils enlèvent sont assignées aux hommes qui présentent avec elles le degré de parenté socialement requis. De même, l'adultère se produit presque toujours avec un cousin de la catégorie prescrite. Par une extension naturelle de ces conceptions qui reposent sur l'équivalence sociale des frères ou sœurs d'un même sexe, un individu hérite de la veuve de son frère aîné et souvent les diverses épouses d'un mari polygyne sont sœurs ou quasi-sœurs.

Sans aucun doute, il existe parmi les Murngin comme chez nous de «mauvais mariages ». Néanmoins, en ce qui concerne certains parents, la prohibition est absolue et, à ce qu'il semble, jamais transgressée. En d'autres cas, une forte désapprobation se fait sentir - un homme qui nouerait une intrigue avec la «fille de sa sœur » (qui en fait pourrait être la fille de sa cousine au troisième degré), serait comparé à un chien et la femme risquerait de recevoir une sérieuse volée.

En outre, dans la limite des unions permises, on distingue un certain idéal. Un homme peut avoir plusieurs épouses, mais il ne doit pas entretenir de rapports sexuels avec d'autres femmes; et une femme est censée normalement se contenter d'un seul conjoint, son mari. Les relations sociales entre époux comportent, en outre, des droits et des devoirs déterminés. La femme rapporte les fruits sauvages et le petit gibier, l'homme fournit les poissons, les tortues, les marsouins et les dugongs. Au point de vue sentimental, l'attachement pour les enfants constitue un lien entre eux et, outre ce facteur, on décèle les marques d'une affection réciproque rappelant l'amour romanesque.

Nous ne trouvons dans tout ceci pas la moindre trace de promiscuité ou de mariage par groupe. Les relations de parenté sont élargies au point qu'une femme qui allaite deux bébés peut être secondée dans cette tâche par sa sœur; et, en général, c'est auprès de la tante maternelle que l'enfant va chercher la nourriture et les soins. C'est cependant un développement tout naturel de la pratique de la polygynie sororale. Mais en dépit du principe de l'équivalence entre frères et sœurs, le groupe de famille immédiat est différencié. Ainsi un mari sans enfants observe les tabous alimentaires qui prennent fin avec la paternité « mais l'enfant doit être le sien et non celui d'un frère ». (Nous discuterons plus loin du sens à donner dans ce texte à l'expression « le sien ».) C'est le père qui détermine pour son fils le type d'initiation, C'est lui qui se met à sa droite lors de certaines danses, c'est lui enfin qui lui enseigne la routine cérémonielle. Bref, l'homme manifeste vis-à-vis de « ses » enfants un intérêt particulier.

Les Murngin reconnaissent donc l'unité de famille, mais cela ne signifie pas qu'elle corresponde à notre type familial. Le frère et la sœur ne sont jamais sur un pied de familiarité aisée. Ils ne dorment jamais dans le même campement et ne s'adressent pas la parole. A côté de ces tabous, nous remarquons une attitude de servi- 
abilité mutuelle qui n'a rien pour nous étonner. Un frère fera des présents à sa sœur pour son fils et son mari. Deux frères coopéreront dans une entreprise économique et marqueront un certain sentiment de communauté dans les biens matériels. Ces derniers incluent dans une certaine mesure les femmes aussi mais diverses restrictions écartent la possibilité d'un communisme sexuel sans frein, même entre frères. Aucun frère cadet ne s'appropriera une belle-sœur sans autorisation. Le frère aîné a des droits de priorité sur les filles de son oncle maternel. Lorsqu'il s'est ainsi procuré deux épouses, son cadet a un droit moral sur la plus âgée des filles qui restent, et le père de cette dernière peut engager le premier mari à renoncer à ses prérogatives légales. Même ici il existe donc un droit coutumier et non la licence complète. Cependant, cet usage oblige le cadet à adopter une certaine attitude, inconnue chez nous, à l'égard de son aîné qui lui fournira une compagne de son vivant ou à sa mort.

A cette description de la famille, un nouveau trait vient s'ajouter, c'est le tabou, observé dans toute l'Australie, défendant tous rapports sociaux entre un homme et sa belle-mère. Pourtant, en dépit de l'usage élargi de termes comme " père ", " frère ", etc., s'appliquant à des parents assez éloignés, le groupe de famille immédiat se sépare nettement du reste de la communauté. Un homme qui veut se marier cherche tout d'abord à épouser la « vraie » fille du « vrai » frère de sa « vraie » mère; et l'oncle provoque le ressentiment s'il marie sa fille à un neveu éloigné.

Nous avons vu que le statut social d'un homme marié dépend du fait qu'il a ses « propres » enfants. La distinction établie entre parents proches et éloignés, à l'intérieur d'une même catégorie, se retrouve ici aussi. Des « frères » éloignés peuvent se tendre des pièges, se tuer ou tout au moins se soupçonner mutuellement d'adultère potentiel; mais entre de vrais frères règnent la confiance et une affection qui ne se dément jamais. Ainsi, dans les périodes de licence rituelle, ce sont les « frères » éloignés et non les vrais qui échangent temporairement leurs femmes. Nous retombons donc à chaque pas sur la preuve évidente que, dans le sentiment de l'indigène, les relations de proche parenté sont une chose sui generis. La famille qui en résulte est une unité bilatérale puisque l'enfant entretient des relations avec le côté maternel comme avec le côté paternel de sa parenté.

L'état décrit par Warner n'est pas unique, mais typiquement australien. L'étude synthétique de Malinowski ${ }^{6}$ sur les anciens auteurs et le résumé encore plus récent de Radcliffe-Brown ${ }^{7}$ ne nous laissent aucun doute sur ce point. Dans toute l'Australie, l'équivalent le plus proche de notre unité politique, l'État, est une « lignée paternelle » localisée ou " horde », possédant et exploitant en commun un territoire déterminé. Un tel groupe a comme noyau principal un certain nombre de frères avec leurs fils, les fils de leurs fils et ainsi de suite. Les femmes du groupe sortent normalement d'une autre horde, constituée de façon similaire. Parmi les enfants, les

6 MALINOWSKI, Bronislaw, The Family among the Australian Aborigines; a Sociological Study (Londres, 1913).

7 RADCLIFFE-BROWN, A. R., "The Social Organization of Australian Tribes » (0ceania Monographs, NI 1, 1931). Voir pp. 4, 6, Il et suiv., 103, 107. 
garçons restent dans le groupe, acquérant dès la petite enfance cette connaissance économique intime du territoire héréditaire, indispensable à leur survivance. Les filles vont se marier ailleurs et ne représentent donc que des éléments temporaires du groupe dans lequel elles sont nées. Cependant, à l'intérieur de cette horde nettement délimitée, les indigènes reconnaissent une unité sociale plus petite, la famille individuelle comprenant les parents et les enfants. " La fonction importante de la famille, dit Radcliffe-Brown, c'est de nourrir et d'élever les enfants. Elle est basée sur la coopération de l'homme et de la femme, le premier fournissant la viande, la seconde l'alimentation végétale, si bien que, la question des enfants mise à part, un homme sans femme est dans une situation désagréable, puisqu'il n'a personne pour l'approvisionner régulièrement en légumes, pour lui chercher le bois, etc. Cet aspect économique de la famille est des plus importants et explique en partie la polygynie australienne. Je crois que, dans l'esprit des indigènes eux-mêmes, cet aspect du mariage se rattachant à la subsistance est beaucoup plus important que le fait que l'homme et la femme sont des partenaires sexuels... les relations sexuelles entre homme et femme ne constituent pas davantage par elles-mêmes le mariage en Australie qu'elles ne le font dans notre société. »

Je crois que le tableau que nous dépeignent nos meilleurs auteurs des conditions en Australie peut être généralisé et s'appliquer aux races humaines récentes. J'écrivais, il y a quelques années : "La famille bilatérale est une unité absolument universelle de la société humaine ${ }^{8}$. " Ce sont de bien grands mots, mais je les considère toujours comme conformes aux faits. Il n'est qu'une région dans le monde où il soit possible de déceler des phénomènes tendant à limiter cette affirmation. En certaines parties de l'Océanie où l'adoption joue un rôle extraordinaire, on observe que les enfants partagent plus ou moins leur temps entre deux maisons, participant ainsi simultanément à deux groupes familiaux. J'ai écrit à propos de ces faits dont j'ai pris récemment connaissance : «Sous cette forme extrême, cette coutume (de l'adoption) modifie inévitablement l'universalité de la famille individuelle ${ }^{9}$. » Remarquons en passant que ces exceptions se rencontrent dans des sociétés horticoles hautement développées qu'on ne peut sans doute pas considérer comme fournissant des exemples de coutumes primitives, et que ces exceptions reposent sur un usage qui est, par définition, dérivé.

La prééminence de la famille en général ne peut naturellement pas être démontrée sans que soient passées successivement en revue les diverses sociétés primitives, pour autant que la place disponible nous le permet. Je voudrais cependant faire ressortir un phénomène assez significatif en Amérique du Nord. Si nous étudions les systèmes de parenté des peuples les moins développés de ce continent, soit les tribus de chasseurs dépourvues d'une organisation politique, sociale et rituelle complexe, nous voyons qu'ils ont presque uniformément une terminologie différente pour les membres immé-

Voir p. 83.

LOWIE, R. H., « Adoption, Primitive », in Encyclopaedia of the Social Sciences, 1 (1930) : pp. 459-460. 
diats de la famille et les parents plus lointains. Donc, alors que les Australiens établissent cette distinction par le comportement, les indigènes les moins évolués de l'Amérique du Nord vont jusqu'à exprimer cette différence dans leurs vocabulaires : un père n'est pas seulement traité d'une autre façon qu'un oncle; il est aussi désigné d'une appellation distincte. De même, un frère n'est pas inclus dans le ternie s'appliquant au cousin, et ainsi de suite. Le fait que la plupart des tribus non horticoles de l'Arctique à la Californie septentrionale et au Nevada n'identifient pas ces divers parents, nous incline fortement à croire que l'unité de famille est reconnue précisément par les peuples les plus bas dans l'échelle culturelle, au nord du Mexique. Il semble que la famille ait joui à une période très ancienne d'un ascendant indiscutable et que son importance ait été ensuite modifiée, mais jamais supprimée, par d'autres formes d'organisation. Ainsi, en Australie, l'identification partielle des frères et sœurs de même sexe limite le caractère individuel de la famille, bien que nous ayons la preuve incontestable de la persistance de celle-ci.

Les termes désignant des unités sociales telles que la " famille » peuvent conduire à des erreurs. C'est pourquoi je vais essayer d'indiquer la somme des faits empiriques qui peuvent se ranger sous cette dénomination. Je précise d'abord que la famille biologique n'est pas nécessairement identique à son équivalent social. Un auteur brillant, Margaret Mead, m'a récemment attribué la croyance en une omniprésence sociale de la famille biologique. Elle y oppose l'opinion plus exacte de RadcliffeBrown qui, tout en prenant le groupe biologique comme point de départ essentiel pour une étude de l'organisation sociale, « donne toute l'importance qui leur est due aux développements plus complexes de beaucoup de sociétés primitives ${ }^{10}$. " Au fond, il n'y a pas conflit. Le point essentiel, c'est que Radcliffe-Brown et moi mettons tous deux en garde contre le danger d'attacher trop de poids à l'aspect biologique de l'unité. « Bilatéral » et " biologique » ne sont pas des termes synonymes. Lorsqu'un Australien parle de son " propre » père, il ne pense pas nécessairement à son procréateur, mais à l'homme adulte que, depuis l'enfance, il a associé avant tous les autres à un certain comportement émotionnel, à une activité économique se rapportant à la maison, etc. J'ai fait remarquer ailleurs en m'appuyant sur Rivers que, parmi les Toda de l'Inde méridionale, la polyandrie rend souvent fort malaisé de déterminer la parenté. Mais les indigènes ne se soucient nullement de la paternité biologique : le mari qui accomplit un certain rite durant la grossesse de sa femme devient le père légitime de tous les enfants engendrés par cette femme, jusqu'à ce qu'un autre mari célèbre cette cérémonie. « La paternité biologique est complètement négligée, car un homme mort depuis longtemps est considéré comme le père d'un enfant tant qu'un autre homme n'aura pas célébré le rite essentiel ${ }^{11}$. »

Ainsi, dans quelques tribus sud-africaines, un homme revendique comme son propre rejeton légitime l'enfant d'une femme dûment achetée, même si elle a vécu durant des années en union adultère avec un amant. Ce qui importe, c'est la parenté

10 MEAD, Margaret, « Family, Primitive », ibid., 6 (1931) : pp. 65-67.

11 Voir p. 58. 
légale et non biologique. Voici donc ce que signifie l'omniprésence de la famille bilatérale : virtuellement partout, un homme qui n'est pas nécessairement le procréateur et une femme qui n'est pas nécessairement la mère adoptent vis-à-vis d'un ou de plusieurs enfants certaines relations préférentielles et forment ainsi une unité distincte à l'intérieur d'un groupe social plus vaste.

Le fait qu'il est possible et même relativement fréquent qu'aux relations de parenté biologique s'en substituent d'autres, est l'une des révélations les plus étonnantes que l'ethnographie puisse offrir à la science voisine, la psychologie. Il réduit, en effet, définitivement à néant la croyance en un instinct paternel. A la place de celui-ci, il nous faut mettre une tendance beaucoup plus vague des mâles adultes à s'attacher à des enfants de leur espèce.

L'indifférence dont font preuve les Toda et les Bantou relativement à l'identité du procréateur suffit à différencier leur conception de la famille de la notion traditionnelle qu'en possède la civilisation occidentale. Notre insistance à ne reconnaître pour les nôtres que les enfants que nous avons dûment engendrés doit paraître à ces indigènes une pédanterie physiologique ridiculement déplacée. L'évaluation du statut des enfants peut reposer sur des considérations toutes différentes. Parmi les Indiens du nord-ouest de la Californie, l'équivalent de notre bâtard est l'enfant dont le père n'a pas payé pour sa femme la somme habituelle et qui, affligé de cette tare, ne pourra jamais entrer dans la maison des hommes.

Socialement néanmoins, le type familial n'est que modérément modifié par l'indifférence relative aux liens physiologiques, car, dans les exemples cités, il ne se produit que la substitution au père d'un individu mâle incarnant le principe paternel. Il continuera donc à exister un lien social appartenant à la même catégorie que nos propres relations de parents à enfants. La modification se fera plus fortement sentir dans le cas d'une organisation clanique utérine. Dans une telle institution, le lien avec le père et sa famille est toujours reconnu, mais les enfants se rattachent à certains égards au groupe de la mère et plus spécifiquement portent le nom de son clan à elle et non de celui du père. C'est de cette manière que prennent naissance toute une série de sentiments, de droits et de devoirs légaux qui entrent en compétition avec les liens proprement parentaux et parfois même ouvertement en conflit. Ce faisant, ils se heurtent aussi inévitablement à la famille en tant qu'unité sociale autonome.

Cette opposition est surtout marquée là où règne l'avunculat. L'oncle maternel usurpe à nos yeux beaucoup des fonctions du père et les neveux et nièces utérins occupent auprès de lui la place que nous réservons aux enfants. Ainsi c'est lui et non le père qui dispose de la main de la jeune fille; c'est lui et non le père qui donne aux jeunes garçons certaines instructions; et bien qu'en quelques tribus africaines patrilinéaires le fils hérite des femmes du père, à l'exception de sa propre mère, certains groupes utérins en Mélanésie et en Amérique permettent au neveu d'épouser la veuve du frère de sa mère. Pour prendre un exemple concret, un Dobu en Mélanésie ne peut pas léguer à son fils son nom, sa terre, son statut et ses arbres fruitiers; ils passent 
automatiquement au fils de la sœur. Le père est autorisé, il est vrai, à enseigner à son fils les formules magiques qu'il connaît, mais il est obligé de le faire avec son neveu utérin ${ }^{12}$.

Néanmoins, les coutumes avunculaires ne suppriment pas le père sociologique. Dans la région même d'où je viens de tirer mon exemple, Malinowski a fait la preuve de l'affection profonde unissant le père au fils et a dépeint de la façon la plus saisissante le sombre et tragique conflit qui met aux prises le sentiment paternel et le devoir avunculaire ${ }^{13}$.

Il est des phénomènes d'un autre ordre et qui modifient également le type familial : ils peuvent être rangés sous l'appellation commune de "dichotomie sexuelle ». Celle-ci se manifeste de plusieurs manières. En Australie, chez les Murngin, nous avons trouvé le tabou du frère et de la sœur, qui exclut donc d'emblée une des formes les plus typiques d'intimité familiale dans notre civilisation. Mais nous avons aussi vu que cette coutume ne rompt pas le lien qui relie entre eux frères et sœurs: ils ne peuvent pas se parler, mais ils s'aident mutuellement, et le frère est très attentif à certains devoirs envers ses sœurs. Une autre variété de dichotomie sépare le mari et la femme. En beaucoup de communautés, en Amérique du Sud par exemple, et en Océanie, les conjoints ne mangent jamais ensemble, ce qui nous paraît presque inconcevable. Les habitants des îles Banks en Mélanésie vont encore plus loin. Parmi ces derniers, tout homme adulte a virtuellement acheté le droit d'entrer dans la maison des hommes qui est strictement tabou aux femmes et où les hommes se reposent, travaillent, mangent ou dorment, rendant visite à intervalles à leurs femmes. En dépit de cette institution, la famille se maintient; le mari exerce sur sa femme des droits déterminés et lui est attaché ainsi qu'aux enfants par certains devoirs ${ }^{14}$. Nous pouvons dire de façon générale que la dichotomie sexuelle se produisant universellement dans la répartition des tâches est un facteur favorisant l'unité de famille, car cette division accroît évidemment les avantages du ménage commun.

Il est encore un autre trait modifiant de façon significative les relations familiales. Il peut y avoir ségrégation selon l'âge et le statut ainsi que selon le sexe; ces deux formes peuvent aussi se combiner. Parmi les Massaï de l'Afrique orientale, les célibataires occupent une hutte séparée où viennent les rejoindre les jeunes filles du village et ils s'unissent avec elles apparemment ad libitum. C'est de la promiscuité dans le sens populaire mais non scientifique du terme. En effet, les Massaï s'abstiennent soigneusement d'avoir des relations sexuelles soit avec des parentes, soit avec leurs futures femmes, c'est-à-dire avec les jeunes filles auxquelles ils ont été fiancés dès l'enfance. Ceci ne fait qu'accentuer encore la persistance du concept de famille.

12 (1) FORTUNE, Reo, Sorcerers of Dobu; the Social Anthropology of the Dobu Islanders of the Western Pacific (Routledge, 193 1), p. 15.

13 MALINOWSKI, Bronislaw, Crime and Custom in Savage Society (Londres et New York, 1926).

14 RIVERS, W. H. R., The History of Melanesian Society (Cambridge, 1914), 1 : pp. 60-143; CODRINGTON, R. H., The Melanesians : Studies in Their Anthropology and Folk-lore (Oxford, 1891), p. 101 et suiv. 
Malgré la licence qui règne dans la maison des célibataires, les jeunes gens et les jeunes filles sont nettement censés se marier après avoir jeté leur gourme. A la liberté prémaritale, succède la régularité de la vie de famille ${ }^{15}$.

En d'autres régions, par exemple en certaines parties de l'Australie, seuls les garçons sont séparés des couples mariés. Cela se produit d'ordinaire après une cérémonie d'initiation, parfois à l'âge de sept ans. Les garçons relativement jeunes sont ainsi en quelque mesure soustraits à l'influence des parents et soumis à l'enseignement de membres plus âgés de leur propre génération. A Samoa, les célibataires sont séparés des couples en groupes distincts d'hommes et de femmes. Les hommes non mariés travaillent la terre, préparent les aliments pour les chefs de plusieurs ménages et remplissent des tâches communales nécessaires. Le groupe féminin correspondant comprend les veuves et les femmes non mariées; il semble avoir pour origine la coutume selon laquelle la fille favorite du chef dort avec des compagnes du même âge et des chaperons plus âgés ${ }^{16}$. De même, parmi les habitants des îles Banks, le club des hommes était fractionné en divers degrés dont on achetait le titre de membre. Il y avait donc séparation non seulement des conjoints, mais aussi des pères et des fils: un homme ne mangeait normalement ni avec sa femme ni avec ses enfants, et la mère était séparée de ses fils dès que ceux-ci entraient dans la maison des hommes, soit presque toujours avant l'adolescence.

Ainsi non seulement j'admets, mais encore je souligne, la diversité des types de famille dans les sociétés humaines récentes. Cette différenciation, toutefois, ne change virtuellement rien au fait que le mari, la femme et l'enfant constituent une unité sociale définie, distincte des autres unités, analogues ou non, de leur communauté.

De peur que l'étrangeté de quelques institutions primitives ne nous fasse perdre le sens de la perspective, rappelons-nous quels sont les changements historiques qu'a subis le concept de « famille » dans les diverses civilisations. Les Chinois, certes, ne sont pas dépourvus du sentiment de la famille, mais celui-ci s'accompagne chez eux de notions, étrangères pour nous, sur les devoirs de la femme, la polygynie et le concubinage. Les patriarches de l'Ancien Testament étaient, eux aussi, polygynes, mais personne ne conteste la prépondérance de la famille à l'époque biblique. On dit actuellement beaucoup d'absurdités sur la destruction de la famille par la civilisation industrielle, Pourtant les liens légaux entre parents et enfants, maris et femmes, sont toujours clairement reconnus. Ce qui s'est produit, c'est une altération de l'idéal familial dans une grande partie de la population. Que ce soit en bien ou en mal, le passage de la campagne à la ville, l'importance croissante des facteurs économiques, l'idéologie individualiste et l'abandon partiel des traditions religieuses, tout cela a contribué à modifier les relations qu'implique le concept de famille. Dans la seconde moitié du XVIIIe siècle, le Dr Samuel Johnson, ce parangon de la piété chrétienne,

15 MERKER, M., Die Masai. Ethnographische Monographie eines ostafrikanischen Semitenvolkes (Berlin, 1910), pp. 44, 84.

16 MEAD, Margaret, "Social Organization of Manua ", Bernice Pauahi Bishop Museum of Polynesian Ethnology and Natural History, Memoirs, Bulletin 76 (1930) : pp. 14, 92 et suiv. 
formula en principe que « les épouses sages ne se préoccupent pas de l'infidélité de leurs maris ». Par contre, il considérait une femme qui rendait la pareille à son mari volage comme «toute désignée pour un bordel ». Je crois que ces idées ne sont plus aussi universellement en vigueur. Ce que je voudrais faire remarquer, c'est qu'entre les défenseurs d'une double morale et ceux qui veulent l'égalité des sexes, il y a la même différence que celle qui les sépare des Murngin ou des habitants des îles Banks. Les seuls iconoclastes qui s'écarteraient de la pratique commune seraient ceux qui élèveraient leurs enfants dans des fermes communales et qui ne toléreraient que des liaisons sexuelles tout à fait temporaires. De telles sociétés ont, en effet, été signalées mais avec plus de commentaires indignés que de preuves réelles.

Voici en résumé quelques conclusions d'intérêt général :

1) Nous ne savons absolument rien du comportement sexuel du prédécesseur immédiat des hominidés modernes, si ce n'est qu'il se conformait très probablement à la norme généralisée des primates. Celle-ci implique en particulier la non-existence d'une saison de rut, si les conclusions de Miller sont exactes.

2) Quoique nous ne puissions décrire la vie sexuelle des proto-hominidés, il exista à coup sûr une phase de promiscuité, c'est-à-dire d'activité sexuelle non limitée socialement. Par définition, en effet, les limites sociales sont une caractéristique de la civilisation. C'est pourquoi la promiscuité, au sens scientifique du terme, précéda toute culture.

3) Toutes les tribus les plus primitives du monde (Andaman, Bochimans, Australiens, Fuégiens, Paiute) réagissent violemment contre l'inceste avec les proches parents. Il est donc extrêmement probable que ce sentiment remonte à la plus haute antiquité.

4) Je ne crois pas, cependant, comme je le faisais autrefois, que l'inceste répugne instinctivement à l'homme. D'une part, il existe suffisamment de preuves légales que les pays occidentaux présentent des cas relativement fréquents d'inceste entre père et fille; et si un dixième seulement de ce qu'avance la psychanalyse est à retenir, le complexe d'Oedipe reste un facteur avec lequel il faut compter. En ce qui regarde l'inceste entre frères et sœurs, nous avons pour le moins trois exemples historiques où le prétendu instinct était délibérément enfreint: l'Égypte antique, le Pérou et Hawaï. Dans chacune de ces sociétés aristocratiques, on considérait qu'aucune épouse ne convenait au souverain mieux que sa propre sœur, la seule femme évidemment qui fût de lignée aussi illustre.

Il nous faut donc considérer l'aversion pour l'inceste comme une adaptation culturelle ancienne; dans toutes les sociétés, certains individus l'enfreignent potentiellement ou réellement, et quelques sociétés évoluées l'ont expressément négligée dans l'intérêt d'un sens hypertrophié de la lignée aristocratique. 
5) L'instinct paternel n'existe pas. Aucun individu ne peut reconnaître par l'instinct que c'est bien lui le procréateur de l'enfant que met au monde sa femme. Or, ainsi qu'on peut le prouver, en de nombreuses et diverses sociétés, les primitifs ignorent tout à fait et de plein gré la question de la parenté physiologique, en donnant toute l'importance à la parenté sociologique. Le sentiment maternel semble reposer sur une base plus solide. En fait, la pression des considérations économiques ou le désir d'éviter la honte d'une naissance illégitime peuvent être plus forts. Parmi les Murngin, « la femme tue parfois son nouveau-né s'il en a suivi d'autres de trop près et qu'elle n'ait pas assez de lait à lui donner ». Ici comme en bien d'autres communautés primitives, la crainte superstitieuse qu'inspirent les jumeaux entraîne la mise à mort immédiate de l'un d'eux.

Ce qui est naturellement universel et nécessaire à la survivance du groupe, c'est l'intérêt générique des adultes pour les enfants. Toutefois, ce sentiment, comme nous venons de le voir, ne se manifeste pas uniformément chez tous les individus de l'espèce, mais peut être ignoré en vertu d'une force supérieure, celle d'un rationalisme utilitaire ou d'un irrationalisme idéologique.

6) Toute société fait une distinction entre la cohabitation simple et la forme approuvée par la société de cohabitation relativement permanente qu'on appelle mariage. Il n'est pas superflu de faire remarquer que, de même qu'il existe une paternité sociale sans notion de procréation, il y a souvent un état social de mariage sans relations physiologiques. Un homme peut hériter d'une femme si âgée qu'elle est inapte au commerce sexuel ou indésirable; néanmoins, elle prendra part avec les autres femmes de la maison aux occupations réservées à leur sexe et aura droit aux soins et à la protection du maître. Pour citer un exemple concret, parmi les Manyika de l'Afrique orientale, la femme devient la propriété du fils aîné de sa sœur aînée. « Il ne cohabite pas avec elle, mais exerce un contrôle absolu sur ses faits et gestes. Il peut la garder dans son kraal où elle accomplit pour lui les tâches habituelles d'une épouse. Elle n'a pas de mari reconnu et est autorisée à prendre un amant ou même plusieurs. » Fait intéressant à noter, les enfants de telles unions ne sont pas sous la tutelle de leur père biologique, mais à la garde de celui qui a hérité de leur mère. C'est ce dernier et lui seul qui reçoit pour les filles le prix d'achat et fournit aux jeunes garçons de quoi se procurer une femme ${ }^{17}$.

7) A part quelques modifications secondaires ou des déviations rares et fortement localisées, la famille basée sur le mariage est un phénomène tout à fait général chez les spécimens connus d'Homo sapiens. Presque partout, un homme remplissant les fonctions sociales de père et de mari s'établit avec une femme faisant office de mère et d'épouse, et ils subviennent en commun aux besoins du ménage et de leurs enfants, que ceux-ci soient leurs rejetons véritables ou reconnus tels par une fiction légale. Comme ce type d'institution est fréquent précisément parmi les tribus les moins évoluées, nous pouvons supposer que chez l'Homo sapiens il remonte à une haute

17 BULLOCK, Charles, The Mashona; the Indigenous Natives of S. Rhodesia (Londres, 1928), p. 65. 
antiquité. Mais à quel moment de son histoire est-il apparu et dans quelle mesure estil antérieur à l'homme? c'est ce que nul ne sait. 


\section{BIBLIOGRAPHIE}

$\underline{\text { Retour à la table des matières }}$

Note. - Cette bibliographie contient tous les ouvrages cités au cours de ce livre. Les abréviations suivantes indiquent les périodiques :

Amer. Anth. Amer. Anth., Mem. A. M. N. H.

A. M. N. H., A. M. N. H.,Hd.

A. M. N. H.,

B. A. E.

B. A. E., Bull. Can. Geol. Sur., Mem.

Can. Geol. Sur., Mus.

Can. Sum. Rept.

Field Mus.

J. A. F. L.
American Anthropologist.

Memoirs of the American Anthropological Association. Anthropological Papers of the American Museum of Natural History.

Bull. Bulletin of the American Museum of Natural History. Handbook Series of the American Museum of Natural History.

Mem. Memoirs of the American Museum of Natural History.

Annual Report of the Bureau of (American) Ethnology. Bulletin, Bureau of American Ethnology.

Memoir, Canada Department of Mines, Geological Survey. Museum Bulletin, Canada Department of Mines, Geological Survey.

Summary Report of the Geological Survey, Canada. Anthropological Series, Field Museum of Natural History. Journal of American Folk-Lore. 
J. A. I.

Reports
Journal of the (Royal) Anthropological Institute of Great Britain and Ireland.

Reports of the Cambridge Anthropological Expedition. 
U. Cal.

University of California Publications in American Archœology and Ethnology.

Z. vgl. R.

Zeitschrift für vergleichende Rechts-wissenschaft.

ADAIR, J. The History Of the American Indians. Londres, 1775.

ALLDRIDGE, T. J. A transformed Colony : Sierra Leone. Lon 1910.

Annual Archaeological Report. Toronto, 1906.

ARCIN, A. La Guinée française. 1907.

BADEN-POWELL, B. H. The Indian Village Community. Londres, 1896.

BANDELIER, A. On the Distribution and Tenure of Lands and the Customs with respect to Inheritance, among the Ancient Mexicans. Reports of the Peabody Museum, 11, No 2, 385-448. 1878.

BARRETT, S. A. Ceremonies of the Pomo Indians. U. Cal., XII, 397-441. 1917.

BARTON, R. F. Ifugao Law. U. Cal., XV, 1-127. 1919.

BLEEK, W. I. et LLOYD, L. C. Specimens of Bushman Folk-Lore. Londres, 1911.

BOAS, F. The Central Eskimo. 6 B. A. E., 409-669. 1888.

- The Social Organization and Secret Societies of the Kwakiutl Indians. Report of the U. S. National Museum for 1895. 315-733. 1897.

— The Eskimo of Baffin Land and Hudson Bay. A. M. N. H., Bull., XV. 1907.

- The Mind of Primitive Man. New York, 1911.

(a) Tsimshian Mythology. 31 B. A. E. 1916.

(b) The Origin of Totemism. Amer. Anth. XVIII, 319-326. 1916.

BOGORAS, F. The Chukchee. New York, 1910.

BUCK, P. H. Samoan Material Culture. Bernice P. Bishop Museum, Bulletin 75. Honolulu, 1930.

CASTREN, M. A. Reiseerinnerungen aus den Jahren 1838-1844. Saint-Pétersbourg, 1853.

CODRINGTON, R. H. The Melanesians : Studies in their Anthropology and FolkLore. Oxford, 1891.

COLE, F. C. The Wild Tribes of Davao District. Field Mus. XII, 49-203. 1913.

COURANT, M. En Chine. Paris, 1901.

CRANZ, D. Historie von Grönland. Barby, 1765.

CUNOW, H. Die Verwandtschafts-Organisationen der Australneger. Stuttgart, 1894.

— Zur Urgeschichte der Ehe und Familie. Stuttgart, 1912.

CZAPLICKA, M. A. Aboriginal Siberia. Oxford, 1914.

DESPLAGNES, L. Le plateau central nigérien. 1907.

DIXON, R. B. The Northern Maidu. A. M. N. H., Bull. XVII, 119-346. 1905.

— The Shasta. Ibid., 381-498. 1907. 
DORSEY, J. 0. Omaha Sociology. 3 B. A. E., 211-370.1884.

ELLIS, A. B. The Ewe-speaking Tribes of the Slave Coast of West Africa. Londres, 1890.

ELLIS, Wm. Polynesian Researches. Londres, 1831.

ERDLAND, P. A. Die Marshall-Insulaner. Anthropos-Bibliothek. Münster i. W., 1914.

FERGUSON, W. S. The Zulus and the Spartans. Harvard African Studies, II, 197234. 1918.

FLETCHER, A. et LA FLESCHE, F. The Omaha Tribe. 27 B. A. B. 1911.

Franciscains (Pères). An Ethnologic Dictionary of the Navaho Language. St.Michaels, 1910.

FRAZER, J. The Aborigines of New South Wales. Sydney, 1892.

FRAZER, J. G. Totemism and Exogamy. 4 vol. Londres, 1910.

— The Golden Bough. 3e édition, partie II. Londres, 1911.

— Psyche's Task. Londres, 1912.

FREIRE-MARRECO, B. Tewe Kinship Terms from the Pueblo of Hano. Amer. Anth., XVI, 269-287.1914.

FREUD, S. Ueber einige Uebereinstimmungen im Seelenleben der Wilden und der Neurotiker. Imago, 17-33 ; 213-227. 1913 ; 1-21, 357-408.1912-1913.

FROBENIUS, L. Und Afrika sprach. 1. Berlin-Charlottenburg, 1913. FROBENIUS, Kulturtypen aus dem Westsudan. Berlin, 1910.

GIFFORD, E. W. Miwok Moieties. U. Cal., XII, 139-194. 1916.

- Clans and Moieties in Southern California. U. Cal., XIV, 155-219. 1918.

GODDARD, P. E. Life and Culture of the Hupa. U. Cal., 1, 1-88. 1903. - Indians of the Southwest. A. M. N., Hd. 1913.

GOLDENWEIWSER, A. A. Totemism : an Analytical Study. J. A. F. L., XXIII, 179293. 1910.

— On Iroquois Work. Can. Sum. Rept., 464-475. 1912.

- On Iroquois Work. Can. Sum. Rept., 365-373. 1913.

— Form and Content in Totemism. Amer. Anth., XX, 280-295. 1918.

GURDON, P. R. T. The Khasis. Londres, 1907.

HAHN, Ed. Das Alter der wirtschaftlichen Kultur der Menschheit. Heidelberg, 1905.

HARTLAND, E. S. Matrilineal Kinship and the Question of its Priority. Amer. Anth. Mem., IV, 1-90. 1917.

HAWKES, E. W. The «Inviting-in » Feast of the Alaskan Eskimo. Can. Geol. Sur., Mem. 45. 1913.

— The Labrador Eskimo. Can. Geol. Sur., Mem. 91. 1916. 
HEARNE, S. Journey from Prince of Wales Fort in Hudson's Bay to the Northern Ocean. Londres, 1795.

HOBHOUSE, L. T. Morais in Evolution. Londres, 1915.

HOCART, A. M. The Fijian Custom of Tauvu. J. A. I., XLIII, 101-108. 1913.

- Chieftainship and the Sister's Son In the Pacific. Amer. Anth., 631-646. 1915.

HODSON, T. C. The Naga Tribes of Manipur. Londres, 1911.

HOLLIS, A. C. The Masai. Oxford, 1905.

— The Nandi. Oxford, 1909.

HOWITT, A. W. The Native of South-east Australia. Londres, 1904.

Jesuit Relations and Allied Documents. 1896-1901. Édité par Reuben Gold Thwaites, 71 volumes.

JOCHELSON, W. Material Culture and Social Organization of the Koryak. A. M. N. H., Mem. X. 1908.

— The Yukaghir and the Yukaghirized Tungus. A. M. N. H., Mem. XIII. 1910.

JUNOD, H. A. The Life of a South African Tribe. 2 vol. Neuchâtel, 1912.

KEYSSER, Ch. Aus dem Leben der Kai-Leute, In Neuhauss, R., Deutsch NeuGuinea, III. Berlin, 1-242,1911.

KINGSLEY, M. H. Travels in West Africa. Londres, 1904.

KIRCHHOFF, P. Die Verwandtschaftsorganisation der Urwaldstämme Südamerikas. Zeitsch. f. Ethn., vol. 63, 85-193. 1931.

— Verwandtschaftsbezelchnungen und Verwandtenheirat. Ibid. vol. 64, 41-71. 1932.

KOHLER, J. Urgeschichte der Ehe. Z. vgl. R., XII. 1897. KRAEMER, A. Die Samoa-Inseln. Stuttgart, 1902.

KRAUSE, A. Die Tlinkit-Indianer. Jena, 1885.

KROEBER, A. L. The Arapaho. A. M. N. H., Bull., XVIII, 1-229, 279-454. 1904.

— Ethnology of the Gros Ventre. A. M. N. H., 1, 145-281. 1908.

(a) Zuñi Kin and Clan. A. M. N. H., XVIII, 39-205. 1917.

(b) The Tribes of the Pacific Coast of North America. XIXe Congrès international des Américanistes, 385-401. 1917.

LABOURET, H. Les tribus du rameau Lobi. Paris, 1931.

LANG, A. Custom and Myth. Londres, 1885. 
LAUFER, B. Preliminary Notes on Explorations among the Amoor Tribes. Amer. Anth., 297-338. 1900.

— The Beginnings of Porcelain in China. Field Mus., XV, 79-177. 1917.

LEBNER, St. Bukama, in Neuhauss, Deutsch Neu-Guinea, 111, 379-485. Berlin, 1911.

LOEB, E. M. Tribal Initiations and Secret Societies. U. C. 25,249-288. 1929.

LOWIE, R. H. Social Life of the Crow Indians. A. M. N. H., IX, 179-248. 1912.

— Societies of the Crow, Hidatsa and Mandan Indians. A. M. N. H., M, 145-358. 1913.

- Exogamy and the Classificatory System of Relationship. Amer. Anth., 223239. 1915.

- Plains Indians Age-Societies : Historical and Comparative Summary. A. M. N. H., XI, 877-984. 1916.

(a) Notes on the Social Organizations and Customs of the Mandan, Hidatsa and Crow Indians. A. M. N. H., XXI, 1-99. 1917.

(b)Culture and Ethnology. New York, 1917.

(a) The Matrilineal Complex. U. Cal., XVI, 29-45. 1919.

(b)Family and Sib. Amer Anth 28-40. 1919.

MacLEAN, Colonel. A Compendium of Kafir Laws and Customs. Imprimé par le Gouvernement de British Kafraria. Mount Coke, 1858.

MAINE, H. Ancient Law. Londres, 1861.

MALINOWSKI, B. The Family among the Australian Aborigines. Londres, 1913.

— Crime and Custom in Savage Society. Londres, 1926.

MAN, E. H. On the Aboriginal Inhabitants of the Andaman Islands. Londres, 1883.

MARETT, R. R. Anthropology. Home University Library. 1912.

MARKHAM. A History of Peru. Chicago, 1892.

MARTIN, R. Die Inlandstämme der Malayischen Halbinsel. Jena, 1905.

MAUSS, M. Essai sur le don, forme archaïque de l'échange. Année sociologique, 1923-1924.

MERKER, M. Die Masai. Berlin, 1910.

MOONEY, J. The Cheyenne Indians. Amer. Anth., Mem., 1, 361-442. 1907.

MORGAN, L. H. Systems of Consanguinity and Affinity of the Human Family. Smithsonian Contributions to Knowledge, XVII. 1871.

— Ancient Society. New York, 1871. 
MURDOCH, J. Ethnological Results of the Point Barrow Expedition. 9 B. A. E., 19441. 1892.

NELSON, E. W. The Eskimo about Bering Strait. 18 B. A. B., 19-518. 1899.

NOPCSA, Fr. Albanien ; Bauten, Trachten und Geräte Nordalbaniens. Berlin et Leipzig, 1925.

PARKINSON, R. Dreissig Jahre in der Südsee. Stuttgart, 1907.

PARSONS, E. C. Avoidance in Melanesia. J. A. F. L., XXIX, 282-292. 1916.

PHILBRICK, F. S. (Traducteur Huebner, R.). A History of Germanie

Private Law. Boston, 1918.

POWERS, S. Tribes of California. Contributions to North American Ethnology, III. 1877.

RADCLIFFE-BROWN, A. R. Three Tribes of Western Australia. J. A. I., XLIII, 143194.1913.

RADIN, P. The Ritual and Significance of the Winnebago Medicine Dance. J. A. F. L., XXIV, 149-208.1911.

The Social Organization of the Winnebago Indians, an Interpretation. Can. Geol. Sur., Mus., No 10. 1915.

RADLOFF, W. Aus Sibirien. 1. Leipzig. 1893.

Reports of the Cambridge Anthropological Expedition to Torres Straits. Vol. V et VI. Cambridge, 1904, 1908.

RESTGREPO, Vicente. Los Chibchas antes de la conquista española. Bogota, 1895.

RIVERS, W. H. R. The Todas. Londres, 1906.

(a) Kinship and Social Organization. Londres, 1914.

(b) The History of Melanesian Society. 2 vol. Cambridge, 1914.

— « Marriage », in Hastings' Encyclopaedia of Religion and Ethics. VIII. 1915.

ROCKHILL, W. W. The Land of the Lamas. New York, 1891.

RPSCOE, J. The Bahima : a Cow Tribe of Enkole in the Uganda Protectorate. J. A. 1., 93-118. 1907.

— The Baganda. Londres, 1911.

ROTH, W. E. North Queensland Ethnography. Bulletin 5. Brisbane, 1903.

- North Queensland Ethnography. Bulletin 8. Brisbane, 1906.

- An Inquiry into the Animism and Folk-Lore of the Guiana Indians. 30 B. A. E., 117-384. 1915.

ROUTLEDGE, W. S. et K. With a Prehistoric People. Londres, 1910.

RUSSELL, F. The Pima Indians. 26 B. A. E., 17-389.1908.

SAPIR, E. Some Aspects of Nootka Language and Culture. Amer. Anth., 15-28. 1911. 
- A Girl's Puberty Ceremonial among the Nootka Indians. Transactions, Royal Society of Canada, 3e série, 67-80. 1913.

— The Social Organization of the West Coast Tribes. Ibid., 355-374. 1915.

SCHINZ, W. Deutsch-Südwest-Afrika. Oldenburg, 1891.

SCHMIDT, M. Indianerstudien in Zentralbrasilien. Berlin, 1905.

SCHULTZE, L. Aus Namaland und Kalahari. Jena, 1907.

SCHURTZ, H. Altersklassen und Männerbünde. Berlin, 1902.

SELIGMAN, C. G. et B. Z. The Veddas. Cambridge, 1911.

SKEAT, W. W. et BLAGDEN, C. 0. Pagan Races of the Malay Peninsula. 2 vol. Londres, 1906.

SKINNER, A. Social Life and Ceremonial Bundles of the Menomini Indians. A. M. N. H., XIII, 1-165. 1913.

SPECK, F. G. Ethnology of the Yuchi. University of Pennsylvania, Anthropological Publications of the University Museum, 1, 1-154. 1909.

(a) Family Hunting Territories. Can. Geol. Sur., Mem., 70. 1915.

(b) The Family Hunting Band as the Basis of Algonkian Social Organization. Amer. Anth., 289-305. 1915.

- Kinship Terms and the Family Band among the Northeastern Algonkians. Amer. Anth., 143 et suiv. 1918.

SPENCER, B. et GILLEN, F. J. The Native Tribes of Central Australia. Londres, 1899.

— The Northern Tribes of Central Australia. Londres, 1904.

SPIETH, J. Die Ewe-Stämme. Berlin, 1906.

SPINDEN, H. J. The Nez Percé Indians. Amer. Anth., Mem., II, 165-274.1908.

- Ancient Civilizations of Mexico and Central America. A. M. N. H., Hd., 1917.

STACK, E. et LYALL, C. The Mikirs. Londres, 1908.

STAIR, J. B. Old Samoa. Oxford, 1897.

STEVENSON, M. C. The Zuñi Indians. 23 B. A. E. 1909.

SWANTON, J. R. (a) Contributions Io the Ethnology of the Haida. A. M. N. H., Mem., VIII. 1905.

(b) The Social Organization of the American Tribes. Amer. Anth., 663-673. 1905.

- A Reconstruction of the Theory of Social Organization. Boas Anniversary Volume, 166-178. New York, 1906.

- Social Condition, Beliefs and Linguistic Relationship of the Tlingit Indians. 26 B. A. E., 391-485.1908. 
— Indian Tribes of the Lower Mississippi Valley. B. A. E., Bull. 43. 1911.

- A Foreword on the Social Organization of the Creek Indians. Amer. Anth., 593-599. 1912.

TAFEL, A. Meine Tibetreise. Stuttgart, 1914.

TALBOT, P. A. In the Shadow of the Bush. Londres, 1912.

TEGGART, F. J. The Processes of History. New-Haven, 1918.

TEIT, J. The Thompson Indians of British Columbia. A. M. N. H., Mem., II, 1900.

— The Shuswap. Ibid., IV. 1909.

TRALBITZER, W. The Ammassalik Eskimo. Copenhague, 1914.

THEAL, G. Mc. History and Ethnography of Africa south of the Zambesi. Londres, 1907.

THOMSON, Basil. The Fijians. Londres, 1908.

THURNWALD, R. Das Rechtsleben der Eingeborenen der deutschen Südseeinseln. Berlin, 1910.

— Ermittlungen über Eingeborenenrechte der Südsee. Z. vgl. R. XXIII, 309-364.

— Die Menschliche Gesellschaft. 1934.

TORDAY, E. et JOYCE, T. A. Les Bushongo. Bruxelles, 1910.

TREGEAR, E. The Maori Race. Wangani, 1904.

TURNER, B. Samoa. Londres, 1884.

TYLOR, E. B. On a Method of Investigating the Development of Institutions ; applied to Laws of Marriage and Descent. J. A. I., XVIII, 245-272. 1889.

— The Matriarchal Family System. Nineteenth Century, XL, 81-96. 1896.

VON DEN STEINEN, K. Unter den Naturvölkern Zentral-Brasillens. Berlin, 1897. WAITZ, Th. et GERLAND, G. Anthropologie der Naturvölker. VI. Leipzig, 1872. WEBSTER, H. Primitive Secret Societies. New York, 1908.

WEULE, K. Wissenschaftliche Ergebnisse meiner ethnographischen Forschungsreise in den Südosten Deutsch-Ostafrikas. Berlin, 1908.

WHIFFEN, T. The Northwest Amazons. New York, 1915.

WILSON, G. L. Agriculture of the Hidatsa Indians. University of Minnesota, Studies in the Social Sciences, No 9. Minneapolis, 1917.

WISSLER, C. The Social Life of the Blackfoot Indians. A. M. N. H., VII, 1-64. 1911. ZAHN, H. Die Jabim, in Neuhauss, Deutsch Neu-Guinea, III, 289-394. 